Portland State University

PDXScholar

\title{
Design and Experimental Investigation of 500kV Current Transformer Seismic Retrofit Utilizing Structure Rocking and Supplemental Damping with Self-Centering
}

Ilya S. Palnikov

Portland State University

Follow this and additional works at: https://pdxscholar.library.pdx.edu/open_access_etds

Part of the Civil and Environmental Engineering Commons Let us know how access to this document benefits you.

\section{Recommended Citation}

Palnikov, Ilya S., "Design and Experimental Investigation of 500kV Current Transformer Seismic Retrofit Utilizing Structure Rocking and Supplemental Damping with Self-Centering" (2017). Dissertations and Theses. Paper 3673.

https://doi.org/10.15760/etd.5557

This Thesis is brought to you for free and open access. It has been accepted for inclusion in Dissertations and Theses by an authorized administrator of PDXScholar. Please contact us if we can make this document more accessible: pdxscholar@pdx.edu. 
Design and Experimental Investigation of 500kV Current Transformer Seismic Retrofit Utilizing Structure Rocking and Supplemental Damping with Self-Centering

\author{
by
}

Ilya S. Palnikov

A thesis submitted in partial fulfillment of the requirements for the degree of

\title{
Master of Science
}

in

Civil and Environmental Engineering

\author{
Thesis Committee: \\ Peter Dusicka, Chair \\ Franz Rad \\ Leon Kempner \\ Evan Kristof
}

\section{Portland State University}




\section{AbSTRACT}

Electrical substations perform a key role in electrical transmission and distribution; the ability for a substation to remain functional during and after a seismic event contributes significantly to the resilience of the clients supplied. Many legacy components currently installed in the main grid substations were designed with minimal consideration of lateral loads and are not qualified per IEEE693. One of the more critical high-voltage substation components that are vulnerable to earthquake damage is the $500 \mathrm{kV}$ freestanding current transformer (CT). The CT is particularly vulnerable due to the slenderness and mass distribution of the component. Current transformers are typically constructed from a combination of aluminum and brittle porcelain. Two novel retrofit measures were investigated utilizing base rocking and supplemental damping to reduce the seismic amplification in the CT while also potentially providing postearthquake self-centering capability. The retrofit measures utilize both shift in system frequency and energy dissipation through supplemental damping to reduce seismic demands on the CT. The purpose of the research was to conceptually develop, detail design, analyze and experimentally validate the retrofit measures. A desired feature of the retrofit measures was for minimal or no residual displacement following the seismic event, which was implemented in the retrofit through a preloaded centering mechanism. Based on the analyses and experiments, the proposed retrofit measures exhibited significantly decreased demands on the CT and true self-centering. 


\section{ACKNOWLEDGEMENTS}

I would first like to thank my advisor and committee chair, Dr. Peter Duscika for the experience and knowledge which was gained through the time spent in the iStar Laboratory. For the opportunity to get involved in research and contributing to society. Also, I would like to thank Dr. Peter Dusicka for assisting with funding through a GRA.

The research team acknowledges Bonneville Power Administration for making the research possible through their funding, input and expertise. Particularly we would like to thank Mr. Michael Riley and Dr. Leon Kempner for their guidance, suggestions, and technical contributions.

I would like to acknowledge Dr. Franz Rad and Evan Kristoff, J.D., for their questions and suggestions used to improve this thesis.

Additional appreciation is due to the staff and faculty at the University of Nevada, Reno (UNR) for accommodating part of the work to be conducted at their facility. We would like to thank Dr. Patrick Laplace for technical assistance and Dr. Sherif Elfass for administrative assistance at UNR. Also, we would like to thank Todd Lyttle and Chad Lyttle from UNR as well as Aaron Smith from PSU for lab floor assistance during the experimental portion at the University of Nevada, Reno.

Finally, I would like to thank my family for the support, love, care and guidance during my studies at Portland State University. I am thankful for their contributions to my success and support in both my undergrad and graduate studies. I am thankful for my wife's love and encouragement during the course of my entire education. 


\section{TABLE OF CONTENTS}

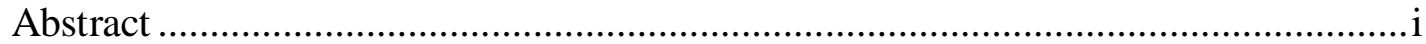

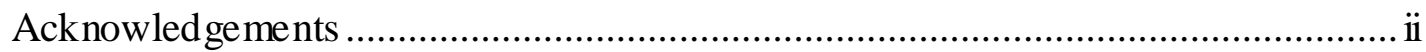

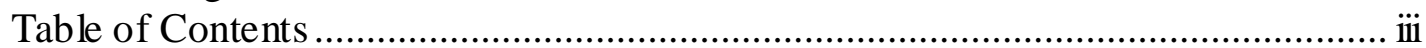

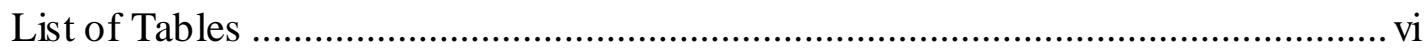

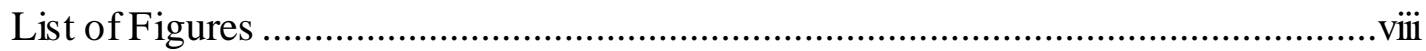

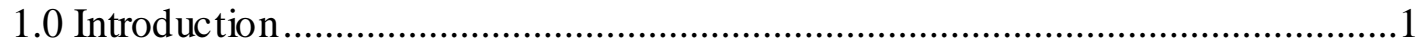

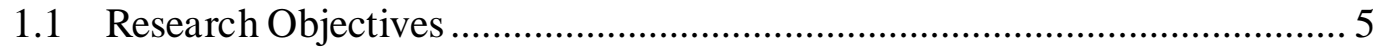

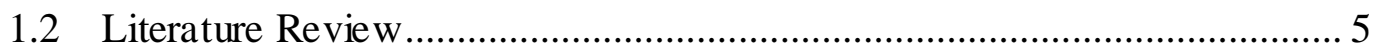

1.2.1 Design Procedure for Controlled Rocking of Self-Centering Steel

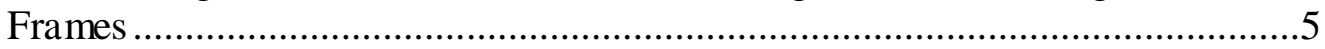

1.2.2 Displacement-Based Design of Precast Walls with Additional

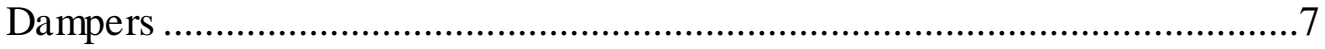

1.2.3 Post-Tensioned Moment Connections with a Bottom Flange Device for Seismic Resistant Self-Centering MRFs

1.2.4 Self-Centering Seismic Lateral Force Resisting System: High Performance Structures for the City of Tomorrow ..........................................10

1.2.5 Mechanism of Energy Absorption in Special Devices for use in Earthquake Resistant Structures .............................................................12

1.2.6 Experimenta1/Numerical Study of U-Shape Flexural Plate (UFP)

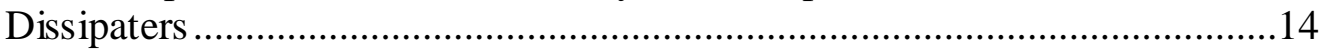

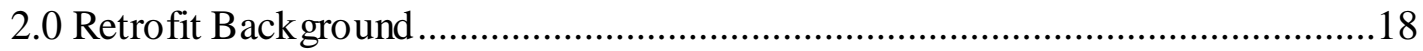

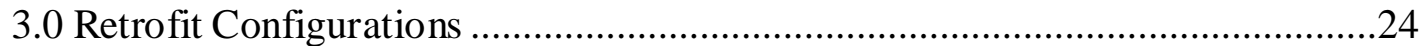

3.1 Pre-Tension Members .............................................................................. 25

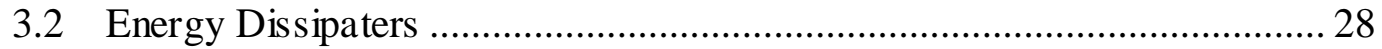

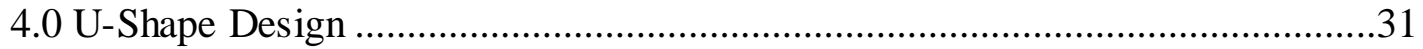

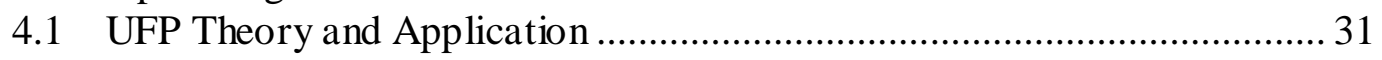

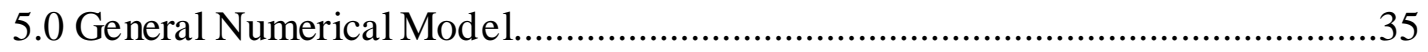

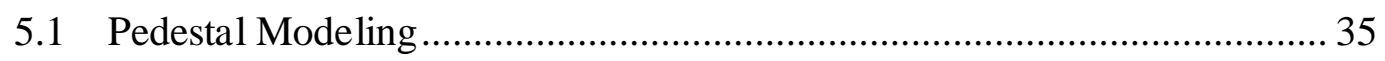

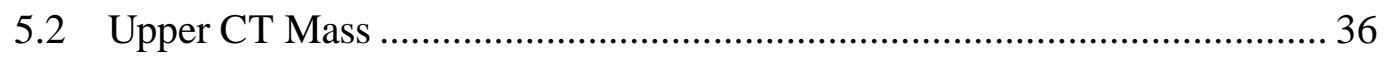

5.3 Foundation and Friction Modeling ....................................................... 38

5.4 Pre-Tension Model Application........................................................ 40

5.5 Hysteretic Device Modeling ............................................................. 42

5.6 Viscous Damper Modeling .................................................................. 46

6.0 Parametric Study on Hysteretic Retrofit Parameters .....................................49

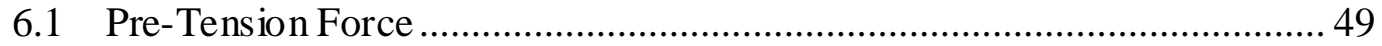

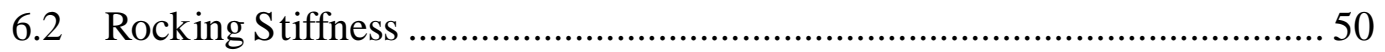

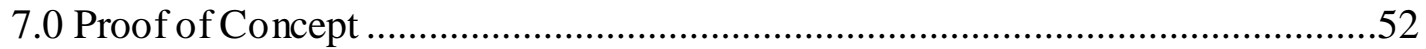




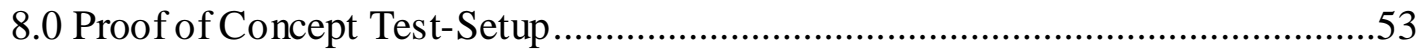

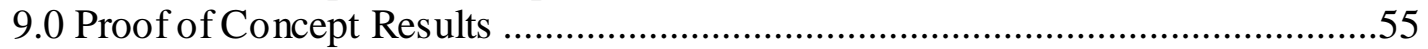

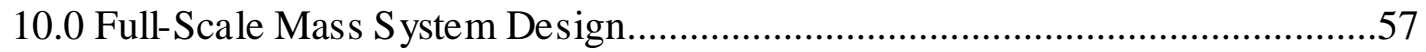

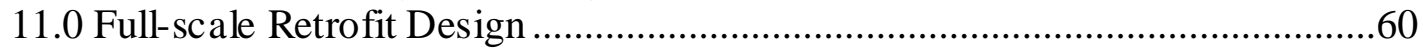

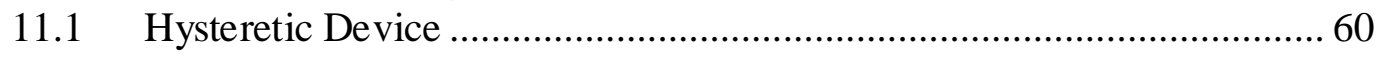

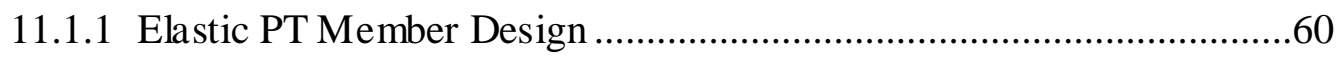

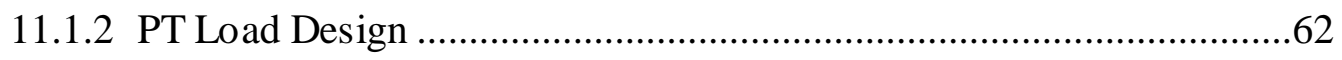

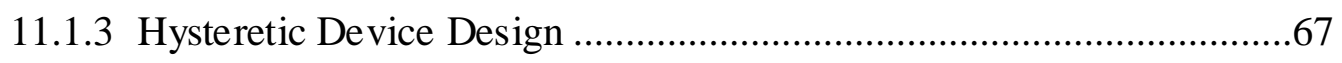

11.1.4 SAP2000 Hysteretic Device Calibration ...............................................69

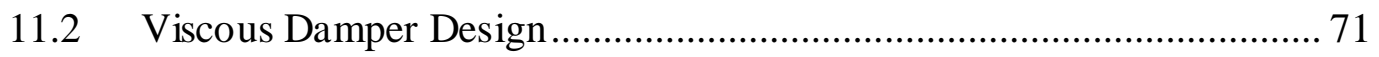

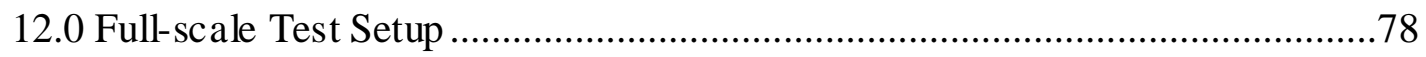

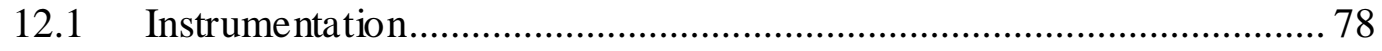

12.2 Fabricated Pedestal.......................................................................... 84

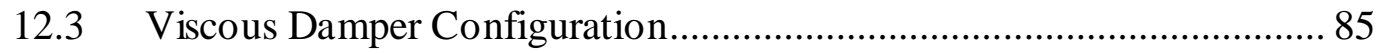

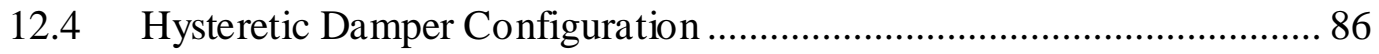

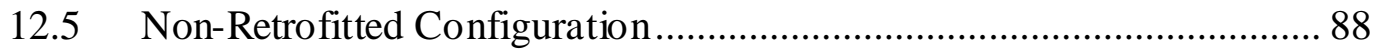

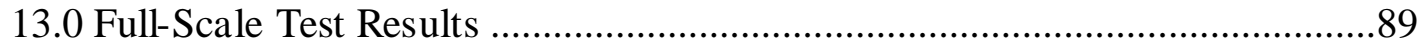

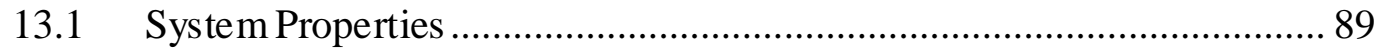

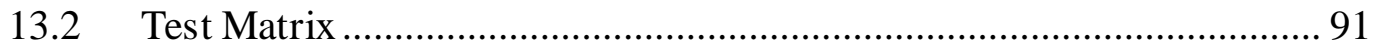

13.3 Viscous Damper Retrofit Results ...................................................... 92

13.3.1 Viscous Damper Table Response Spectrum..........................................92

13.3.2 Viscous Damper System Damping \& Free Vibration ............................95

13.3.3 Viscous Damper System Response ......................................................96

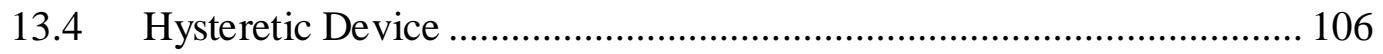

13.4.1 Hysteretic Device Table Response Spectrum.....................................106

13.4.2 Hysteretic Device System Damping \& Free Vibration .......................109

13.4.3 Hysteretic Device System Response .................................................110

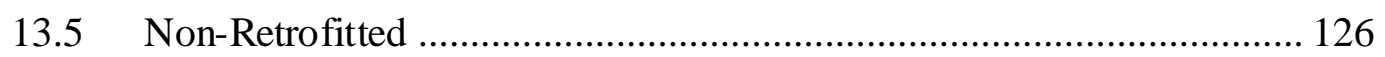

13.5.1 Non-Retrofitted Table Response Spectrum.......................................126

13.5.2 Non-Retrofitted System Damping \& Free Vibration............................128

13.5.3 Non-Retrofitted System Response .....................................................129

14.0 Numerical Model vs. Experimental Results ....................................................134

14.1 Viscous Damper Experimental Comparison to Numerical ................... 134

14.2 Hysteretic Experimental Comparison to Numerical .............................. 136

14.3 Non-Retrofitted Experimental Comparison to Numerical ..................... 138 


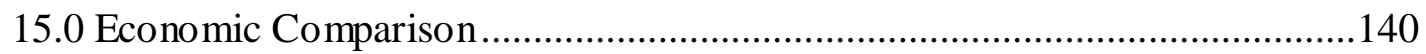

16.0 Full-scale Retrofit Comparison and Summary ..........................................142

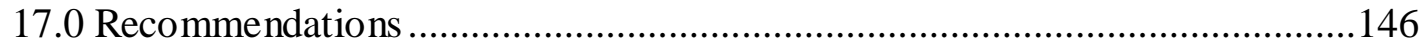

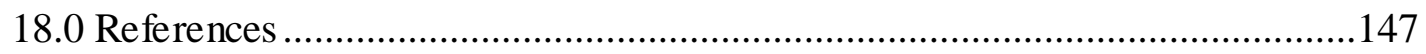

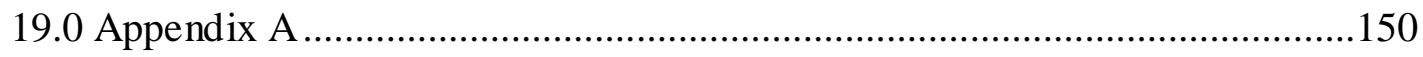

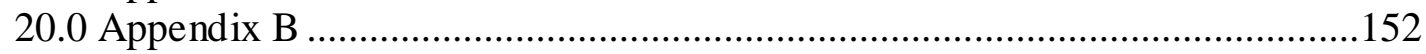

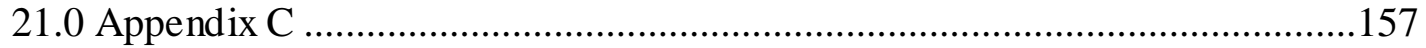

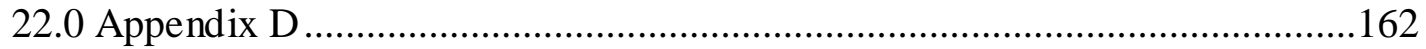

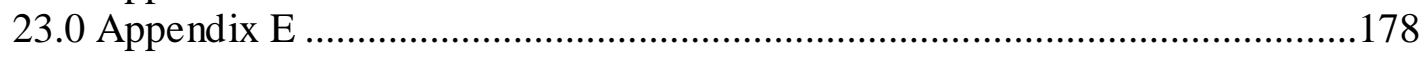

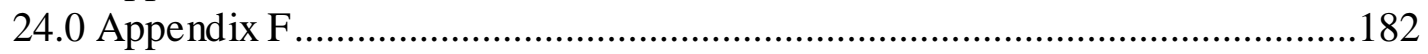

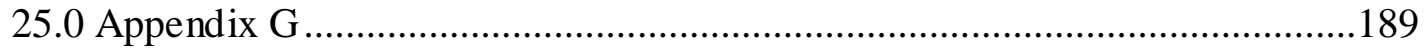

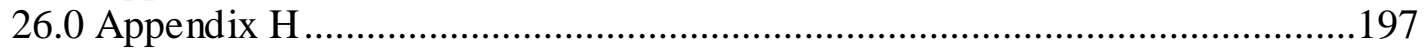

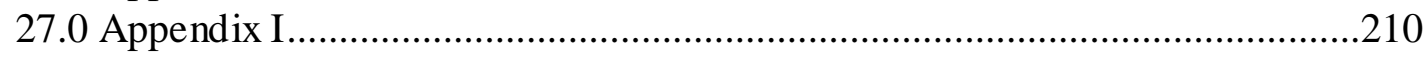

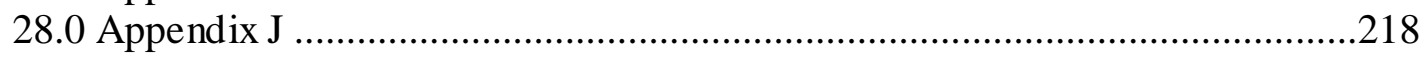

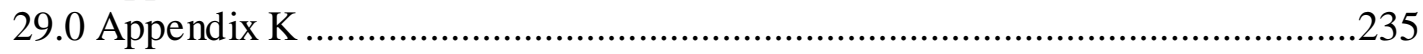

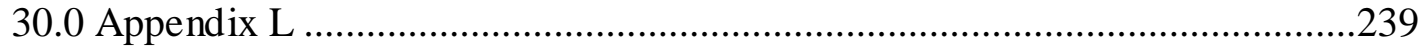




\section{LIST OF TABLES}

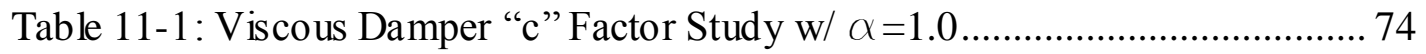

Table 11-2: Viscous Damper "c" Factor Study w/ $\alpha=0.7$..................................... 76

Table 12-1: Accelerometer and String Pot Locations .......................................... 83

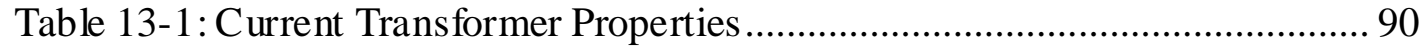

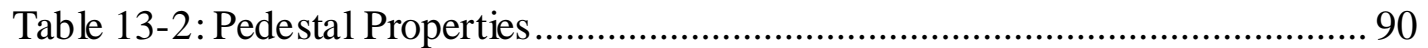

Table 13-3: System Properties ....................................................................... 91

Table 13-4: Viscous Damper -- Retrofit Damping and Fundamental Frequency.... 96

Table 13-5: Viscous Damper - System X Absolute Maximum Acceleration ......... 97

Table 13-6: Viscous Damper - System Y Absolute Maximum Acceleration ......... 98

Table 13-7: Viscous Damper - System Z Absolute Maximum Acceleration ......... 98

Table 13-8: Viscous Damper - System Absolute Maximum Reactions.................. 99

Table 13-9: Viscous Damper - System Maximum Displacement 20-65\% Motions

Table 13-10: Viscous Damper - System Maximum Displacement 70-100\% Motions 100

Table 13-11: Viscous Damper - System Residual Displacement 20-65\% Motions

Table 13-12: Viscous Damper - System Residual Displacement 70-100\% Motions

Table 13-13: Viscous Damper - Pedestal Absolute Maximum Strain ......... 101

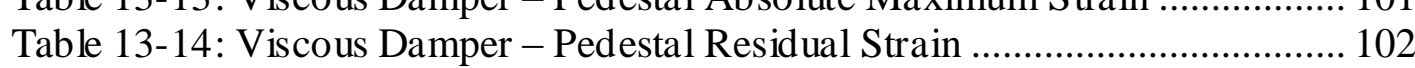

Table 13-15: Viscous Damper - Damper Absolute Maximum Displacement ...... 102

Table 13-16: Viscous Damper - Damper Residual Displacement ....................... 102

Table 13-17: Viscous Damper - Damper Maximum Force .................................... 103

Table 13-18: Viscous Damper - Damper Bracket Slip ........................................ 104

Table 13-19: Viscous Damper - Maximum PT Force .......................................... 104

Table 13-20: Viscous Damper - \% PT Force Loss Post Motion............................ 105

Table 13-21: Viscous Damper - Pedestal Rocking Uplift .................................... 105

Table 13-22: Viscous Damper - Maximum Base Plate Slip ................................. 106

Table 13-23: Viscous Damper - Relative Base Plate Movement Pre-Post Motion

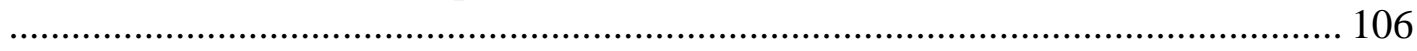

Table 13-24: UFP Retrofit Damping and Fundamental Frequency ...................... 110

Table 13-25: Hysteretic Device - System X Absolute Maximum Acceleration ... 111

Table 13-26: Hysteretic Device - System Y Absolute Maximum Acceleration ... 112

Table 13-27: Hysteretic Device - System Z Absolute Maximum Acceleration ... 114

Table 13-28: Hysteretic Device - 20-65\% System Absolute Maximum Reactions

Table 13-29: Hysteretic Device - 70-100\% System Absolute Maximum Reactions

Table 13-30: Hysteretic Device - System Maximum Displacement 20-70\% Motions

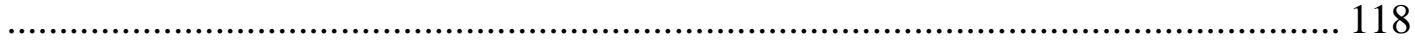

Table 13-31: Hysteretic Device - System Maximum Displacement 75-100\%

Motions 118 
Table 13-32: Hysteretic Device - System Residual Displacement 20-70\% Motions 118

Table 13-33: Hysteretic Device - System Residual Displacement 75-100\% Motions

Table 13-34: Hysteretic Device - Pedestal Absolute Maximum Strain ................. 119

Table 13-35: Hysteretic Device - Pedestal Residual Strain ................................... 120

Table 13-36: Hysteretic Device - UFP Bracket Slip ............................................ 120

Table 13-37: Hysteretic Device - UFP Absolute Maximum Displacement ........... 121

Table 13-38: Hysteretic Device - UFP Residual Displacement .............................. 121

Table 13-39: Hysteretic Device - UFP Set Maximum Force …………………....... 122

Table 13-40: Hysteretic Device - Maximum PT Force ......................................... 122

Table 13-41: Hysteretic Device - \% PT Force Loss Post Motion............................ 123

Table 13-42: Hysteretic Device - Pedestal Rocking Uplift ................................... 124

Table 13-43: Hysteretic Device - Maximum Base Plate Slip ............................... 124

Table 13-44: Hysteretic Device - Relative Base Plate Movement Pre-Post Motion

Table 13-45: Non-Retrofitted System Damping and Fundamental Frequency ..... 129

Table 13-46: Non-Retrofitted - System X Absolute Maximum Acceleration ...... 129

Table 13-47: Non-Retrofitted - System Y Absolute Maximum Acceleration ...... 130

Table 13-48: Non-Retrofitted - System Z Absolute Maximum Acceleration....... 130

Table 13-49: Non-Retrofitted - 20-50\% System Absolute Maximum Reactions . 131

Table 13-50: Non-Retrofitted - System Maximum Displacement 20-50\% Motions

Table 13-51: Non-Retrofitted - System Residual Displacement 20-50\% Motions 132

Table 13-52: Non-Retrofitted - Pedestal Absolute Maximum Strain .................... 132

Table 13-53: Non-Retrofitted - Pedestal Residual Strain..................................... 132

Table 13-54: Initial Anchor Loads .................................................................... 133

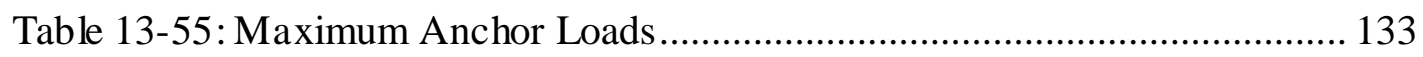

Table 15-1: Viscous Damper Retrofit Cost Summary ………………….............. 140

Table 15-2: Hysteretic Device Retrofit Cost Summary ........................................... 141

Table 16-1: Damping and Fundamental Frequency Comparison .......................... 142 


\section{LIST OF FIGURES}

Figure 1-1: 500kV Current Transformer......................................................... 2

Figure 1-2: IEEE693-0.5g PGA Design Spectrum (IEEE, 2006)............................ 4

Figure 1-3: Rocking Shear Wall Theoretical Diagram ..................................... 8

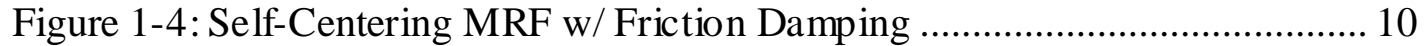

Figure 1-5: Life Time of Double Strip Test Apparatus (UFP) .............................. 14

Figure 1-6: NZ2014 Testing Configuration and Results ...................................... 15

Figure 1-7: NZ2014 Numerical and Experimental UFP Results .......................... 16

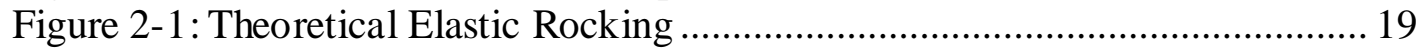

Figure 2-2: UFP Theoretical Hysteretic Behavior ............................................. 21

Figure 2-3: Theoretical Rocking w/ Supplemental Damping ............................... 22

Figure 3-1: Rendering of Provided CT Pedestal..................................................... 24

Figure 3-2: Proposed CFRP Configuration............................................................. 26

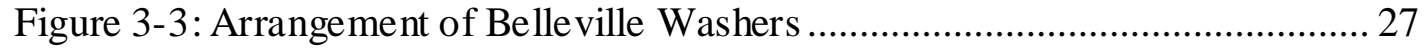

Figure 3-4: BeS Stiffness and Displacement Relationship ................................... 27

Figure 3-5: Belleville Washer Dimensional Specifications ................................... 28

Figure 3-6: Base Retrofit w/ Taylor Viscous Dampers ..................................... 29

Figure 3-7: Hysteretic Device Base Retrofit ......................................................... 29

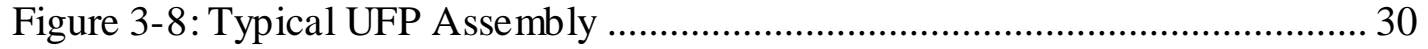

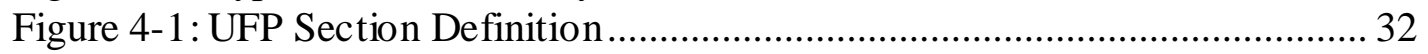

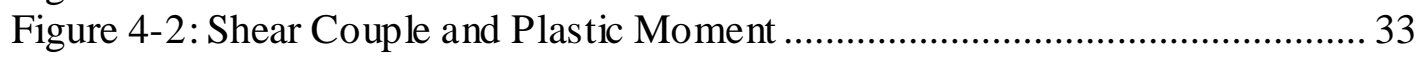

Figure 5-1: Pedestal SAP2000 Shell Model ........................................................ 35

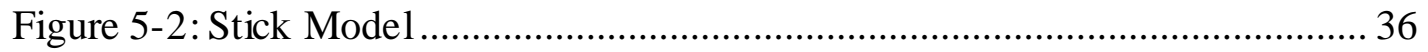

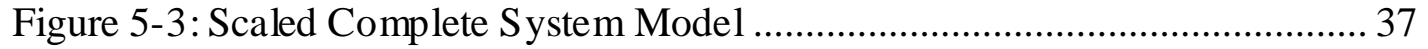

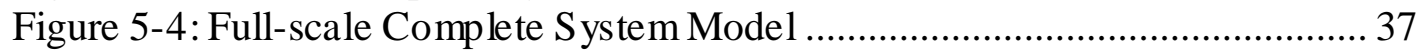

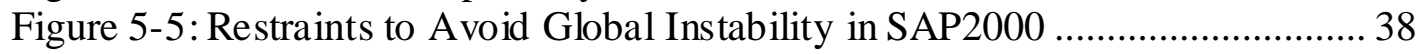

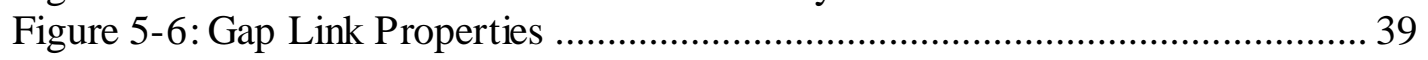

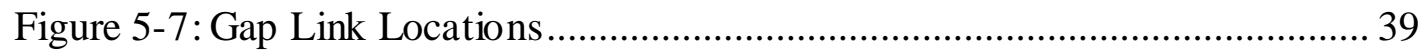

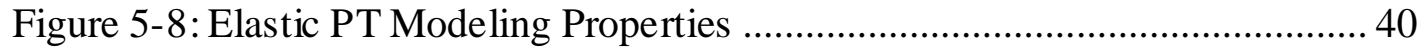

Figure 5-9: PT Location on S AP2000 Model .......................................................... 41

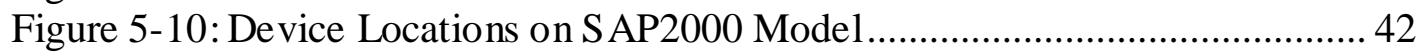

Figure 5-11: Wen- link Property Data .................................................................. 43

Figure 5-12: CSI Example 6-008 Wen-link Definition .......................................... 44

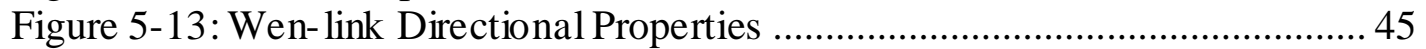

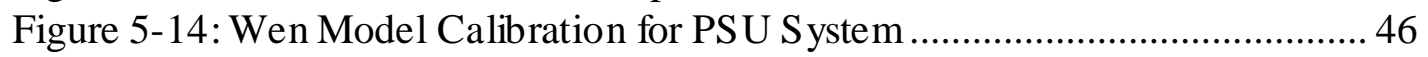

Figure 5-15: Damper- Exponential Link Properties ............................................ 47

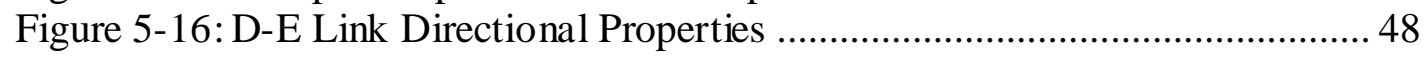

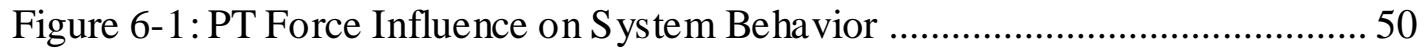

Figure 6-2: Rocking Stiffness Influence on System Behavior .............................. 51

Figure 7-1: Scaled Mass System........................................................................... 52

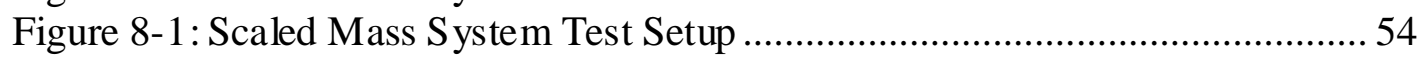

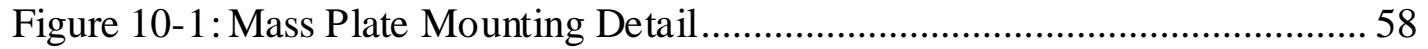

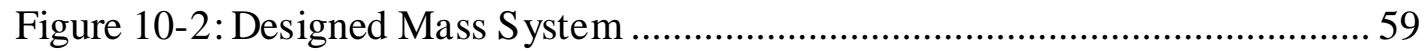

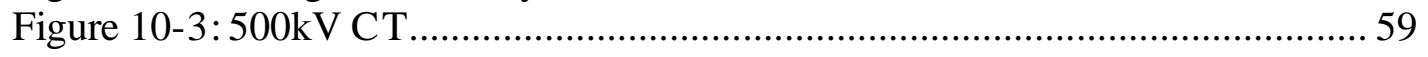

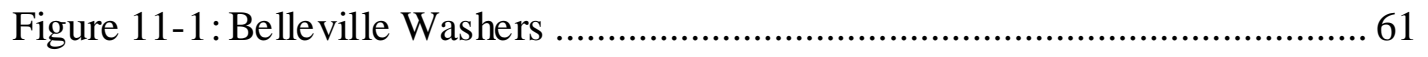




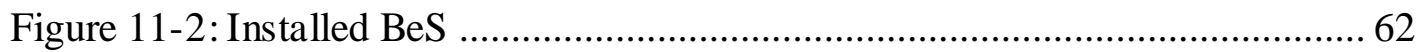

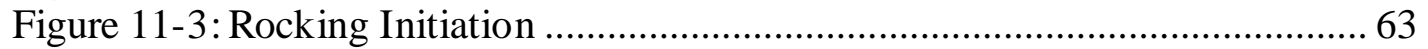

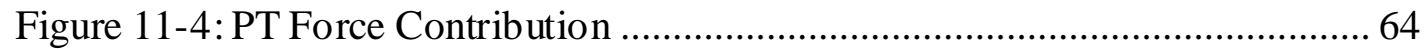

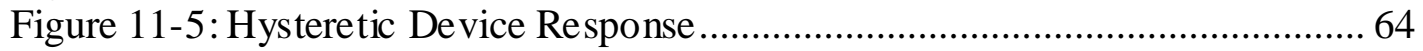

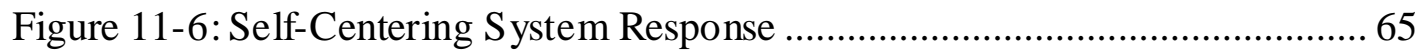

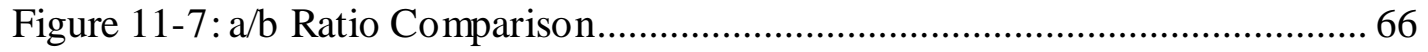

Figure 11-8: Hysteretic Device Design Procedure ……………………….............. 68

Figure 11-9: Full-Scale Wen-link Properties ..................................................... 70

Figure 11-10: Full-Scale Model Wen-link Response ………………....................... 70

Figure 11-11: Viscous Damper $\alpha$ Influence of Device Force................................... 72

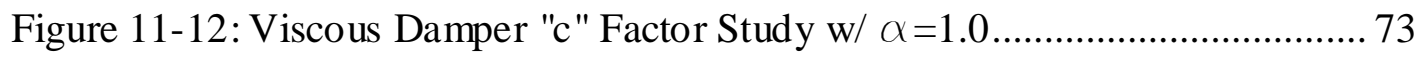

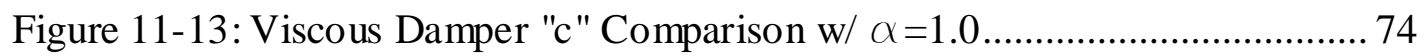

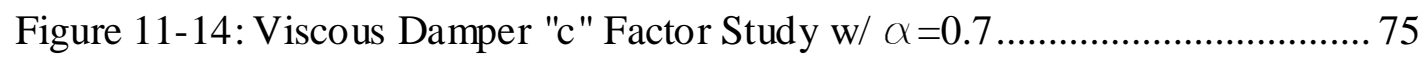

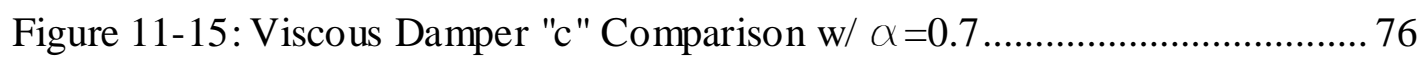

Figure 11-16: Viscous Damper Design Procedure ……………………………..... 77

Figure 12-1: LVDT Locations on Pedestal Base …………................................... 79

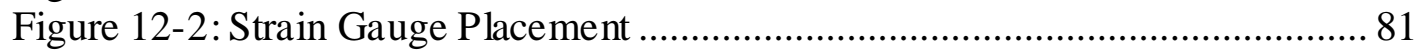

Figure 12-3: Lumped Mass Method .................................................................. 82

Figure 12-4: Accelerometer \& String Pot Locations ............................................... 82

Figure 12-5: Instrumented Specimen …………………................................... 84

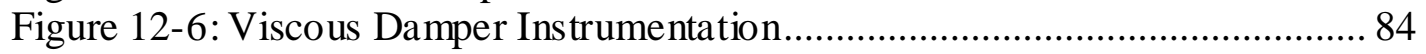

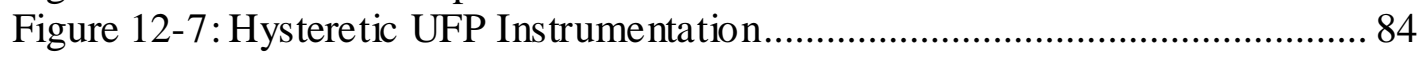

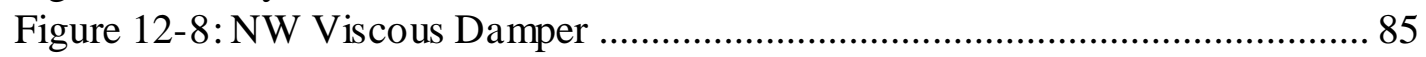

Figure 12-9: Retrofitted Base w/ Taylor Viscous Dampers...................................... 86

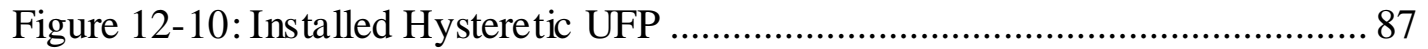

Figure 12-11: Retrofitted Base w/ Hysteretic Device ……….................................. 87

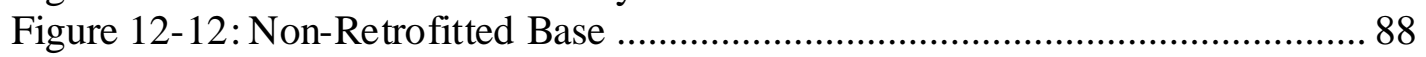

Figure 13-1: Response Spectrum X (Viscous Damper) ........................................... 93

Figure 13-2: Response Spectrum Y (Viscous Damper)............................................ 94

Figure 13-3: Response Spectrum Z (Viscous Damper) ………………………..... 95

Figure 13-4: 100\% 0.5 g IEEE693 Y-System Response w/ Viscous Dampers ...... 103

Figure 13-5: Hysteretic Device - Response Spectrum X ....................................... 107

Figure 13-6: Hysteretic Device - Response Spectrum Y ……………………....... 108

Figure 13-7: Hysteretic Device - Response Spectrum Z ...................................... 108

Figure 13-8: Hysteretic Device - System X Absolute Maximum Acceleration Plot

Figure 13-9: Hysteretic Device - System Y Absolute Maximum Acceleration Plot

Figure 13-10: Hysteretic Device - System Y Absolute Maximum Acceleration Plot

Figure 13-11: Non-Retrofitted - Response Spectrum X....................................... 127

Figure 13-12: Non-Retrofitted- Response Spectrum Y ........................................... 127

Figure 13-13: Non-Retrofitted- Response Spectrum Z ......................................... 128 
Figure 14-1: Viscous Damper - 100\% - X Experimental vs. Numerical Comparison

Figure 14-2: Viscous Damper -100\% - Y Experimental vs. Numerical Comparison

Figure 14-3: Hysteretic Device - 100\% - X Experimental vs. Numerical

Comparison

Figure 14-4: Hysteretic Device - 100\% - Y Experimental vs. Numerical

Comparison 138

Figure 14-5: Non-Retrofitted - 40\% - X Experimental vs. Numerical Comparison 139

Figure 14-6: Non-Retrofitted - 40\% - Y Experimental vs. Numerical Comparison

Figure 15-1: Viscous Damper Retrofit Cost .................................................... 140

Figure 15-2: Hysteretic Device Retrofit Cost .................................................. 141

Figure 16-1:X - Retrofitted System Compared to Non-Retrofitted System ......... 143

Figure 16-2: Y - Retrofitted System Compared to Non-Retrofitted System ......... 144

Figure 19-1: Trench Support Pedestal ............................................................. 150

Figure 19-2: 226-293 Trench 500kV Current Transformer Specifications ........... 151

Figure 25-1: Viscous Damper Retrofit X-Damping 20-50\% ............................... 189

Figure 25-2: Viscous Damper Retrofit X-Damping 60-75\% ............................... 190

Figure 25-3: Viscous Damper Retrofit X-Damping 85-95\% .............................. 191

Figure 25-4: Viscous Damper Retrofit X-Damping 100\% …............................... 192

Figure 25-5: Viscous Damper Retrofit Y-Damping 20-50\% .............................. 193

Figure 25-6: Viscous Damper Retrofit Y-Damping 60-75\% .............................. 194

Figure 25-7: Viscous Damper Retrofit Y-Damping 80-95\% ............................... 195

Figure 25-8: Viscous Damper Retrofit Y-Damping 100\% .................................. 196

Figure 26-1: 20\% 0.5 g IEEE693 X-System Response w/ Viscous Dampers ........ 197

Figure 26-2: 20\% 0.5 g IEEE693 Y-System Response w/ Viscous Dampers ........ 197

Figure 26-3: 20\% 0.5 g IEEE693 NE Viscous Damper Response ........................ 198

Figure 26-4: 20\% 0.5 g IEEE693 SE Viscous Damper Response .......................... 198

Figure 26-5:20\% 0.5 g IEEE693 SW Viscous Damper Response......................... 199

Figure 26-6: 20\% 0.5 g IEEE693 NW Viscous Damper Response ........................ 199

Figure 26-7: 50\% 0.5 g IEEE693 X-System Response w/ Viscous Devices .......... 200

Figure 26-8:50\% 0.5 g IEEE693 Y-System Response w/ Viscous Devices .......... 200

Figure 26-9: 50\% 0.5 g IEEE693 NE Viscous Damper Response ........................ 201

Figure 26-10: 50\% 0.5 g IEEE693 SE Viscous Damper Response ....................... 201

Figure 26-11:50\% 0.5 g IEEE693 SW Viscous Damper Response ...................... 202

Figure 26-12: 50\% 0.5 g IEEE693 NW Viscous Damper Response ..................... 202

Figure 26-13: 75\% 0.5 g IEEE693 X-System Response w/ Viscous Devices........ 203

Figure 26-14: 75\% 0.5 g IEEE693 Y-System Response w/ Viscous Devices........ 203

Figure 26-15: 75\% 0.5 g IEEE693 NE Viscous Damper Response ...................... 204

Figure 26-16: 75\% 0.5 g IEEE693 SE Viscous Damper Response ....................... 204

Figure 26-17: 75\% 0.5 g IEEE693 SW Viscous Damper Response ...................... 205

Figure 26-18: 75\% 0.5 g IEEE693 NW Viscous Damper Response ..................... 205

Figure 26-19: 100\% 0.5g IEEE693 X-System Response w/ Viscous Dampers .... 206 
Figure 26-20: 100\% 0.5 g IEEE693 Y-System Response w/ Viscous Dampers .... 206 Figure 26-21: 100\% 0.5g IEEE693 X-System Response w/ Viscous Dampers .... 207 Figure 26-22: 100\% 0.5g IEEE693 Y-System Response w/ Viscous Dampers .... 207 Figure 26-23: 100\% 0.5g IEEE693 NE Viscous Damper Response .................... 208 Figure 26-24: 100\% 0.5 g IEEE693 SE Viscous Damper Response ..................... 208 Figure 26-25: 100\% 0.5 g IEEE693 SW Viscous Damper Response .................... 209 Figure 26-26: 100\% 0.5g IEEE693 NW Viscous Damper Response .................... 209 Figure 27-1: Hysteretic Device Retrofit X-Damping 20-50\% ............................ 210 Figure 27-2: Hysteretic Device Retrofit X-Damping 60-75\% ............................ 211

Figure 27-3: Hysteretic Device Retrofit X-Damping 80-95\% ............................ 212

Figure 27-4: Hysteretic Device Retrofit X-Damping 100\% ............................... 213

Figure 27-5: Hysteretic Device Retrofit Y-Damping 20-50\% ............................ 214

Figure 27-6: Hysteretic Device Retrofit Y-Damping 60-75\% ............................. 215

Figure 27-7: Hysteretic Device Retrofit Y-Damping 80-95\% ............................ 216

Figure 27-8: Hysteretic Device Retrofit Y-Damping 100\% ............................... 217

Figure 28-1: 20\% 0.5 g IEEE693 X-System Response w/ Hysteretic Device........ 218

Figure 28-2: 20\% 0.5 g IEEE693 X-System Response w/ Hysteretic Device........ 218

Figure 28-3: 20\% 0.5 g IEEE693 NE Hysteretic Device Response ...................... 219

Figure 28-4: 20\% 0.5 g IEEE693 SE Hysteretic Device Response ....................... 219

Figure 28-5:20\% 0.5 g IEEE693 SW Hysteretic Device Response ...................... 220

Figure 28-6: 20\% 0.5 g IEEE693 NW Hysteretic Device Response ..................... 220

Figure 28-7: 50\% 0.5 g IEEE693 X-System Response w/ Hysteretic Device........ 221

Figure 28-8: 50\% 0.5 g IEEE693 Y-System Response w/ Hysteretic Device........ 221

Figure 28-9: 50\% 0.5 g IEEE693 NE Hysteretic Device Response ..................... 222

Figure 28-10: 20\% 0.5 g IEEE693 SE Hysteretic Device Response ..................... 222

Figure 28-11:50\% 0.5 g IEEE693 SW Hysteretic Device Response .................... 223

Figure 28-12: 50\% 0.5 g IEEE693 NW Hysteretic Device Response ................... 223

Figure 28-13: 75\% 0.5 g IEEE693 X-System Response w/ Hysteretic Device...... 224

Figure 28-14: 75\% 0.5 g IEEE693 Y-System Response w/ Hysteretic Device...... 224

Figure 28-15: 75\% 0.5 g IEEE693 NE Hysteretic Device Response ................... 225

Figure 28-16: 75\% 0.5 g IEEE693 SE Hysteretic Device Response ..................... 225

Figure 28-17: 75\% 0.5 g IEEE693 SW Hysteretic Device Response .................... 226

Figure 28-18: 75\% 0.5 g IEEE693 NW Hysteretic Device Response ................... 226

Figure 28-19: 100\% 0.5g IEEE693 X-System Response w/ Hysteretic Device .... 227

Figure 28-20: 100\% 0.5 g IEEE693 X-System Response w/ Hysteretic Device.... 227

Figure 28-21: 100\% 0.5g IEEE693 Y-System Response w/ Hysteretic Device.... 228

Figure 28-22: 100\% 0.5g IEEE693 Y-System Response w/ Hysteretic Device .... 228

Figure 28-23: 100\% 0.5 g IEEE693 NE Hysteretic Device Response .................. 229

Figure 28-24: 100\% 0.5 g IEEE693 SE Hysteretic Device Response ................... 229

Figure 28-25: 100\% 0.5 g IEEE693 SW Hysteretic Device Response .................. 230

Figure 28-26: 100\% 0.5 g IEEE693 NW Hysteretic Device Response .................. 230

Figure 28-27: 100\% 0.5g IEEE693 X-System Response w/ Virgin Hysteretic

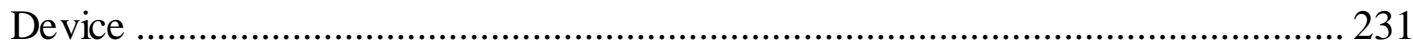

Figure 28-28: 100\% 0.5g IEEE693 X-System Response w/ Virgin Hysteretic

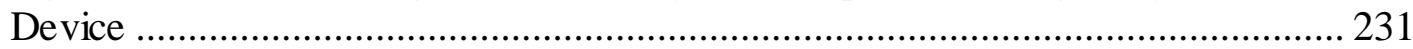


Figure 28-29: 100\% 0.5g IEEE693 Y-System Response w/ Virgin Hysteretic Device

Figure 28-30: 100\% 0.5g IEEE693 Y-System Response w/ Virgin Hysteretic

Device 232

Figure 28-31: 100\% 0.5g IEEE693 NE Virgin Hysteretic Device Response 233

Figure 28-32: 100\% 0.5 g IEEE693 SE Virgin Hysteretic Device Response 233

Figure 28-33: 100\% 0.5 g IEEE693 NE SW Virgin Hysteretic Device Response . 234 Figure 28-34: 100\% 0.5 g IEEE693 NW Virgin Hysteretic Device Response ...... 234 Figure 29-1: Non- Retrofitted X-Damping 20-50\% ....................................... 235

Figure 29-2: Non- Retrofitted X-Damping 50\% ............................................... 236

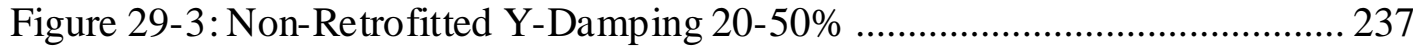

Figure 29-4: Non- Retrofitted Y-Damping 50\% …............................................. 238

Figure 30-1:20\% 0.5 g IEEE693 Non-Retrofitted X-System Response ................ 239

Figure 30-2: 20\% 0.5 g IEEE693 Non-Retrofitted Y-System Response ................ 239

Figure 30-3: 40\% 0.5 g IEEE693 Non-Retrofitted X-System Response ................ 240

Figure 30-4: 40\% 0.5 g IEEE693 Non-Retrofitted Y-System Response ................ 240 


\subsection{INTRODUCTION}

The ability for a substation to function prior, during, and after a seismic event significantly influences individual consumers. Resilience of substations in a seismic event allows the consumers to utilize electricity rapidly after a natural disaster. Previous analysis of the individual components in the $115 \mathrm{kV}, 230 \mathrm{kV}$, and $500 \mathrm{kV}$ substations indicated that the current transformers used in the $500 \mathrm{kV}$ main grid substations was extremely vulnerable to lateral forces. As part of the main grid system, interruption in service would affect many individual consumers. Current transformers typically have long lead times and are expensive to replace if damaged. Shown in Figure 1-1 is a typical $500 \mathrm{kV}$ current transformer used in a substation located in Wilsonville, Oregon. Typically, a 500kV CT weighs 5000 1.-7000 lb and has a total height of $27 \mathrm{ft}-30 \mathrm{ft}$ when assembled on a pedestal. The center of gravity of a typical $500 \mathrm{kV}$ CT is $20 \mathrm{ft}-24 \mathrm{ft}$ from the pedestal base. 


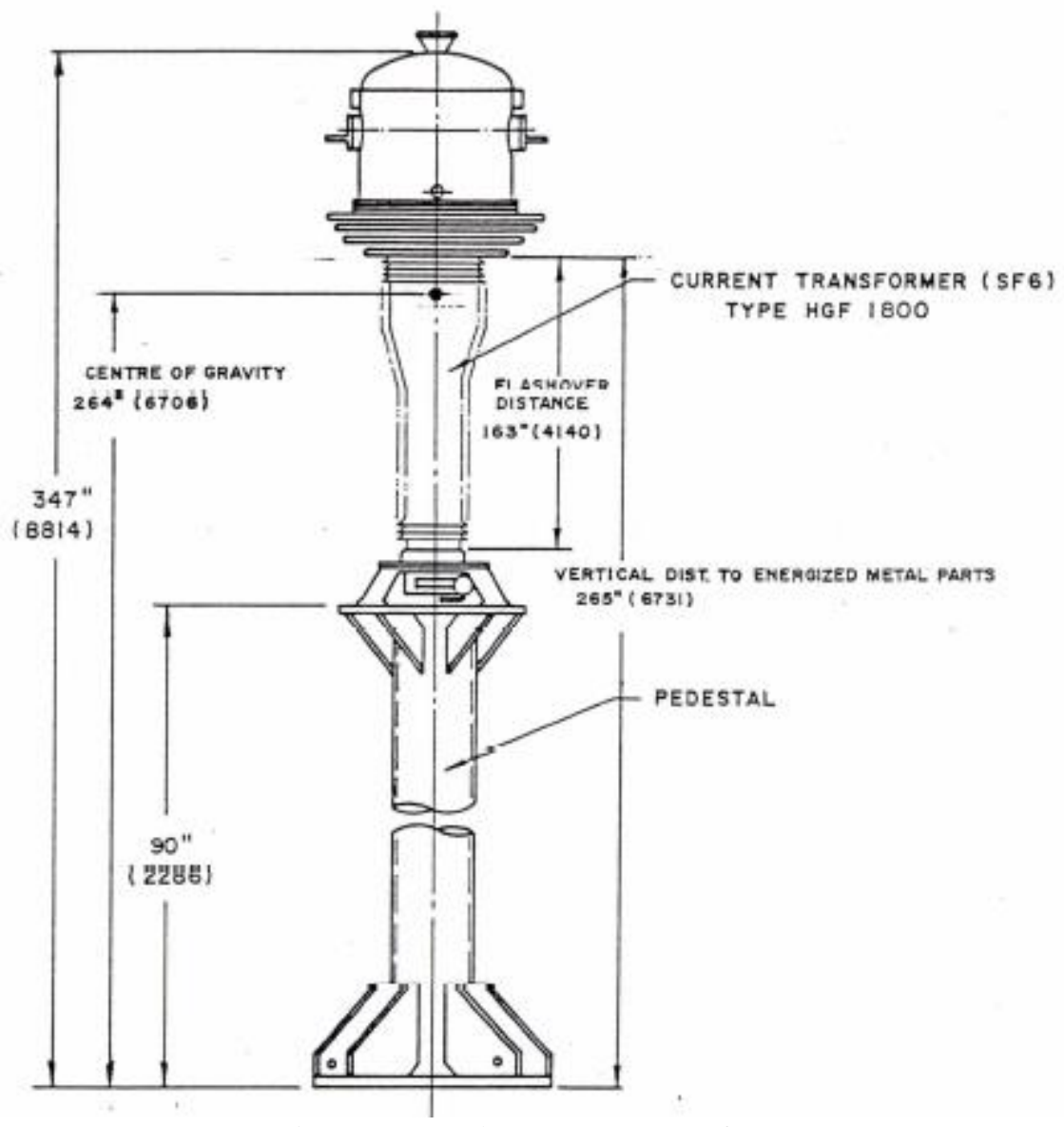

Figure 1-1: $500 \mathrm{kV}$ Current Transformer

If an as-built $500 \mathrm{kV} \mathrm{CT}$ is exposed to IEEE693 qualification motion, the forces transmitted to the base of the bolted structure base will result in pedestal yielding and likely cause porcelain fracture. Various studies on rocking reinforced concrete shear walls, steel braced frames, and various forms of beam-column connections have shown promising results by decreasing forces. A combination of rocking, self-centering, and energy dissipation is proposed as a retrofit measure to mitigate structure damage. Rocking is utilized to shorten the natural frequency (elongate the period) of the system. As the system becomes more flexible due to base rocking, energy dissipaters are introduced to the system to control displacements of the structure. Pre-tensioning added 
to the base of the CT allows the system to plumb after the motion has seized if all components are sized properly. As gaps form from the movement of the base plate, the system stiffness reduces and frequency shortens. By softening the global stiffness, the fundamental frequency of the system could be reduced and the demands decreased. Examining the $0.5 \mathrm{~g}$ PGA IEEE693 design spectrum shown in Figure 1-2, reveals how decreasing the frequency below $1.1 \mathrm{~Hz}$ leads to sudden decreases in spectral acceleration. Likewise, additional damping further reduces the spectral acceleration of the system. Throughout the work described, 0.5g PGA IEEE693 motion is referred to as the 100\% IEE693 motion. 


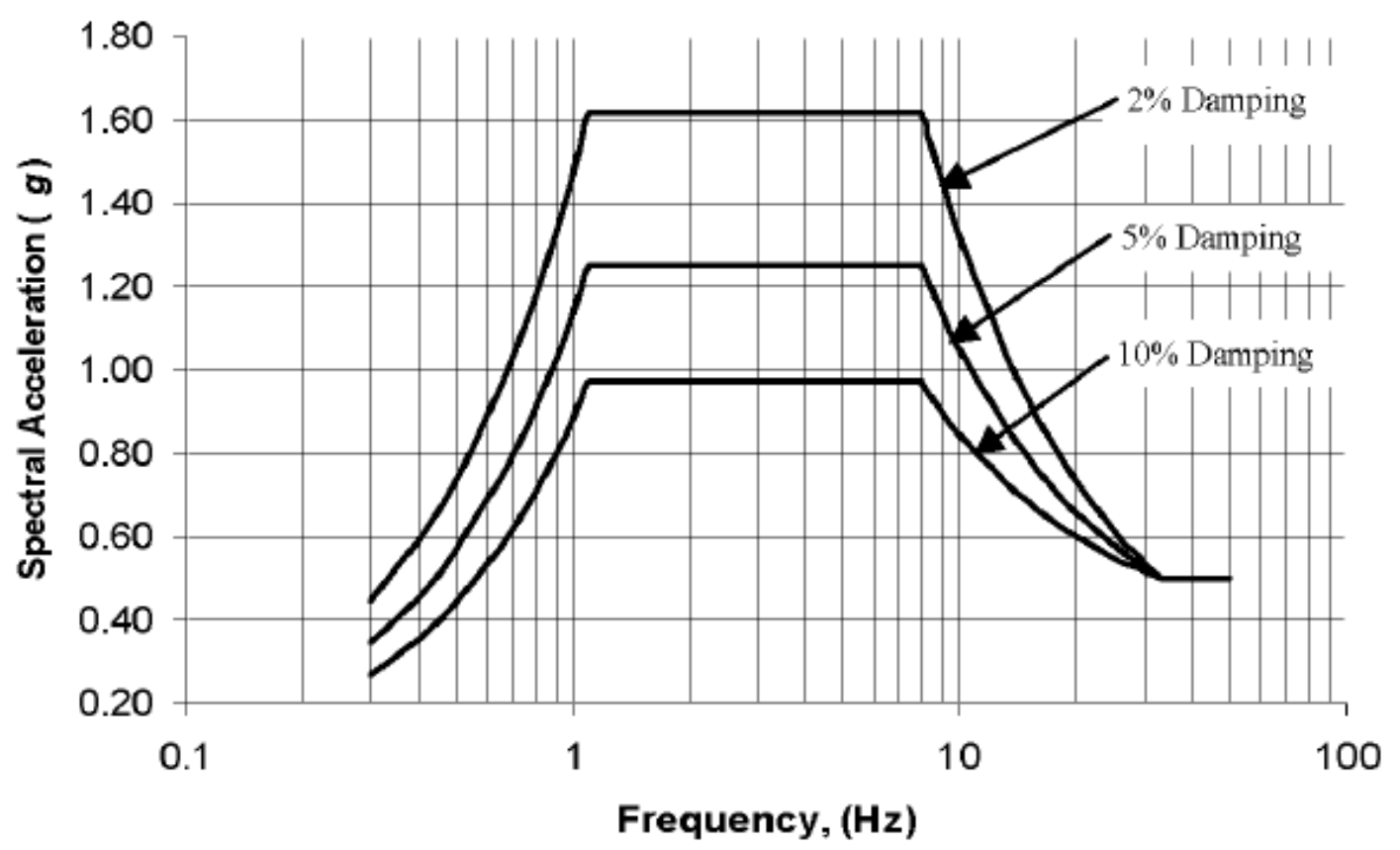

Spectral Accelerations, $S_{\|}(g)$, for Frequencies, $f(\mathrm{~Hz})$ :

$$
\begin{array}{ll}
S_{a}=1.144 \beta f & \text { for } 0.0 \leq f \leq 1.1 \\
S_{a}=1.25 \beta & \text { for } 1.1 \leq f \leq 8.0 \\
S_{a}=(13.2 \beta-5.28) / f-0.4 \beta+0.66 & \text { for } 8.0 \leq f \leq 33 \\
S_{a}=0.5 & \text { for } f>33
\end{array}
$$

$\beta=(3.21-0.68 \ln (d)) / 2.1156$, where $d$ is the percent damping $(2,5,10$, etc. $)$ and $d \leq 20 \%$.

Figure 1-2: IEEE693-0.5g PGA Design Spectrum (IEEE, 2006)

The research conducted evaluates the effectiveness of a self-centering rocking system with supplemental damping. Two primary phases of the research were conducted; the initial phase was a scaled concept validation on a single directional shake table at Portland State University (PSU). The second phase of the work was performed on a fullscale representative CT model at the University of Nevada, Reno (UNR) on a 6 degreeof-freedom shake table (6-DOF). Two types of damping devices were investigated, viscous dampers and a hysteretic device. Taylor Devices fluid viscous dampers designed to the specific application were utilized as the first device type. The hysteretic device 
used was u-shaped flexural plate (UFP). Component level modeling, global modeling, scaled testing, and full-scale testing was conducted to validate the proposed retrofit concepts.

\subsection{Research Objectives}

The primary objective of the research performed is to improve the seismic performance of the $500 \mathrm{kV}$ current transformers by reducing the demands on the CT components. BPA personnel would like the system to have no residual displacement after the seismic event. Two types of energy dissipating devices for the CT retrofit were considered; viscous and hysteretic. The retrofit measure aimed to reduce foundation anchor loads, decrease acceleration of the equipment, reduce forces on the pedestal, and reduce forces at the $\mathrm{CT}$ interface.

\subsection{Literature Review}

Discussed in this section are previous studies and publication on similar controlled rocking self-centering systems. Many studies and implementations of rocking systems have been complete on braced frames and shear wall in order to prevent structural damage and decrease demand on the system.

\subsubsection{Design Procedure for Controlled Rocking of Self-Centering Steel Frames}

The study (Eatherton, 2014) consisted of multiple experimental and computational phases in order to develop a self-centering rocking braced frame. The work summarizes the results obtained from the experimental and computation studies into design concept recommendations. Various arrangements of the pre-tension strands, fuses, and gap opening were shown for various height buildings. The authors state that as frame height 
increases and slenderness decreases the higher modes will have a larger impact on structure response to seismic events.

The proposed design procedure for the fuse and PT design consider the first mode typically controlling base overturning for low-rise and midrise structures. The primary design parameter is the system overturning where the moment due to lateral loading must be less than the resisting PT and fuse resisting force. Key parameters and recommended considerations in design include initial uplift, fuse yielding, PT yielding, loss of selfcentering capacity, and overall strength degradation.

Self-centering is the ability for a system to have minimal residual drift after the inertial loads are no longer present on the system. The authors introduce a self-centering ratio which is a ratio of the moment associated with rocking initiation to the fuse yielding moment. The ratio must be greater than unity for self-centering to occur. Although the ratio provides a metric for determining if self-centering occurs, it neglects effects of increasing PT force during loading and additional moment due to fuse hardening.

The author's design recommendations discuss a global uplift limit state that must be checked to ensure that global uplift will not occur. Global uplift is prevented when the total vertical load, including the PT force and dead load are greater than the upward force from the fuses.

The authors discuss the recommended minimum energy dissipation to avoid strength degradation and excessive structure drift. The energy dissipation ratio is defined as the ratio of the fuse moment to the bi-linear elastic system moment. The author states that previous research $(\mathrm{Seo}, 2005)$ suggests that the ductility demands between an elastic 
perfectly plastic system and inelastic flag-shaped hysteretic system depend on the postyield stiffness of the flag shape system. For system with a post-yield stiffness of $10 \%$ and $20 \%$ of the initial stiffness and energy dissipation ratios of $25 \%$ and $12.5 \%$ respectively, resulted in similar ductility demands to conventional systems. Previous research also suggested that for energy dissipation (ED) ratios above $25 \%$ of the peak drift was less sensitive to ED ratio. The author suggest using a minimum energy dissipation ratio of $25 \%$ unless the analysis to determine the drift demand explicitly considers damping.

Next, the authors discuss PT yield and fracture. The author suggests considering initial preloading of the member along with the expected uplift assuming rigid body motion to determine the strain in the members.

The authors state that the rocking typically only effects the first mode of the system and causes the forces to be reduced. Higher modes are not impacted by the rocking system and must be considered to properly estimate base shears and moments. In previous work on bridges, rocking columns had a significant impact on the system loading due to the high mass of the system, since the high weight of the bridge resulted in both lateral and vertical inertial effects with rocking columns. The authors state that for buildings the vertical inertial effects are less significant since the vertical mass contribution is less than bridges.

\subsubsection{Displacement-Based Design of Precast Walls with Additional Dampers}

The research by Pennucci (2009) tailors previously proposed displacement based design procedures to rocking precast walls with additional energy dissipating devices. 
The proposed procedure is applied to multiple scenarios and validated through numerical analyses.

In order to limit costs of structure retrofit associated with damage due to seismic events in conventional construction, an alternative form of construction with precast concrete walls post-tensioned with partially unbounded cables is discussed in this work. Conventional construction typically results in permanent drift, structural damage, and high repair costs. A comparison between the system response in conventional construction, rocking structure, and rocking structure with supplemental damage is shown in Figure 1-3.

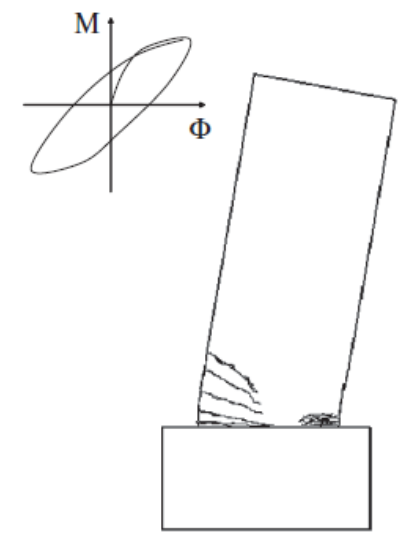

(a) Traditional concrete: - Large energy dissipation - Extensive damage

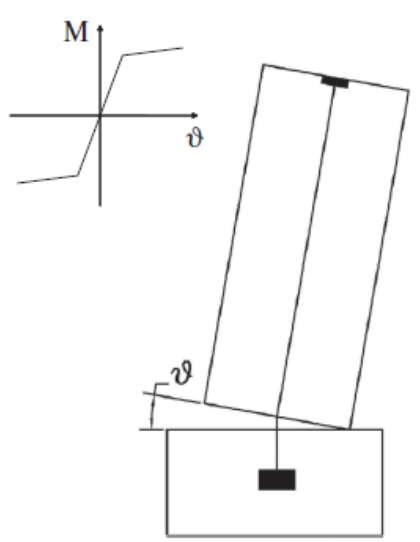

(b) Precast post-tensioned: - No energy dissipation - Negligible residual deformations

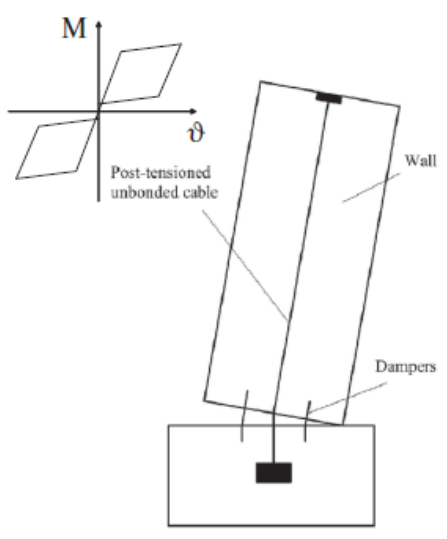

(c) Hybrid wall: - Large energy dissipation - Negligible residual deformations

Figure 1-3: Rocking Shear Wall Theoretical Diagram

Rocking systems avoid plastic hinge formation and allow for concentration of inelasticity in replaceable fuses. The research conducted considers the use of steel dependent yielding dampers. The procedure includes the following steps: select design drift, define equivalent SDOF system, determine equivalent elastic damping, obtain design loads, and design based on capacity. The researchers refer to an a/b metric which 
compares the restoring moment to the device moment used to characterize a systems selfcentering capabilities (defined in Figure 11-7). The authors developed a dampingductility curve for $\lambda=\mathrm{a} / \mathrm{b}=1.25$ used for estimating the equivalent viscous damping of the system. Although the maximum dissipation ratio occurs when $a / b=1.0$ or when the fuse and the restoring moment are equal, previous research indicated suggests that 1.25 be used to account for material over strength. The authors validated the recommended design procedure through numerical modeling and obtained similar results. The researchers recommend additional investigations on the systems response with threedimensional excitation.

\subsubsection{Post-Tensioned Moment Connections with a Bottom Flange Device for Seismic Resistant Self-Centering MRFs}

Lehigh, Princeton, and Purdue Universities collaborated in development of a new earthquake-resistant structural steel moment resisting frame (MRF) through experimental and analytical research (Ricles, 2006). The work consisted of developing a self-centering moment resisting frame (SC-MRF) with additional energy dissipation through a friction device at the bottom beam flange. The authors state that in traditional MRFs, damage occurs to the structure and an alternative to weld construction is discussed. 


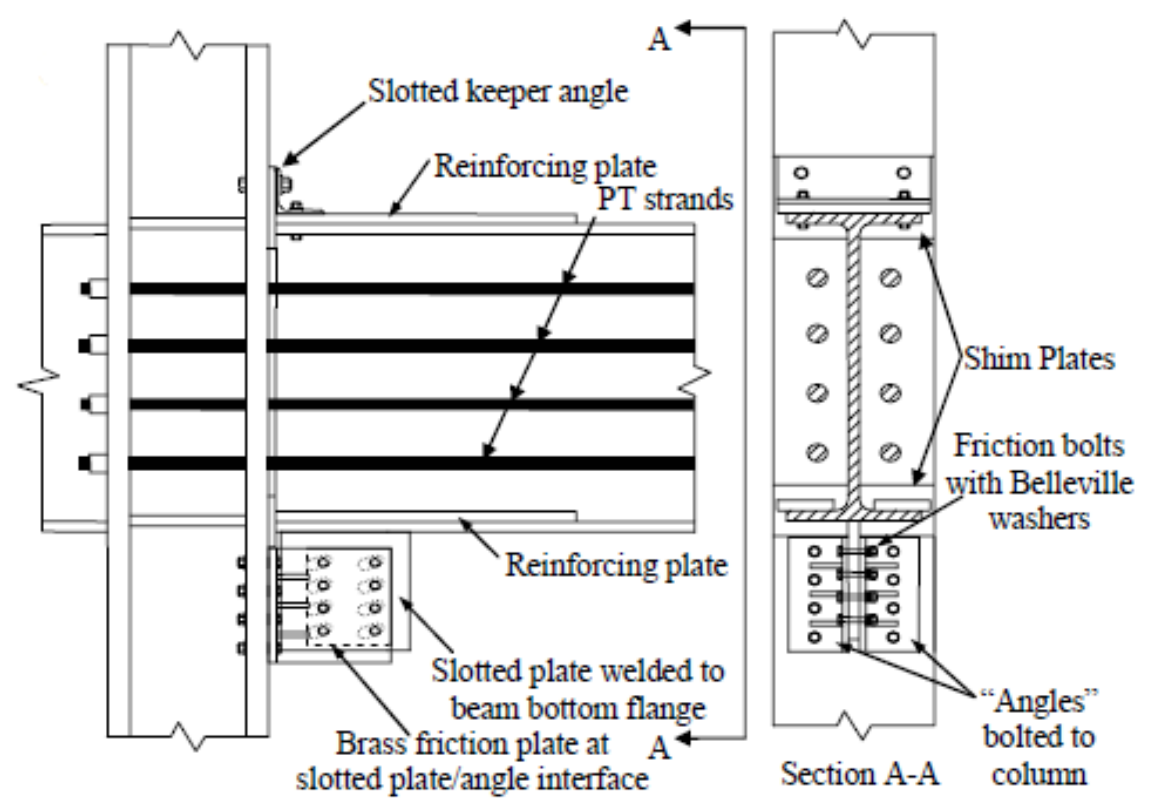

Figure 1-4: Self-Centering MRF w/ Friction Damping

The authors utilized AST B-19 UNS cartridge brass as the friction material which was positioned between two steel angles. The angle-brass friction interfaces were precompressed using Belleville disk spring washers. The self-centering mechanism was achieved through conventional PT strands as shown in Figure 1-4. Rotation at the connection leads to gap opening and displacement in the bottom flange friction device (BFFD). The authors used recommendations made by (Seo, 2005) and used an energy dissipation ratio of 0.25 . The SC-MRF with a bottom flange friction device demonstrated sufficient energy dissipation and self-centering capabilities. Self-centering was achieved without residual drift when the PT strains remained elastic.

\subsubsection{Self-Centering Seismic Lateral Force Resisting System: High Performance Structures for the City of Tomorrow}

Four authors from three educational institutions explain self-centering seismic lateral force resisting systems and discussing the current challenges with self-centering system in (Chancellor, 2014). The authors discuss lateral force resisting systems that 
reduce structural damage by softening the system through gap opening. Yielding devices or friction devices are used to dissipate energy through relative movement due to the gap opening.

The authors discuss conventional lateral systems which rely on damage of structural components in order to dissipate energy. For various conventional system, energy dissipation is achieved though plastic hinge formation, buckling of braces, or crushing and yielding in reinforced structures. Traditional lateral systems typically result in residual displacements after lateral loads are removed and structure damage. The authors discuss the large economic impact that the 6.3 magnitude earthquake had on Christchurch, New Zealand in 2011. The authors claim that the estimated repair costs are $\$ 40$ billion (NZD) not accounting for any economic losses associated with business downtime.

The authors state that conventional design approaches are inefficient in limiting structural damage and residual drift. Introduced by the researchers are the key components for a self-centering seismic lateral resisting system. The academics discuss the restoring force and gap opening mechanisms typically used in self-centering systems, which provide bilinear elastic self-centering but no energy dissipation. Previous research indicates that minimal energy dissipation is required to limit drift in a self-centering system to those of conventional elastic-plastic systems. Most self-centering systems have four limit states outlined by the authors: (1) PT decompression coupled energy dissipating device; (2) PT yielding; (3) limited damage to structural elements; and (4) severe damage to structural elements. 
The investigators discuss four main challenges with self-centering systems, including component compatibility, higher mode effects, collapse assessment, and strategic implementation of self-centering. Due to the gap opening associated with rocking in self-centering system, detailing is of extreme importance for all components of the system. The authors discuss floor diaphragms and other connection complications associated with rocking systems. Limited research and knowledge is know on the effect of higher modes on self-centering systems. The authors state that base rocking only reduces forces in the first mode and additional softening locations along the length of the structure. The authors state that the approach appears promising but additional research must be conducted to determine the effectiveness of a multi-rocking system. The investigators also state that although self-centering systems are designed to reduce forces and minimize residual displacements, extreme earthquakes may cause permanent damage to the restoring mechanism and a safety collapse assessments must be completed. The research also suggest performing life-cycle costs analysis to determine whether a selfcentering system is worth the premium detail and construction costs compared to those of conventional systems.

\subsubsection{Mechanism of Energy Absorption in Special Devices for use in Earthquake Resistant Structures}

A paper by Kelly, Skinner, and Heine (1972) investigated methods for designing structures that dissipate kinetic energy due to ground motion. The authors state concern regarding conventional design where damage to structural components results in member capacity reductions. The authors propose adding additional members into structures for 
the sole purpose of energy absorption. The academics explore rolling strips, torsion of square and rectangular bars, and the flexure of short thick beams.

The authors state that torsion was the most effective energy dissipating mechanism. Plastic strains from $3 \%$ to $12 \%$ and energy dissipation of $2000 \mathrm{lb} \mathrm{in} / \mathrm{in}^{3}-7500 \mathrm{lb}$ in/in $\mathrm{in}^{3}$ per cycle was achieved in plastic torsion of mild steel. The devices dissipating energy through plastic torsion had lifespan within 100 cycles to 1000 cycles. In addition, the researchers stated that the torsional devise had a gradual decay failure. The authors state that rolling strips and flexure of short thick beams dissipate less energy and are less reliable. The devices dissipated $500 \mathrm{lb}-2000 \mathrm{lb}$ in/in ${ }^{3}$ per cycle and had lifespans between 20 to 200 cycles. The rolling strips and flexure of short thick beams are more compact than the torsional devices.

The researchers tested a range of steel strip geometries made of mild steel and stainless steel. The strips were all $9 \mathrm{~mm}(0.354 \mathrm{in}$.) wide and ranged in thickness from $0.75 \mathrm{~mm}(0.029 \mathrm{in}$.$) to 2.0 \mathrm{~mm}(0.079 \mathrm{in}$.). Four diameters were used for the bent strips, 9 mm (0.354 in.), $11 \mathrm{~mm}(0.43$ in.), $13 \mathrm{~mm}(0.511 \mathrm{in.})$, and $15 \mathrm{~mm}$ (0.59 in.). All plates were cold rolled and tested under displacement control. The devices would kink and then completely fracture. The academics state that the stroke and the level of maximum strain dictate the lifespan of the device. Device stroke should be near $\pi \mathrm{R}$ to maximum the energy dissipation, but should not exceed $\pi \mathrm{R}$. A summary provided by the authors relates the maximum strain, normalized stroke and lifespan shown in Figure 1-5. The researchers also discuss the results obtained from torsional and flexural dissipaters which are not 
implemented in the research discussed due to the required compactness of the energy dissipating devices.

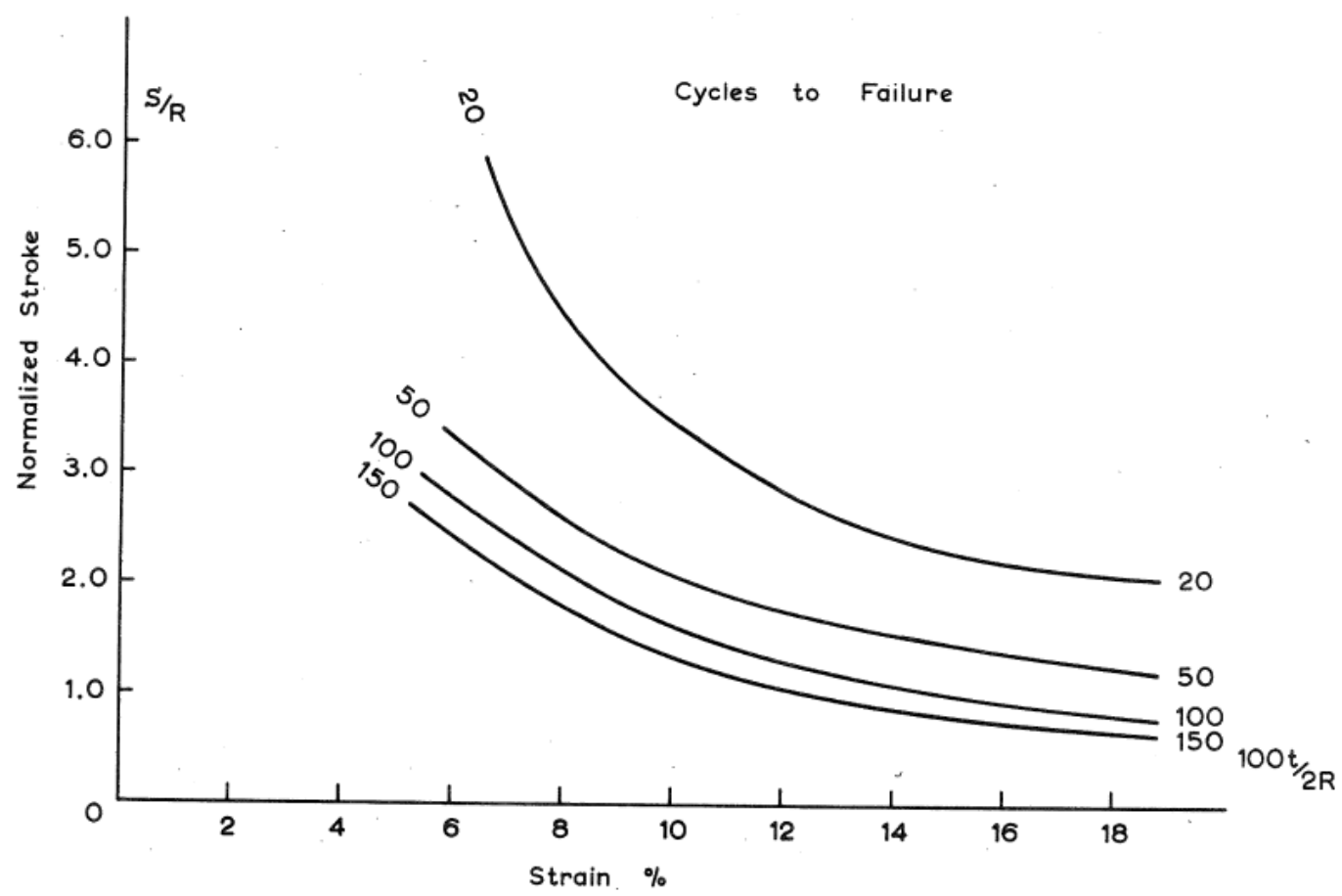

Figure 1-5: Life Time of Double Strip Test Apparatus (UFP)

\subsubsection{Experimental/Numerical Study of U-Shape Flexural Plate (UFP) Dissipaters}

The Christchurch earthquake resulted in increase in interest in structures that have low damage according to (Baird, 2014). Low damage structures minimize the need of repairs after events and allow for immediate occupancy. Low damage structures concentrate all non-linear behavior into replaceable components rather than relying on structural member yielding for energy dissipation. The authors use U-shape flexural plate (UFP) dissipaters because they are simple to design, inexpensive, versatile, and replaceable. The researchers state that limited information on UFPs is available regarding initial and post-yield stiffness. The author summarizes the work performed in Kelly, 
Skinner, and Heine (1972) where the equations for UFP capacity and max strain were derived.

The authors apply Castgliano's Second Theorem in order to derive the initial stiffness of a UFP. Shown in (1) is the derived equation the author presents.

$$
k_{0}=\frac{F_{y}}{\Delta_{y}}=\frac{16 E b_{u}}{27 \pi}\left(\frac{t_{u}}{D_{u}}\right)^{3}
$$

The academics performed a series of experimental tests using ACI loading recommendations. The loading protocol consisted of multiple amplitude displacements, three cycles at each amplitude and a maximum amplitude of $82.5 \mathrm{~mm}$. The experimental results were compared to the model outputs developed in the research. The UFPs had the following properties $d_{i}=120 \mathrm{~mm}, b=8 \mathrm{~mm}, F_{y}=6.4 \mathrm{kN}, F_{p}=9.6 \mathrm{kN}$ and a leg length of 100 mm. The maximum strain for the UFP geometry was determined to be $6.3 \%$. The authors state that they expected more than 150 cycles for the specified geometry based on the limited data summarized in Kelly, Skinner, and Heine (1972). Testing Configuration and Results are shown in Figure 1-6.
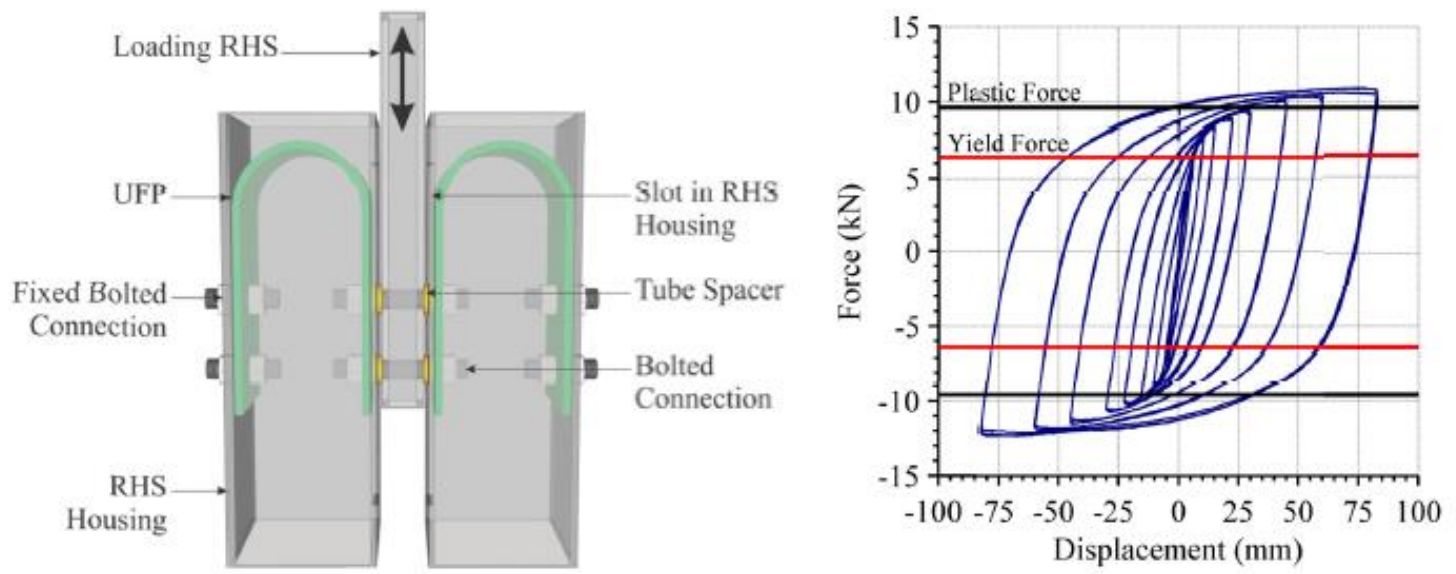

Figure 1-6: NZ2014 Testing Configuration and Results 
ABAQUS finite element models were constructed with similar geometries to the experimental UFPs. The model utilized tetrahedral elements with isotropic yielding and cyclic hardening. The model material properties were based on a tensile coupon of the steel used for the UFPs. Shown in Figure 1-7 are the results obtained by the reseachers. The authors accurately modeled the post-yield stiffness and maximum forces in the UFP. The model did not accurately capture the Bauschinger effect in the steel, resulting in a larger energy area than determined from experimental results.
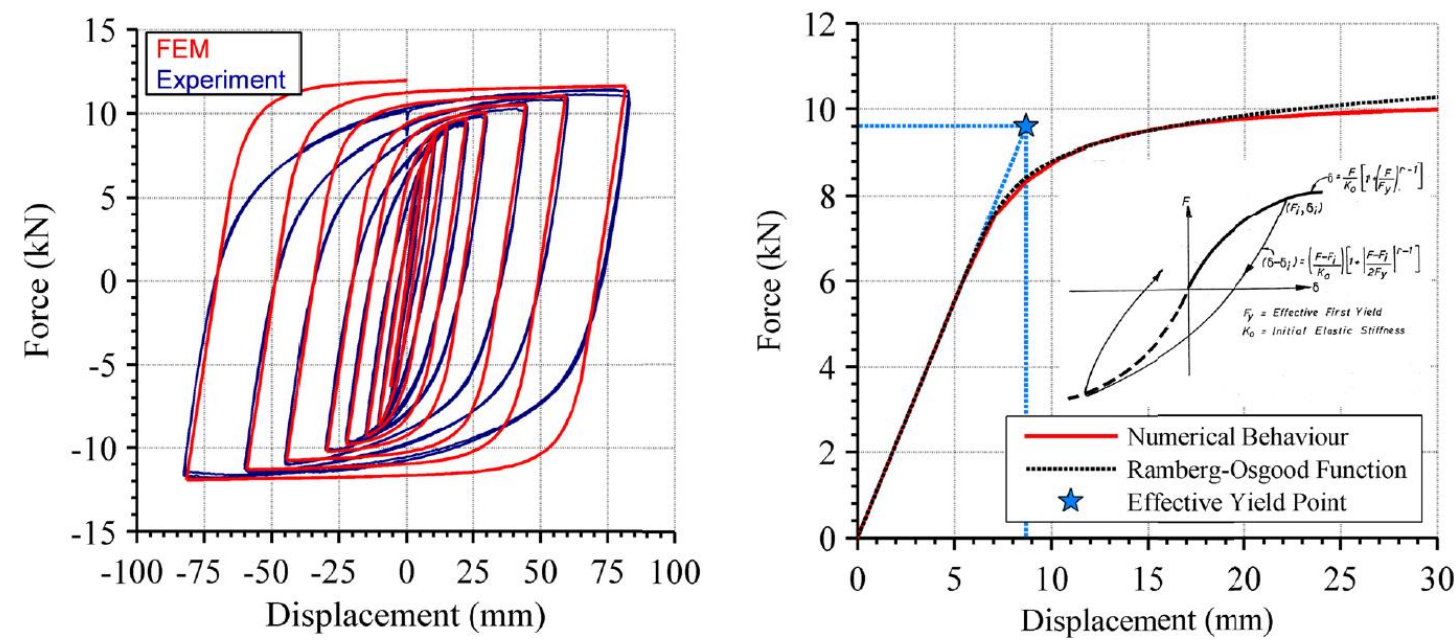

Figure 1-7: NZ2014 Numerical and Experimental UFP Results

A UFP parametric investigation was conducted using finite element modeling that was developed based on the experimental results. Plate thickness and diameter of the UFP were investigated to determine the influence on yield force, yield displacement, initial stiffness and post-yield stiffness. The Ramberg-Osgood function was used to fit the results and found to have an excellent representation of the behavior as shown in Figure 1-7. Based on the results obtained from numerical analysis, the author suggests the relationship shown in (2) for defining the Ramberg-Osgood $\mathrm{R}$ factor. 


$$
R=7.1 \ln \left(\frac{t_{u}}{D_{u}}\right)+29.5
$$

The researchers state that high levels of accuracy were observed between the experimental and numerical results. Also, the post-yield stiffness was well represented with the Ramberg-Osgood function and the recommend function is proposed for determine the $\mathrm{R}$ factor. 


\subsection{RETROFIT BACKGROUND}

Based on the objectives of this research, a self-centering rocking system with supplemental damping is proposed to reduce demands on the $500 \mathrm{kV}$ Current

Transformers. The proposed system utilizes reduction in stiffness, energy dissipation, and elastic pre-tensioned members (PT) to reduce demands on the CT while eliminating residual displacements.

The retrofitted CT consists of three primary components: elastic structure, elastic pre-tensioned members, and energy dissipating device which significantly influence the behavior of the system during a seismic event.

Understanding the fundamentals of the elastic and non-linear components is the key to designing a self-centering rocking system. Illustrated in Figure 2-1, is the idealized bilinear elastic load-deformation curve of a rocking system's response. When a system is pre-tensioned with elastic members, the system stiffness could be assumed to be equal to the bolted stiffness structure until uplift occurs. The initial stiffness, $K_{f}$, is the flexural stiffness of the bolted structure shown in the diagram from (a) to (b) and on the reversal cycle from (g) to (i). Before the pre-tensioned components are decompressed, the flexural stiffness controls the system response. The force required for uplift to occur is controlled by adjusting the pre-tension force. For the work conducted, two metrics were used to determine the decompression load, wind load and target maximum acceleration. Once the decompression force is overcome, any additional load causes uplift and results in system frequency shift. When uplift occurs (b), the stiffness reduces to $K_{P T}$ based on the stiffness of the pre-tension members. By reducing the global stiffness, the demand on the structure's components is reduced, but displacements increase. The equivalent stiffness of 
the system varies with displacement, typically equivalent stiffness decreases as the displacement increases.

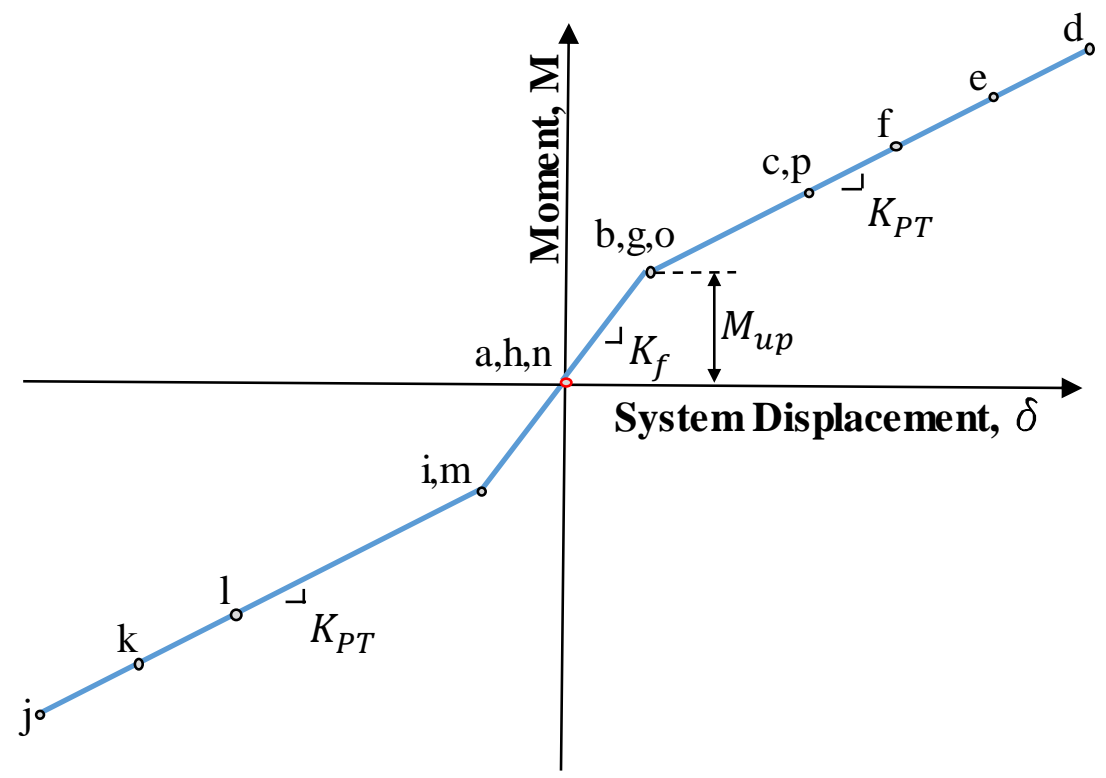

Figure 2-1: Theoretical Elastic Rocking

As the equivalent stiffness of the structure decreases, the displacement of the system increases. Energy dissipating devices are introduced to reduce system displacements and concentrate the non-linearities of the systems response into the energy dissipating devices. Two types of energy dissipating devices were investigated, Taylor Devices fluid viscous dampers and hysteretic yielding devices.

A fluid viscous damper dissipates energy by forcing fluid through orifices from one chamber to another. As the fluid travels through the orifices, the kinetic energy from the relative velocity is converted to thermal energy which is absorbed and dissipated by the damper. The size of the orifices control the amount of force produced. Viscous dampers are velocity dependent, the force developed is proportional to the relative velocity of the damper end constrains. Viscous damper coefficients are based on three main parameters, 
velocity at the location of the device, global displacement, and device force at max velocity. Each parameter is interconnected and the properties are typically iterated until a desirable medium is met for all the design parameters. General equation used for determining the force in the device is defined in (3).

$$
\begin{aligned}
F= & C \cdot v^{\alpha} \\
& F=\text { Damper Force }(\mathrm{lb}) \\
& C=\text { Damping Constant }(\mathrm{lb}-\mathrm{sec} / \mathrm{in}) \\
& v=\text { Velocity }(\mathrm{in} / \mathrm{sec}) \\
& \alpha=\text { Velocity Exponent }(0.3<\alpha<1.0)
\end{aligned}
$$

After a seismic event, when relative velocity is near zero, self-centering could be achieved by the pre-tensioned members. Viscous dampers act out of phase to the structure's displacement. Unlike velocity dependent viscous dampers, hysteretic devices develop residual forces in the devices after the lateral inertial loads are no longer present, due to elasto-plastic deformation, which must be considered when designing the selfcentering system. Also, when removing yielded devices, the developed residual forces must be considered.

Hysteretic devices use material non-linearity, yielding of material to dissipate energy. Many forms of hysteretic devices have been evaluated for stable and repeatable properties. Used in the research, are u-shape flexural plates which yield when relative displacement between the two legs occurs, the plate steel is rolled and energy is dissipated.

The UFP is designed based on the initial pre-tension force and structure weight to ensure self-centering after a seismic event. If the hysteretic device capacity is larger than 
the restoring force, the system will no longer be self-centering and residual displacement will result.

Illustrated in Figure 2-2 is the UFP's moment response as a function of displacement. It is important to note that the hysteretic device does not begin to experience relative displacement until uplift begins at (b). After uplift occurs, the UFP undergoes elastic behavior until yield (c) where the stiffness is denoted $K_{d}$. The post yield stiffness is defined as $K_{y}$ where the stiffness may vary due to fatigue and other factors during cyclic loading. The diagram illustrates the hysteretic devices global contribution to moment.

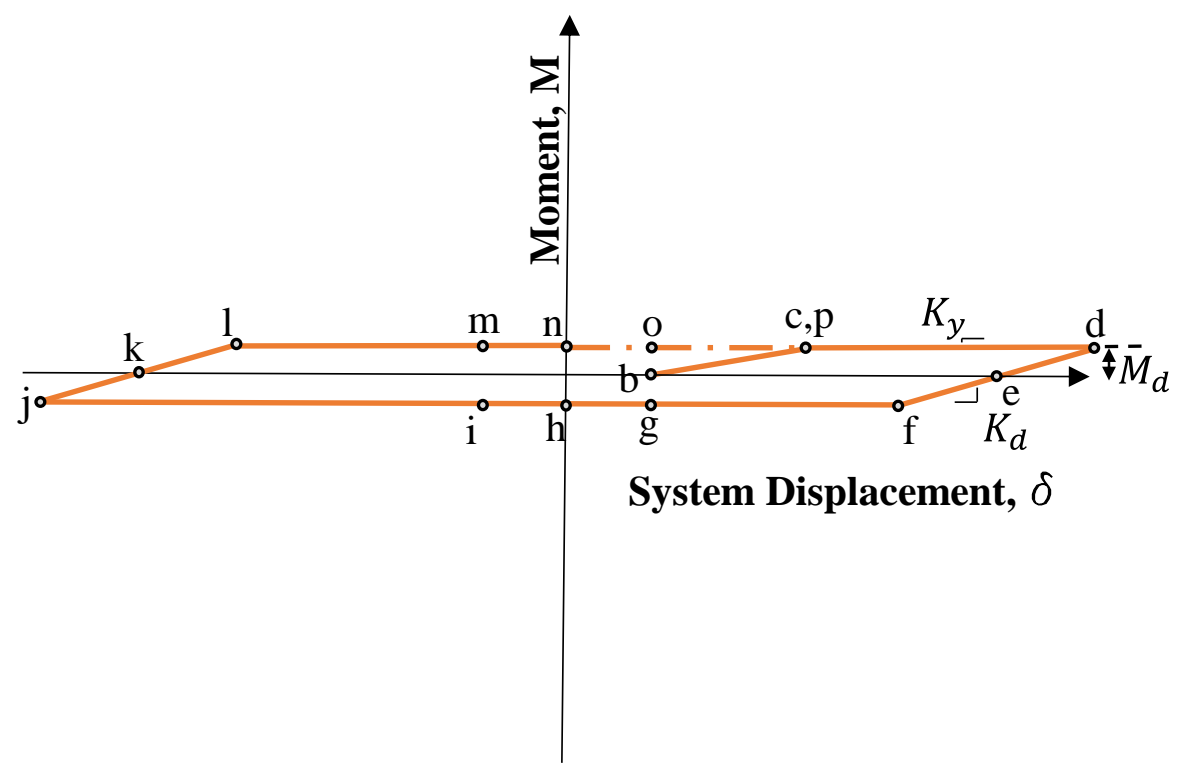

Figure 2-2: UFP Theoretical Hysteretic Behavior

Combining the rocking behavior with hysteretic damping, the system response is expected to be as shown in Figure 2-3. Starting at (a) the system undergoes cantilever fixed base deformation until the design uplift moment is achieved (b). The initial cantilever stiffness is defined as $K_{1}$. At (b) the PT has been decompressed and uplift 
initiates. From (b) to (c) the stiffness of the system is a combination of damper yield stiffness $\left(K_{d}\right)$ and PT stiffness $\left(K_{P T}\right)$ defined as $K_{2}$. From (c) to (d) the plastic damper deforms with stiffness $\left(K_{y}\right)$ and PT elongates elastically $\left(K_{P T}\right)$ defined as $K_{3}$. UFPs typically present relatively low post-yield stiffness compared to their initial stiffness.

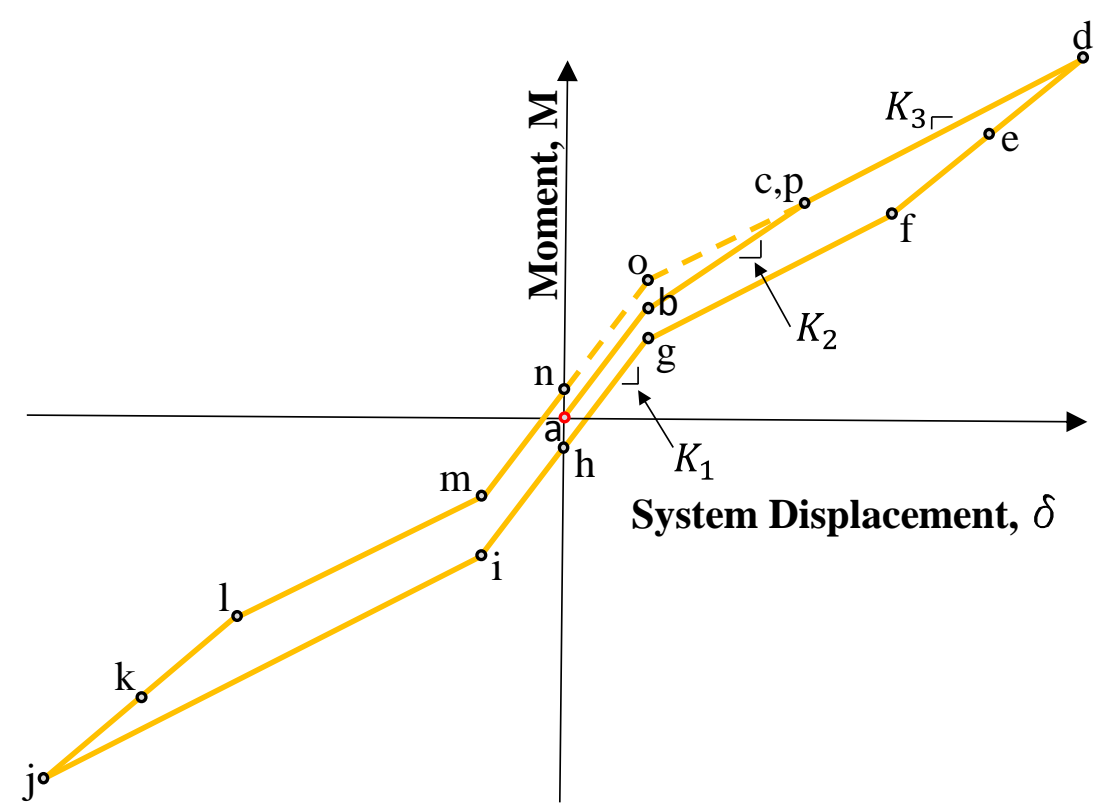

Figure 2-3: Theoretical Rocking w/ Supplemental Damping

When the system is unloaded, (d) to (f), the system deforms following $K_{2}$. Once the damper has yielded in the opposite direction (f), the system follows $K_{3}$ until the base plate and damper are in their initial positions (g). The system then is allowed to recover its flexural deformation (g) to (h) following $K_{1}$. Although no external force is present at (h) the hysteretic devices produce locked-moments in the system when returned to its initial position by the PT members.

The moment produced by the PT elements must always be greater than the moment required to yield the dampers back to their original position. If the elastic moment is less 
than the moment required to yield the fuse elements back to their original position, the system will not plumb.

Two main differences are expected in the dynamic characteristics of the viscous dampers and the hysteretic devices. While both devices are expected to reduce displacement of the system, the viscous devices are expected to be more effective at small levels of excitation. At low excitations, the hysteretic devices will remain elastic and are expected to minimally contribute to damping. Taylor viscous dampers dissipate energy whenever any relative velocity is present on the shaft. Another important difference is viscous dampers are out of phase with structural stresses. Out of phase damping typically leads to lower demand on structural members. 


\subsection{RETROFIT CONFIGURATIONS}

Legacy current transformers are typically installed on pedestals supplied by the manufacturer of the electrical component. Both the pedestal and the porcelain CT were designed with minimal considerations to lateral loads. The proposed retrofit aims to reduce demand on all components of the current transformer. Reduced demand in anchors, support pedestal, and porcelain members are expected from the retrofitted CT.

Supplied by BPA was a typical support pedestal used to install 500kV CTs, complete details on the pedestal are shown in Figure 19-1. The support structure has a 1$1 / 8$ " base plate that is 30 " square. Each corner of the base plate has $1-15 / 16$ " holes drilled for anchoring the support. Two stiffeners are located 4" apart from each other. Shown in Figure 3-1, is the type of CT pedestal provided. The pedestal has 8 existing stiffeners which are utilized for mounting brackets for both the viscous and hysteretic devices. The retrofit method could be applied to other pedestal geometries although custom brackets and other modifications to the retrofit will be required.

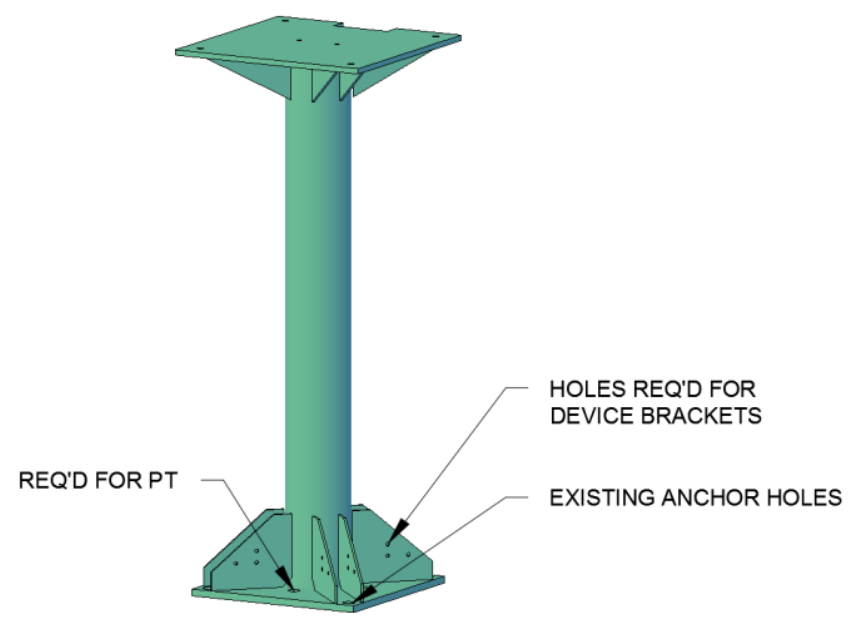

Figure 3-1: Rendering of Provided CT Pedestal 


\subsection{Pre-Tension Members}

Numerous material types were considered for the elastic self-centering mechanism of the CT retrofit. Factors that dictated the material or device used to generate the system restoring force included the pedestal geometry, stiffness of the material, and ease of application. Conventional pre-tensioning strands were initially investigated but their high stiffness and maximum elongation did not meet the desirable traits for the application. A desired characteristic of the elastic self-centering device is low stiffness while accommodating the displacement demands of the system.

Next, Carbon Fiber Reinforced Polymer (CFRP) Bar Aslan 200/250 series pretension bars were investigated because of two specific properties that the material exhibits: the high ultimate stress and the modulus of elasticity. A high ultimate stress along with a lower modulus of elasticity allows for a smaller area of PT to be used and longer material elongation to occur. The smaller area allows for a reduced axial stiffness and overall reduction in stiffness once the system begins to rock. Aslan 200/250 material has a modulus of elasticity of $18,000 \mathrm{ksi}$, ultimate strain of $1.67 \%-1.75 \%$ and a guaranteed tensile strength of $300 \mathrm{ksi}-315 \mathrm{ksi}$ (Aslan FRP, 2011).

Typically, steel PT strands could reach strains of $0.8-1 \%$ at yield and have a modulus of elasticity of 27,000 ksi-28,000 ksi. The CFRP material could handle forces of $24 \%$ more than steel with similar axial stiffness; likewise, the CFRP members could elongate $12 \%$ more than an equivalently stiff steel member assuming a yield strain of 0.008 for steel pre-tension strands. Shown in Figure 3-2 was the original retrofit concept utilizing CFRP. 


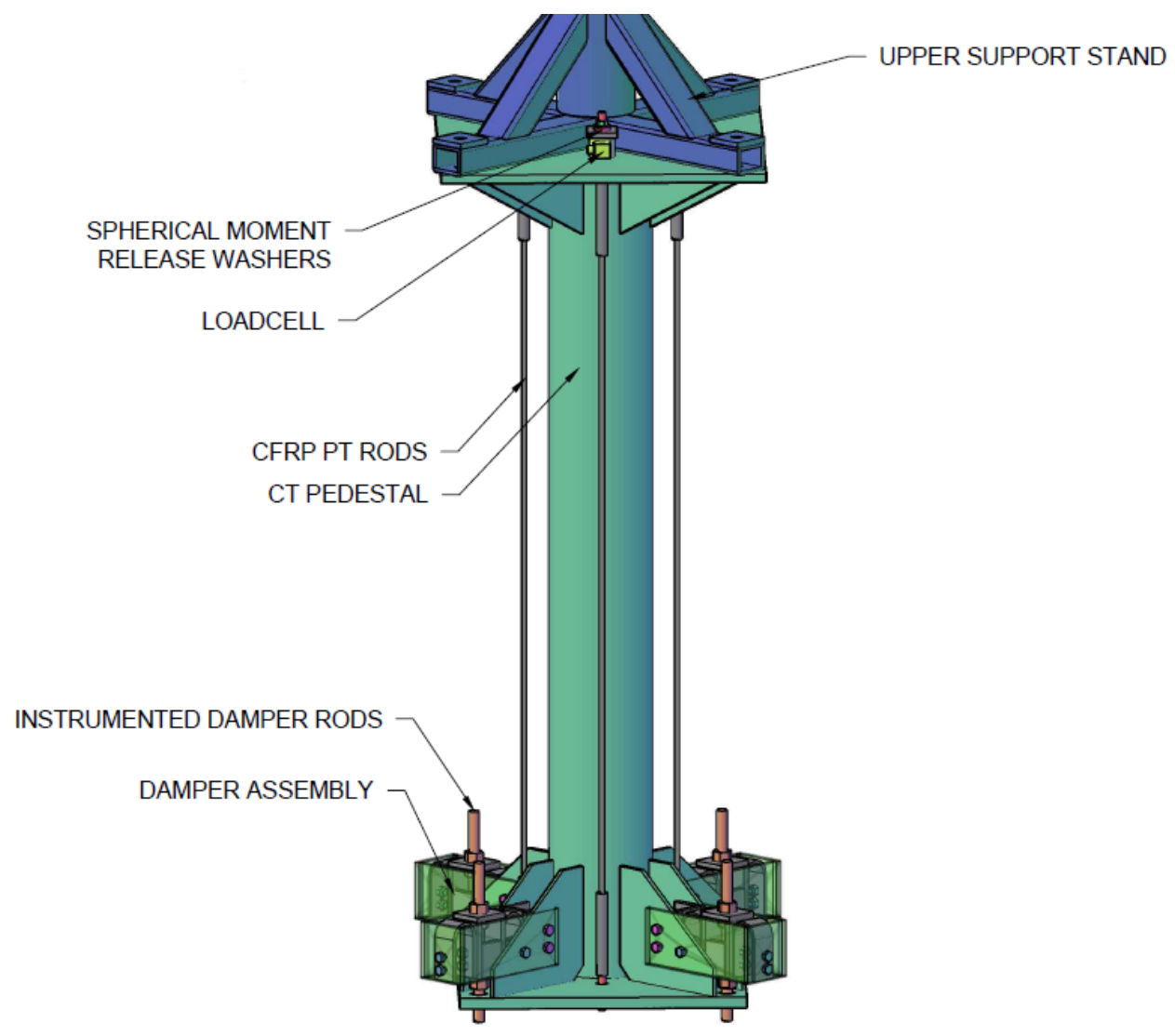

Figure 3-2: Proposed CFRP Configuration

CFRP presented two properties which made CFRP an unattractive choice for the retrofit, brittle behavior and difficulty of implementation. Belleville Spring Washers (BeS) were chosen as the restoring device for the retrofit because of their stiffness and displacement versatility and ease of application in the design. BeS washers are spherically shaped washers which could be arranged in parallel or series arrangements to achieve the desired stroke and stiffness. Obtained from the Solon Manufacturing Co. catalog is Figure 3-3 and Figure 3-4 which shows the various arrangements of Belleville Spring Washers to achieve the desirable stiffness. Solon also provides design guides for using Belleville Spring Washers to maintain bolt preload. 
A. Single, one spring.

B. Parallel, all springs stacked the same way.

C. Series, all springs stacked opposing each other.

D. Parallel-Series, a combination of the two.

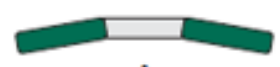

A

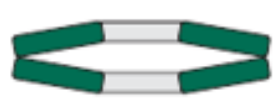

C

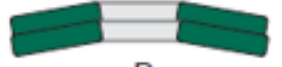

B

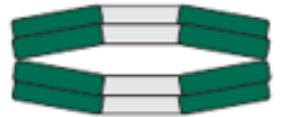

D

Figure 3-3: Arrangement of Belleville Washers

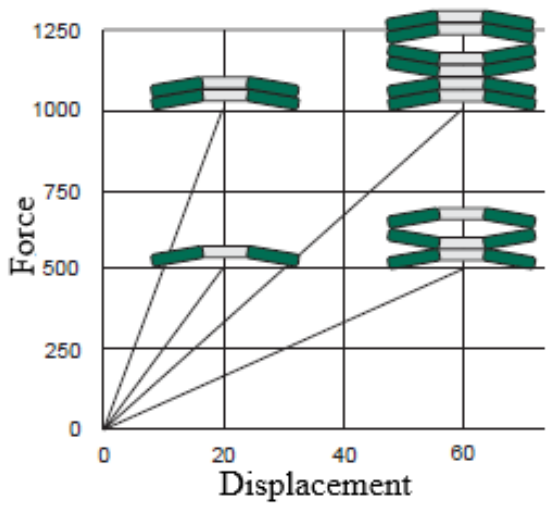

Figure 3-4: BeS Stiffness and Displacement Relationship

Belleville Spring Washers are linear for $90 \%$ of their total flat load. After $90 \%$, the stiffness of the washer set starts to increase until the flat load is achieved. At the flat load the threaded rod which is used to transfer the load to the foundation will feel the load directly. Stiffness of the system significantly increases if the system experiences displacements larger than the BeS could occupy. One major advantage of the BeS system compared to the CFRP strands is that collapse is prevented when the displacement exceeds the designed displacement. Typical specifications for Belleville washers are shown in Figure 3-5 obtained from Solon Mfg. 


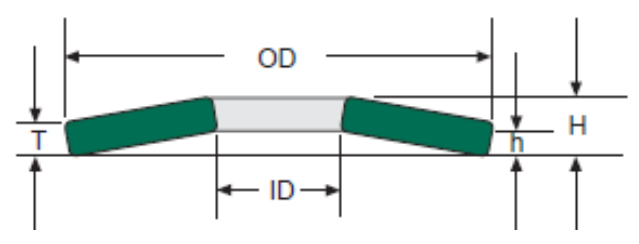

Figure 3-5: Belleville WasherDimensional Specifications

\subsection{Energy Dissipaters}

Once rocking initiates, the elastic member's pre-tension force is surpassed, the pedestal will exhibit relative displacement to the anchors used in the fixed base condition. Relative displacement between the existing anchors and the pedestal allows for energy dissipation. Dampers and UFPs are activated, providing supplemental damping, i.e. energy dissipation, as the relative displacement occurs.

The self-centering concept developed for potential retrofit of CT equipment concentrates non-linear energy dissipating devices on each corner of the existing pedestal. Shown in Figure 3-6 is the proposed viscous device arrangement which was evaluated in this research. When rocking occurs, relative displacement between the anchors and the pedestal cause movement in the damper's shaft and energy dissipation occurs. Detailed drawings of the viscous damper application are located in Appendix B. When uplift occurs, the base plate reacting on the bottom of the BeS washers compresses the washers and stores elastic energy. The stored elastic energy is used to plumb the system after the motion has seized. 


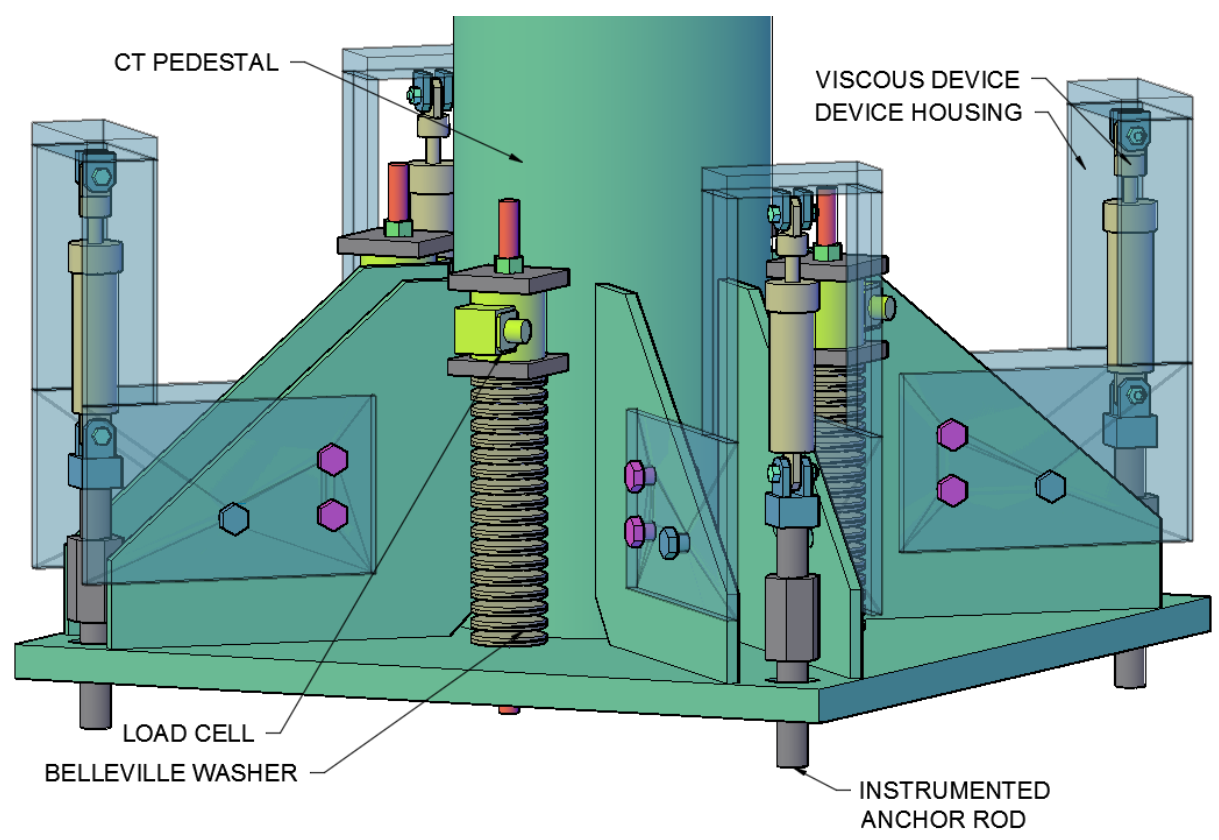

Figure 3-6: Base Retrofit w/ Taylor Viscous Dampers

Illustrated in Figure 3-7 is the proposed position and application of the second type of energy dissipating device, u-shape flexural plates. The proposed orientation shown in Figure 3-7 is composed of device sets at each corner of the pedestal.

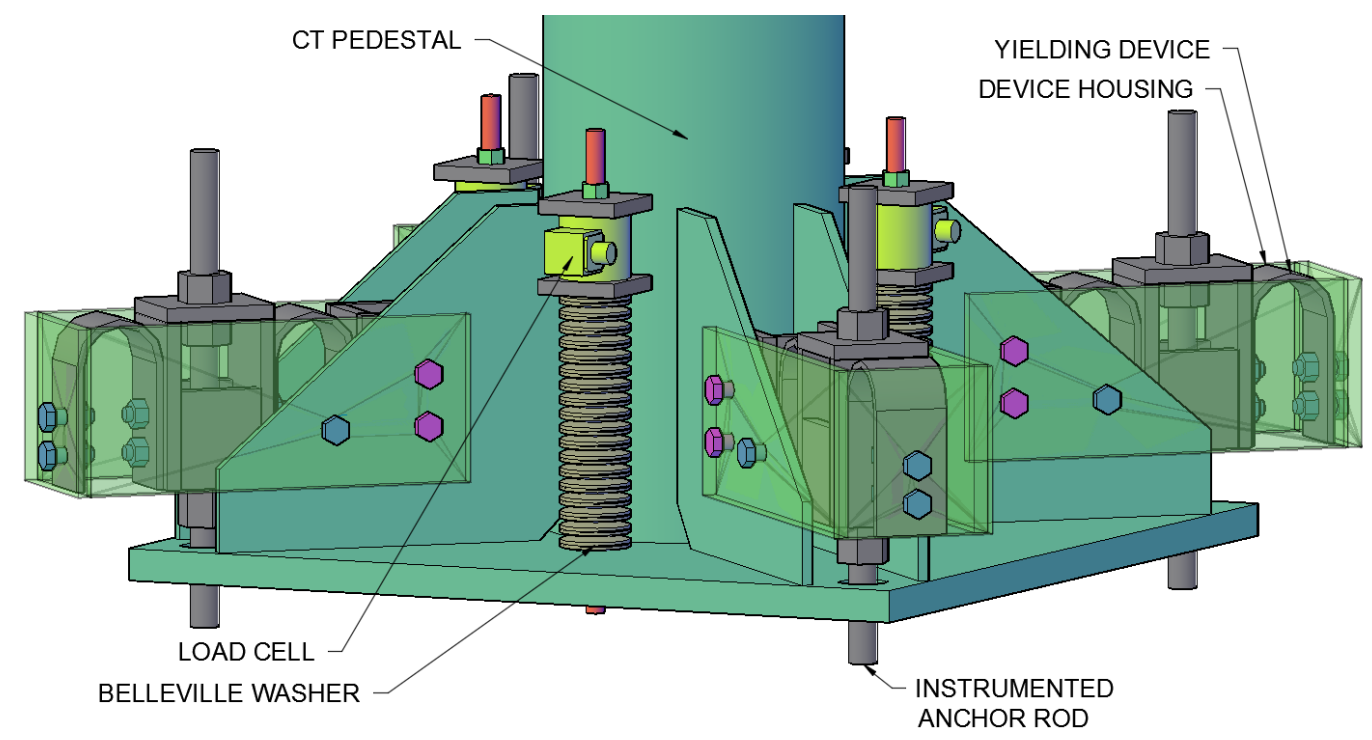

Figure 3-7: Hysteretic Device Base Retrofit 
A set is comprised of two u-shape flexural plates and is positioned so the reaction block utilizes the existing anchoring holes in the CT pedestal base plate. Shown in Figure 3-8 is a subassembly of a UFP set. The reaction block has a nut and washer on both the top and the bottom of the top plate. The top plate is welded to the sides of the reaction block; the UFPs are slip-critically bolted to the reaction block. The dual-nut arrangement allows for the u-shapes to be yielded in both directions of motion. Detailed drawings and dimensions of the retrofit are located in Appendix C.

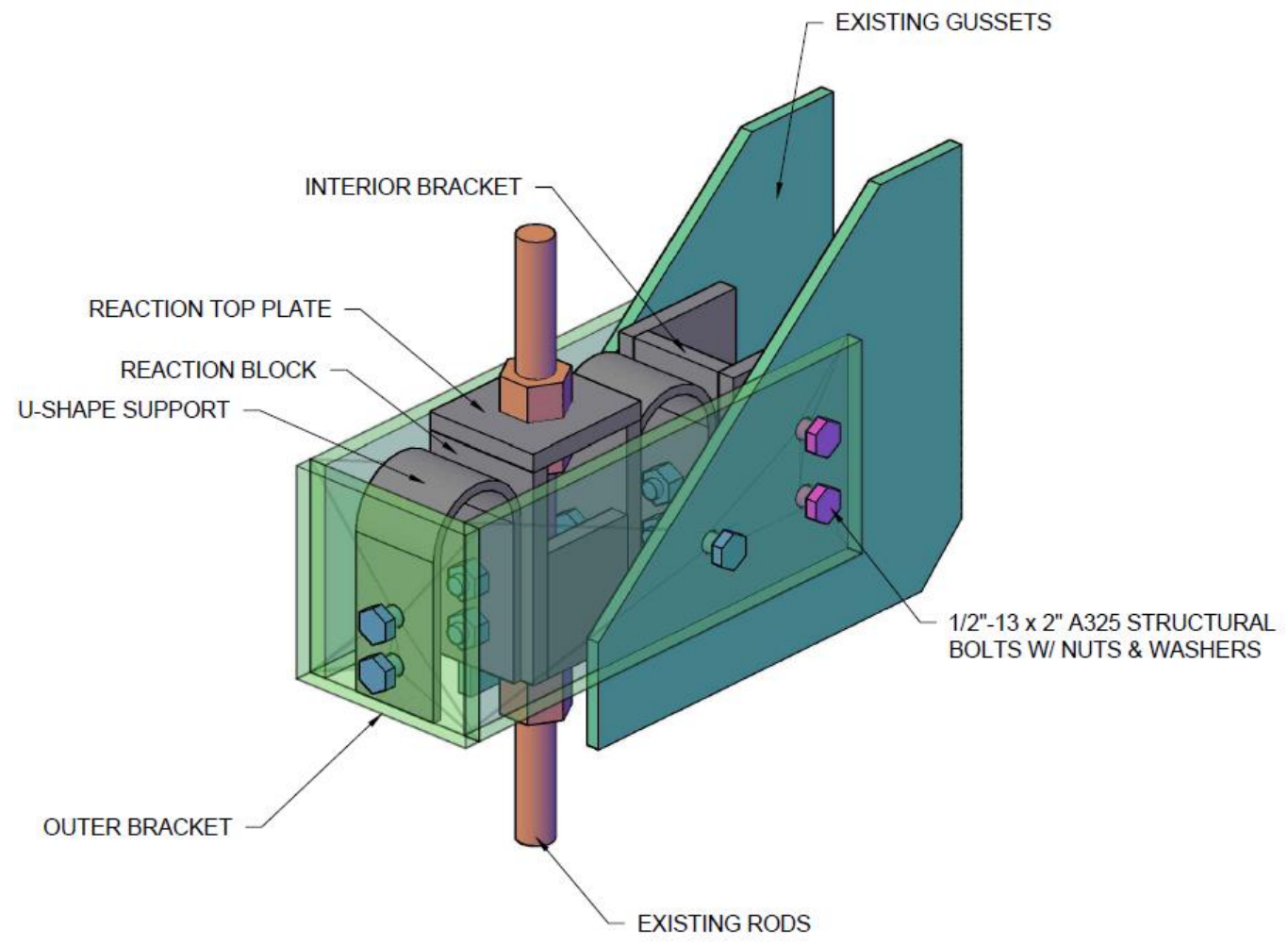

Figure 3-8: Typical UFP Assembly 


\subsection{U-SHAPE DESIGN}

\subsection{UFP Theory and Application}

Various researchers have studied energy dissipation through u-shape flexural plates where mechanical strains are the primary source of dissipated energy. The advantage of UFP elements is their ability to undergo large deformations while maintaining a similar capacity. Fabrication of UFPs requires little specialty skills and allow for effective energy dissipation at a low cost. The UFPs are positioned in a location where a stiffer member allows the radius to roll as the sides of the UFP move parallel to each other. The member forms two plastic hinges at the location of contact with the stiffer elements. The capacity of the damper could be calculated by relating the plastic moment to the shear force required to cause the entire section to yield. Typical parameters used to define the shape of the UFP are presented in Figure 4-1.

As defined in the figure:

$t_{u}=$ thickness of the steel plate

$D_{u}=$ diameter to the center of the plate

$r_{u}=$ radius to the center of the plate

$b_{u}=$ width of the plate 


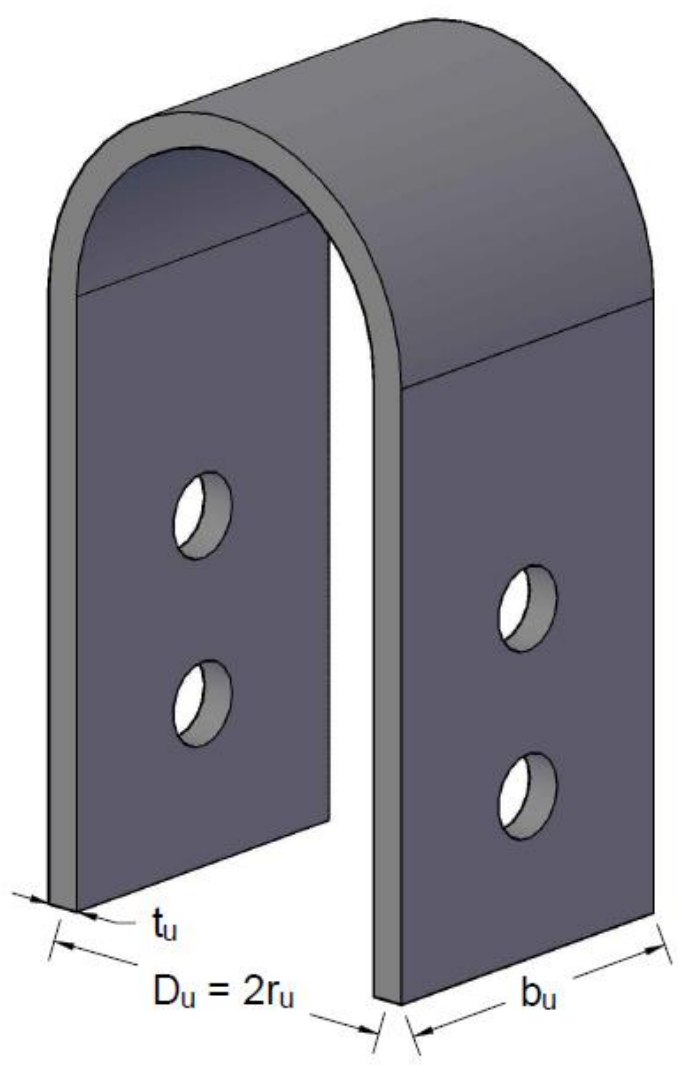

Figure 4-1: UFP Section Definition

The plastic moment, where the entire rectangular section has yielded can be defined by (4). The capacity of the UFP was analytically derived by researchers in the early 1970's by relating the shear couple to the plastic moment (Kelly, Skinner, \& Heine, 1972).

$$
M_{p}=\sigma_{y} Z_{U R P}=\frac{\sigma_{y} b_{u} t_{u}^{2}}{4}
$$

Illustrated in Figure 4-2 is the shear couple which must be equal to $2 M_{p}$ in order for rolling of the plate to occur. Using the previously derived plastic moment (4) and summing the moments we could relate the shear force to the plastic moment as presented in (5). 


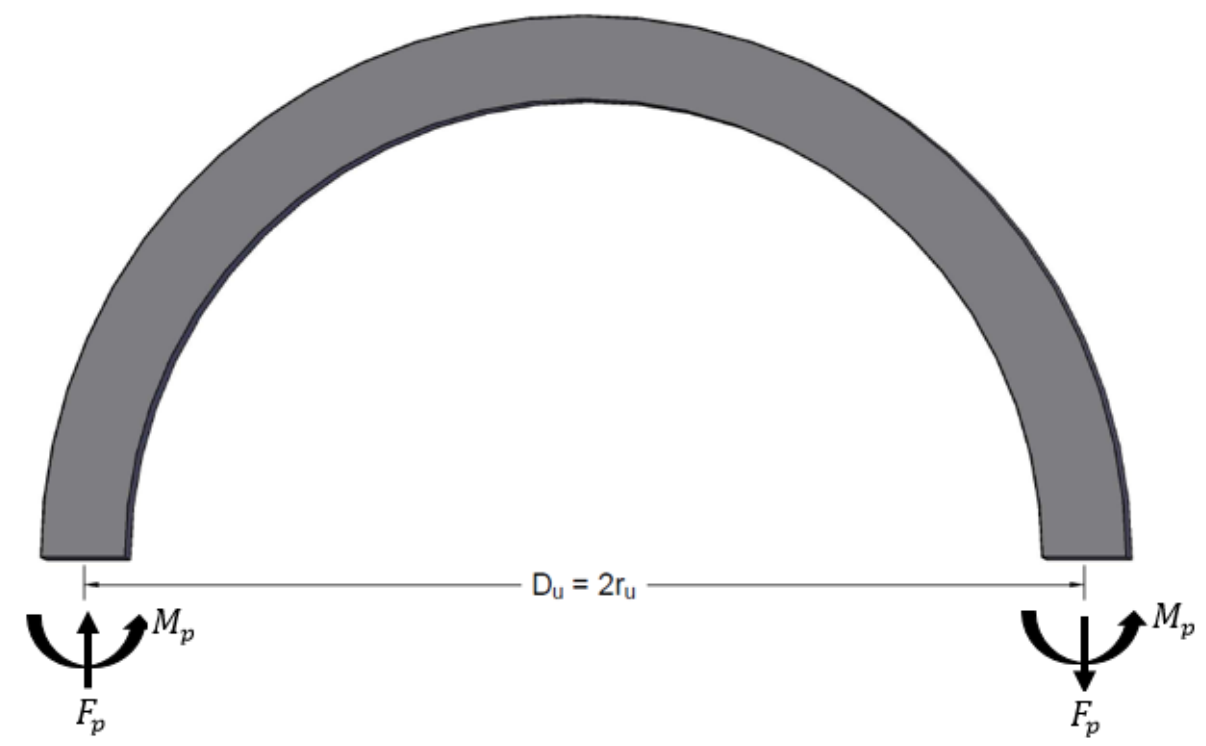

Figure 4-2: Shear Couple and Plastic Moment

$$
F_{p}=\frac{2}{D_{u}} \cdot M_{p}=\frac{2}{D_{u}} \cdot \frac{\sigma_{y} b_{u} t_{u}^{2}}{4}=\frac{\sigma_{y} b_{u} t_{u}^{2}}{2 D_{u}}
$$

The same approach could be utilized to determine the yield force and yield moment of the UFP. For a rectangular section, the elastic section modulus shown in (6), could replace the plastic section modulus to determine the yield force and yield moment. Following the same methodology as described above for plastic properties, (7) to (10) show the relationship between shear couple, yield moment, and yield force. For a rectangular UFP section, the yield moment and plastic moment are related by a $2 / 3$ factor. Shown in (10) is the derivation for the relationship between yield force and plastic force of the UFP.

$$
S_{U F P}=\frac{b_{u} t_{u}^{2}}{6}
$$




$$
M_{y}=\sigma_{y} S_{U R P}=\frac{\sigma_{y} b_{u} t_{u}^{2}}{6}
$$

$$
2 M_{y}=D_{u} F_{y}
$$

$$
F_{y}=\frac{2}{D_{u}} \cdot M_{y}=\frac{2}{D_{u}} \cdot \frac{\sigma_{y} b_{u} t_{u}^{2}}{6}=\frac{\sigma_{y} b_{u} t_{u}^{2}}{3 D_{u}}
$$

$$
\frac{F_{y}}{F_{p}}=\frac{\frac{\sigma_{y} b_{u} t_{u}^{2}}{3 D_{u}}}{\frac{\sigma_{y} b_{u} t_{u}^{2}}{2 D_{u}}}=\frac{2}{3}
$$

Recent research on UFP application have been conducted using u-shape devices for energy dissipation in Cross Laminated Timber (CLT) walls (Gu, Pang, \& Schiff, 2015) and precast concrete panel systems (Schultz, R., Tadros, \& Huo, 1994). Similar approaches utilizing URPs are to be extended in the research outline. The benefits of rocking, along with supplemental damping with UFPs, are to be explored for the vulnerable current transformer. 


\subsection{GeNeral NuMERICAL MODEL}

\subsection{Pedestal Modeling}

The actual Trench current transformer, model: 226-293 used as basis for the work performed has a fundamental frequency of $3.78 \mathrm{~Hz}$. A shell model of the pedestal was constructed in SAP2000 to determine the flexibility of the support structure, Figure 5-1. The shell model incorporated the base and stiffeners of the support structure. Joints were shared between the stiffeners and the mounting plates to account for the stiffness increase due to the stiffeners.

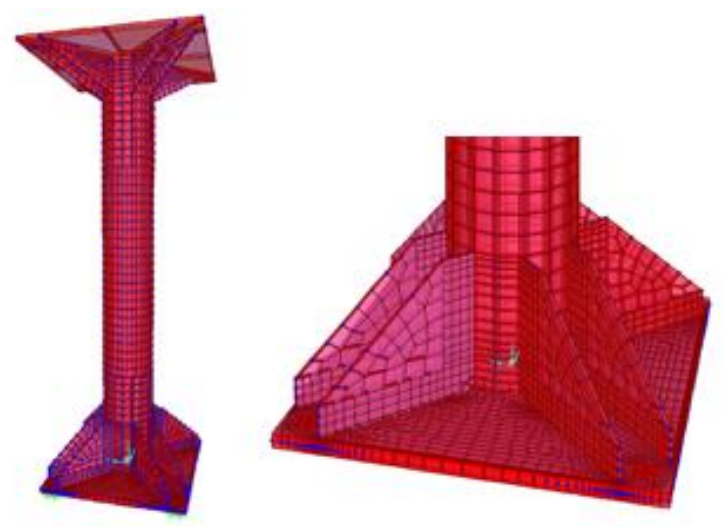

Figure 5-1: Pedestal SAP2000 Shell Model

In order to reduce the runtime of a non-linear direct integration model, an equivalent stick model was constructed. Stiffeners were modeled by rigidity end length offsets. A rigid zone factor of 1 was used along with the appropriate stiffener length to account for the significant increase in stiffness at the stiffener locations. To properly model the stiffener's contribution to the base plate stiffness, the stick model contained $\mathrm{x}$ beams at the top and bottom of the pedestal which were calibrated by the shell model. After similar stiffness was achieved by the two models, additional masses were added to the top and bottom of the stick model to account for the plate weight. Shown in Figure 
5-2 is the representative stick model of the CT pedestal. After combining the $3.78 \mathrm{~Hz} \mathrm{CT}$ with the $20.37 \mathrm{~Hz}$ pedestal, the system fundamental frequency was $1.22 \mathrm{~Hz}$.

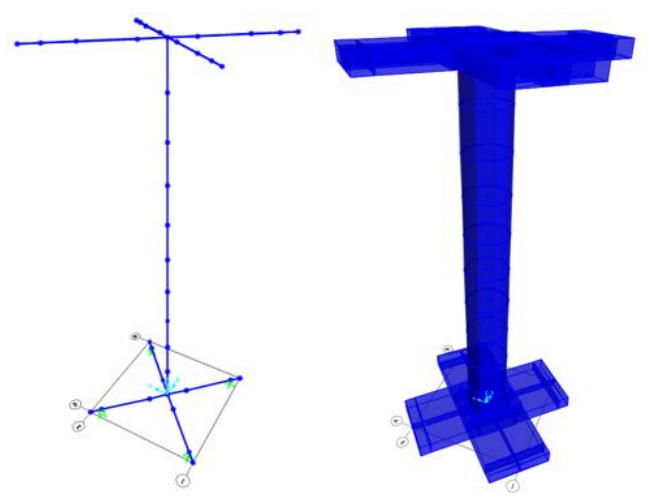

Figure 5-2: Stick Model

\subsection{Upper CT Mass}

Scaled system CT mass was constructed in SAP2000 using beam elements along with lumped masses. A single lumped mass concentrated the mass plates at the proper location. Since the initial phases of the research focused on a SDOF system, no mass distribution was required to properly size the dampers and UFP. The retrofit devices were only sized for the full-scale system due to the limited project funds. Shown in Figure 5-3 is the SAP2000 model used to estimate the system response prior to testing. The scaled system was designed with a $5000 \mathrm{lbs}$. lumped mass at $12 \mathrm{ft}$. from the structures base. 


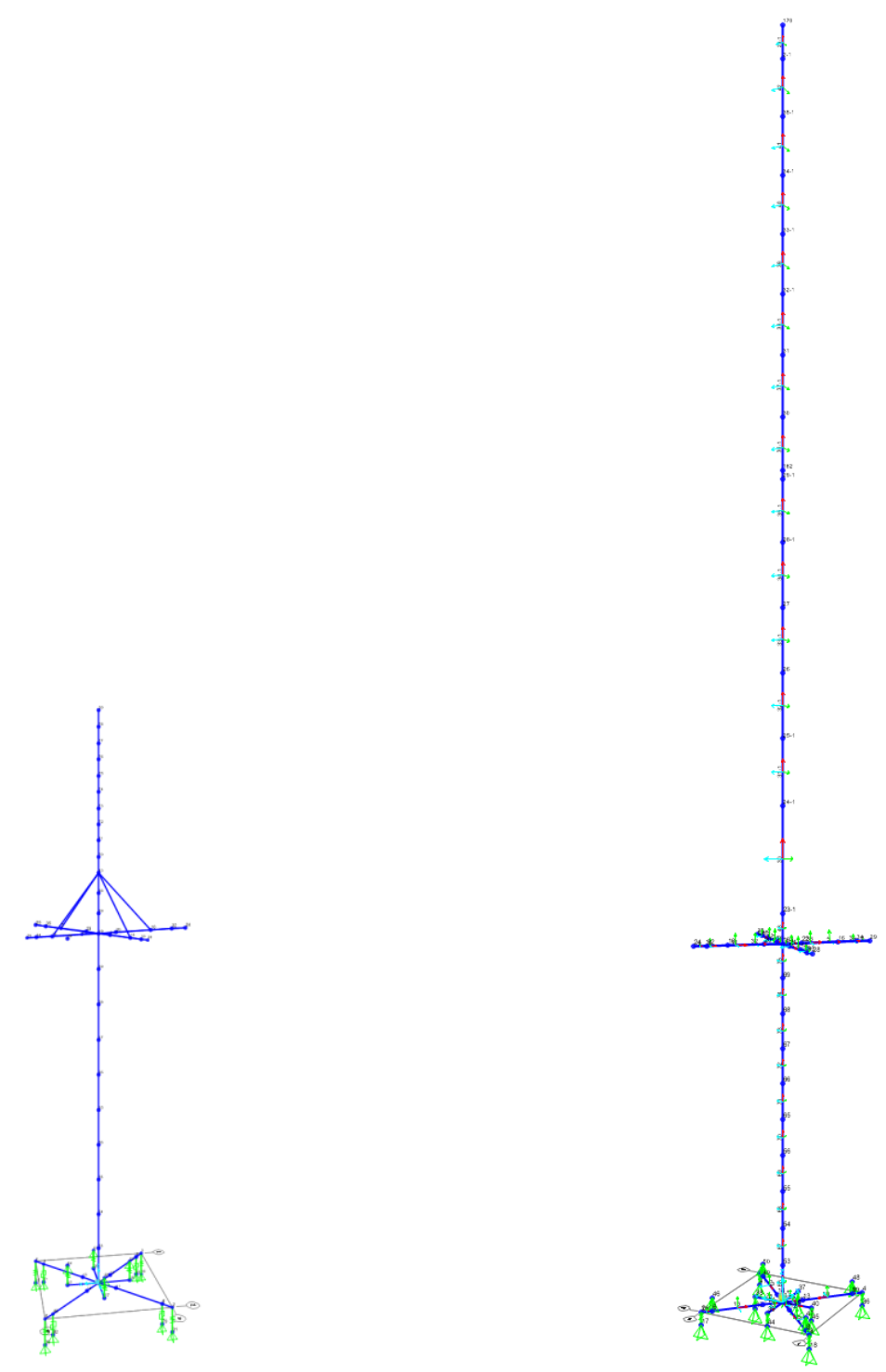

Figure 5-3: Scaled Complete System Model

Figure 5-4: Full-scale Complete System Model

The full-scale system considered distributed masses corresponding to the seismic qualification of the 226 Trench current transformer and the designed mass system. Discussed in section 10.0, Full-Scale Mass System Design, is the procedure used to design the mass which was applied to the SAP2000 model. The model was iterated until the proper mass distribution was achieved. Shown in Figure 5-4 is the model constructed in SAP2000 to represent the full-scale system. 


\subsection{Foundation and Friction Modeling}

With a rocking system, base sliding has to be controlled or eliminated in order to avoid global instability. In addition, z-displacement only could occur in one direction when the system undergoes uplift. SAP2000 does not have the capabilities to model friction between surfaces or components. Hand calculations were performed to verify that rocking or tipping would occur prior to base sliding. Next, in order to have a proper response in SAP2000 not allowing sliding at the base, u1, u2, and r3 degrees of freedom were restrained at the base center. Shown in Figure 5-5 is the node definition to eliminate global instability.

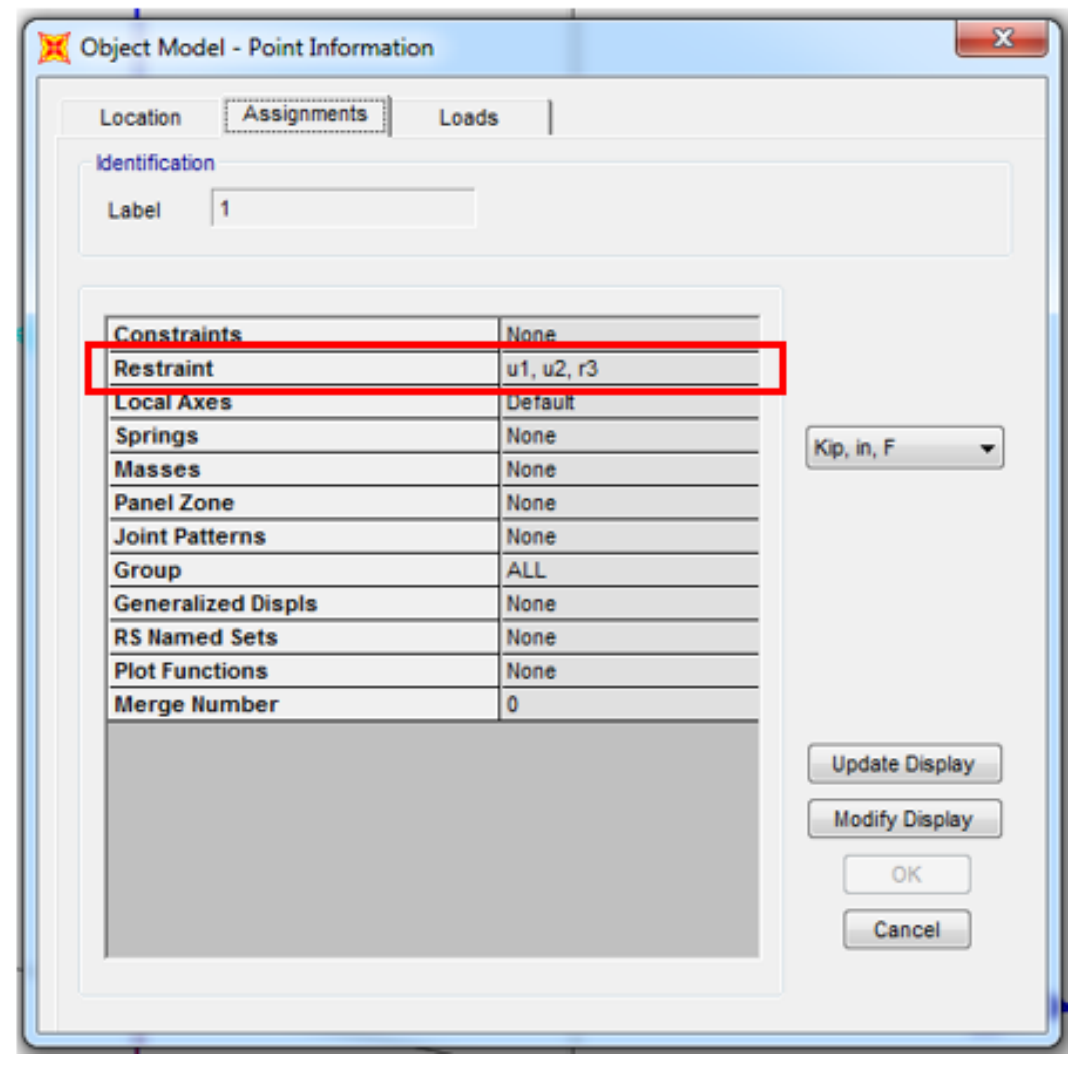

Figure 5-5: Restraints to Avoid Global Instability in SAP2000

To model the base support plate/foundation interface of the structure, non-linear gap elements were defined and implemented. Gap links are compression-only acting 
springs. The gap elements were defined to act in the ul direction along the link element and had a high stiffness in order to avoid any downward displacement along the edges of the structure. Generally, a minimum of 10 times the stiffness of the stiffest element is recommended to define a gap element. Shown in Figure 5-6 are the properties used to define the gap elements and Figure 5-7 shows the location of the gap elements on the model.

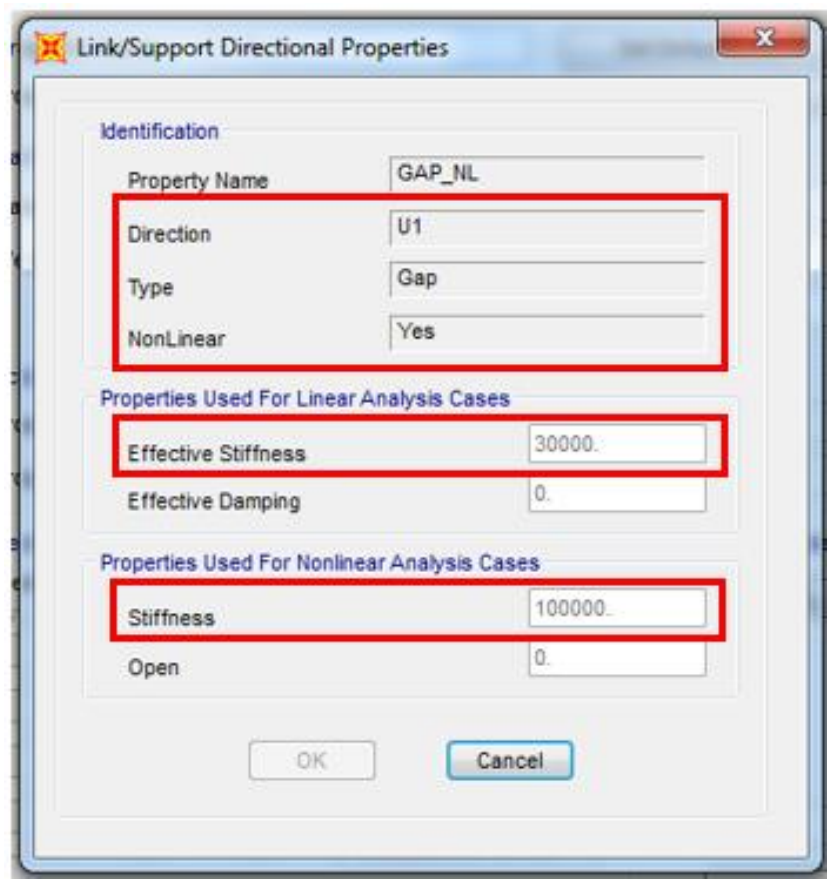

Figure 5-6: Gap Link Properties

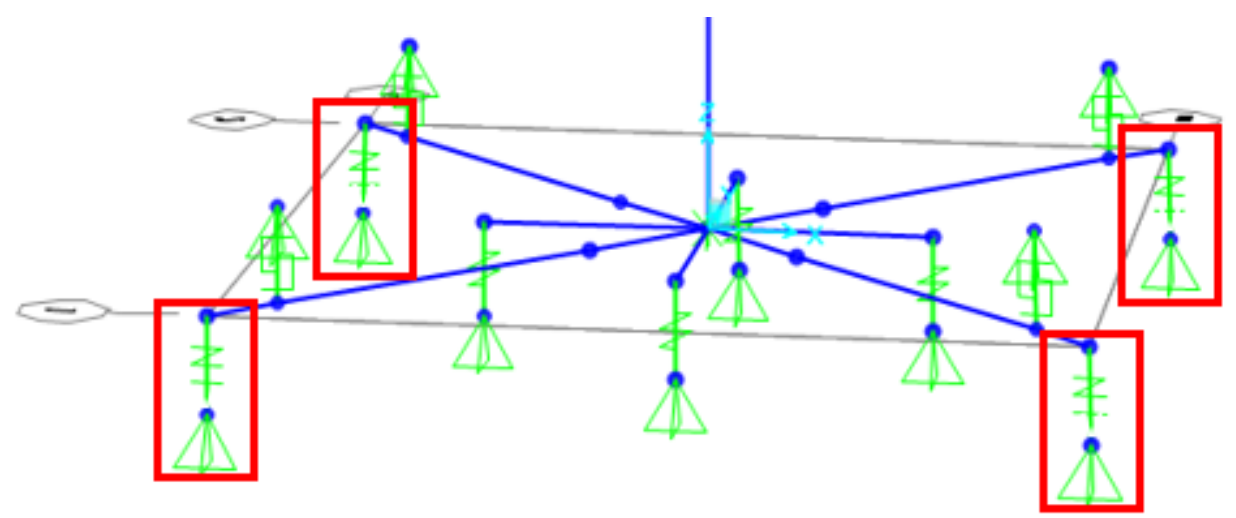

Figure 5-7: Gap Link Locations 


\subsection{Pre-Tension Model Application}

Belleville springs, used for pre-tensioning the pedestal base, were modeled using linear link elements. Properties for the linear link were defined in the u1 direction along the length of the link. Using the determined linear load and linear displacement based on the specified type/number of BeS washers, the stiffness of the linear PT could be determined and defined in the model. The type/number defines the configuration, capacity, and stiffness. Based on the full-scale system, a linear stiffness for the PT was determined to be 7.395kips/in. Shown in Figure 5-8 are the properties specified for the PT elastic elements. Discussed in 11.1.1, Elastic PT Member Design, are the assumptions made in determining the required BeS configuration and stiffness.

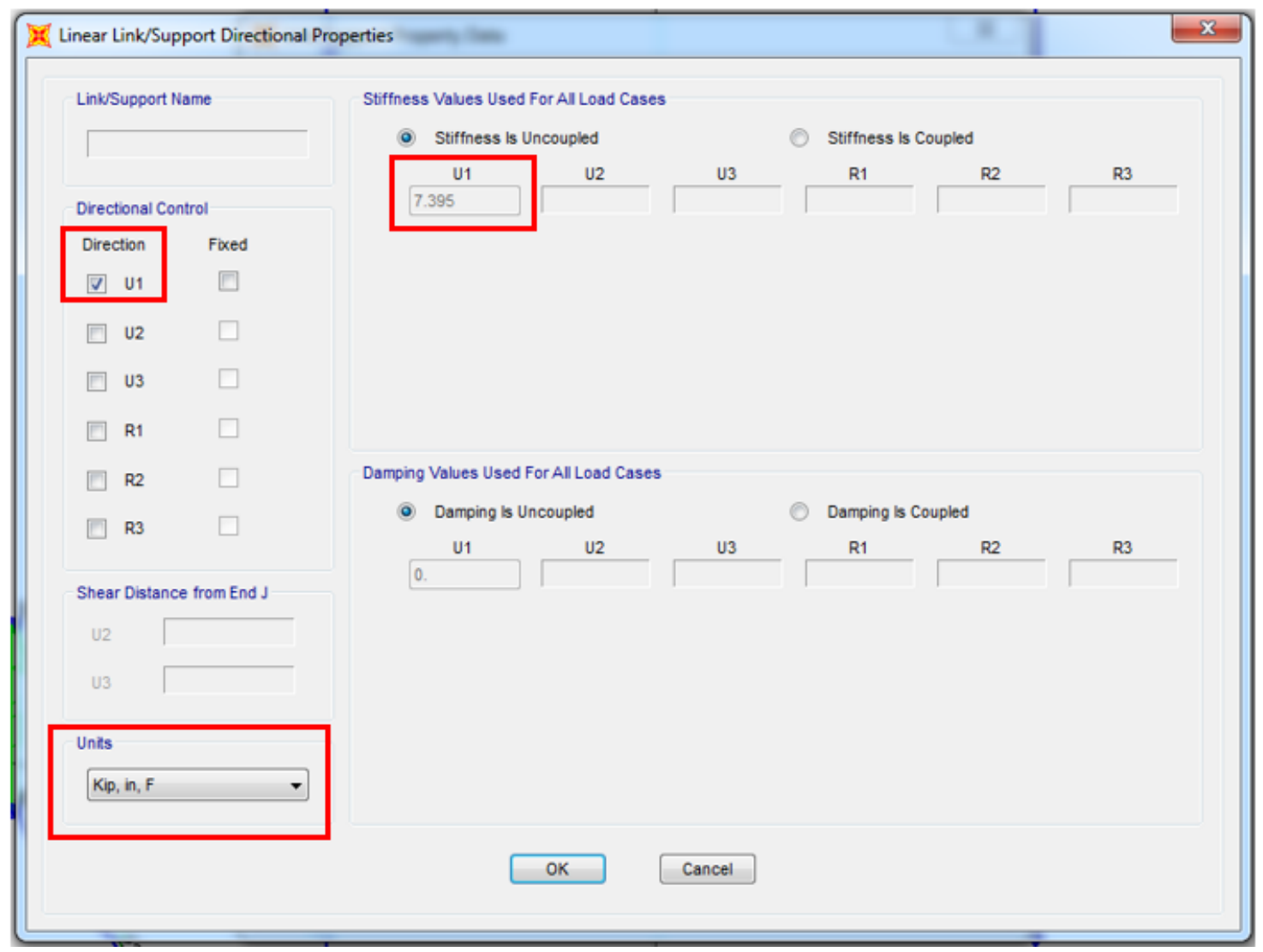

Figure 5-8: Elastic PT Modeling Properties 
PT was positioned near the center of the base plate to maximize the contribution of each BeS stack, limit the required stroke, and minimize post-rocking stiffness. When rocking occurs about an edge or corner, the BeS stack located furthest from the rocking point will experience the largest displacement. PT members were placed at a 8 " radius from the base center point at each quadrant. The center node of the pedestal base plate was utilized to connect rigid links from the PT location to the base center as shown in Figure 5-9. Rigid links were utilized because negligible base plate deformation was expected. The pre-tension application point lies between the base plate stiffeners and the HSS pedestal, increasing the flexural stiffness of the base plate significantly.

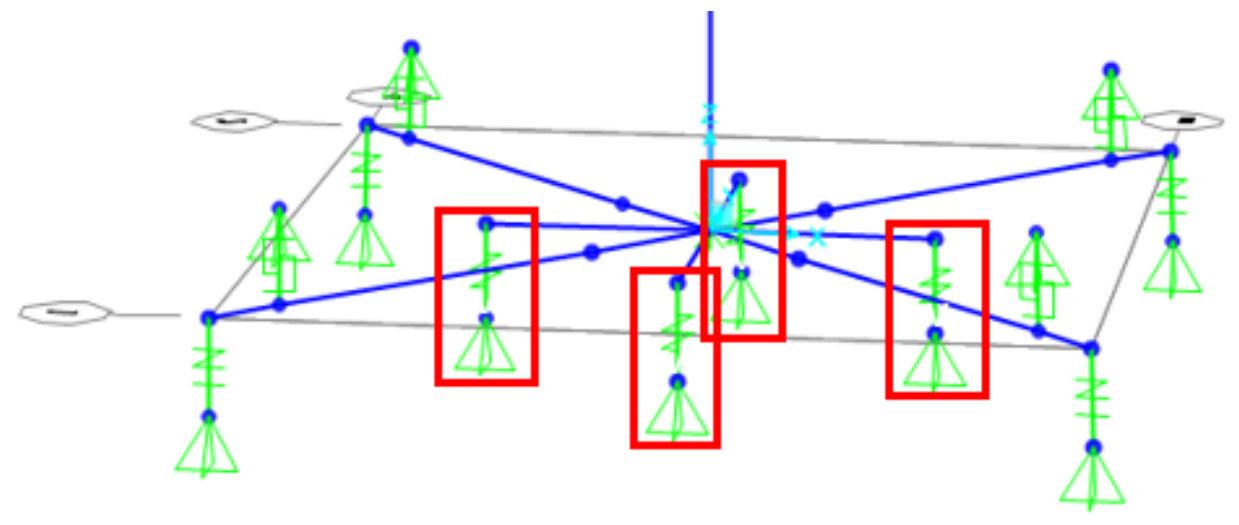

Figure 5-9: PT Location on SAP2000 Model

The model defines the BeS system without bounds or limits which must be considered post analysis. The maximum BeS linear displacement must not be exceeded in order to obtain representative results. Linear link elements have an infinite stroke and improper use of the model could lead to significant error in system response. 


\subsection{Hysteretic Device Modeling}

Hysteretic UFP sets were modeled in SAP2000 using non-linear Wen-links. The location of the UFPs are shown in Figure 5-10, the same locations were used for the viscous model retrofit.

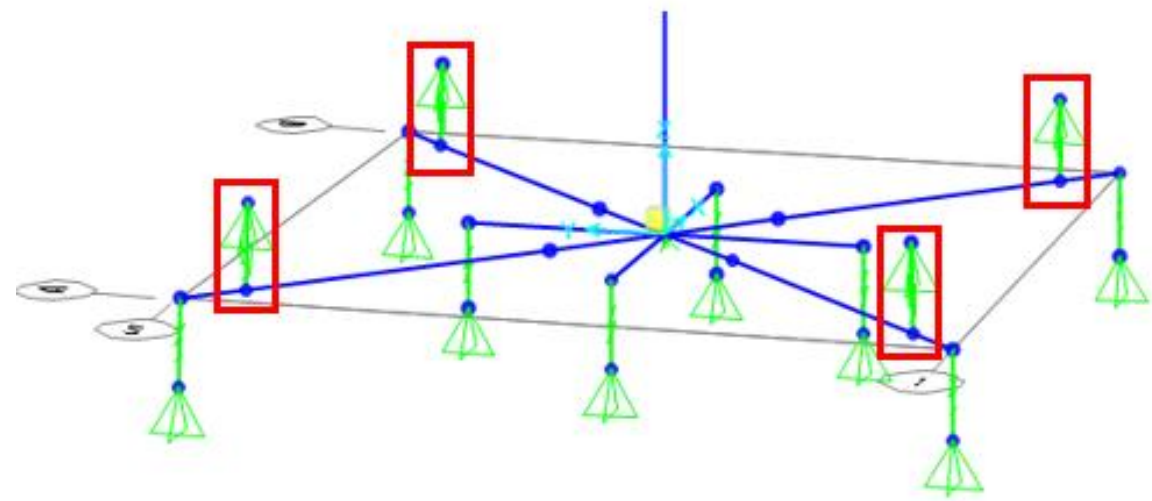

Figure 5-10: Device Locations on SAP2000 Model

The Wen-link defines uniaxial plasticity through 6 core properties. Wen-link definition includes effective stiffness, effective damping, stiffness, yield strength, post yield stiffness ratio, and yielding exponent. Shown in Figure 5-11 and Figure 5-13 are the required property specifications. 


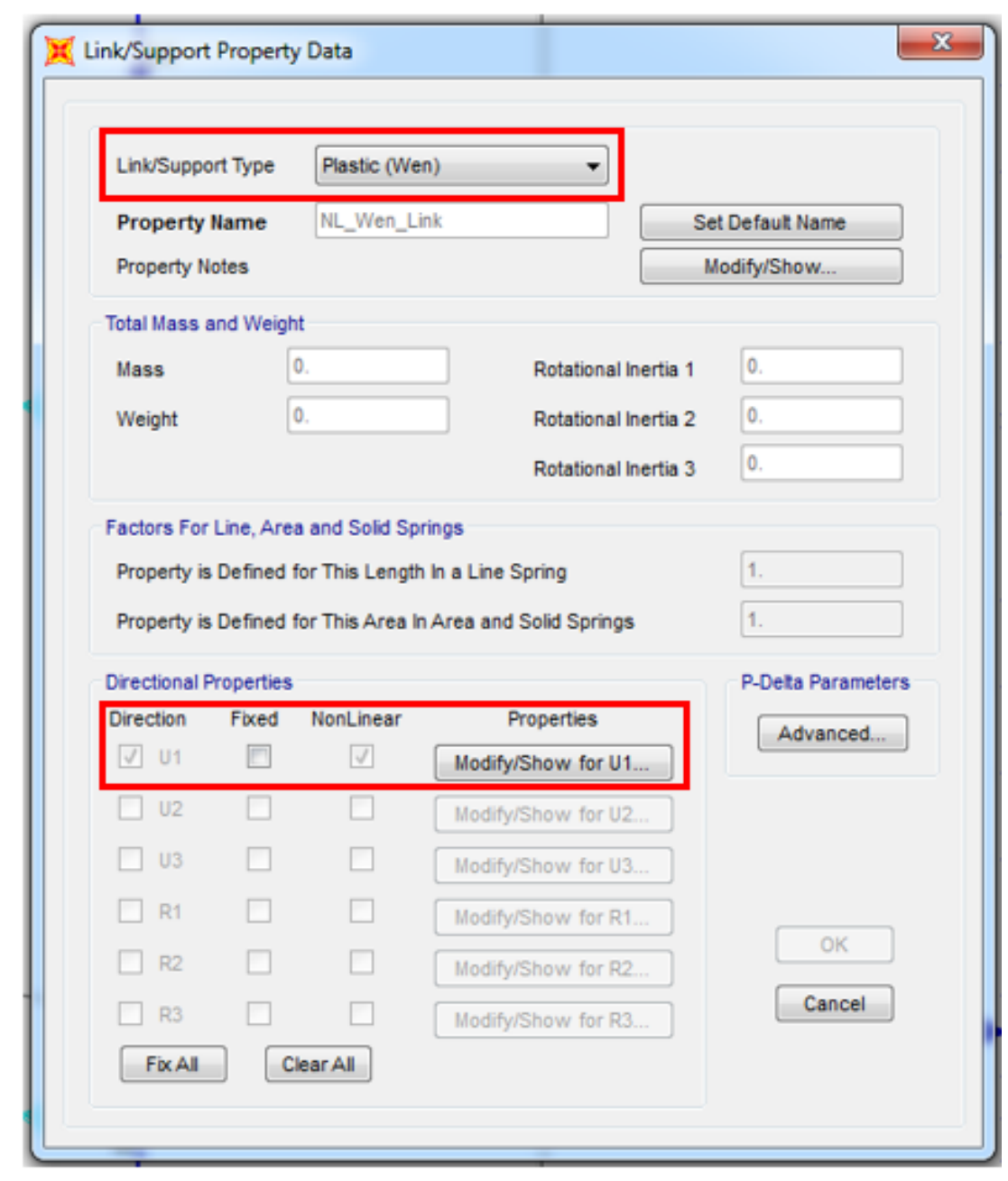

Figure 5-11: Wen-link Property Data

Directional properties for the retrofit were defined for each UFP set. A UFP set was comprised of two UFP members at each quadrant. The UFP members were positioned in parallel with each other and their stiffness and strength properties were cumulative. Based on the geometry of the UFP, the initial stiffness and yield strength could be determined using the methods discussed in section 4.0, U-Shape Design. The Wen-link definition required amplified yield strength to properly model the UFP of interest. Obtained from (CSI, 2016) is the CSI definition of the Wen-link shown in Figure 5-12. Yield strength is defined as the slope transition point " $y$ " and the yield exponent "e" 
dictates how rapid the transition occurs. Listed in section 4.0, U-Shape Design, is the equation to determine the yield force of a single UFP. The yield force is defined as the initial yield or the transition point from linear to non-linear in section 4.0. Since the CSI and theoretical definitions of the yield force are inconsistently defined, the true yield force must be amplified to properly define the Wen-link.

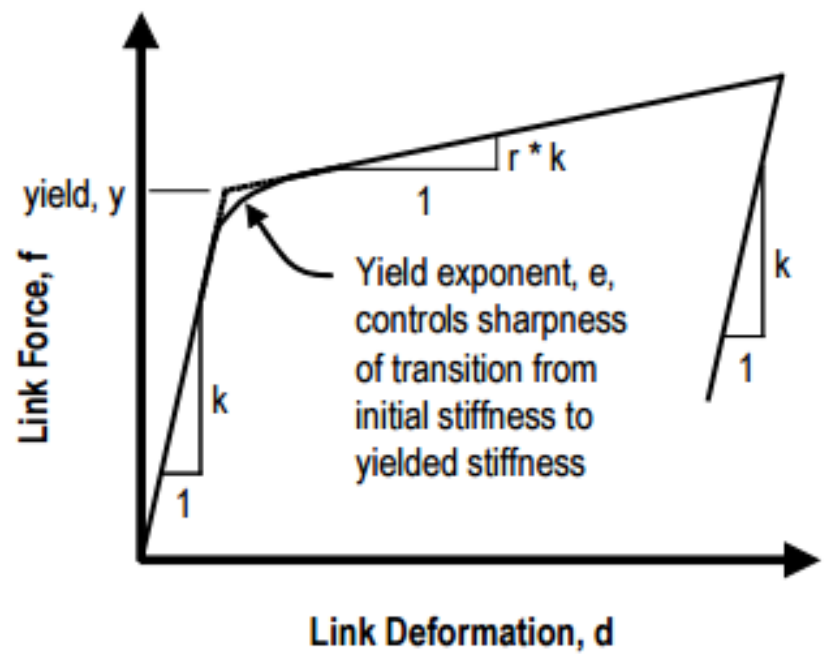

Figure 5-12: CSI Example 6-008 Wen-link Definition

Initially, iterations on the yielding exponent were conducted to match component level test behavior to the component model output. After the shape of the transition was properly defined, iterations of the yield strength were conducted to match the ultimate strength at the required displacement. The post-yield stiffness ratio was determined from scaled component level testing and used for both system models. Also, Wen-links have no fatigue properties, stroke limitations, or maximum force definitions so the final output of the model must fall within the design limitations of the hysteretic device. 


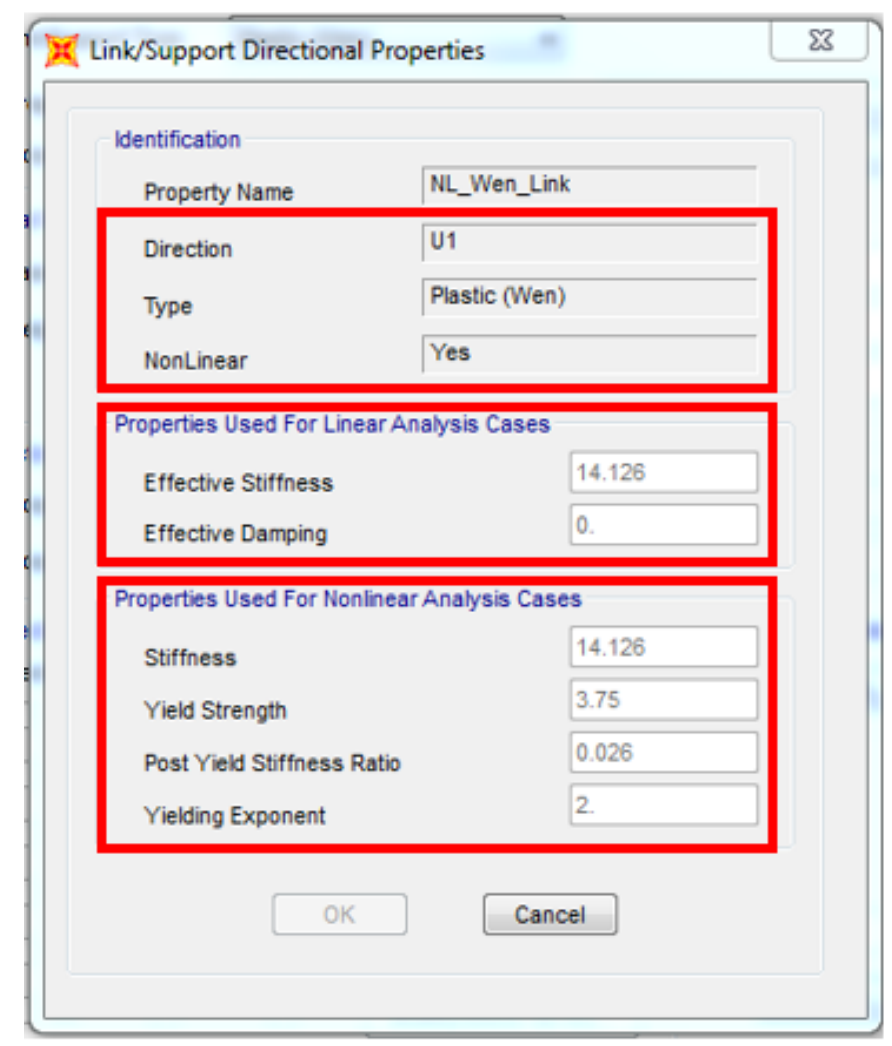

Figure 5-13: Wen-link Directional Properties

Component level testing was completed utilizing FEMA461 and IEEE693 seismic protective device protocol. The component level test results were used to calibrate the Wen-link as previously described. The backbone constructed from the component hysteresis results was used to define the Wen-link. Shown in Figure 5-14 are the component level test results, backbone curve, and SAP2000 Wen-link response for the PSU system UFP set. 


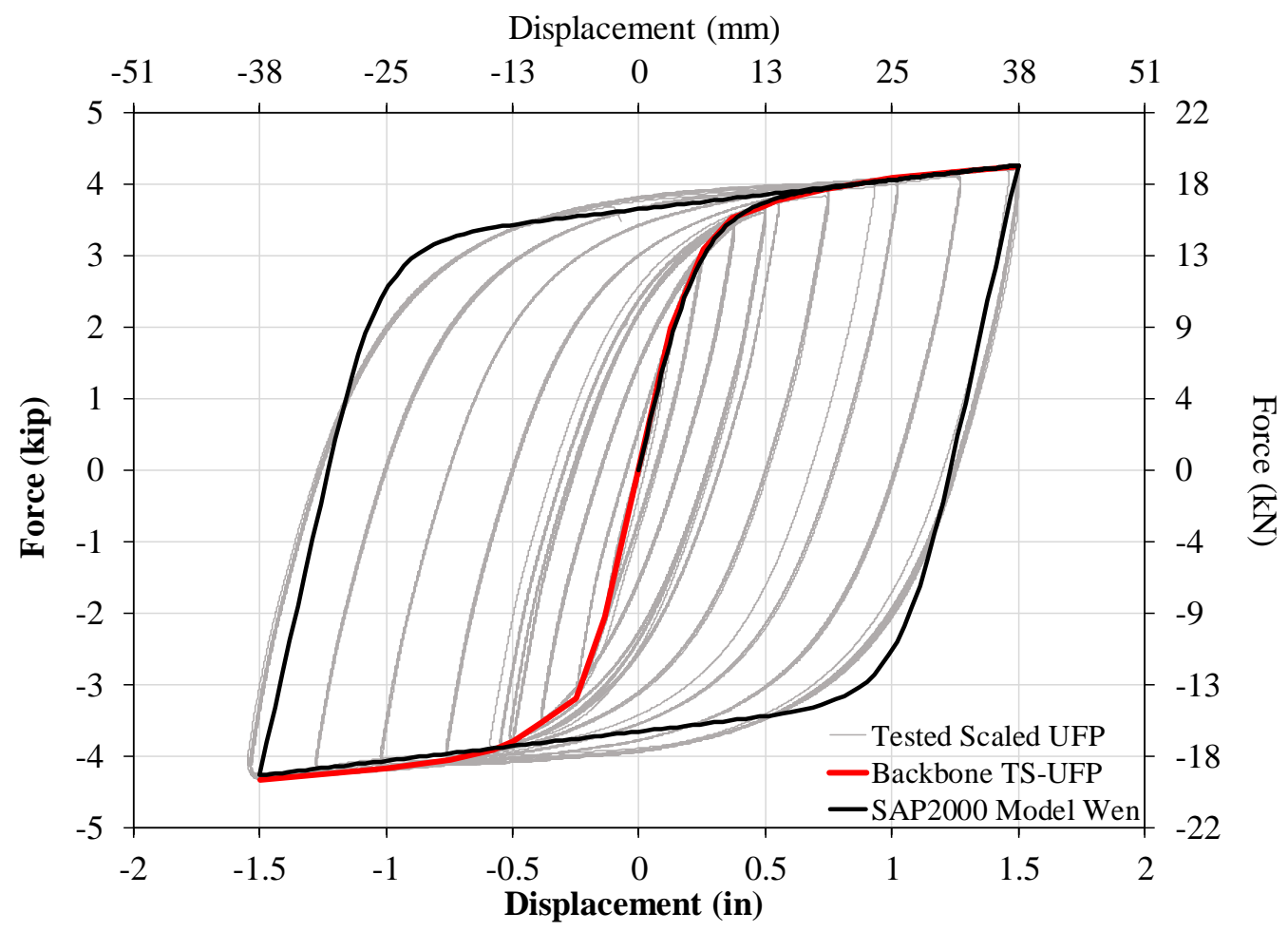

Figure 5-14: Wen Model Calibration for PSU System

\subsection{Viscous Damper Modeling}

Viscous dampers are modeled in SAP2000 using damper-exponential non-linear links. Depending on the orientation of the link element, the directional properties could be specified. A sample property assignment of the damper-exponential link is shown in Figure 5-15 and Figure 5-16. The designer typically specifies the exponent and force constant and the device stiffness is obtained from the manufacturer. Only one damper type was utilized in the work performed and was designed based on the full-scale system. Discussed in 11.2, Viscous Damper Design, are the methods for determining the specified damper design for the retrofit. 


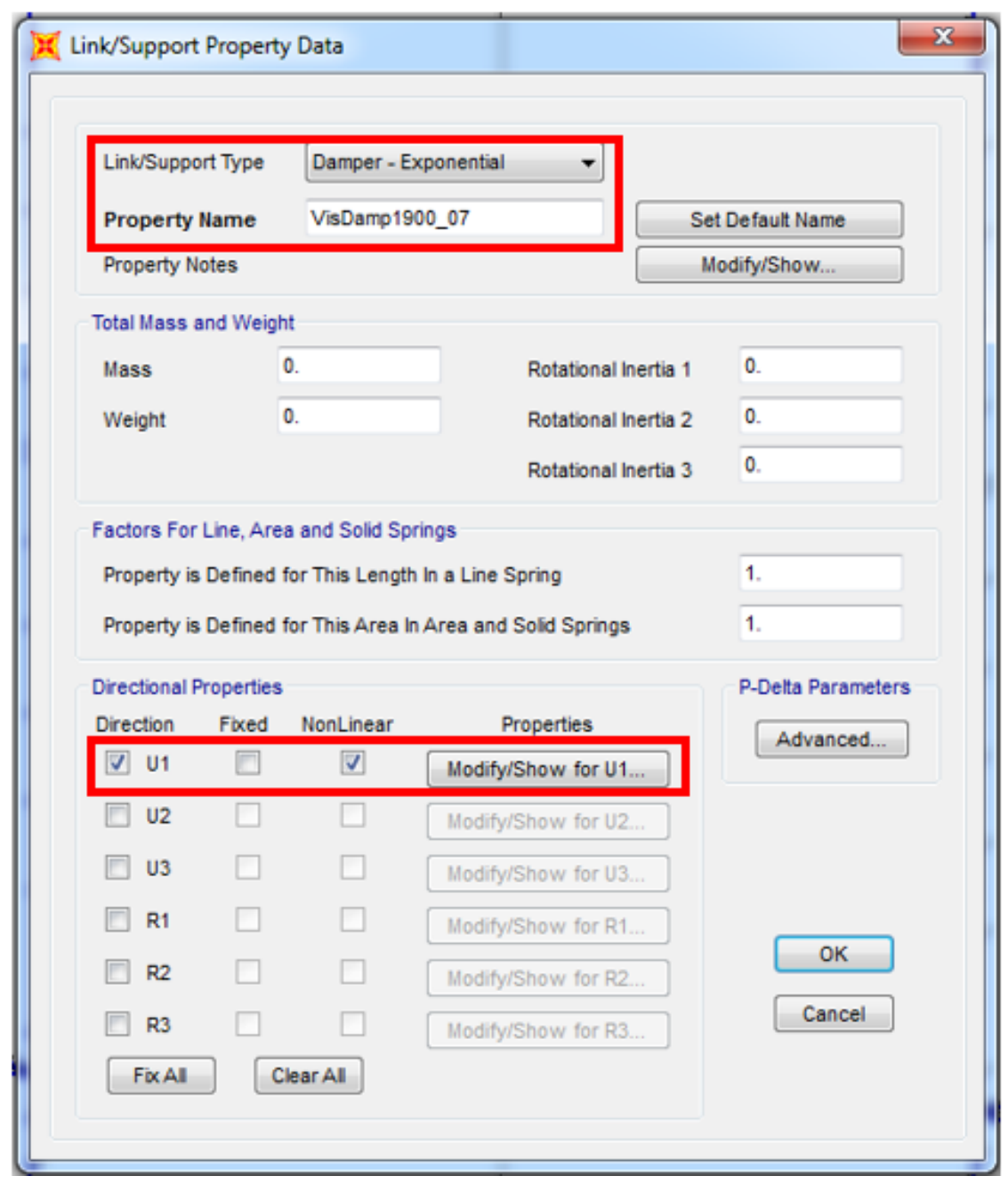

Figure 5-15: Damper- Exponential Link Properties 


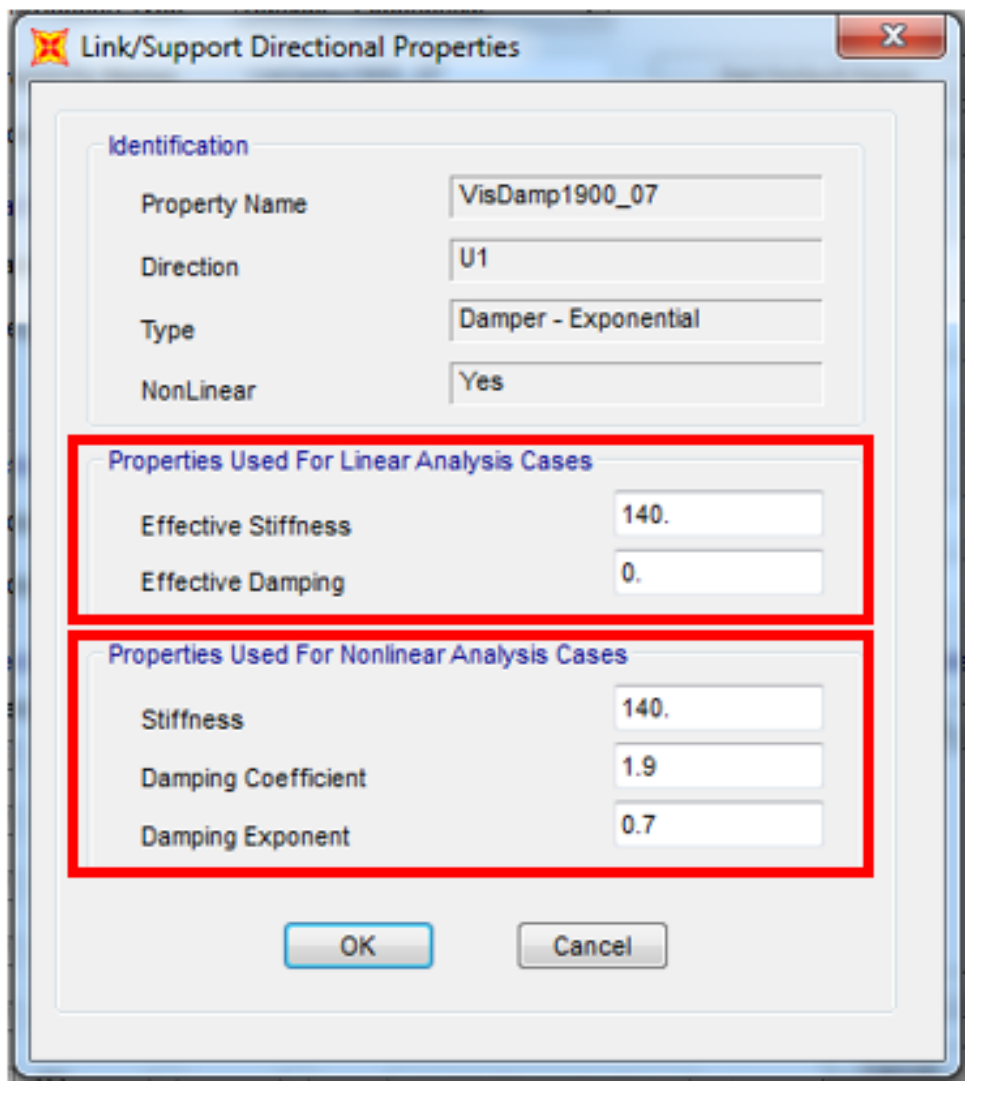

Figure 5-16: D-E Link Directional Properties 


\subsection{Parametric Study on Hysteretic Retrofit Parameters}

Parametric studies were performed on an early design of the rocking system. The trends obtained show the influence of the initial pre-tensioning force and the rocking stiffness. Although the stiffness of the actual CT is not represented in this parametric study, the trends and influence of important factors are demonstrated.

A model was constructed in SAP2000 using the scaled system geometry to explore how each component of the retrofit influences the behavior of the system. The parametric studies investigated pre-tension force and rocking stiffness independently to supplement full-scale system design. When investigating each parameter, the parameter of interest was varied throughout a range while all other system properties were held constant.

\subsection{Pre-Tension Force}

Pre-tension force controls when rocking initiates and contributes significantly to the systems equivalent stiffness. As the pre-tension force is increased, the system experiences less uplift. If the pre-tension force produces a moment larger than the moment due to lateral seismic loading, the system will be non-rocking and effectively will have a bolted base stiffness. Figure 6-1 shows the influence of varying the PT force from 1kip-7 kips on a rocking system with hysteretic devices. Generally, the pre-tension force had the largest influence on system displacement. The system experiences similar magnitude base shear values for all the PT force values investigated. For a case where the base does not experience rocking, the base shear will be significantly higher following the initial stiffness throughout the entire duration of the displacement. When uplift occurs, device displacement and yielding occurs. When PT force is lower, lesser load is required to cause uplift and rocking. 


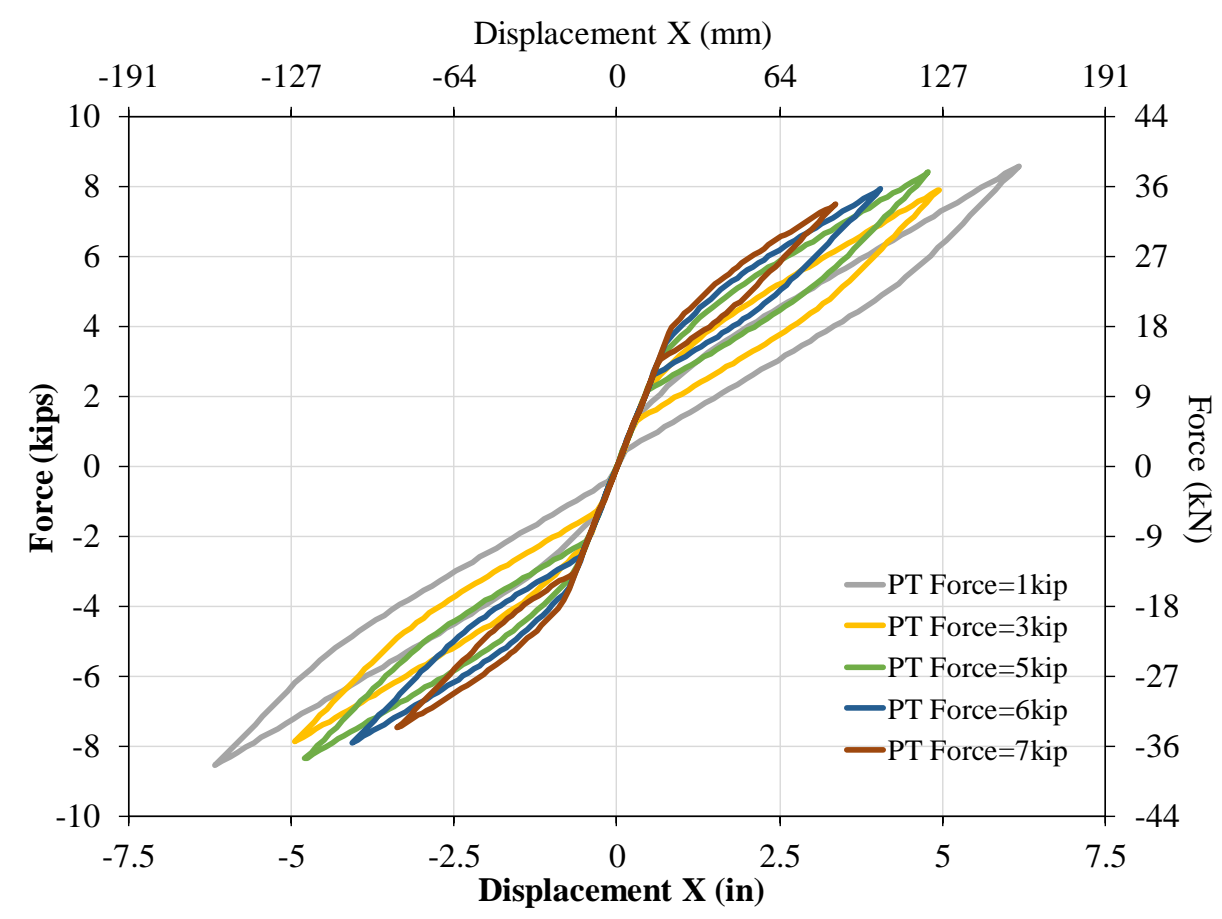

Figure 6-1: PT Force Influence on System Behavior

\subsection{Rocking Stiffness}

Next, the influence of rocking stiffness was investigated. Rocking stiffness could be varied multiple ways: change in elastic PT stiffness, change in PT location, and change in base size. For the parametric study, the base size or rocking location was altered in order to see how rocking stiffness influences system behavior. Reducing rocking stiffness caused the system to have larger displacements. The base shear trends were not clear and no conclusions could be obtained. For the full-scale system, rocking stiffness is expected to have a greater impact on the systems base shear. The spectral acceleration would shift off the response spectrum plateau, due to the lower fundamental frequency, and demands would decrease significantly. Shown in Figure 6-2 are the results obtained from SAP2000 for the base size variation parametric study. The results shown are push-over results, using the maximum displacement obtained from a time history analysis of each case. The 
push-over results allow for clearer representation of trends while capturing maximum displacements from the time history analysis.

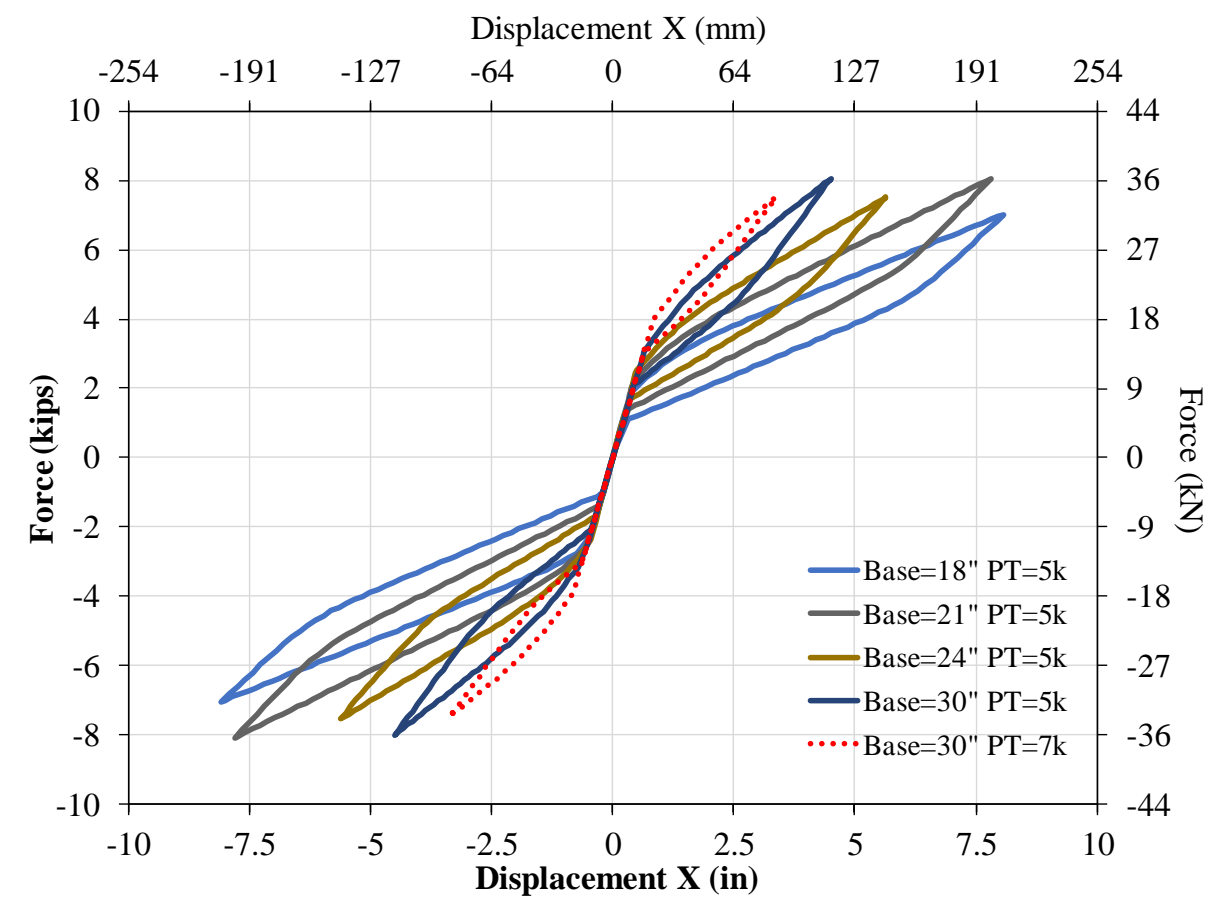

Figure 6-2: Rocking Stiffness Influence on System Behavior 


\subsection{PROOF OF CONCEPT}

After completing numerical analysis and testing on individual components of the retrofit system, a set of proof of concept tests were conducted to validate the retrofit method. A scaled model system was designed and tested on a single-directional shake table at the iStar Laboratory located at the Portland State University campus. Laboratory height limitations allowed a maximum CT mass system height of 12 feet.

Due to the height limitation and flexibility of the system, a system with a similar property to the actual CT could not be achieved. To achieve a similar fundamental frequency, the required section would have insufficient strength. Using the maximum allowable lab clearance and a reduced mass of $5000 \mathrm{lbs}$., an upper support was designed based on strength. The system tested had a fundamental frequency of $2.93 \mathrm{~Hz}$ and a concentrated $5000 \mathrm{lb}$. mass located 12 feet above the CT base. The designed system located on the shake table is shown in Figure 7-1. A complete set of fabrication drawings for the scaled upper mass is located in Appendix D.

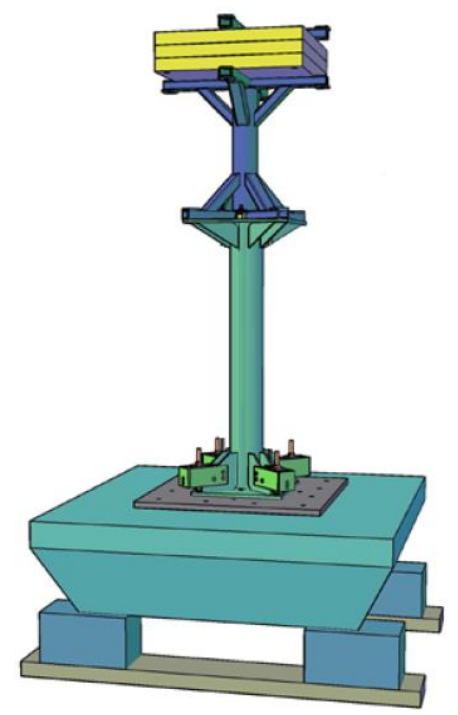

Figure 7-1: Scaled Mass System 


\subsection{Proof OF CONCePT TeST-SETUP}

Do to the costs associated with testing components on a 6-DOF shake table, and procuring energy dissipation devices, a scaled mass was tested to ensure proper system performance prior to performing full-scale system research. The scaled system utilized PT and viscous dampers from the full-scale system design which were not tailored to the specific dynamic properties of the scaled system. Two key differences between the scaled system and the full-scaled system were the fundamental frequency and mass distribution. As previously mentioned, the benefits of the retrofit were expected to be significantly greater in the full-scale system based on the definition of the IEEE693 design spectrum and system frequency.

Design of the PT force and UFP include the same procedures outlined for the fullscale system in 11.1.2, PT Load Design, and 11.1.3, Hysteretic Device Design, respectively. The final PT load was determined to be 2.35 kips for each BeS stack. The final UFP had the following properties: $F y=0.96 \mathrm{kips}, F p=1.43 \mathrm{kips}, F u=1.86 \mathrm{kips}$, and an initial stiffness of $k=7.91 \mathrm{kips} / \mathrm{in}$. Detailed drawings for the u-shape geometry used for testing with the scaled mass are shown in Appendix D. Since the system was a SDOF system, the key metrics for comparison were acceleration at C.G. and displacement at C.G.. Other instrumentation capturing device displacement, base uplift, and strains at the pedestal were implemented during tests, but have little meaningful value when comparing to the full-scale system. The proof of concept aimed to show reduced demand on the structure while maintaining reasonable displacements. Also, the proof of concept was implemented to identify potential problems with instrumentation application and retrofit 
tolerances. Presented in Figure 8-1 is the test setup with hysteretic devices and a safety catch system.

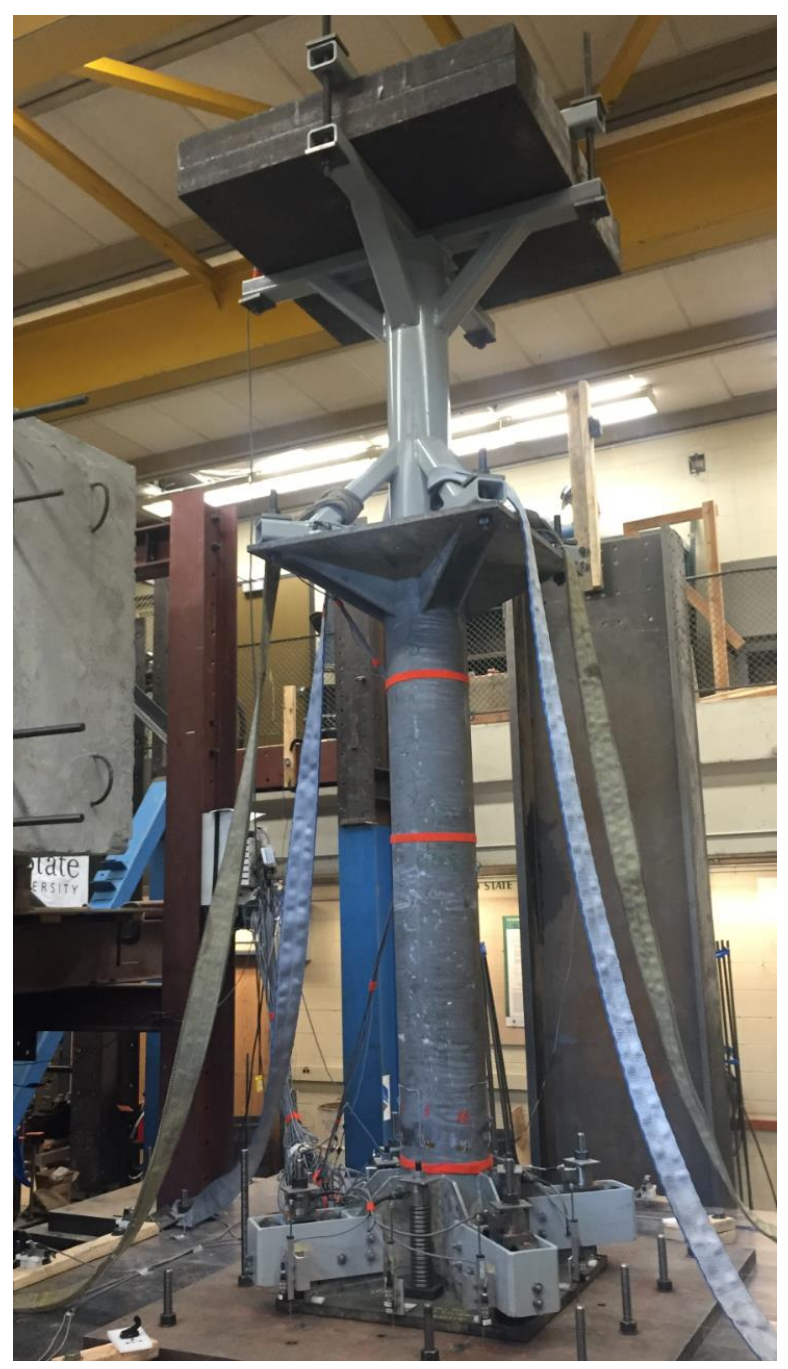

Figure 8-1: Scaled Mass System Test Setup 


\subsection{Proof of Concept Results}

As previously stated, the BeS PT configuration and viscous dampers were designed for the full-scale system. The results obtained were used primarily to show that structure demands could be decreased through the designed retrofit approach. Based on the data obtained, the $100 \%$ IEEE693 motion response of each of the systems are discussed. Also, 55\% IEEE693 motions is discussed since the non-retrofitted system could only be tested at $55 \%$ due to the high demand on the pedestal.

The rocking system with viscous dampers had a maximum C.G. displacement of 3.28 inches when exposed to the $100 \% 0.5 \mathrm{~g}$ IEEE693 motion. The C.G. acceleration was $0.67 \mathrm{~g}$ and the maximum pedestal strain was $601 \mu \varepsilon$. For the $55 \%$ motion the system had a maximum C.G. displacement of 1.65 inches and a maximum acceleration of $0.45 \mathrm{~g}$. The maximum strain of $405 \mu \varepsilon$ was measured in the north quadrant of the system.

Next, results obtained for the scaled system with hysteretic energy dissipating devices and self-centering are discussed. For the $100 \%$ motion, the system experienced a relative displacement of 6.25 inches and a maximum C.G. acceleration of $0.93 \mathrm{~g}$. The higher accelerations at the mass location caused larger strains in the pedestal also. The largest pedestal strain was $825 \mu \varepsilon$. When the $55 \% 0.5 \mathrm{~g}$ IEEE693 motion excited the system, the system experienced 3.25 inches of displacement at C.G.. The C.G. acceleration was $0.7 \mathrm{~g}$ causing $690 \mu \varepsilon$ in the extreme location of the pedestal.

Lastly, the non-retrofitted scaled system with a 55\% $0.5 \mathrm{~g}$ IEEE693 input motion is discussed. The excitation resulted in 2.32 inches of displacement at the C.G. of the system. The C.G. experienced $2.04 \mathrm{~g}$ of accelerations and the pedestal $1422 \mu \varepsilon$. 
Comparing the three scenarios for the 55\% 0.5g IEEE693 excitation, the viscous damper resulted in the lowest system demand and displacements. The viscous dampers along with the self-centering mechanism reduced the displacement by $30 \%$ compared to the non-retrofitted case. While the viscous dampers reduced the system displacement, the hysteretic devices were unable to tame the displacements and a $40 \%$ increase in displacements was present in the system response.

Both retrofit methods resulted in significant decreases of acceleration at the mass location and pedestal strains. The viscous damper retrofit case presented a $78 \%$ reduction in mass acceleration while the hysteretic devices reduced the mass acceleration by $54 \%$. For all retrofit cases, the system presented repeatable self-centering capabilities. 


\subsection{Full-Scale MASS System Design}

A full-scale representative mass was designed to validate the rocking retrofits. Using a completely steel mass system eliminated the safety concerns associated with testing brittle porcelain. In order to simulate an actual current transformer, the center of mass, mass distribution, height, and fundamental frequency were targeted for the mass system.

BPA substations contain a wide variety of 500kV CT models. Each model varies in mass, mass distribution, and height. Generally, 500kV current transformers are roughly 7000lb, $20 \mathrm{ft} .-22 \mathrm{ft}$. in height, and sit on a 90 inch pedestal. A 226-293 Trench CT was used as the basis for the work performed and a representative mass was designed based on the seismic qualification and drawings of the 226-293 CT.

Current Transformer Type HGF 1800 Model 226-293 has a mass of 3280 kg (7231 lb.) and a fundamental frequency of $3.78 \mathrm{~Hz}$ without the pedestal. According to Trench, the Center of Gravity is $3870 \mathrm{~mm}$ (152.36 in.) from the pedestal-CT assembly base. Based on pedestal specification drawings, the pedestal C.G. was determined to be 43.24 inches from the base. Using the manufacturer provided CT-pedestal C.G. of 152.36 inches, CTpedestal weight of $8257 \mathrm{lbs}$., and calculated pedestal C.G. of 43.24 inches and a weight of 1026 lbs., the CT C.G. was determined. The CT only weight of $7231 \mathrm{lbs}$. was determined to act at 217.63 inches from the pedestal base.

A circular section was used to provide the similar stiffness properties in all directions of the equivalent CT mass. Mass was distributed based on geometry of the CT and distribution was iterated until the C.G. location was near 152.36 inches. After the 
final iteration, the C.G. location of the designed mass system was 149.74 inches, total weight of $7240 \mathrm{lb}$., and a fundamental frequency of 3.79Hz. An HSS16x0.375 was used to achieve the stiffness and the mass was considered to provide no stiffness contribution to the HSS section. The mass consisted of steel plates attached to the HSS sections with plate washer spacers. The plate washer spacers allowed flexure in the HSS section without having the mass plates bear on the circular section. The gap provided by the plate washers was intended to eliminate any stiffness contribution of the mass plates. Shown in Figure 10-1 is a diagram showing the method used to attach the mass plates to the HSS section.

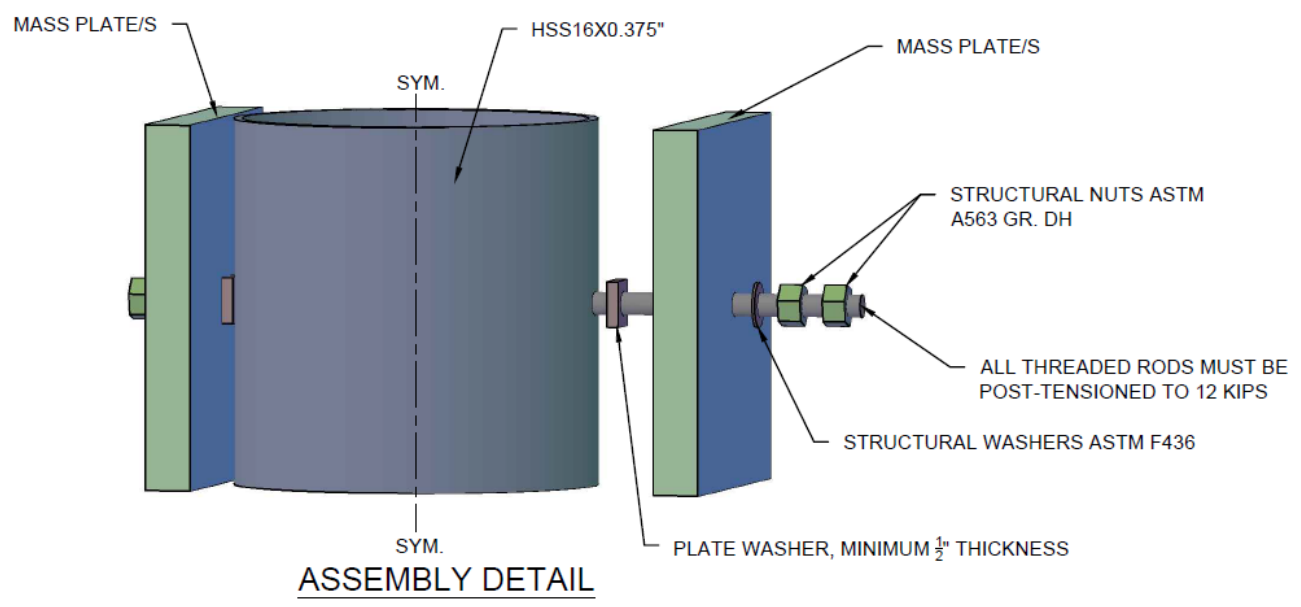

Figure 10-1: Mass Plate Mounting Detail

After completing the final iteration of the mass system, SAP2000 analysis indicated that the combined $3.79 \mathrm{~Hz}$ representative $\mathrm{CT}$ and the $20.37 \mathrm{~Hz}$ pedestal resulted in a system frequency of $1.25 \mathrm{~Hz}$. Complete details on the designed mass system are located in Appendix F. Shown in Figure 10-2 is the designed mass system. A schematic showing the plate locations, number of plates at each location, and details is shown in Appendix F. 
The mass distribution was determined using a combination of the CT profile and the location of the C.G. Figure 10-3 shows a typical CT, the shown current transformer is not the exact model used to construct the representative mass system, but shows the vast nature of the $\mathrm{CT}$.

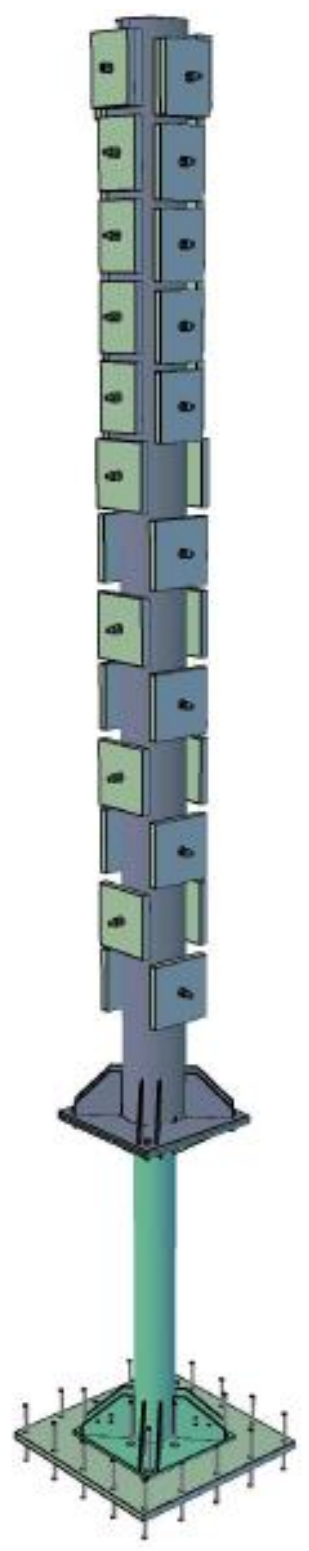

Figure 10-2: Designed Mass System

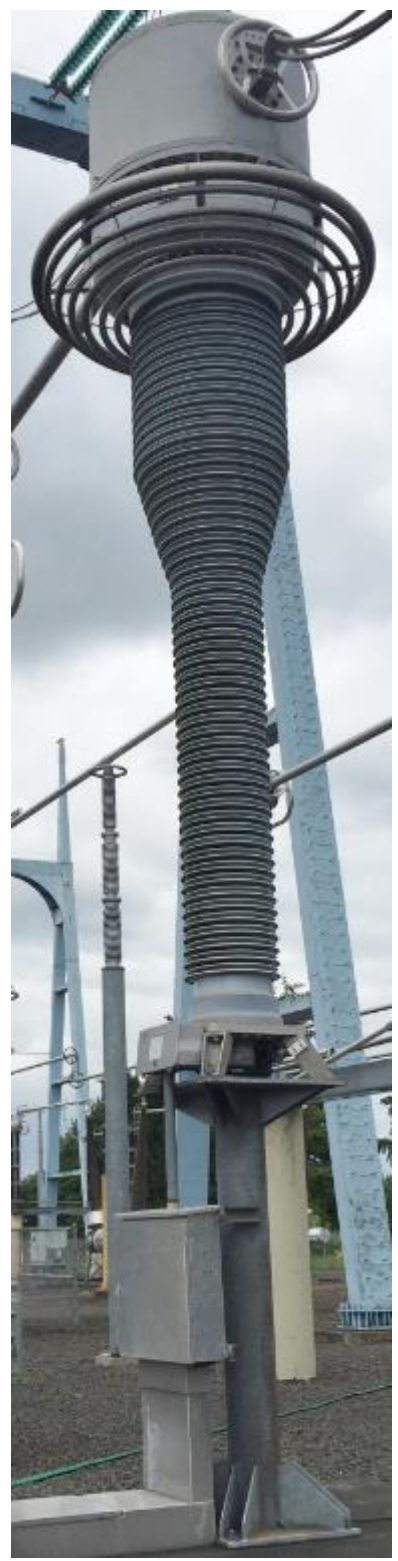

Figure 10-3: 500kV CT 


\subsection{FULL-SCALE RETROFIT DESIGN}

When designing a rocking system, many considerations must be made to achieve self-centering with a sufficient amount of energy dissipation. Key parameters that dictate the behavior of the system are: base geometry, BeS pre-tension force, restoring forces, and resisting forces. As previously mentioned, the viscous devices have little influence on not achieving self-centering because they are velocity dependent. Altering the pretensions load in the viscous configuration will change the equivalent stiffness of the system as it undergoes motion because the amount of force required to initiate rocking will change. Hysteretic devices must be deformed to their initial position by the PT and self-weight in order to achieve self-centering.

\subsection{Hysteretic Device}

The key components, elastic PT types, preload in PT members, and hysteretic devices, must be properly designed for a rocking system with hysteretic energy dissipaters to be self-centering. Discussed are the methods used to design the tested selfcentering system and recommended procedure.

\subsubsection{Elastic PT Member Design}

Design of the PT system must be completed prior to designing the energy dissipating devices to ensure the restoring moment is greater than the resisting moment in the system. In a rocking system, a low stiffness is desired to minimize the increase in load on the system after rocking is initiated. The BeS system must have the displacement capacity due to initial PT load and displacement due to rocking. Converting the IEEE693 design spectrum to a displacement spectrum, the displacement of the non-retrofitted system could be determined. Assuming the displacement of the elastic system is equal to 
the retrofitted system the amount of uplift at the base could be determined. A displacement of 10.1 inches at C.G. was determined based on the displacement spectrum, frequency of the system, and $2 \%$ damping. Using the system geometric properties, a maximum uplift of 1.07 inches was calculated at the location of the PT.

Using 6H187177 stainless Belleville Washers, a design uplift of 1.75 inches was used to determine the required amount of elastic washers. Based on the linear displacement of the $6 \mathrm{H} 187177 \mathrm{BeS}$ and total stroke required, it was determined that 36 Belleville washers were required in each stack to accommodate 1.728 inches. Shown in Figure 11-1 are the washers required for the retrofit. Based on the BeS type, the flat load could be obtained from the manufacture and the stiffness of the BeS stack could be determined. For the $6 \mathrm{H} 187177 \mathrm{BeS}$ the linear load is $12,780 \mathrm{lb}$. The stiffness of the BeS stack is $7.4 \mathrm{kip} /$ in used for the PT system. Next, the PT load was determined and remaining stroke after pre-tensioning must be determined. Shown in Figure 11-2 is the elastic-restoring system installed on the pedestal base.

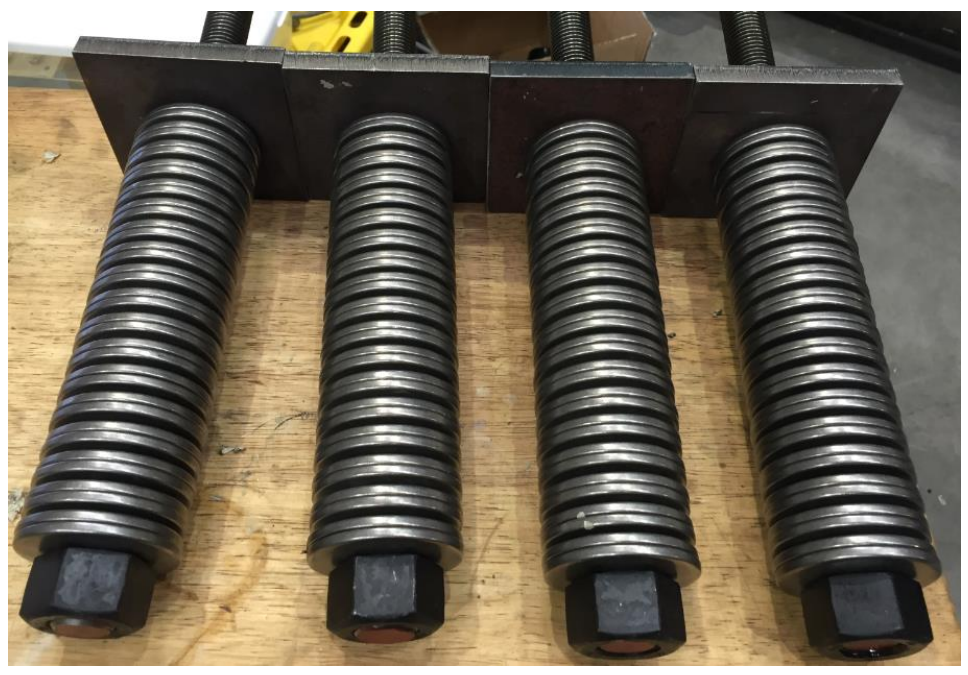

Figure 11-1: Belleville Washers 


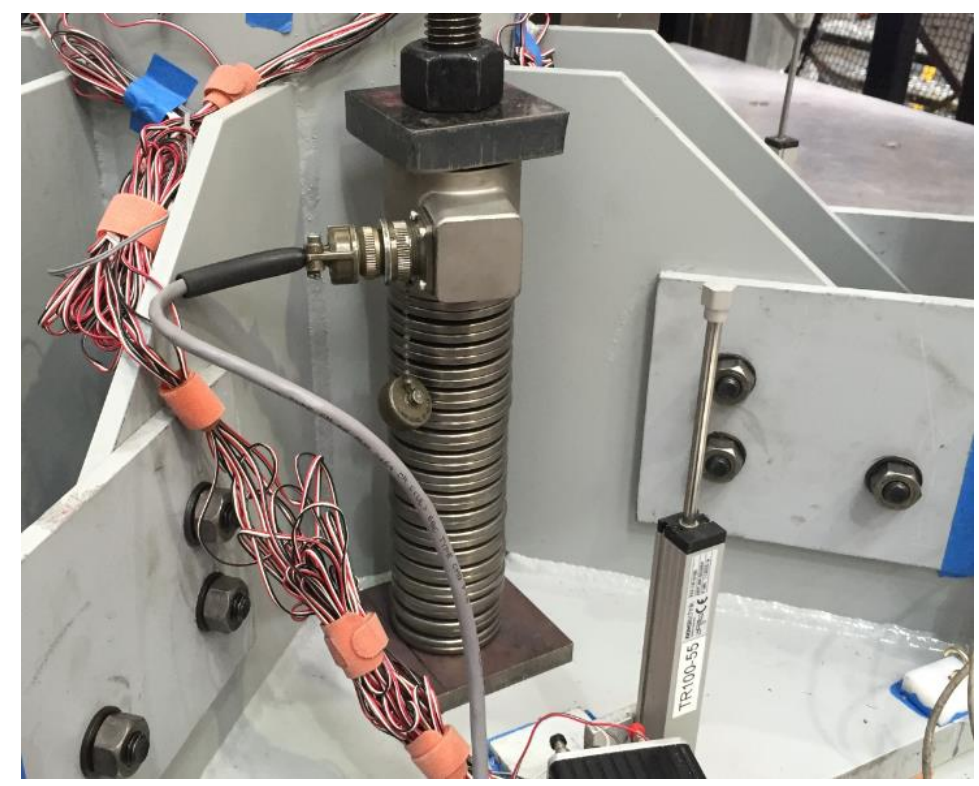

Figure 11-2: Installed BeS

\subsubsection{PT Load Design}

The second consideration that must be made in design of the PT system is the lateral force which causes uplift. Premature uplift is undesirable because premature displacement in the hysteretic devices may cause fatigue in the hysteretic fuse.

Three criterions were established to determine the load at which uplift initiation would occur. The first criteria limited the allowable C.G. displacement to the expected displacement in the non-retrofitted elastic system exerted to $0.5 \mathrm{~g}$ IEEE693 motion. The second criterion was a target C.G. acceleration of $0.5 \mathrm{~g}$ under the $0.5 \mathrm{~g}$ IEEE693 motion. Since period shift occurs during the rocking, reducing the frequency would result in a significant decrease in spectral acceleration but slight amplification is still expected. And finally, the retrofit PT was designed so that rocking does not occur at lateral loads at and below the retrofit design wind load. Figure 11-3 summarizes the three conditions used to determine the rocking force. Shown in the figure are two rocking forces, each rocking force $\left(F_{R}\right)$ are found independently using the lateral wind and $0.5 \mathrm{~g}$ criteria along with the 
expected elastic displacement $\left(\Delta_{\text {Total }}\right)$. The stiffness of the PT and elastic structure must be known to determine the rocking force. The larger of $F_{R 1}$ and $F_{R 2}$ calculated is recommended to be used for design. For the lateral wind loading case, the $F_{R}$ load is known from the wind load calculation and the final load is determined. For the $0.5 \mathrm{~g}$ seismic case, the final load is known and the rocking load must be determined. It is important to note that all stiffness values must be at the global scale applied at the C.G.. The figure shows the contributions of the elastic system, not the non-linear devices, which are considered separately for design.

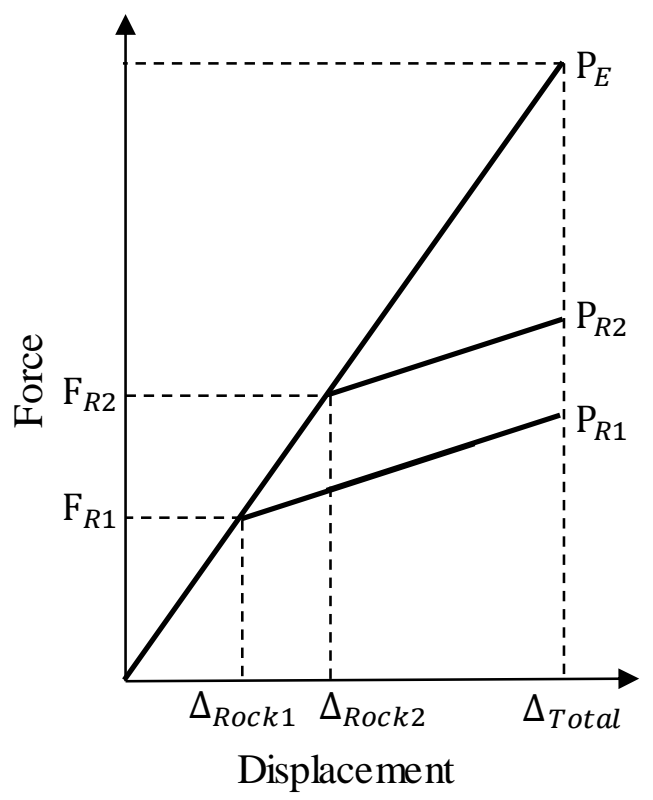

Figure 11-3: Rocking Initiation

The $0.5 \mathrm{~g}$ seismic criterion is controlled by two factors, force contribution of the elastic system and device force contribution. Hysteretic devices could be sized in such a manner that insufficient energy dissipation occurs or where the device force is too large and self-centering cannot be achieved. Similar to Figure 11-3, Figure 11-4 shows the 
contribution of the PT to global force. Denoted "a" on the figure is the maximum force due to PT at the expected displacement.

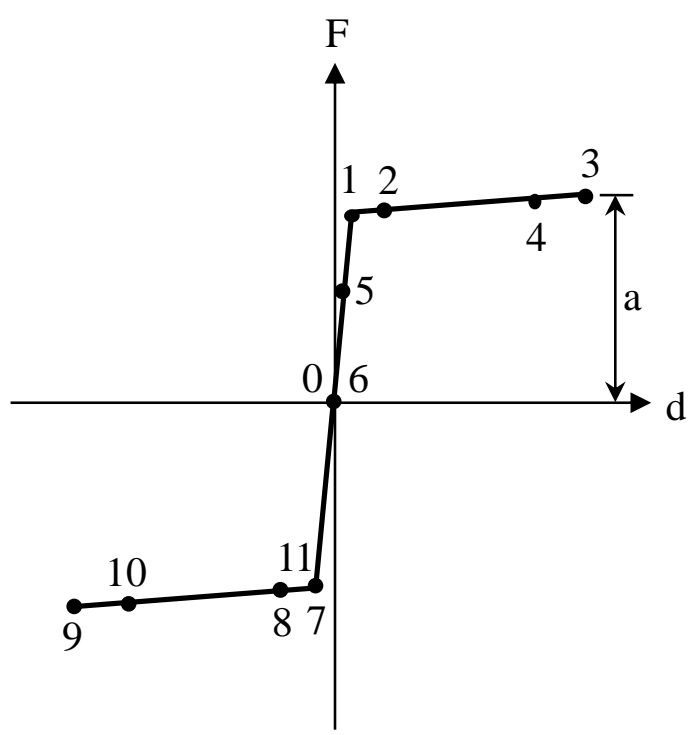

Figure 11-4: PT Force Contribution

The idealized bi-linear behavior of the hysteretic device response due to uplift is shown in Figure 11-5. Shown in the figure as "b" is the maximum force due to the hysteretic devices at maximum uplift.

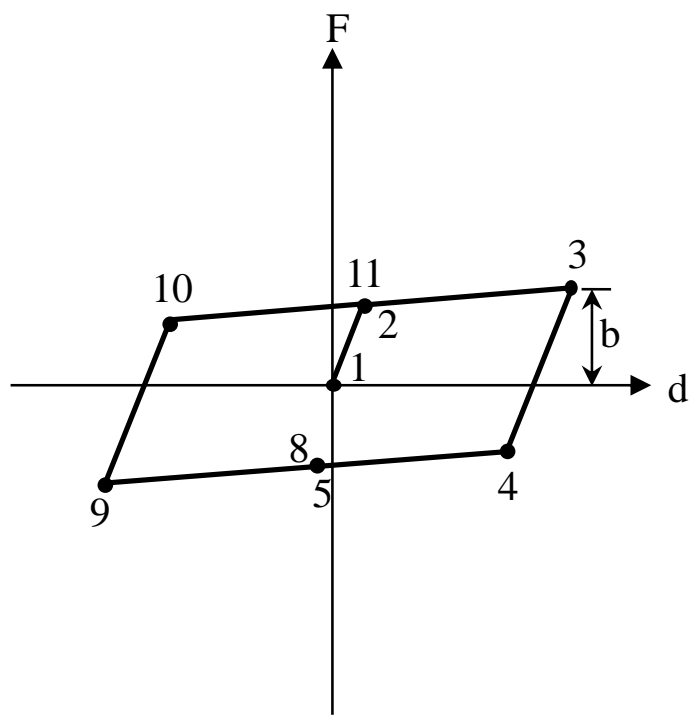

Figure 11-5: Hysteretic Device Response 
Combining the contribution of the PT and the UFP response is shown in Figure 11-6. The maximum force of the system is a combination of the elastic system's force and device force. The ratio of $\mathrm{a} / \mathrm{b}$ is a self-centering ratio used to relate the contribution of the non-linear system to the elastic system. Any system with an a/b ratio less than unity will not self-center.

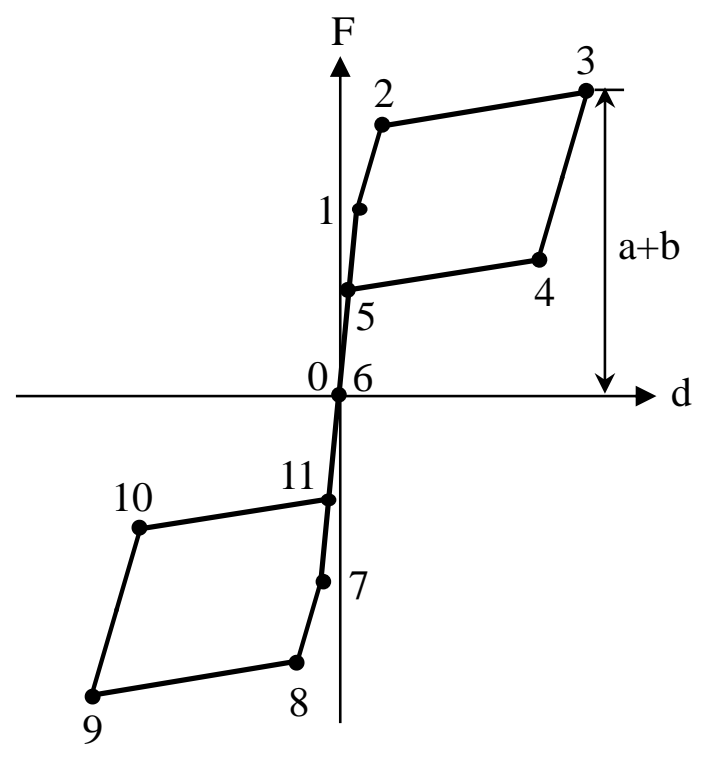

Figure 11-6: Self-Centering System Response

Based on previous work conducted on rocking systems, Pampanin suggests an $(\mathrm{a} / \mathrm{b})$ ratio of 1.25 for rocking systems (Pampanin, 2001). Figure 11-7 compares how the a/b ratio changes the amount of energy dissipation and self-centering ability. When the flag shape is eliminated, the system is no longer self-centering. 

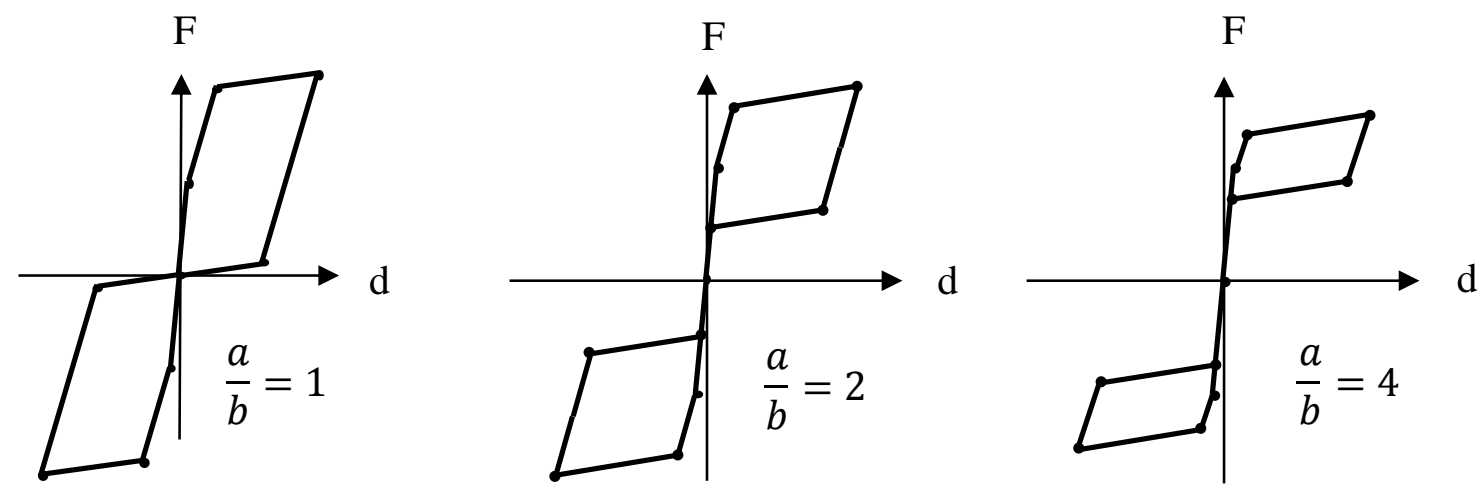

Figure 11-7: a/b Ratio Comparison

Using the recommended $\mathrm{a} / \mathrm{b}=1.25$, the contribution of moment due to PT for the $0.5 \mathrm{~g}$ seismic criteria could be determined. Also, the wind load lateral force could be determined using typical ASCE procedures. The greater of the two values will control the uplift force. The uplift force could be calculated using the PT locations and assuming rocking about the base edge. Assuming the base plate does not deform, the force in each PT member is proportional to the distance away from the rocking location.

Based on the pedestal and system geometry an equivalent force at the C.G. was determined for both uplift criterions. For the $0.5 \mathrm{~g}$ criteria, the equivalent force was 1028 lbs. and for the wind criteria $648 \mathrm{lbs}$. The moment produced by the larger force was used to determine the required pre-tension force based on the $4 \mathrm{BeS}$ stack locations. A pretension force of 3.73 kips was determined to eliminate rocking prior to the design wind loading and achieve near $0.5 \mathrm{~g}$ acceleration in the system. Using the BeS stack stiffness of $7.4 \mathrm{kip} / \mathrm{in}$, a total of $0.5 \mathrm{inch}$ is expected to be lost in the BeS stack stroke due to initial pre-tensioning. A total stroke of 1.73 inches is available in the elastic washers, allowing for 1.2 inches of uplift to occur. Based on the rigid body calculations, the system 
demands 1.07 inches of uplift which could be achieved with the specified BeS stack. Rocking is expected to occur slightly above the design load due to self-weight. Selfweight is neglected in the restoring force because large displacements will result in minimal contribution of self-weight in restoring moment. Considering self-weight and the elastic mechanism, a force of 1.6 kips must be applied at the C.G. to initiate rocking.

\subsubsection{Hysteretic Device Design}

UFP design consists of many assumptions and could be conducted many ways. The described method uses a SDOF approach and iterates with a numerical model in order to fine-tune the device properties. Summarized in Figure 11-8 is the proposed design iteration for initial hysteretic device sizing. 


\section{Hysteretic Device Proposed Design Method}

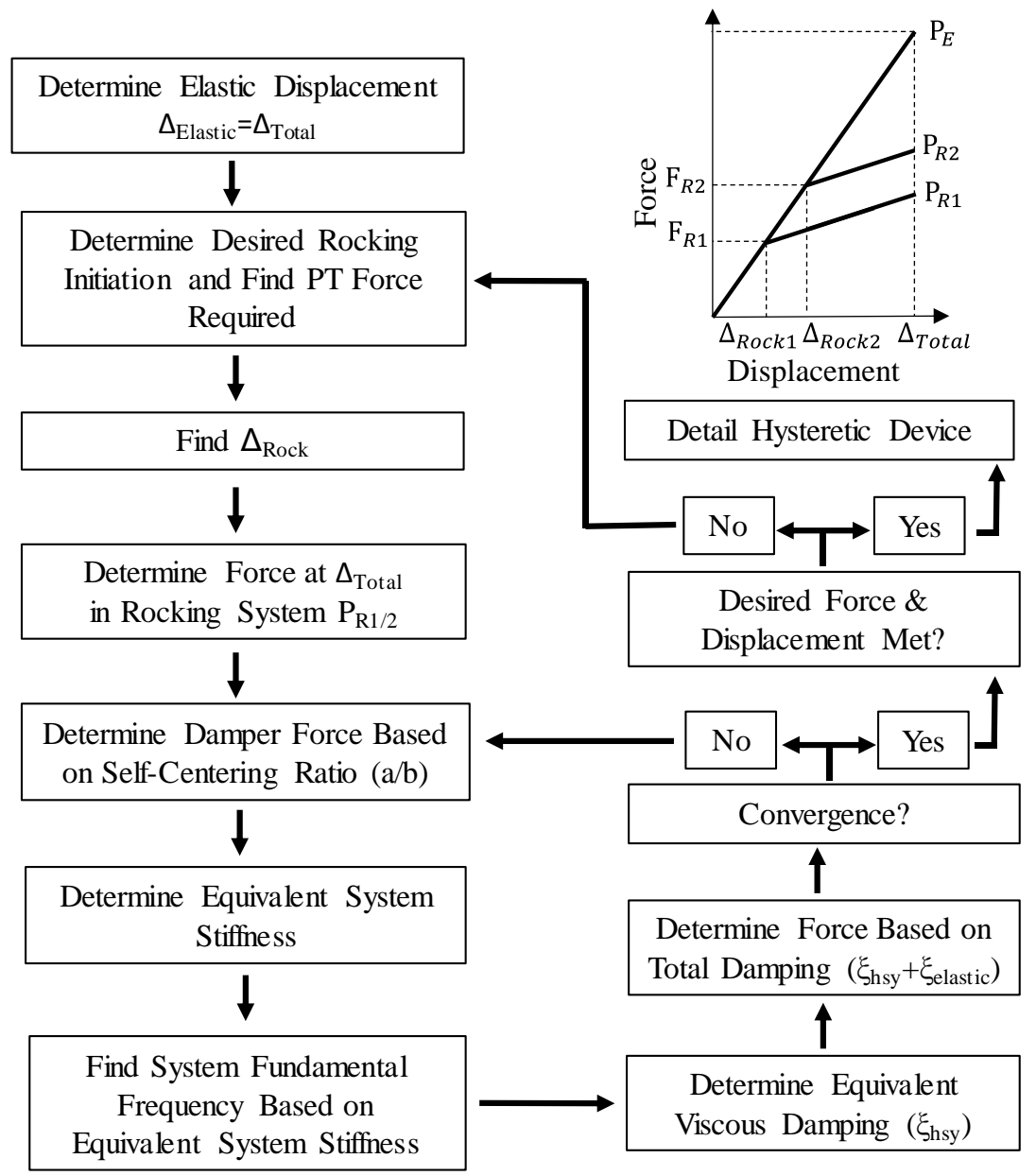

Figure 11-8: Hysteretic Device Design Procedure

The initial 4 steps in the design procedure are discussed in 11.1.1 and 11.1.2.

Previous research conducted by Pampanin suggest an $\mathrm{a} / \mathrm{b}$ ratio of 1.25 for sufficient energy dissipation is recommend to account for material over-strength (Pampanin, 2001). Next, the system rocking stiffness and fixed base stiffness must be determined. Using the determined preload force the amount of deflection prior to rocking could be determined. Assuming the elastic system displaces the same amount as the rocking system, the rocking displacement could be calculated. The PT moment at the expected maximum 
displacement could be determined. After calculating the PT contribution to moment resistance, the a/b ratio could be utilized to compute the device moment resistance. The equivalent stiffness could be determined at the maximum displacement in order to estimate the rocking frequency. Using the values obtained, calculate equivalent viscous damping and iterate using the new frequency and damping ratio. Continue iterations until system displacements converge. Using the location of the devices and the required moment contributions from the devices, assume all devices are at their maximum capacity and determine the required capacity of each UFP.

\subsubsection{SAP2000 Hysteretic Device Calibration}

UFP modeling for the full-scale system utilized the methods discussed in 5.5, Hysteretic Device Modeling, and 11.1.3, Hysteretic Device Design. Shown in Figure 11-9 are the properties used to define the Wen-link used for modeling the full-scale testing. The UFP for the full-scale system had a width of 2.25 inches, an outer diameter of 3.25 inches, a plate thickness of 5/16 inch, and fabricated from ASTM A572 Gr.50 steel. Theoretically, the designed UFP should have the following nominal properties: $F_{y}=1.37$ kips, $F_{p}=2.06$ kips, $F_{u}=2.67$ kips, and kinitial=14.8kip/in. Shown in Figure 11-10 is the output from the calibrated SAP2000 model for a UFP set comprised of 2 UFP devices. The final UFP design was designed for $\mathrm{a} / \mathrm{b}$ ratio of 1.5 rather than 1.25 because the model results showed little flag shape behavior. 


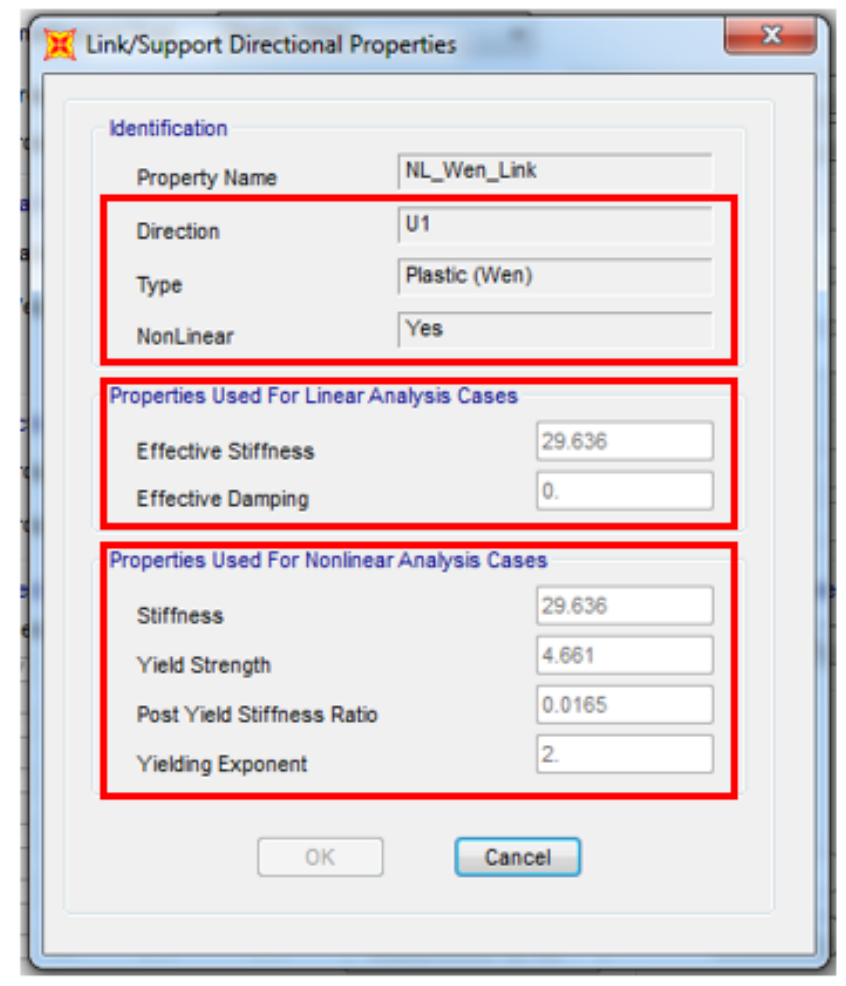

Figure 11-9: Full-Scale Wen-link Properties

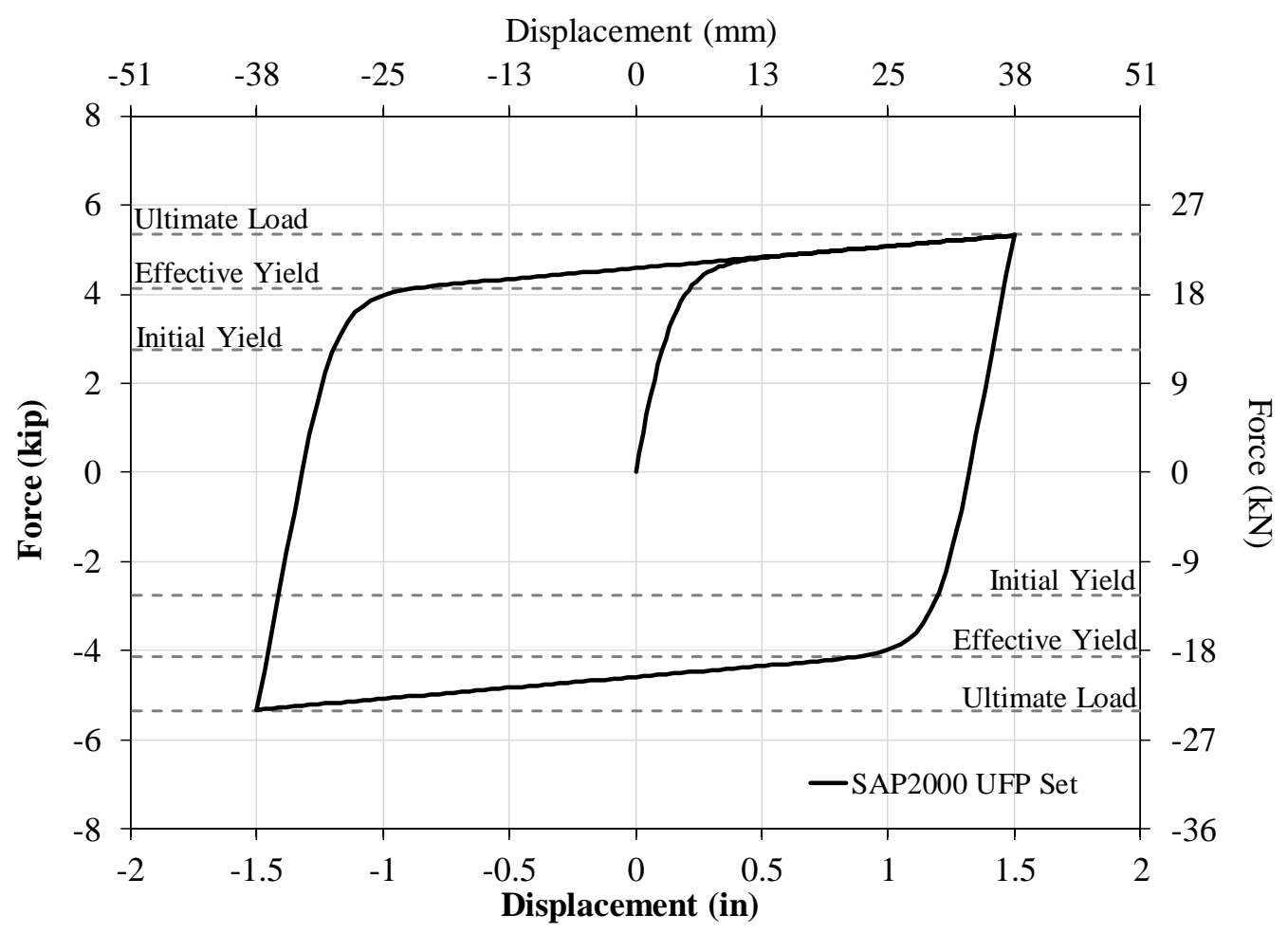

Figure 11-10: Full-Scale Model Wen-link Response 


\subsection{Viscous Damper Design}

Various methods could be used to determine the required properties of the viscous dampers. All methods include approximating the maximum damper velocity and the desired output force at the maximum velocity. Due to the costs associated with rebuilding and procuring viscous dampers, only one set of Taylor Devices viscous dampers was purchased based on the full-scale requirements. The full-scale dampers were utilized at the scaled testing conducted at the iStar Laboratory to verify that all components were properly functioning and no tolerance or constructability issues were present in the designed base retrofit.

Typically, linear viscous dampers are not used in seismic mitigations because the force continues to increase linearly with increases in velocity. Uncertainties in damper velocities could cause overloading in structural members. With non-linear dampers, $0.3 \leq \alpha<1$, the force in the damper increases rapidly at low velocities but levels off at higher velocities. Figure 11-11 shows comparison between a linear damper and two nonlinear dampers. Taylor Device's recommends initial iterations of damper properties based on a linear damper. After initial properties are established, refinements with lower exponents could be made to optimize system performance. 


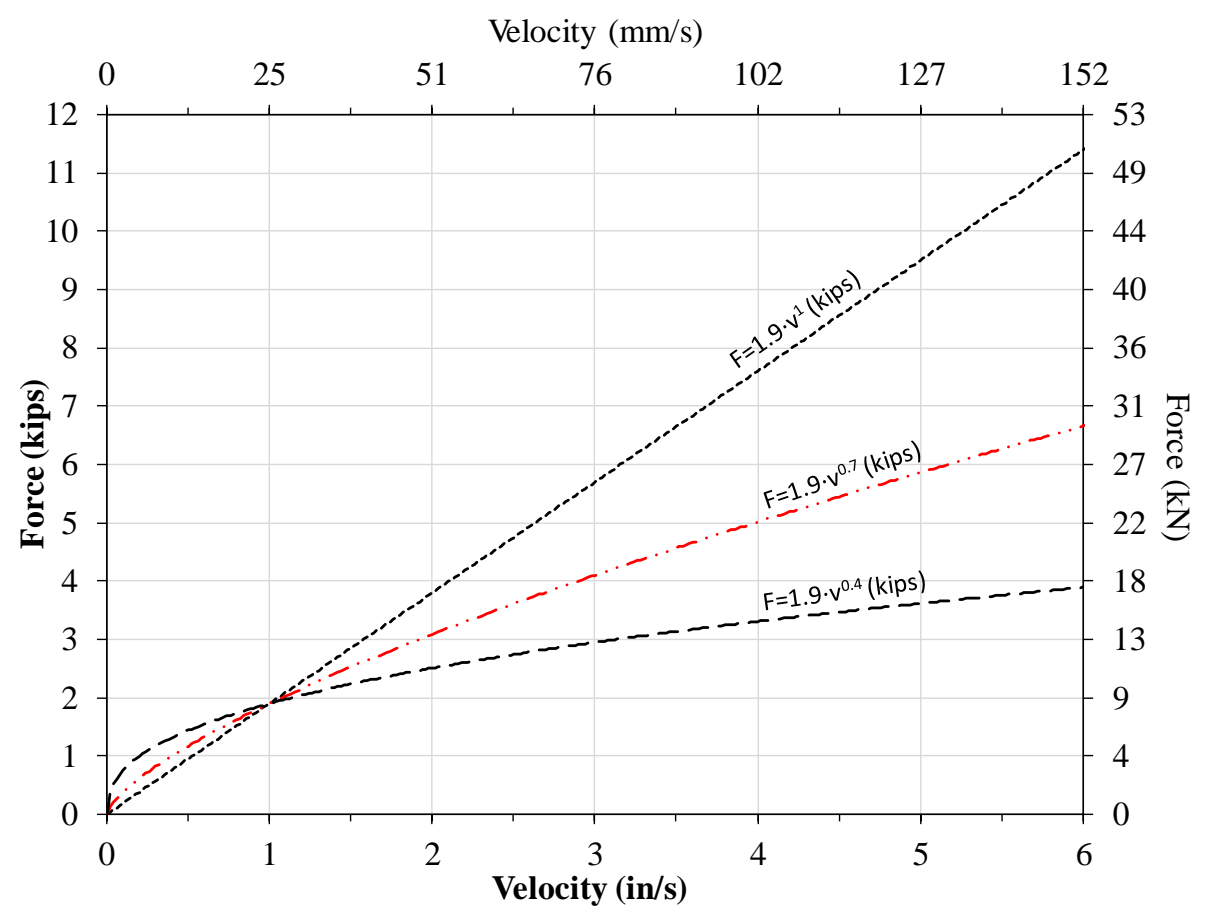

Figure 11-11: Viscous Damper $\alpha$ Influence of Device Force

General application of damper-exponential link elements is described in 5.0, General Numerical Model section. Using the properties of the D-series double acting stainless steel Taylor Devices damper with a total stroke of 2 inches, iterations were conducted to determine the optimal alpha and "c" factor for the full-scale retrofit configuration. Taylor Devices stated that the units have a stiffness of 140 kips/in and an alpha factor of 0.6 to 1 is recommended for the units.

Shown in Figure 11-12 is the systems response to a linear viscous damper with varying "c" variable. A snapshot of two "c" values (800 and 1600) from Figure 11-12 is shown in Figure 11-13. Increasing the "c" value led to decrease in system displacements for the cases investigated. A summary table of system parameters for the various "c" values for $\alpha=1.0$ is shown in Table 11-1. 


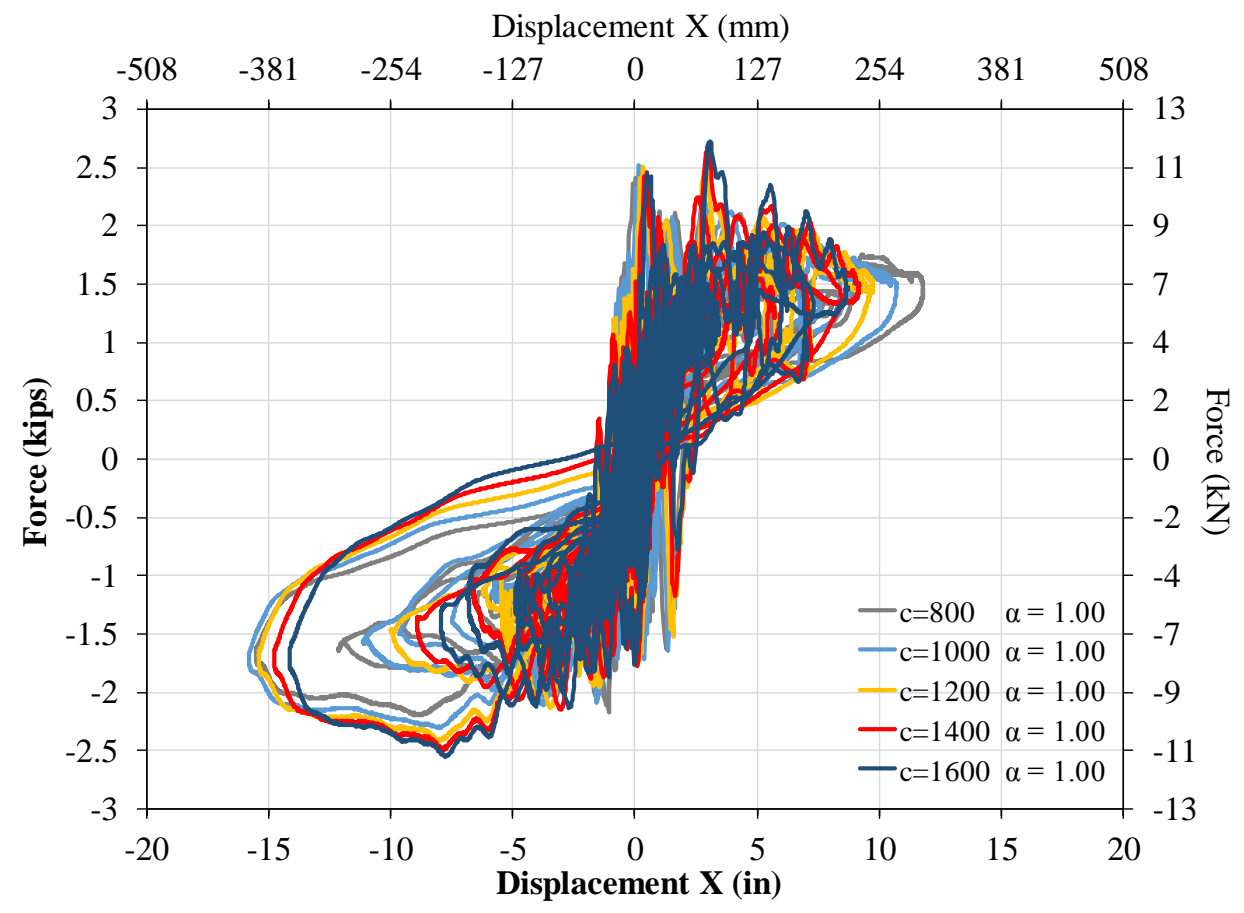

Figure 11-12: Viscous Damper "c" Factor Study w/ $\alpha=1.0$ 


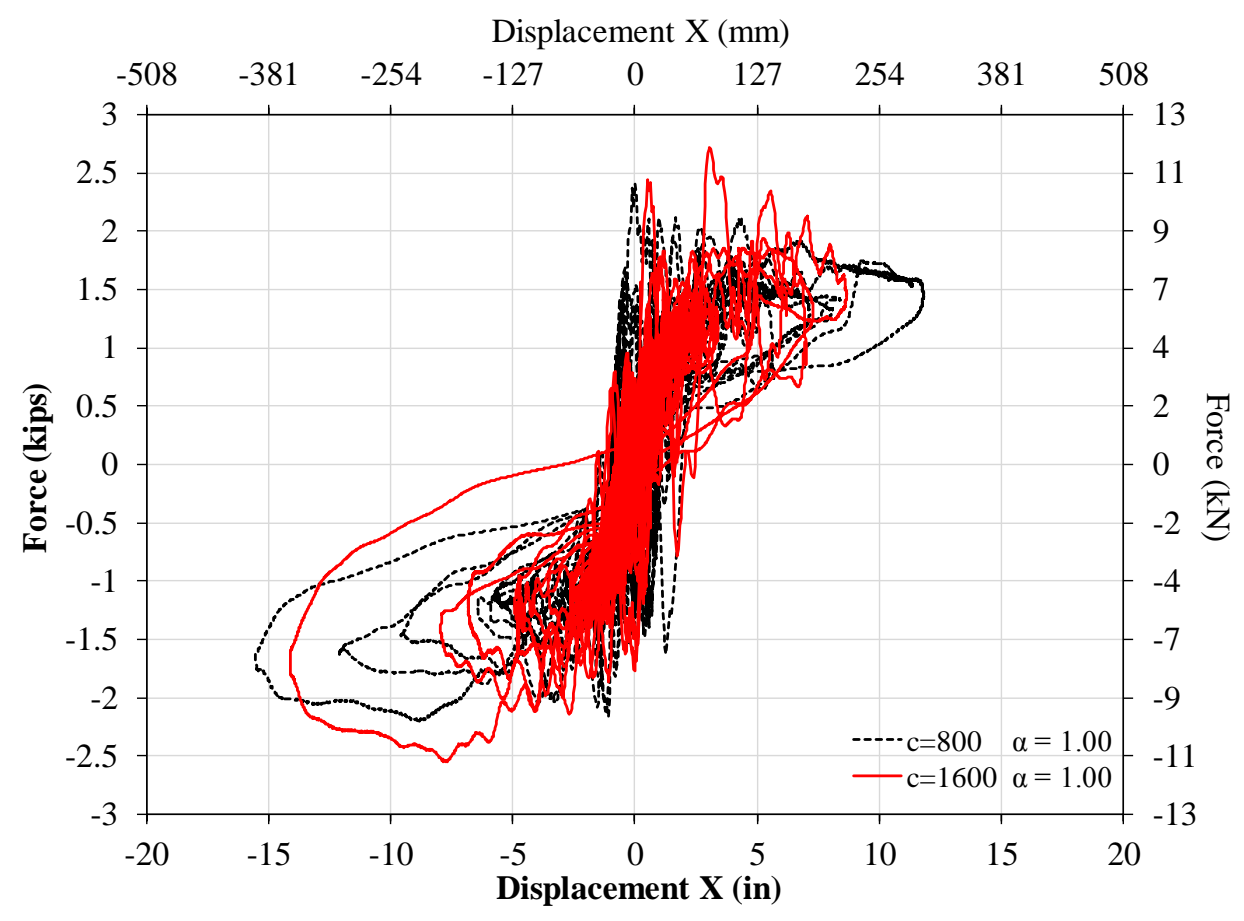

Figure 11-13: Viscous Damper "c" Comparison w/ $\alpha=1.0$

Table 11-1: Viscous Damper “c” Factor Study w/ $\alpha=1.0$

\begin{tabular}{|c|c|c|c|c|c|c|c|c|}
\hline \multicolumn{2}{|c|}{$\mathrm{F}=\mathrm{c} \cdot \mathrm{v}^{\alpha}(\mathrm{Ib})$} & \multicolumn{7}{|c|}{ Results from SAP2000 Models } \\
\hline$\alpha$ & c & $\begin{array}{c}\text { Damper- } v_{\max } \\
(\mathrm{in} / \mathrm{s})\end{array}$ & $\begin{array}{c}\text { Damper- } F_{\max } \\
\text { (lb) }\end{array}$ & $\begin{array}{c}\text { Damper- } \delta_{\max } \\
\text { (in) }\end{array}$ & B.S. $F_{\max }(k i p)$ & C.G. $d_{\max }$ (in.) & C.G. $a_{\max }\left(i n / s^{2}\right)$ & C.G. $a_{\max }(g)$ \\
\hline 1 & 800 & 5.33 & 3769 & 0.89 & 2.41 & 15.55 & 397 & 1.03 \\
\hline 1 & 1000 & 4.89 & 4211 & 0.80 & 2.52 & 15.83 & 404 & 1.05 \\
\hline 1 & 1200 & 4.52 & 4516 & 0.71 & 2.51 & 15.41 & 387 & 1.00 \\
\hline 1 & 1400 & 3.96 & 4763 & 0.66 & 2.64 & 14.78 & 440 & 1.14 \\
\hline 1 & 1600 & 3.50 & 5005 & 0.62 & 2.72 & 14.15 & 371 & 0.96 \\
\hline
\end{tabular}

Note: B.S. is the base shear

Similar to the figures presented for linear viscous dampers, Figure 11-14 is a summary of the influence the "c" factor has on the systems base shear and C.G. displacement when $\alpha=0.7$. 


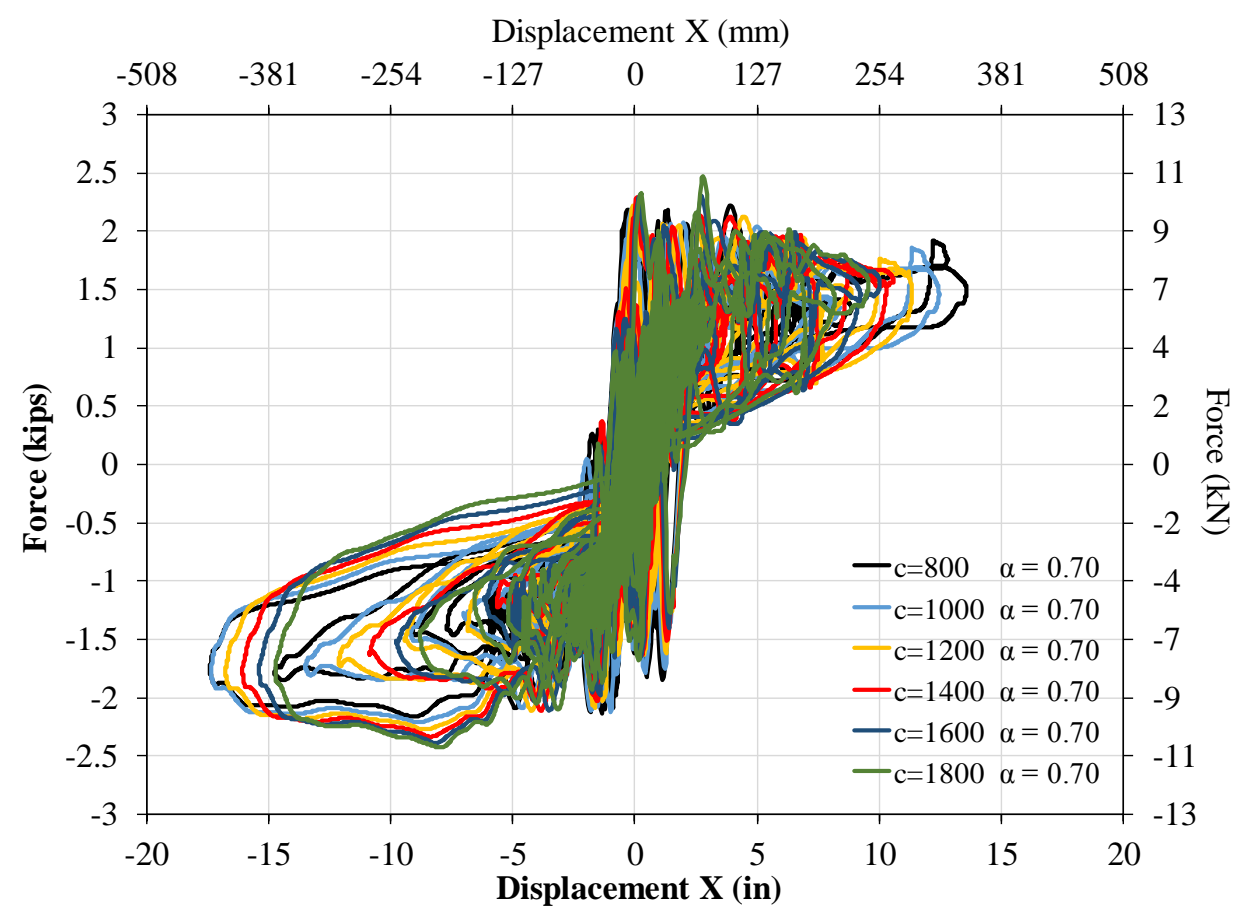

Figure 11-14: Viscous Damper "c" Factor Study w/ $\alpha=0.7$

A complete table of key system parameters is presented in Table 11-2. To show the trends more clearly, a reduced data set, only $c=1000$ and $c=1800$, is shown in Figure 11-15. Between the tabulated results and the figures of the response, increasing the "c" value generally results in smaller system displacements. Also, as "c" increases the maximum velocity seen in the system also reduces. Maximum force developed in the device increased as "c" values were increased. C.G. acceleration tended to decrease with the increase in "c" value, but base shear slightly increased 2.22kips for $\mathrm{c}=800$ and 2.48kips for $\mathrm{c}=1800$. 


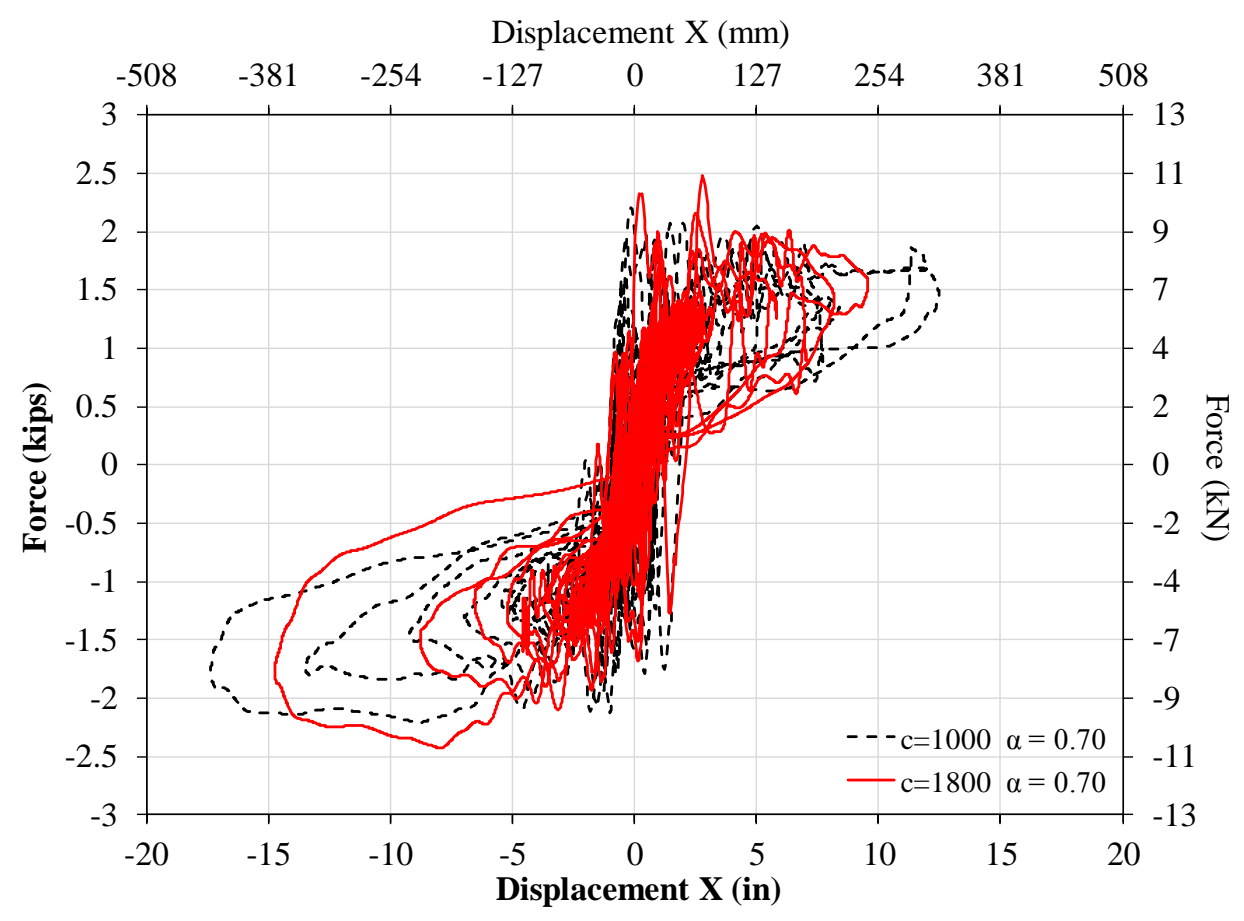

Figure 11-15: Viscous Damper "c" Comparison w/ $\alpha=0.7$

Table 11-2: Viscous Damper "c" Factor Study w/ $\alpha=0.7$

\begin{tabular}{|c|c|c|c|c|c|c|c|c|}
\hline \multicolumn{2}{|c|}{$\mathrm{F}=\mathrm{c} \cdot \mathrm{v}^{\alpha}(\mathrm{Ib})$} & \multicolumn{7}{|c|}{ Results from SAP2000 Models } \\
\hline$\alpha$ & $\mathrm{c}$ & $\begin{array}{c}\text { Damper- } \mathrm{v}_{\max } \\
(\mathrm{in} / \mathrm{s})\end{array}$ & $\begin{array}{c}\text { Damper- } \mathrm{F}_{\max } \\
(\mathrm{lb})\end{array}$ & $\begin{array}{c}\text { Damper- } \delta_{\max } \\
(\mathrm{in})\end{array}$ & B.S. $\mathrm{F}_{\max }(\mathrm{kip})$ & C.G. $\mathrm{d}_{\max }(\mathrm{in})$. & C.G. $\mathrm{a}_{\max }\left(\mathrm{in} / \mathrm{s}^{2}\right)$ & C.G. $\mathrm{a}_{\max }(\mathrm{g})$ \\
\hline 0.7 & 800 & 6.03 & 2754 & 1.01 & 2.22 & 17.43 & 427 & 1.11 \\
\hline 0.7 & 1000 & 5.56 & 3212 & 0.94 & 2.21 & 17.39 & 424 & 1.10 \\
\hline 0.7 & 1200 & 5.15 & 3591 & 0.85 & 2.27 & 16.78 & 414 & 1.07 \\
\hline 0.7 & 1400 & 4.79 & 3920 & 0.78 & 2.33 & 16.08 & 400 & 1.04 \\
\hline 0.7 & 1600 & 4.46 & 4209 & 0.71 & 2.39 & 15.39 & 379 & 0.98 \\
\hline 0.7 & 1800 & 4.13 & 4449 & 0.69 & 2.48 & 14.73 & 369 & 0.96 \\
\hline
\end{tabular}

Note: B.S. is the base shear

Comparing the values obtained for the linear and non-linear viscous damper investigated, generally the linear viscous damper resulted in higher base shear forces, damper forces, and acceleration. A non-linear viscous damper with a $\alpha=0.7$ was used for the specified damper for the retrofit application. Taylor devices recommended $\alpha=0.6$ to $\alpha=1.0$ for the specific damper. The damper obtained for the retrofit had the following properties: $\mathrm{c}=1900 \mathrm{lbs}, \alpha=0.7,2$-inch total stroke, double acting, and slack-free moment- 
free ends. The damper was to exert $5015 \mathrm{lbs}$. at $4 \mathrm{in} / \mathrm{sec}$ and had a safety factor of 1.4 at $7500 \mathrm{lbs}$. Approved drawings for the specified viscous damper are attached in Appendix B.

Shown in Figure 11-16 is a summary of the design procedure used to size the viscous damper. Approaches are available that estimate the velocity of a system prior to numerical iterations but the method shown uses modeling technique to converge on an optimal device.

\section{Viscous Damper Design Method}

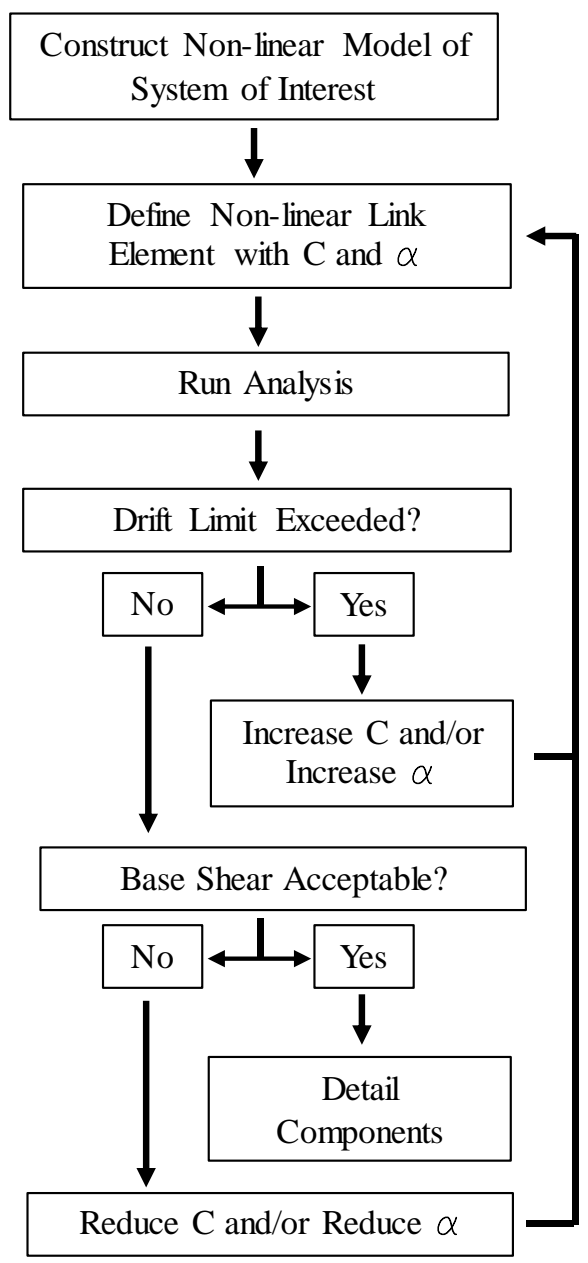

Figure 11-16: Viscous Damper Design Procedure 


\subsection{Full-scale TeSt SetuP}

\subsection{Instrumentation}

Full-scale testing of the CT representative mass model was conducted at the University of Nevada, Reno (UNR), on a 6 degree-of-freedom (6-DOF) shake table. The tQke IEEE693 motion in $\mathrm{X}, \mathrm{Y}$, and Z directions was utilized to evaluate the effectiveness of the retrofit. A total of 155 instrumentation channels were used to monitor the behavior of the system. The instrumentation included 29 displacement transducers, 8 load cells, 8 strain gauges, 16 3-D accelerometers, and 62 6-DOF table transducers.

Key metrics captured by the instrumentation included: relative global displacement of the system, uplift on each side of the base plate, device force and displacement, anchor loads, and shear/moment at various locations of the specimen. In addition to the instrumentation, a total of 7 video cameras were used for visual observations of the system behavior.

Relative displacement determined at 3 key locations was computed for each test case. The locations of interest were top of the assembly, C.G. of the assembly, and top of the pedestal. At each location $\mathrm{X}$ and $\mathrm{Y}$ relative displacements were obtained by taking the difference in the measured displacement and the table displacement.

LVDTs located on each side of the base plate measured base plate uplift. All base plate LVDTs were placed with minimal distance between the base plate and the transducer in order to eliminate measurement corrections. Also, each device mounting assembly was instrumented with three LVDTs to measure the displacement of the device relative to the shake table and slip in the bracket connected to the pedestal. Two LVDTs 
were used on each mounting assembly in order to calculate the true displacement of the energy dissipating device due to tri-axial excitation. Shown in Figure 12-1 is a diagram showing the locations of the transducers.

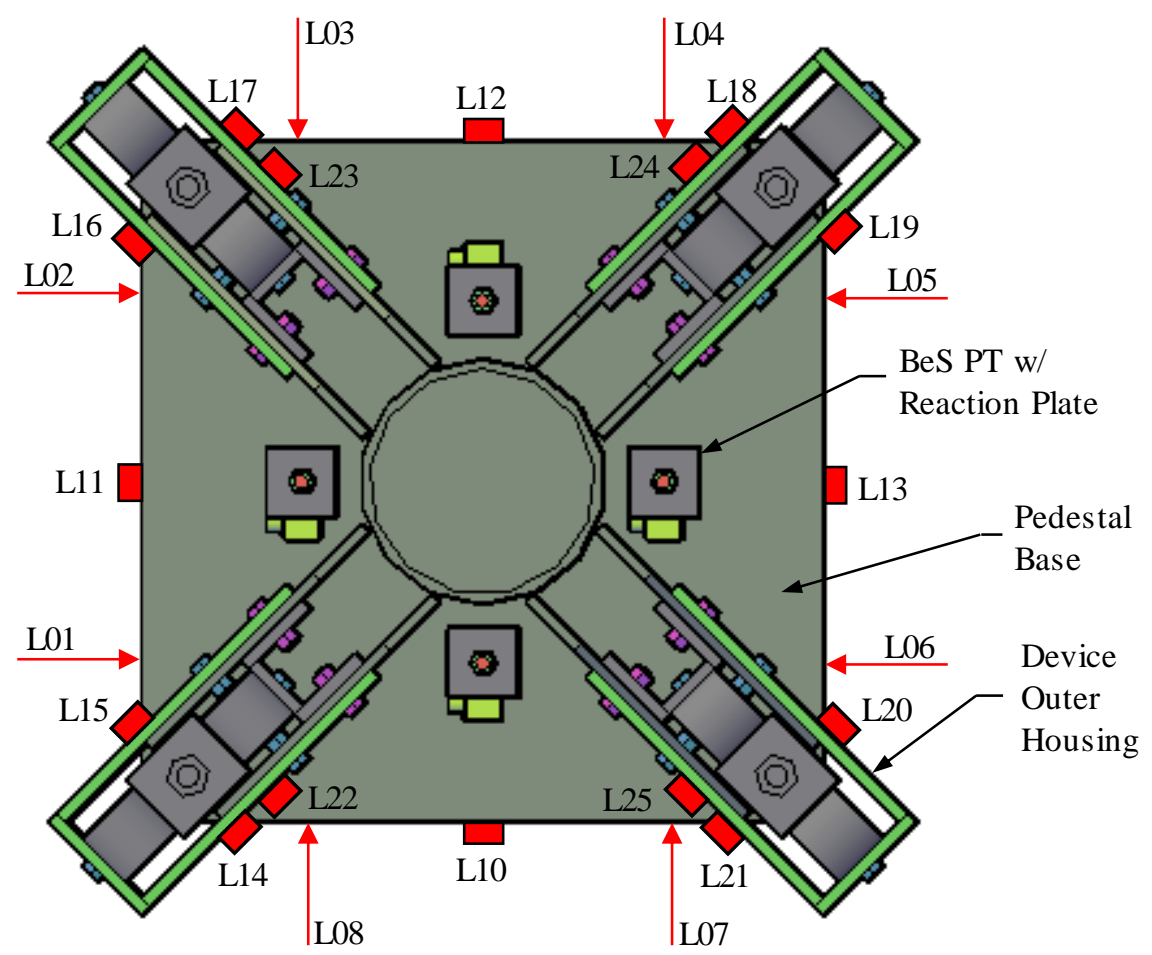

Figure 12-1: LVDT Locations on Pedestal Base

Labeled L01-L08 are LVDTs which measured horizontal movement of the pedestal base. Denoted L10-L13 are LVDTs measuring vertical uplift of the pedestal base. Instruments labeled L14-L21 were used to determine the displacement in the device. Each pair of LVDTs on the device assemblies (L14 \& L15, L16 \& L17, L18 \& L19, L20 \& L21) were used to determine the displacement at the location of the anchoring rod. L14-L21 measured relative displacement between the device outer housing and the shake table. Also, slip in the bracket relative to the pedestal was measured using L22-L25. Shown in Figure 12-1 are the instruments installed on the hysteretic retrofit case, the 
same instrumentation was implemented on the viscous device retrofit. The non-retrofitted base consisted of the same instrumentation less any device transducers, L14-L25.

Load cells were used to measure forces in foundation anchors. Between the various test cases the anchors were used for various applications. For the retrofitted cases, the 1" anchor rods were used to transfer load from the energy dissipation devices to the foundation. Also, additional anchors are required for the retrofitted case for the PT load to transfer to the foundation. Load cells monitored PT loads, device loads, and bolt yielding during testing.

The $\mathrm{CT}$ is a multi-degree of freedom (MDOF) system requiring a higher quantity of sensors to accurately capture the reactions due to the structure dynamics. Shear and moment at key components on the test specimen allow for a simple comparison between the various test cases. Base moment was determined using two independent methods.

The first method utilized 8 strain gauges located 16 inches from the base plate on the HSS pedestal. Four primary gauges were located on the $\mathrm{X}$ and $\mathrm{Y}$ axis. An additional gauge was placed at four points between the quarter point gauges. Shown in Figure 12-2 are the locations of the strain gauges used to determine moment in the pedestal. A calibration factor between moment and strain was determined and used to determine base moment from the strain gauge readings. The $\mathrm{CT}$ representative mass was bolted to the shake table adaptor plate and lateral loads were applied at the top of the specimen. The cantilever load and strain was measured directly, the moment was determined and a calibration factor for each strain gauge was determined. Since only 8 strain gauges were 
instrumented on the specimen, only base moment could be obtained from the strain gauge data.

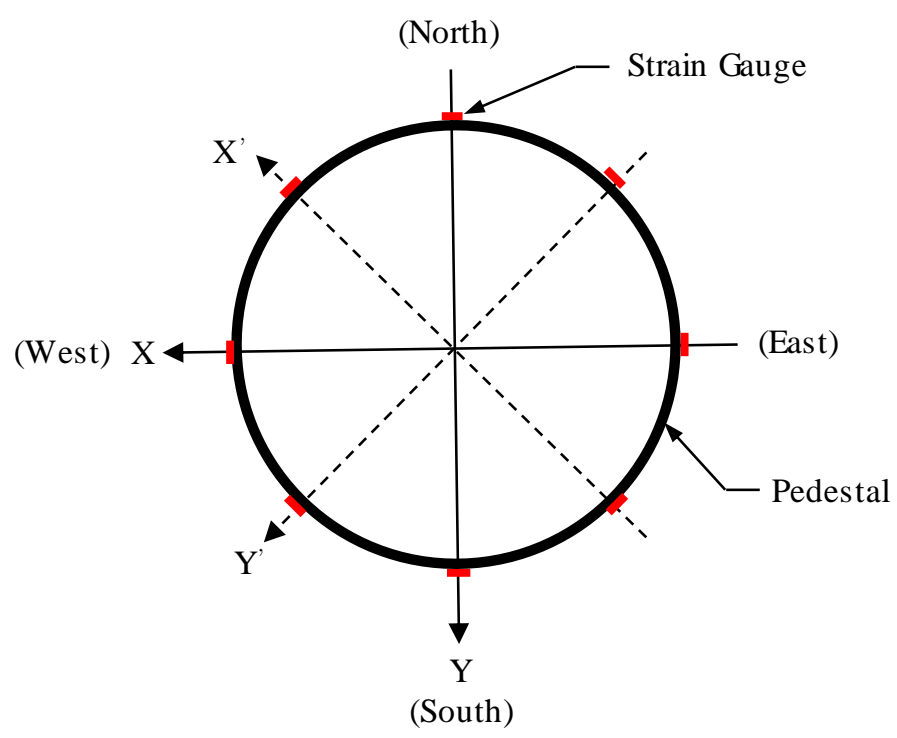

Figure 12-2: Strain Gauge Placement

The second more versatile method used for determining shear and moment on the specimen was acceleration data. A total of 16 tri-axial accelerometers were distributed along the test specimen. Figure 12-4 shows the location of the accelerometers used to determine moment and shear. Figure 12-4 could be used in conjunction with Table 12-1 to determine the exact location of specific accelerometers and string pots. Shear and moment were determined by lumping masses at each accelerometer location. The lumped masses were multiplied by the accelerometer reading in order to obtain the lateral force at the location of interest. Shear and moment at any location along the specimen could be determined by summing the contributions of each node above the location of interest. Figure 12-3 graphically shows the lumped mass method tailored to the test specimen. Although the described method is not exact, the results at the base yielded almost identical results to those obtained from the direct strain gauge reading. 


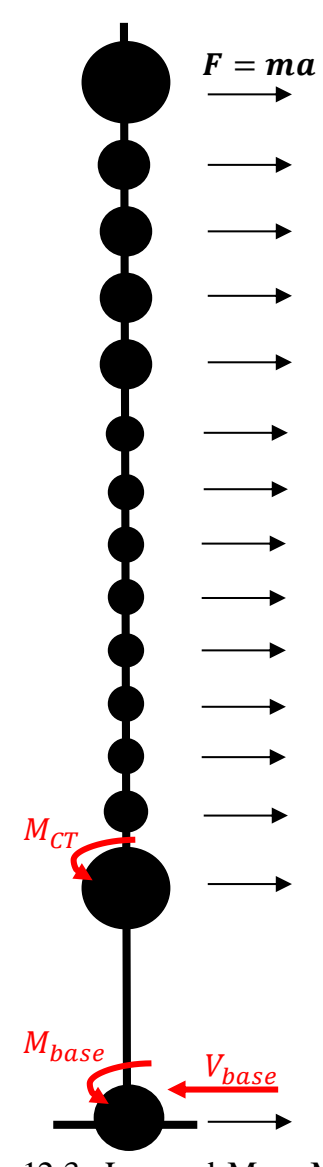

Figure 12-3: Lumped Mass Method

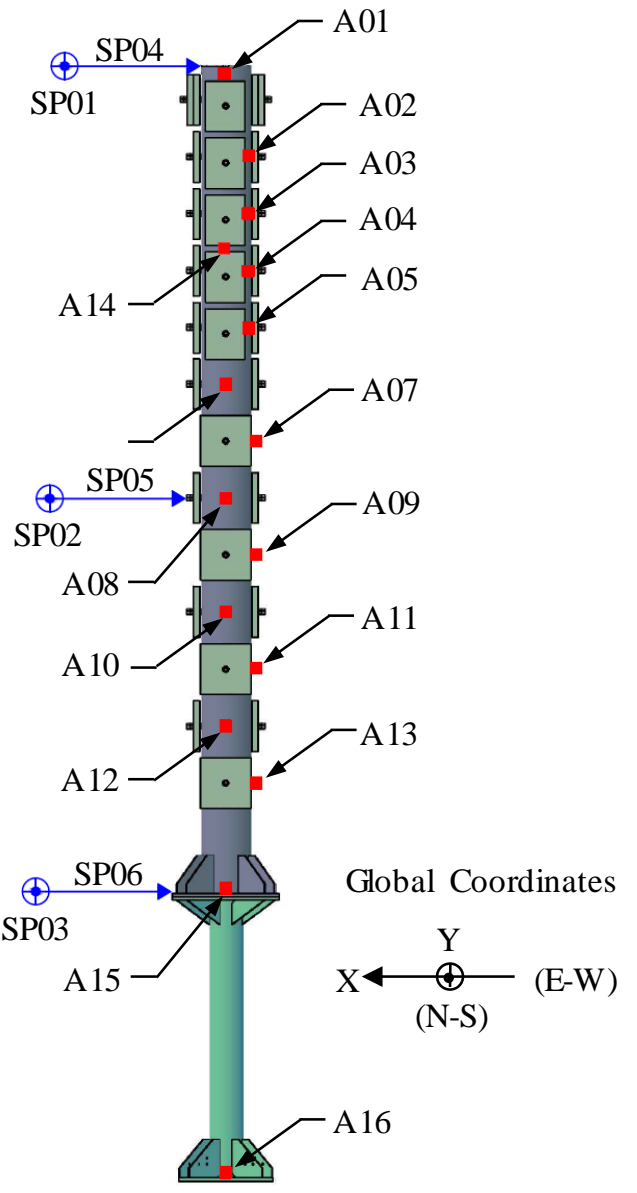

Figure 12-4: Accelerometer \& String Pot Locations 
Table 12-1: Accelerometer and String Pot Locations

\begin{tabular}{|c|c|c|c|c|c|}
\hline & \multirow[t]{2}{*}{ Instrument } & \multirow[t]{2}{*}{ Location (in.) } & \multicolumn{3}{|c|}{$\begin{array}{c}\text { Measured } \\
\text { Direction }\end{array}$} \\
\hline & & & $x$ & $Y$ & Z \\
\hline \multirow{16}{*}{ 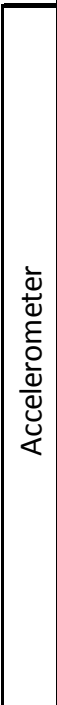 } & $\mathrm{A} 01$ & 351 & $x$ & $x$ & $x$ \\
\hline & $\mathrm{A} 02$ & 322 & $x$ & $x$ & $x$ \\
\hline & $\mathrm{A} 03$ & 304.5 & $x$ & $x$ & $x$ \\
\hline & $\mathrm{A} 04$ & 285.5 & $x$ & $x$ & $x$ \\
\hline & A05 & 268.5 & $x$ & $x$ & $x$ \\
\hline & A06 & 254 & $x$ & $\mathrm{x}$ & $x$ \\
\hline & $\mathrm{A} 07$ & 235 & $x$ & $x$ & $x$ \\
\hline & A08 & 214.5 & $x$ & $x$ & $x$ \\
\hline & A09 & 199 & $x$ & $x$ & $x$ \\
\hline & $\mathrm{A} 10$ & 182 & $x$ & $x$ & $x$ \\
\hline & A11 & 163 & $x$ & $x$ & $x$ \\
\hline & A12 & 146 & $x$ & $x$ & $x$ \\
\hline & A13 & 127 & $x$ & $x$ & $x$ \\
\hline & A14 & 294.5 & $\mathrm{x}$ & $x$ & $x$ \\
\hline & A15 & 91 & $x$ & $x$ & $x$ \\
\hline & A16 & 1.125 & $x$ & $x$ & $x$ \\
\hline \multirow{6}{*}{ 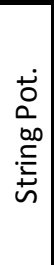 } & SP01 & 352.5 & & $x$ & \\
\hline & SP02 & 216.5 & & $x$ & \\
\hline & SP03 & 91.5 & & $x$ & \\
\hline & SP04 & 352.5 & $x$ & & \\
\hline & SP05 & 215 & $x$ & & \\
\hline & SP06 & 91.5 & $x$ & & \\
\hline
\end{tabular}

Shown in Figure 12-5 is a completely instrumented specimen. Accelerometers could be clearly seen in the image along the length of the CT representative mass.

Illustrated in Figure 12-6 and Figure 12-7 is the base instrumentation for the viscous and hysteretic retrofitted cases. 


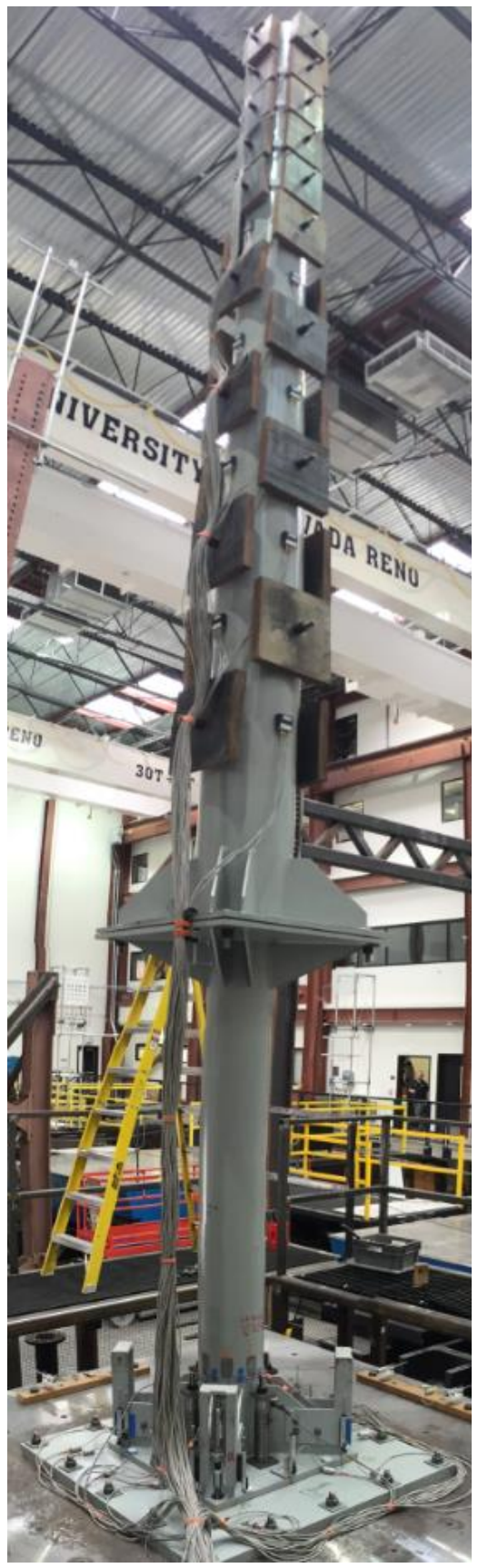

Figure 12-5: Instrumented Specimen

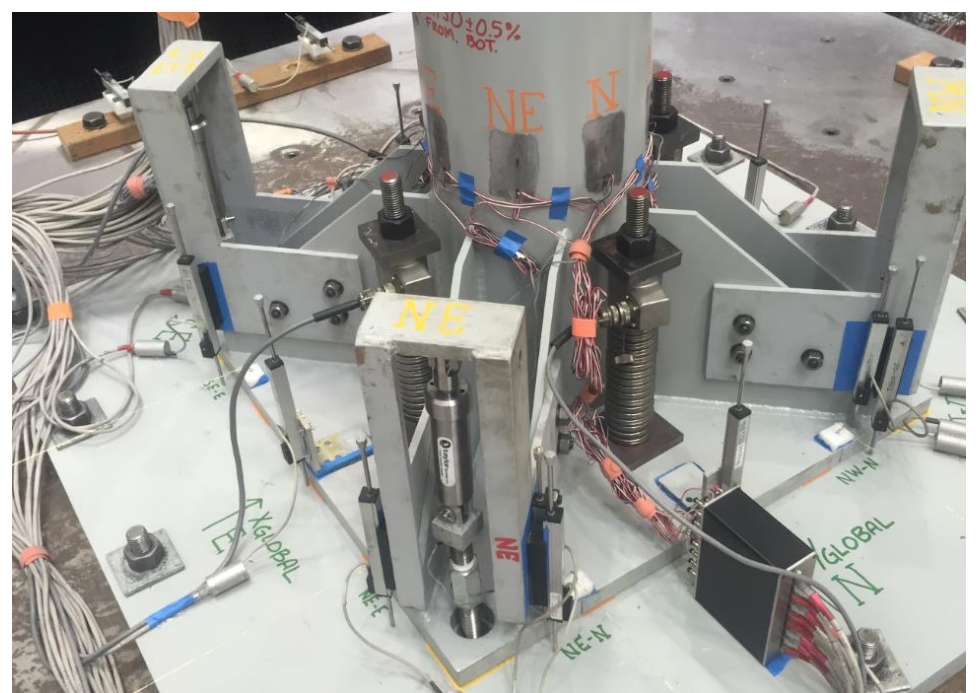

Figure 12-6: Viscous Damper Instrumentation

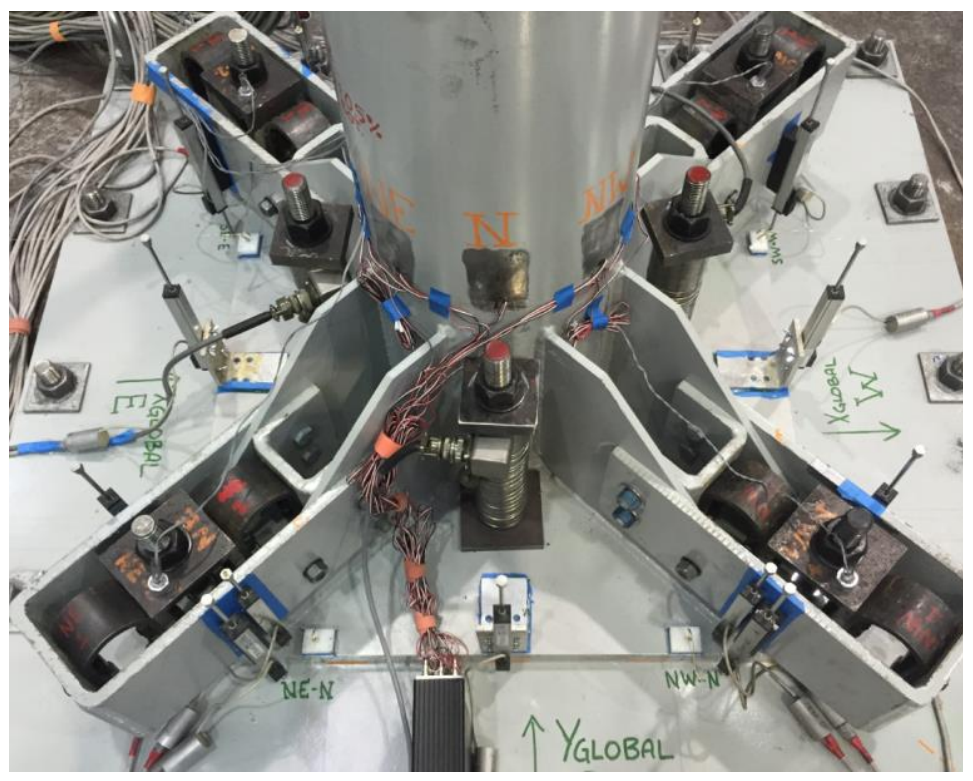

Figure 12-7: Hysteretic UFP Instrumentation

\subsection{Fabricated Pedestal}

A pedestal was fabricated for full-scale testing which included all the additional holes required for the retrofit. The retrofit requires 24 holes to be drilled in order to attach the device housing to the stiffeners. Also, 4 holes are required in the base plate for the 
elastic-self-centering mechanism. A pedestal manufactured by Trench was used in conjunction with the manufacturer's specifications to make detailed drawings of the required modified pedestal. Provided in Appendix A are the Trench drawings for the pedestal provided. Detailed fabrication draws for the modified pedestal are located in Appendix D.

\subsection{Viscous Damper Configuration}

Installed Taylor viscous dampers are shown in Figure 12-8 and Figure 12-9. In order to measure the load in the damper, an instrumented coupler was used to obtain device force readings. Reduced rod length and coupler elimination is suggested in field installation to reduce bending in the anchoring rods.

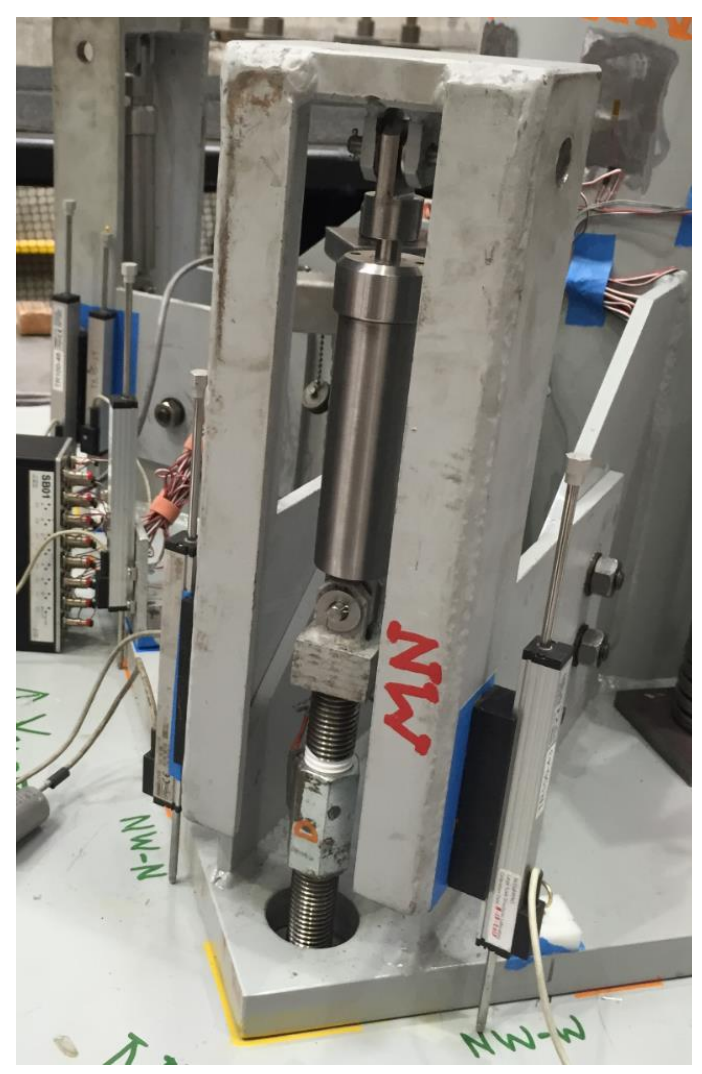

Figure 12-8: NW Viscous Damper 


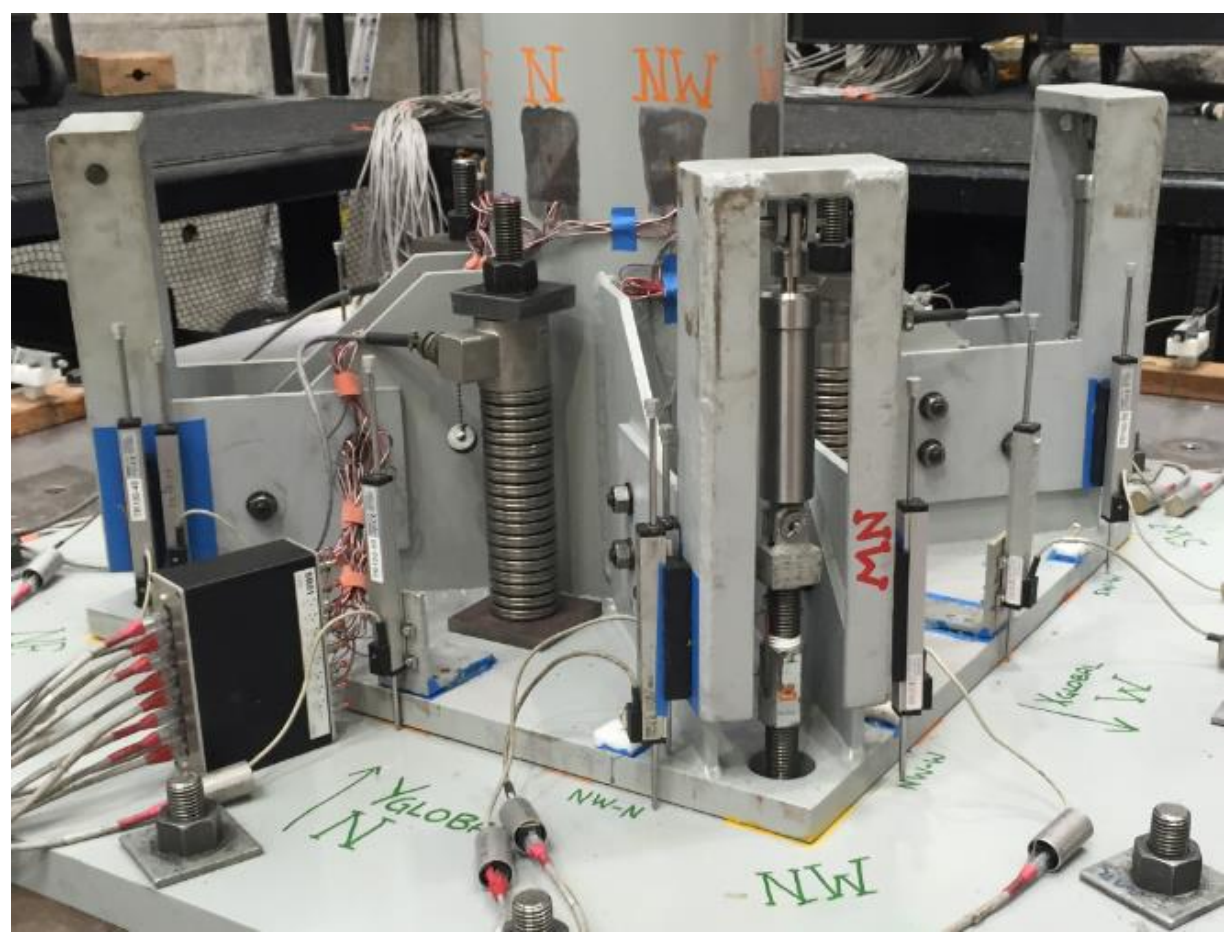

Figure 12-9: Retrofitted Base w/ Taylor Viscous Dampers

\subsection{Hysteretic Damper Configuration}

Application of the UFP system is shown in Figure 12-10 and Figure 12-11. It is important that all sides of the device are able to displace without reacting on the outer walls of the assembly. Centering the UFP unit is recommended when installing the device. Also, all connections must be slip-critical to maximize energy dissipation and minimize slip in the system. 


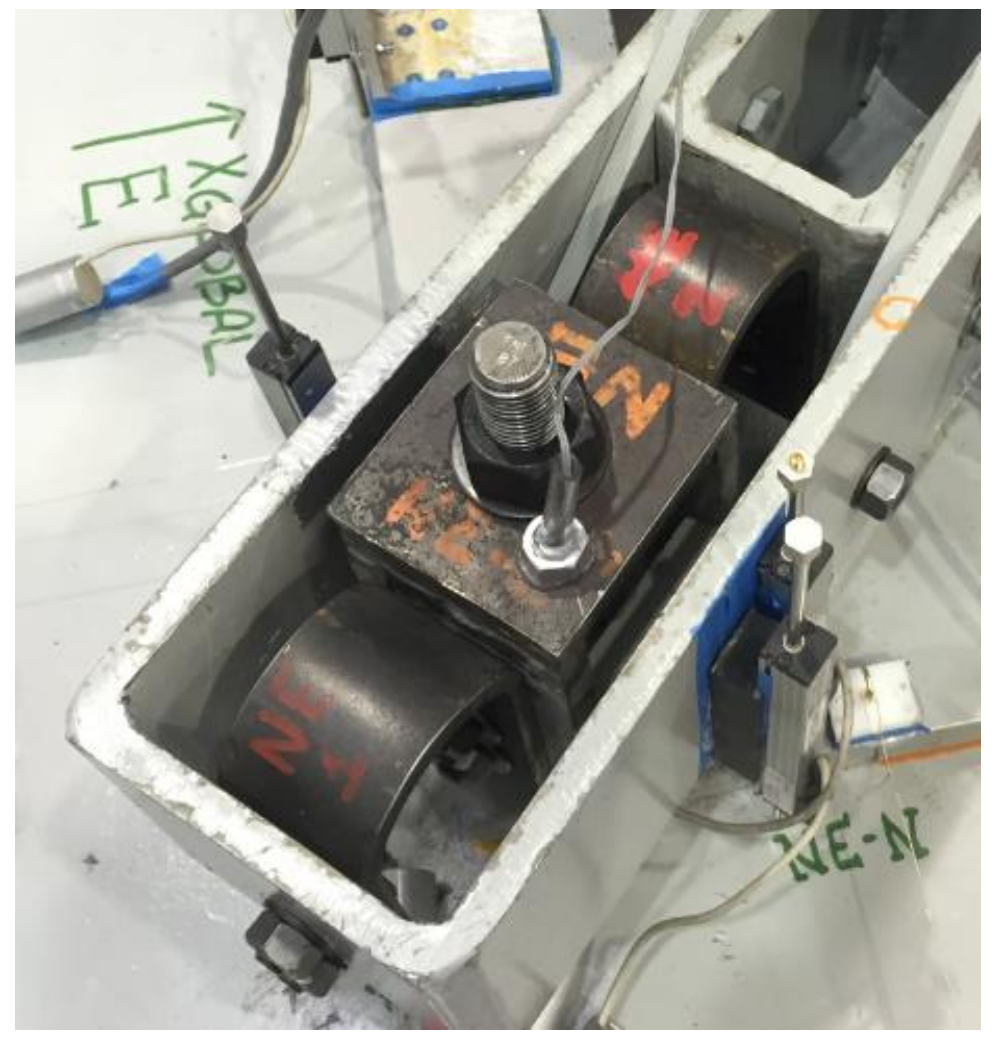

Figure 12-10: Installed Hysteretic UFP

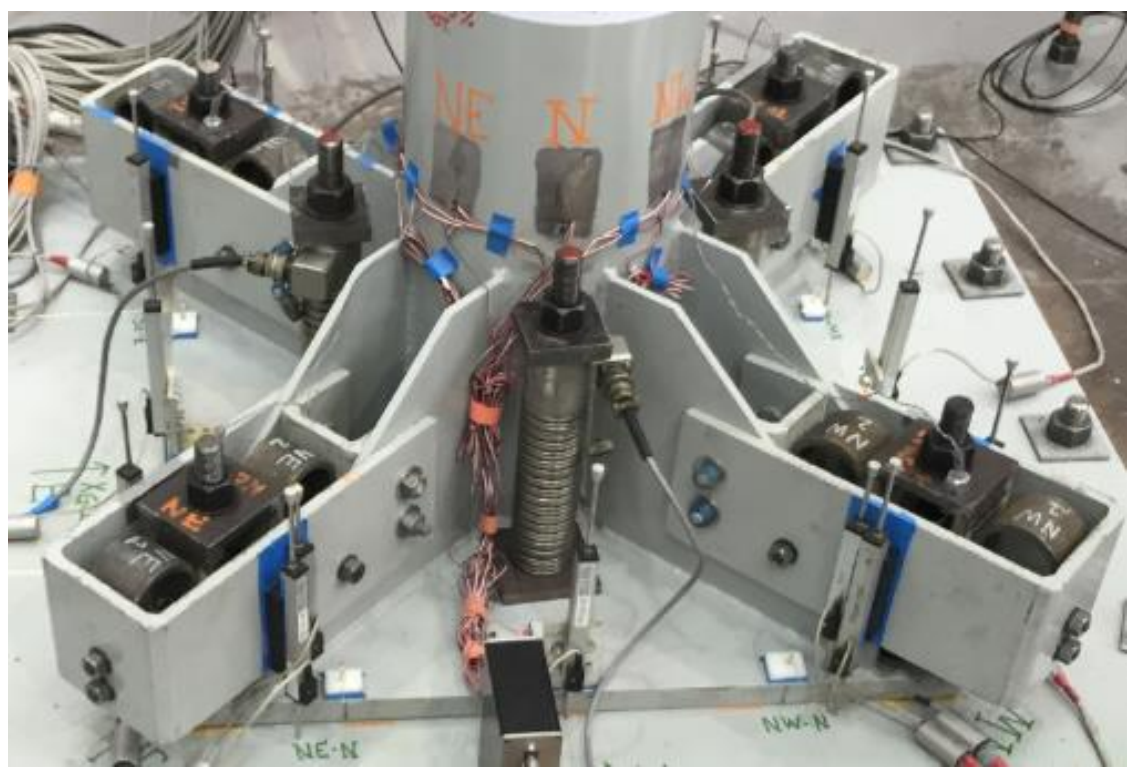

Figure 12-11: Retrofitted Base w/ Hysteretic Device 


\subsection{Non-Retrofitted Configuration}

Shown in Figure 12-12 is the pedestal base without any retrofit devices. The additional holes required for the retrofitted cases were considered to have minimal influence on the dynamic properties of the non-retrofitted case.

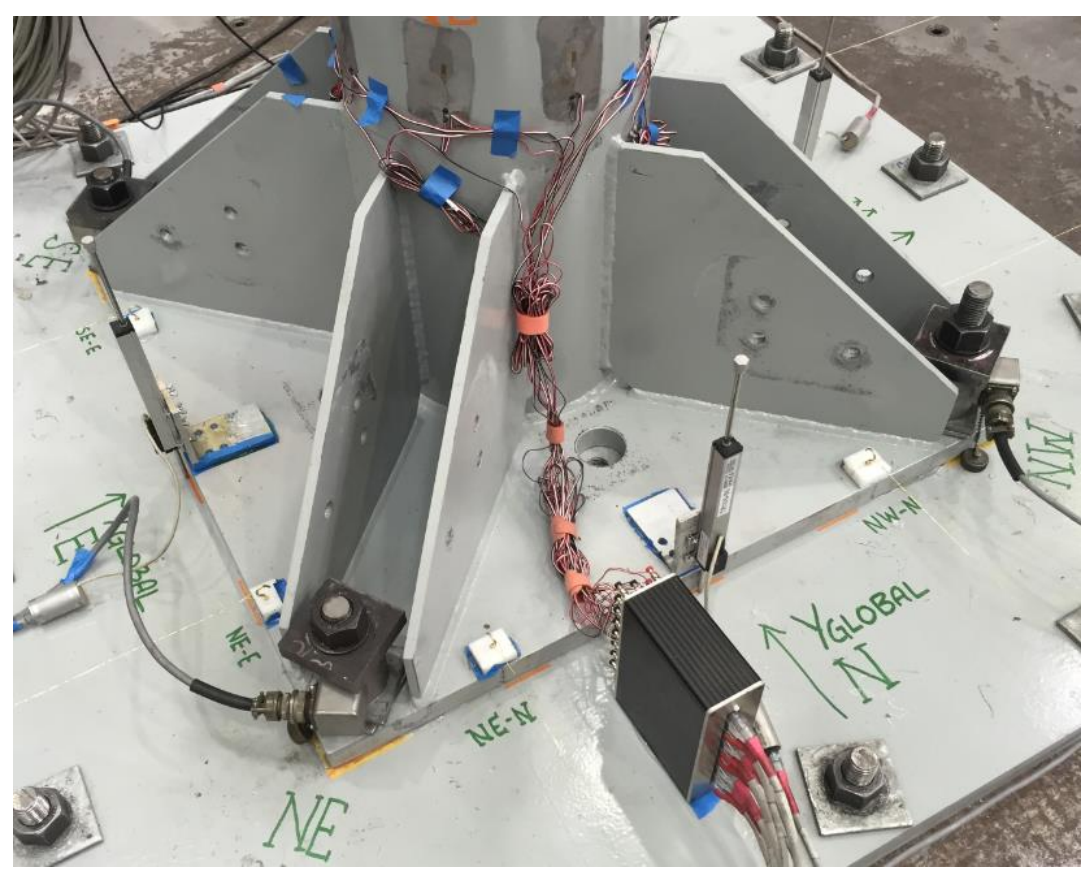

Figure 12-12: Non-Retrofitted Base 


\subsection{Full-Scale Test Results}

After the experimental portion of the full-scale mass system was complete, trends, benefits, and conclusions were determined from the data obtained. Summarized in the following section is the system's response to both types of energy dissipating devices investigated, viscous and hysteretic. In addition, the non-retrofitted base case results are compared to show how acceleration, base reactions, and anchor loads compare to the retrofitted cases.

\subsection{System Properties}

Prior to testing the retrofitted system, the "as-built" system properties were compared to design and equipment properties. Summarized in Table 13-1, Table 13-2, and Table 13-3 are comparisons between the "as-built" mass system, design values, and manufacturer specifications for the actual CT.

Comparing the values for the current transformer, the most variation was in the system weight, shown in Table 13-1. The "as-built" mass system had a weight of 6752 lbs. while the actual 226-293 Type 1800 CT had a weight of $7231 \mathrm{lbs}$. The estimated weight of the designed CT mass was $7240 \mathrm{lbs}$., which is $6.7 \%$ more than the "as-built" weight of the system. The center of gravity varies by $1.7 \%$ between the actual CT and the "as-built" mass system. The largest difference occurred in the natural frequency of the "as-built" mass system, 18\% difference between the manufacturer seismic qualification and the free vibration of the upper mass. Proper anchoring of the upper mass was hard to achieve because the CT mass had a hole spacing different than the lab floor spacing. The clamping likely caused more flexibility in the mass system reducing the fundamental frequency of the "as-built" mass system. Comparing the "as-built" CT-pedestal 
properties, the combined system exhibited behaviors that were very similar to the actual CT on a pedestal.

Table 13-1: Current Transformer Properties

\begin{tabular}{|c|c|c|c|}
\hline \multicolumn{4}{|c|}{ Current Transformer 226-293 Type HGF 1800 Properties } \\
\hline Property & $\begin{array}{c}\text { Manufacture Spec of } \\
\text { Actual Equipment }\end{array}$ & $\begin{array}{c}\text { Calculated for Mass } \\
\text { System }\end{array}$ & $\begin{array}{c}\text { "As-Built" Mass } \\
\text { System }\end{array}$ \\
\hline Center of Gravity (in.) & 152.36 & 149.74 & 149.75 \\
\hline Weight of CT (lb) & 7231 & 7240 & 6752 \\
\hline Natural Frequency (Hz) & 3.78 & 3.79 & 3.10 \\
\hline
\end{tabular}

Next, the pedestal weight and center of gravity was determined and compared to the values specified by the manufacturer drawings. The manufacturer only provides an estimated pedestal weight but center of mass is not specified. Based on the pedestal drawings, a center of mass was calculated, 43.24 inches from the pedestal base. The "asbuilt" pedestal had a C.G. that was $0.6 \%$ less than the calculated C.G.. The weight of the pedestal differed by $2.4 \%$ between the "as-built" and manufacture drawings specifications.

Table 13-2: Pedestal Properties

\begin{tabular}{|c|c|c|c|}
\hline \multicolumn{4}{|c|}{ Pedestal Properties } \\
\hline Property & Manufacture Spec & Calcualted & "As-Built" \\
\hline Center of Gravity (in.) & N/A & 43.24 & 43 \\
\hline Weight of Pedestal (lb.) & 1035 & 1026 & 1010 \\
\hline
\end{tabular}

After assembling the pedestal and upper mass, the systems properties were compared to the results obtained from SAP2000 modeling. The manufacture of the current transformer did not specify a pedestal-CT fundamental frequency that could be compared to the "as-built" system. Using individual component C.G. and mass values, a system C.G. of 217.6 inches was determined for the pedestal-CT system. The "as-built" system had a C.G. of 215.25 inches resulting in a $1 \%$ difference. The combined pedestal- 
CT "as-built" system had a $6 \%$ smaller mass than the sum of the component weights specified by the manufacturer.

Table 13-3: System Properties

\begin{tabular}{|c|c|c|c|c|}
\hline \multicolumn{5}{|c|}{ CT-Pedestal System } \\
\hline Property & Manufacture Spec & $\begin{array}{c}\text { Calculated based on } \\
\text { Manf. Components }\end{array}$ & $\begin{array}{c}\text { Calculated Designed } \\
\text { System }\end{array}$ & "As-Built" \\
\hline System C.G. (in.) & N/A & 217.63 & 213.78 & 215.25 \\
\hline System Total Weight (lb.) & N/A & 8256.55 & 7840 & 7762 \\
\hline Natural Frequency (Hz) & N/A & N/A & 1.24 & 1.24 \\
\hline
\end{tabular}

Minor differences were observed between the manufacturer's specifications and field properties. All the difference presented by the "as-built" system is considered negligible. Elastic damping of the actual CT is unknown and is likely higher than that of the steel mass system damping. Based on the properties obtained, the designed mass system demonstrates similar properties to the actual 226-293 Type 1800 CT Trench current transformer.

\subsection{Test Matrix}

Each retrofit device was evaluated using $20-100 \%$ of the $0.5 \mathrm{~g}$ IEEE693 motion. Prior to each motion, implementation of a system identification series provided the ability for detecting undesired system deterioration and alteration. The system identification series consisted of X-pulse, Y-pulse, Z-pulse, and XYZ white noise. Two increment steps were used in motions scaling, for low amplitude motions up to $60 \%, 10 \%$ increments were utilized. After the $60 \%$ motions, 5\% increment steps were used until $100 \% 0.5 \mathrm{~g}$ motions were achieved.

An extended test matrix was implemented on the hysteretic device retrofit. In order to determine if the repeated increases in amplitude effected the device performance an 
additional test with virgin hysteretic devices was conducted at 100\% 0.5g IEEE693. Also, actual ground records for a crustal and subduction earthquake were used to excite the system. The 6.4 magnitude subduction record that centered in Curicó, Maule, Chile was utilized in $100 \%, 120 \%, 140 \%, 160 \%$, and $200 \%$ amplitudes. Next, a crustal input motion then excited the pedestal-CT system. The Capitola, California earthquake record was scaled by $100 \%, 120 \%$ and $140 \%$.

\subsection{Viscous Damper Retrofit Results}

\subsubsection{Viscous Damper Table Response Spectrum}

Prior to evaluating the effects of the retrofit, a comparison between the shake table feedback and IEEE693 design spectrum was conducted. For equipment seismic qualification, the ground motion must envelope the design spectrum. The retrofits introduced aimed to reduce structure demands and not necessarily qualify the equipment. Shown in Figure 13-1,

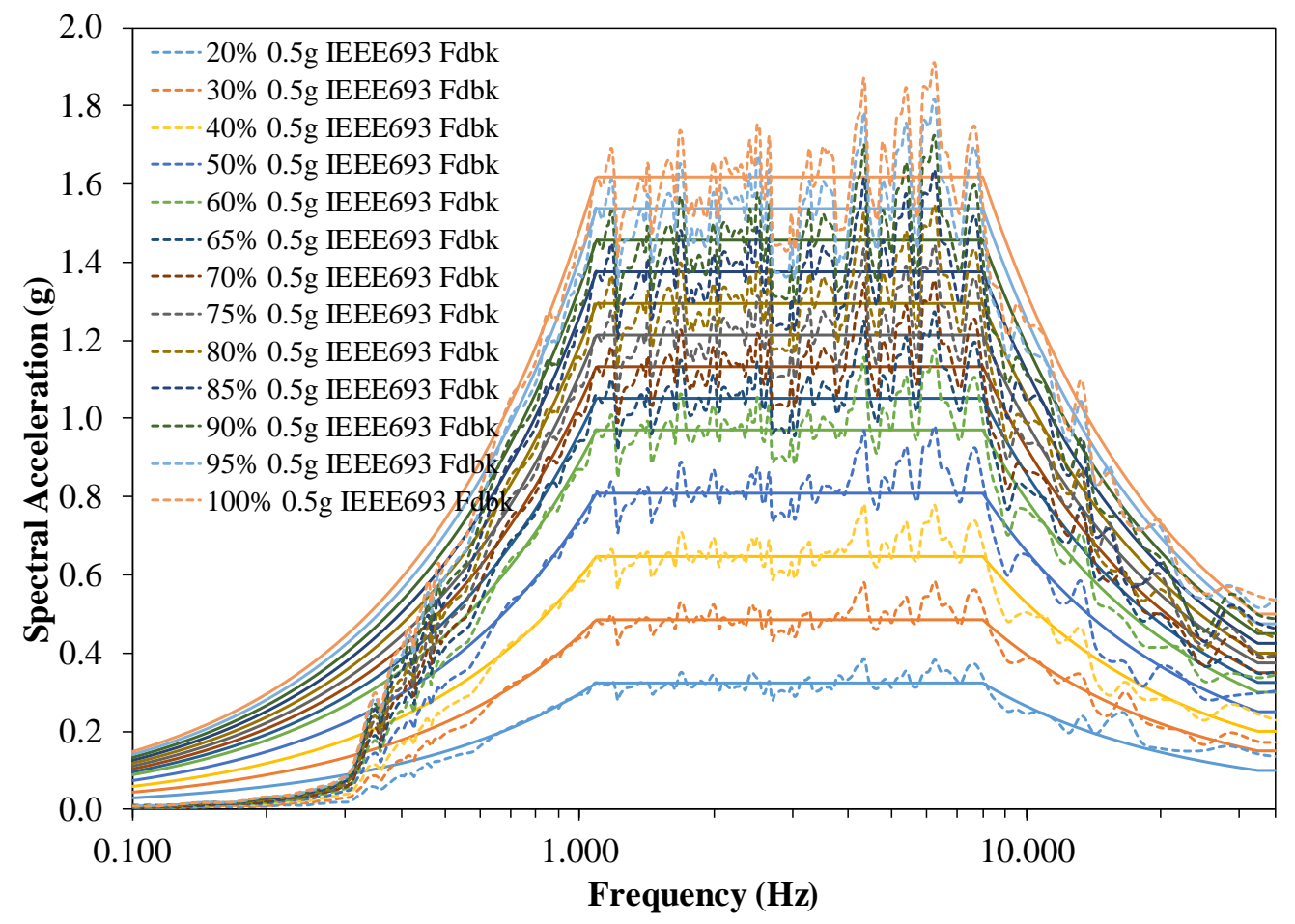

Figure 13-2, and Figure 13-3 is the table response and design spectrum. Low

frequencies are considerably undershot in all directions of the motion. For the two 
horizontal directions, $\mathrm{X}$ and $\mathrm{Y}$, only frequencies between $0.5 \mathrm{~Hz}-1.3 \mathrm{~Hz}$ are of importance.

The non-retrofitted system has a fundamental frequency of $1.24 \mathrm{~Hz}$; reduction in frequency results from system rocking in the retrofitted cases is expected.

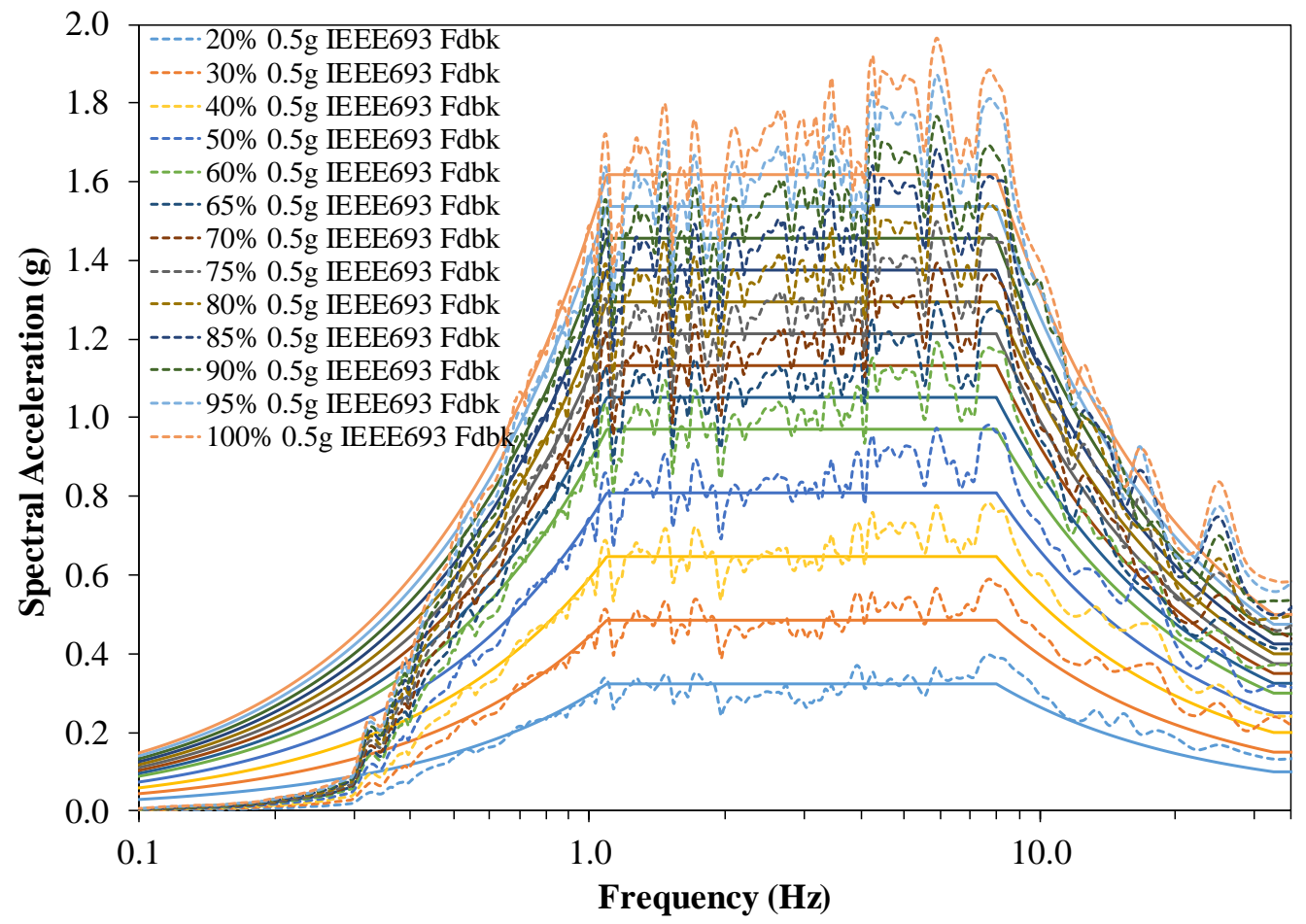

Figure 13-1: Response Spectrum X (Viscous Damper) 


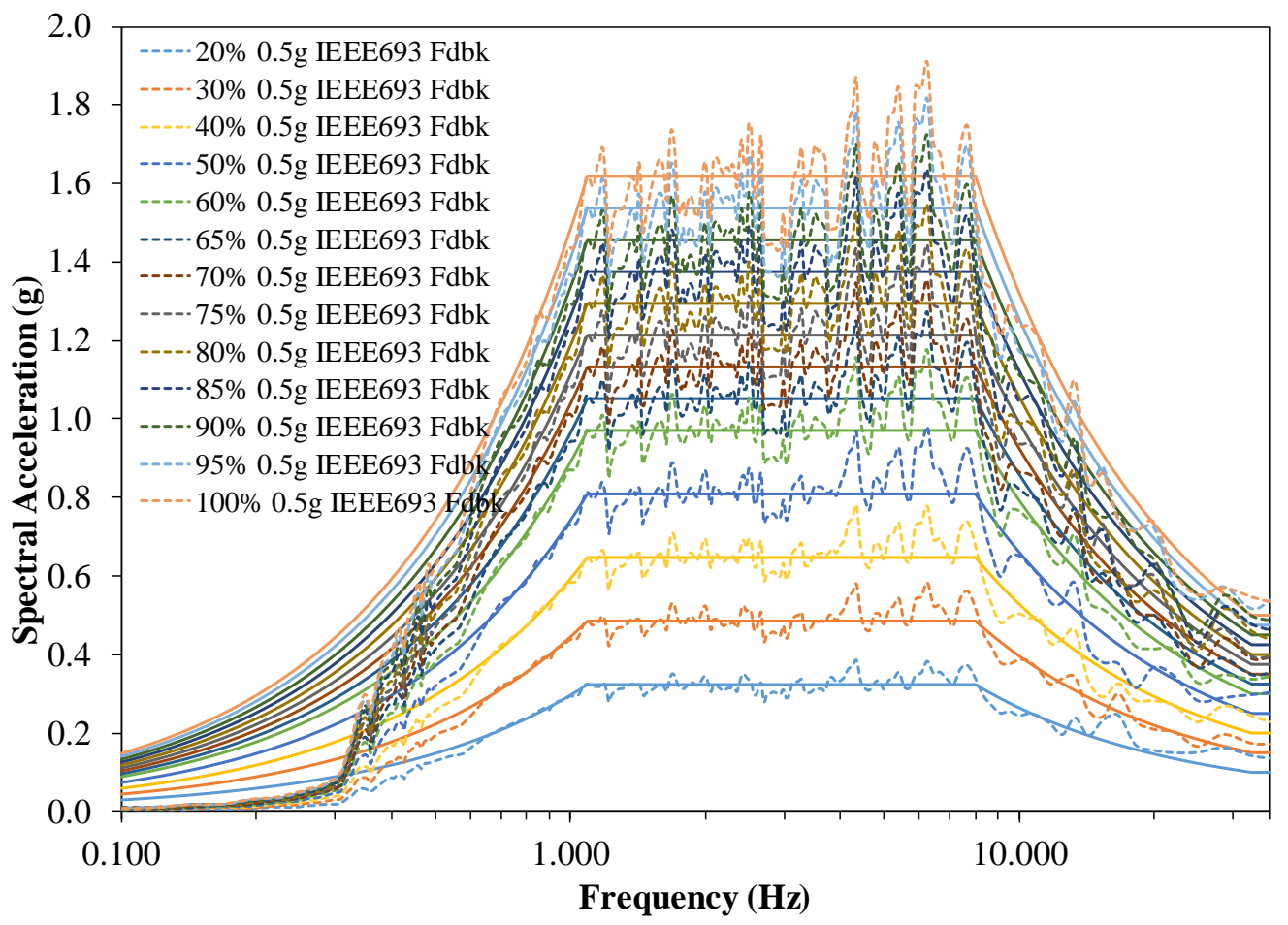

Figure 13-2: Response Spectrum Y (Viscous Damper)

For the $\mathrm{Z}$ direction of the motion, all low frequency content is significantly

undershot. All frequencies below $10 \mathrm{~Hz}$ are of little importance for the system because of its high vertical stiffness. The table response envelopes the $0.5 \mathrm{~g}$ IEEE693 spectrum at all frequencies from $10 \mathrm{~Hz}$ and above. 


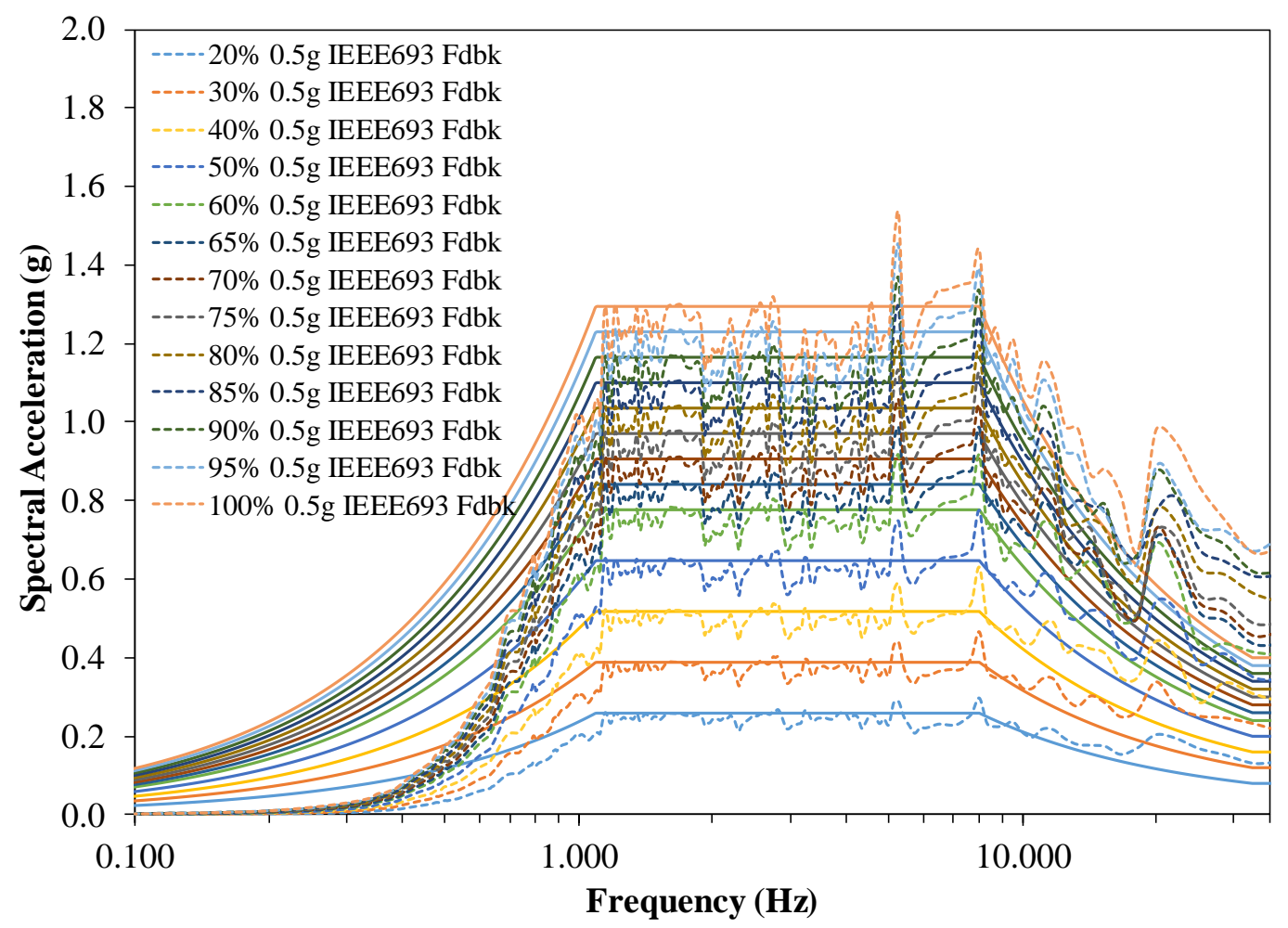

Figure 13-3: Response Spectrum Z (Viscous Damper)

\subsubsection{Viscous Damper System Damping \& Free Vibration}

Utilizing pulse and white noise excitations, system properties were determined prior to each ground motion. Using Fourier analysis the fundamental frequency was determined and recorded in Table 13-4. In addition, elastic damping was calculated using logarithmic decrement and recorded in Table 13-4. Free vibration plots for each test case are located in Appendix G. The system with viscous dampers had an average fundamental frequency of $0.86 \mathrm{~Hz}$ in the $\mathrm{X}$-direction and $0.75 \mathrm{~Hz}$ in the Y-direction. The system had $1 \%-2.5 \%$ damping in the horizontal directions with an average of $1.22 \%$ in the $\mathrm{X}$-direction and $1.84 \%$ in the Y-direction. 
Table 13-4: Viscous Damper -- Retrofit Damping and Fundamental Frequency

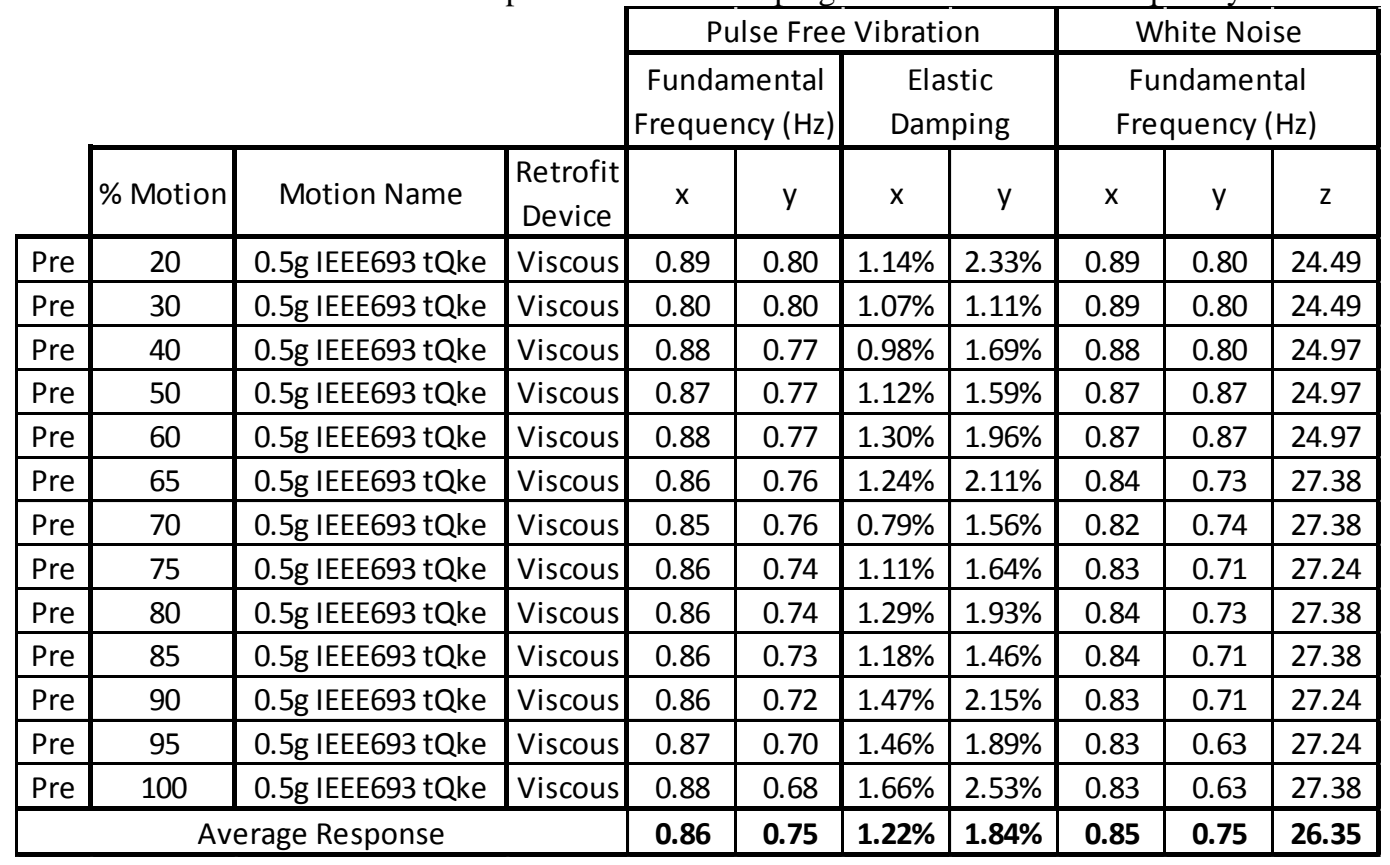

\subsubsection{Viscous Damper System Response}

Summarized in this section is the system response to various amplitudes of the $0.5 \mathrm{~g}$ IEEE693 synthetic ground motion. Metrics of importance include maximum accelerations along the specimen, system forces, and system displacements. In initial phases of the retrofit development, utilizing a SDOF approach simplified calculations and the design procedure. Evaluating the full-scale system's response, solely considering a lumped mass misrepresents the system's behavior.

A summary of the maximum accelerations measured along the specimen length are reported in Table 13-5, Table 13-6, and Table 13-7. The tables present absolute maximum accelerations for each tested IEEE693 amplitude. Maximum acceleration magnitudes are reported for each accelerometer location along the specimen's length. Generally, the viscous damper retrofitted system experienced no vertical amplifications. The input vertical excitation was equal to the amplitude of the response acceleration at 
the top of the specimen. Amplification trends are present in the lateral components of the acceleration data. Throughout the data obtained for the viscous retrofit case, the top three accelerometer nodes present higher acceleration magnitudes than the rest of the system. For all accelerometers located below 304-1/2 inches, the acceleration magnitudes were similar with exception of the base accelerometer.

Table 13-5: Viscous Damper - System X Absolute Maximum Acceleration

\begin{tabular}{|c|c|c|c|c|c|c|c|c|c|c|c|c|c|c|c|}
\hline & \multicolumn{13}{|c|}{$\%$ of IEEE693 0.5g Motion } & & \\
\hline & $20 \%$ & $30 \%$ & $40 \%$ & $50 \%$ & $60 \%$ & $65 \%$ & $70 \%$ & $75 \%$ & $80 \%$ & $85 \%$ & $90 \%$ & $95 \%$ & $100 \%$ & & \\
\hline \multirow{16}{*}{ 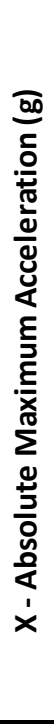 } & 0.23 & 0.37 & 0.47 & 0.58 & 0.66 & 0.67 & 0.71 & 0.84 & 0.85 & 0.82 & 0.85 & 0.78 & 1.02 & 351 & \multirow{16}{*}{ 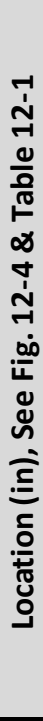 } \\
\hline & 0.18 & 0.29 & 0.37 & 0.42 & 0.49 & 0.51 & 0.56 & 0.61 & 0.59 & 0.58 & 0.62 & 0.64 & 0.82 & 322 & \\
\hline & 0.16 & 0.26 & 0.34 & 0.38 & 0.45 & 0.47 & 0.49 & 0.56 & 0.53 & 0.54 & 0.59 & 0.60 & 0.75 & $3041 / 2$ & \\
\hline & 0.14 & 0.24 & 0.30 & 0.35 & 0.4 & .43 & 0.44 & 0.46 & 0.45 & 0.50 & 0.56 & 0.57 & 0.69 & 285 & \\
\hline & 0.15 & 0.21 & 0.27 & 0.34 & 0.4 & 0.42 & 0.43 & 0.46 & 0.45 & 0.51 & 0.56 & 0.59 & .72 & $2681 / 2$ & \\
\hline & 0.14 & 0.22 & 0.28 & 0.34 & 0.39 & 0.44 & 0.45 & 0.50 & 0.47 & 0.52 & 0.57 & 0.58 & 0.72 & 254 & \\
\hline & 0.15 & 0.25 & 0.29 & 0.35 & 0.43 & 0.42 & 0.46 & 0.52 & 0.45 & 0.56 & 0.66 & 0.64 & 0.69 & 235 & \\
\hline & 0.16 & 0.23 & 0.28 & 0.38 & 0.38 & 0.44 & 0.45 & 0.55 & 0.46 & 0.60 & 0.71 & 0.62 & 0.71 & $2141 / 2$ & \\
\hline & 0.17 & 0.25 & 0.31 & 0.39 & 0.40 & 0.44 & 0.46 & 0.57 & 0.47 & 0.62 & 0.77 & 0.66 & 0.66 & 199 & \\
\hline & 0.18 & 0.24 & 0.30 & 0.42 & 0.43 & 0.46 & 0.48 & 0.61 & 0.48 & 0.67 & 0.88 & 0.67 & 0.68 & 182 & \\
\hline & 0.17 & 0.24 & 0.30 & 0.39 & 0.47 & 0.47 & 0.50 & 0.58 & 0.54 & 0.68 & 0.95 & 0.73 & 0.69 & 163 & \\
\hline & 0.16 & 0.22 & 0.29 & 0.41 & 0.48 & 0.47 & 0.50 & 0.52 & 0.56 & 0.69 & 0.88 & 0.73 & 0.72 & 146 & \\
\hline & 0.17 & 0.21 & 0.30 & 0.42 & 0.49 & 0.47 & 0.50 & 0.51 & 0.59 & 0.64 & 0.81 & 0.84 & 0.81 & 127 & \\
\hline & 0.18 & 0.22 & 0.32 & 0.41 & 0.45 & 0.48 & 0.51 & 0.53 & 0.68 & 0.71 & 0.92 & 0.89 & 0.81 & 91 & \\
\hline & 0.14 & 0.18 & 0.24 & 0.30 & 0.35 & 0.39 & 0.41 & 0.44 & 0.47 & 0.49 & 0.51 & 0.53 & 0.63 & $11 / 8$ & \\
\hline & 0.12 & 0.18 & 0.24 & 0.29 & 0.34 & 0.37 & 0.40 & 0.44 & 0.45 & 0.48 & 0.49 & 0.52 & 0.55 & 0 & \\
\hline
\end{tabular}


Table 13-6: Viscous Damper - System Y Absolute Maximum Acceleration

\begin{tabular}{|c|c|c|c|c|c|c|c|c|c|c|c|c|c|c|c|}
\hline & \multicolumn{13}{|c|}{$\%$ of IEEE693 0.5g Motion } & & \\
\hline & $20 \%$ & $\%$ & $\%$ & 5 & $60 \%$ & $65 \%$ & $70 \%$ & $75 \%$ & $80 \%$ & $85 \%$ & $90 \%$ & $5 \%$ & 1 & & \\
\hline \multirow{16}{*}{ 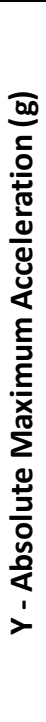 } & 0.31 & 0.41 & 0.49 & 0.57 & 0 & 0.73 & 32 & 0.82 & 1.0 & 1.15 & 1.04 & 1.06 & 1.06 & 351 & \multirow{16}{*}{ 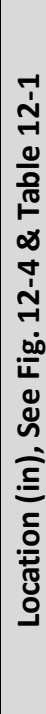 } \\
\hline & 0.26 & 0.36 & 0.41 & 0.51 & -2 & 0.60 & 0.62 & 0.7 & 0.7 & 0.92 & 0.87 & 0.83 & 0.80 & 22 & \\
\hline & 0.23 & 0.31 & 0.39 & 0.45 & 0.48 & 0.53 & 0.58 & 0.61 & 0.45 & 0.73 & 0.72 & 0.70 & 0.70 & $3041 / 2$ & \\
\hline & 0.20 & .28 & 0.35 & 0.41 & 0.45 & 0.49 & 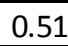 & 0.5 & 0.5 & 0.60 & 0.61 & 0.61 & 0.65 & $2001 / 2$ & \\
\hline & 0.18 & 0.26 & 0.32 & 0.39 & 0.4 & 0.4 & .48 & 0.5 & 0.5 & 0.57 & 0.57 & 0.57 & 0.6 & $2681 / 2$ & \\
\hline & 0.18 & .26 & 0.33 & 0.37 & .4 & 0.42 & .40 & 0.45 & 0.5 & 0.55 & 0.59 & 0.56 & 0.67 & 2 & \\
\hline & 0.19 & 0.26 & 0.34 & 0.36 & 0.44 & 0.45 & 0.49 & 0.52 & 0.5 & 0.61 & 0.62 & 0.55 & 0.67 & 235 & \\
\hline & 0.19 & 0.27 & 0.34 & 0.39 & 0.51 & 0.54 & 0.58 & 0.53 & 0.5 & 0.61 & 0.65 & 0.62 & 0.76 & $2141 / 2$ & \\
\hline & 0.19 & 0.28 & 0.33 & 0.39 & 0.4 & 0.54 & 0.59 & 0.51 & 0.5 & 0.66 & 0.61 & 0.60 & 0.67 & 19 & \\
\hline & 0.20 & 0.28 & 0.33 & 0.41 & 0.52 & 0 & 0.64 & 0. & 0.5 & 0.66 & 0.65 & 0.62 & 0.67 & 1 & \\
\hline & 0.19 & 0.27 & 0.33 & 0.4 & 0 & 0 & 0 & 0 & & 0.68 & 0.61 & 0.63 & 06 & 1 & \\
\hline & 0.19 & 0.2 & 0.33 & 0. & 0.52 & 0. & 0 & 0 & & 0.64 & 0.59 & 0.58 & 0.05 & 146 & \\
\hline & 0.18 & 0.27 & 0.32 & 0.42 & 0. & 0.53 & 0.01 & 0.60 & 0.6 & 0.73 & 0.62 & 0.57 & 0.68 & 127 & \\
\hline & 0.17 & 0.22 & 0.32 & 0.41 & 0.4 & 0.52 & 0.59 & 0.71 & 0 & 0.74 & 0.78 & 0.72 & 0.76 & 91 & \\
\hline & 0.14 & 0.18 & 0.24 & 0.30 & 0.35 & 0.39 & 0.35 & 0.3 & 0. & 0.43 & 0.44 & 0.47 & 0.57 & $11 / 8$ & \\
\hline & 0.11 & 0.14 & 0.20 & 0.24 & 0.30 & 0.32 & 0.35 & 0.35 & 0.3 & 0.40 & 0.43 & 0.44 & 0.46 & 0 & \\
\hline
\end{tabular}

Table 13-7: Viscous Damper - System Z Absolute Maximum Acceleration

\begin{tabular}{|c|c|c|c|c|c|c|c|c|c|c|c|c|c|c|c|}
\hline & \multicolumn{13}{|c|}{$\%$ of IEEE693 0.5g Motion } & & \\
\hline & $20 \%$ & $30 \%$ & $40 \%$ & $50 \%$ & $60 \%$ & $65 \%$ & $70 \%$ & $75 \%$ & $80 \%$ & $85 \%$ & $90 \%$ & $95 \%$ & $100 \%$ & & \\
\hline \multirow{16}{*}{ 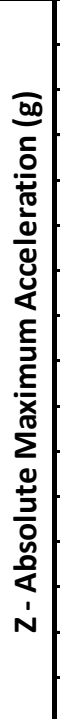 } & 0.13 & 0.20 & 0.27 & 0.33 & 0.40 & 0.43 & 0.49 & 0.52 & 0.55 & 0.57 & 0.64 & 0.65 & 0.67 & 351 & \multirow{16}{*}{ 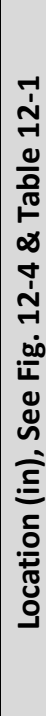 } \\
\hline & 0.13 & 0.21 & 0.28 & 0.33 & 0.41 & 0.45 & 0.50 & 0.51 & 0.56 & 0.59 & 0.64 & 0.66 & 0.67 & 322 & \\
\hline & 0.13 & 0.22 & 0.28 & 0.33 & 0.44 & 0.44 & 0.46 & 0.51 & 0.58 & 0.59 & 0.63 & 0.64 & 0.67 & $3041 / 2$ & \\
\hline & 0.13 & 0.21 & 0.28 & 0.34 & 0.41 & 0.45 & 0.50 & 0.51 & 0.56 & 0.58 & 0.63 & 0.65 & 0.66 & $2851 / 2$ & \\
\hline & 0.14 & 0.21 & 0.28 & 0.33 & 0.42 & 0.45 & 0.47 & 0.51 & 0.57 & 0.60 & 0.64 & 0.65 & 0.67 & $2681 / 2$ & \\
\hline & 0.13 & 0.20 & 0.28 & 0.33 & 0.40 & 0.44 & 0.49 & 0.51 & 0.55 & 0.57 & 0.64 & 0.64 & 0.68 & 254 & \\
\hline & 0.14 & 0.21 & 0.29 & 0.34 & 0.42 & 0.45 & 0.49 & 0.51 & 0.56 & 0.59 & 0.64 & 0.66 & 0.68 & 235 & \\
\hline & 0.13 & 0.20 & 0.29 & 0.33 & 0.41 & 0.44 & 0.49 & 0.50 & 0.55 & 0.58 & 0.64 & 0.65 & 0.69 & $2141 / 2$ & \\
\hline & 0.13 & 0.21 & 0.28 & 0.33 & 0.40 & 0.44 & 0.49 & 0.51 & 0.55 & 0.58 & 0.63 & 0.65 & 0.68 & 199 & \\
\hline & 0.13 & 0.20 & 0.28 & 0.34 & 0.41 & 0.45 & 0.49 & 0.52 & 0.55 & 0.59 & 0.64 & 0.64 & 0.69 & 182 & \\
\hline & 0.15 & 0.21 & 0.28 & 0.33 & 0.40 & 0.45 & 0.48 & 0.51 & 0.54 & 0.58 & 0.64 & 0.64 & 0.68 & 163 & \\
\hline & 0.14 & 0.19 & 0.28 & 0.33 & 0.41 & 0.46 & 0.51 & 0.51 & 0.55 & 0.58 & 0.63 & 0.66 & 0.68 & 146 & \\
\hline & 0.13 & 0.19 & 0.28 & 0.34 & 0.39 & 0.45 & 0.50 & 0.51 & 0.53 & 0.57 & 0.62 & 0.65 & 0.67 & 127 & \\
\hline & 0.14 & 0.21 & 0.28 & 0.33 & 0.39 & 0.43 & 0.48 & 0.51 & 0.54 & 0.57 & 0.60 & 0.65 & 0.67 & 91 & \\
\hline & 0.13 & 0.20 & 0.27 & 0.33 & 0.38 & 0.41 & 0.45 & 0.46 & 0.50 & 0.54 & 0.56 & 0.60 & 0.64 & $11 / 8$ & \\
\hline & 0.12 & 0.19 & 0.26 & 0.31 & 0.37 & 0.40 & 0.44 & 0.46 & 0.50 & 0.53 & 0.56 & 0.60 & 0.64 & 0 & \\
\hline
\end{tabular}

Using the methods discussed in 12.1, Instrumentation, accelerometer

measurements allowed for base shear and moment at specific locations of the system to

be determined. Summarized in Table 13-8 are moment and shear values at two locations 
on the specimen. The first area of interest is the maximum moment in the pedestal base and the second location of interested is the pedestal-CT interface. The maximum moment seen between both measurement methods was $1079 \mathrm{kip}$-in. for the $100 \% 0.5 \mathrm{~g}$ IEEE693 motion. Only the acceleration methods discussed in 12.1, Instrumentation, allowed calculating base shear in the specimen because of the strain gauge orientation on the specimen. The maximum base shear in the primary direction was 4 kips associated with a moment of 1039 kip-in. A base shear of 5.2 kips is estimated in the X'-direction associated with the maximum moment. Based on the section modulus and standard $42 \mathrm{ksi}$ grade steel, the expected nominal yield moment is $1205 \mathrm{kip}$-in. The maximum moment exerted on the CT interface was 681 kip-in.

Table 13-8: Viscous Damper - System Absolute Maximum Reactions

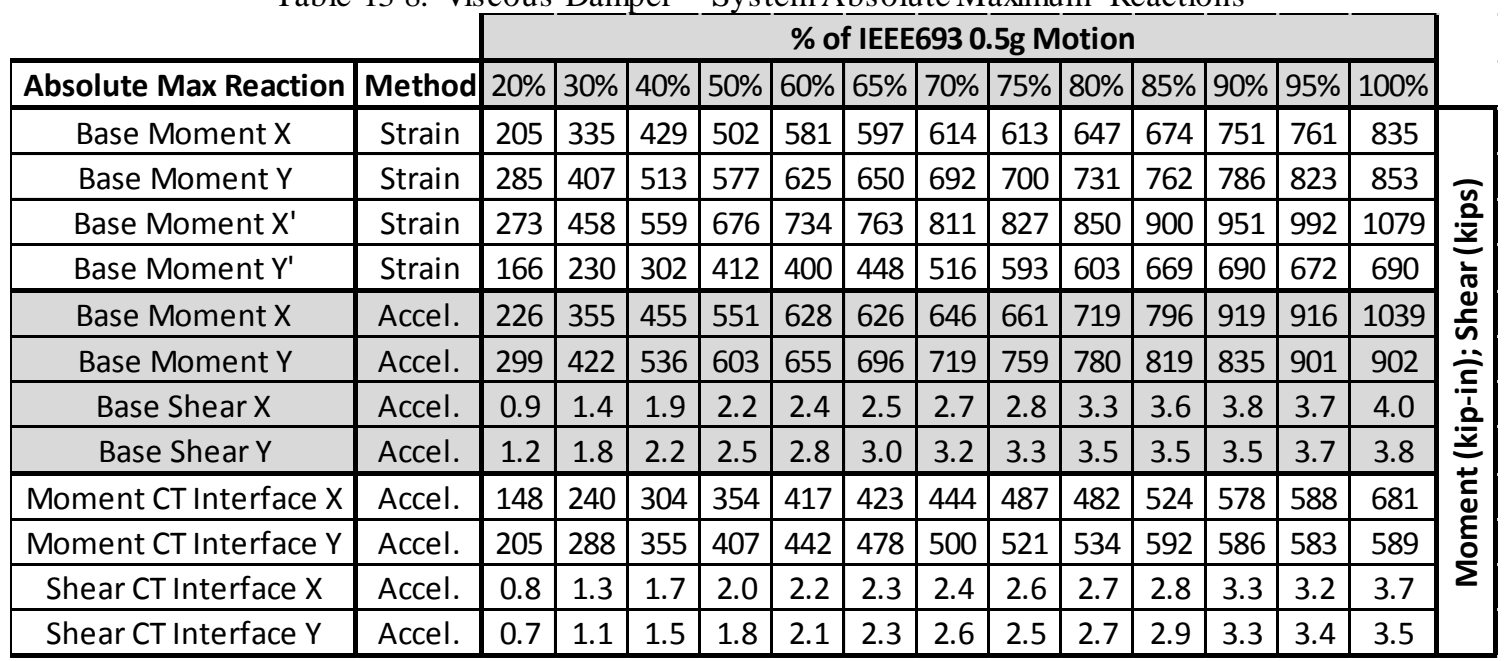

(See Figure 12-2, Strain Gauge Placement for $\left.(X, Y) \&\left(X^{\prime}, Y^{\prime}\right)\right)$

System relative displacement was another important factor used for determining the effectiveness of the retrofit measure. Shown in Table 13-9 and Table 13-10 are the relative displacements between the point of interest and the shake table. Top of Pedestal, 
modeled CT C.G., and top of specimen measurements are reported. For the $100 \%$ motion, the top of the modeled CT experienced a 21.7 inch maximum displacement.

Table 13-9: Viscous Damper - System Maximum Displacement 20-65\% Motions

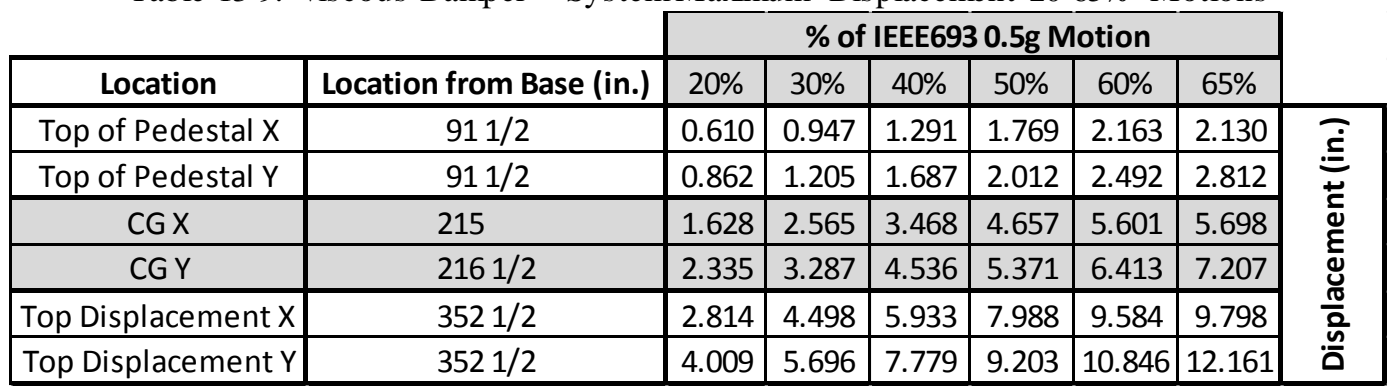

Table 13-10: Viscous Damper - System Maximum Displacement 70-100\% Motions

\begin{tabular}{|c|c|c|c|c|c|c|c|c|c|}
\hline & & \multicolumn{7}{|c|}{ \% of IEEE693 0.5g Motion } & \\
\hline Location & Location from Base (in.) & $70 \%$ & $75 \%$ & $80 \%$ & $85 \%$ & $90 \%$ & $95 \%$ & $100 \%$ & \\
\hline Top of Pedestal X & $911 / 2$ & 2.202 & 2.847 & 3.493 & 3.876 & 4.508 & 4.738 & 5.137 & $\bar{\varepsilon}$ \\
\hline Top of Pedestal Y & $911 / 2$ & 3.024 & 3.441 & 3.867 & 4.119 & 4.421 & 4.638 & 4.945 & \\
\hline CGX & 215 & 5.899 & 7.155 & 8.710 & 9.669 & 11.210 & 11.759 & 12.819 & \\
\hline CG Y & $2161 / 2$ & 7.757 & 8.785 & 9.875 & 10.510 & 11.293 & 11.847 & 12.612 & प्र \\
\hline Top Displacement X & $3521 / 2$ & 10.139 & 12.186 & 14.766 & 16.360 & 18.970 & 19.867 & 21.707 & 읍 \\
\hline Top Displacement Y & $3521 / 2$ & 13.078 & 14.827 & 16.690 & 17.741 & 19.048 & 19.985 & 21.239 & $\ddot{0}$ \\
\hline
\end{tabular}

To investigate the true self-centering abilities of the designed retrofit, post-motion system position is reported in Table 13-11 and Table 13-12. The largest residual displacements of 0.22 inch is present for the $100 \%$ motion. The residual displacement equates to a $0.035^{\circ}$ angle. The tested system with viscous dampers presented true selfcentering with negligible residual displacements.

Table 13-11: Viscous Damper - System Residual Dis placement 20-65\% Motions

\begin{tabular}{|c|c|c|c|c|c|c|c|c|}
\hline & & \multicolumn{6}{|c|}{$\%$ of IEEE693 0.5g Motion } & \\
\hline Location & Location from Base (in.) & $20 \%$ & $30 \%$ & $40 \%$ & $50 \%$ & $60 \%$ & $65 \%$ & \\
\hline Top of Pedestal X & $911 / 2$ & -0.008 & 0.005 & -0.005 & 0.002 & 0.016 & 0.023 & \\
\hline Top of Pedestal $Y$ & $911 / 2$ & 0.002 & 0.008 & 0.009 & 0.015 & 0.006 & 0.011 & \\
\hline CGX & 215 & -0.032 & 0.039 & -0.010 & 0.014 & 0.038 & 0.056 & \\
\hline CG Y & $2161 / 2$ & -0.005 & 0.030 & 0.005 & 0.020 & 0.006 & 0.016 & ע \\
\hline Top Displacement X & $3521 / 2$ & -0.048 & 0.068 & 0.000 & 0.022 & 0.065 & 0.078 & \\
\hline Top Displacement Y & $3521 / 2$ & -0.034 & 0.046 & -0.018 & 0.009 & 0.003 & -0.010 & 光 \\
\hline
\end{tabular}


Table 13-12: Viscous Damper - System Residual Displacement 70-100\% Motions

\begin{tabular}{|c|c|c|c|c|c|c|c|c|c|}
\hline & & \multicolumn{7}{|c|}{ \% of IEEE693 0.5g Motion } & \\
\hline Location & Location from Base (in.) & $70 \%$ & $75 \%$ & $80 \%$ & $85 \%$ & $90 \%$ & $95 \%$ & $100 \%$ & \\
\hline Top of Pedestal X & $911 / 2$ & 0.019 & 0.000 & -0.002 & 0.020 & 0.051 & 0.055 & 0.069 & $\overline{\dot{\varepsilon}}$ \\
\hline Top of Pedestal Y & $911 / 2$ & 0.016 & 0.013 & 0.006 & 0.017 & 0.008 & 0.000 & 0.010 & \\
\hline CG $X$ & 215 & 0.033 & 0.015 & 0.008 & 0.022 & 0.131 & 0.101 & 0.129 & \\
\hline CG Y & $2161 / 2$ & 0.026 & 0.018 & 0.017 & 0.039 & 0.022 & 0.020 & 0.022 & \\
\hline Top Displacement X & $3521 / 2$ & 0.042 & 0.013 & 0.036 & 0.029 & 0.213 & 0.163 & 0.220 & $\frac{0}{n}$ \\
\hline Top Displacement Y & $3521 / 2$ & -0.008 & 0.021 & 0.017 & -0.005 & 0.007 & -0.001 & 0.019 & \\
\hline
\end{tabular}

Pedestal strain gauges allow for determining yielding initiation in the support pedestal. Nominal yielding is to occur at $42 \mathrm{ksi}$ or 1450 micro strain in extreme fibers of the pedestal. All motions over $90 \%$ caused strains in the pedestal beyond 1450 micro strain in at least a single strain gauge on the pedestal. A complete summary of absolute maximum strain readings are presented in Table 13-13.

Table 13-13: Viscous Damper - Pedestal Absolute Maximum Strain

\begin{tabular}{|c|c|c|c|c|c|c|c|c|c|c|c|c|c|}
\cline { 2 - 12 } & \multicolumn{10}{c|}{ \% of IEEE63 0.5g Motion } \\
\hline Pedestal Strain $(\mu \varepsilon)$ & $20 \%$ & $30 \%$ & $40 \%$ & $50 \%$ & $60 \%$ & $65 \%$ & $70 \%$ & $75 \%$ & $80 \%$ & $85 \%$ & $90 \%$ & $95 \%$ & $100 \%$ \\
\hline North Gauge & 358 & 525 & 666 & 731 & 789 & 822 & 872 & 877 & 873 & 877 & 896 & 947 & 1011 \\
\hline South Gauge & 296 & 448 & 570 & 626 & 730 & 766 & 819 & 886 & 966 & 1002 & 1026 & 1076 & 1109 \\
\hline NE Gauge & 219 & 285 & 356 & 485 & 620 & 663 & 723 & 714 & 735 & 924 & 1091 & 1061 & 1072 \\
\hline SW Gauge & 235 & 320 & 458 & 653 & 620 & 702 & 820 & 943 & 960 & 1072 & 1112 & 1056 & 1087 \\
\hline East Gauge & 190 & 308 & 429 & 519 & 595 & 578 & 586 & 617 & 705 & 729 & 814 & 813 & 894 \\
\hline West Gauge & 257 & 435 & 558 & 658 & 755 & 781 & 805 & 804 & 762 & 693 & 769 & 795 & 870 \\
\hline SE Gauge & 393 & 722 & 883 & 1013 & 1115 & 1135 & 1205 & 1243 & 1266 & 1308 & 1409 & 1474 & 1635 \\
\hline NW Gauge & 410 & 672 & 865 & 1068 & 1165 & 1205 & 1279 & 1305 & 1346 & 1426 & 1508 & 1575 & 1726 \\
\hline
\end{tabular}

Residual strain from each ground motion provides a metric for comparing the demand exerted on the pedestal during the motion with the specific retrofit. Shown in Table 13-14 are the residual strains in the pedestal for each ground motion. All motions under $90 \%$ had residual strains up to $8 \mu \varepsilon$. The slight shift in strain readings is likely due to slight reposition in the mass positions after each ground motion. 
Table 13-14: Viscous Damper - Pedestal Residual Strain

\begin{tabular}{|c|c|c|c|c|c|c|c|c|c|c|c|c|c|}
\cline { 2 - 17 } & \multicolumn{10}{c|}{ \% of IEEE93 0.5g Motion } \\
\hline Pedestal Strain $(\mu \varepsilon)$ & $20 \%$ & $30 \%$ & $40 \%$ & $50 \%$ & $60 \%$ & $65 \%$ & $70 \%$ & $75 \%$ & $80 \%$ & $85 \%$ & $90 \%$ & $95 \%$ & $100 \%$ \\
\hline North Gauge & 2 & -6 & 3 & 2 & 0 & 0 & 3 & 2 & 1 & 5 & 7 & 10 & 16 \\
\hline South Guage & -4 & 4 & 1 & 6 & -1 & 2 & 1 & 5 & 9 & 10 & 6 & 6 & 12 \\
\hline NE Gauge & 9 & -6 & 2 & -1 & -2 & -2 & -2 & -1 & 2 & 2 & 9 & 0 & 7 \\
\hline SW Gauge & -6 & 12 & 2 & 5 & 2 & 1 & 2 & 4 & 2 & 1 & -3 & 2 & 4 \\
\hline East Gauge & 7 & -6 & 0 & -5 & 5 & 1 & 0 & 2 & 3 & 5 & 11 & 6 & 8 \\
\hline West Gauge & -6 & 10 & 1 & 6 & 6 & 4 & 7 & 6 & 3 & 4 & 4 & 7 & 14 \\
\hline SE Gauge & 3 & -4 & 0 & 0 & 3 & 3 & 3 & 6 & 7 & 5 & 11 & 13 & 24 \\
\hline NW Gauge & -2 & 4 & 4 & 9 & 7 & 4 & 8 & 5 & 4 & 8 & 12 & 16 & 28 \\
\hline
\end{tabular}

Maximum uplift of the damper was determined for each ground motion, Table

13-15. The largest uplift experienced by the NW viscous damper measured 1.29 inches.

All quadrants of the base had similar magnitude uplift values ranging from 1.07 inches -

1.29 inches. In all cases, the system returned to its initial position and the devices had no residual displacements, Table 13-16.

Table 13-15: Viscous Damper - Damper Absolute Maximum Displacement

\begin{tabular}{|c|c|c|c|c|c|c|c|c|c|c|c|c|c|}
\hline & \multicolumn{13}{|c|}{$\%$ of IEEE693 0.5g Motion } \\
\hline $\begin{array}{c}\text { Device } \\
\text { Dispalcement }\end{array}$ & $20 \%$ & $30 \%$ & $40 \%$ & $50 \%$ & $60 \%$ & $65 \%$ & $70 \%$ & $75 \%$ & $80 \%$ & $85 \%$ & $90 \%$ & $95 \%$ & $100 \%$ \\
\hline NE & 0.12 & 0.15 & 0.25 & 0.35 & 0.51 & 0.59 & 0.64 & 0.76 & 0.87 & 0.92 & 1.01 & 1.04 & 1.11 \\
\hline SE & 0.12 & 0.19 & 0.31 & 0.44 & 0.58 & 0.62 & 0.68 & 0.73 & 0.81 & 0.90 & 1.02 & 1.08 & 1.18 \\
\hline SW & 0.13 & 0.18 & 0.29 & 0.36 & 0.44 & 0.47 & 0.51 & 0.55 & 0.71 & 0.80 & 0.97 & 1.00 & 1.07 \\
\hline NW & 0.14 & 0.20 & 0.32 & 0.45 & 0.58 & 0.65 & 0.70 & 0.81 & 0.92 & 0.99 & 1.11 & 1.19 & 1.29 \\
\hline
\end{tabular}

Table 13-16: Viscous Damper - Damper Residual Displacement

\begin{tabular}{|c|c|c|c|c|c|c|c|c|c|c|c|c|c|}
\hline & \multicolumn{13}{|c|}{$\%$ of IEEE693 $0.5 \mathrm{~g}$ Motion } \\
\hline $\begin{array}{c}\text { Device } \\
\text { Dispalcement }\end{array}$ & $20 \%$ & $30 \%$ & $40 \%$ & $50 \%$ & $60 \%$ & $65 \%$ & $70 \%$ & $75 \%$ & $80 \%$ & $85 \%$ & $90 \%$ & $95 \%$ & $100 \%$ \\
\hline $\mathrm{NE}$ & 0.00 & 0.00 & 0.00 & 0.00 & 0.00 & 0.00 & 0.00 & 0.00 & 0.00 & 0.00 & 0.01 & 0.01 & 0.01 \\
\hline SE & 0.00 & 0.00 & 0.00 & 0.00 & 0.00 & 0.00 & 0.00 & 0.00 & 0.00 & 0.00 & 0.01 & 0.01 & 0.01 \\
\hline SW & 0.00 & 0.00 & 0.00 & 0.00 & 0.00 & 0.00 & 0.00 & 0.00 & 0.00 & 0.00 & -0.01 & -0.01 & -0.01 \\
\hline NW & 0.00 & 0.00 & 0.00 & 0.00 & 0.00 & 0.00 & 0.00 & 0.00 & 0.01 & 0.01 & 0.00 & 0.01 & 0.01 \\
\hline
\end{tabular}

Also, force displacement curves were constructed for $20 \%, 50 \%, 75 \%$, and $100 \%$

motions. Shown in Figure 13-4 is a force-displacement hysteresis for the Y-direction of 
the $100 \%$ test case. The system presents frequency reduction shift due to stiffness reduction after rocking is initiated, energy dissipation, and self-centering. A complete set of force-displacement curves for each device on the retrofit is located in Appendix $\mathrm{H}$. The largest damper force was present in the NW device, 7.43 kips. Since the viscous devices are velocity dependent and non-linear, the load is not directly proportional to the displacement felt by the device.

Table 13-17: Viscous Damper - Damper Maximum Force

\begin{tabular}{|c|c|c|c|c|c|c|c|c|c|c|c|c|c|}
\cline { 2 - 14 } \multicolumn{1}{c|}{} & \multicolumn{10}{c|}{ \% of IEEE693 0.5g Motion } \\
\hline $\begin{array}{c}\text { Device } \\
\text { Force (kips) }\end{array}$ & $20 \%$ & $30 \%$ & $40 \%$ & $50 \%$ & $60 \%$ & $65 \%$ & $70 \%$ & $75 \%$ & $80 \%$ & $85 \%$ & $90 \%$ & $95 \%$ & $100 \%$ \\
\hline NE Device & 1.87 & 2.42 & 3.02 & 3.62 & 4.44 & 4.70 & 5.14 & 5.27 & 5.73 & 6.06 & 6.02 & 6.21 & 6.42 \\
\hline SE Device & 1.70 & 1.97 & 2.78 & 3.60 & 4.26 & 4.47 & 4.70 & 4.98 & 5.19 & 4.97 & 5.46 & 5.40 & 5.39 \\
\hline SW Device & 1.71 & 2.15 & 2.85 & 3.40 & 3.84 & 4.04 & 4.44 & 4.51 & 4.65 & 4.77 & 5.72 & 5.70 & 6.26 \\
\hline NW Device & 2.14 & 3.16 & 3.51 & 4.25 & 4.99 & 5.41 & 6.35 & 6.74 & 7.70 & 7.23 & 7.80 & 7.30 & 7.43 \\
\hline
\end{tabular}

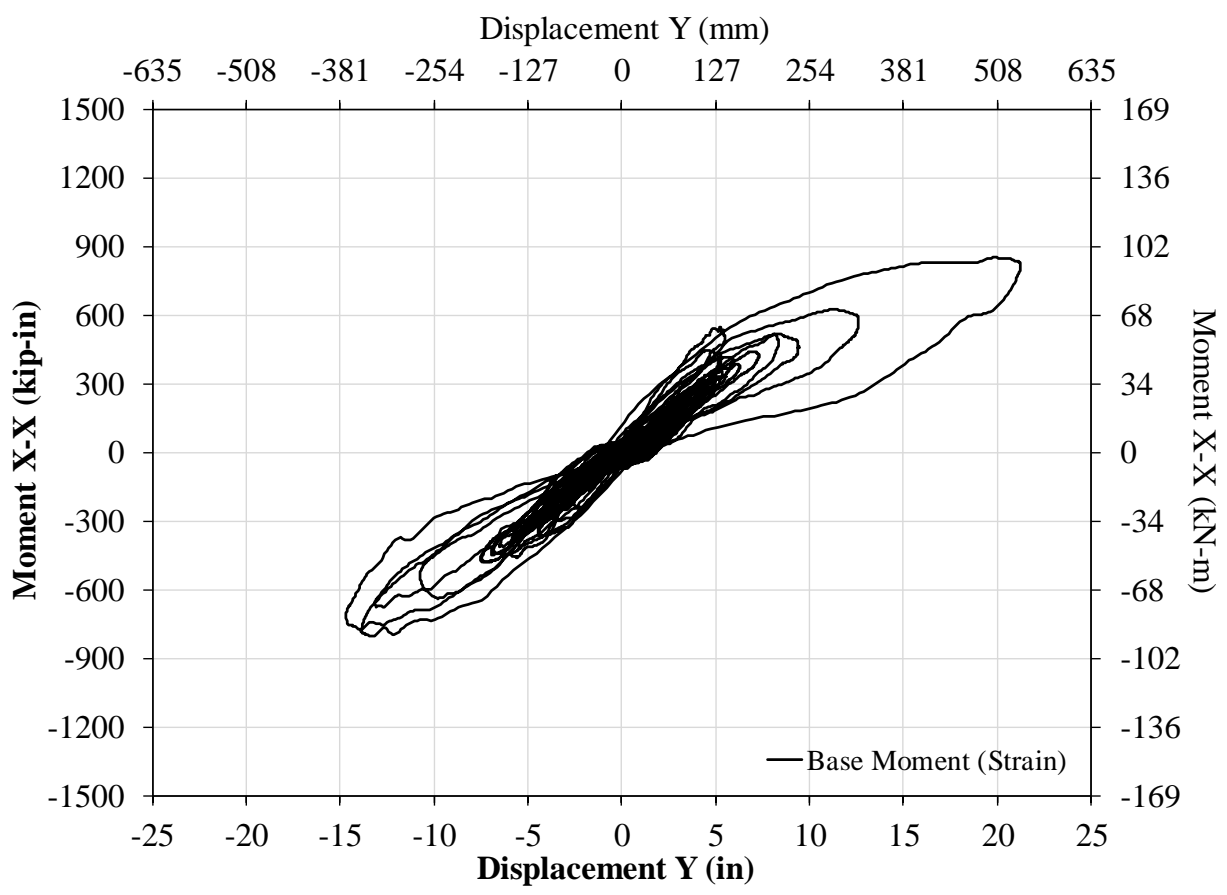

Figure 13-4: 100\% 0.5g IEEE693 Y-System Response w/ Viscous Dampers 
All device assembly bracket connections are designed to be slip-critical, slip is undesirable because slip reduces the relative displacement between the energy-dissipating device and the rocking structure. The reduced relative displacement decreases the effectiveness of the device and typically leads to larger accelerations and global displacements. Summarized in Table 13-18 is the maximum slip in the device assembly bracket which occurred during the ground motion. All slip values were minimal but retorqueing bolts between motions could have reduced slip magnitudes.

Table 13-18: Viscous Damper - Damper Bracket Slip

\begin{tabular}{|c|c|c|c|c|c|c|c|c|c|c|c|c|c|}
\cline { 2 - 12 } \multicolumn{1}{c|}{} & \multicolumn{10}{c|}{ \% of IEE693 0.5g Motion } \\
\hline Device Slip (in.) & $20 \%$ & $30 \%$ & $40 \%$ & $50 \%$ & $60 \%$ & $65 \%$ & $70 \%$ & $75 \%$ & $80 \%$ & $85 \%$ & $90 \%$ & $95 \%$ & $100 \%$ \\
\hline NE-N & 0.001 & 0.002 & 0.003 & 0.005 & 0.011 & 0.013 & 0.015 & 0.018 & 0.022 & 0.025 & 0.025 & 0.024 & 0.026 \\
\hline SE-S & 0.002 & 0.003 & 0.005 & 0.006 & 0.007 & 0.008 & 0.010 & 0.011 & 0.013 & 0.013 & 0.018 & 0.017 & 0.020 \\
\hline SW-S & 0.064 & 0.003 & 0.072 & 0.075 & 0.008 & 0.010 & 0.012 & 0.011 & 0.010 & 0.011 & 0.010 & 0.013 & 0.013 \\
\hline NW-N & 0.002 & 0.003 & 0.005 & 0.004 & 0.006 & 0.007 & 0.012 & 0.017 & 0.024 & 0.023 & 0.031 & 0.029 & 0.033 \\
\hline
\end{tabular}

Each BeS set was preloaded to 3.73 kips as previously discussed in the full-scale design section. During each motion, the maximum force transferred through the elastic springs into the foundation was determined and reported in Table 13-19. The maximum force transferred though the pre-tensioning was 11.4 kips, which was below $12.8 \mathrm{kip}$ maximum linear load.

Table 13-19: Viscous Damper - Maximum PT Force

\begin{tabular}{|c|c|c|c|c|c|c|c|c|c|c|c|c|c|}
\cline { 2 - 13 } & \multicolumn{10}{c|}{ \% of IEEE93 0.5g Motion } \\
\hline PT Force (kips) & $20 \%$ & $30 \%$ & $40 \%$ & $50 \%$ & $60 \%$ & $65 \%$ & $70 \%$ & $75 \%$ & $80 \%$ & $85 \%$ & $90 \%$ & $95 \%$ & $100 \%$ \\
\hline PT North & 4.07 & 4.34 & 5.32 & 6.01 & 6.89 & 7.51 & 7.51 & 8.65 & 9.29 & 9.61 & 10.12 & 10.33 & 11.38 \\
\hline PT East & 4.18 & 4.49 & 4.84 & 5.57 & 6.23 & 6.37 & 6.59 & 6.54 & 6.91 & 7.29 & 8.07 & 8.53 & 9.16 \\
\hline PT South & 4.45 & 4.76 & 5.50 & 6.13 & 6.76 & 7.00 & 7.48 & 7.64 & 7.98 & 8.23 & 8.51 & 8.71 & 9.13 \\
\hline PT West & 4.15 & 4.59 & 4.89 & 5.68 & 6.24 & 6.06 & 6.33 & 7.29 & 8.36 & 9.01 & 10.13 & 10.83 & 11.44 \\
\hline
\end{tabular}

Loss in pre-tensioning force is of extreme importance because of its influence on rocking initiation, self-centering, and system behavior. Percent of force variation between 
the initial pre-tension load and the final pre-tension load are shown in Table 13-20. Pretension force loss was usually below $2 \%$, some cases presented pre-tension force decreases ranging from $4 \%-7 \%$. Two instances showed a pre-tension member loosing roughly $7 \%$ of the force after excitation.

Table 13-20: Viscous Damper - \% PT Force Loss Post Motion

\begin{tabular}{|c|c|c|c|c|c|c|c|c|c|c|c|c|c|}
\hline \multirow[b]{2}{*}{$\begin{array}{c}\text { PT Force Loss } \\
\text { (kips) }\end{array}$} & \multicolumn{13}{|c|}{$\%$ of IEEE693 $0.5 \mathrm{~g}$ Motion } \\
\hline & $20 \%$ & $30 \%$ & $40 \%$ & $50 \%$ & $60 \%$ & $65 \%$ & $70 \%$ & $75 \%$ & $80 \%$ & $85 \%$ & $90 \%$ & $95 \%$ & $100 \%$ \\
\hline PT North & $0.2 \%$ & $-0.4 \%$ & $-0.8 \%$ & $-0.8 \%$ & $-1.4 \%$ & $-1.0 \%$ & $-1.7 \%$ & $-1.8 \%$ & $-1.2 \%$ & $-6.8 \%$ & $-1.7 \%$ & $-1.9 \%$ & $-4.0 \%$ \\
\hline PT East & $-0.8 \%$ & $0.4 \%$ & $0.0 \%$ & $-1.2 \%$ & $-1.0 \%$ & $0.0 \%$ & $-1.4 \%$ & $-0.4 \%$ & $-0.4 \%$ & $-3.7 \%$ & $0.6 \%$ & $-0.4 \%$ & $0.2 \%$ \\
\hline PT South & $-0.9 \%$ & $0.0 \%$ & $-1.3 \%$ & $-1.2 \%$ & $-0.6 \%$ & $-0.2 \%$ & $-1.9 \%$ & $-0.6 \%$ & $-0.6 \%$ & $-4.8 \%$ & $-1.0 \%$ & $-2.3 \%$ & $-1.9 \%$ \\
\hline PT West & $-1.3 \%$ & $-0.9 \%$ & $-0.4 \%$ & $-1.5 \%$ & $-1.5 \%$ & $-0.4 \%$ & $-1.6 \%$ & $-1.7 \%$ & $-2.1 \%$ & $-7.1 \%$ & $-4.3 \%$ & $-4.0 \%$ & $-4.0 \%$ \\
\hline
\end{tabular}

As the motion amplitude increased, the system rocking and displacement also increased. Displayed in Table 13-21 are the maximum uplifts experienced by each side of the pedestal base plate. The largest uplift occurred on the north side of the base plate for the $0.5 \mathrm{~g}$ IEEE693 motion. The system experienced 1.326 inches of vertical displacement on the north side of the pedestal base.

Table 13-21: Viscous Damper - Pedestal Rocking Uplift

\begin{tabular}{|c|c|c|c|c|c|c|c|c|c|c|c|c|c|}
\cline { 2 - 13 } \multicolumn{1}{c|}{} & \multicolumn{10}{c|}{ \% of IEEE693 0.5g Motion } \\
\hline Uplift (in.) & $20 \%$ & $30 \%$ & $40 \%$ & $50 \%$ & $60 \%$ & $65 \%$ & $70 \%$ & $75 \%$ & $80 \%$ & $85 \%$ & $90 \%$ & $95 \%$ & $100 \%$ \\
\hline Base PL Uplift N & 0.139 & 0.180 & 0.304 & 0.423 & 0.599 & 0.693 & 0.747 & 0.874 & 0.996 & 1.069 & 1.177 & 1.233 & 1.326 \\
\hline Base PL Uplift E & 0.093 & 0.160 & 0.226 & 0.356 & 0.476 & 0.501 & 0.512 & 0.505 & 0.498 & 0.567 & 0.706 & 0.757 & 0.863 \\
\hline Base PL Uplift S & 0.140 & 0.206 & 0.335 & 0.442 & 0.562 & 0.606 & 0.659 & 0.688 & 0.737 & 0.774 & 0.824 & 0.847 & 0.906 \\
\hline Base PL Uplift W & 0.101 & 0.171 & 0.235 & 0.369 & 0.477 & 0.447 & 0.440 & 0.664 & 0.851 & 0.957 & 1.148 & 1.207 & 1.304 \\
\hline
\end{tabular}

In order to anchor the system to the shake table, a steel adaptor plate was utilized to attach all foundation anchors. Usually, steel-to-steel contact provides less surface friction than a steel-to-concrete interface. The friction force developed in actual implementation of the retrofit is expected to be greater than that presented in the experimental stages of this study. The largest base slip exhibited by the system was 0.17 
inch. The maximum residual base slip displacement was 0.12 inch manifesting in the $100 \%$ motion. A report of the maximum and residual base plate slip results are presented in Table 13-22 and Table 13-23.

Table 13-22: Viscous Damper - Maximum Base Plate Slip

\begin{tabular}{|c|c|c|c|c|c|c|c|c|c|c|c|c|c|}
\cline { 2 - 14 } \multicolumn{1}{c|}{} & \multicolumn{10}{c|}{ \% of IEE693 0.5g Motion } \\
\hline Device Uplift (in.) & $20 \%$ & $30 \%$ & $40 \%$ & $50 \%$ & $60 \%$ & $65 \%$ & $70 \%$ & $75 \%$ & $80 \%$ & $85 \%$ & $90 \%$ & $95 \%$ & $100 \%$ \\
\hline Base PL Slip NE-X & 0.01 & 0.01 & 0.02 & 0.04 & 0.05 & 0.05 & 0.06 & 0.05 & 0.06 & 0.08 & 0.08 & 0.12 & 0.13 \\
\hline Base PL Slip SE-X & 0.01 & 0.03 & 0.03 & 0.04 & 0.06 & 0.08 & 0.08 & 0.08 & 0.08 & 0.09 & 0.12 & 0.13 & 0.13 \\
\hline Base PL Slip SW-X & 0.01 & 0.02 & 0.03 & 0.03 & 0.04 & 0.06 & 0.06 & 0.06 & 0.08 & 0.07 & 0.11 & 0.14 & 0.16 \\
\hline Base PL Slip NW-X & 0.01 & 0.02 & 0.03 & 0.05 & 0.07 & 0.06 & 0.07 & 0.08 & 0.11 & 0.13 & 0.15 & 0.16 & 0.15 \\
\hline Base PL Slip NE-Y & 0.02 & 0.03 & 0.04 & 0.05 & 0.06 & 0.08 & 0.09 & 0.10 & 0.12 & 0.13 & 0.14 & 0.14 & 0.16 \\
\hline Base PL Slip SE-Y & 0.02 & 0.02 & 0.03 & 0.04 & 0.05 & 0.05 & 0.05 & 0.06 & 0.07 & 0.09 & 0.11 & 0.17 & 0.17 \\
\hline Base PL Slip SW-Y & 0.02 & 0.02 & 0.04 & 0.06 & 0.07 & 0.08 & 0.09 & 0.10 & 0.11 & 0.10 & 0.11 & 0.12 & 0.14 \\
\hline Base PL Slip NW-Y & 0.02 & 0.02 & 0.03 & 0.04 & 0.05 & 0.05 & 0.06 & 0.06 & 0.08 & 0.11 & 0.10 & 0.14 & 0.17 \\
\hline
\end{tabular}

Table 13-23: Viscous Damper - Relative Base Plate Movement Pre-Post Motion

\begin{tabular}{|c|c|c|c|c|c|c|c|c|c|c|c|c|c|}
\cline { 2 - 13 } \multicolumn{1}{c|}{} & \multicolumn{10}{c|}{ \% of IEE693 0.5g Motion } \\
\hline Device Uplift (in.) & $20 \%$ & $30 \%$ & $40 \%$ & $50 \%$ & $60 \%$ & $65 \%$ & $70 \%$ & $75 \%$ & $80 \%$ & $85 \%$ & $90 \%$ & $95 \%$ & $100 \%$ \\
\hline Base PL Slip NE-X & 0.00 & 0.00 & 0.00 & -0.01 & -0.01 & -0.02 & -0.03 & -0.01 & -0.01 & -0.08 & -0.06 & -0.11 & -0.12 \\
\hline Base PL Slip SE-X & 0.00 & 0.00 & 0.00 & 0.01 & 0.01 & 0.00 & 0.00 & 0.02 & 0.05 & 0.06 & 0.08 & 0.11 & 0.11 \\
\hline Base PL Slip SW-X & 0.00 & 0.00 & 0.00 & -0.01 & -0.01 & 0.00 & 0.00 & -0.02 & -0.05 & -0.06 & -0.08 & -0.11 & -0.11 \\
\hline Base PL Slip NW-X & 0.00 & 0.00 & 0.00 & 0.01 & 0.01 & 0.02 & 0.03 & 0.01 & 0.01 & 0.07 & 0.05 & 0.10 & 0.12 \\
\hline Base PL Slip NE-Y & 0.00 & -0.01 & -0.01 & 0.00 & 0.01 & 0.00 & 0.01 & 0.01 & 0.03 & 0.05 & 0.07 & 0.11 & 0.10 \\
\hline Base PL Slip SE-Y & 0.00 & 0.01 & 0.01 & 0.00 & -0.01 & 0.00 & -0.01 & -0.01 & -0.03 & -0.06 & -0.08 & -0.13 & -0.12 \\
\hline Base PL Slip SW-Y & 0.00 & 0.00 & 0.01 & 0.02 & 0.01 & 0.01 & 0.02 & 0.02 & 0.02 & 0.08 & 0.06 & 0.10 & 0.12 \\
\hline Base PL Slip NW-Y & 0.00 & -0.01 & -0.01 & -0.02 & -0.01 & -0.01 & -0.02 & -0.02 & -0.03 & -0.08 & -0.07 & -0.11 & -0.12 \\
\hline
\end{tabular}

\subsection{Hysteretic Device}

\subsubsection{Hysteretic Device Table Response Spectrum}

A response spectrum for each IEEE693 input motion was constructed to show the discrepancies between the design spectrum and the table feedback. As previously mentioned, the work conducted aimed to reduce component demands and not necessarily seismically qualify the equipment. For the two horizontal directions, $\mathrm{X}$ and $\mathrm{Y}$, only frequencies between $0.5 \mathrm{~Hz}-1.3 \mathrm{~Hz}$ are of importance. The non-retrofitted system has a fundamental frequency of $1.24 \mathrm{~Hz}$; reduction in frequency due to rocking is expected in 
the retrofitted cases. Shown in Figure 13-5 and Figure 13-6 are the horizontal component response spectrums. Both horizontal components have minor undershooting when comparing the table response to the design spectrum. Shown in Figure 13-3 is the response spectrum for the vertical direction, which envelopes all high frequencies of interest.

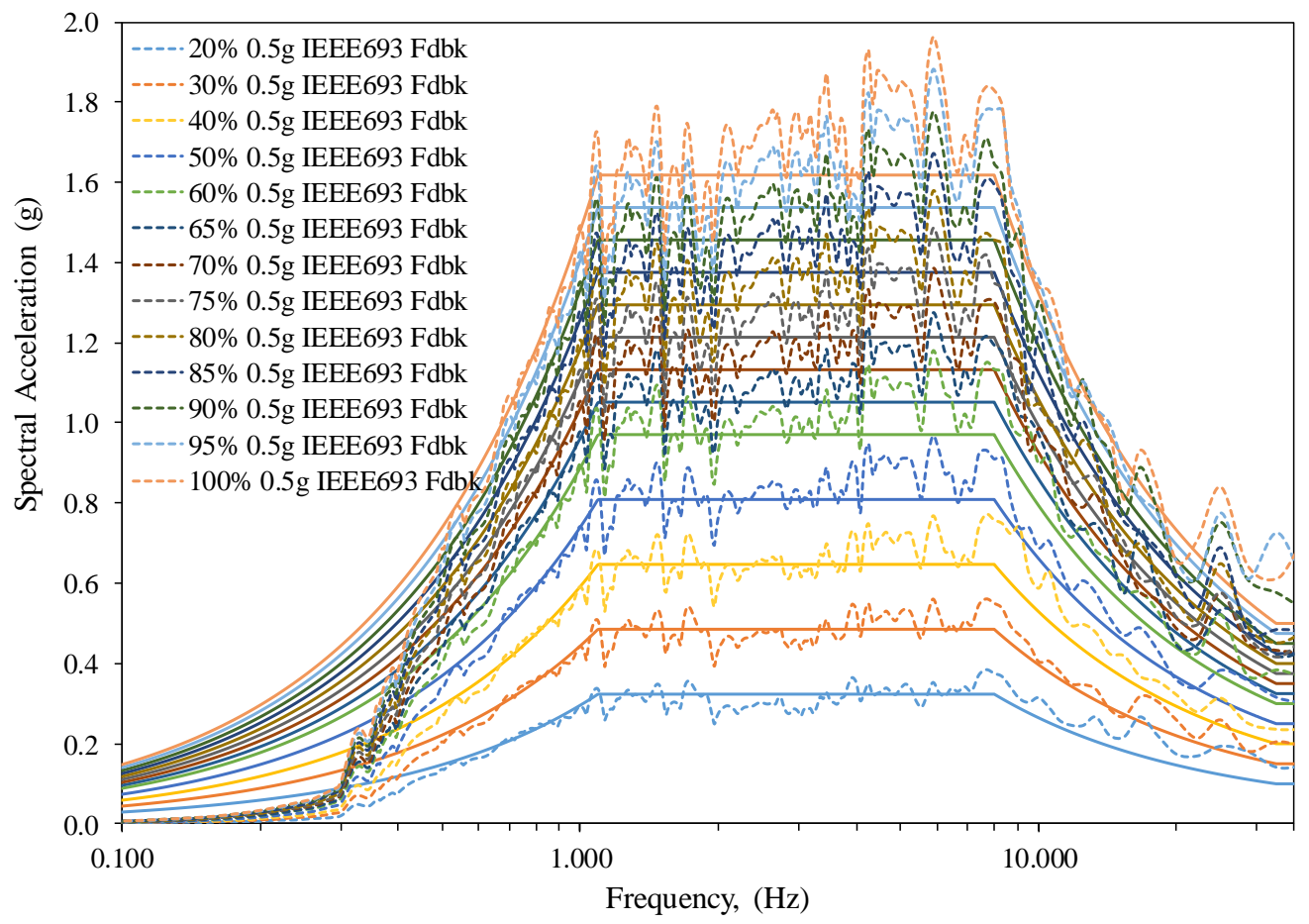

Figure 13-5: Hysteretic Device - Response Spectrum X 


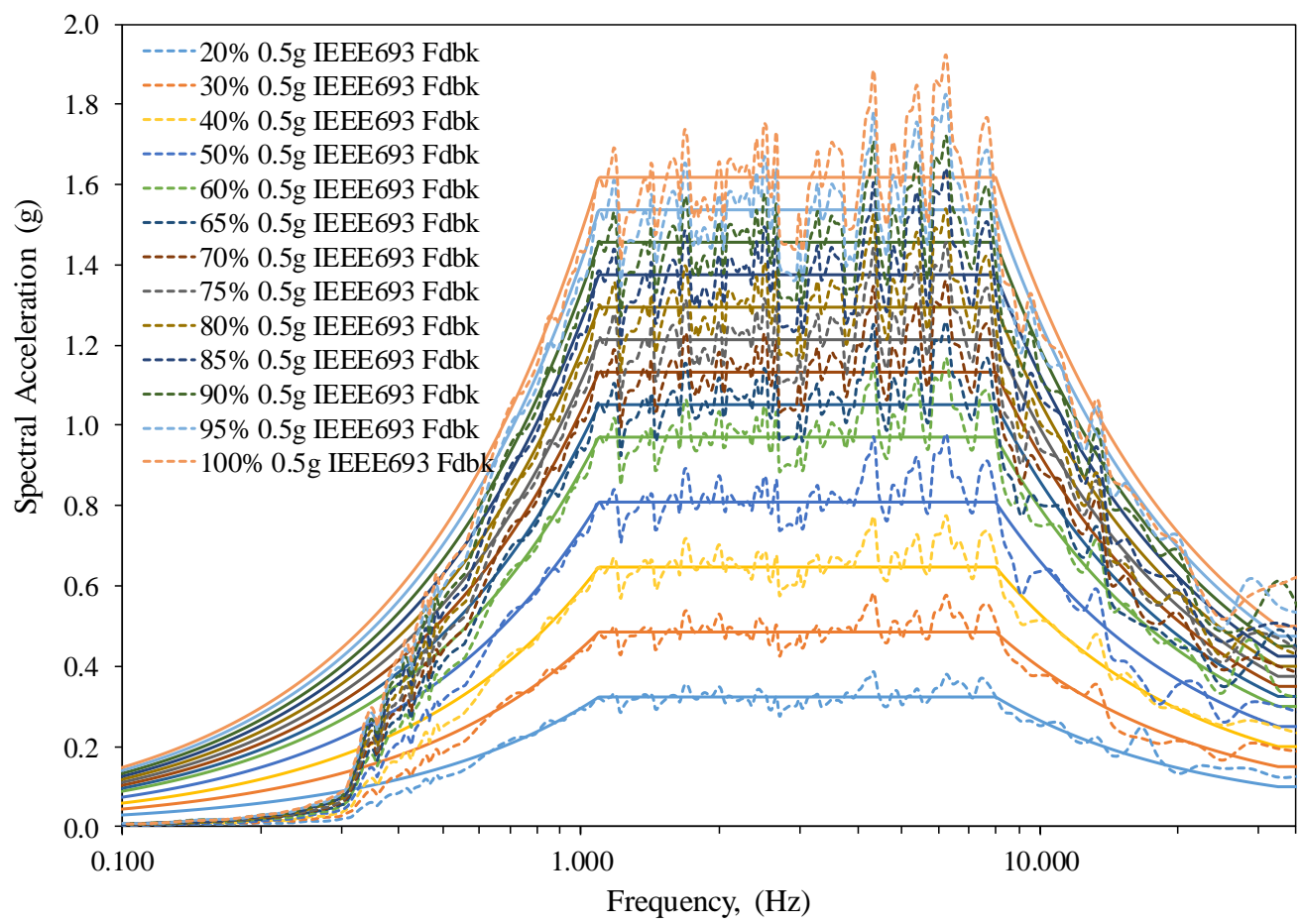

Figure 13-6: Hysteretic Device - Response Spectrum Y

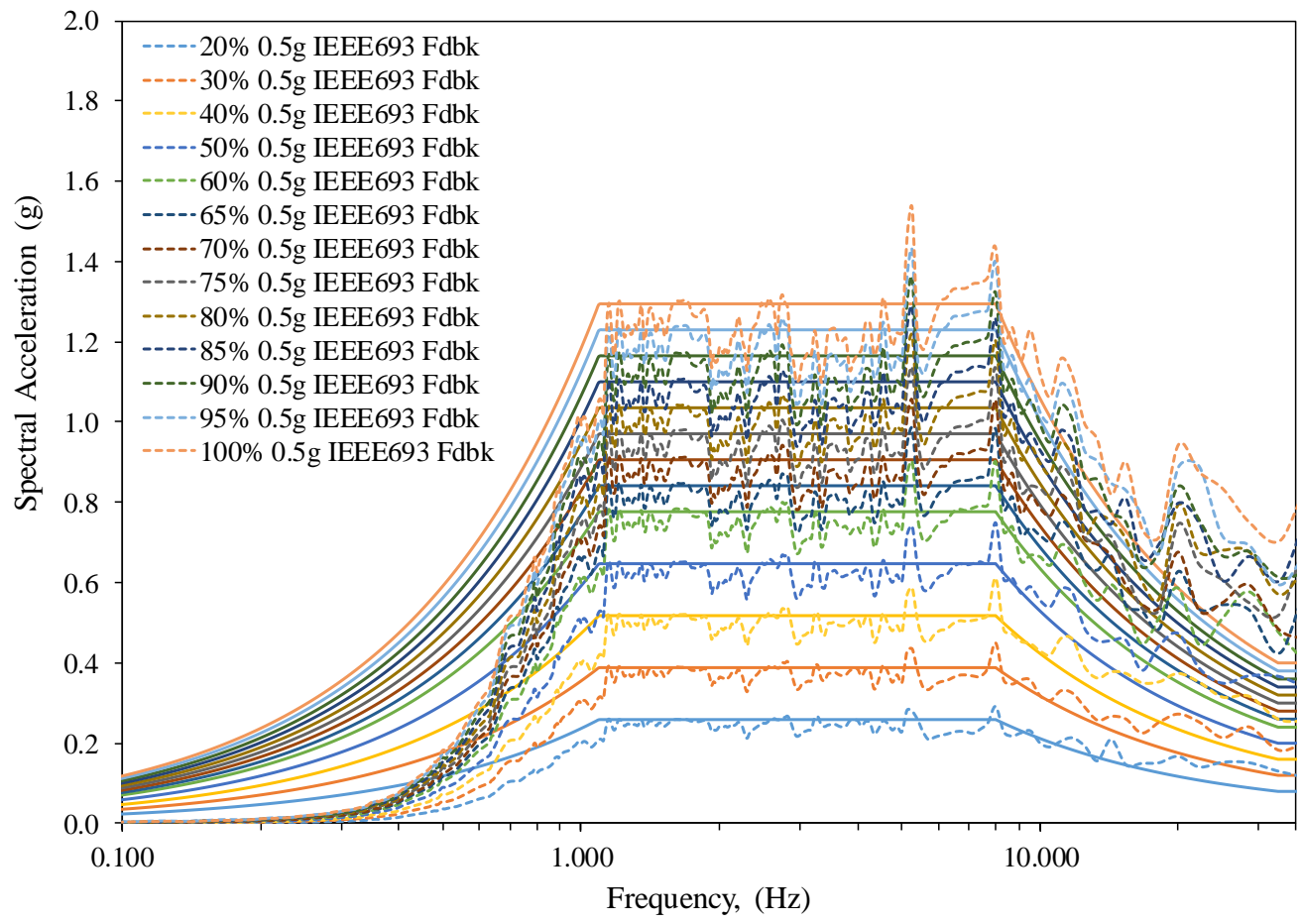

Figure 13-7: Hysteretic Device - Response Spectrum Z 


\subsubsection{Hysteretic Device System Damping \& Free Vibration}

Utilizing the same methods discussed in 13.3.2, Viscous Damper System Damping \& Free Vibration, the elastic damping and fundamental frequency was determined for the rocking system with hysteretic devices. The average fundamental frequency in the Xdirection was $0.80 \mathrm{~Hz}$ using a pulse excitation and $0.78 \mathrm{~Hz}$ using white noise excitation. Similarly, the average fundamental frequency for the Y-direction was $0.70 \mathrm{~Hz}$ using a pulse excitation and $0.68 \mathrm{~Hz}$ using white noise. Elastic damping $\mathrm{X}$ and $\mathrm{Y}$ components were $0.66 \%$ and $0.87 \%$ respectively. A complete summary of the system identification properties are presented in Table 13-24. 
Table 13-24: UFP Retrofit Damping and Fundamental Frequency

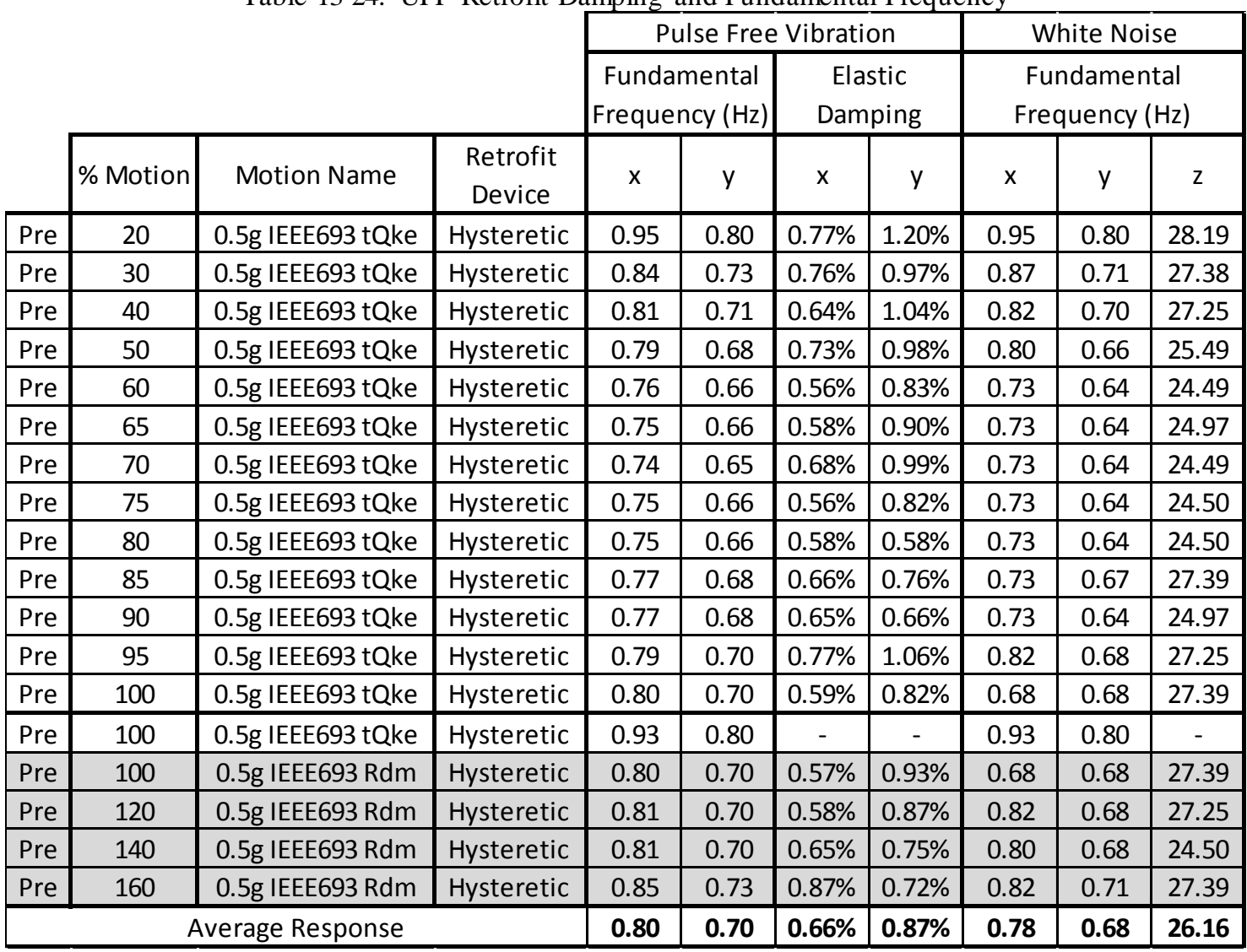

Supplemental figures demonstrating the damping for each case shown in Table 13-24 are provided in Appendix G. The calculated damping values are integrated into the plots through the bounded curves in Appendix G.

\subsubsection{Hysteretic Device System Response}

Similar to the metrics discussed for the viscous damper retrofit, this section summarizes the results obtained from testing for the rocking system with hysteretic devices. Summarized in Table 13-25, Table 13-26, and Table 13-27 are the absolute maximum acceleration values obtained during testing. Magnitudes are shown for each accelerometer location along the specimen length. The magnitudes do not all occur at the same instance during the ground motion but are maximums presented during the ground 
motion. The 100\% 0.5g IEEE693 motions were performed twice, one case considered virgin hysteretic devices denoted “*”, while the other $100 \%$ case was conducted post all preceding motion levels.

Table 13-25: Hysteretic Device - System X Absolute Maximum Acceleration

\begin{tabular}{|c|c|c|c|c|c|c|c|c|c|c|c|c|c|c|c|c|}
\hline & \multicolumn{14}{|c|}{ \% of IEEE693 0.5g Motion } & & \\
\hline & $20 \%$ & $30 \%$ & $40 \%$ & $50 \%$ & $60 \%$ & $65 \%$ & $70 \%$ & $75 \%$ & $80 \%$ & $85 \%$ & $90 \%$ & $95 \%$ & $100 \%$ & $100 \% *$ & & \\
\hline \multirow{16}{*}{ 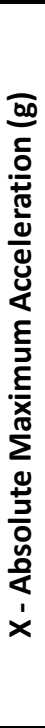 } & 0.51 & 0.70 & 0.72 & 0.79 & 1.10 & 1.14 & 1.09 & 1.01 & 1.12 & 1.06 & 1.09 & 1.16 & 1.13 & 1.22 & 351 & \\
\hline & 0.42 & 0.54 & 0.59 & 0.61 & 0.68 & 0.81 & 0.82 & 0.78 & 0.81 & 0.81 & 0.81 & 0.88 & 0.92 & 0.96 & 2 & \multirow{15}{*}{$\subseteq$} \\
\hline & 0.39 & 0.48 & 0.54 & 0.54 & 0.63 & 0.69 & 0.68 & 0.70 & 0.68 & 0.71 & 0.76 & 0.81 & 0.85 & 0.87 & $3041 / 2$ & \\
\hline & 0.34 & 0.40 & 047 & 0.48 & 0.55 & 0 . & 6 & 0.59 & 0.56 & 0.59 & 0.65 & 0.68 & 0.76 & 0.72 & $1 / 2$ & \\
\hline & 0.32 & 0.39 & 0.43 & .46 & 0.53 & (0.0. & 54 & 0.60 & 0.54 & 0.00 & 0.62 & 0.69 & 0.71 & & & \\
\hline & 0.31 & 0.41 & 0.43 & 0.48 & 0.55 & 0.57 & 0.56 & 0.63 & 0.58 & 0.65 & 0.66 & 0.69 & 0.77 & 0.73 & $\angle 34$ & \\
\hline & 0.30 & 0.42 & 0.44 & 0.49 & 0.67 & 0.64 & 0.60 & 0.64 & 0.64 & 0.67 & 0.66 & 0.73 & 0.78 & 0.76 & 235 & \\
\hline & 0.28 & 0.43 & 0.43 & 0.49 & 0.58 & 0.67 & 0.67 & 0.65 & 0.66 & 0.69 & 0.67 & 0.74 & 0.72 & 0.74 & $2141 / 2$ & \\
\hline & 0.30 & 0.45 & 0.43 & 0.49 & 0.68 & 0.71 & 0.71 & 0.71 & 0.72 & 0.72 & 0.71 & 0.76 & 0.73 & 0.76 & 199 & \\
\hline & 0.30 & 0.47 & 0.43 & 0.50 & 0.68 & 0.72 & 0.78 & 0.81 & 0.67 & 0.75 & 0.73 & 0.74 & 0.79 & 0.84 & 182 & \\
\hline & 0.30 & 0.50 & 0.40 & 0.56 & 0.71 & 0.73 & 0.79 & 0.79 & 0.71 & 0.80 & 0.82 & 0.79 & 0.82 & 0.87 & 163 & \\
\hline & 0.28 & 0.49 & 38 & 0.58 & 0.64 & 0. & 81 & 0.72 & 0.74 & 7 & 3 & 0.79 & 91 & 88 & 146 & \\
\hline & 0.26 & 0.47 & 0.38 & 0.62 & 0.71 & 0.68 & 0.76 & 0.72 & 0.76 & 0.83 & 0.83 & 0.78 & 0.89 & 0.84 & 127 & \\
\hline & 0.23 & 0.49 & 0.41 & 0.66 & 0.85 & 0.73 & 0.72 & 0.78 & 0.89 & 0.86 & 0.86 & 0.91 & 1.09 & 0.98 & 91 & \\
\hline & 0.14 & 0.18 & 0.24 & 0.29 & 0.36 & 0.38 & 0.40 & 0.54 & 0.50 & 0.50 & 0.53 & 0.53 & 0.73 & 0.60 & $11 / 8$ & \\
\hline & 0.14 & 0.18 & 0.23 & 0.28 & 0.34 & 0.38 & 0.38 & 0.40 & 0.42 & 0.45 & 0.50 & 0.52 & 0.56 & 0.56 & 0 & \\
\hline
\end{tabular}

*virgin hysteretic device

Figure 13-8 show graphically the maximum acceleration results from Table 13-25 $(20 \%, 50 \%, 75 \%, 100 \%$, and $100 \% *)$. From the figure, visible increases in maximum acceleration are seen up to 151 inches from the system base and from 228 inches-304-1/2 inches. The graphic demonstrates how using just a single acceleration magnitude location near the CG (215-1/4”) will significantly misrepresent the system's response. If actual acceleration is compared along the specimen length for the same time instance, not only are magnitudes of acceleration different along the specimen, but the direction of acceleration also varies as the system undergoes higher modes. 


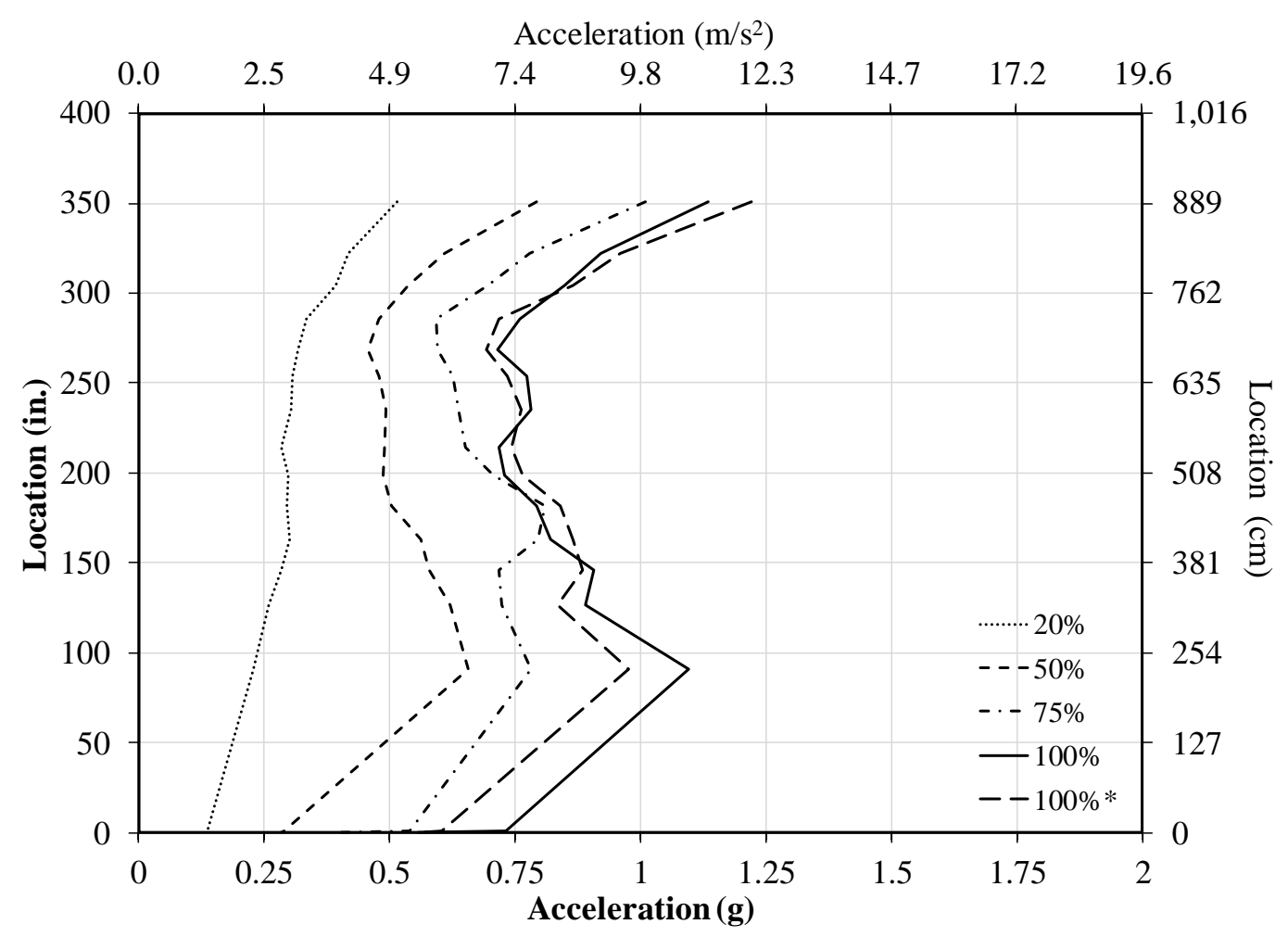

Figure 13-8: Hysteretic Device - System X Absolute Maximum Acceleration Plot

Table 13-26: Hysteretic Device - System Y Absolute Maximum Acceleration $\%$ of IEEE693 $0.5 \mathrm{~g}$ Motion

\begin{tabular}{|l|l|l|l|l|l|l|l|l|l|l|l|l|l|}
\hline $20 \%$ & $30 \%$ & $40 \%$ & $50 \%$ & $60 \%$ & $65 \%$ & $70 \%$ & $75 \%$ & $80 \%$ & $85 \%$ & $90 \%$ & $95 \%$ & $100 \%$ & $100 \% *$ \\
\hline
\end{tabular}

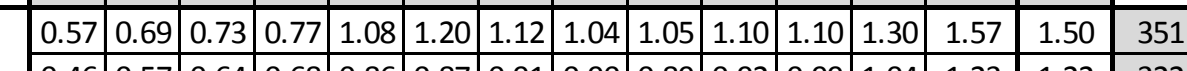

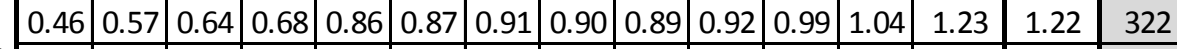

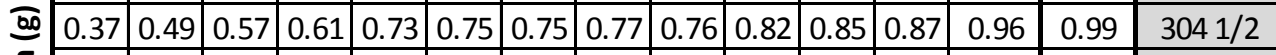

으 \begin{tabular}{llllllllllll|l|l|l|l|l|}
\hline & 0.32 & 0.40 & 0.50 & 0.55 & 0.66 & 0.66 & 0.65 & 0.69 & 0.70 & 0.72 & 0.76 & 0.76 & 0.77 & 0.81 & $2851 / 2$ \\
\hline
\end{tabular}

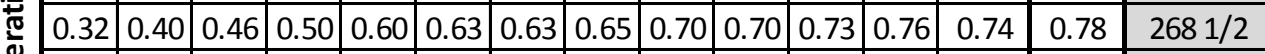

\begin{tabular}{llllllll|l|l|l|l|l|l|l|l|}
\hline & 0.33 & 0.41 & 0.45 & 0.48 & 0.63 & 0.63 & 0.63 & 0.66 & 0.81 & 0.70 & 0.72 & 0.78 & 0.79 & 0.81 & 254 \\
\hline
\end{tabular}

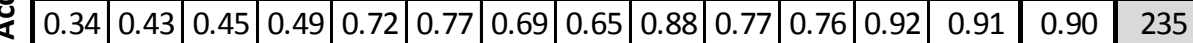

\begin{tabular}{|l|l|l|l|l|l|l|l|l|l|l|l|l|l|l|}
\hline 0.34 & 0.44 & 0.49 & 0.57 & 0.79 & 0.90 & 0.78 & 0.80 & 0.90 & 0.80 & 0.86 & 1.00 & 1.08 & 0.97 & $2141 / 2$ \\
\hline
\end{tabular}

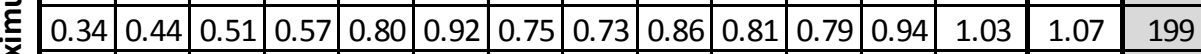

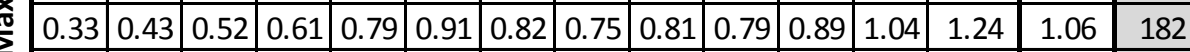

\begin{tabular}{|l|l|l|l|l|l|l|l|l|l|l|l|l|l|l}
0.33 & 0.44 & 0.56 & 0.61 & 0.85 & 0.87 & 0.79 & 0.77 & 0.73 & 0.80 & 0.82 & 0.96 & 1.10 & 1.06 & 163 \\
\hline
\end{tabular}

\begin{tabular}{|l|l|l|l|l|l|l|l|l|l|l|l|l|l|l}
\hline 0.31 & 0.42 & 0.54 & 0.64 & 0.88 & 0.92 & 0.83 & 0.83 & 0.79 & 0.79 & 0.85 & 0.91 & 1.09 & 1.11 & 146 \\
\hline
\end{tabular}

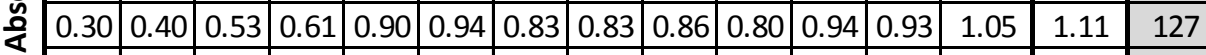

$>$
$>$

\begin{tabular}{|l|l|l|l|l|l|l|l|l|l|l|l|l|l|l|}
0.14 & 0.18 & 0.24 & 0.29 & 0.36 & 0.38 & 0.40 & 0.54 & 0.50 & 0.50 & 0.53 & 0.53 & 0.73 & 0.60 & $11 / 8$ \\
\hline
\end{tabular}

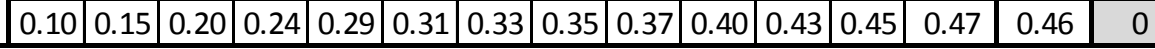

*virgin hysteretic device 
Similar acceleration trends were exhibited by the system in the Y-direction as discussed for the X-direction. Throughout the central portion of the system the acceleration maximums are near constant, while the values at the top of the structure and near the CT-pedestal interface significantly increase. The maximum magnitudes for $20 \%$, $50 \%, 75 \%, 100 \%$ and $100 \% *$ from Table 13-26 are shown in Figure 13-9.

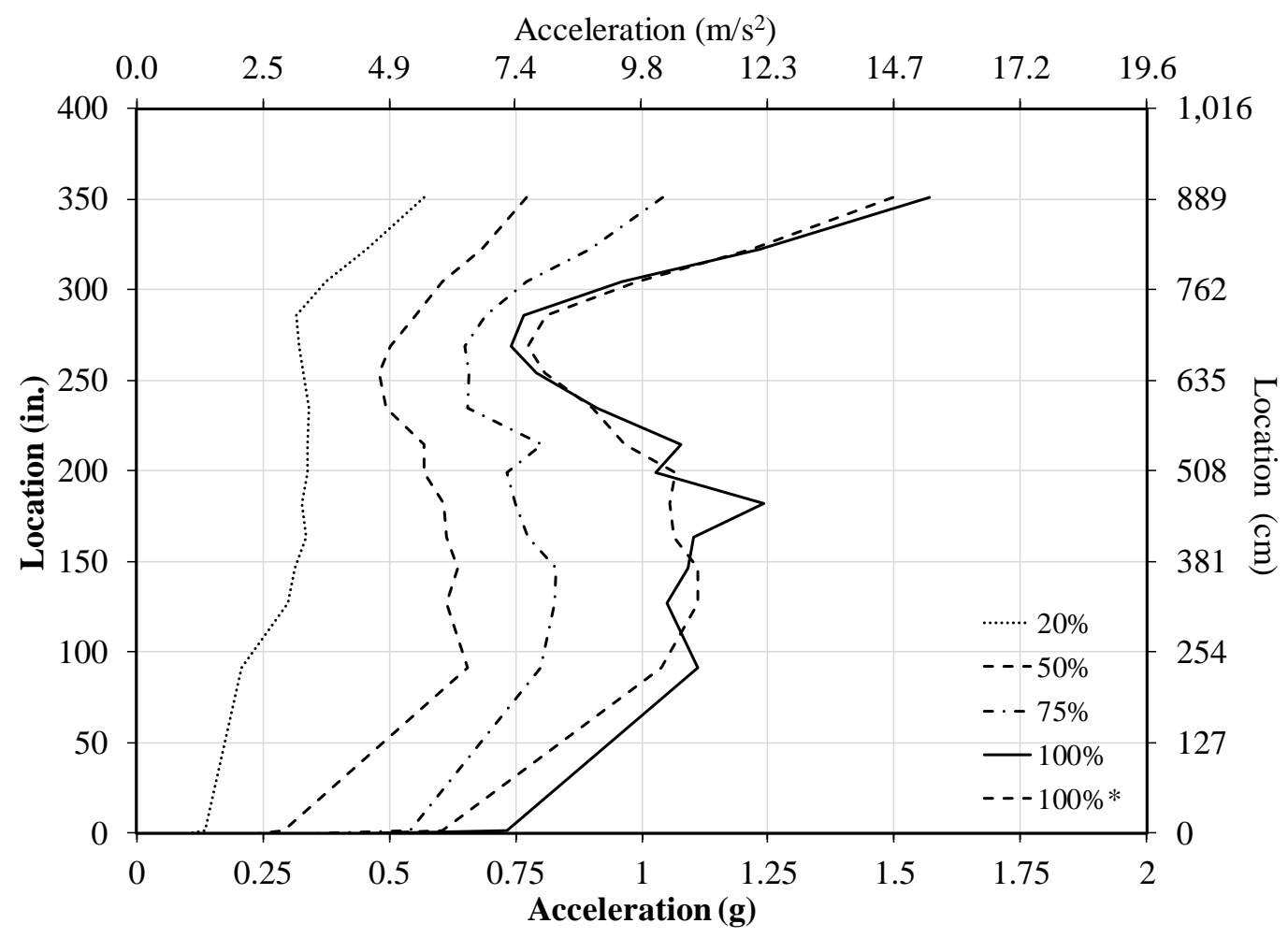

Figure 13-9: Hysteretic Device - System Y Absolute Maximum Acceleration Plot

Slight amplifications were detected in the z-direction accelerations; base accelerations could be compared to accelerations along the length of the specimen using Table 13-27. Due to slight angle change in the accelerometer during rocking, a portion of the acceleration detected is likely due to accelerometer tilt rather than acceleration amplification in the z-direction or base slapping. 
Table 13-27: Hysteretic Device - System Z Absolute Maximum Acceleration

$\%$ of IEEE693 0.5g Motion

\begin{tabular}{|l|l|l|l|l|l|l|l|l|l|l|l|l|l|}
$20 \%$ & $30 \%$ & $40 \%$ & $50 \%$ & $60 \%$ & $65 \%$ & $70 \%$ & $75 \%$ & $80 \%$ & $85 \%$ & $90 \%$ & $95 \%$ & $100 \%$ & $100 \% *$ \\
\hline
\end{tabular}

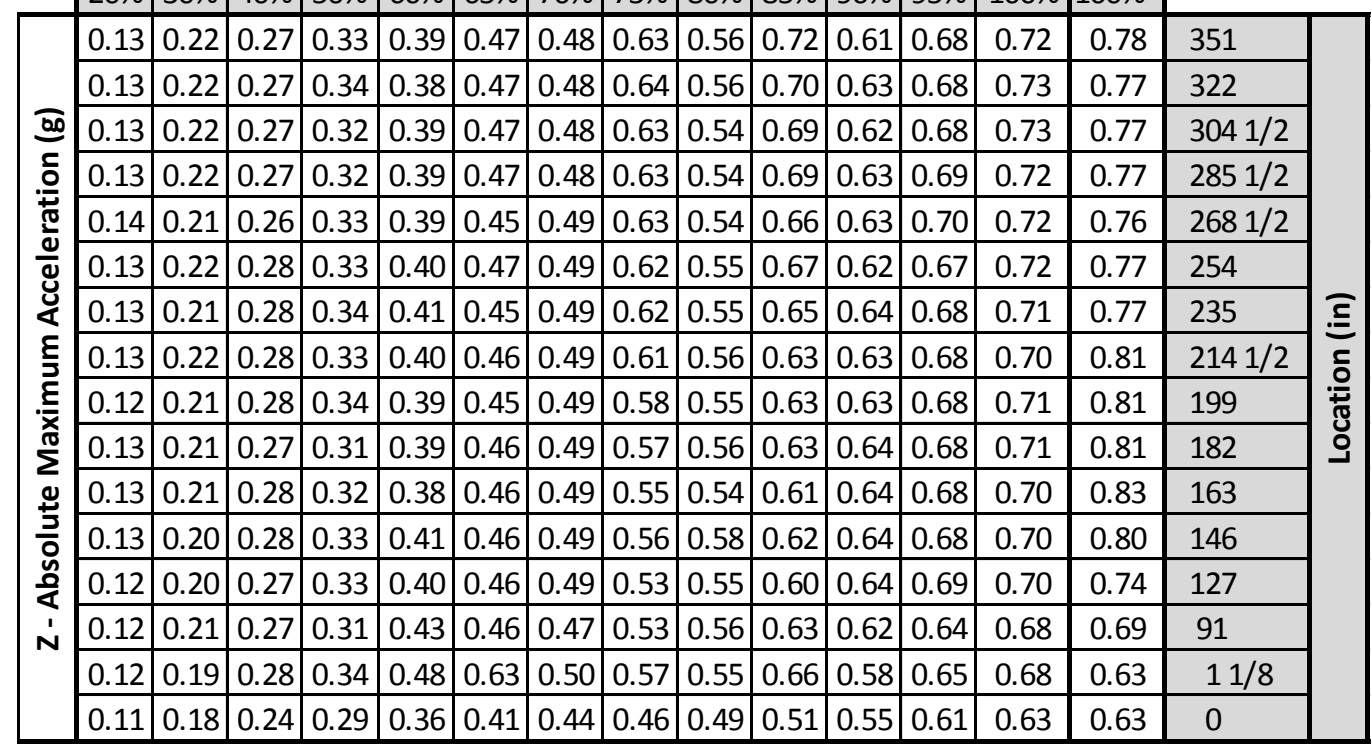

*virgin hysteretic device

A visual representation of $20 \%, 50 \%, 75 \%, 100 \%$, and $100 \% *$ z-direction

acceleration magnitudes are presented in Figure 13-10. At low level excitations, minimal amplification is exhibited by the system. As the amplitude of the input motion increases, larger increases in acceleration occur in the z-direction. The largest increase in acceleration is $29 \%$ seen in the virgin $100 \% 0.5 \mathrm{~g}$ IEEE693 test case. 


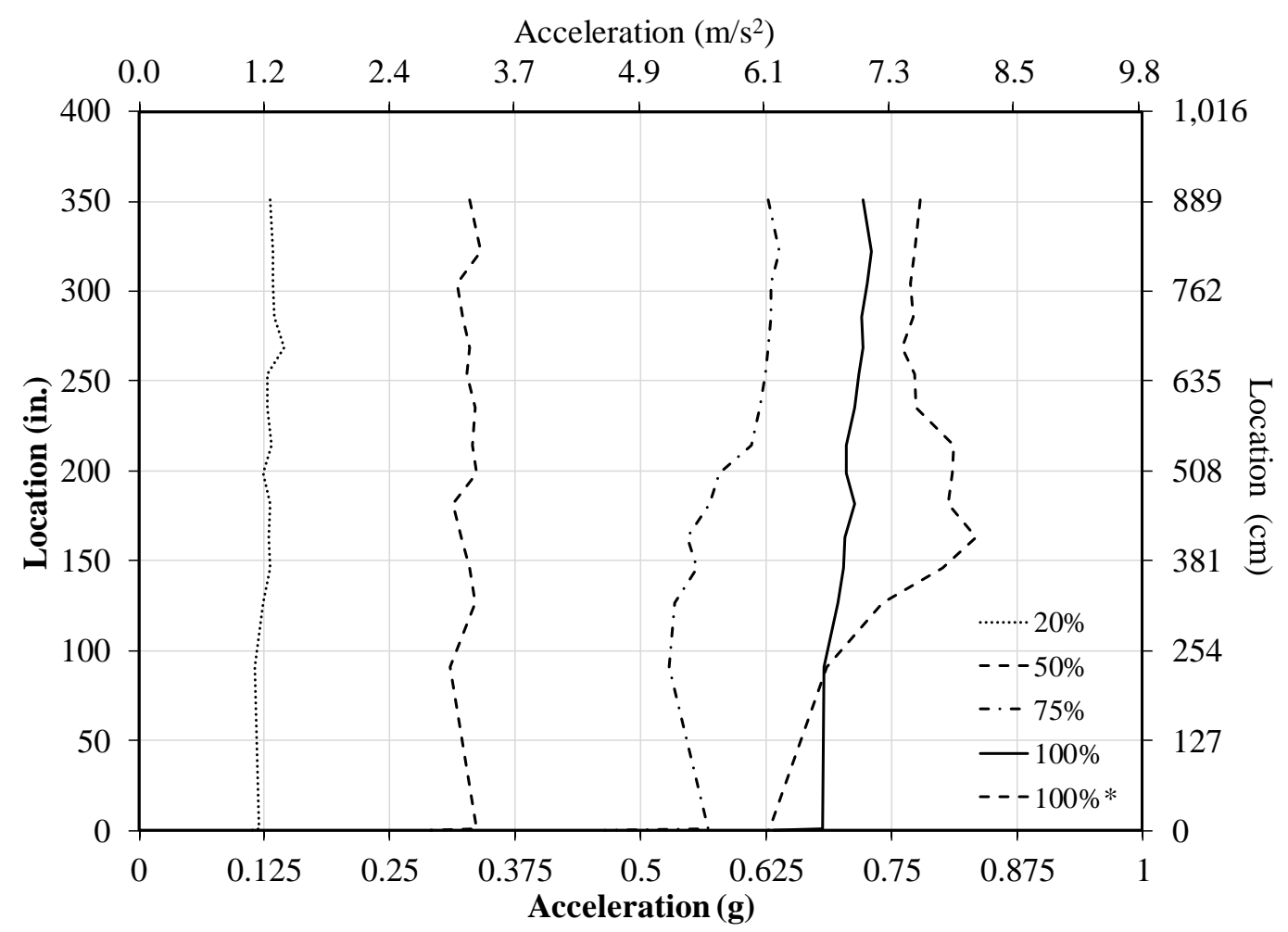

Figure 13-10: Hysteretic Device - System Y Absolute Maximum Acceleration Plot

Shear and moment measured directly and indirectly provide a more informative metric compared to maximum instantaneous acceleration because of higher mode effects. Using each acceleration reading along the specimen, base shear and moment for each instance of the motion was determined using the methods discussed in 12.1, Instrumentation. Both the direct strain and indirect acceleration approaches presented similar magnitude shear and moment values. For the $100 \%$ motions, the virgin device test case presented lower moments. The maximum X-moment and Y-moment using the strain method was 1070 kip-in and 1095 kip-in respectively for the non-virgin devices. Using the same method for the virgin cases, the maximum X-moment was 984 kip-in and 1047 kip-in for the virgin $100 \%$ motion. Utilizing the indirect acceleration method for the nonvirgin case, the maximum X-moment was 1174 kip-in and 1134 kip-in for the Y- 
direction. For the virgin case, the indirect method suggested a maximum X-moment of 1098 kip-in and maximum y-moment of 1109 kip-in. Generally, the mass-acceleration method resulted in 5\%-10\% greater moment magnitudes for the cases discussed. Using the nominal yield strength and section modulus, yield initiation is expected at 1188 kipin. Maximum flexure resulted in the X'-direction with a moment of 1436 kip-in where pedestal yielding would occur even with the retrofit.

Table 13-28: Hysteretic Device - 20-65\% System Absolute Maximum Reactions

\begin{tabular}{|c|c|c|c|c|c|c|c|c|}
\hline & & \multicolumn{6}{|c|}{$\%$ of IEEE693 0.5g Motion } & \\
\hline $\begin{array}{c}\text { Absolute Max } \\
\text { Reaction }\end{array}$ & Method & $20 \%$ & $30 \%$ & $40 \%$ & $50 \%$ & $60 \%$ & $65 \%$ & \\
\hline Base Moment X & Strain & 481 & 578 & 647 & 669 & 796 & 831 & \multirow{12}{*}{ 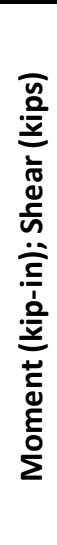 } \\
\hline Base Moment $Y$ & Strain & 465 & 584 & 719 & 795 & 879 & 934 & \\
\hline Base Moment X' & Strain & 518 & 609 & 779 & 789 & 917 & 999 & \\
\hline Base Moment $Y^{\prime}$ & Strain & 403 & 556 & 698 & 537 & 541 & 544 & \\
\hline Base Moment X & Accel. & 513 & 616 & 725 & 757 & 863 & 872 & \\
\hline Base Moment $\mathrm{Y}$ & Accel. & 498 & 621 & 762 & 819 & 923 & 977 & \\
\hline Base Shear X & Accel. & 2.0 & 2.7 & 3.0 & 3.1 & 3.5 & 3.5 & \\
\hline Base Shear $Y$ & Accel. & 2.1 & 2.7 & 3.1 & 3.3 & 4.2 & 4.3 & \\
\hline CT-Inter. X Moment & Accel. & 354 & 432 & 500 & 490 & 580 & 607 & \\
\hline CT Inter. Y Moment & Accel. & 342 & 435 & 523 & 559 & 656 & 671 & \\
\hline CT Inter. X Shear & Accel. & 1.8 & 2.4 & 2.7 & 2.7 & 3.2 & 3.2 & \\
\hline CT Inter. Y Shear & Accel. & 0.7 & 1.2 & 1.6 & 1.9 & 2.1 & 2.4 & \\
\hline
\end{tabular}

(See Figure 12-2, Strain Gauge Placement for $(\mathrm{X}, \mathrm{Y}) \&\left(\mathrm{X}^{\prime}, \mathrm{Y}^{\prime}\right)$ ) 
Table 13-29: Hysteretic Device - 70-100\% System Absolute Maximum Reactions

\begin{tabular}{|c|c|c|c|c|c|c|c|c|c|c|}
\hline & & \multicolumn{8}{|c|}{$\%$ of IEEE693 0.5g Motion } & \\
\hline $\begin{array}{c}\text { Absolute Max } \\
\text { Reaction }\end{array}$ & Method & $70 \%$ & $75 \%$ & $80 \%$ & $85 \%$ & $90 \%$ & $95 \%$ & $100 \%$ & $100 \% *$ & \\
\hline Base Moment X & Strain & 825 & 821 & 771 & 839 & 887 & 962 & 1070 & 984 & \\
\hline Base Moment $Y$ & Strain & 947 & 996 & 1005 & 1021 & 1093 & 1055 & 1095 & 1047 & \\
\hline Base Moment X' & Strain & 1046 & 1121 & 1158 & 1223 & 1348 & 1325 & 1436 & 1341 & $\frac{2}{z}$ \\
\hline Base Moment $Y^{\prime}$ & Strain & 605 & 706 & 801 & 882 & 946 & 932 & 910 & 921 & $\frac{1}{2}$ \\
\hline Base Moment X & Accel. & 872 & 856 & 878 & 941 & 996 & 1050 & 1174 & 1098 & \\
\hline Base Moment $Y$ & Accel. & 955 & 1005 & 1043 & 1046 & 1125 & 1082 & 1134 & 1109 & $\ddot{\bar{c}}$ \\
\hline Base Shear X & Accel. & 3.4 & 3.5 & 3.6 & 4.1 & 4.2 & 4.4 & 4.9 & 4.7 & \\
\hline Base Shear Y & Accel. & 4.3 & 4.4 & 4.3 & 4.7 & 4.8 & 5.0 & 5.2 & 5.1 & \\
\hline CT-Inter. X Moment & Accel. & 596 & 616 & 598 & 618 & 667 & 705 & 796 & 768 & \\
\hline CT Inter. Y Moment & Accel. & 669 & 692 & 688 & 706 & 758 & 755 & 788 & 805 & $\Sigma$ \\
\hline CT Inter. X Shear & Accel. & 3.2 & 3.2 & 3.3 & 3.6 & 3.7 & 3.9 & 4.4 & 4.1 & \\
\hline CT Inter. Y Shear & Accel. & 2.6 & 2.9 & 2.9 & 3.3 & 3.1 & 3.7 & 4.2 & 4.9 & \\
\hline
\end{tabular}

(See Figure 12-2, Strain Gauge Placement for $(\mathrm{X}, \mathrm{Y}) \&\left(\mathrm{X}^{\prime}, \mathrm{Y}^{\prime}\right)$ )

Relative displacement between the shake table and key locations on the specimen were determined and summarized in Table 13-30 and Table 13-31. The virgin 100\% case had a maximum displacement of 22.38 inches at the top of the structure and 13.02 inches at the C.G. location. For the same motion, with non-virgin UFPs, the system experienced 24.56 inches of displacement at the top of the structure and 14.26 inches at the C.G.. The tables summarize the maximum displacement in both directions of motion at the CTpedestal interface, C.G. of the specimen, and top of the specimen. A complete set of moment-displacement plots for $20 \%, 50 \%, 75 \%$, and $100 \%$ motions are located in Appendix J. 
Table 13-30: Hysteretic Device - System Maximum Displacement 20-70\% Motions

\begin{tabular}{|c|c|c|c|c|c|c|c|c|c|}
\hline & & \multicolumn{7}{|c|}{$\%$ of IEEE693 $0.5 \mathrm{~g}$ Motion } & \\
\hline Location & $\begin{array}{c}\text { Location from } \\
\text { Base (in.) }\end{array}$ & $20 \%$ & $30 \%$ & $40 \%$ & $50 \%$ & $60 \%$ & $65 \%$ & $70 \%$ & \\
\hline Top of Pedestal X & $911 / 2$ & 1.29 & 1.62 & 2.11 & 2.27 & 2.70 & 2.85 & 2.78 & \\
\hline Top of Pedestal Y & $911 / 2$ & 1.11 & 1.43 & 2.05 & 2.69 & 3.29 & 3.53 & 3.70 & \pm \\
\hline CGX & 215 & 3.64 & 4.52 & 5.81 & 6.12 & 7.40 & 7.78 & 7.59 & 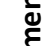 \\
\hline CG Y & $2161 / 2$ & 3.16 & 4.08 & 5.65 & 7.26 & 8.78 & 9.45 & 9.91 & 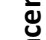 \\
\hline Top of Specimen X & $3521 / 2$ & 6.38 & 7.92 & 10.15 & 10.64 & 12.87 & 13.51 & 13.14 & $\frac{\pi}{0}$ \\
\hline Top of Specimen $Y$ & $3521 / 2$ & 5.51 & 7.15 & 9.79 & 12.47 & 15.10 & 16.26 & 17.03 & $\ddot{\Delta}$ \\
\hline
\end{tabular}

Table 13-31: Hysteretic Device - System Maximum Displacement 75-100\% Motions

\begin{tabular}{|c|c|c|c|c|c|c|c|c|c|}
\hline \multicolumn{2}{|c|}{ 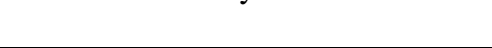 } & \multicolumn{7}{|c|}{$\%$ of IEEE693 0.5g Motion } & \\
\hline Location & $\begin{array}{c}\text { Location from } \\
\text { Base (in.) } \\
\end{array}$ & $75 \%$ & $80 \%$ & $85 \%$ & $90 \%$ & $95 \%$ & $100 \%$ & $100 \% *$ & \\
\hline Top of Pedestal X & $911 / 2$ & 2.70 & 3.26 & 3.75 & 4.33 & 4.82 & 5.52 & 5.02 & \multirow{6}{*}{ 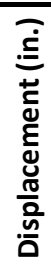 } \\
\hline Top of Pedestal Y & $911 / 2$ & 3.85 & 4.10 & 4.37 & 4.67 & 4.91 & 5.26 & 5.07 & \\
\hline CGX & 215 & 7.32 & 8.51 & 9.76 & 11.20 & 12.43 & 14.26 & 12.93 & \\
\hline CGY & $2161 / 2$ & 10.32 & 10.73 & 11.38 & 12.14 & 12.76 & 13.64 & 13.02 & \\
\hline Top of Specimen X & $3521 / 2$ & 12.71 & 14.77 & 16.90 & 19.34 & 21.43 & 24.56 & 22.38 & \\
\hline Top of Specimen $Y$ & $3521 / 2$ & 17.78 & 18.37 & 19.38 & 20.60 & 21.60 & 23.05 & 21.88 & \\
\hline
\end{tabular}

The rocking system with hysteretic devices presented true self-centering with

minimal permanent drift. One instance presented a permanent drift value of 0.14 inch that was considered negligible compared to the system maximum displacement amplitude and structure height. All residual displacements are summarized in Table 13-32 and Table 13-33.

Table 13-32: Hysteretic Device - System Residual Displacement 20-70\% Motions

\begin{tabular}{|c|c|c|c|c|c|c|c|c|c|}
\hline & & \multicolumn{7}{|c|}{$\%$ of IEEE693 0.5g Motion } & \\
\hline Location & $\begin{array}{c}\text { Location from } \\
\text { Base (in.) } \\
\end{array}$ & $20 \%$ & $30 \%$ & $40 \%$ & $50 \%$ & $60 \%$ & $65 \%$ & $70 \%$ & \\
\hline Top of Pedestal X & $911 / 2$ & -0.03 & -0.01 & 0.00 & 0.01 & 0.02 & 0.01 & 0.00 & \\
\hline Top of Pedestal Y & $911 / 2$ & 0.00 & 0.01 & 0.04 & -0.02 & 0.01 & 0.01 & 0.00 & Ez \\
\hline CGX & 215 & -0.08 & -0.03 & 0.00 & 0.03 & 0.04 & 0.02 & 0.00 & 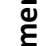 \\
\hline CG Y & $2161 / 2$ & -0.03 & 0.01 & 0.08 & -0.05 & 0.02 & 0.01 & -0.01 & 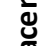 \\
\hline Top of Specimen X & $3521 / 2$ & -0.14 & -0.06 & 0.01 & 0.06 & 0.07 & 0.01 & 0.00 & $\frac{5}{2}$ \\
\hline Top of Specimen Y & $3521 / 2$ & 0.02 & -0.01 & 0.00 & 0.00 & 0.01 & 0.02 & 0.02 & 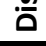 \\
\hline
\end{tabular}


Table 13-33: Hysteretic Device - System Residual Displacement 75-100\% Motions

\begin{tabular}{|c|c|c|c|c|c|c|c|c|c|}
\hline & & \multicolumn{7}{|c|}{$\%$ of IEEE693 0.5g Motion } & \\
\hline Location & $\begin{array}{c}\text { Location from } \\
\text { Base (in.) }\end{array}$ & $75 \%$ & $80 \%$ & $85 \%$ & $90 \%$ & $95 \%$ & $100 \%$ & $100 \% *$ & \\
\hline Top of Pedestal X & $911 / 2$ & 0.01 & 0.00 & 0.02 & 0.01 & 0.00 & -0.01 & 0.01 & ‡̇ \\
\hline Top of Pedestal Y & $911 / 2$ & 0.00 & 0.01 & 0.01 & 0.02 & 0.01 & 0.01 & 0.04 & \\
\hline $\operatorname{CGX}$ & 215 & 0.01 & -0.02 & 0.02 & 0.02 & 0.00 & -0.03 & 0.02 & zot \\
\hline CGY & $2161 / 2$ & 0.01 & 0.00 & 0.04 & 0.02 & 0.03 & 0.03 & 0.12 & صِّ \\
\hline Top of Specimen X & $3521 / 2$ & 0.00 & -0.03 & 0.03 & 0.03 & 0.01 & -0.05 & 0.01 & $\frac{0}{2}$ \\
\hline Top of Specimen Y & $3521 / 2$ & -0.01 & 0.00 & 0.01 & -0.01 & 0.00 & 0.01 & -0.02 & 5 \\
\hline
\end{tabular}

The pedestal was instrumented with 8 strain gauges as outlined in 12.1,

Instrumentation. Most strain pairs presented values less than the yield strain of $1450 \mu \varepsilon$, but the NW-SE strain pair. The NW-SE strain pair experienced strains of $1892 \mu \varepsilon-$ $2240 \mu \varepsilon$ for the $0.5 \mathrm{~g} 100 \%$ IEEE693 motions.

Table 13-34: Hysteretic Device - Pedestal Absolute Maximum Strain

\begin{tabular}{|c|c|c|c|c|c|c|c|c|c|c|c|c|c|c|}
\hline \multirow[b]{2}{*}{$\begin{array}{c}\text { Pedestal Strain } \\
(\mu \varepsilon)\end{array}$} & \multicolumn{14}{|c|}{$\%$ of IEEE693 $0.5 \mathrm{~g}$ Motion } \\
\hline & $20 \%$ & $30 \%$ & $40 \%$ & $50 \%$ & $60 \%$ & $65 \%$ & $70 \%$ & $75 \%$ & $80 \%$ & $85 \%$ & $90 \%$ & $95 \%$ & $100 \%$ & $100 \% *$ \\
\hline North Gauge & 547 & 707 & 917 & 1016 & 1116 & 1182 & 1197 & 1243 & 1252 & 1262 & 1338 & 1277 & 1303 & 1235 \\
\hline South Gauge & 596 & 749 & 928 & 946 & 1003 & 1076 & 1104 & 1134 & 1167 & 1181 & 1241 & 1288 & 1335 & 1253 \\
\hline NE Gauge & 547 & 768 & 1021 & 781 & 742 & 789 & 772 & 909 & 1039 & 1206 & 1322 & 1214 & 1140 & 1274 \\
\hline SW Gauge & 537 & 750 & 1048 & 678 & 807 & 781 & 867 & 1052 & 1220 & 1368 & 1484 & 1454 & 1426 & 1430 \\
\hline East Gauge & 458 & 576 & 656 & 684 & 699 & 724 & 747 & 744 & 777 & 830 & 884 & 960 & 1094 & 1001 \\
\hline West Gauge & 588 & 719 & 798 & 831 & 997 & 1045 & 1041 & 1035 & 975 & 1043 & 1078 & 1078 & 1179 & 1087 \\
\hline SE Gauge & 745 & 883 & 1161 & 1161 & 1332 & 1341 & 1375 & 1519 & 1605 & 1737 & 1820 & 1923 & 2134 & 1892 \\
\hline NW Gauge & 758 & 873 & 1086 & 1188 & 1368 & 1501 & 1584 & 1703 & 1770 & 1894 & 2101 & 2054 & 2240 & 2085 \\
\hline
\end{tabular}

The high strains above $1450 \mu \varepsilon$ caused minor pedestal yielding which could be seen by examining the residual pedestal strains shown in Table 13-35. For all motions above $70 \%$ where the max strain values exceeded the yielding strain, the sensors exhibited larger residual strains. 
Table 13-35: Hysteretic Device - Pedestal Residual Strain

\begin{tabular}{|c|c|c|c|c|c|c|c|c|c|c|c|c|c|c|}
\hline & \multicolumn{14}{|c|}{$\%$ of IEEE693 0.5g Motion } \\
\hline $\begin{array}{c}\text { Pedestal Strain } \\
(\mu \varepsilon)\end{array}$ & $20 \%$ & $30 \%$ & $40 \%$ & $50 \%$ & $60 \%$ & $65 \%$ & $70 \%$ & $75 \%$ & $80 \%$ & $85 \%$ & $90 \%$ & $95 \%$ & $100 \%$ & $100 \% *$ \\
\hline North Gauge & 6 & 0 & 5 & 0 & -1 & 3 & 7 & 8 & 12 & 17 & 30 & 7 & 24 & 3 \\
\hline South Gauge & 3 & -1 & 4 & -2 & -3 & -2 & 0 & 2 & 8 & 10 & 8 & 17 & 42 & 2 \\
\hline NE Gauge & -4 & -1 & -4 & -5 & -2 & 0 & 2 & 1 & 1 & 0 & 13 & -2 & 17 & -3 \\
\hline SW Gauge & -2 & -3 & -1 & -1 & 1 & -2 & -3 & -2 & 0 & 5 & 5 & 0 & 8 & -2 \\
\hline East Gauge & -4 & 2 & -1 & 0 & 0 & 1 & 0 & 0 & -2 & 7 & 11 & 20 & 27 & 6 \\
\hline West Gauge & -6 & 1 & 0 & 5 & 7 & 4 & 1 & 7 & 9 & 20 & 32 & 10 & 17 & 3 \\
\hline SE Gauge & 6 & 4 & 4 & 1 & 1 & 0 & 0 & 1 & 0 & 14 & 16 & 32 & 45 & 18 \\
\hline NW Gauge & 4 & 2 & 4 & 5 & 3 & 5 & 5 & 16 & 19 & 32 & 58 & 23 & 37 & 17 \\
\hline
\end{tabular}

Examining bracket slip allows for a better understanding of the device

displacement results. Shown in Table 13-36 are the individual device bracket slip values.

When bracket slip occurs, the UFP is not deformed and efficient energy dissipation does

not occur. Examining the SW-S device, slip initiated at early stages and continued

throughout the testing sequence. Torque verification was not performed on the slip-

critical bracket, leading to slip in the bracket. In reality, torque verification would not

likely happen between an initial subduction zone (mega thrust) event and the aftershocks.

The rocking system with hysteretic devices was tested with 3 out of 4 dampers fully

engaged.

Table 13-36: Hysteretic Device - UFP Bracket Slip

\begin{tabular}{|c|c|c|c|c|c|c|c|c|c|c|c|c|c|c|}
\hline \multirow[b]{2}{*}{$\begin{array}{c}\text { Device Slip } \\
\text { (in.) }\end{array}$} & \multicolumn{14}{|c|}{$\%$ of IEEE693 $0.5 \mathrm{~g}$ Motion } \\
\hline & $20 \%$ & $30 \%$ & $40 \%$ & $50 \%$ & $60 \%$ & $65 \%$ & $70 \%$ & $75 \%$ & $80 \%$ & $85 \%$ & $90 \%$ & $95 \%$ & $100 \%$ & $100 \% *$ \\
\hline NE-N & 0.004 & 0.009 & 0.012 & 0.008 & 0.009 & 0.013 & 0.014 & 0.014 & 0.015 & 0.015 & 0.016 & 0.016 & 0.017 & 0.015 \\
\hline SE-S & 0.005 & 0.006 & 0.013 & 0.016 & 0.018 & 0.019 & 0.019 & 0.017 & 0.016 & 0.016 & 0.017 & 0.019 & 0.023 & 0.023 \\
\hline SW-S & 0.015 & 0.023 & 0.032 & 0.039 & 0.049 & 0.064 & 0.066 & 0.072 & 0.081 & 0.097 & 0.158 & 0.306 & 0.399 & 0.371 \\
\hline NW-N & 0.003 & 0.004 & 0.009 & 0.011 & 0.012 & 0.013 & 0.013 & 0.013 & 0.012 & 0.013 & 0.017 & 0.019 & 0.022 & 0.018 \\
\hline
\end{tabular}

The bracket slip is reflected in the UFP displacement results presented in Table 13-37. The bracket connection continued to loosen as demonstrated by the device 
displacement data. Comparing the magnitude of displacement in all devices for the $100 \%$ motion, the SW device displacements was roughly $30 \%$ of the SE and NW displacement, and $50 \%$ of the NE displacement.

Table 13-37: Hysteretic Device - UFP Absolute Maximum Displacement

\begin{tabular}{|c|c|c|c|c|c|c|c|c|c|c|c|c|c|c|}
\hline & \multicolumn{14}{|c|}{$\%$ of IEEE693 0.5g Motion } \\
\hline $\begin{array}{c}\text { Device } \\
\text { Dispalcement }\end{array}$ & $20 \%$ & $30 \%$ & $40 \%$ & $50 \%$ & $60 \%$ & $65 \%$ & $70 \%$ & $75 \%$ & $80 \%$ & $85 \%$ & $90 \%$ & $95 \%$ & $100 \%$ & $100 \% *$ \\
\hline $\mathrm{NE}$ & 0.132 & 0.198 & 0.360 & 0.369 & 0.512 & 0.599 & 0.671 & 0.732 & 0.819 & 0.885 & 0.954 & 1.012 & 1.101 & 1.069 \\
\hline SE & 0.155 & 0.211 & 0.373 & 0.507 & 0.756 & 0.869 & 0.972 & 1.045 & 1.180 & 1.267 & 1.362 & 1.512 & 1.602 & 1.553 \\
\hline SW & 0.204 & 0.252 & 0.349 & 0.451 & 0.579 & 0.610 & 0.641 & 0.649 & 0.678 & 0.659 & 0.614 & 0.534 & 0.574 & 0.500 \\
\hline NW & 0.218 & 0.283 & 0.479 & 0.494 & 0.671 & 0.784 & 0.863 & 0.933 & 1.063 & 1.220 & 1.399 & 1.520 & 1.698 & 1.589 \\
\hline
\end{tabular}

After each motion was complete, the self-centering mechanism along with the self-weight plumbed the system. The hysteretic devices were yielded back to their original, however possessing residual forces, to plumb the system. Demonstrated in Table 13-38 is the systems ability to deform the UFPs back to their original orientation and selfcenter the system.

Table 13-38: Hysteretic Device - UFP Residual Displacement

\begin{tabular}{|c|c|c|c|c|c|c|c|c|c|c|c|c|c|c|}
\hline \multirow[b]{2}{*}{$\begin{array}{c}\text { Device } \\
\text { Dispalcement }\end{array}$} & \multicolumn{14}{|c|}{$\%$ of IEEE693 $0.5 \mathrm{~g}$ Motion } \\
\hline & $20 \%$ & $30 \%$ & $40 \%$ & $50 \%$ & $60 \%$ & $65 \%$ & $70 \%$ & $75 \%$ & $80 \%$ & $85 \%$ & $90 \%$ & $95 \%$ & $100 \%$ & $100 \% *$ \\
\hline NE & -0.010 & -0.002 & 0.010 & -0.001 & 0.006 & 0.003 & -0.001 & 0.000 & -0.002 & 0.003 & 0.001 & 0.001 & -0.002 & 0.015 \\
\hline SE & -0.001 & 0.000 & 0.004 & 0.012 & 0.008 & 0.004 & 0.003 & 0.002 & 0.002 & 0.004 & 0.004 & 0.008 & 0.011 & 0.012 \\
\hline SW & 0.007 & 0.002 & 0.000 & 0.008 & 0.014 & 0.006 & 0.012 & 0.008 & 0.006 & 0.024 & 0.068 & 0.069 & 0.037 & -0.011 \\
\hline NW & 0.003 & 0.002 & 0.012 & 0.000 & 0.007 & 0.007 & 0.004 & 0.005 & 0.005 & 0.009 & 0.013 & 0.015 & 0.019 & 0.027 \\
\hline
\end{tabular}

Device forces were measured directly through the anchoring rod for the UFP sets. Modeling and design assumed pure flexural contribution of the UFP. Under rocking, slight torsional resistance and axial resistance could have contributed to increased the device force. While under dynamic motion, torsion, axial, and flexure may contribute to UFP resistance, when re-centering the system, combined shear-flexure action controls the 
UFP response. The UFP maximum force ranged from 7 kips-8.3 kips in the 4 devices; all device maximum forces are reported in Table 13-39.

\begin{tabular}{|c|c|c|c|c|c|c|c|c|c|c|c|c|c|c|}
\hline \multirow[b]{2}{*}{$\begin{array}{c}\text { Device } \\
\text { Force (kips) } \\
\end{array}$} & \multicolumn{14}{|c|}{$\%$ of IEEE693 0.5g Motion } \\
\hline & $20 \%$ & $30 \%$ & $40 \%$ & $50 \%$ & $60 \%$ & $65 \%$ & $70 \%$ & $75 \%$ & $80 \%$ & $85 \%$ & $90 \%$ & $95 \%$ & $100 \%$ & $100 \% *$ \\
\hline NE Device & 5.07 & 5.21 & 6.23 & 6.29 & 6.65 & 7.01 & 7.11 & 7.18 & 7.32 & 7.28 & 7.35 & 7.38 & 7.60 & 7.90 \\
\hline SE Device & 5.04 & 5.92 & 6.61 & 6.73 & 7.35 & 7.66 & 7.34 & 7.00 & 6.73 & 6.83 & 7.00 & 7.11 & 7.28 & 7.95 \\
\hline SW Device & 3.56 & 3.96 & 4.97 & 4.59 & 5.31 & 5.36 & 5.52 & 5.46 & 5.42 & 5.47 & 6.23 & 6.32 & 7.00 & 7.09 \\
\hline NW Device & 4.33 & 5.06 & 6.14 & 6.32 & 6.89 & 7.01 & 7.24 & 7.27 & 7.32 & 7.59 & 7.50 & 7.48 & \begin{tabular}{|l|}
7.71 \\
\end{tabular} & 8.32 \\
\hline
\end{tabular}

The BeS linear load for the specified Belleville spring is 12.76 kips. Since one damper was minimally engaging, the BeS system experienced higher forces than expected. For the $100 \%$ motions with non-virgin UFPs, the PT force exceeded 12.76 kips causing the BeS washers to permanently deform and pre-tension force to be lost. The largest loss in pre-tension force was $8.3 \%$ in the $100 \%$ non-virgin test case. Shown in Table 13-40 is the maximum force experienced by the PT member. Table 13-41 compares the initial PT load to the final PT load,. Decreases in PT load are caused by slight shifting in the system base or PT yielding. Although the load exceeded the linear load, the design specified is expected to perform without exceedance with all 4 UFP sets engaged.

Table 13-40: Hysteretic Device - Maximum PT Force

\begin{tabular}{|c|c|c|c|c|c|c|c|c|c|c|c|c|c|c|}
\cline { 2 - 14 } \multicolumn{1}{c|}{} & \multicolumn{10}{c|}{ \% of IEEE693 0.5g Motion } \\
\hline PT Force (kips) & $20 \%$ & $30 \%$ & $40 \%$ & $50 \%$ & $60 \%$ & $65 \%$ & $70 \%$ & $75 \%$ & $80 \%$ & $85 \%$ & $90 \%$ & $95 \%$ & $100 \%$ & $100 \% *$ \\
\hline PT North & 4.60 & 4.89 & 5.68 & 6.09 & 6.86 & 7.71 & 8.29 & 8.83 & 9.50 & 9.92 & 10.45 & 10.85 & 11.45 & 11.20 \\
\hline PT East & 4.41 & 4.71 & 5.30 & 5.85 & 7.06 & 7.42 & 7.41 & 7.80 & 8.45 & 8.97 & 9.48 & 10.40 & 11.08 & 10.95 \\
\hline PT South & 4.38 & 4.90 & 5.65 & 6.78 & 8.01 & 8.56 & 8.94 & 9.18 & 9.93 & 10.31 & 10.58 & 11.53 & 11.85 & 11.64 \\
\hline PT West & 4.72 & 5.06 & 6.00 & 6.19 & 6.56 & 6.81 & 7.11 & 7.93 & 8.69 & 9.69 & 10.72 & 11.81 & 13.02 & 12.61 \\
\hline
\end{tabular}


Minimal loss in PT force was presented for the system when exposed to motions below $80 \%$. Less than $3 \%$ PT loss was exhibited by all motions from $20 \%-80 \%$. For motions above $80 \%$, generally, less than $5 \%$ PT loss was presented by the system, although one instance exceeded $5 \%$ loss.

Table 13-41: Hysteretic Device - \% PT Force Loss Post Motion

\begin{tabular}{|c|c|c|c|c|c|c|c|c|c|c|c|c|c|c|}
\hline & \multicolumn{14}{|c|}{$\%$ of IEEE693 $0.5 \mathrm{~g}$ Motion } \\
\hline $\begin{array}{c}\text { PT Force Loss } \\
\text { (kips) }\end{array}$ & $20 \%$ & $30 \%$ & $40 \%$ & $50 \%$ & $60 \%$ & $65 \%$ & $70 \%$ & $75 \%$ & $80 \%$ & $85 \%$ & $90 \%$ & $95 \%$ & $100 \%$ & $100 \% *$ \\
\hline PT North & $-1.4 \%$ & $-0.4 \%$ & $-0.6 \%$ & $-0.6 \%$ & $-1.2 \%$ & $-2.0 \%$ & $-1.6 \%$ & $-1.2 \%$ & $-1.2 \%$ & $-3.3 \%$ & $-2.0 \%$ & $-3.4 \%$ & $-4.2 \%$ & $-3.3 \%$ \\
\hline PT East & $-1.9 \%$ & $-0.4 \%$ & $-0.6 \%$ & $0.0 \%$ & $-1.2 \%$ & $-0.6 \%$ & $0.0 \%$ & $-0.6 \%$ & $-1.2 \%$ & $-1.9 \%$ & $-1.2 \%$ & $-2.9 \%$ & $-3.1 \%$ & $-1.4 \%$ \\
\hline PT South & $0.0 \%$ & $-0.4 \%$ & $-1.5 \%$ & $-0.6 \%$ & $-2.1 \%$ & $-1.9 \%$ & $-0.8 \%$ & $-1.0 \%$ & $-1.4 \%$ & $-2.7 \%$ & $-2.2 \%$ & $-4.8 \%$ & $-4.2 \%$ & $-2.5 \%$ \\
\hline PT West & $-1.1 \%$ & $0.0 \%$ & $-0.9 \%$ & $-1.1 \%$ & $-0.7 \%$ & $-0.9 \%$ & $-0.6 \%$ & $-1.7 \%$ & $-0.9 \%$ & $-2.5 \%$ & $-3.0 \%$ & $-4.3 \%$ & $-8.3 \%$ & $-3.9 \%$ \\
\hline
\end{tabular}

Displacement in the system increased as the input motion amplitude was increased. System displacement primarily consisted of flexure and rotation of the structure due to rocking. The maximum base uplift was manifested by the structure for the non-virgin $100 \% 0.5 \mathrm{~g}$ IEEE693 motion, see Table 13-42. A vertical uplift of 1.49 inches was measured by the LVDT located in the center of the west base plate edge. The north, east, and south sides measured 1.37 inches, 1.13 inches, and 1.28 inches respectively. Using virgin hysteretic devices, uplift reductions of $2 \%-8 \%$ were exhibited by the system. Using virgin UFPs changes the behavior of the initial loop of the hysteresis, starting at zero rather than maximum negative pre-compressed load. The differences in device response could easily be distinguished when comparing the forcedisplacement curves in Appendix $\mathbf{J}$. 
Table 13-42: Hysteretic Device - Pedestal Rocking Uplift

\begin{tabular}{|c|c|c|c|c|c|c|c|c|c|c|c|c|c|c|}
\cline { 2 - 13 } \multicolumn{1}{c|}{} & \multicolumn{10}{c|}{ \% of IEEE63 0.5g Motion } \\
\hline Uplift (in.) & $20 \%$ & $30 \%$ & $40 \%$ & $50 \%$ & $60 \%$ & $65 \%$ & $70 \%$ & $75 \%$ & $80 \%$ & $85 \%$ & $90 \%$ & $95 \%$ & $100 \%$ & $100 \% *$ \\
\hline Base PL Uplift N & 0.18 & 0.24 & 0.40 & 0.45 & 0.65 & 0.78 & 0.86 & 0.93 & 1.02 & 1.10 & 1.19 & 1.26 & 1.37 & 1.34 \\
\hline Base PL Uplift E & 0.14 & 0.21 & 0.32 & 0.41 & 0.63 & 0.67 & 0.65 & 0.63 & 0.72 & 0.80 & 0.89 & 1.03 & 1.13 & 1.10 \\
\hline Base PL Uplift S & 0.17 & 0.23 & 0.38 & 0.56 & 0.76 & 0.85 & 0.92 & 0.96 & 1.06 & 1.10 & 1.15 & 1.25 & 1.28 & 1.21 \\
\hline Base PL Uplift W & 0.20 & 0.27 & 0.43 & 0.46 & 0.52 & 0.56 & 0.58 & 0.70 & 0.85 & 0.99 & 1.17 & 1.31 & 1.49 & 1.37 \\
\hline
\end{tabular}

While slight base plate translations occur during the motion, no residual base plate displacement was presented for any of the test cases. A contributing factor to the horizontal displacements measured was the base rocking. The UFPs out-of-plane stiffness helped prevent base translation. Summarized in Table 13-43 are the magnitudes of the maximum translation measured on all corners of the pedestal base plate. Shown in Table 13-44 are the residual translations, where all values measured were in the instrument noise range.

Table 13-43: Hysteretic Device - Maximum Base Plate Slip

\begin{tabular}{|c|c|c|c|c|c|c|c|c|c|c|c|c|c|c|}
\cline { 2 - 13 } \multicolumn{1}{c|}{} & \multicolumn{10}{c|}{ \% of IEEE693 0.5g Motion } \\
\hline Device Uplift (in.) & $20 \%$ & $30 \%$ & $40 \%$ & $50 \%$ & $60 \%$ & $65 \%$ & $70 \%$ & $75 \%$ & $80 \%$ & $85 \%$ & $90 \%$ & $95 \%$ & $100 \%$ & $100 \% *$ \\
\hline Base PL Slip NE-X & 0.02 & 0.02 & 0.03 & 0.04 & 0.06 & 0.06 & 0.05 & 0.05 & 0.06 & 0.06 & 0.06 & 0.06 & 0.07 & 0.07 \\
\hline Base PL Slip SE-X & 0.04 & 0.03 & 0.04 & 0.04 & 0.06 & 0.08 & 0.08 & 0.08 & 0.08 & 0.08 & 0.09 & 0.11 & 0.13 & 0.14 \\
\hline Base PL Slip SW-X & 0.03 & 0.03 & 0.05 & 0.06 & 0.04 & 0.05 & 0.07 & 0.06 & 0.09 & 0.08 & 0.10 & 0.12 & 0.14 & 0.11 \\
\hline Base PL Slip NW-X & 0.02 & 0.03 & 0.04 & 0.05 & 0.06 & 0.07 & 0.07 & 0.07 & 0.08 & 0.08 & 0.10 & 0.12 & 0.14 & 0.12 \\
\hline Base PL Slip NE-Y & 0.04 & 0.04 & 0.05 & 0.05 & 0.06 & 0.08 & 0.09 & 0.09 & 0.10 & 0.11 & 0.12 & 0.13 & 0.15 & 0.14 \\
\hline Base PL Slip SE-Y & 0.03 & 0.03 & 0.04 & 0.05 & 0.08 & 0.09 & 0.10 & 0.10 & 0.11 & 0.13 & 0.13 & 0.14 & 0.15 & 0.13 \\
\hline Base PL Slip SW-Y & 0.02 & 0.03 & 0.05 & 0.07 & 0.09 & 0.10 & 0.10 & 0.10 & 0.11 & 0.12 & 0.12 & 0.13 & 0.12 & 0.12 \\
\hline Base PL Slip NW-Y & 0.02 & 0.03 & 0.04 & 0.05 & 0.05 & 0.07 & 0.07 & 0.09 & 0.10 & 0.10 & 0.12 & 0.12 & 0.14 & 0.12 \\
\hline
\end{tabular}


Table 13-44: Hysteretic Device - Relative Base Plate Movement Pre-Post Motion $\%$ of IEEE693 $0.5 \mathrm{~g}$ Motion

\begin{tabular}{|c|c|c|c|c|c|c|c|c|c|c|c|c|c|c|}
\hline Device Uplift (in.) & $20 \%$ & $30 \%$ & $40 \%$ & $50 \%$ & $60 \%$ & $65 \%$ & $70 \%$ & $75 \%$ & $80 \%$ & $85 \%$ & $90 \%$ & $95 \%$ & $100 \%$ & $100 \% *$ \\
\hline Base PL Slip NE-X & 0.00 & 0.00 & 0.00 & 0.00 & 0.00 & 0.00 & 0.00 & -0.01 & 0.00 & -0.01 & 0.00 & 0.00 & -0.01 & 0.00 \\
\hline Base PL Slip SE-X & -0.02 & -0.01 & -0.01 & 0.00 & -0.01 & -0.01 & 0.00 & -0.01 & 0.00 & -0.01 & 0.00 & 0.00 & 0.00 & -0.01 \\
\hline Base PL Slip SW-X & 0.02 & 0.01 & 0.01 & 0.00 & 0.01 & 0.01 & 0.00 & 0.01 & 0.00 & 0.01 & 0.00 & 0.00 & 0.00 & 0.02 \\
\hline Base PL Slip NW-X & 0.00 & 0.00 & 0.00 & 0.00 & 0.01 & 0.00 & 0.00 & 0.01 & 0.00 & 0.01 & 0.00 & 0.00 & 0.01 & 0.01 \\
\hline Base PL Slip NE-Y & -0.03 & -0.02 & -0.02 & -0.02 & -0.01 & 0.00 & -0.01 & 0.00 & -0.01 & 0.00 & 0.00 & 0.00 & 0.00 & 0.01 \\
\hline Base PL Slip SE-Y & 0.03 & 0.02 & 0.02 & 0.02 & 0.01 & 0.00 & 0.01 & 0.00 & 0.01 & 0.00 & 0.00 & -0.01 & 0.00 & 0.00 \\
\hline Base PL Slip SW-Y & 0.01 & 0.01 & 0.02 & 0.01 & 0.00 & 0.00 & 0.00 & 0.00 & 0.01 & 0.00 & 0.00 & 0.00 & 0.01 & 0.00 \\
\hline Base PL Slip NW-Y & -0.01 & -0.01 & -0.01 & -0.01 & 0.00 & 0.00 & -0.01 & 0.01 & -0.01 & 0.00 & 0.00 & 0.00 & -0.01 & 0.01 \\
\hline
\end{tabular}

*virgin hysteretic device 


\subsection{Non-Retrofitted}

\subsubsection{Non-Retrofitted Table Response Spectrum}

The non-retrofitted system was exposed to three complete ground motions prior to pedestal strains exceeding $2500 \mu \varepsilon$. Amplitudes of $20 \%, 30 \%$, and $40 \%$ of the $0.5 \mathrm{~g}$ IEEE693 record were used to excite the system. When the system was exposed to the $50 \% 0.5 \mathrm{~g}$ IEEE693 motion, pedestal strains exceeded $2500 \mu \varepsilon$ set for the shake table limit. Shown in Figure 13-11, Figure 13-12, and Figure 13-13 is a comparison between the $0.5 \mathrm{~g}$ IEEE693 design spectrum and table response spectrum. For the horizontal directions, the table performed well near the fundamental frequency, $1.13 \mathrm{~Hz}-1.18 \mathrm{~Hz}$. More error is seen in the incomplete $50 \%$ motion compared to the other executed motions. Significant undershooting of the design spectrum was produced by the incomplete motion in the vertical direction. 


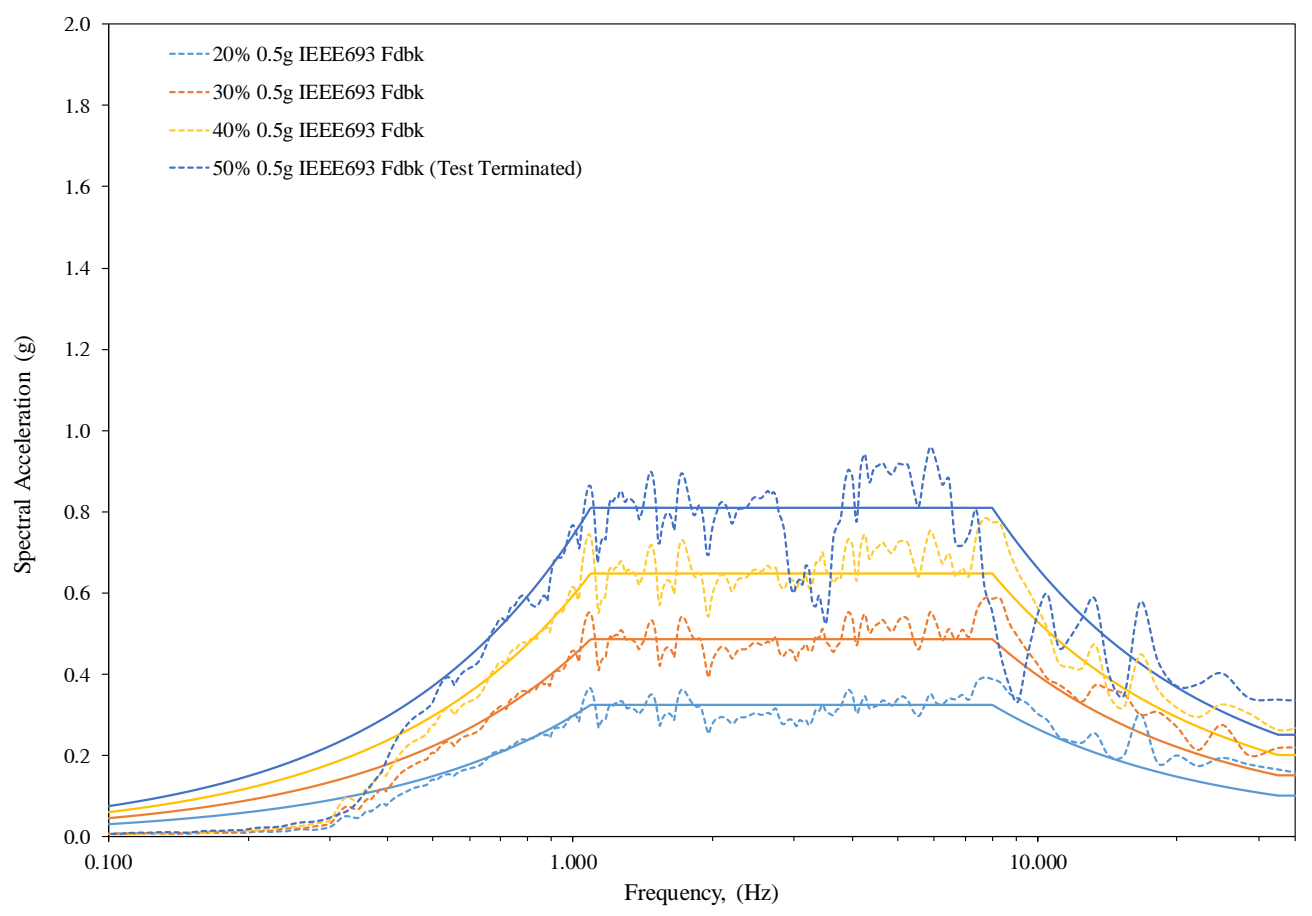

Figure 13-11: Non-Retrofitted - Response Spectrum X

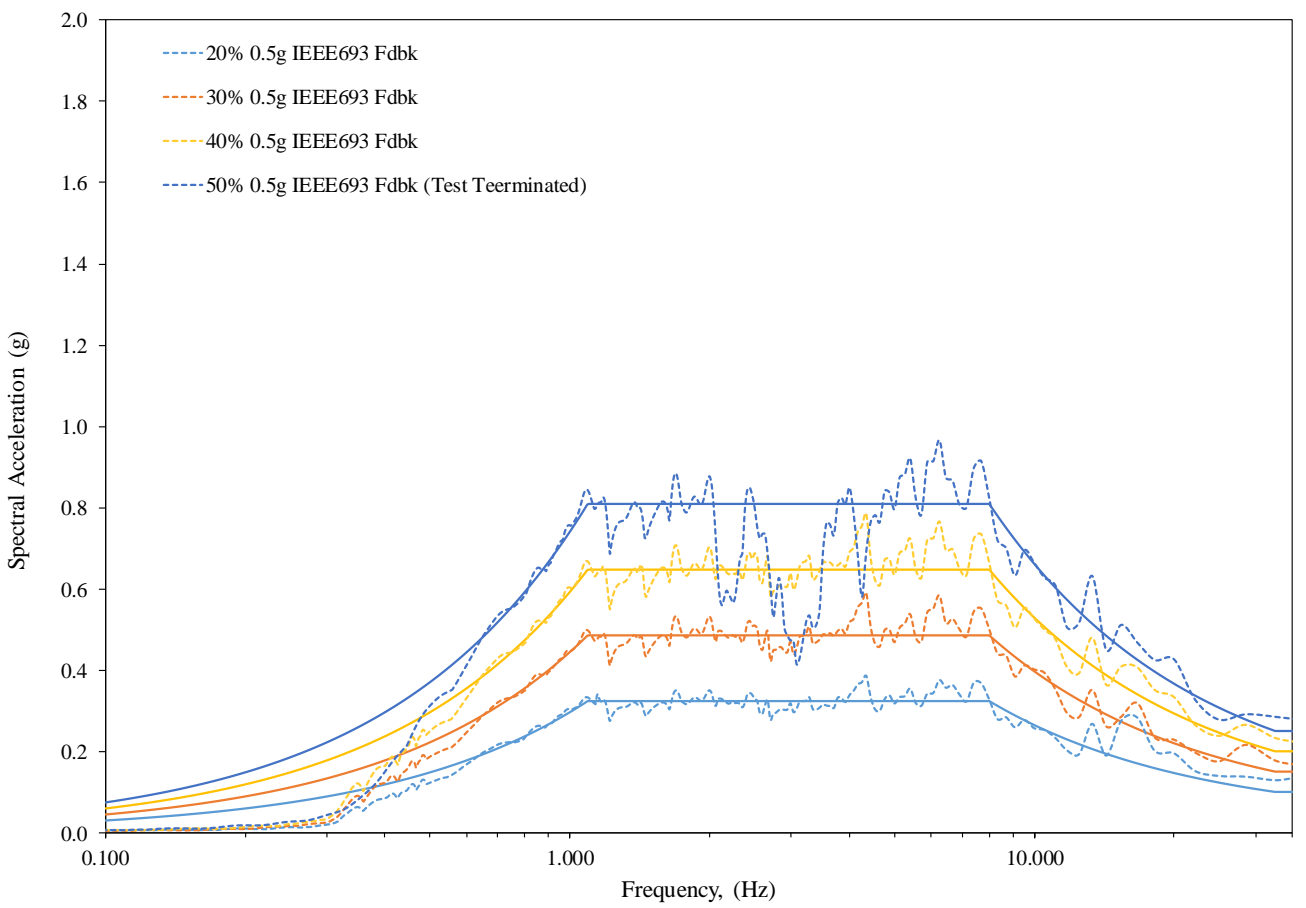

Figure 13-12: Non-Retrofitted- Response Spectrum Y 


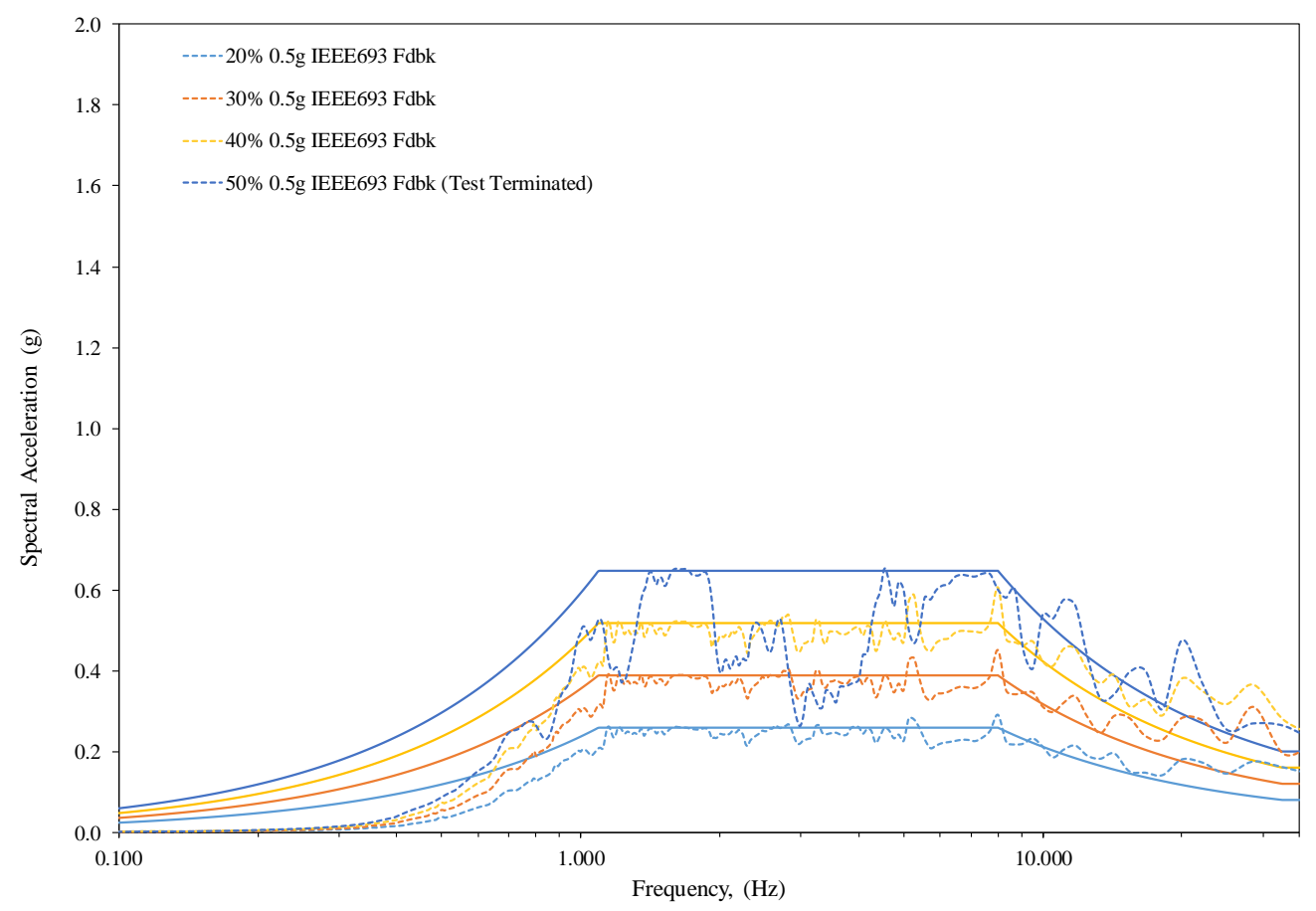

Figure 13-13: Non-Retrofitted- Response Spectrum Z

\subsubsection{Non-Retrofitted System Damping \& Free Vibration}

The non-retrofitted system has a fundamental frequency between $1.13 \mathrm{~Hz}-1.18 \mathrm{~Hz}$ in the horizontal directions. Using pulse excitation, the X-direction fundamental frequency was $1.13 \mathrm{~Hz}$. Similarly, exciting the structure via a pulse, the Y-direction had a fundamental frequency of $1.18 \mathrm{~Hz}$. Using white noise, the structure had a fundamental frequency of $1.18 \mathrm{~Hz}$ in both horizontal directions. The average vertical fundamental frequency was $28.8 \mathrm{~Hz}$. The non-retrofitted mass system has minimal elastic damping, estimated to be $0.32 \%-0.35 \%$. A complete summary of the individual pulses and white noise results are shown in Table 13-45. Free vibration plots for each test case are presented in Appendix K. 
Table 13-45: Non-Retrofitted System Damping and Fundamental Frequency

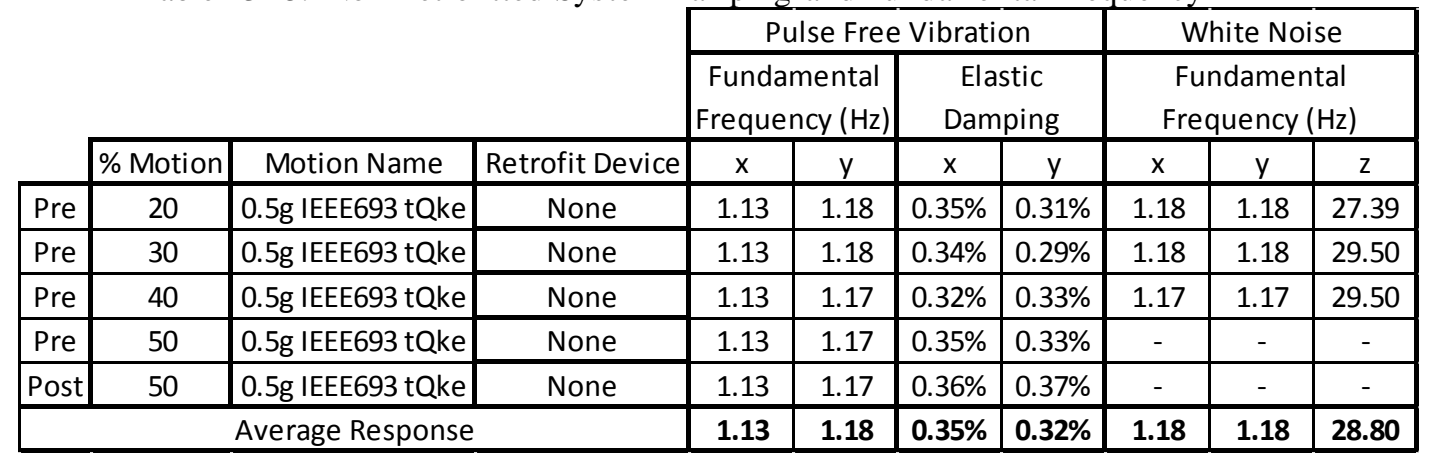

\subsubsection{Non-Retrofitted System Response}

The non-retrofitted system manifested accelerations at the top of the structure exceeding $1 \mathrm{~g}$ for motions $40 \%$ and above. Large amplifications were demonstrated by the system in the horizontal directions. Minimal vertical amplification was produced by the system because of the directional stiffness. Maximum acceleration values are reported in Table 13-46, Table 13-47, and Table 13-48.

Table 13-46: Non-Retrofitted - System X Absolute Maximum Acceleration

\begin{tabular}{|c|c|c|c|c|c|c|}
\hline & \multicolumn{4}{|c|}{$\%$ of IEEE693 0.5g Motion } & & \\
\hline & $20 \%$ & $30 \%$ & $40 \%$ & $50 \%$ & & \\
\hline \multirow{16}{*}{ 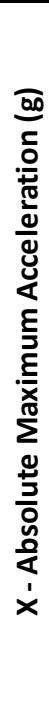 } & 0.67 & 0.77 & 1.12 & 1.38 & 351 & \multirow{16}{*}{ 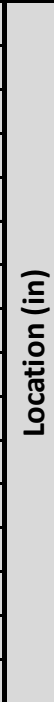 } \\
\hline & 0.60 & 0.67 & 0.95 & 1.18 & 322 & \\
\hline & 0.56 & 0.64 & 0.88 & 1.08 & $3041 / 2$ & \\
\hline & 0.54 & 0.62 & 0.86 & 1.01 & $2851 / 2$ & \\
\hline & 0.49 & 0.57 & 0.79 & 0.95 & $2681 / 2$ & \\
\hline & 0.47 & 0.53 & 0.76 & 0.91 & 254 & \\
\hline & 0.42 & 0.46 & 0.68 & 0.81 & 235 & \\
\hline & 0.39 & 0.45 & 0.67 & 0.81 & $2141 / 2$ & \\
\hline & 0.36 & 0.39 & 0.59 & 0.73 & 199 & \\
\hline & 0.33 & 0.35 & 0.53 & 0.67 & 182 & \\
\hline & 0.27 & 0.31 & 0.52 & 0.61 & 163 & \\
\hline & 0.25 & 0.29 & 0.49 & 0.59 & 146 & \\
\hline & 0.23 & 0.27 & 0.46 & 0.52 & 127 & \\
\hline & 0.19 & 0.23 & 0.39 & 0.43 & 91 & \\
\hline & 0.13 & 0.19 & 0.25 & 0.31 & $11 / 8$ & \\
\hline & 0.14 & 0.18 & 0.25 & 0.31 & 0 & \\
\hline
\end{tabular}


Table 13-47: Non-Retrofitted - System Y Absolute Maximum Acceleration

\begin{tabular}{|c|c|c|c|c|c|c|}
\hline & \multicolumn{4}{|c|}{$\%$ of IEEE693 $0.5 \mathrm{~g}$ Motion } & & \\
\hline & $20 \%$ & $30 \%$ & $40 \%$ & $50 \%$ & & \\
\hline \multirow{16}{*}{ 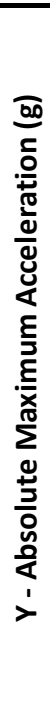 } & 0.31 & 0.41 & 1.06 & 1.06 & 351 & \multirow{16}{*}{$\stackrel{\equiv}{\equiv}$} \\
\hline & 0.26 & 0.36 & 0.83 & 0.80 & 322 & \\
\hline & 0.23 & 0.31 & 0.70 & 0.70 & $3041 / 2$ & \\
\hline & 0.20 & 0.28 & 0.61 & 0.65 & $2851 / 2$ & \\
\hline & 0.18 & 0.26 & 0.56 & 0.65 & $2681 / 2$ & \\
\hline & 0.18 & 0.26 & 0.56 & 0.67 & 254 & \\
\hline & 0.19 & 0.25 & 0.55 & 0.67 & 235 & \\
\hline & 0.19 & 0.27 & 0.62 & 0.76 & $2141 / 2$ & \\
\hline & 0.19 & 0.26 & 0.58 & 0.67 & 199 & \\
\hline & 0.20 & 0.25 & 0.55 & 0.66 & 182 & \\
\hline & 0.19 & 0.25 & 0.59 & 0.68 & 163 & \\
\hline & 0.19 & 0.24 & 0.54 & 0.65 & 146 & \\
\hline & 0.18 & 0.24 & 0.57 & 0.63 & 127 & \\
\hline & 0.17 & 0.22 & 0.72 & 0.73 & 91 & \\
\hline & 0.14 & 0.18 & 0.45 & 0.48 & $11 / 8$ & \\
\hline & 0.10 & 0.14 & 0.44 & 0.46 & 0 & \\
\hline
\end{tabular}

Table 13-48: Non-Retrofitted - System Z Absolute Maximum Acceleration

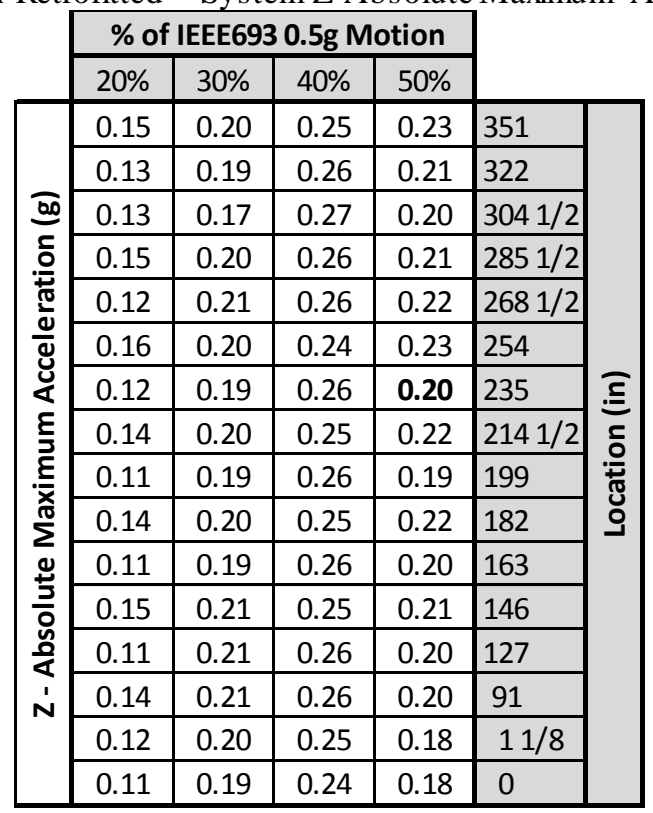

The non-retrofitted case exhibited extremely high moments in all directions, Table 13-49. For the partial $50 \%$ motion maximum moments were as follows: X-moment was 1752 kip-in, the Y-moment was 1231 kip-in, the $\mathrm{X}^{\prime}$-moment was $1059 \mathrm{kip}$-in, and the 
Y'-moment was $2141 \mathrm{kip}$-in. Yielding occurred during the $40 \%$ and $50 \%$, the residual strain values obtained are shown in Table 13-53.

Table 13-49: Non-Retrofitted - 20-50\% System Absolute Maximum Reactions

\begin{tabular}{|c|c|c|c|c|c|c|}
\hline & & $\%$ of & EEE69 & $0.5 \mathrm{~g} \mathrm{M}$ & tion & \\
\hline Absolute Max Reaction & Method & $20 \%$ & $30 \%$ & $40 \%$ & $50 \%$ & \\
\hline Base Moment X & Strain & 768 & 880 & 1338 & 1752 & \\
\hline Base Moment $Y$ & Strain & 847 & 1198 & 1046 & 1231 & $\bar{n}$ \\
\hline Base Moment X' & Strain & 494 & 754 & 913 & 1059 & \\
\hline Base Moment $Y^{\prime}$ & Strain & 941 & 1188 & 1551 & 2141 & $=$ \\
\hline Base Moment X & Accel. & 820 & 938 & 1306 & 1547 & \\
\hline Base Moment $Y$ & Accel. & 902 & 1255 & 1109 & 1207 & $\ddot{\bar{x}}$ \\
\hline Base Shear X & Accel. & 3.1 & 3.5 & 5.0 & 5.9 & \\
\hline Base Shear Y & Accel. & 3.4 & 4.6 & 4.3 & 4.6 & \\
\hline Moment CT Interface X & Accel. & 540 & 621 & 861 & 1040 & \\
\hline Moment CT Interface Y & Accel. & 595 & 834 & 739 & 820 & \\
\hline Shear CT Interface X & Accel. & 2.9 & 3.3 & 4.7 & 5.6 & \\
\hline Shear CT Interface $\mathrm{Y}$ & Accel. & 1.2 & 1.4 & 1.6 & 1.6 & \\
\hline
\end{tabular}

With the bolted base, the flexible system displaced 10.2 inches in the X-direction and 8.0 inches in the Y-direction during the partial 50\% motion. All maximum displacement values for the non-retrofitted test cases are summarized in Table 13-50. After the free vibration stopped, the system presented only 0.045 inch of permanent displacement at the top of the structure. Maximum residual displacements for both horizontal directions at three specimen locations are shown in Table 13-51.

Table 13-50: Non-Retrofitted - System Maximum Displacement 20-50\% Motions

\begin{tabular}{|c|c|c|c|c|c|}
\hline & & \multicolumn{4}{|c|}{$\overrightarrow{f I E}$} \\
\hline Location & $\begin{array}{c}\text { Location from } \\
\text { Base (in.) }\end{array}$ & $20 \%$ & $30 \%$ & $40 \%$ & $50 \%$ \\
\hline Top of Pedestal X & $911 / 2$ & 0.721 & 0.852 & 1.236 & 1.521 \\
\hline Top of Pedestal Y & $911 / 2$ & 0.780 & 1.143 & 1.033 & 1.196 \\
\hline CGX & 215 & 2.608 & 3.044 & 4.480 & 5.454 \\
\hline CG Y & $2161 / 2$ & 2.910 & 4.203 & 3.789 & 4.310 \\
\hline Top Displacement X & $3521 / 2$ & 4.921 & 5.731 & 8.369 & 10.238 \\
\hline Top Displacement Y & $3521 / 2$ & 5.456 & 7.890 & 7.020 & 8.092 \\
\hline
\end{tabular}


Table 13-51: Non-Retrofitted - System Residual Displacement 20-50\% Motions

\begin{tabular}{|c|c|c|c|c|c|c|}
\hline & & $\%$ of & IEEE693 & $0.5 \mathrm{~g} \mathrm{~N}$ & lotion & \\
\hline Location & $\begin{array}{c}\text { Location from } \\
\text { Base (in.) }\end{array}$ & $20 \%$ & $30 \%$ & $40 \%$ & $50 \%$ & \\
\hline Top of Pedestal X & $911 / 2$ & -0.008 & -0.014 & -0.013 & -0.017 & $\overline{\dot{\varepsilon}}$ \\
\hline Top of Pedestal Y & $911 / 2$ & -0.001 & 0.003 & -0.001 & 0.001 & 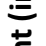 \\
\hline CGX & 215 & -0.014 & -0.037 & -0.035 & -0.023 & 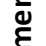 \\
\hline CGY & $2161 / 2$ & 0.001 & 0.001 & 0.010 & 0.011 & ভ্ّ \\
\hline Top Displacement X & $3521 / 2$ & -0.030 & -0.073 & -0.051 & -0.045 & $\frac{\pi}{\frac{2}{n}}$ \\
\hline Top Displacement Y & $3521 / 2$ & 0.007 & -0.022 & 0.020 & -0.008 & 茨 \\
\hline
\end{tabular}

Extremely high strains were obtained in the non-retrofitted system significantly surpassing the nominal yield strain. For the incomplete 50\% $0.5 \mathrm{~g}$ IEEE693 motion, the system had maximum strains ranging from $1168 \mu \varepsilon-2710 \mu \varepsilon$. All maximum strain values are reported in Table 13-52 for the tested cases without retrofit. Permanent strains are reported in Table 13-53 for the test cases.

Table 13-52: Non-Retrofitted - Pedestal Absolute Maximum Strain

\begin{tabular}{|c|c|c|c|c|}
\cline { 2 - 5 } \multicolumn{1}{c|}{} & \multicolumn{4}{c|}{$\%$ of IEEE693 0.5g Motion } \\
\hline $\begin{array}{c}\text { Pedestal Strain } \\
(\mu \varepsilon)\end{array}$ & $20 \%$ & $30 \%$ & $40 \%$ & $50 \%$ \\
\hline North Gauge & 925 & 1367 & 1129 & 1340 \\
\hline South Gauge & 992 & 1388 & 1237 & 1444 \\
\hline NE Gauge & 1127 & 1429 & 1912 & 2710 \\
\hline SW Gauge & 1061 & 1382 & 1833 & 2350 \\
\hline East Gauge & 812 & 941 & 1416 & 1860 \\
\hline West Gauge & 858 & 995 & 1409 & 1835 \\
\hline SE Gauge & 948 & 1344 & 1160 & 1444 \\
\hline NW Gauge & 544 & 818 & 1008 & 1168 \\
\hline
\end{tabular}

Table 13-53: Non-Retrofitted - Pedestal Residual Strain

\begin{tabular}{|c|c|c|c|c|}
\cline { 2 - 5 } \multicolumn{1}{c|}{} & \multicolumn{3}{c|}{$\%$ of IEEE693 0.5g Motion } \\
\hline $\begin{array}{c}\text { Pedestal Strain } \\
(\mu \varepsilon)\end{array}$ & $20 \%$ & $30 \%$ & $40 \%$ & $50 \%$ \\
\hline North Gauge & -1 & 11 & 17 & 43 \\
\hline South Gauge & -2 & -15 & -6 & 11 \\
\hline NE Gauge & 4 & -21 & -26 & -73 \\
\hline SW Gauge & 9 & -26 & -33 & -15 \\
\hline East Gauge & -4 & 1 & 5 & -13 \\
\hline West Gauge & -3 & -8 & 7 & 61 \\
\hline SE Gauge & -13 & 0 & -2 & 9 \\
\hline NW Gauge & -18 & 2 & -13 & -11 \\
\hline
\end{tabular}


The non-retrofitted system produced high anchor loads ranging from 36 kips-62 kips. The overturning moment is resisted through a moment couple at the base of the structure. The anchors were torqued subjecting them to an initial load. The initial loads of the anchors are conveyed in Table 13-54. As the system is excited, the load in the anchors increase when the lateral forces produce a higher overturning moments than the initial pre-loaded anchor couple resists. The maximum experienced anchor loads are shown in Table 13-55.

Table 13-54: Initial Anchor Loads

\begin{tabular}{|c|c|c|c|c|}
\cline { 2 - 5 } \multicolumn{1}{c|}{} & \multicolumn{4}{|c|}{$\%$ of IEEE693 $0.5 \mathrm{~g}$ Motion } \\
\hline $\begin{array}{c}\text { Anchor Force } \\
\text { (kips) }\end{array}$ & $20 \%$ & $30 \%$ & $40 \%$ & $50 \%$ \\
\hline NW & 12.1 & 11.2 & 9.4 & 8.8 \\
\hline SW & 12.0 & 10.1 & 8.7 & 7.2 \\
\hline SE & 23.5 & 21.8 & 19.2 & 17.8 \\
\hline NE & 21.8 & 19.2 & 16.9 & 14.8 \\
\hline
\end{tabular}

Table 13-55: Maximum AnchorLoads

\begin{tabular}{|c|c|c|c|c|}
\hline $\begin{array}{c}\text { Anchor Force } \\
\text { (kips) }\end{array}$ & $20 \%$ & $30 \%$ & $40 \%$ & $50 \%$ \\
\hline NW & 24.8 & 29.9 & 33.2 & 36.0 \\
\hline SW & 34.8 & 41.8 & 50.9 & 57.3 \\
\hline SE & 31.7 & 39.2 & 38.6 & 38.5 \\
\hline NE & 41.1 & 46.3 & 54.2 & 62.1 \\
\hline
\end{tabular}




\subsection{NuMERICAL MODEL VS. EXPERIMENTAL RESUlTS}

Numerical model outputs for XYZ, X-direction, Y-direction, and Z-direction simultaneously, 0.5g PGA IEEE693 motions were compared to the experimental response of the system. For all retrofitted cases, the models underestimated the system displacements. In fabrication, the pedestal stiffener welding caused a concave profile in the surface of the base plate. The concave surface caused premature rocking and a significantly lower initial elastic stiffness. Due to existing gaps around the exterior edge of the base plate, the initial structure stiffness was reduced and no clear transition was exhibited when rocking initiated. Premature rocking due to the base plate geometry resulted in larger system displacements, acting as if the pre-tension force was lower. As introduced in 6.1, Pre-Tension Force, the parametric study suggested that a reduced pretension force results in larger system displacements. Since rocking did not occur at the exterior edge until high lateral forces, less moment was required to cause rocking in the experimental work.

\subsection{Viscous Damper Experimental Comparison to Numerical}

Figure 14-1 and Figure 14-2 illustrate the experimental and numerical momentdisplacement hysteresis for the rocking system with Taylor viscous dampers. From the numerical work, the maximum $\mathrm{X}$-displacement at the top of the structure was 11.25 inches with an associated moment of 636 kip-in. The experimental results had a displacement of 21.71 inches and an associated moment of 834.9 kip-in. The discrepancies in the initial stiffness of the systems are clearly demonstrated in Figure 14-1 and Figure 14-2. The experimental results had a maximum displacement that was 
93\% larger than the numerical model output. The numerical model under-estimated the maximum moment in the pedestal by $31 \%$.

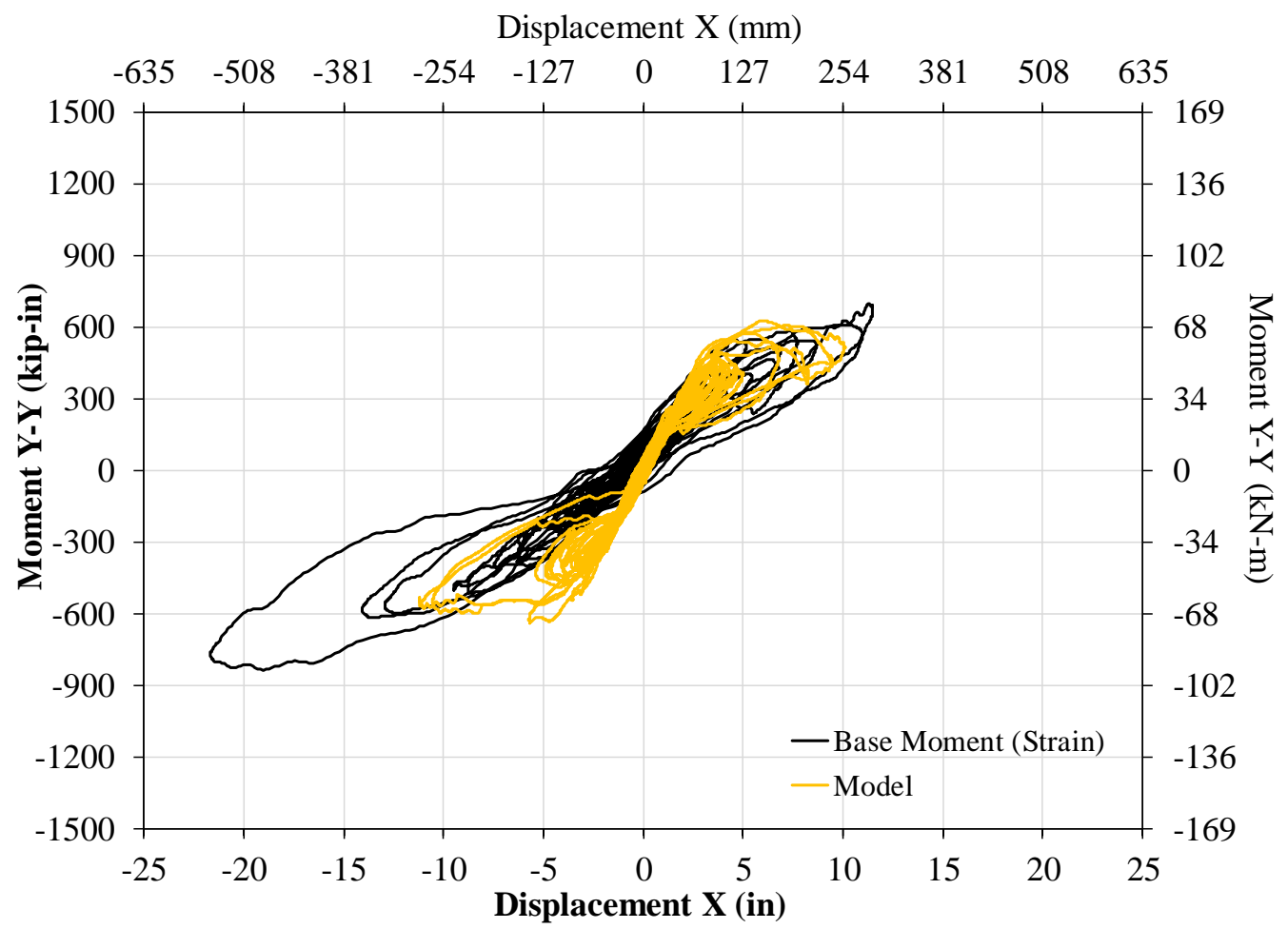

Figure 14-1: Viscous Damper - 100\% - X Experimental vs. Numerical Comparison

Comparing the numerical and experimental response for the Y-direction of the system, less error between the model and the experimental results was present. A maximum moment of 731 kip-in was obtained through SAP2000 analysis compared with the experimental moment of $853 \mathrm{kip}$-in. The experimental maximum moment obtained for the X-X-moment was $18 \%$ greater than the model output. From SAP2000 output, the Y-direction maximum displacement exhibited by the system was 15.91 inches compared to 21.24 inches from experimental instrumentation. System top displacement in the Ydirection was $34 \%$ larger than the estimated displacement by the SAP2000 model. 


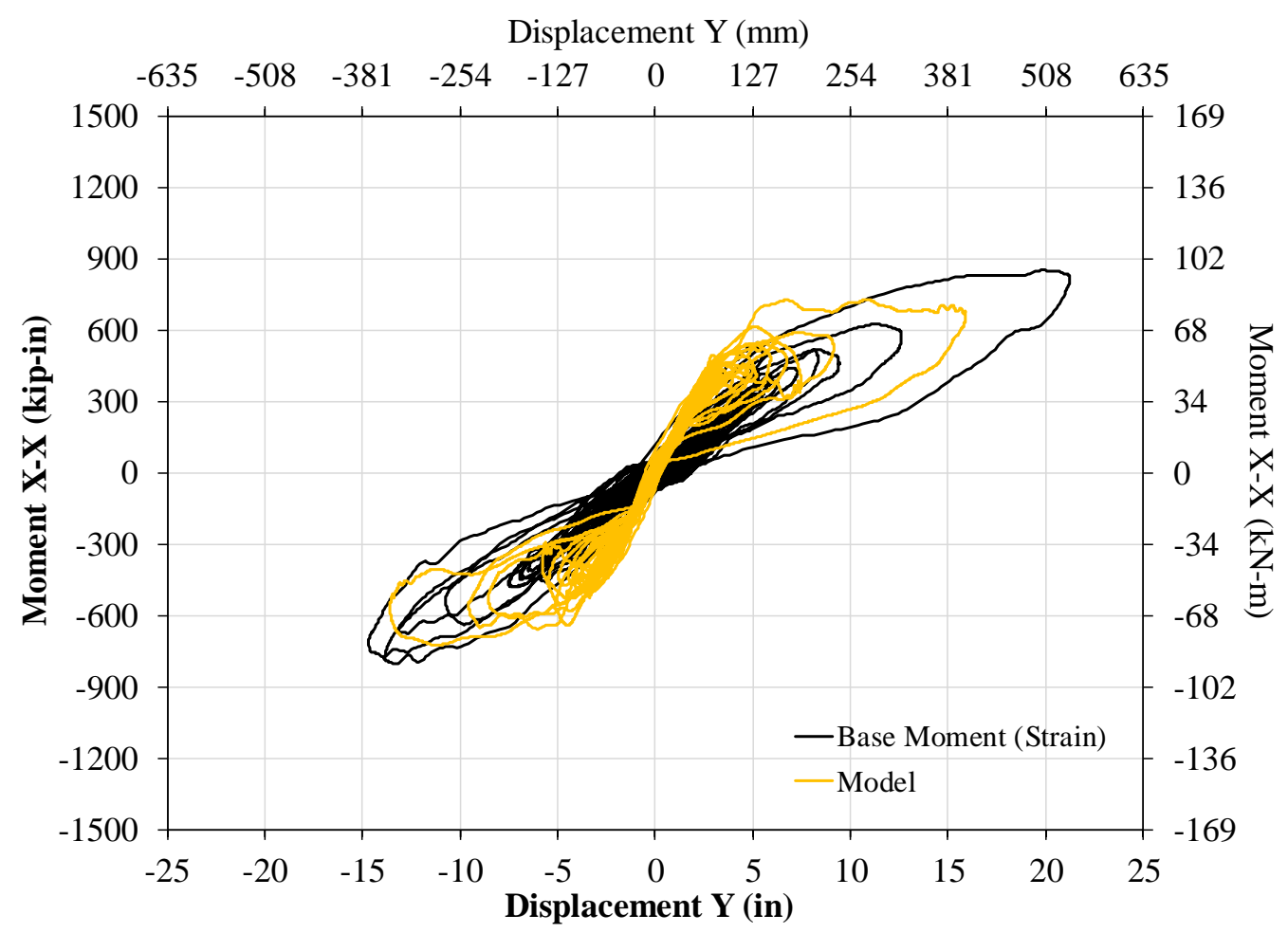

Figure 14-2: Viscous Damper -100\% -Y Experimental vs. Numerical Comparison

\subsection{Hysteretic Experimental Comparison to Numerical}

In addition to the premature rocking due to the base plate geometry, the hysteretic test cases only fully engaged 3 out of 4 of the UFP sets as discussed in 13.4.3, Hysteretic Device System Response. With all four UFP sets fully engaging, energy dissipation would increase and structure drift would be reduced.

Shown in Figure 14-3 and Figure 14-4 are comparisons between the numerical and experimental results for the rocking system with hysteretic energy dissipaters. From numerical analysis, the defined system had a maximum top X-displacement of 14.78 inches and a maximum associated moment of 804 kip-in. The instrumentation from the 3$\mathrm{D}$ virgin UFP test measured 22.38 inches of X-displacement at the specimen top. The 
maximum $\mathrm{Y}-\mathrm{Y}$ base moment measured by the calibrated strain gauges was 984 kipinches.

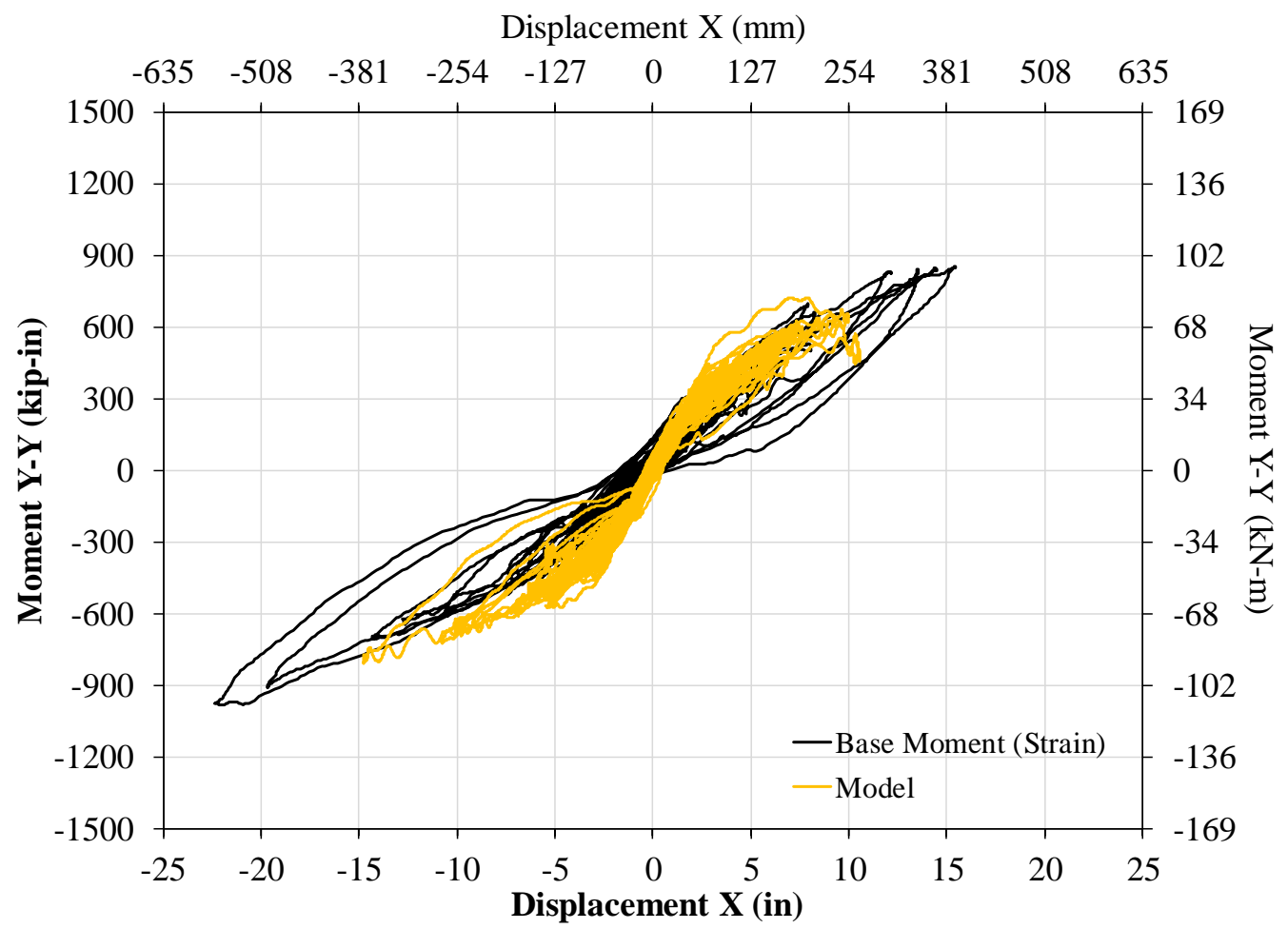

Figure 14-3: Hysteretic Device - 100\% - X Experimental vs. Numerical Comparison

Examining Figure 14-4, the maximum Y-displacement was 16.08 inches and 23.05 inches for the numerical and experimental respectively. The experimental results with 3 out of 4 UFP sets engaging resulted in $43 \%$ larger displacements than the numerical model predicted. The maximum X-X-moments were $810 \mathrm{kip}$-in and $1047 \mathrm{kip}$-in for the numerical and experimental respectively. 


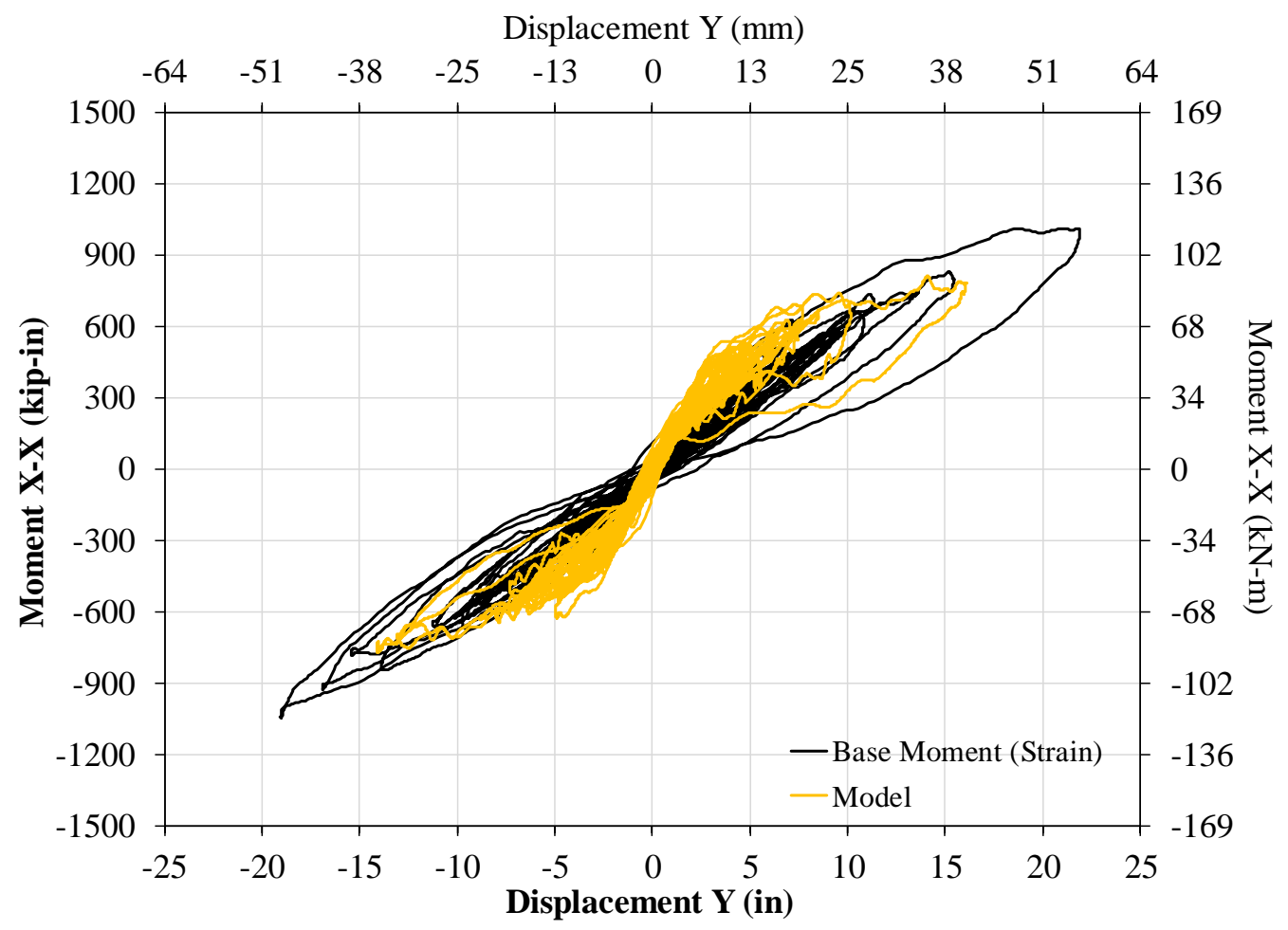

Figure 14-4: Hysteretic Device - 100\% - Y Experimental vs. Numerical Comparison

\subsection{Non-Retrofitted Experimental Comparison to Numerical}

Unlike the retrofitted cases, the bolted non-retrofitted case was not affected by the geometric imperfection of the base plate. The stiffness of the numerical model compared to the experimental results was near identical. Consistently, the numerical model under estimated the maximum displacement and reactions in the system. The numerical models were constructed assuming $2 \%$ elastic damping while the actual full steel structure had less than $0.4 \%$ elastic damping. Both methods for determining moment in the experimental system are plotted along with the numerical output in Figure 14-5 and Figure 14-6. 


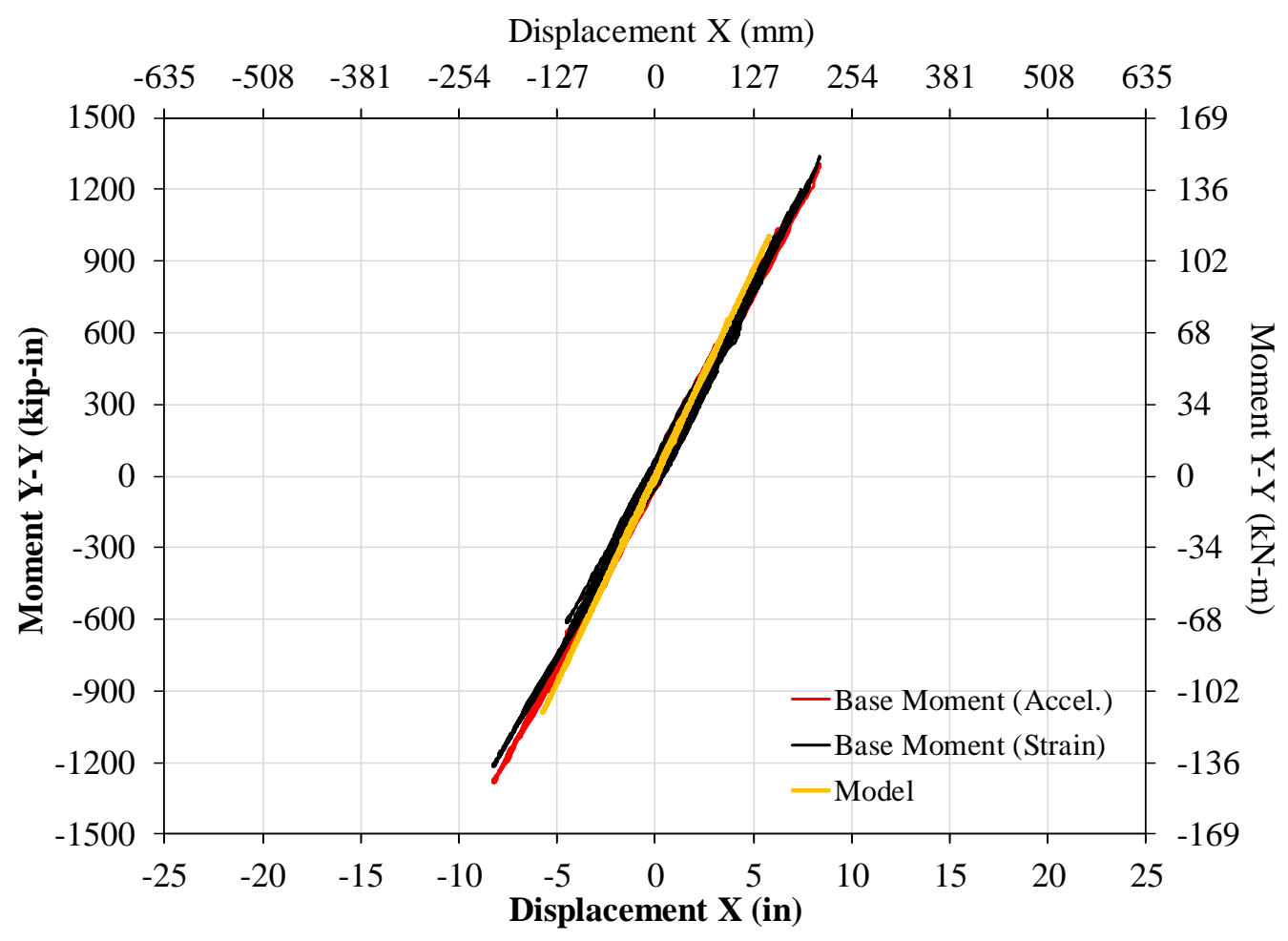

Figure 14-5: Non-Retrofitted - 40\% - X Experimental vs. Numerical Comparison

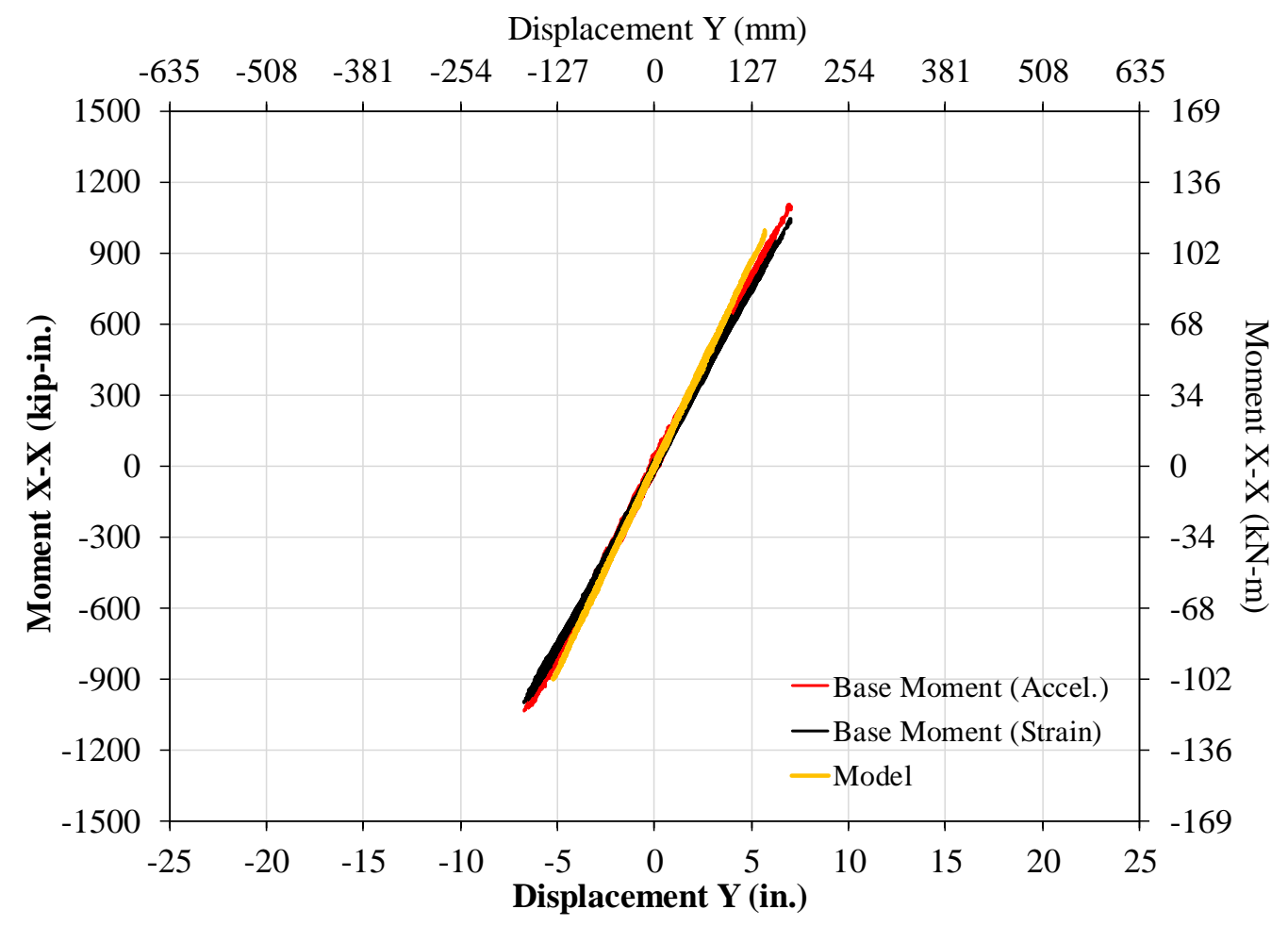

Figure 14-6: Non-Retrofitted - 40\% - Y Experimental vs. Numerical Comparison 


\subsection{ECONOMIC COMPARISON}

Both retrofit measures consistently presented repeatable self-centering behavior. Summarized in the following section are the costs for each current transformer retrofit. Both retrofit measures economic comparisons exclude the cost of installation labor. Labor required for each retrofit installation is near equal and are neglected in the economical comparison.

Portrayed in Figure 15-1 and Table 15-1 are the costs of each component required for the self-centering system with viscous dampers. The total cost of the viscous damper retrofit components is $\$ 16,900$ of which $\$ 10,000$ is the cost of the four Taylor viscous dampers.

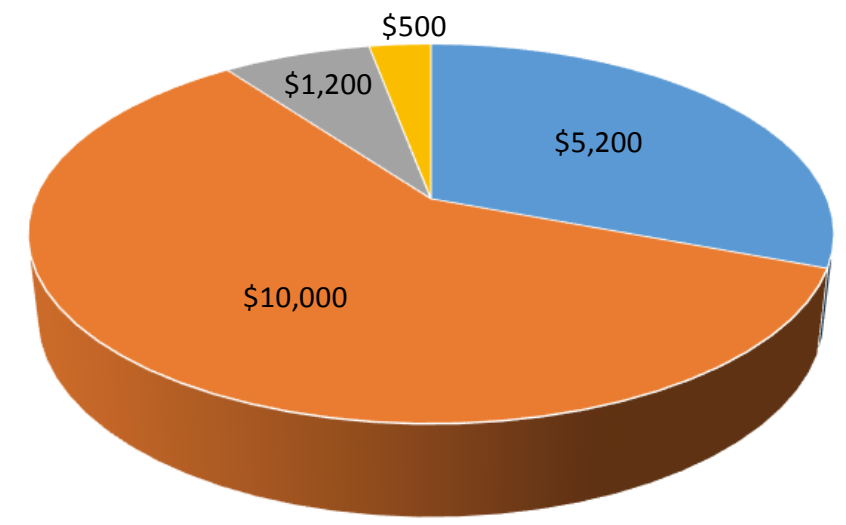

$$
\begin{array}{ll}
\square \text { Brackets and Mounts } & \text { Taylor Viscous Dampers } \\
- \text { Belleville Springs } & \text { Belleville Spring Protective Caps }
\end{array}
$$

Figure 15-1: Viscous Damper Retrofit Cost

Table 15-1: Viscous Damper Retrofit Cost Summary

\begin{tabular}{|l|l|r|}
\hline \multicolumn{1}{|c|}{ Component } & \multicolumn{1}{|c|}{ Additional Details } & Cost \\
\hline Brackets and Mounts & Set of 4 & $\$ 5,200$ \\
\hline Taylor Viscous Dampers & Set of 4 & $\$ 10,000$ \\
\hline Belleville Springs & Set of 144 - 4 Stacks of 36 & $\$ 1,200$ \\
\hline Belleville Spring Protective Caps & Set of 4 & $\$ 500$ \\
\hline \multicolumn{2}{|c|}{ Total Retrofit Cost } & $\$ 16,900$ \\
\hline
\end{tabular}


The self-centering retrofit with hysteretic devices has a material cost of $\$ 3,820$. The attachment brackets cost $47 \%$ of the total retrofit. A complete breakdown of each component of the retrofit is shown in Figure 15-2 and Table 15-2.

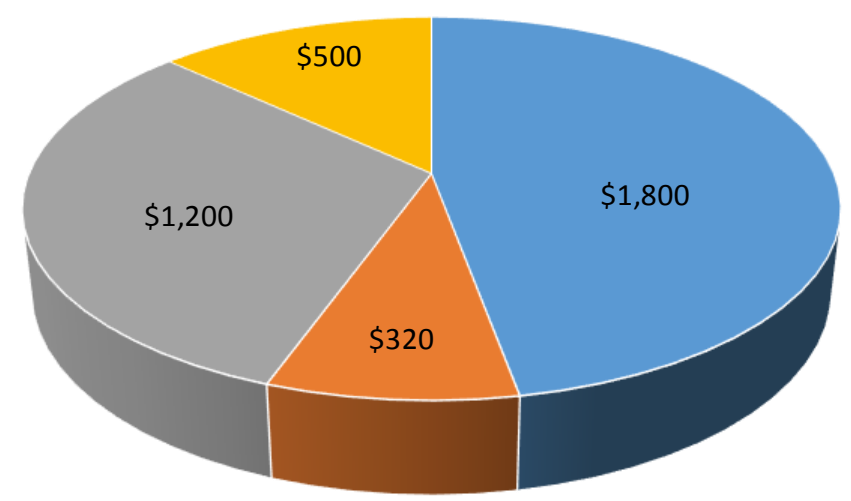

$$
\begin{aligned}
& \text { Brackets and Mounts } \\
& \text { Belleville Springs }
\end{aligned}
$$$$
\text { - UFP Devices }
$$$$
\text { Belleville Spring Protective Caps }
$$

Figure 15-2: Hysteretic Device Retrofit Cost

Table 15-2: Hysteretic Device Retrofit Cost Summary

\begin{tabular}{|l|l|r|}
\hline \multicolumn{1}{|c|}{ Component } & \multicolumn{1}{c|}{ Additional Details } & \multicolumn{1}{c|}{ Cost } \\
\hline Brackets and Mounts & Set of 4 & $\$ 1,800$ \\
\hline UFP Devices & Set of 8 & $\$ 320$ \\
\hline Belleville Springs & Set of 144 - 4 Stacks of 36 & $\$ 1,200$ \\
\hline Belleville Spring Protective Caps & Set of 4 & $\$ 500$ \\
\hline \multicolumn{2}{|c|}{ Total Retrofit Cost } & $\$ 3,820$ \\
\hline
\end{tabular}




\subsection{Full-SCALE RETROFIT COMPARISON AND SUMMARY}

Both retrofit concepts have been shown to demonstrate effectiveness as compared to the non-retrofitted case. Damping and fundamental frequency for all three test cases are shown in Table 16-1. As previously discussed, base plate imperfections caused instant softening of the elastic structure in the retrofitted cases, however that can be readily mitigated with design specifications or installation procedures. Comparing the fundamental frequency in the retrofitted cases to the non-retrofitted case, $25-40 \%$ decrease in fundamental frequency occurs when the system is retrofitted. When the system is retrofitted with viscous dampers, any displacement engages the dampers. With an $1 / 8$ inch gap between the base plate edge and the "foundation", any load on the system engages the dampers. The viscous damper retrofit increases the elastic damping of the system from $0.35 \%-1.22 \%$ in the $\mathrm{X}$-direction and $0.32 \%-1.84 \%$ in the Y-direction. The UFP device is displacement-dependent and energy dissipation occurs only when the yield displacement of 0.093 inch is exceeded. The self-centering system with hysteretic devices presented $0.66 \%$ damping in the $\mathrm{X}$-direction and $0.87 \%$ damping in the Y-direction.

Table 16-1: Damping and Fundamental Frequency Comparis on

\begin{tabular}{|l|c|c|c|c|c|c|c|}
\cline { 2 - 8 } & \multicolumn{2}{c|}{} & \multicolumn{3}{c|}{ Pulse Free Vibration } & \multicolumn{3}{c|}{ White Noise } \\
\cline { 2 - 9 } & \multicolumn{2}{c|}{$\begin{array}{c}\text { Fundamental } \\
\text { Frequency }(\mathrm{Hz})\end{array}$} & $\begin{array}{c}\text { Elastic Damping } \\
(\%)\end{array}$ & \multicolumn{3}{c|}{$\begin{array}{c}\text { Fundamental } \\
\text { Frequency (Hz) }\end{array}$} \\
\hline Retrofit Device & $\mathrm{x}$ & $\mathrm{y}$ & $\mathrm{x}$ & $\mathrm{y}$ & $\mathrm{x}$ & $\mathrm{y}$ & $\mathrm{z}$ \\
\hline Hysteretic & 0.86 & 0.75 & $1.22 \%$ & $1.84 \%$ & 0.85 & 0.75 & 26.35 \\
\hline None Retrofitted & 0.80 & 0.70 & $0.66 \%$ & $0.87 \%$ & 0.78 & 0.68 & 26.16 \\
\hline
\end{tabular}

Both retrofits methods significantly reduced demand on the CT-interface and pedestal moments compared to the non-retrofitted system. Presented in Figure 16-1 and Figure 16-2 are comparisons between the two retrofit types and the non-retrofitted 
structure. The response of the retrofitted cases excited by the 100\% 0.5g PGA IEEE693 tQke motion is compared to the response of the non-retrofitted system excited by a $40 \%$ 0.5g PGA IEEE693 tQke motion. Comparing the viscous damper retrofit to the hysteretic device retrofit, minor differences in system response were presented. Viscous dampers act out of phase with system displacement and generally the shape of the system response is significantly different than that of hysteretic devices. The combination of the selfcentering and elliptical viscous damper response generally results with higher forces near zero displacement. Since the system tested was extremely flexible, the viscous dampers did not significantly outperform the hysteretic devices. The differences are marginal which would not be the case for a stiff system.

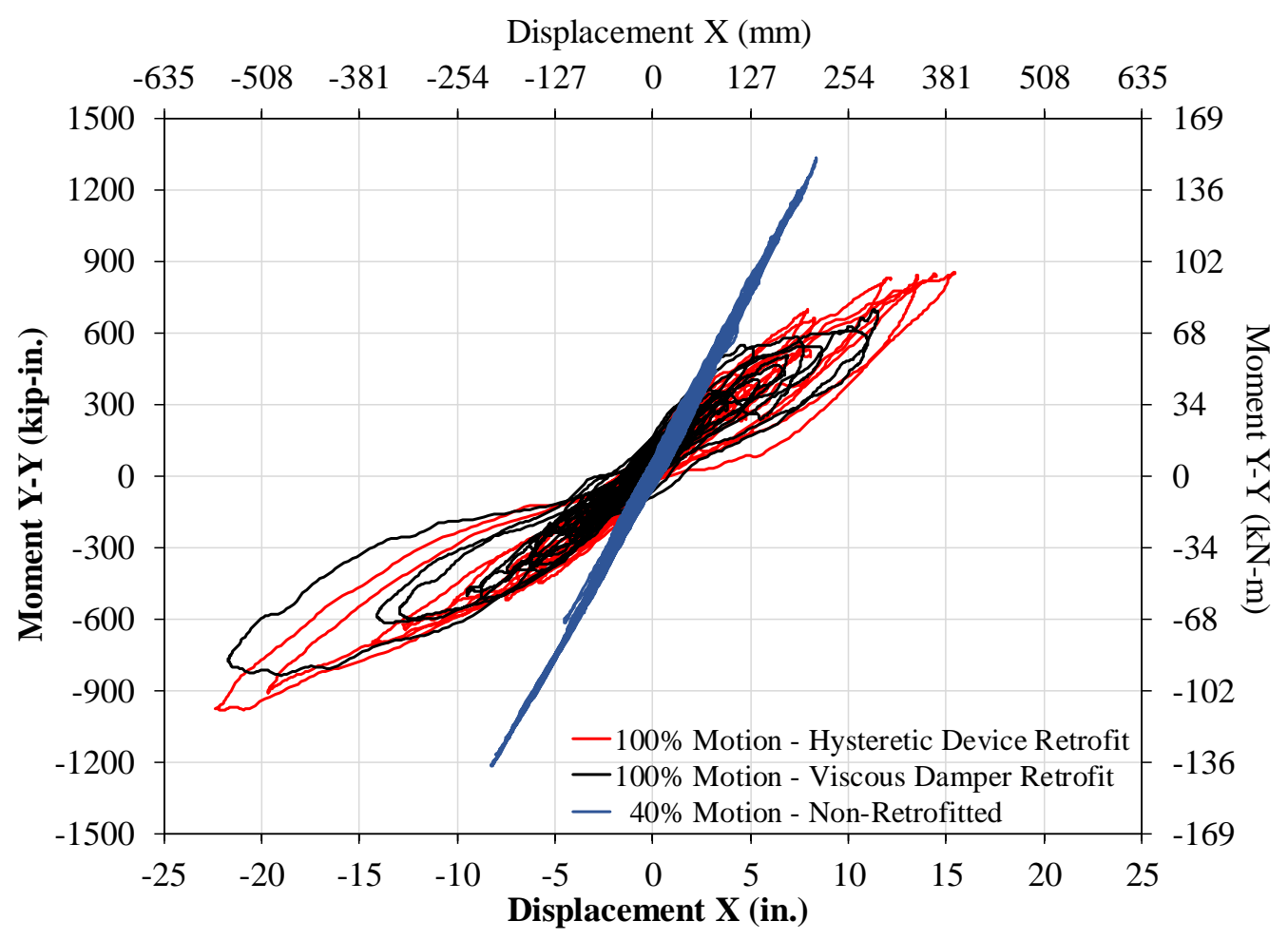

Figure 16-1: X - Retrofitted System Compared to Non-Retrofitted System 


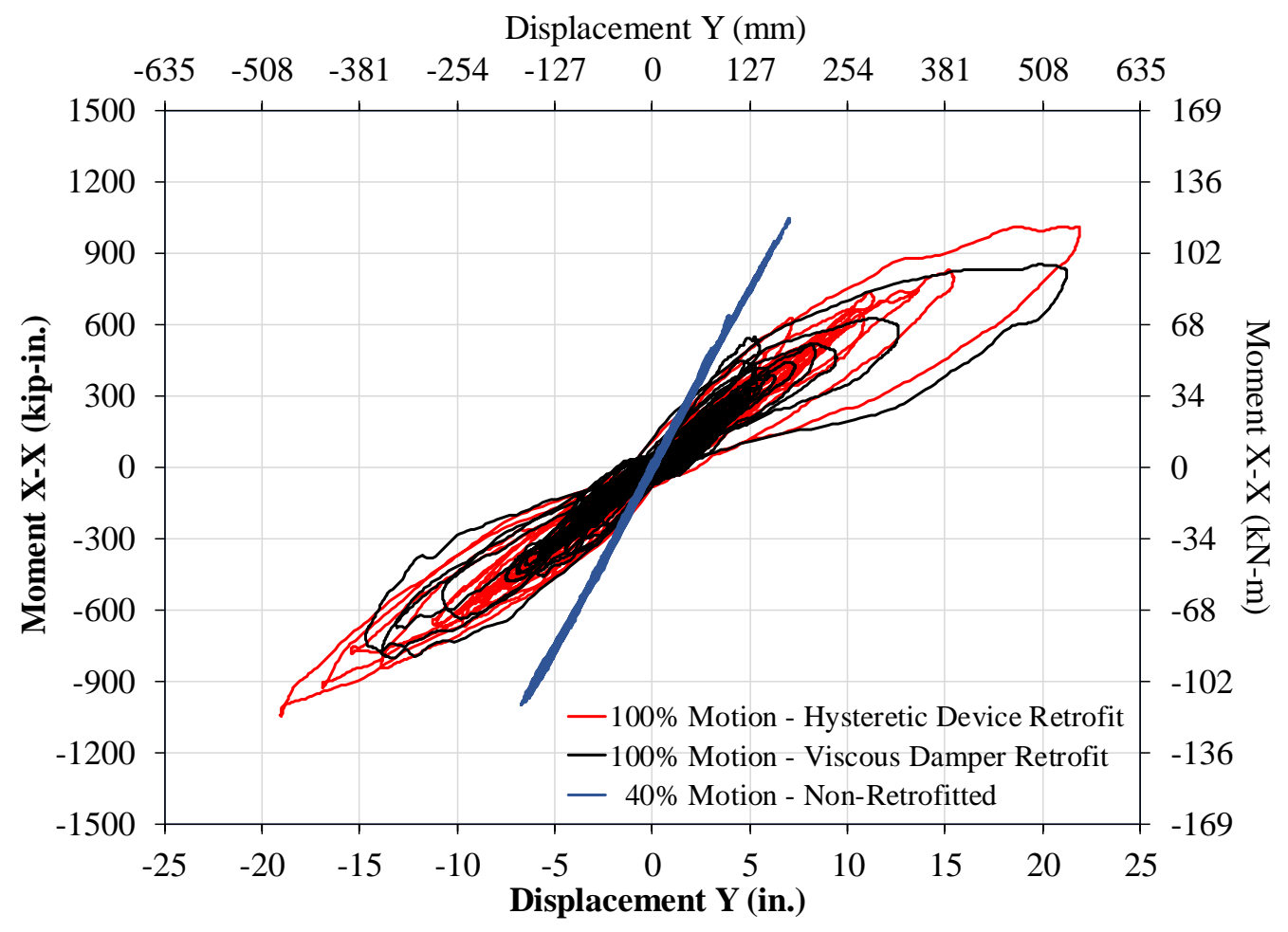

Figure 16-2: Y - Retrofitted System Compared to Non-Retrofitted System

The viscous damper retrofit was most effective in limiting displacement and decreasing system forces, but only marginally compared to the hysteretic retrofit. The UFP performance difference would likely be even less if all 4 UFP sets were fully engaged and energy dissipation was maximized. Comparing the X-direction response (100\% motion), the viscous damper retrofit had a maximum moment of 835 kip-in and a top X-displacement of 21.70 inches, while the hysteretic device retrofit had a maximum base moment of $984 \mathrm{kip}$-in and a top X-displacement of 22.38 inches. The non-retrofitted system had a maximum base moment of $1338 \mathrm{kip}$-in and a maximum X-displacement of 8.37 inches for the $40 \%$ motion. Significant pedestal yielding and damage to the CT is expected if the $100 \%$ motion excites the non-retrofitted system. 
Similar trends were exhibited in the Y-direction system response (100\% motion). The viscous damper retrofit experienced a maximum moment of $853 \mathrm{kip}$-in and a top Ydisplacement of 21.4 inches. The structure retrofitted with UFPs had a maximum measured X-X-moment of $1047 \mathrm{kip}$-in and a Y-displacement of 21.88 inches. The nonretrofitted structure excited by the $40 \%$ motion manifested 1046 kip-in moment and 7.02 inches of top displacement.

Extreme reductions in the anchor forces were presented in the retrofitted cases. The retrofit distributed the loads on the foundation into 8 anchors and a rocking edge compared to the non-retrofitted system which uses a 4 bolt moment couple to resist overturning. For the retrofitted cases, the energy dissipating device anchors are limited to the maximum device capacity. For the $100 \%$ IEEE693 retrofitted cases the device anchor were all below 9 kips while in the non-retrofitted system the anchor loads were 30-54 kips for the $40 \%$ motion. Nominal yield of the anchors is expected for any loads over 28 kips. The rocking structure does impose a concentrated line load along the length of the rocking edge, but the distribution of the load produces little concern.

Viscous damper retrofit showed significantly more benefit for the PSU scaled system than for the full-scale system. The full-scale results suggest that the gain in benefit compared to the high costs associated with viscous damper procurement is minimal. The hysteretic device retrofit economically shows significant favorability at only $23 \%$ the cost of the viscous damper retrofit. 


\subsection{RECOMMENDATIONS}

Current Transformer retrofit is highly effective in mitigating seismic ground motion. The high demands imposed on the CT-pedestal interface and the pedestal base alone, encourage consideration of retrofit. The retrofit aims to reduce loads, but yielding may still occur in the pedestal due to its structural deficiency. The following further investigations are recommended:

- Design of a slack release system to accommodate the displacements at the top of the CT.

- Investigation on options for rerouting electrical connections at the base of the structure.

- Investigating temperature conditions and performance of devices under extreme conditions.

- Self-leveling foundation to reduce and eliminate premature rocking and decrease displacements. Having a foundation that is molded to the actual base plate will reduce premature rocking, increase initial stiffness, and result in more predictable structure response.

- Increase moment capacity of the current transformer pedestal.

- Adding additional gap opening at other locations of the structure to reduce effects of higher modes. Gap openings at the pedestal-CT interface could reduce loads further by limiting the load above the stiff pedestal.

- Investigate potential applicability of the retrofit technology on other types of substation equipment. 


\subsection{REFERENCES}

Aslan FRP. (2011). Carbon Fiber Reinforced Polymer (CFRP) Bar-Aslan 200 Series. Seward: Hughes Brothers, Inc.

Baird, A. S. (2014). Experimental and numerical study of U-shape flexural plate (UFP) dissipators. Auckland: New Zealand Society for Earthquake Engineering 2014 Technical Conference and AGM.

Chancellor, N. B. (2014). Chancellor, N. B., Eatherton, M. R., Roke, D. A., \& Akbaş, T. (2014). Self-centering seismic lateral force resisting systems: high performance structures for the city of tomorrow. Buildings, 4(3), 520-548.

CSI. (2016, January 5). Software Verification EXAMPLE 6-008 LINK - PLASTIC WEN LINK. Retrieved from CSI America:

http:/docs.csiamerica.com/manuals/sap2000/Verification/Analysis/Links/Proble m\%206-008.pdf

Eatherton, M. R. (2014). Design concepts for controlled rocking of self-centering steelbraced frames. Journal of Structural Engineering, 140.11 .

Gu, M. W. (2015). Displacement Design Procedure for Cross Laminated Timber (CLT) Rocking Walls with Sacrificial Dampers. Structures Congress 2015, (pp. 27772791).

Gu, M., Pang, W., \& Schiff, S. (2015). Displacement Design Procedure for Cross Laminated Timber (CLT) Rocking Walls with Sacrificial Dampers. Structures Congress 2015. Clemson: Glenn Department of Civil Engineering. 
IEEE. (2006). Recommended Practice for Seismic Design of Substations (IEEE693). New York.

Kelly, J. M. (1972). Mechanisms of energy absorption in special devices for use in earthquake resistant structures. Bulletin of NZ Society for Earthquake Engineering, 63-88.

Kelly, J. M., Skinner, R. I., \& Heine, A. J. (1972). Mechanisms of Energy Absorption in Special Devices for use in Earthquake Resistant Structures. Bulletin of the New Zealand Society for Earthquake Engineering, 5(3).

Pampanin, S. P. (2001). Analytical modelling of the seismic behaviour of precast concrete frames designed with ductile connections. Journal of Earthquake, 329367.

Pennucci, D. G. (2009). Displacement-Based Design of Precast Walls with Additional Dampers. Journal of Earthquake Engineering 13.S1, 40-65.

Ricles, J. M. (2006). Ricles, J. M., et al. "Post-tensioned moment connections with a bottom flange friction device for seismic resistant self-centering steel MRFs. 4th International conference on earthquake engineering.

Schultz, A., R., M., Tadros, M., \& Huo, X. (1994). Experimental Study of Joint Connections in Precast Concrete Walls. Fifth U.S. National Conference on Earthquake Engineering (p. Vol. II). Oakland: Earthquake Engineering Research Institute. 
Seo, C.-Y. a. (2005). Ductility demands on self-centering systems. ACI Struct. J., 102(2), 275-285. 


\subsection{APPENDIX A}

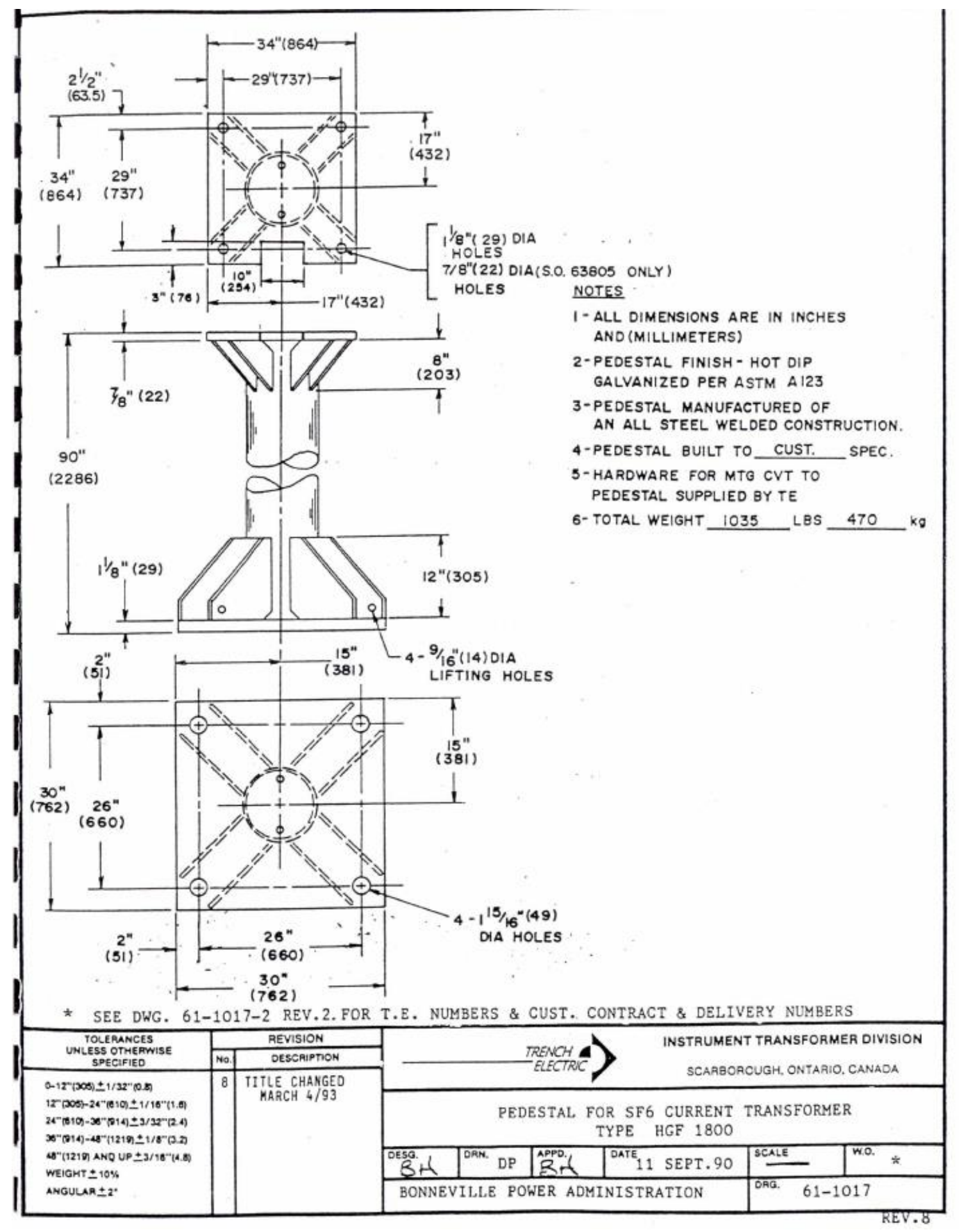

Figure 19-1: Trench Support Pedestal 


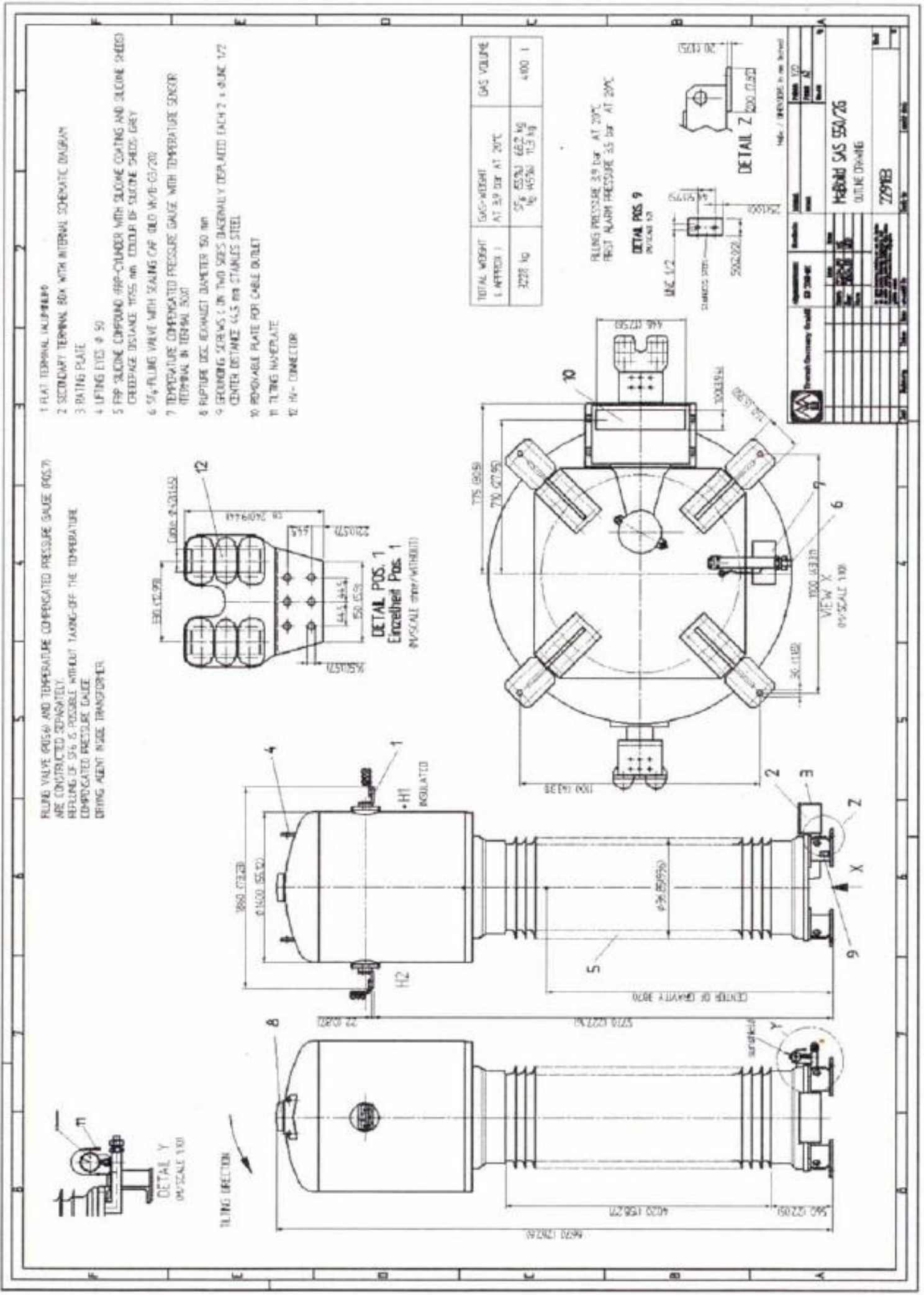

Figure 19-2: 226-293 Trench 500kV Current Transformer Specifications 


\subsection{APPENDIX B}
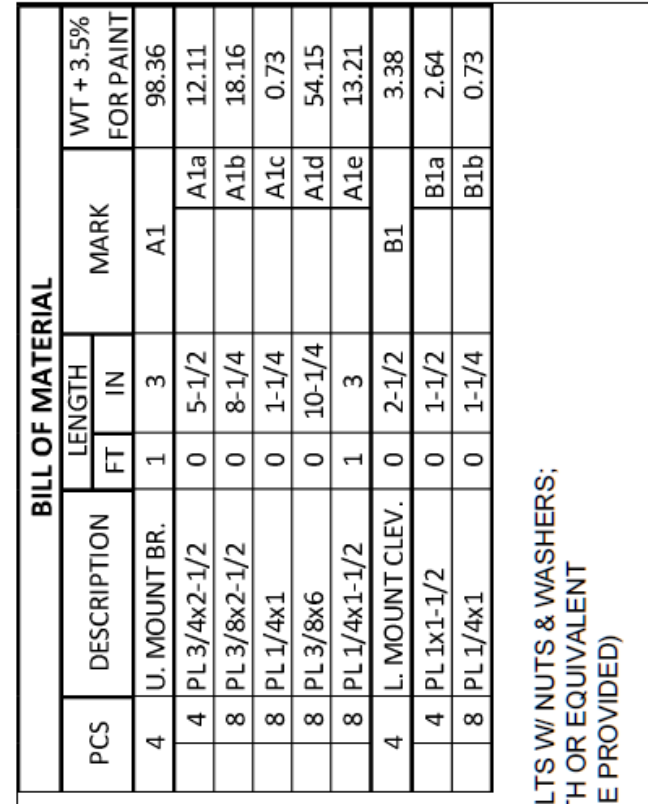

步岗

ํํㅇ잉

这七

ले 또는

ज吉
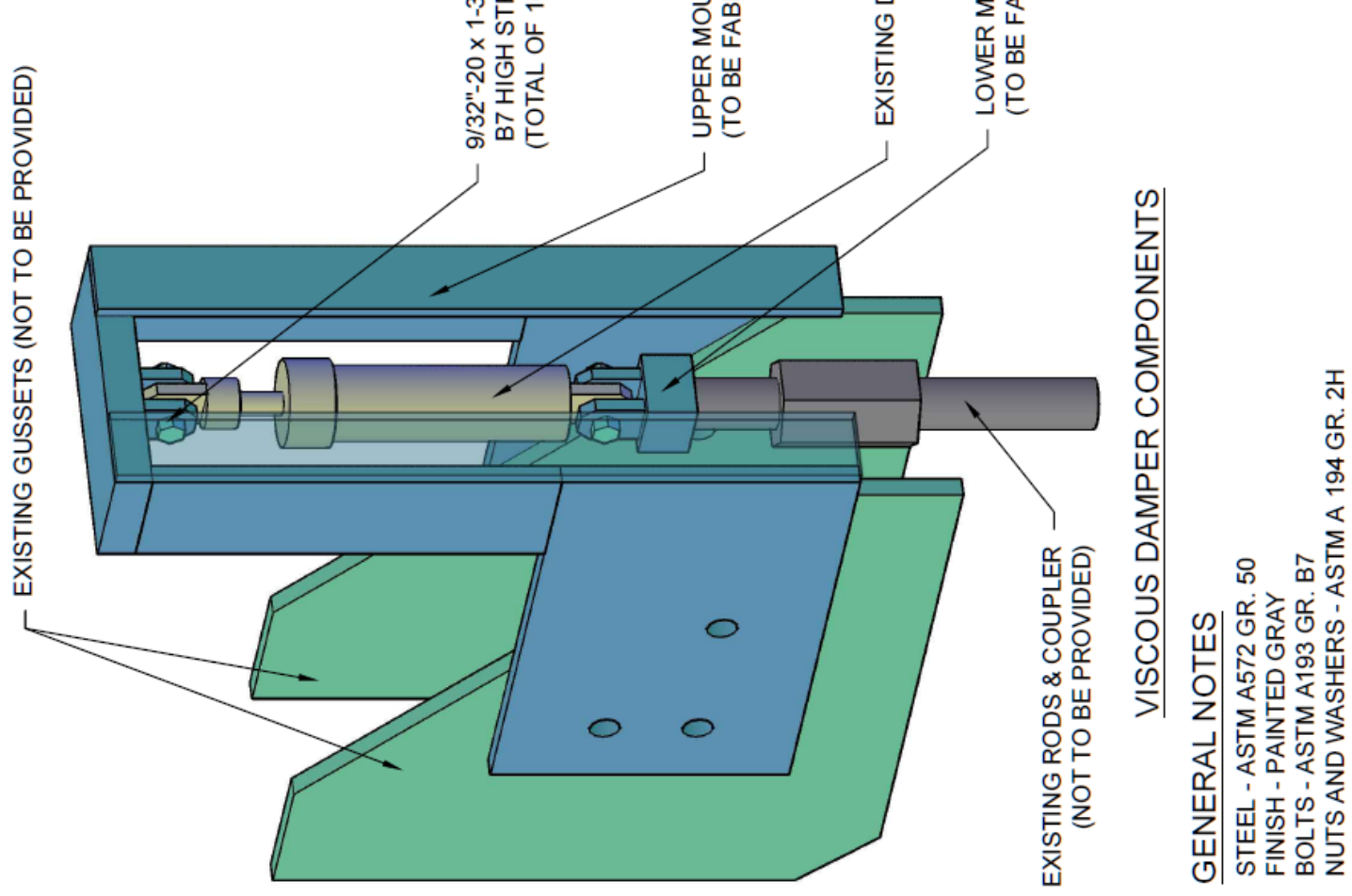


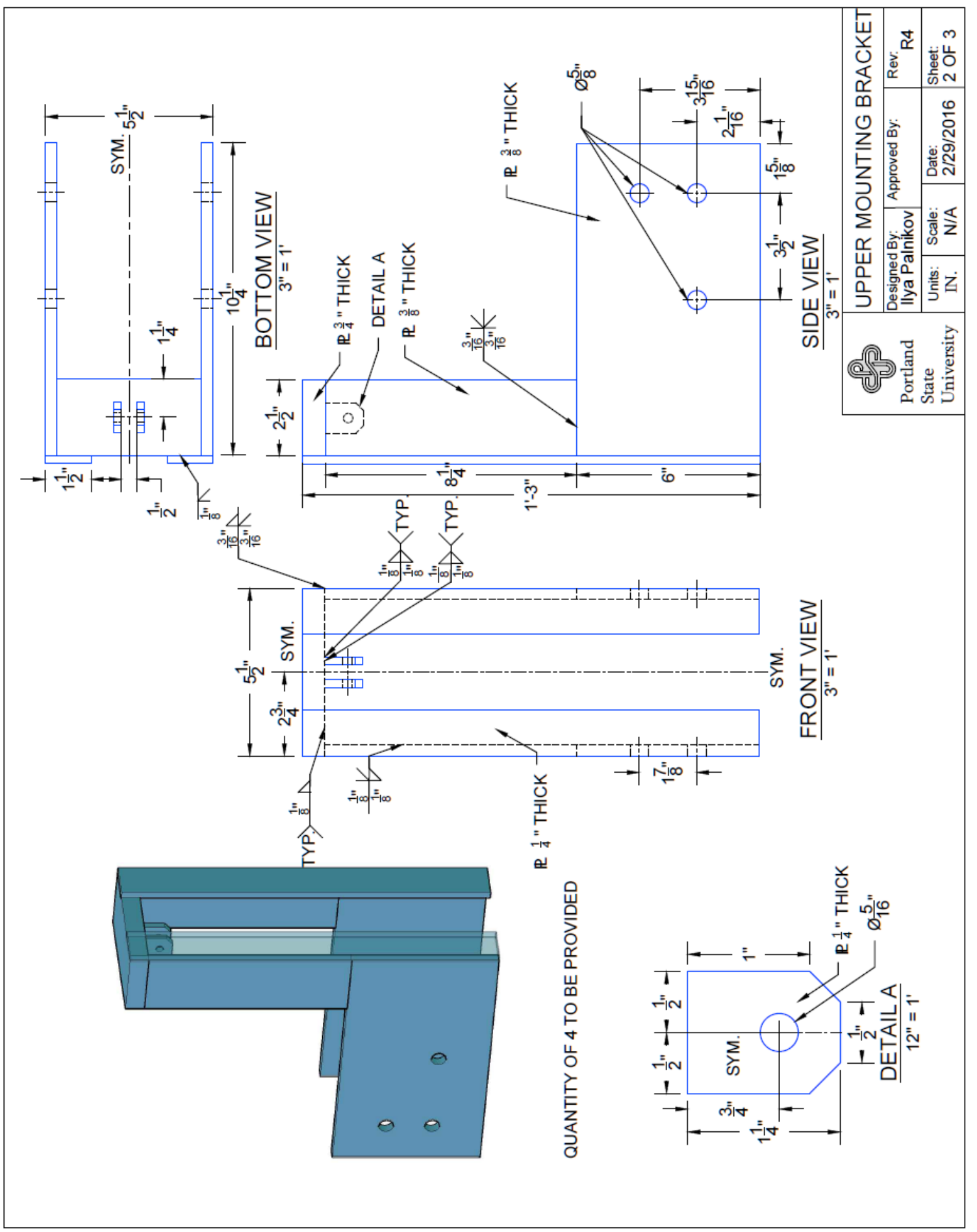




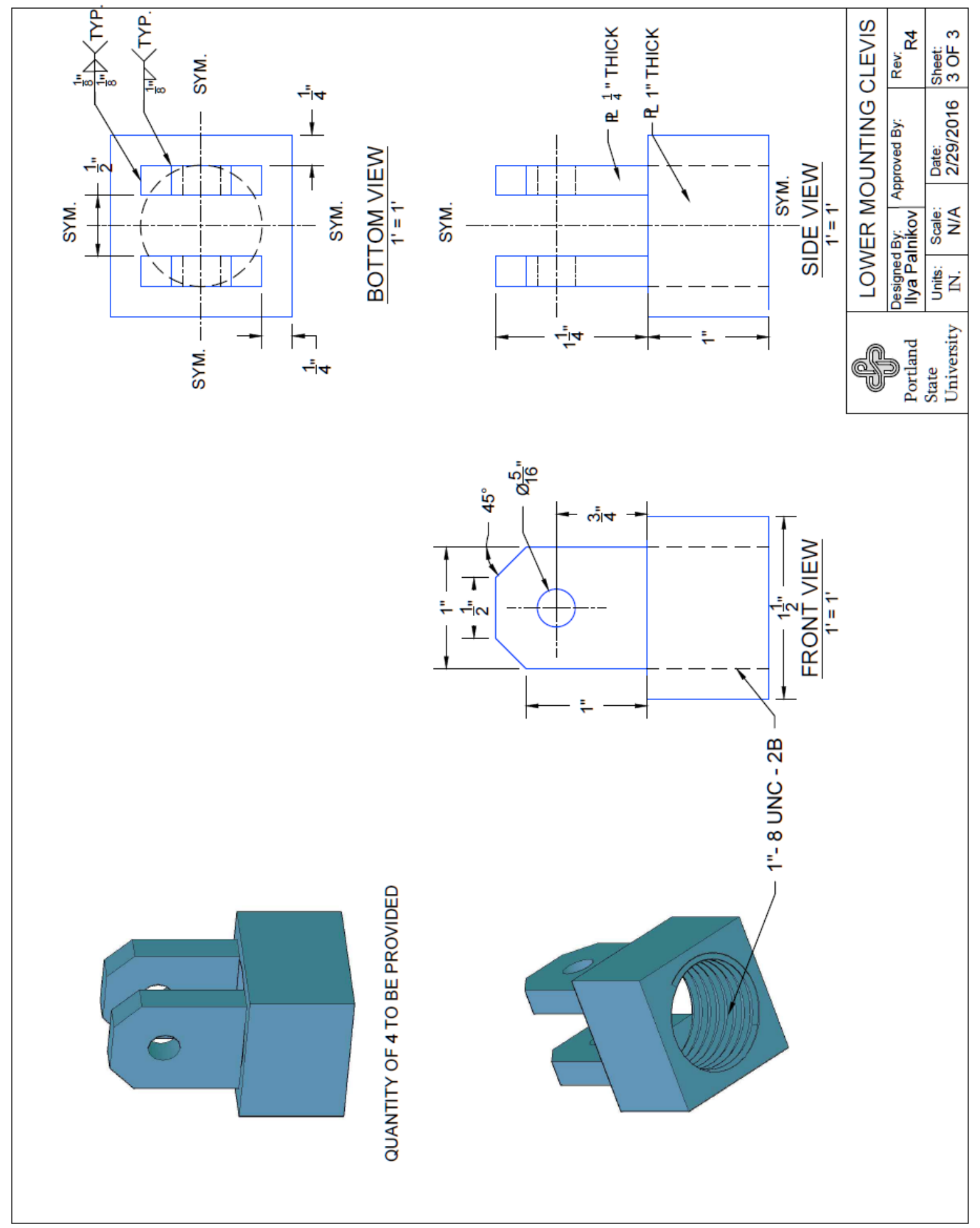




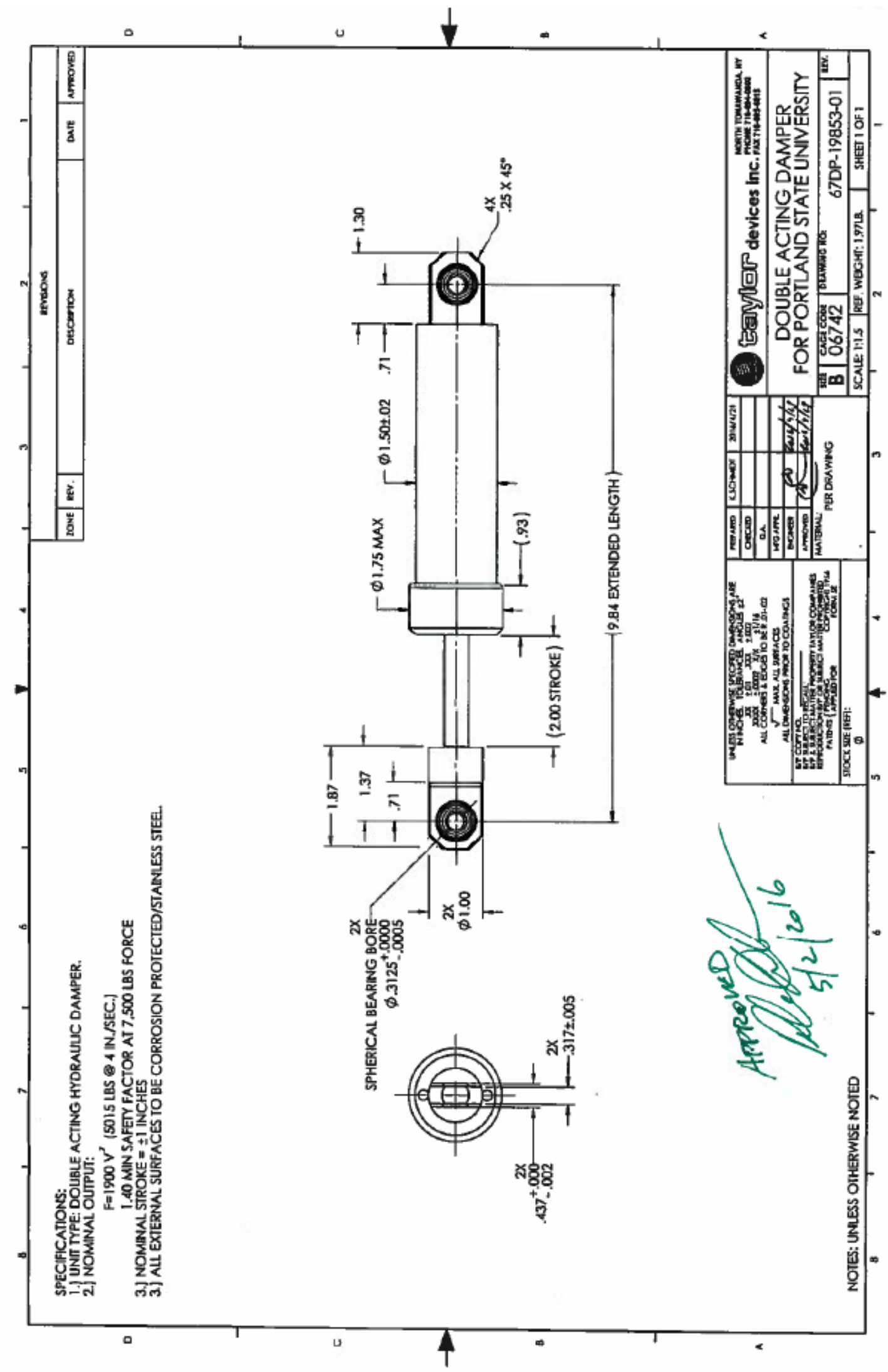




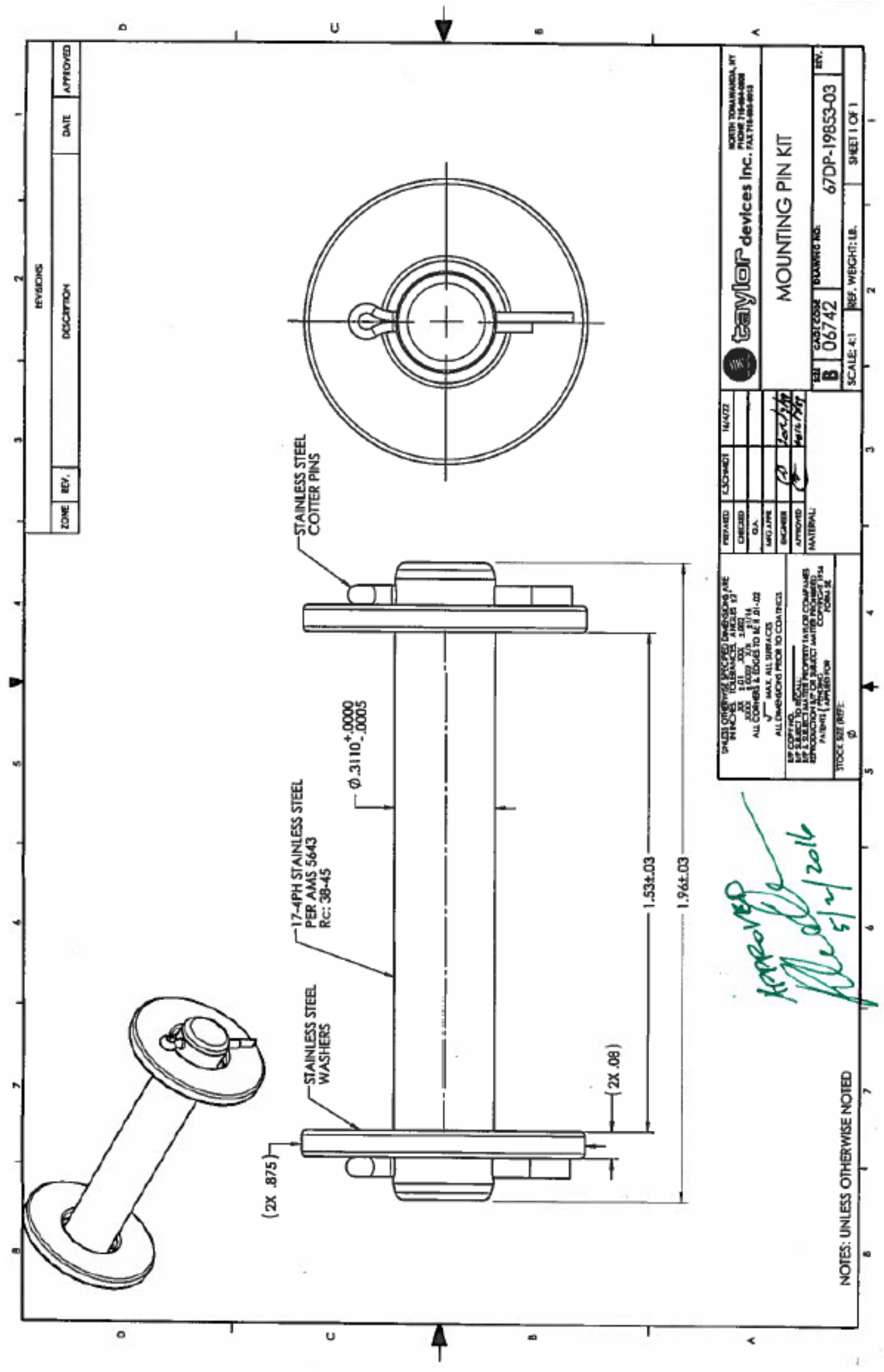


21.0 APPENDIX C

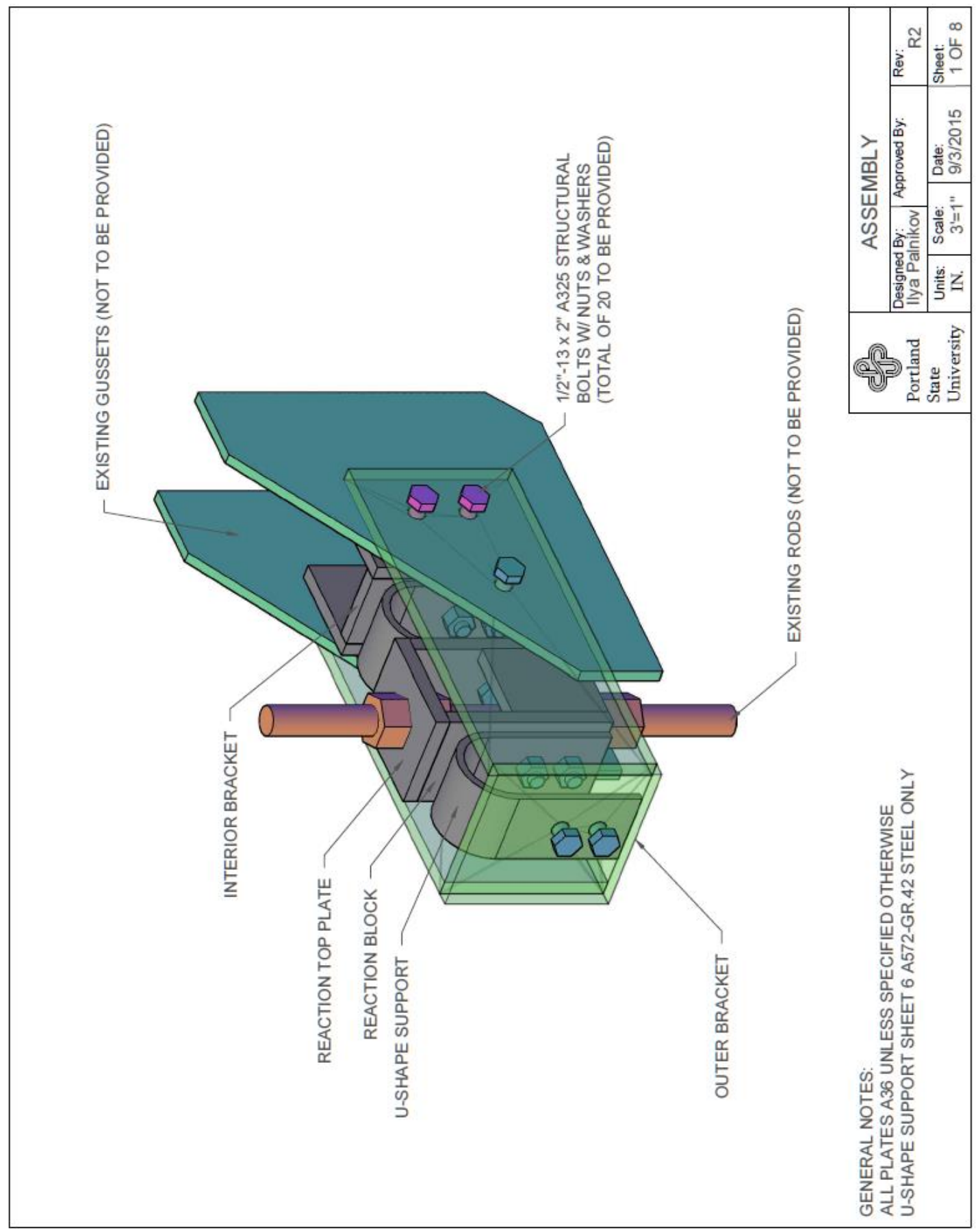




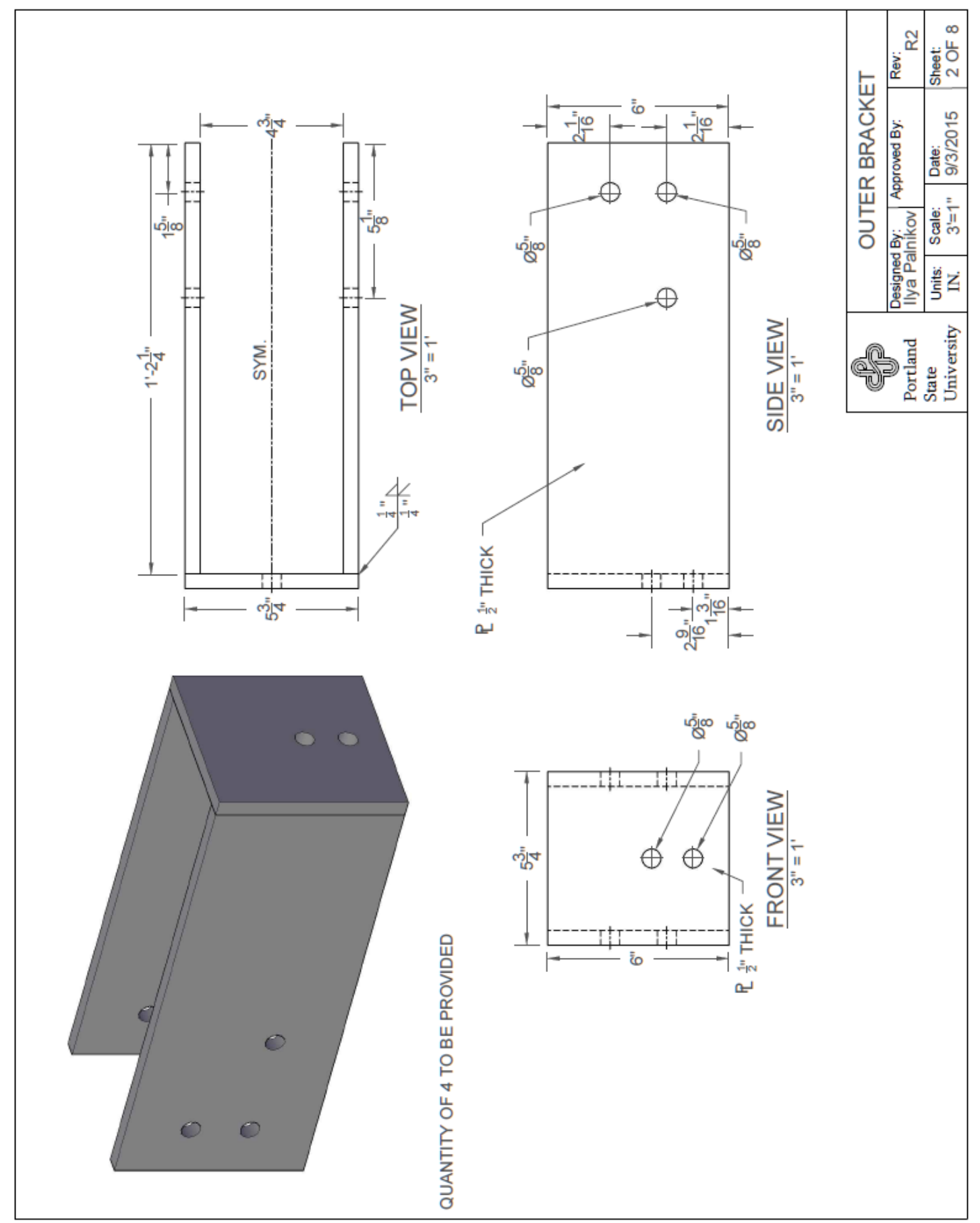




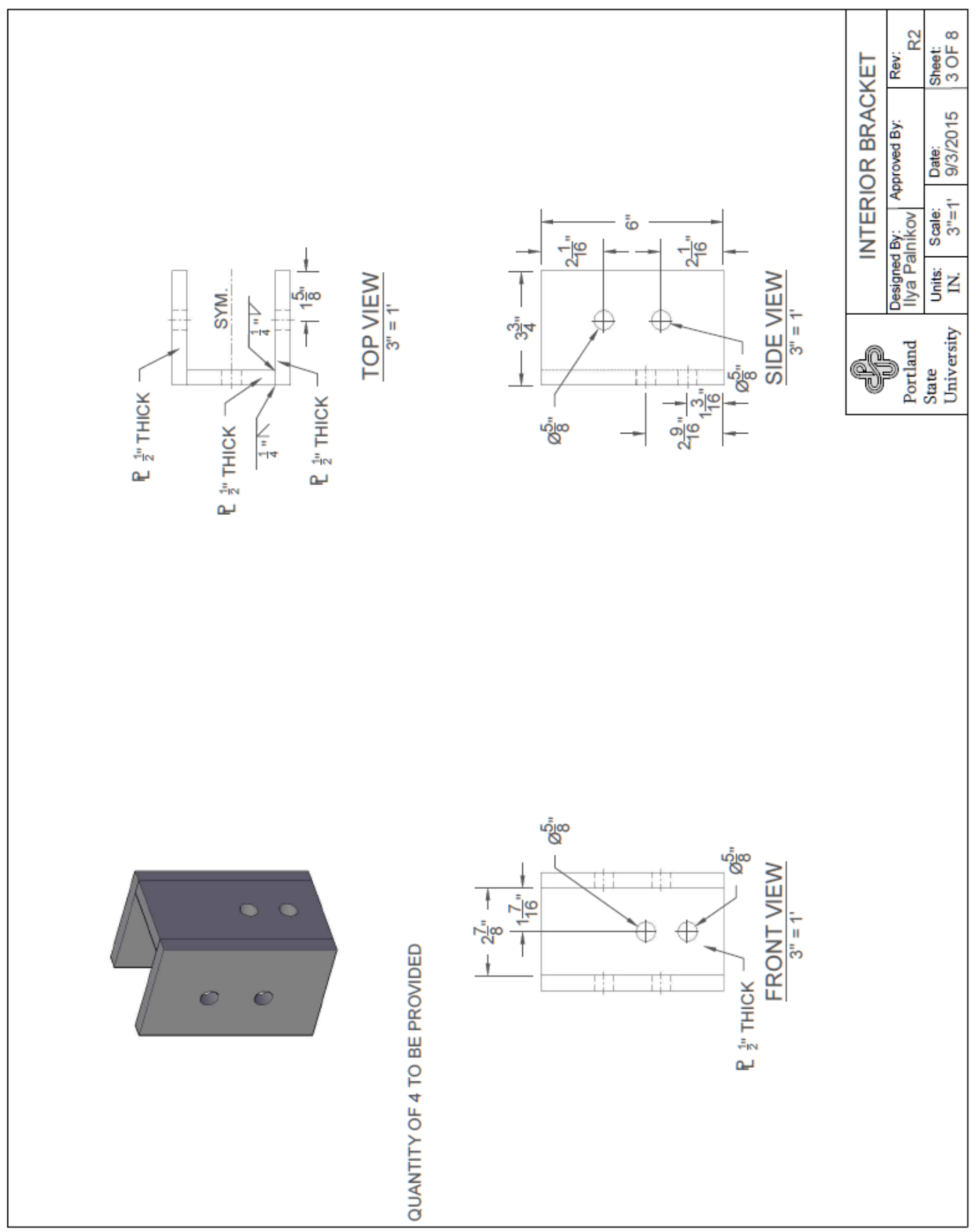




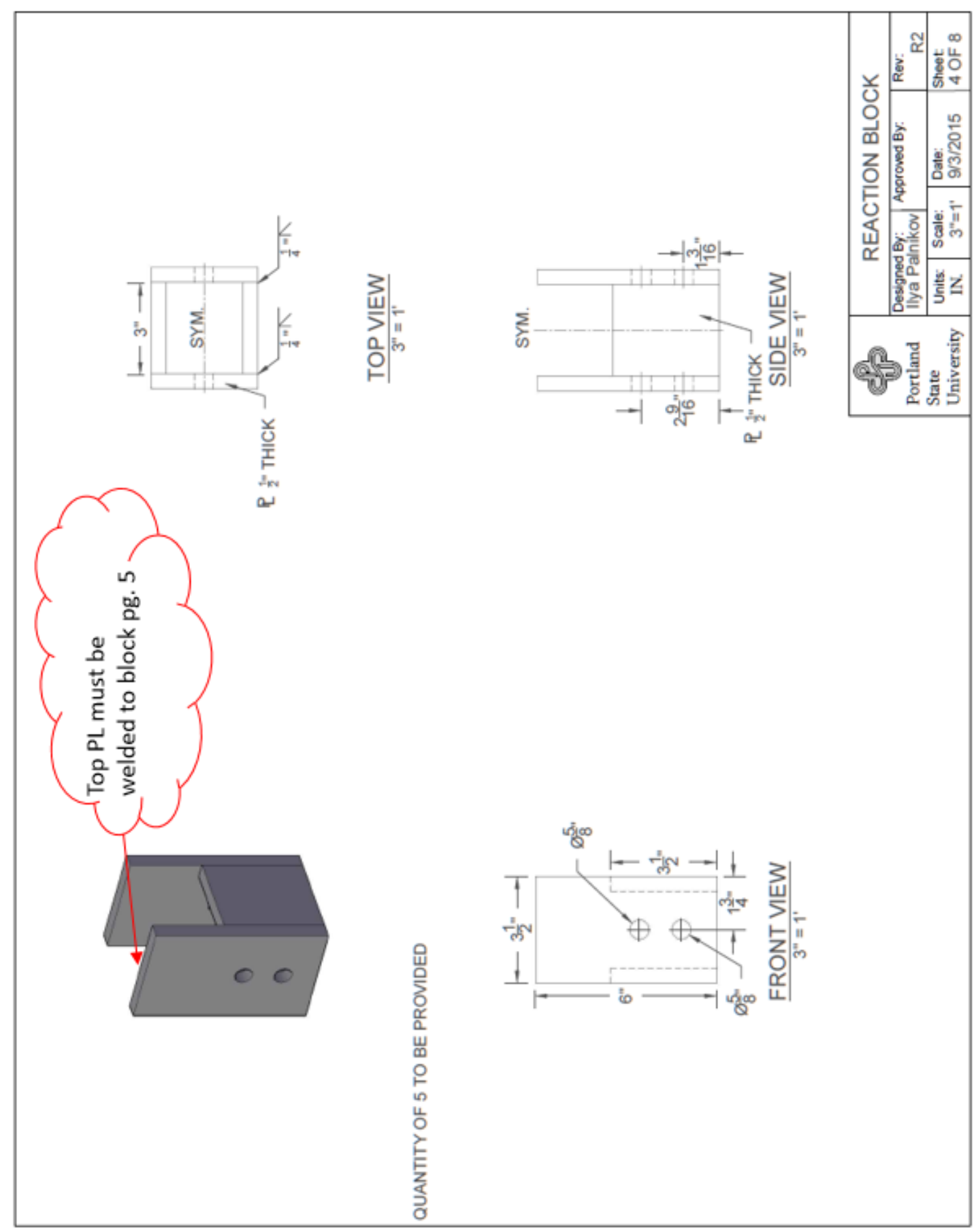




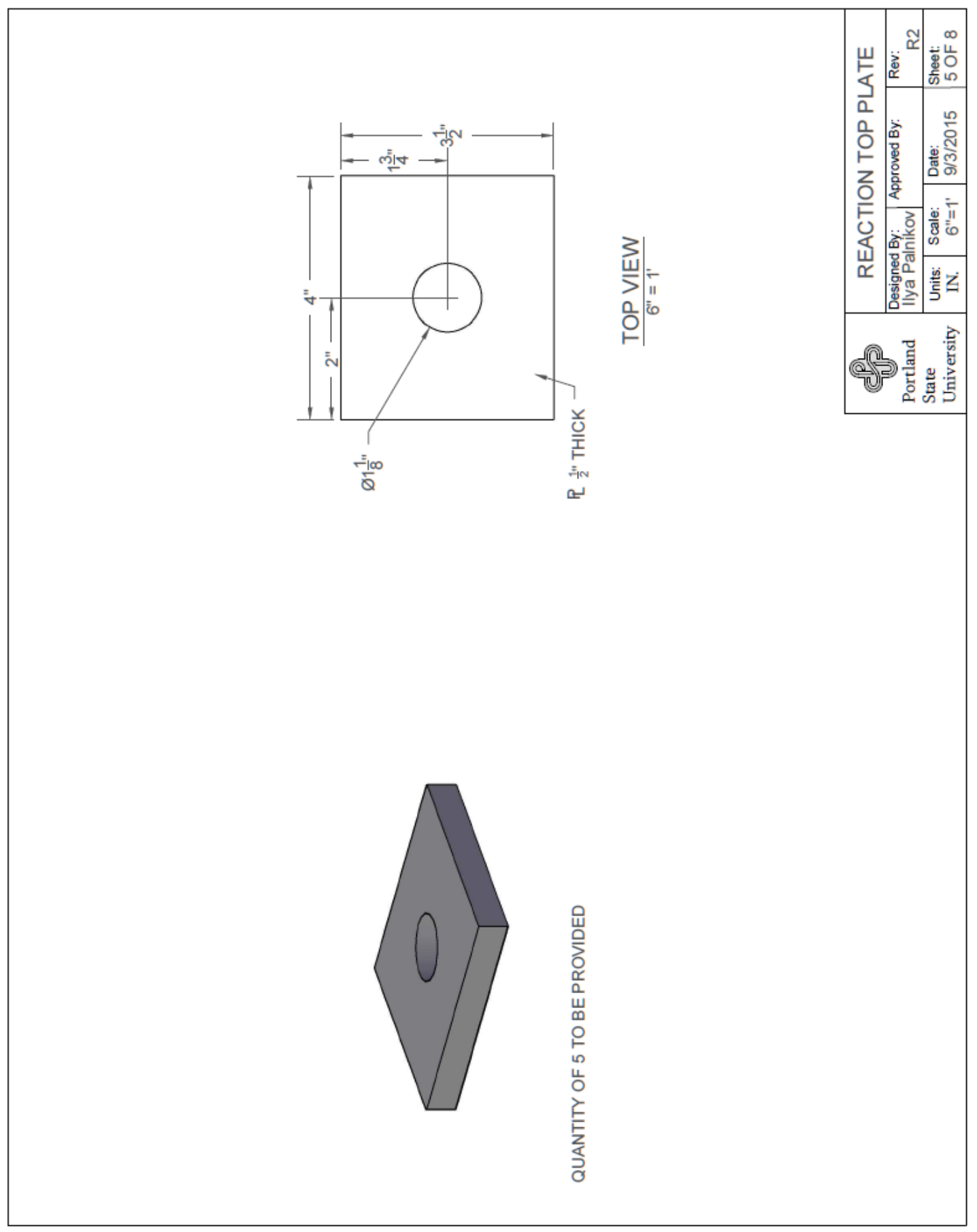




\subsection{APPENDIX D}

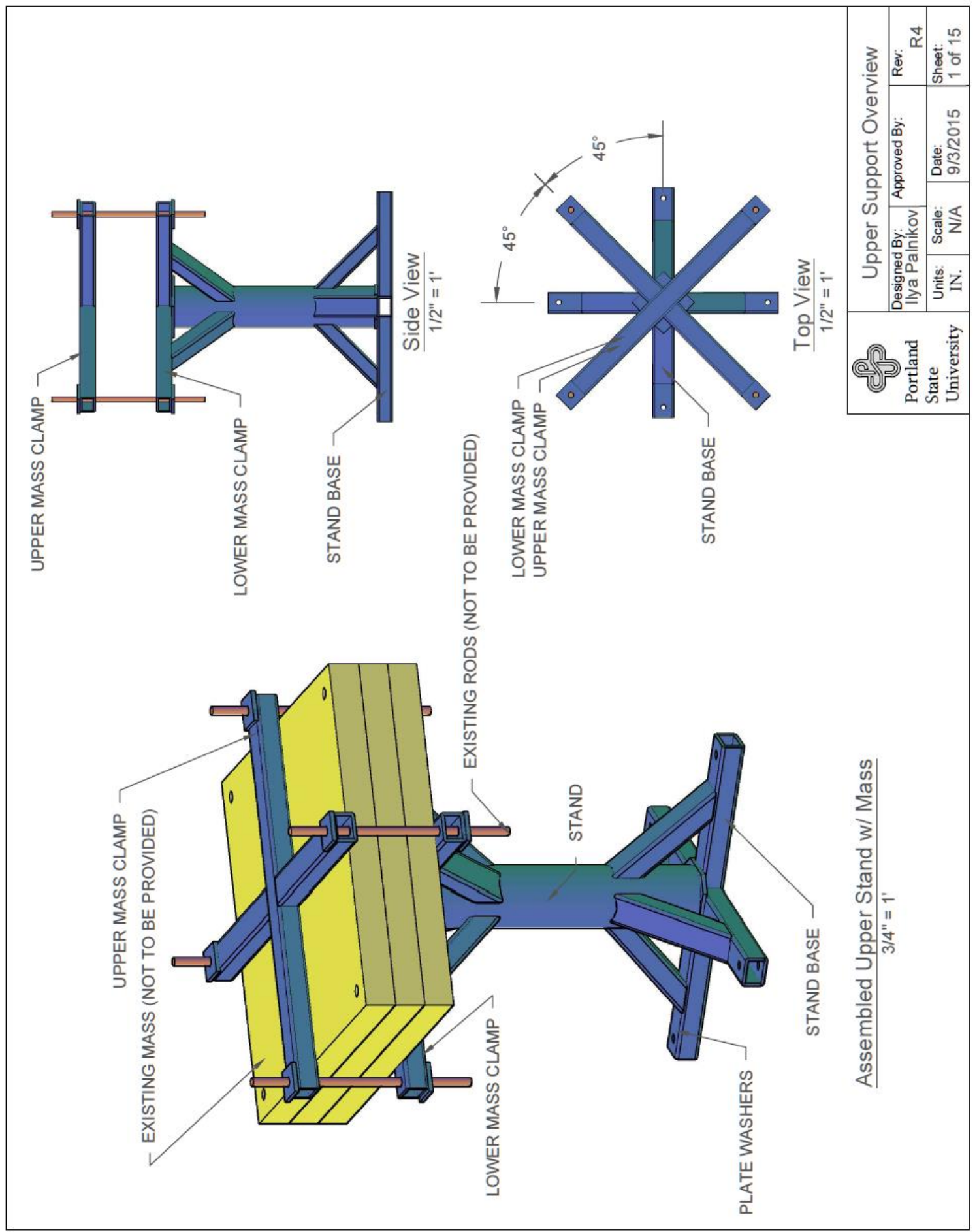




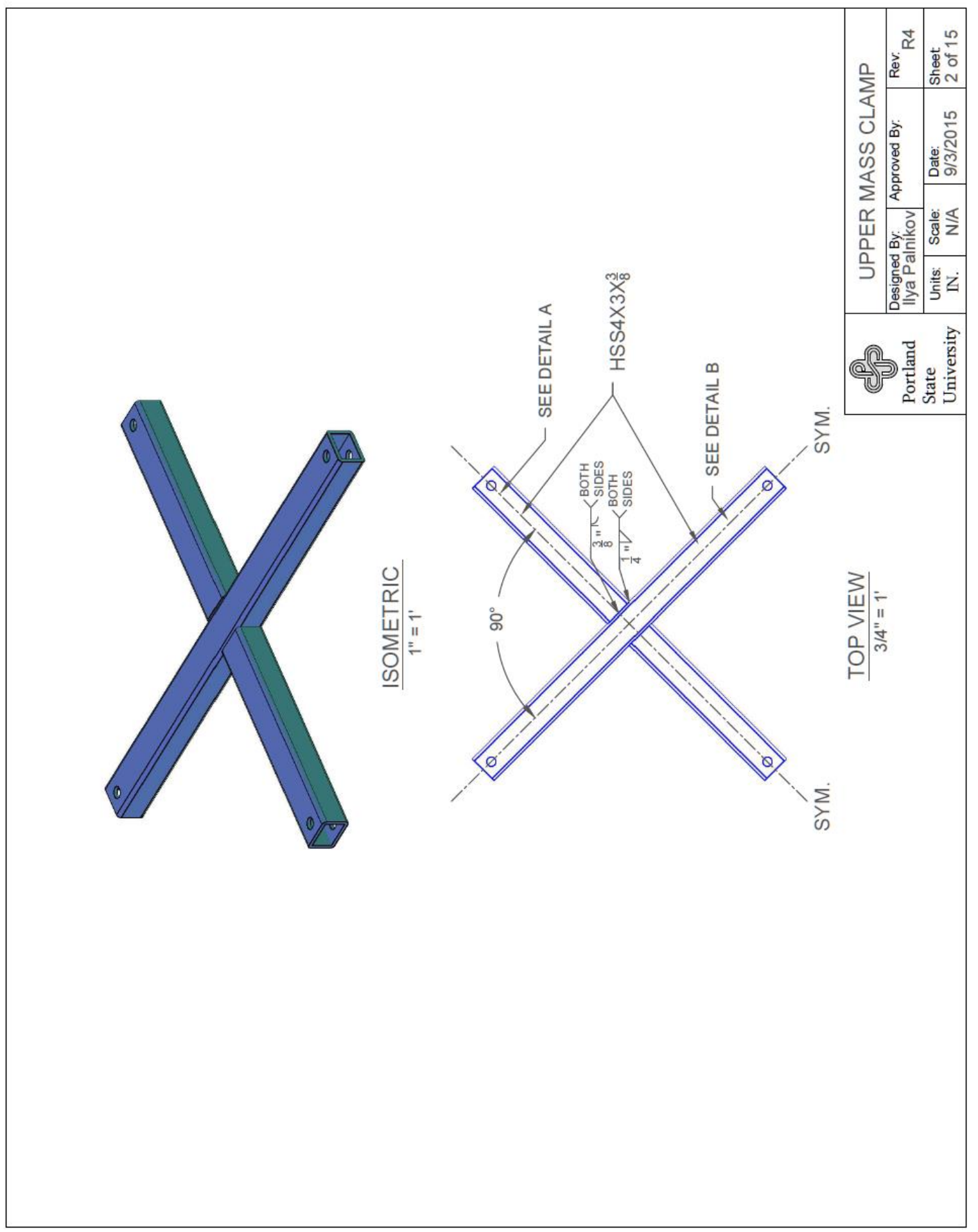




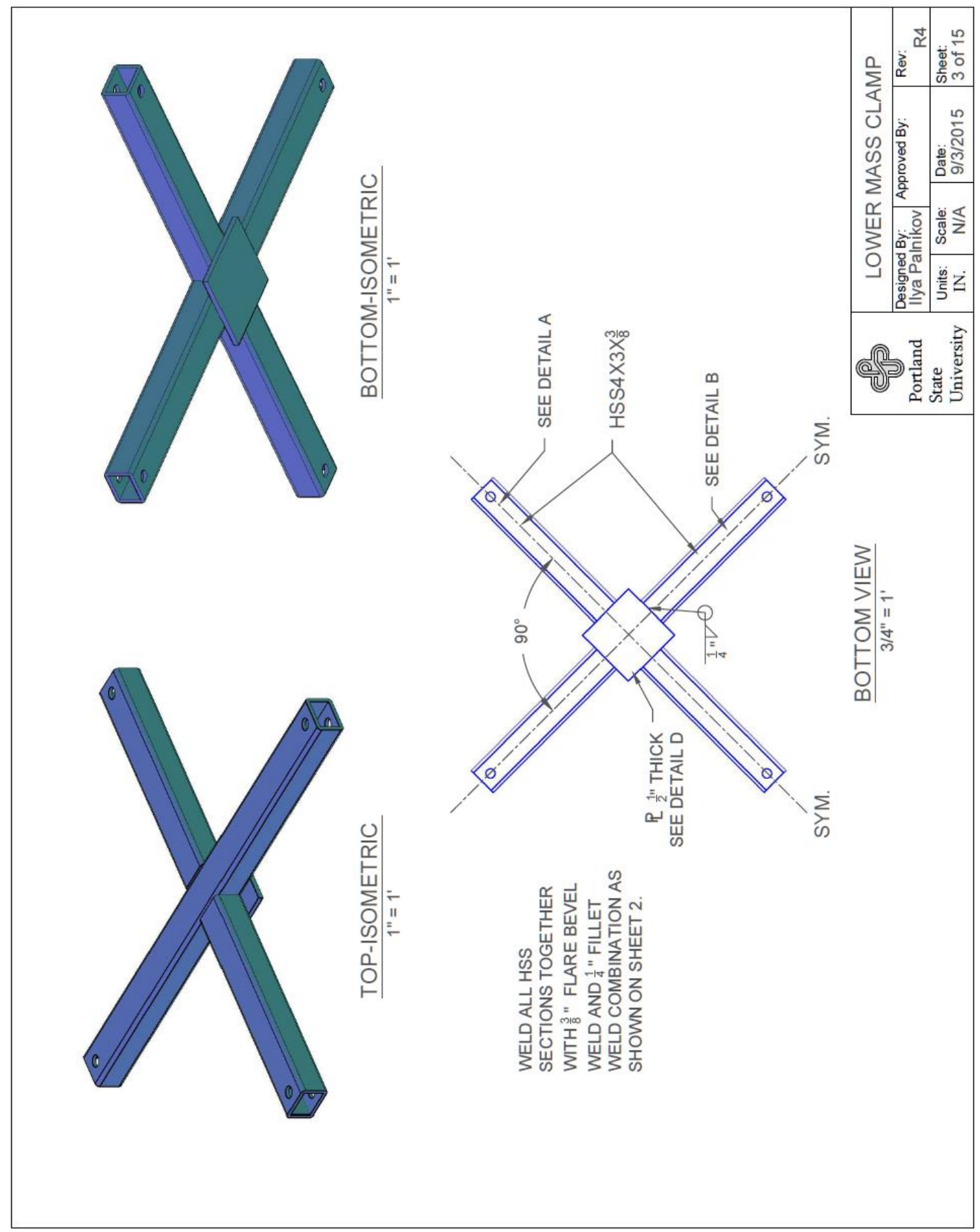




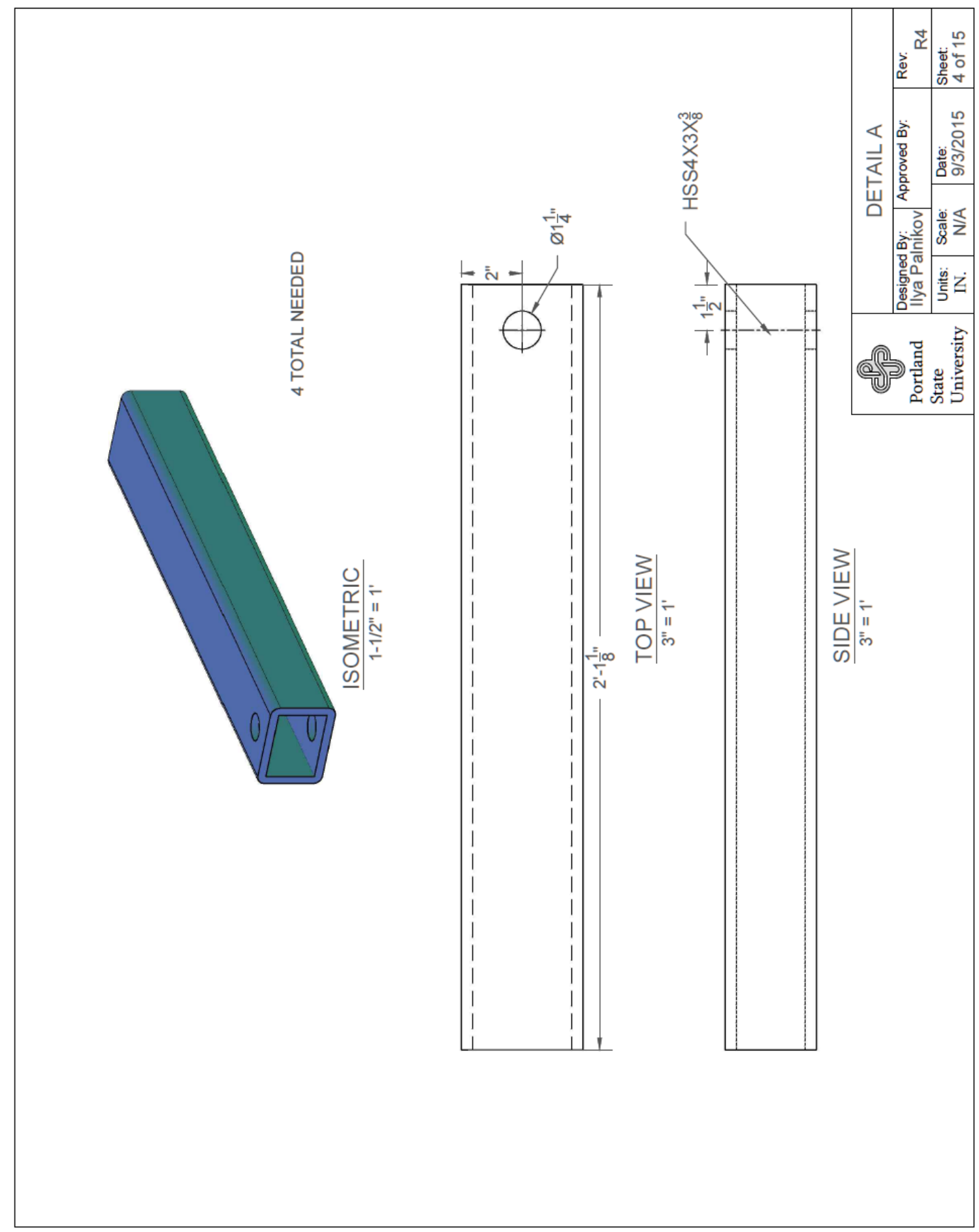




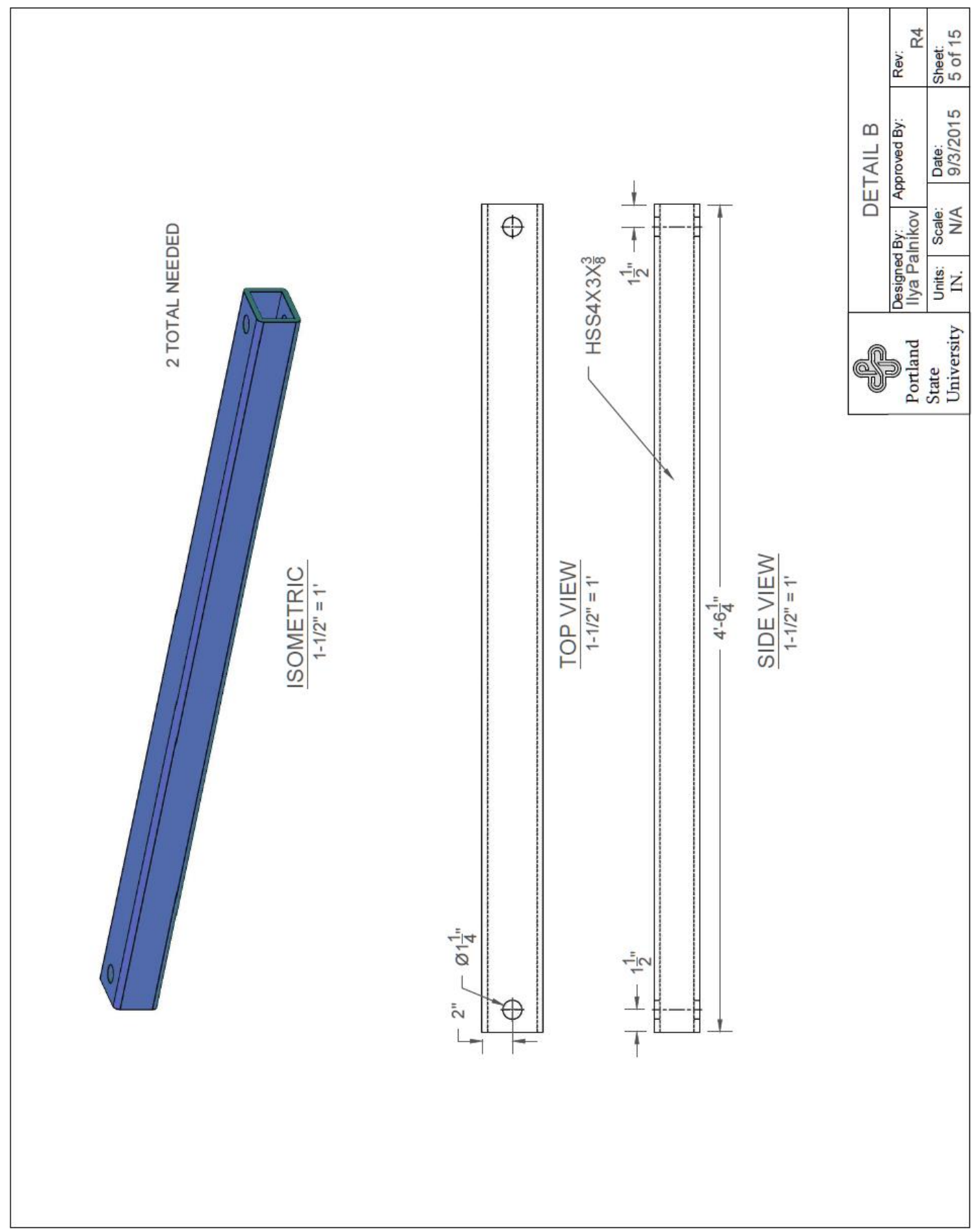




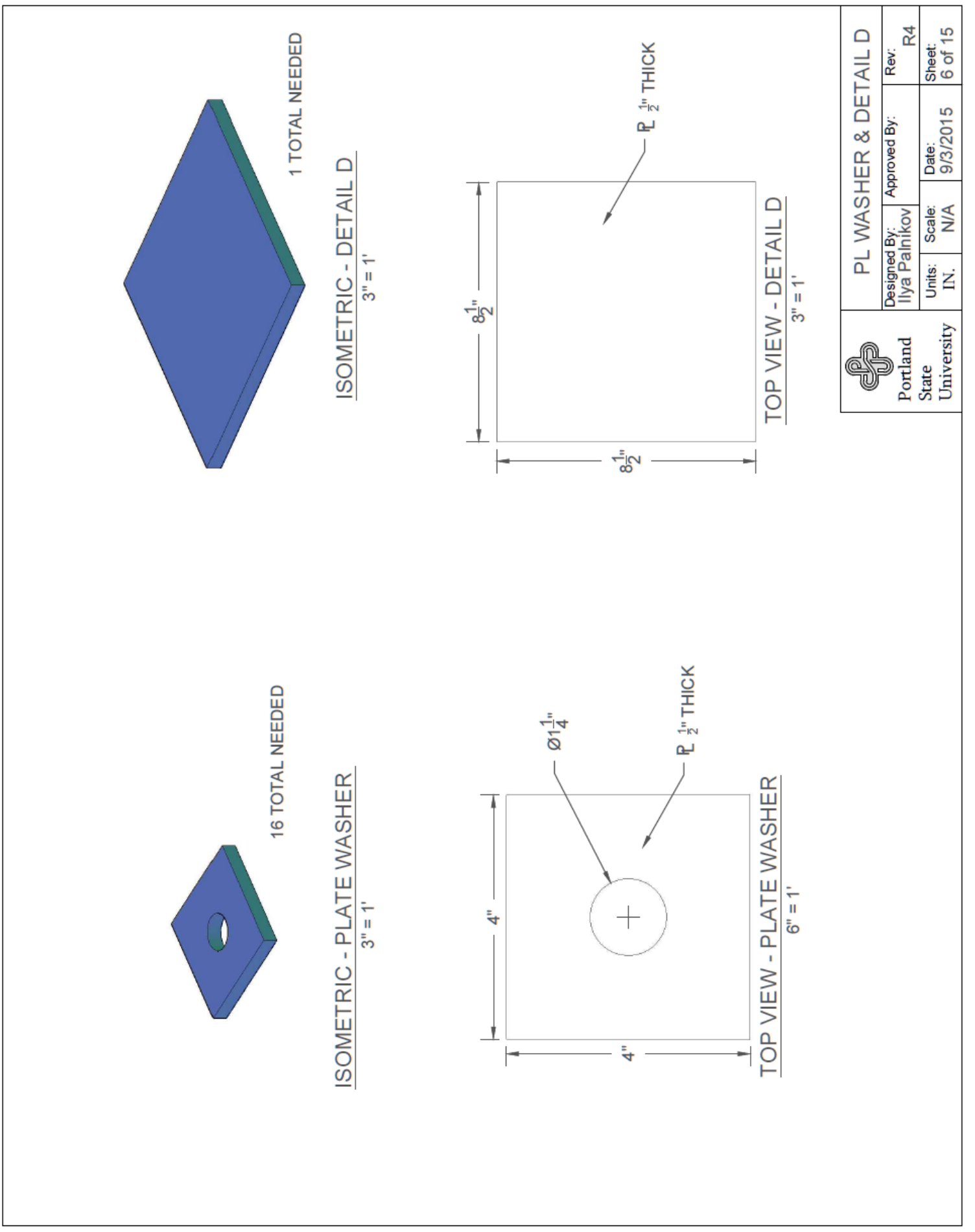




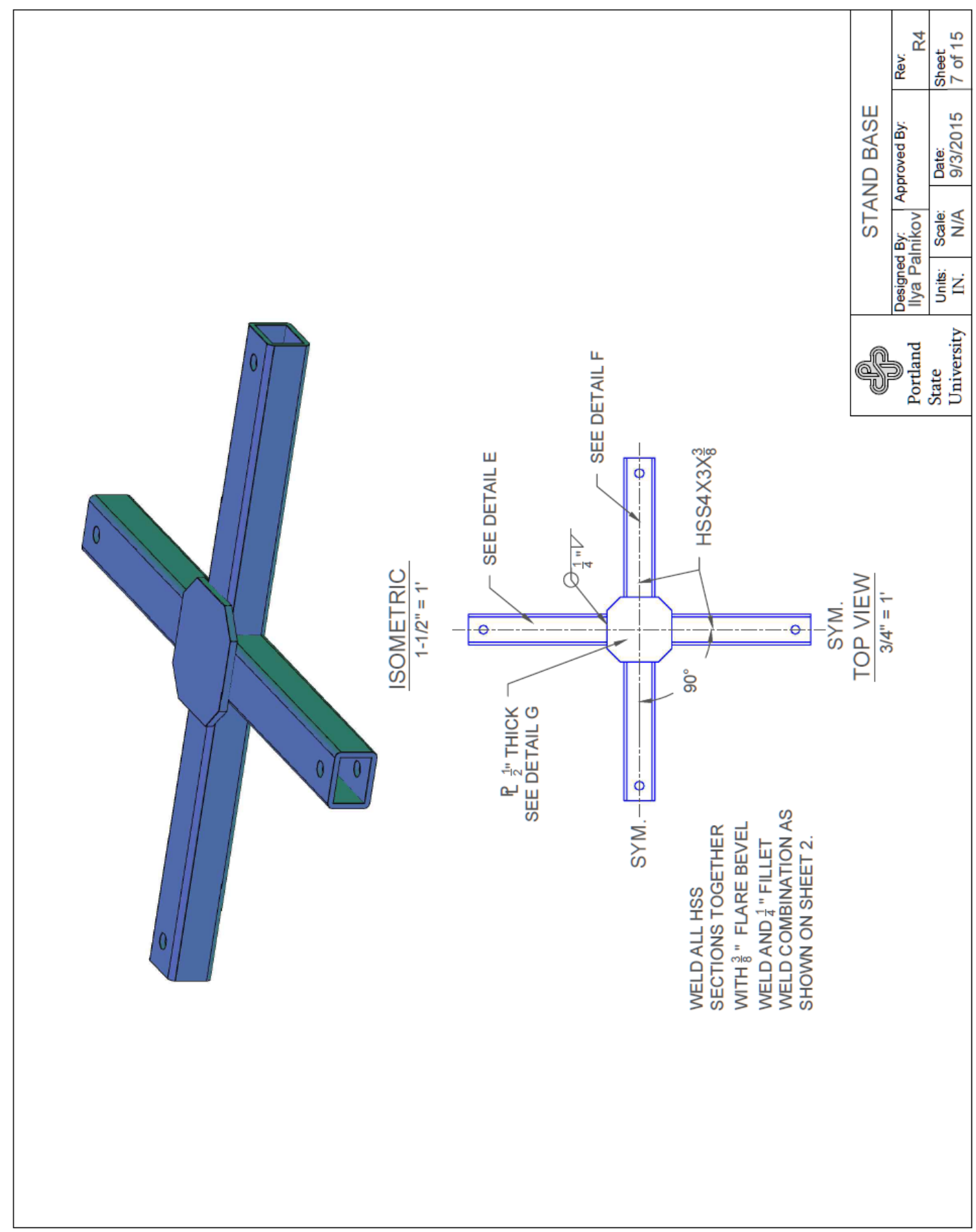




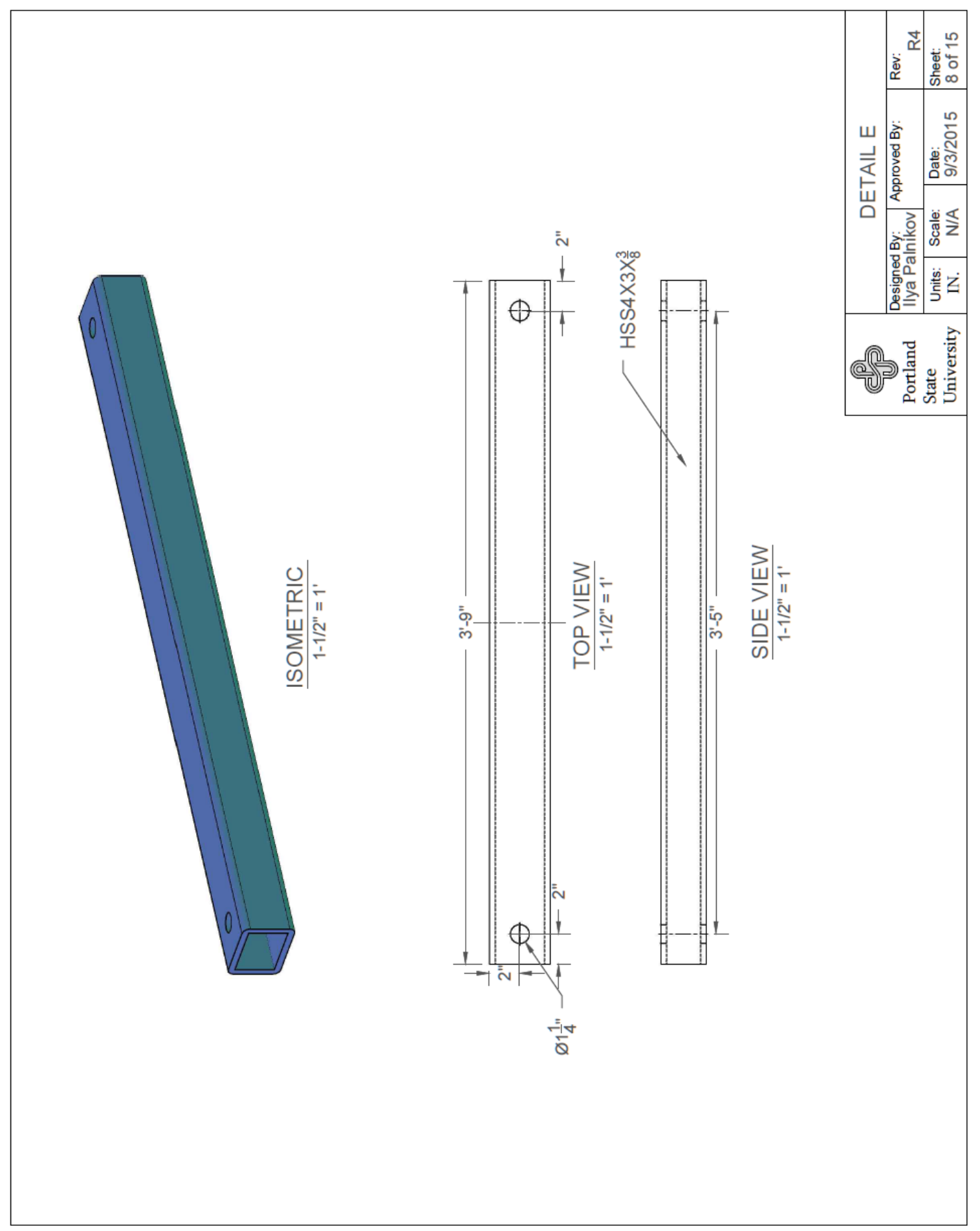




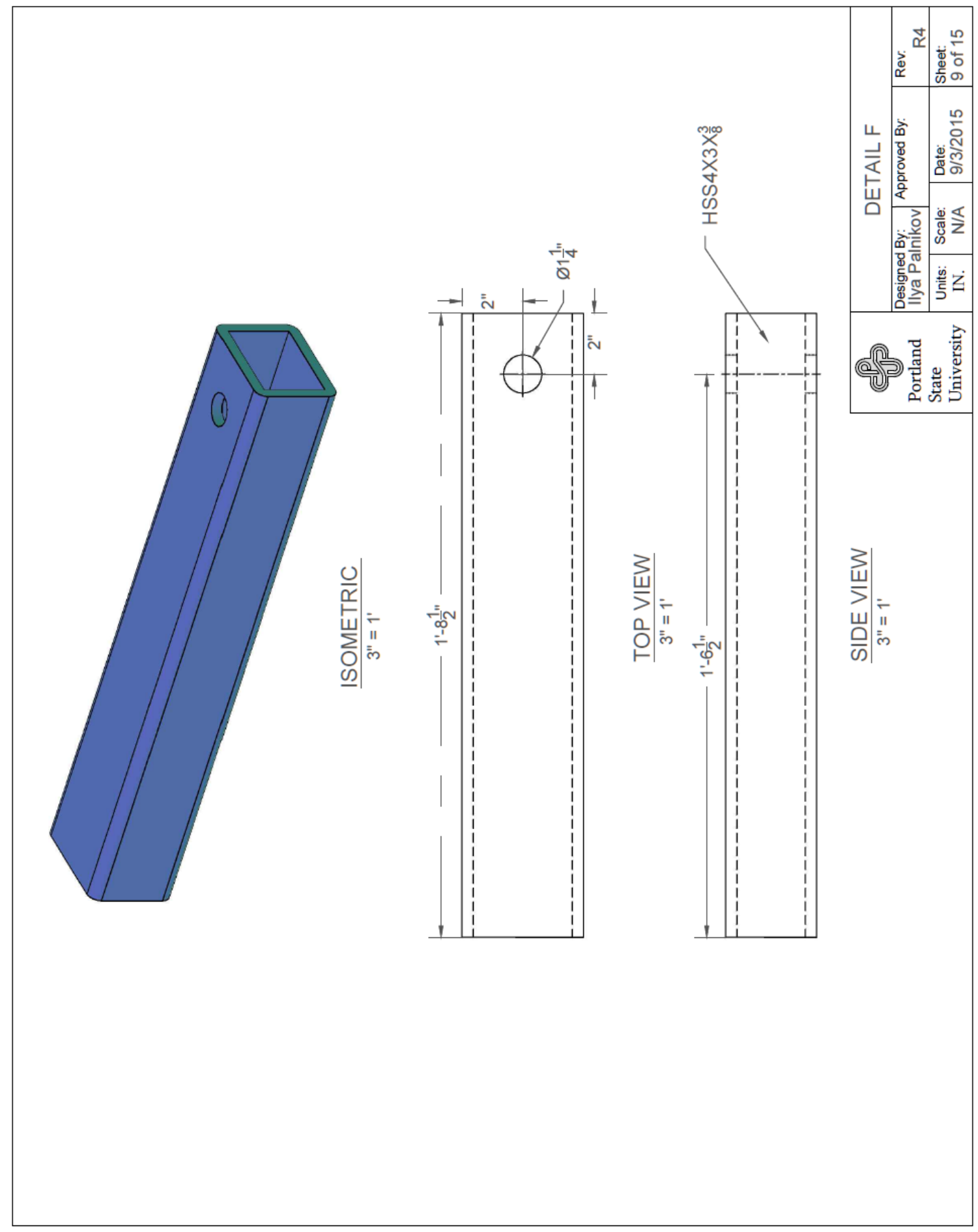




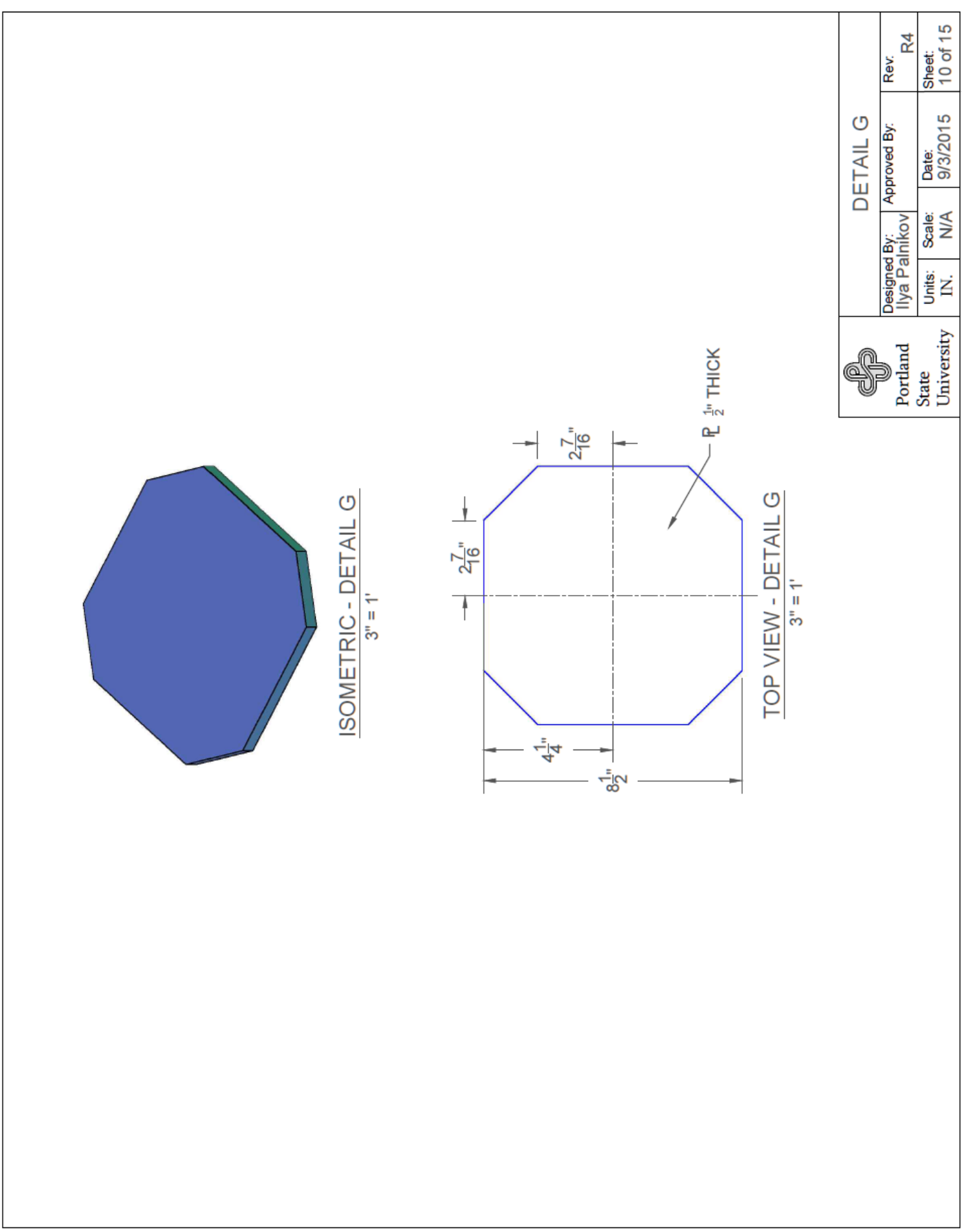




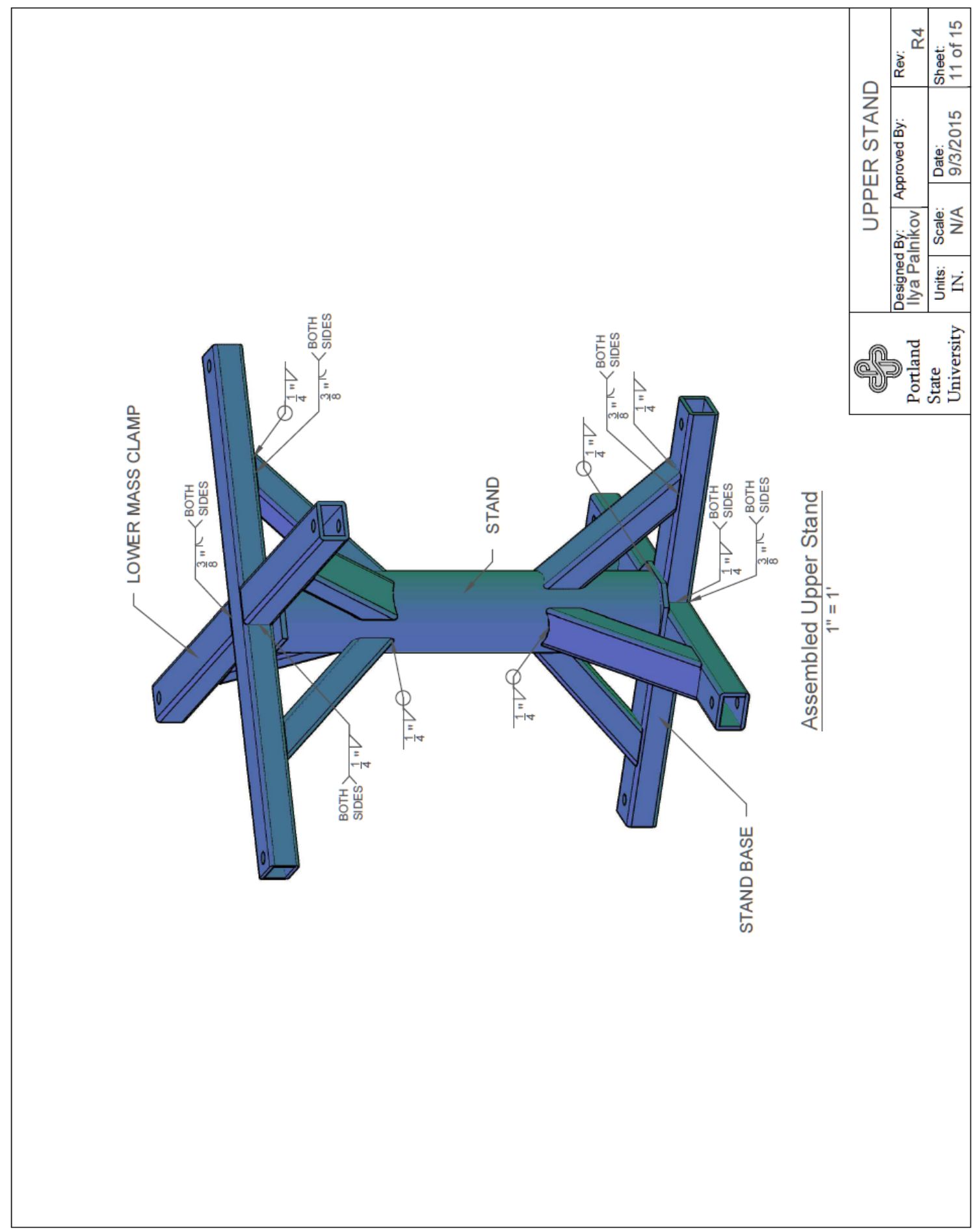




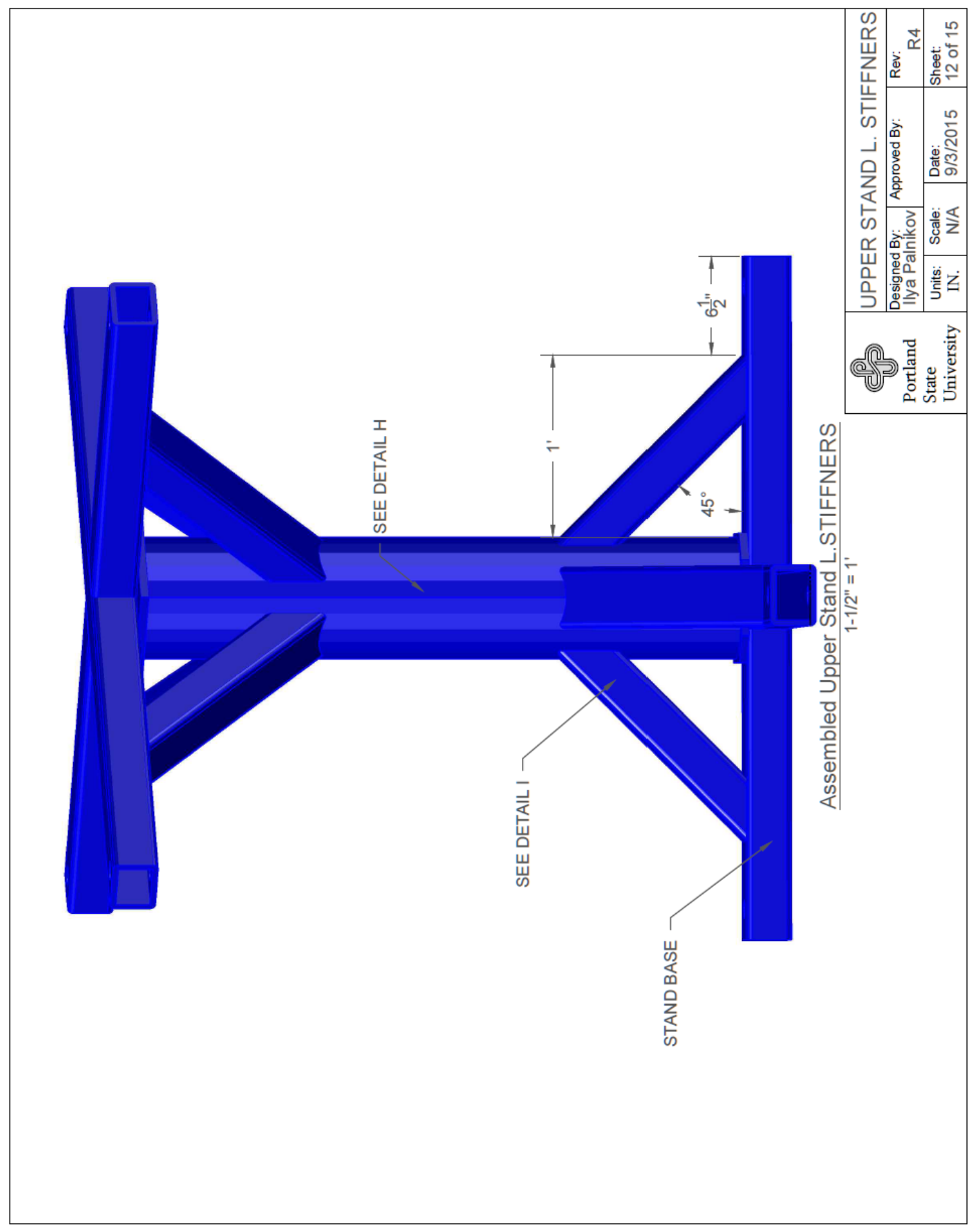




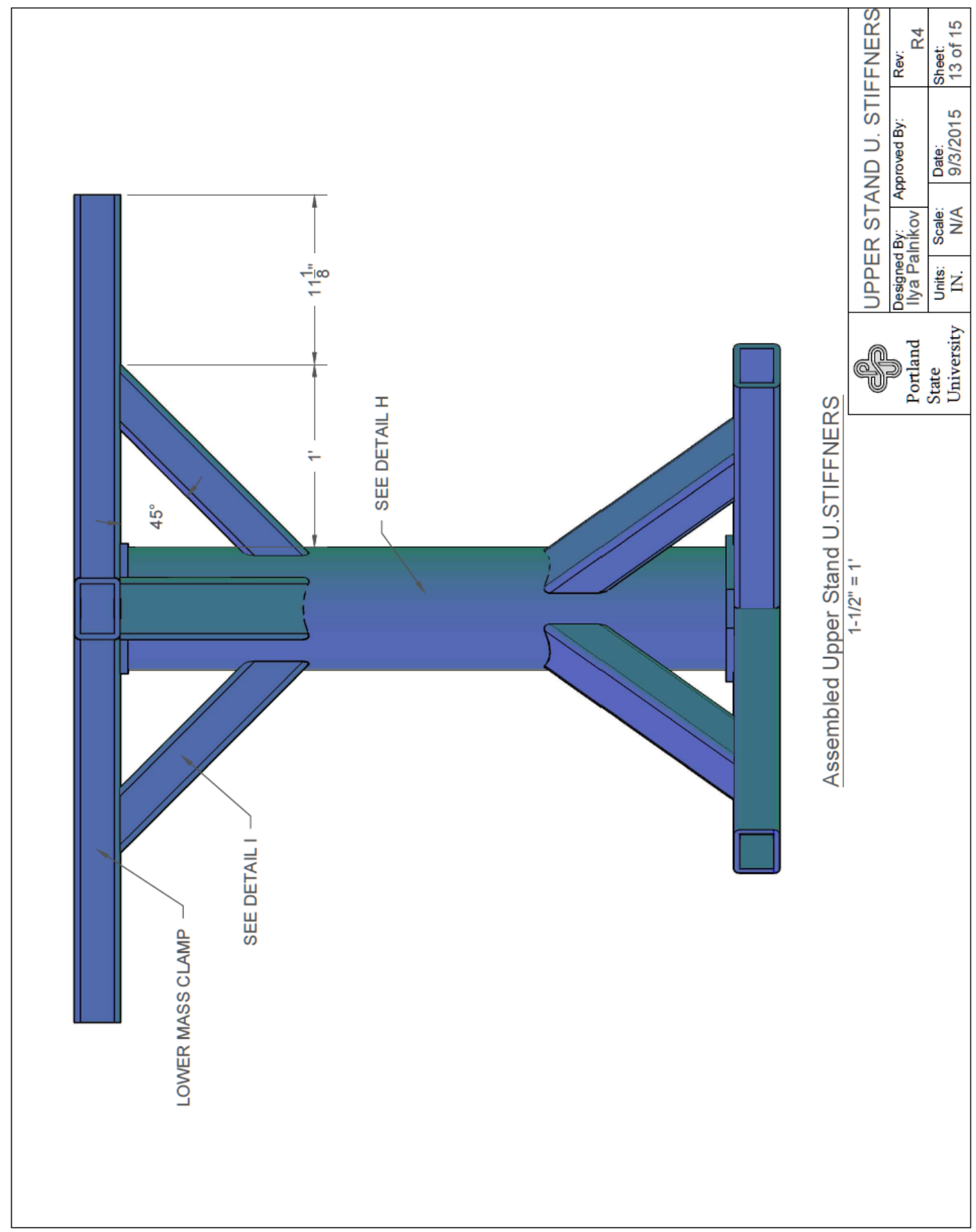




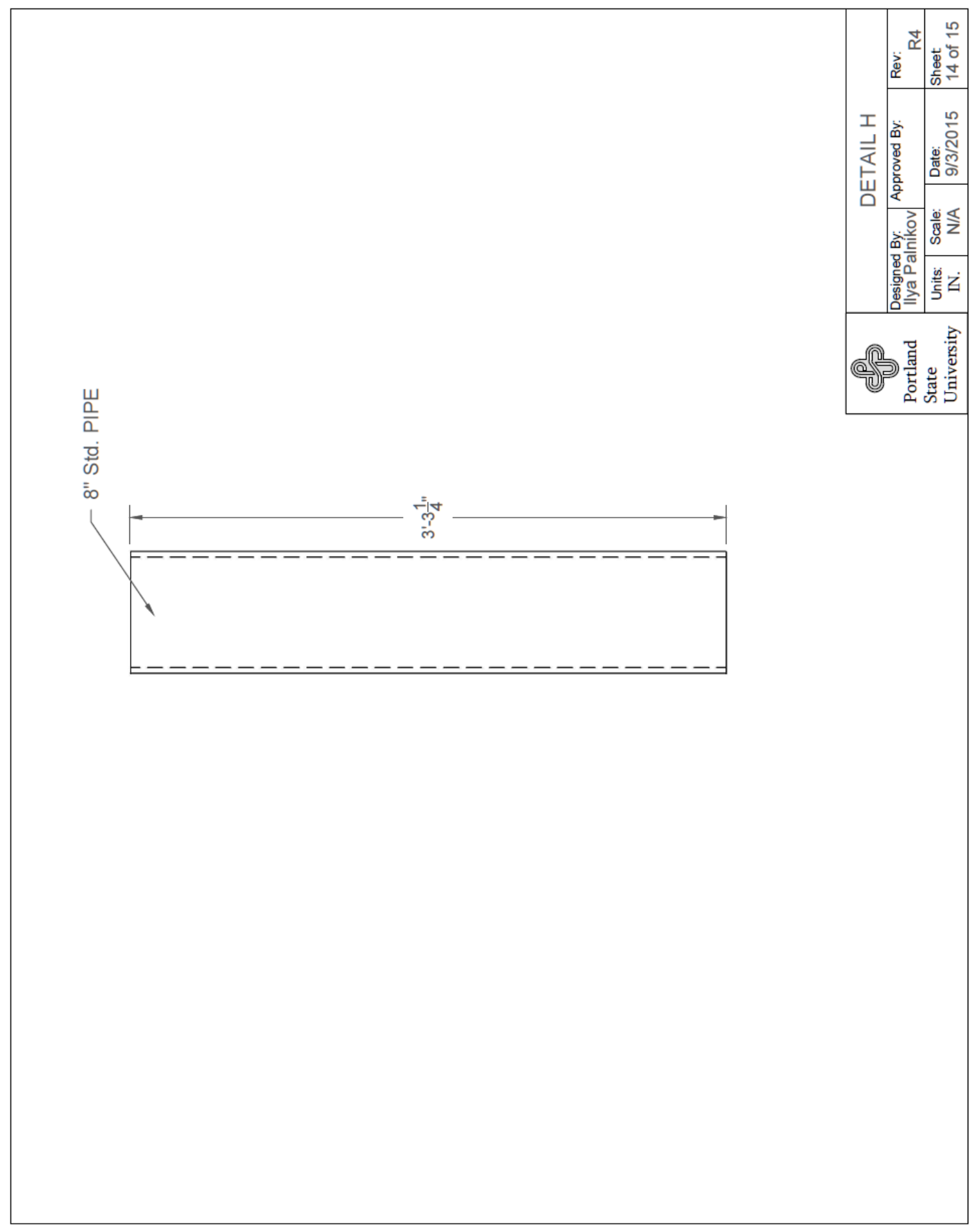




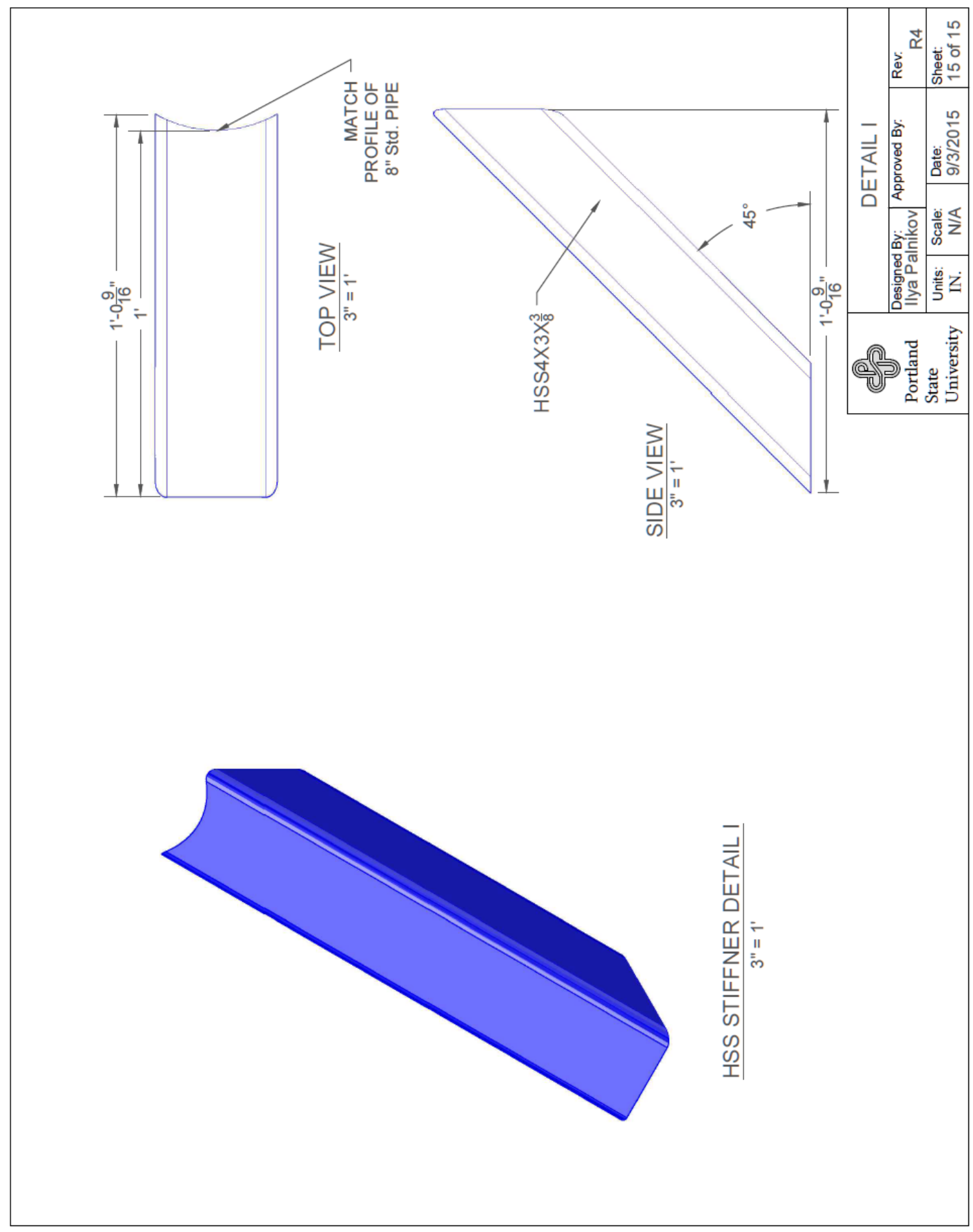




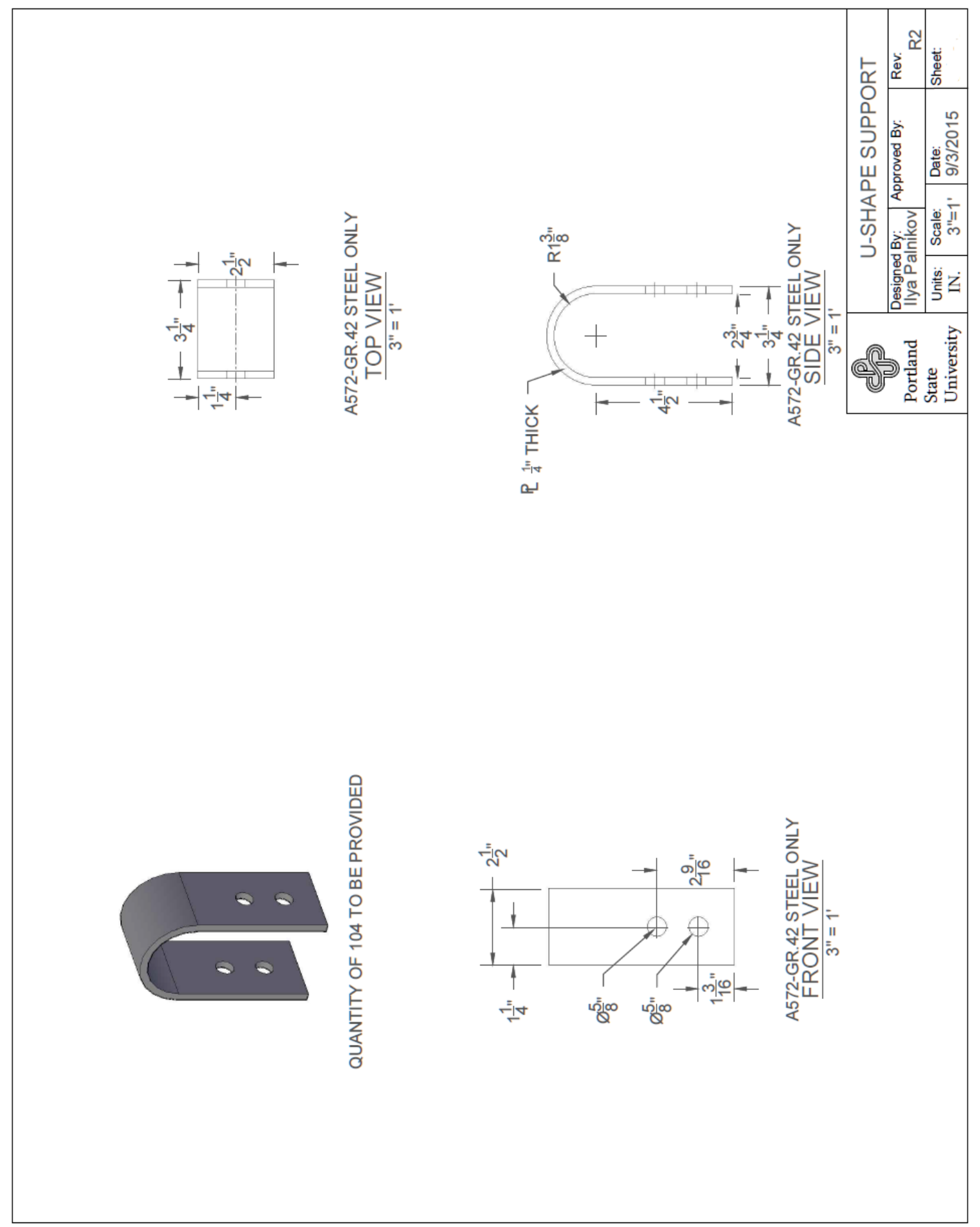




\subsection{APPENDIX E}

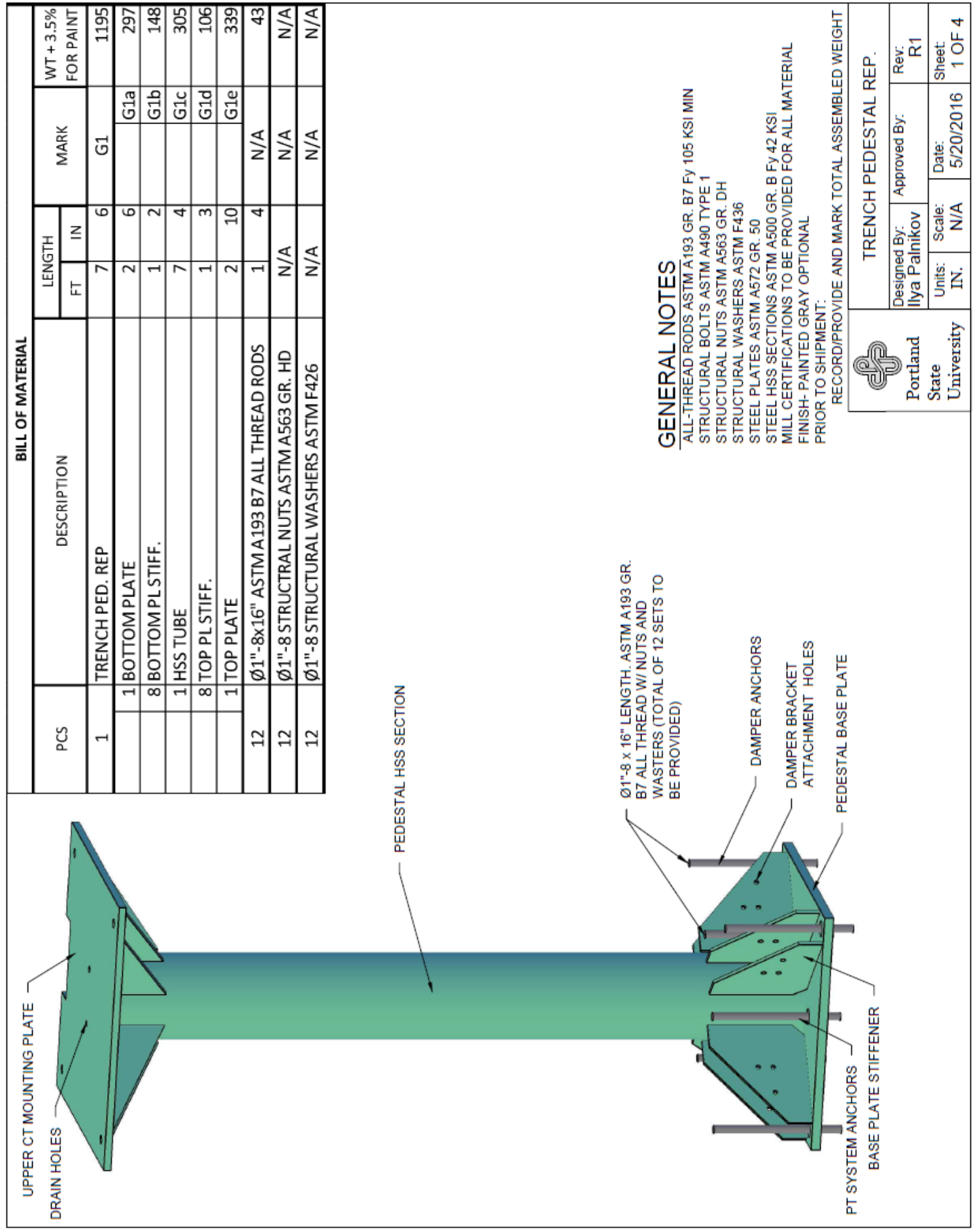




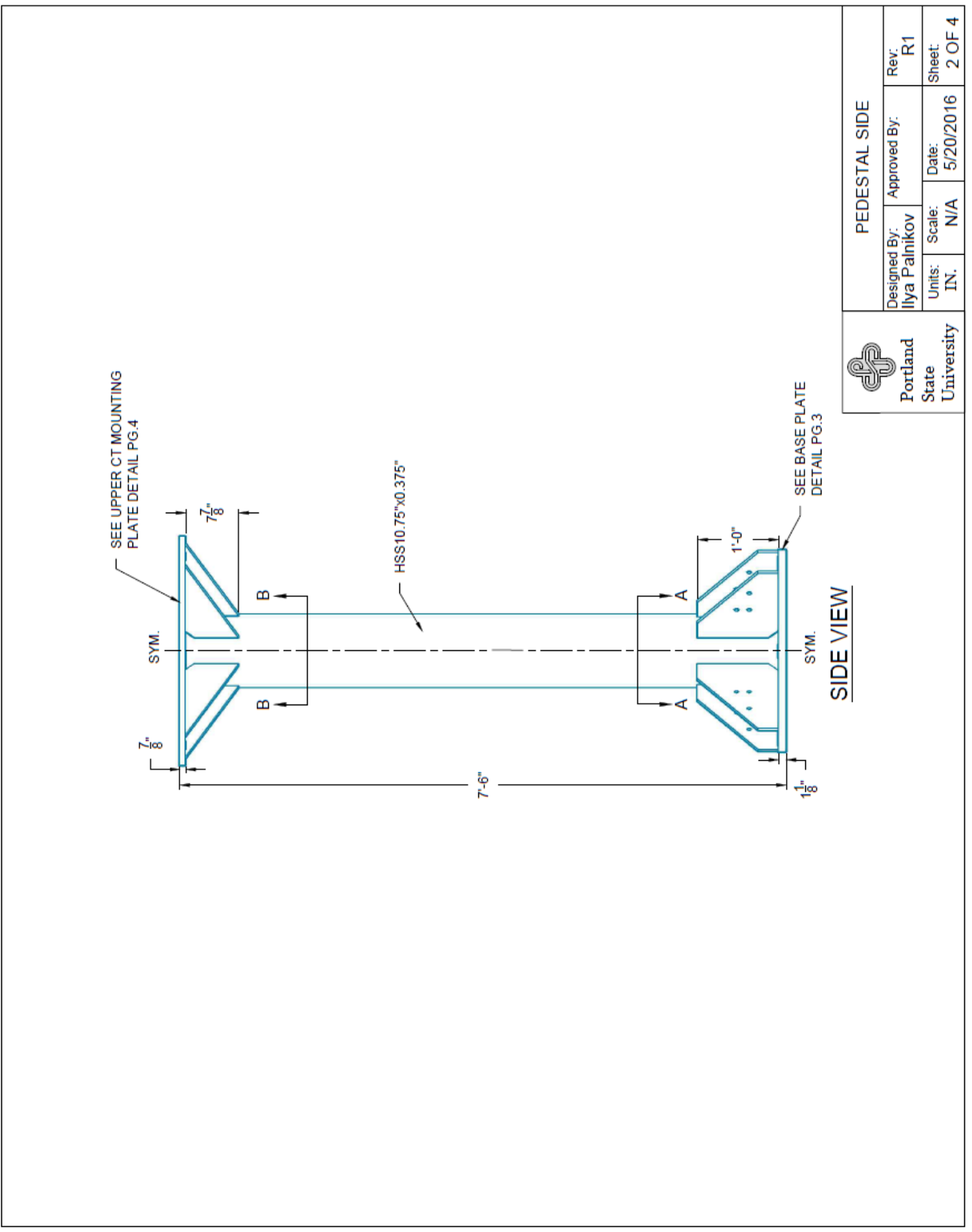




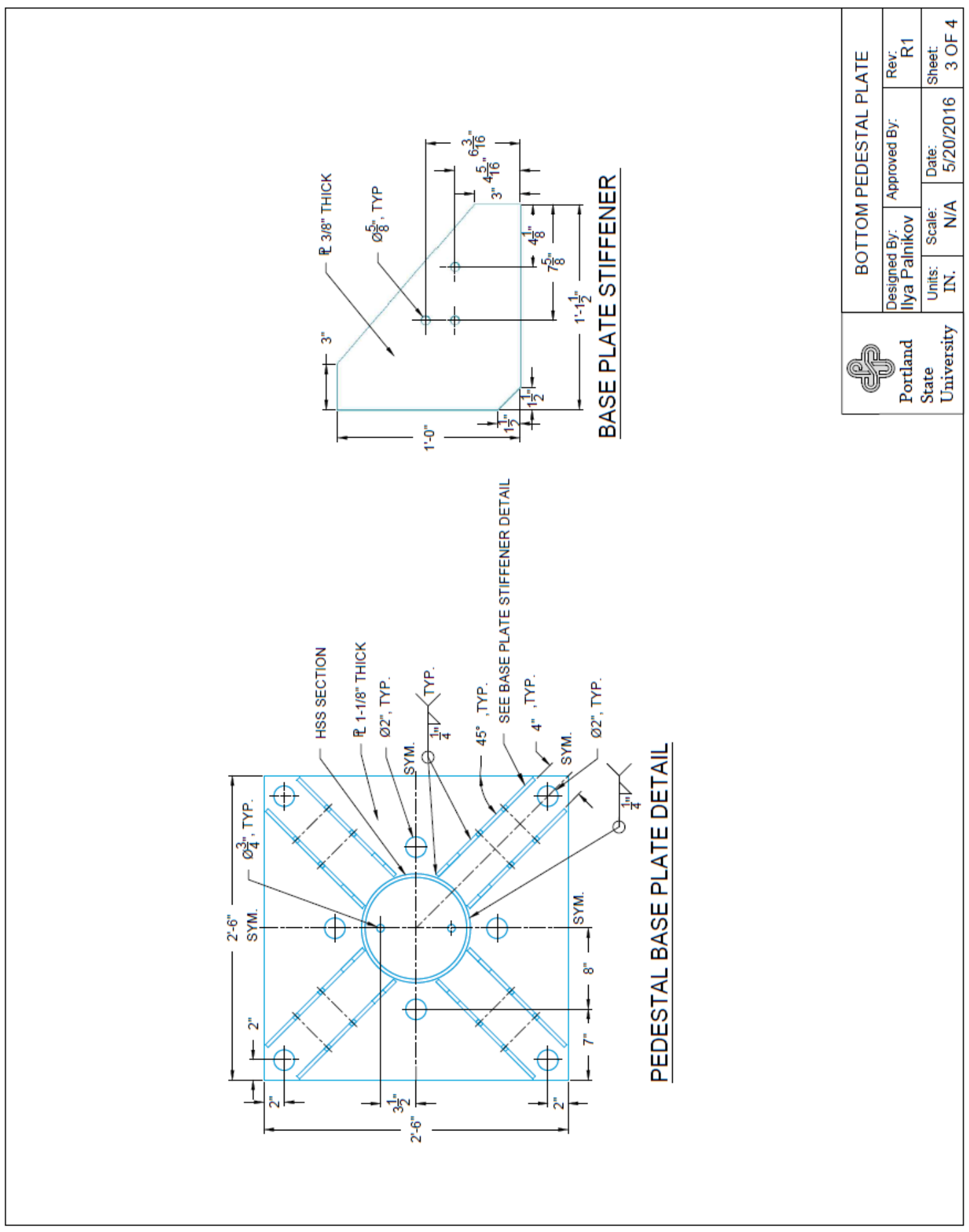




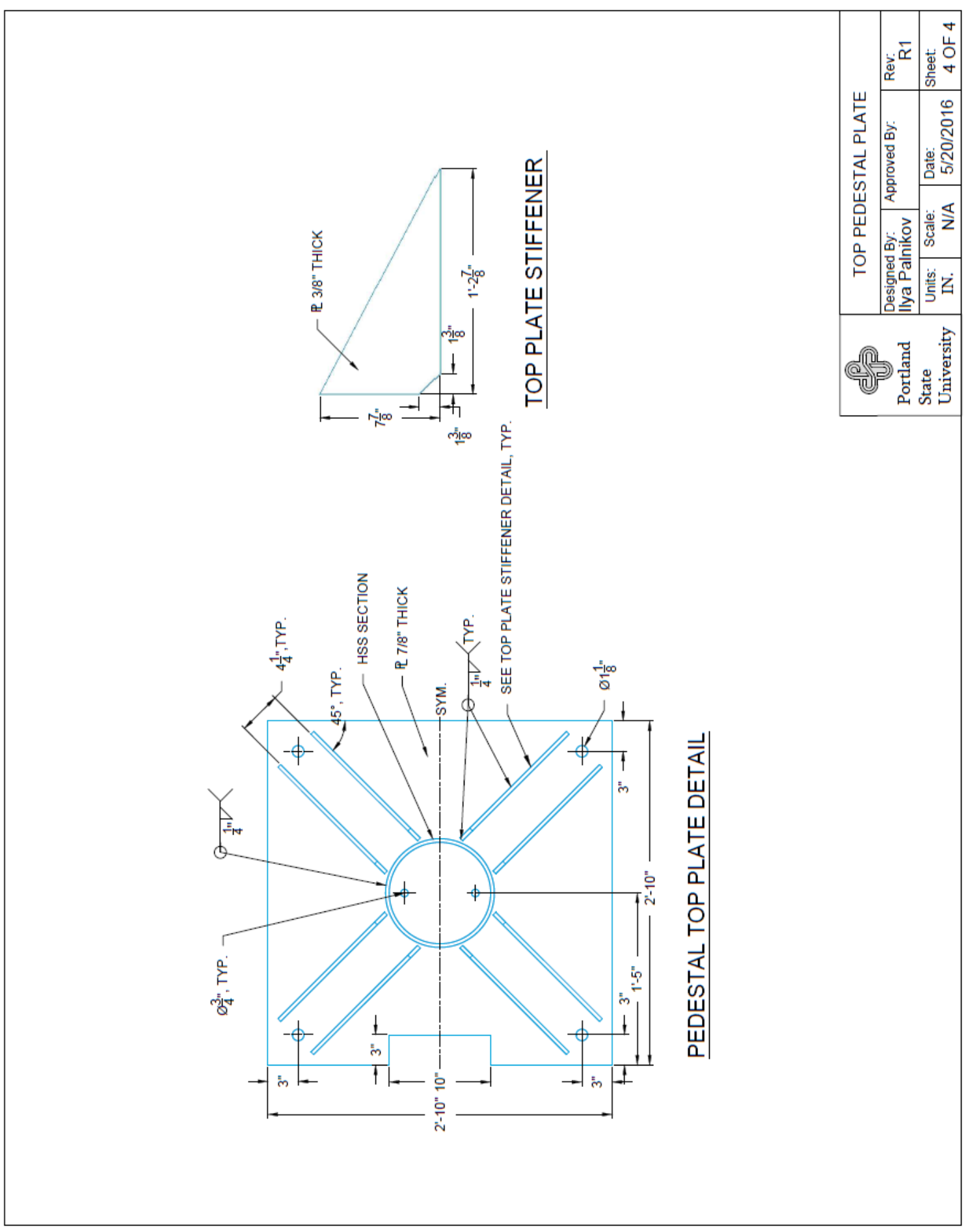




\subsection{APPENDIX F}

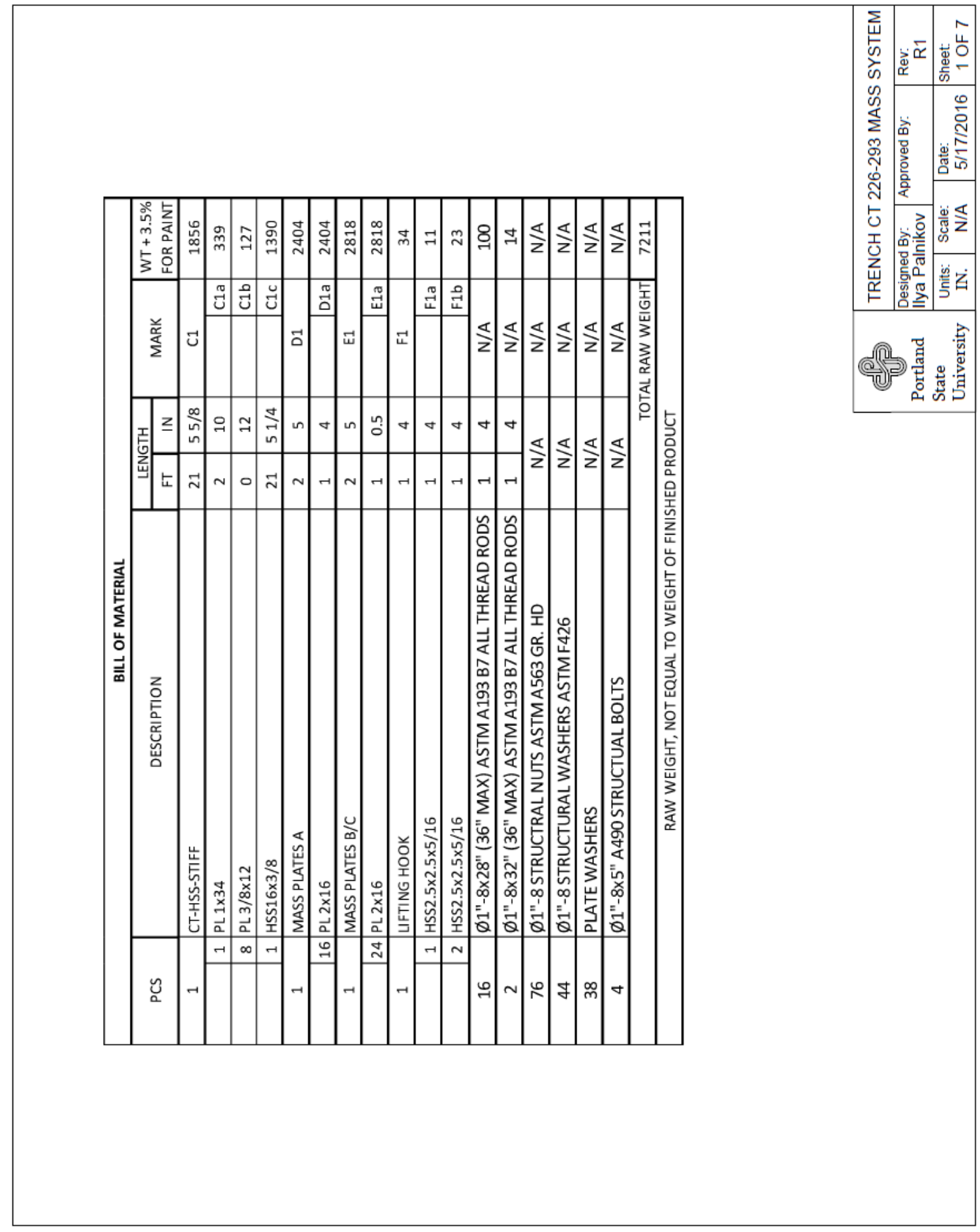




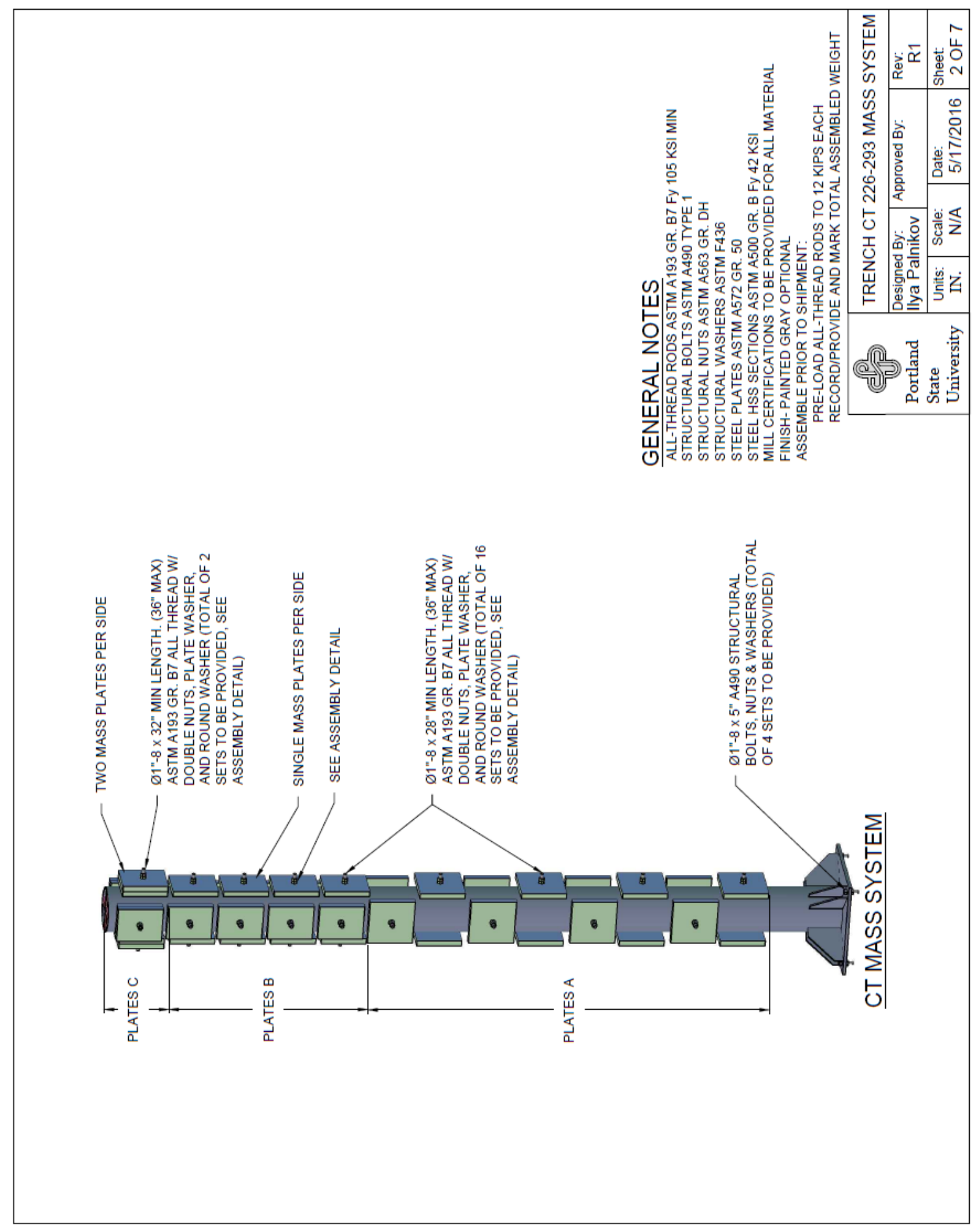




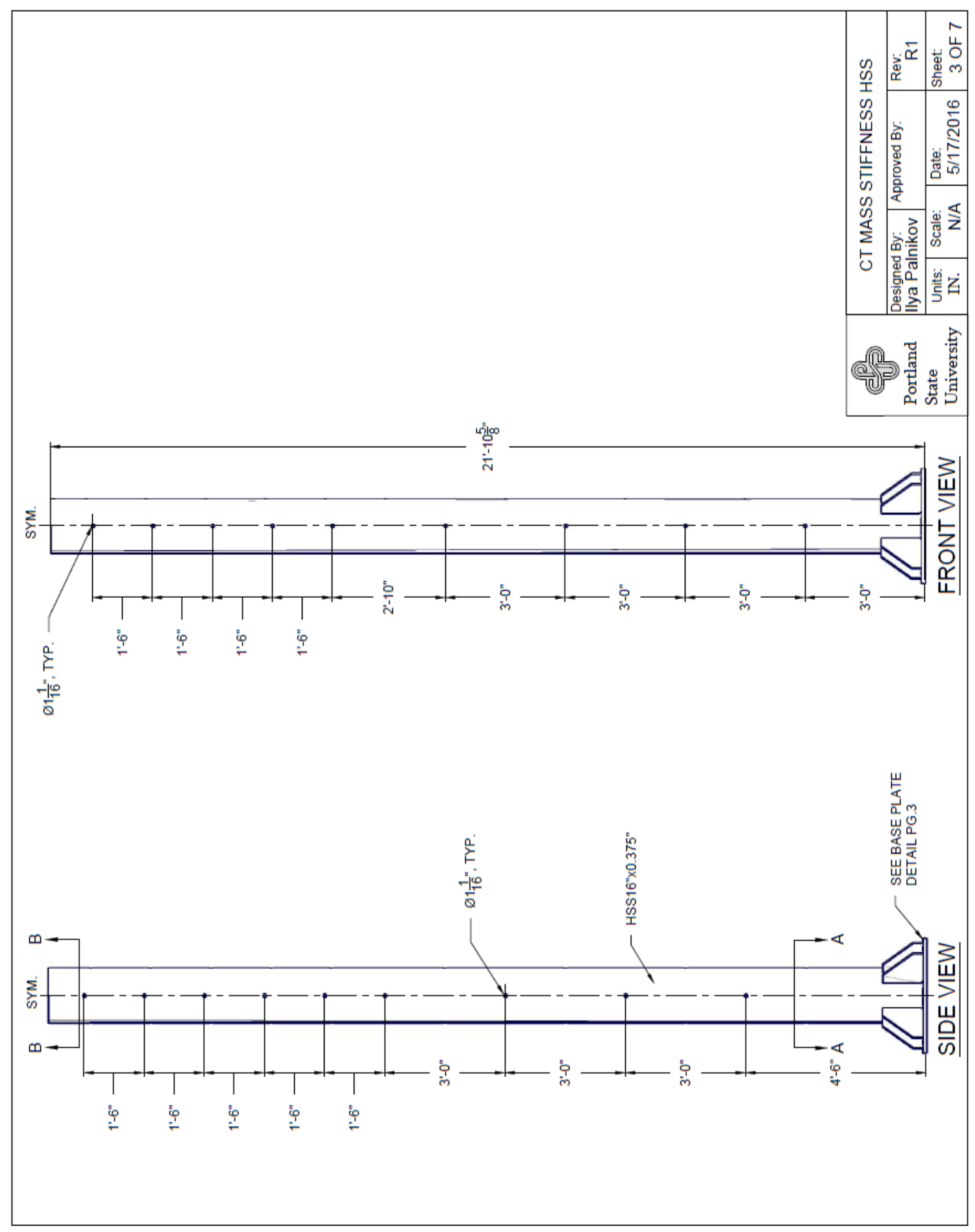




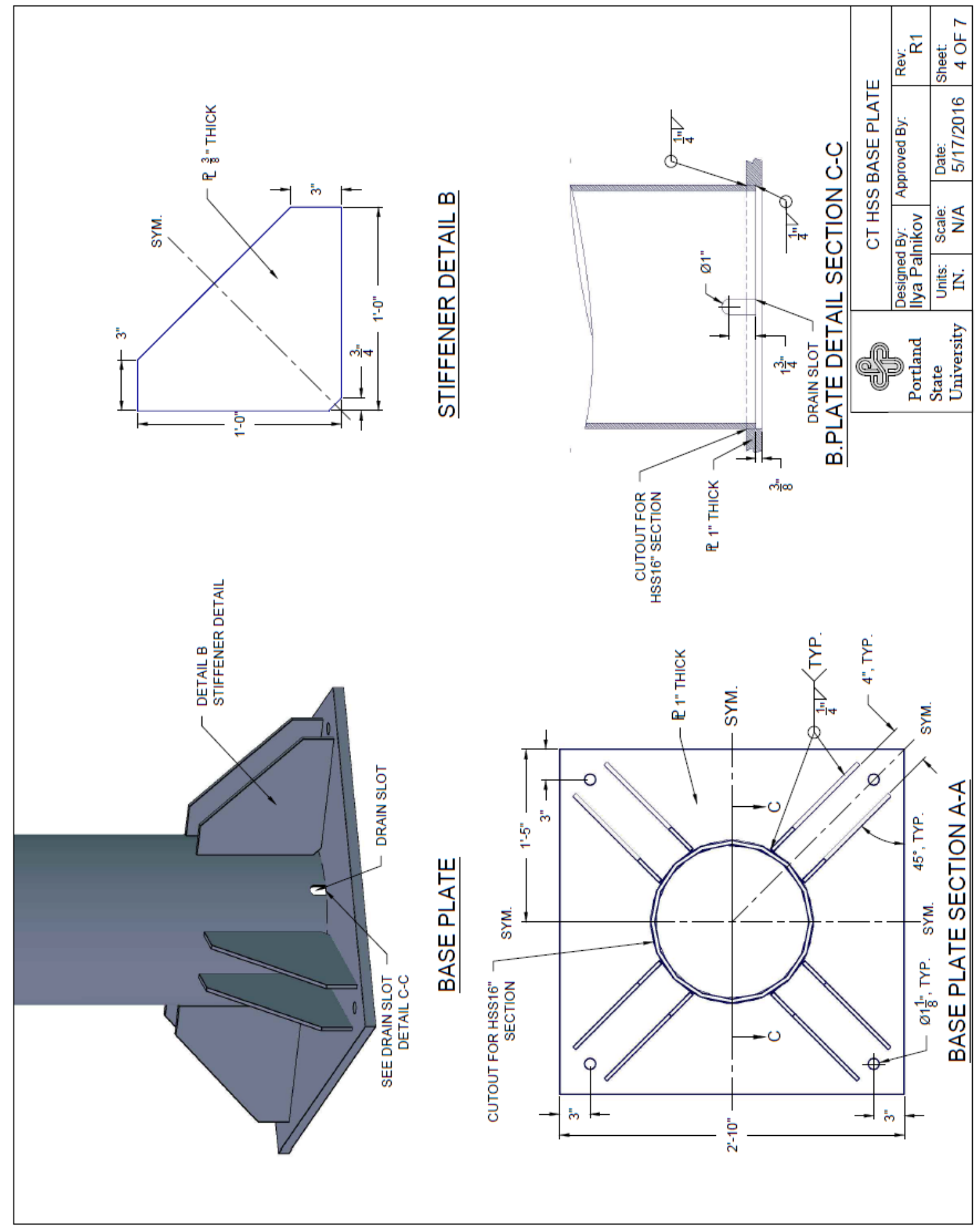




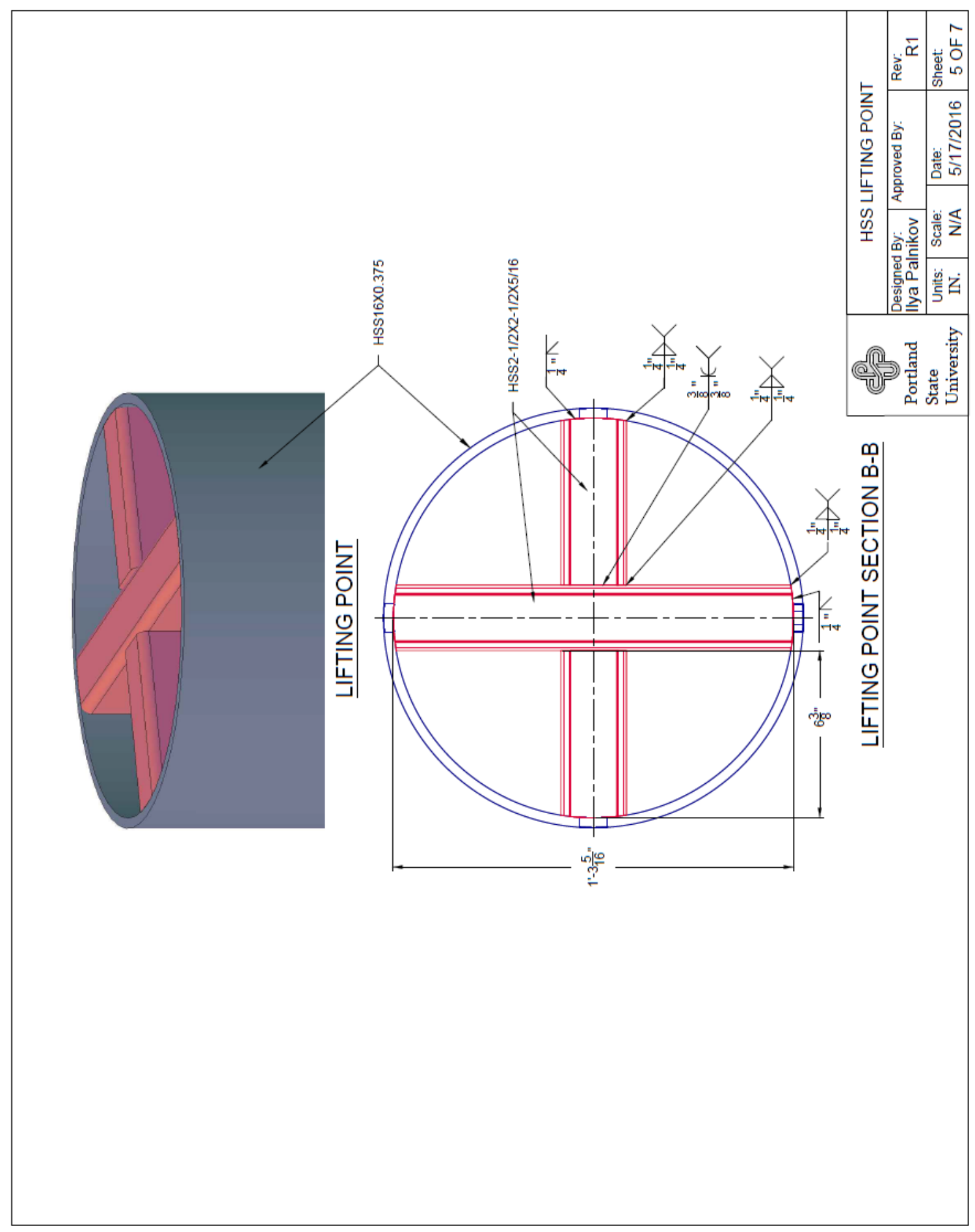




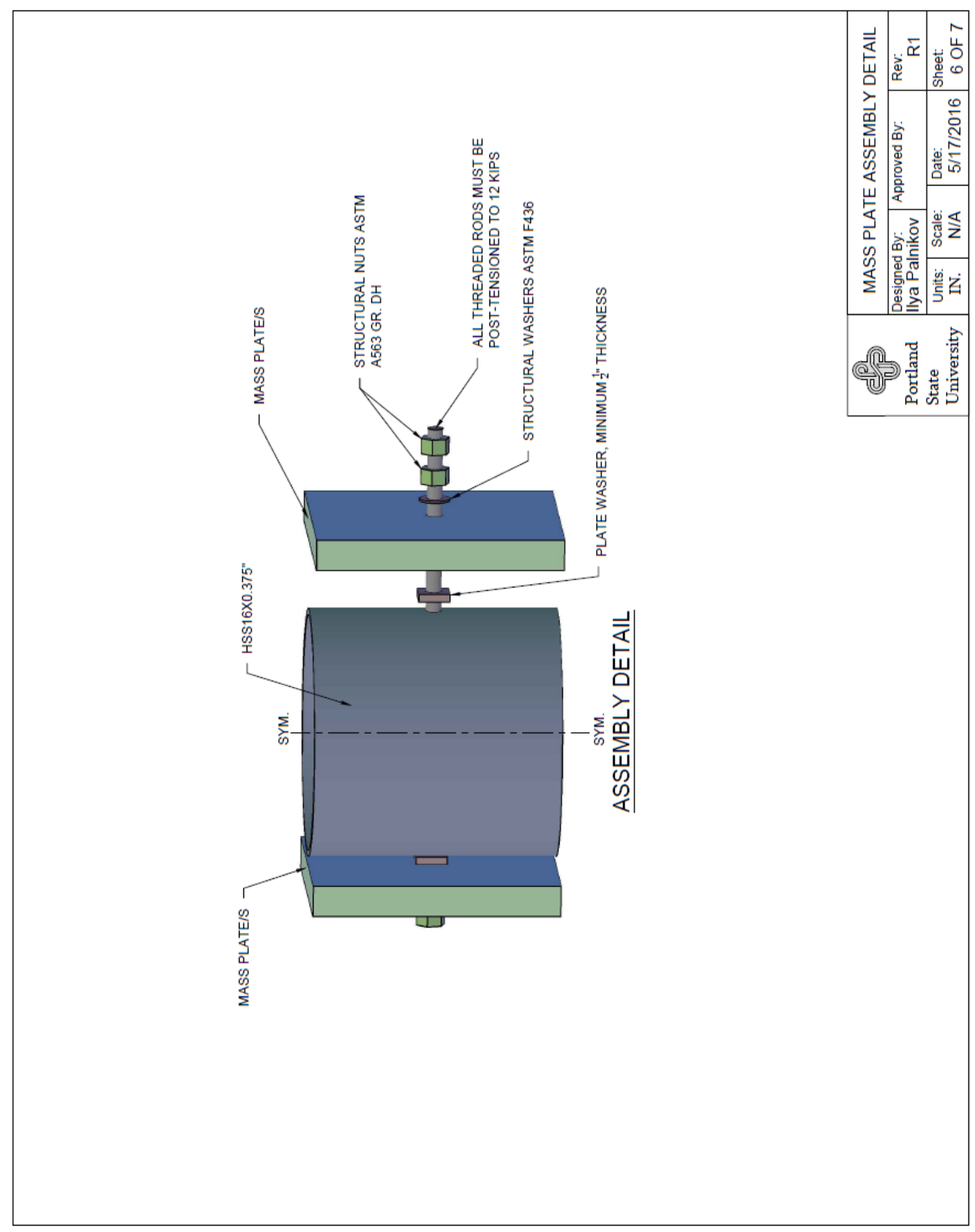




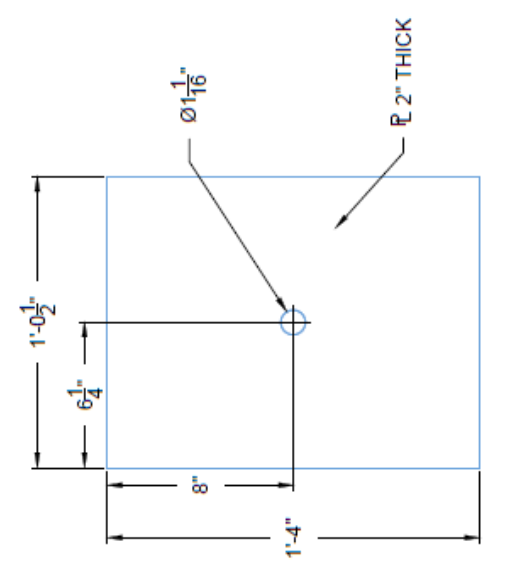

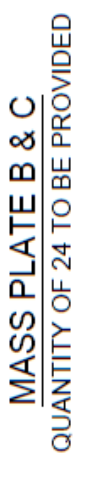
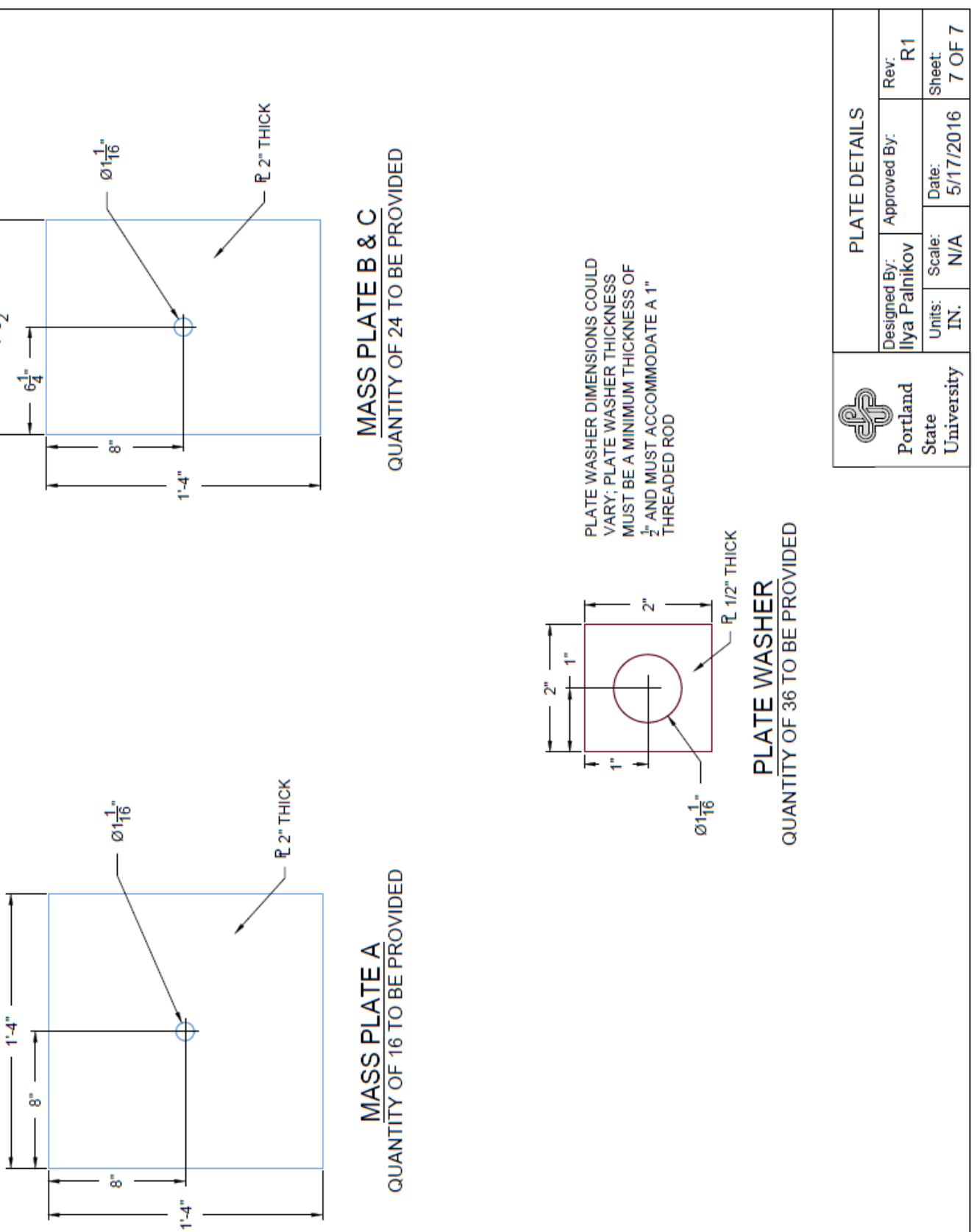

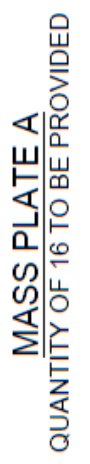




\subsection{APPENDIX G}

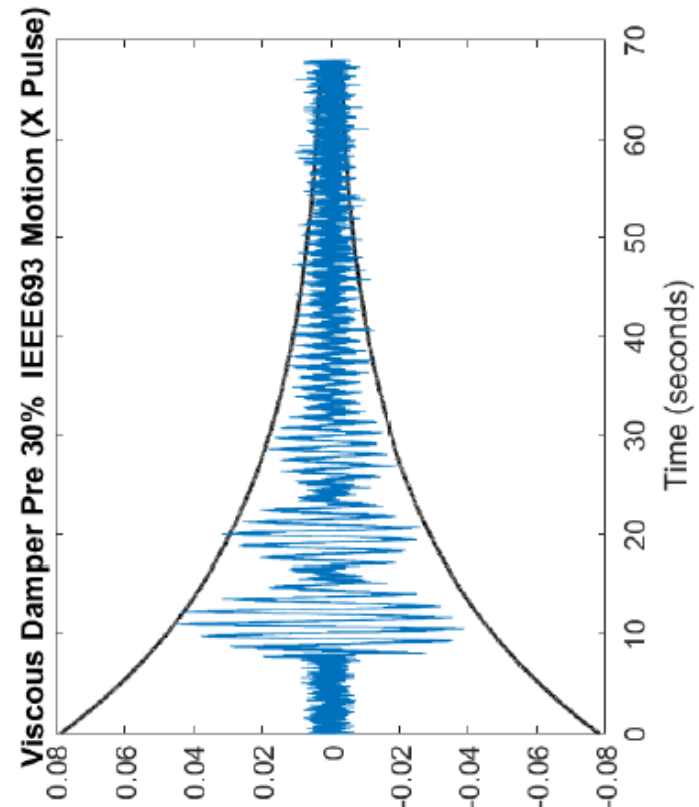

(6) $x$ do $\perp$ te uo!̣ผ

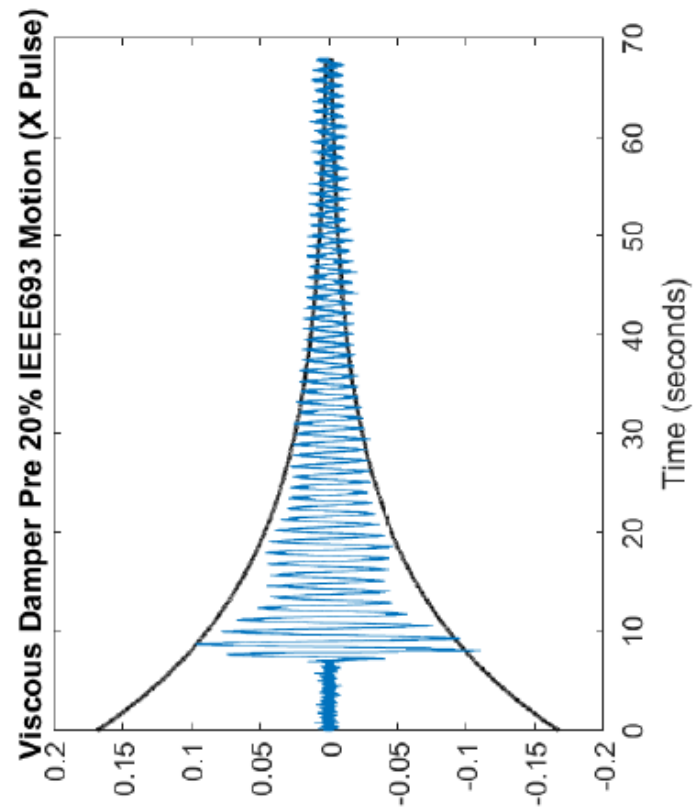

(6) $X$ do $\perp$ te uo!̣eมอวәว $\forall$

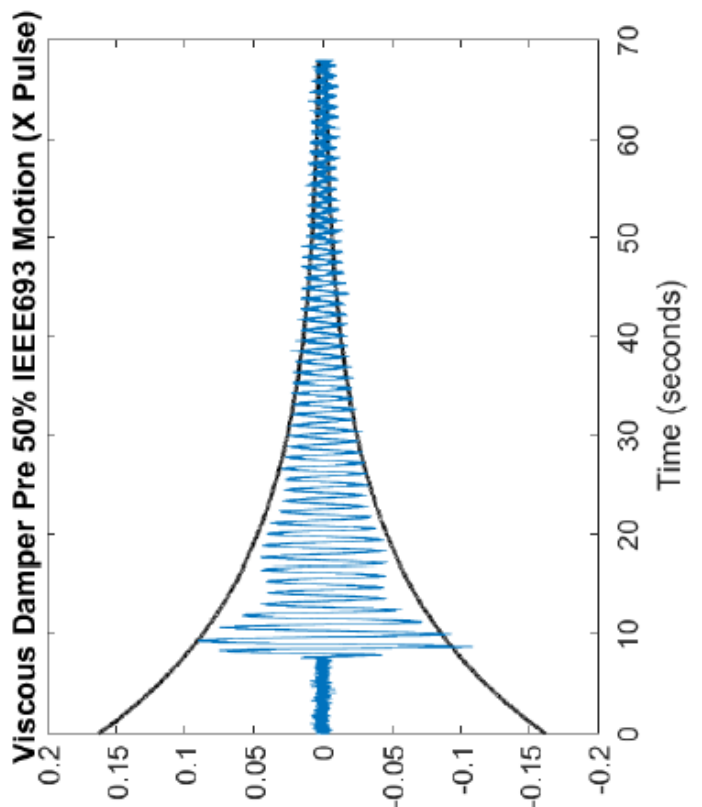

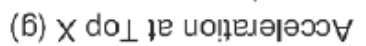

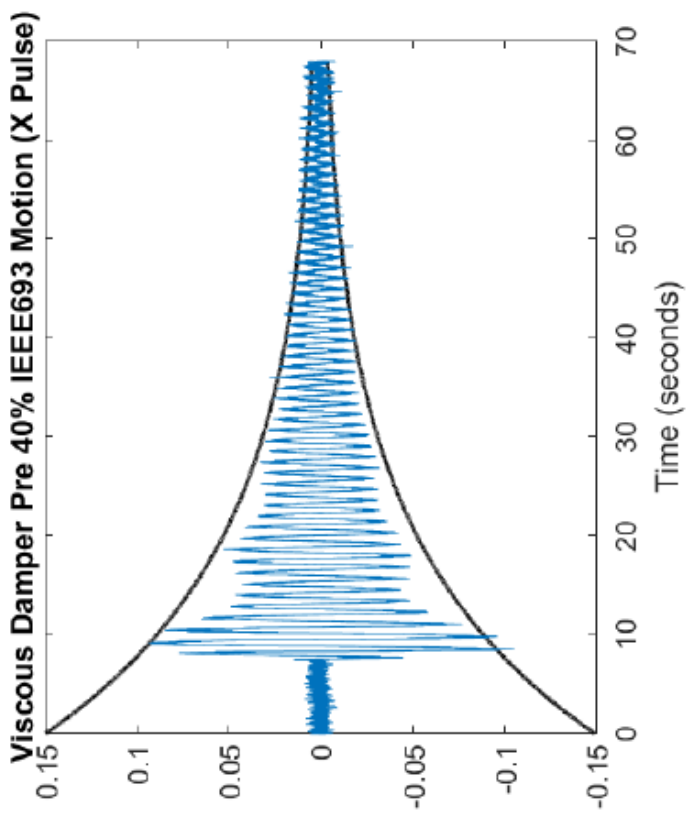

(6) $X$ do $\perp$ fe uo!๋̣อวәอง $\forall$

Figure 25-1: Viscous Damper Retrofit X-Damping 20-50\% 


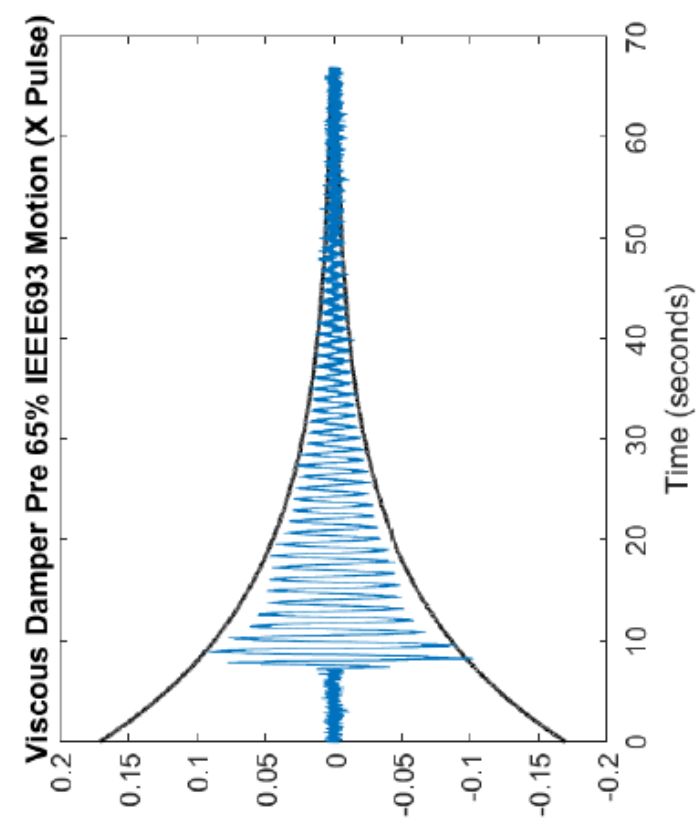

(6) $X$ do $\perp$ te uo!̣eมәอว $\forall$

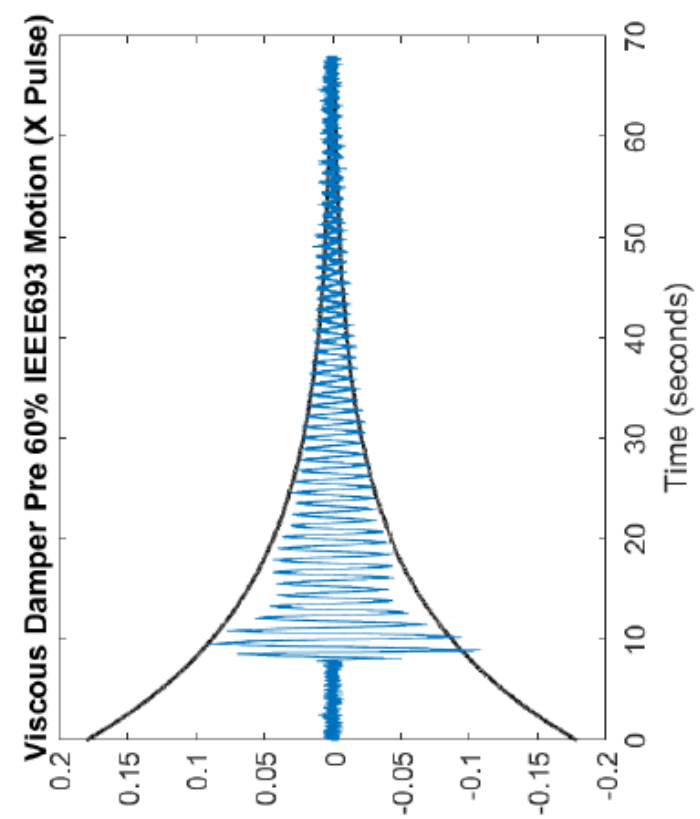

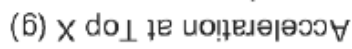

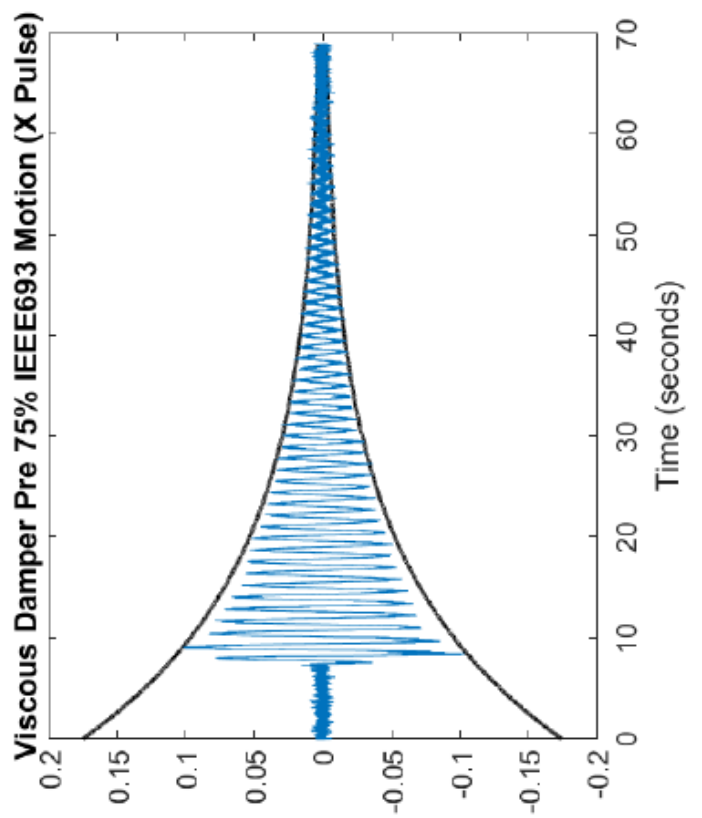

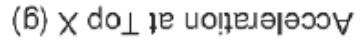

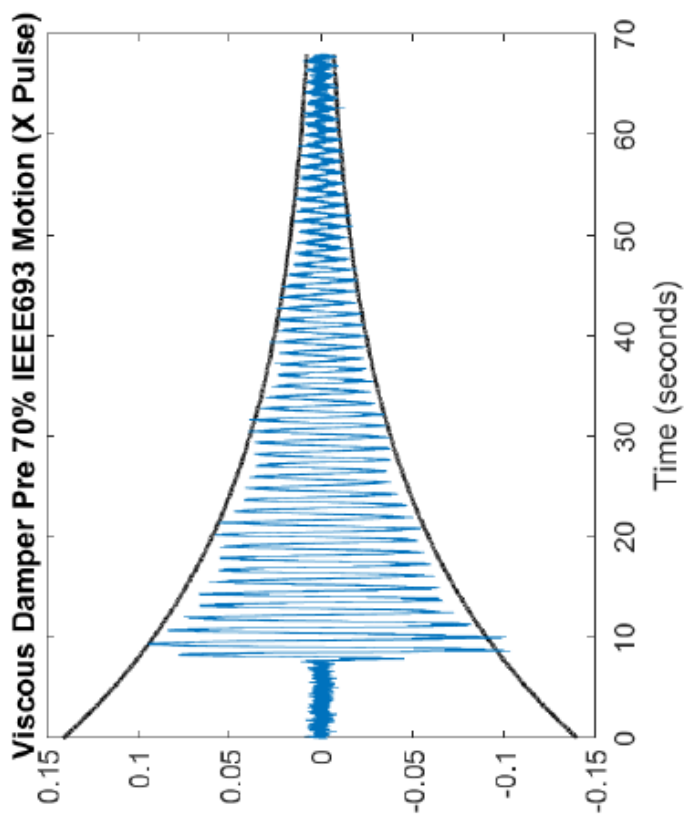

(6) $\mathrm{X}$ do $\perp$ Ie บоำมә|әэо

Figure 25-2: Viscous Damper Retrofit X-Damping 60-75\% 


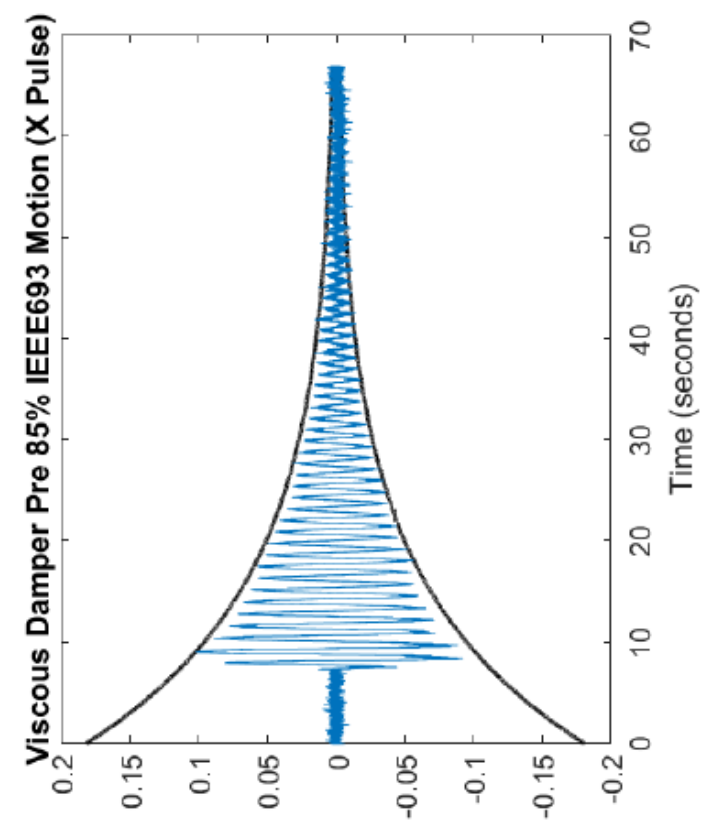

(6) $X$ do $\perp$ fe uo!̣eมə|ววง $\forall$

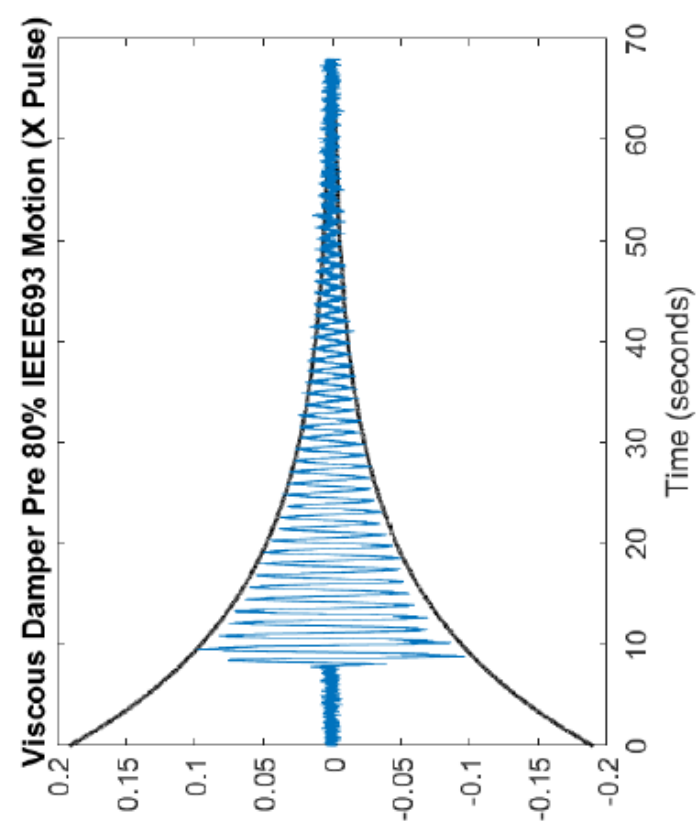

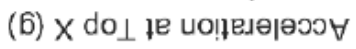

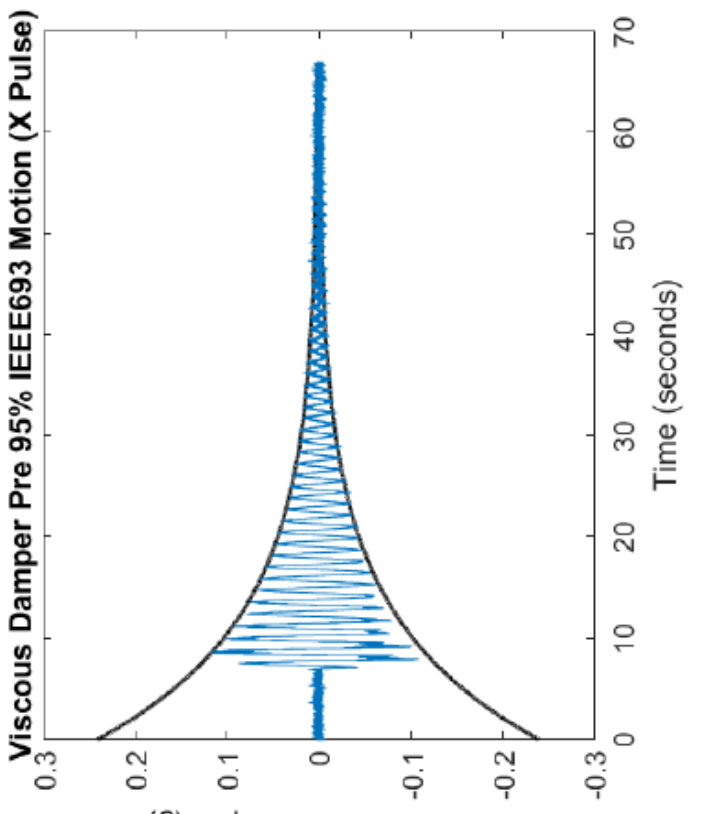

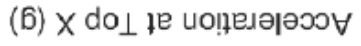

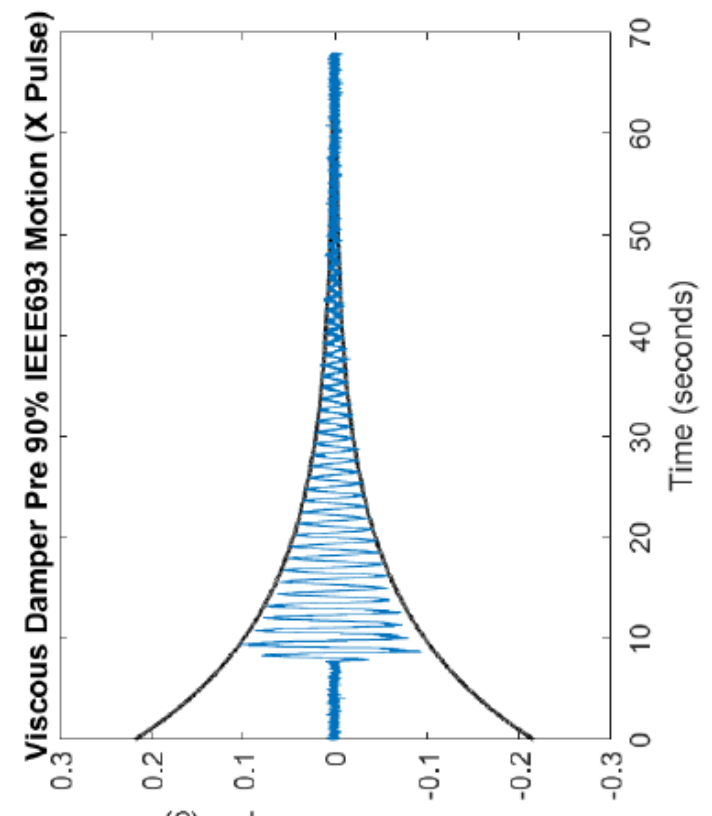

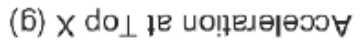

Figure 25-3: Viscous Damper Retrofit X-Damping 85-95\% 


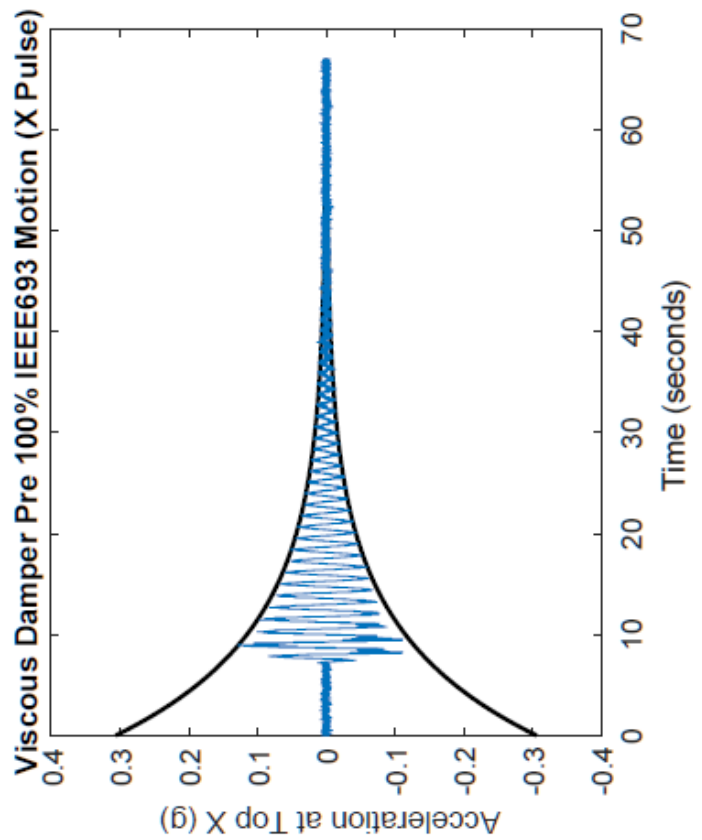

Figure 25-4: Viscous Damper Retrofit X-Damping 100\% 


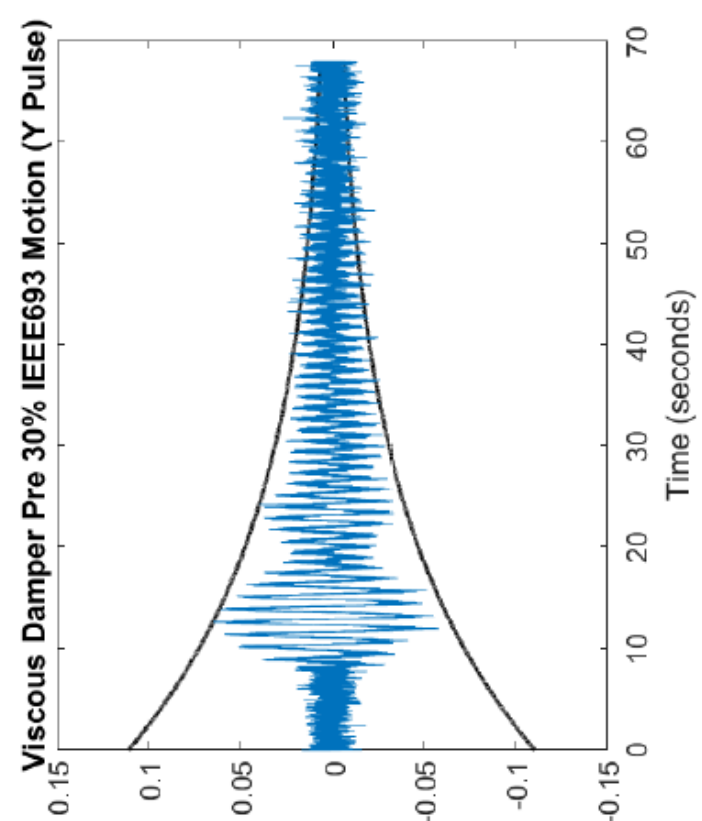

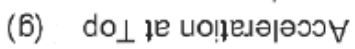

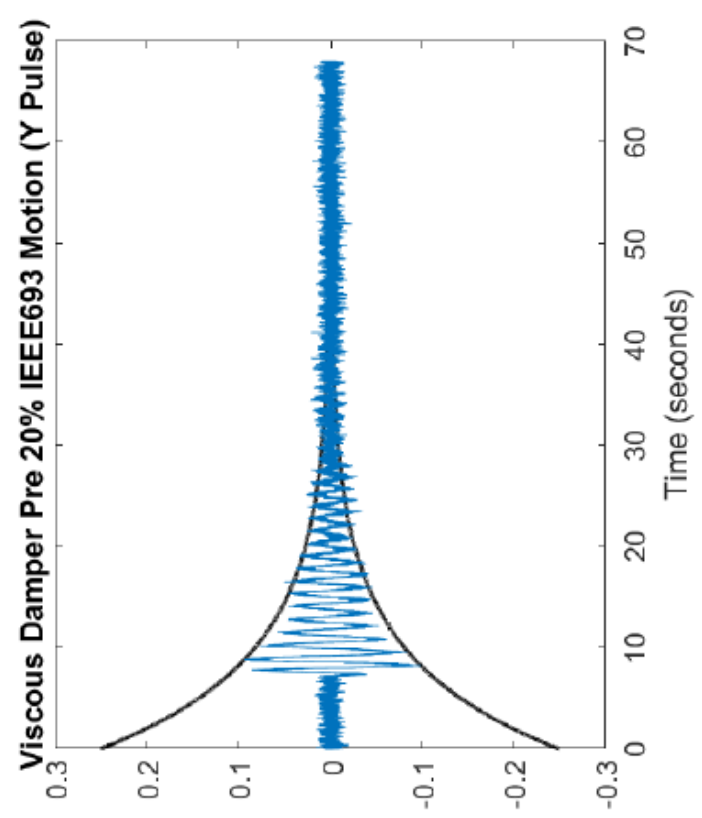

(6) do $\perp$ le บо!ฺะยө|өวว

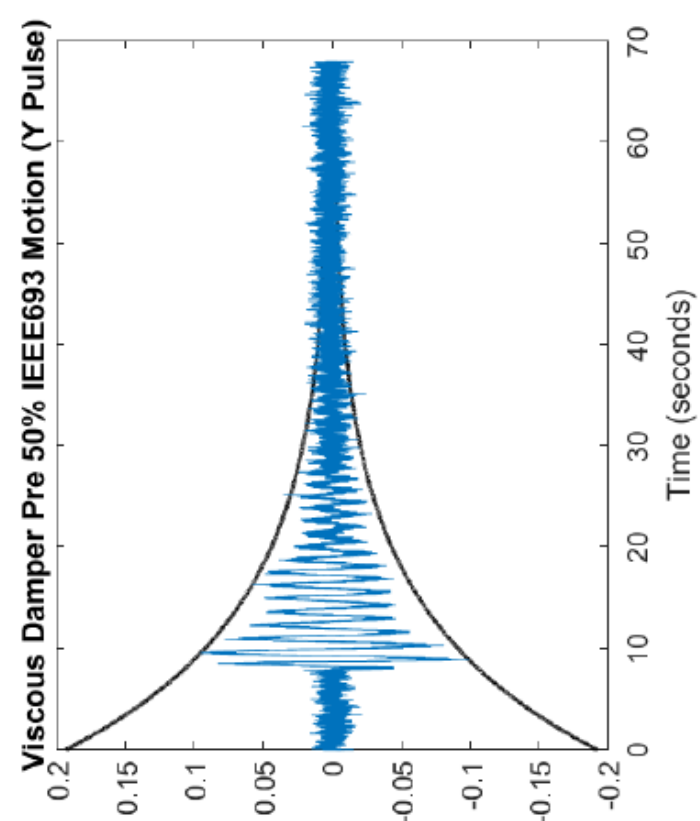

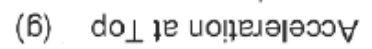

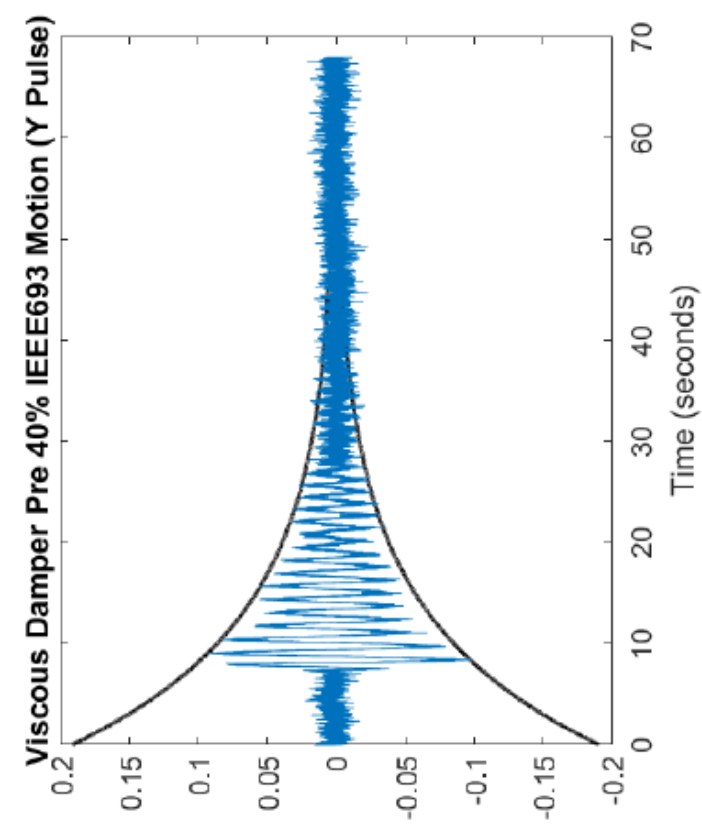

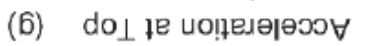

Figure 25-5: Viscous Damper Retrofit Y-Damping 20-50\% 


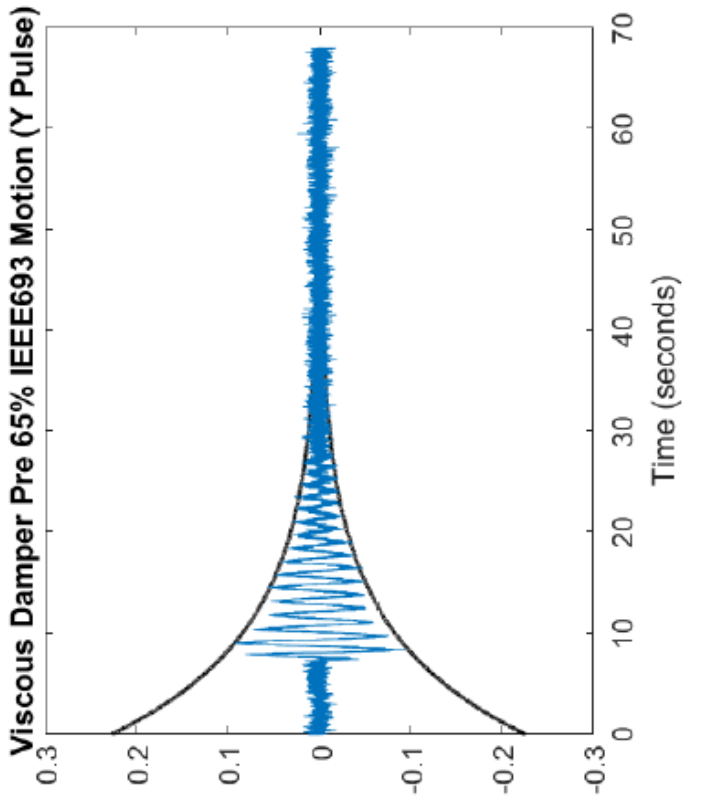

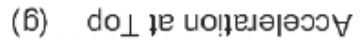

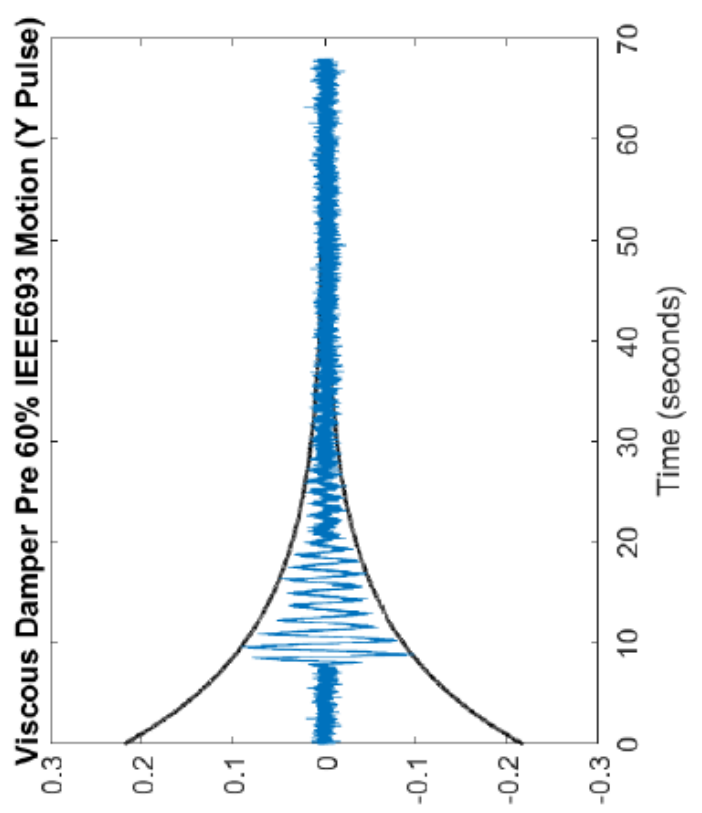

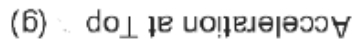

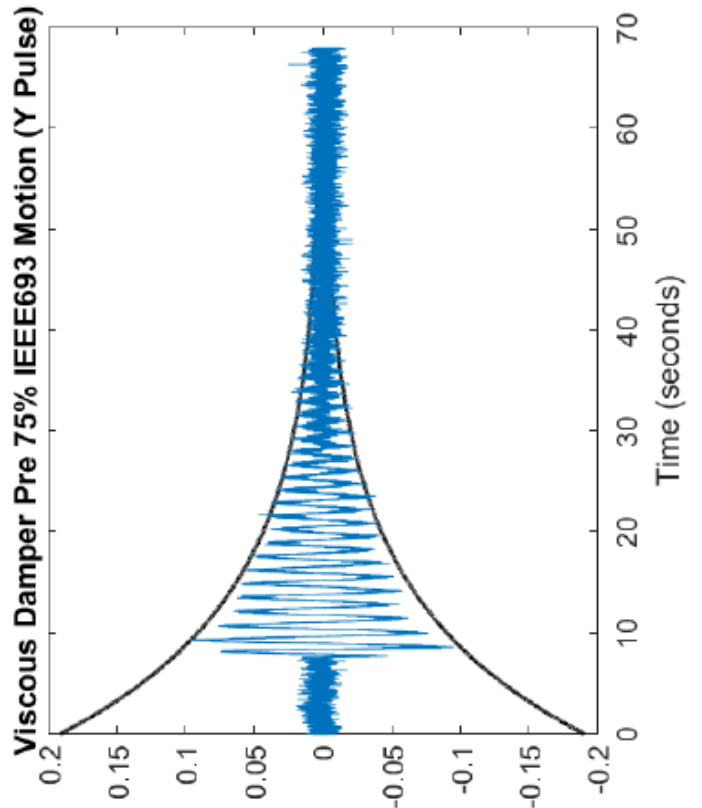

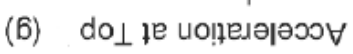

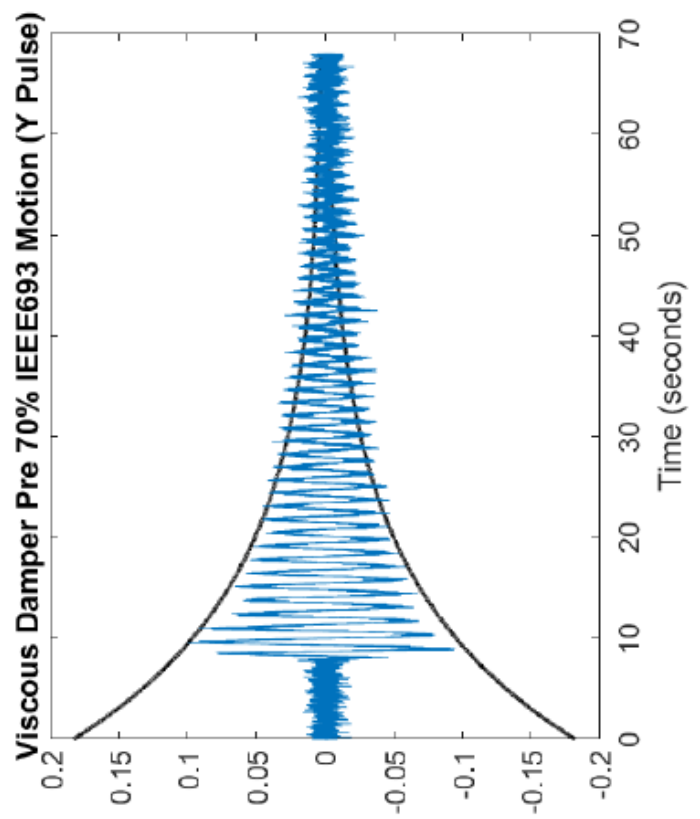

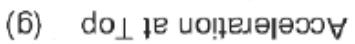

Figure 25-6: Viscous Damper Retrofit Y-Damping 60-75\% 


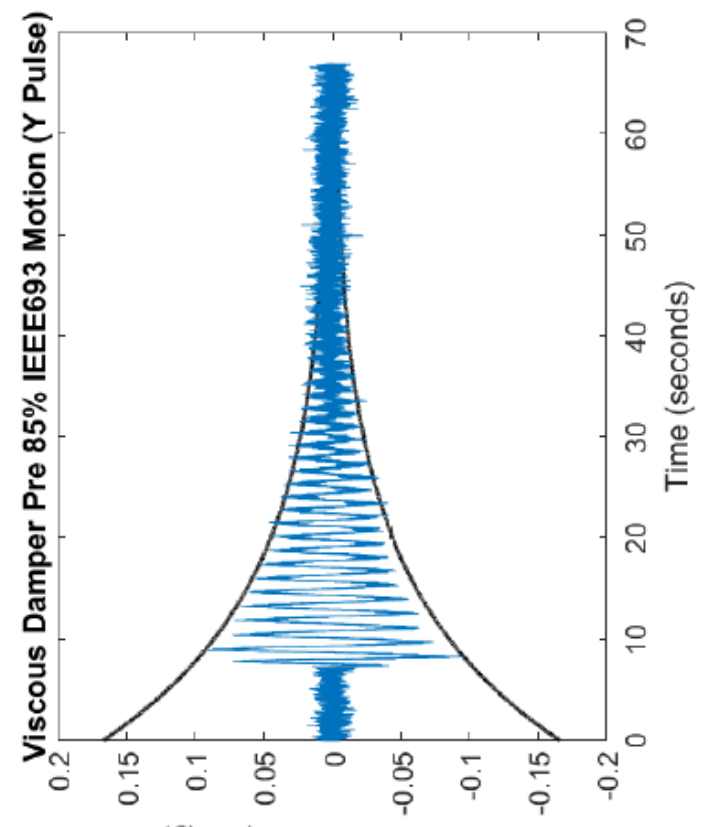

(6) do $\perp$ fe uo!̣eมə|әวง

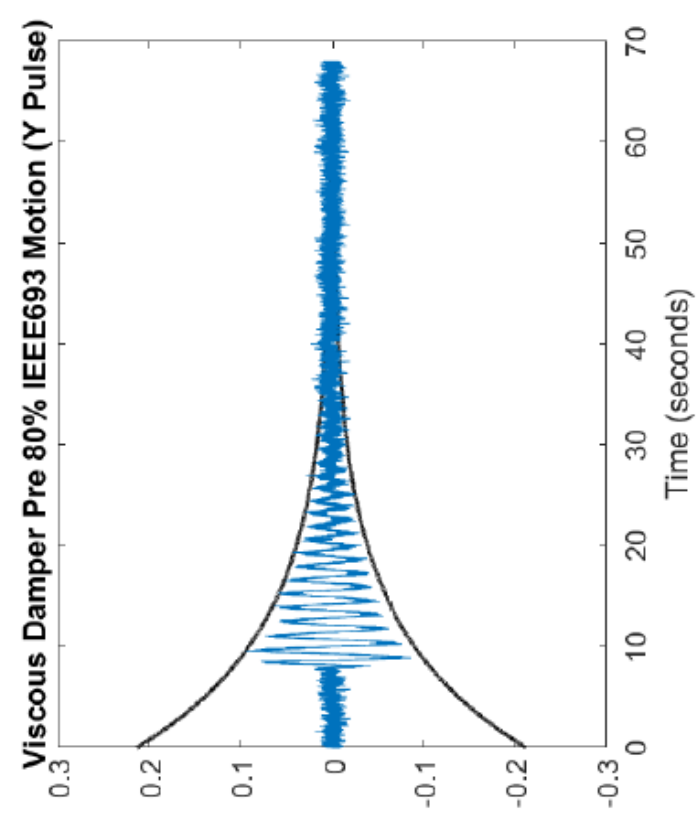

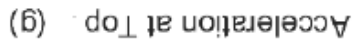

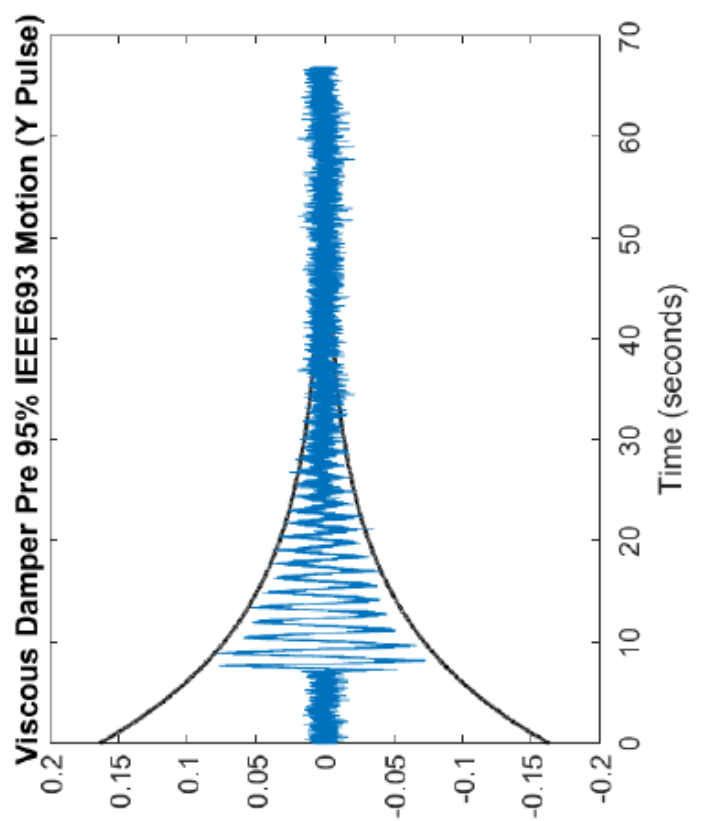

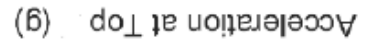

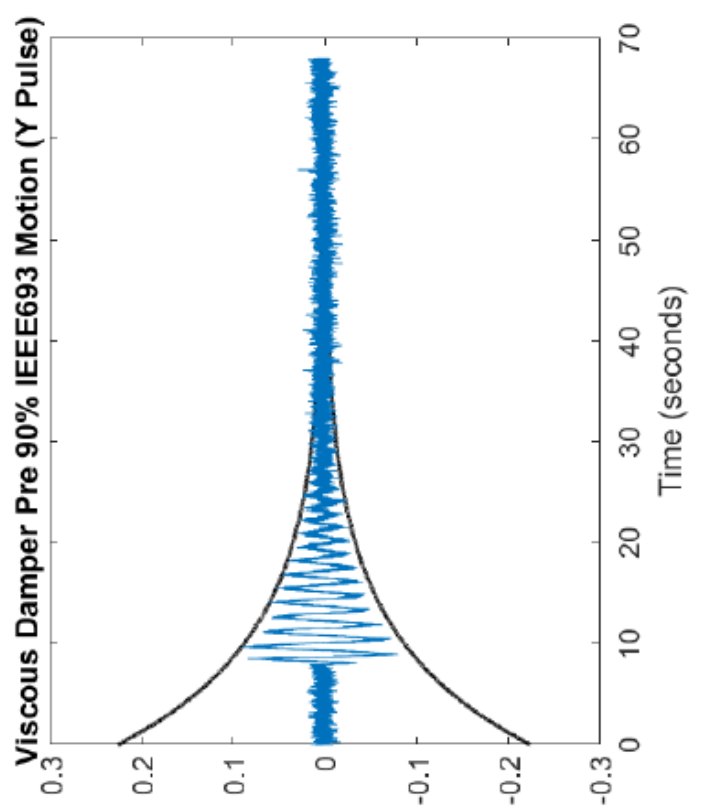

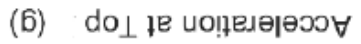

Figure 25-7: Viscous Damper Retrofit Y-Damping 80-95\% 


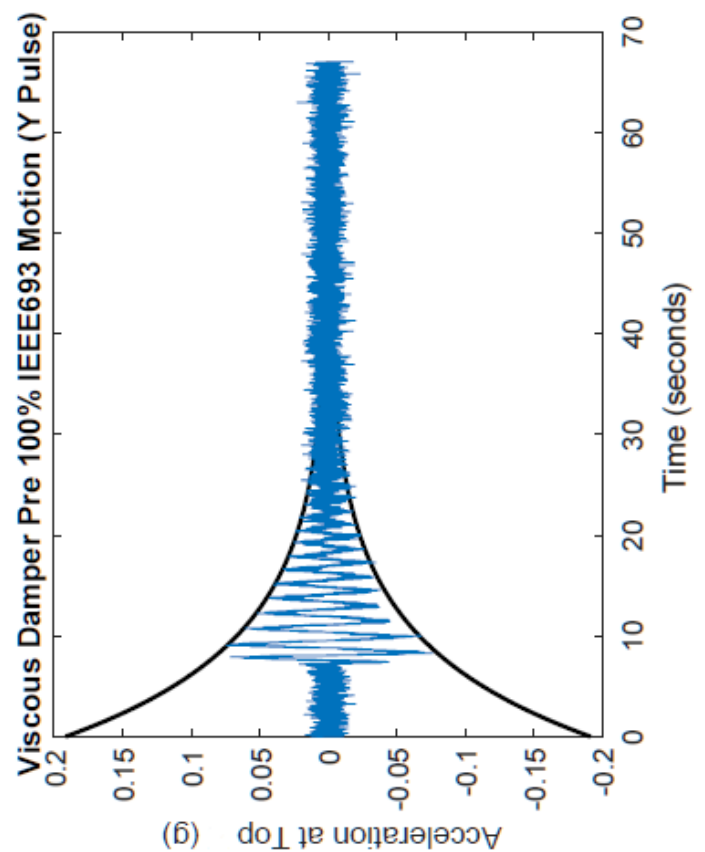

Figure 25-8: Viscous Damper Retrofit Y-Damping 100\% 


\subsection{APPENDIX H}

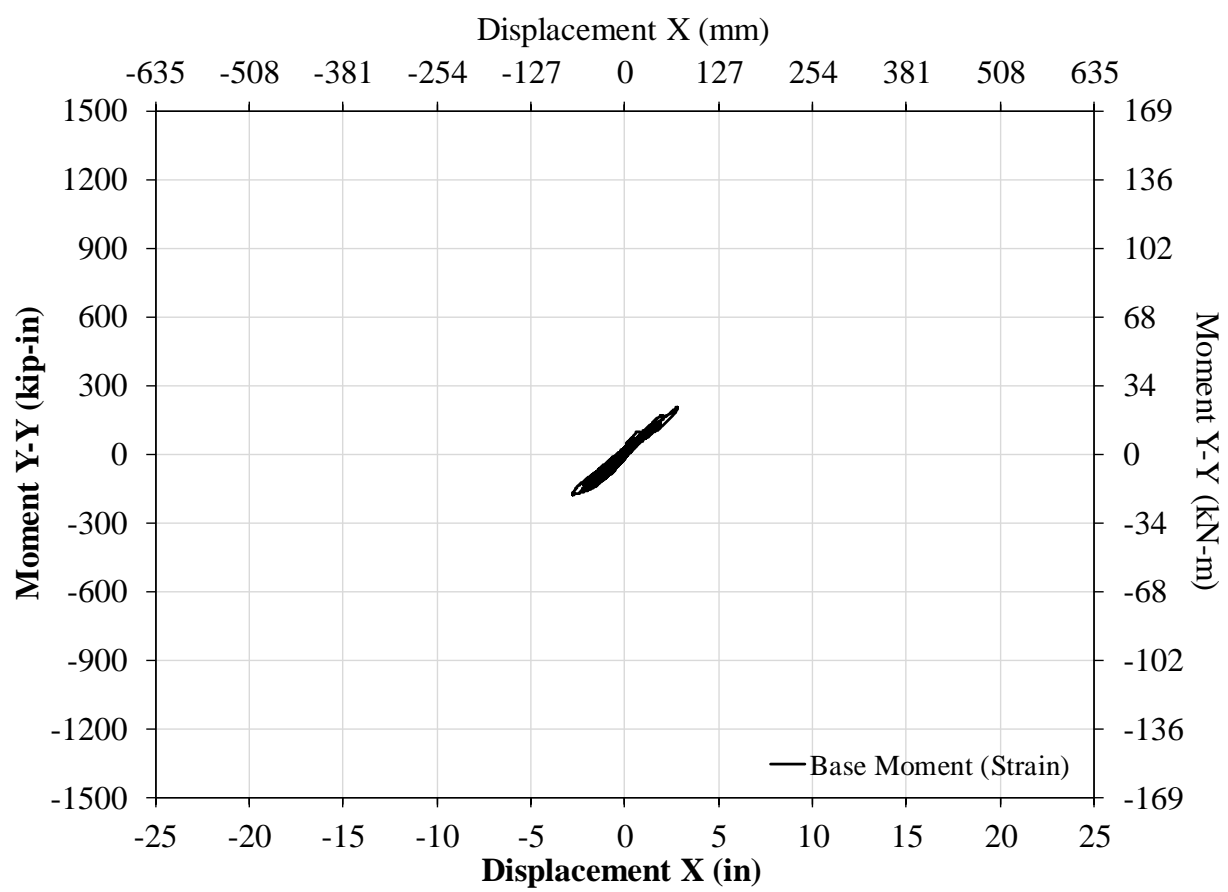

Figure 26-1: 20\% 0.5g IEEE693 X-System Response w/ Viscous Dampers

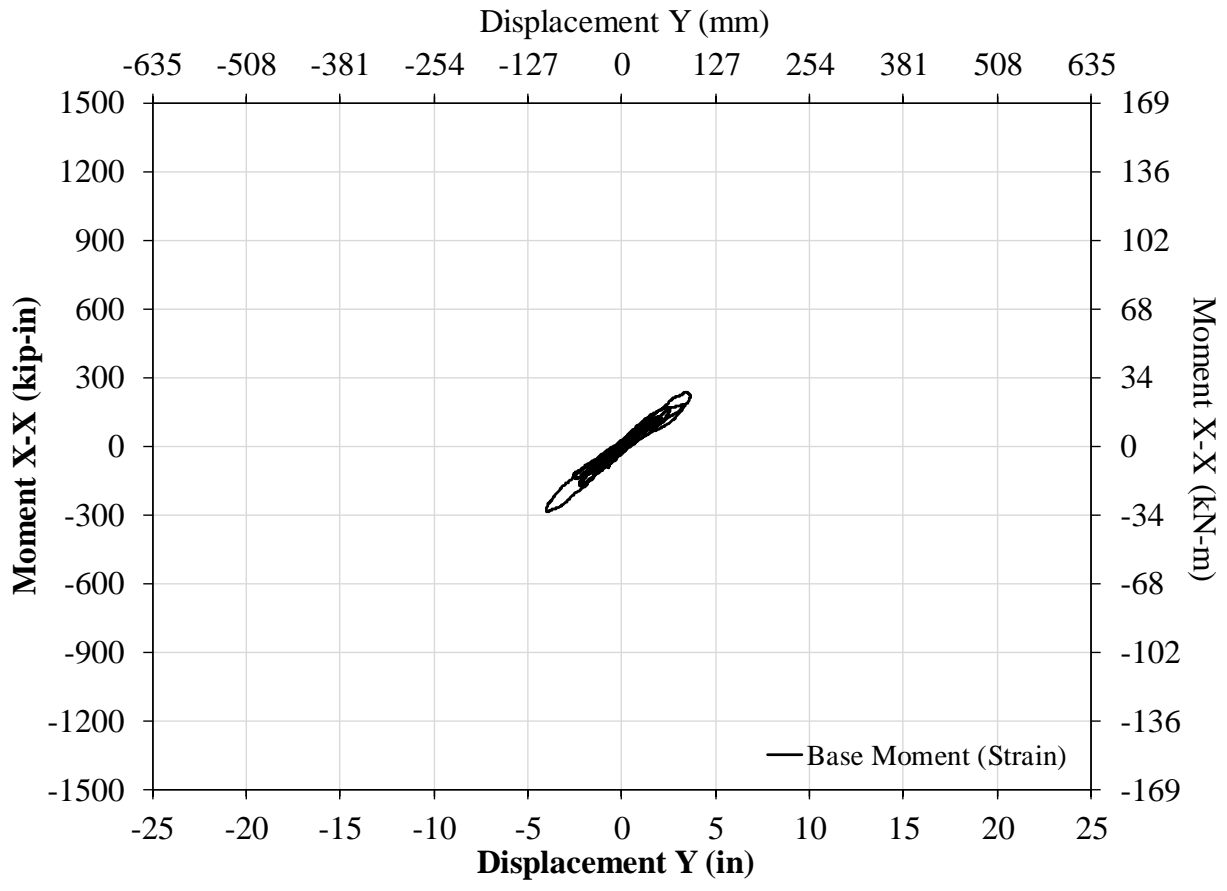

Figure 26-2: 20\% 0.5g IEEE693 Y-System Response w/ Viscous Dampers 


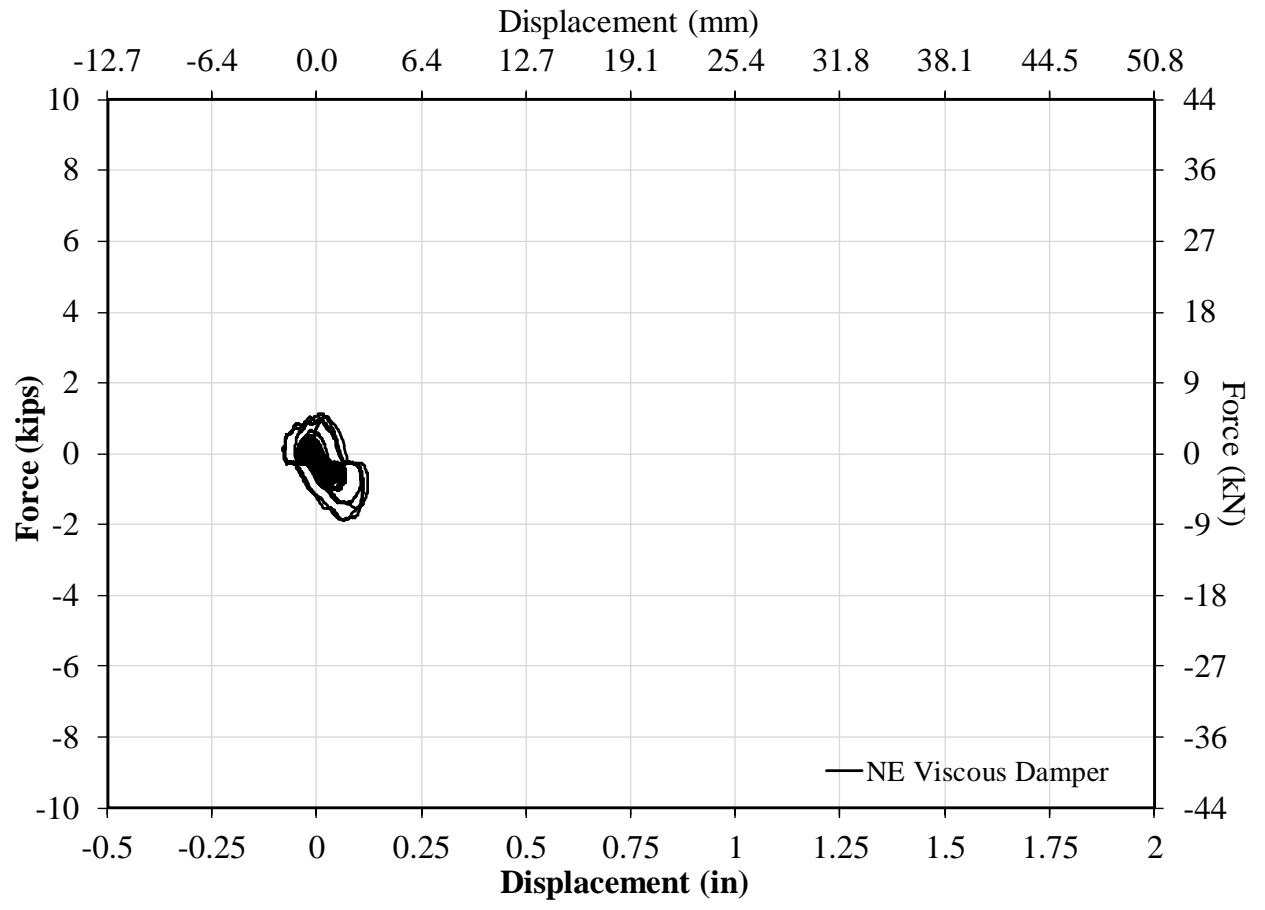

Figure 26-3: 20\% 0.5g IEEE693 NE Viscous Damper Response

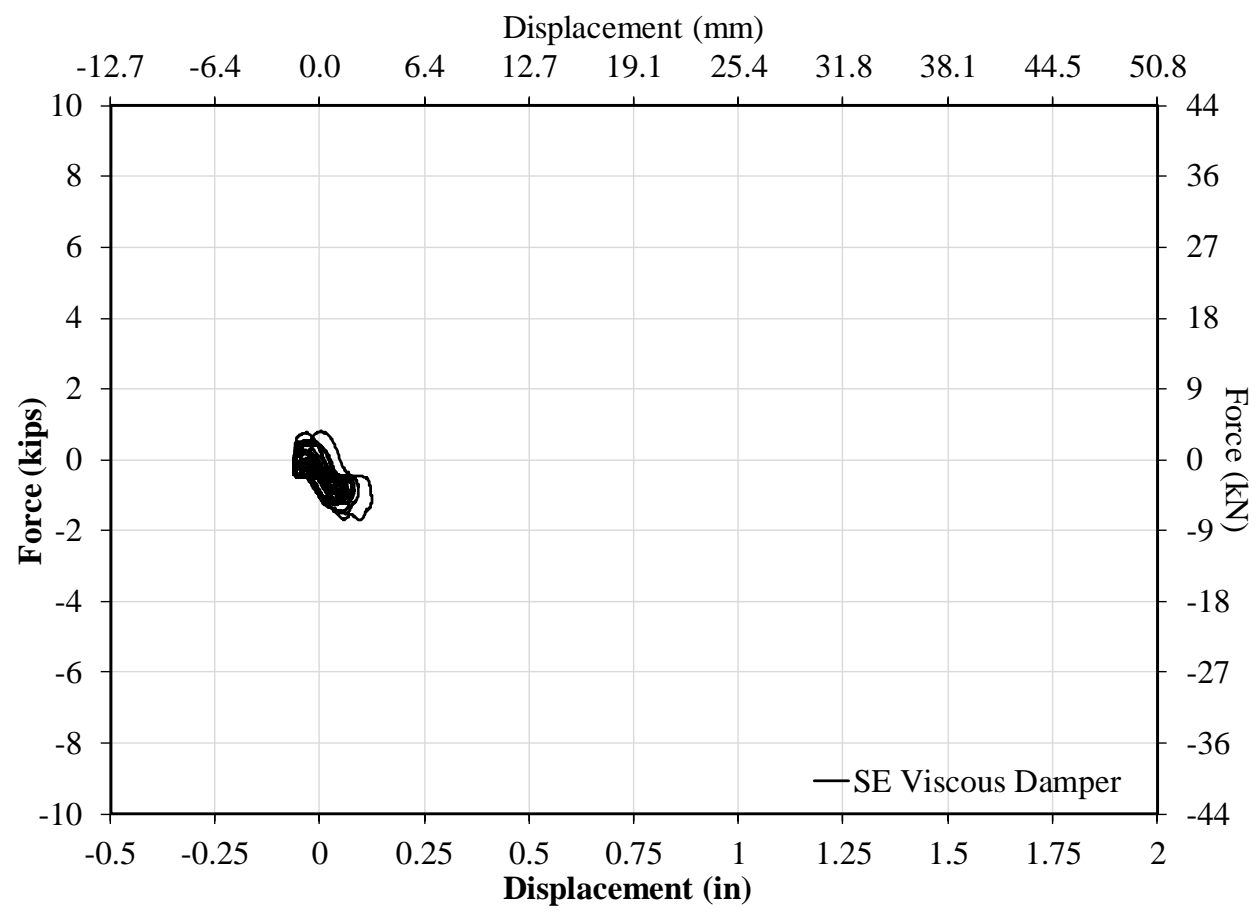

Figure 26-4: 20\% 0.5g IEEE693 SE Viscous Damper Response 


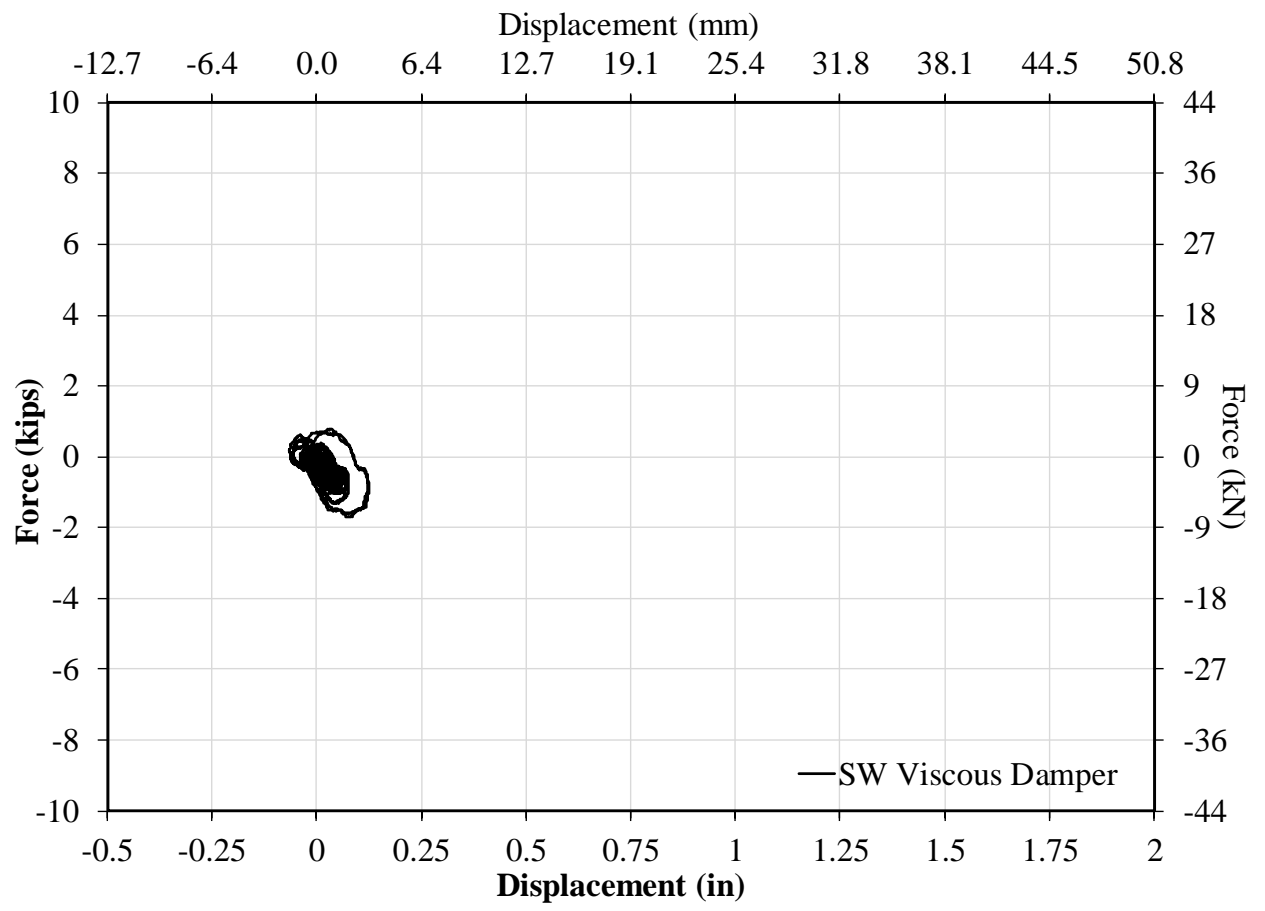

Figure 26-5: 20\% 0.5g IEEE693 SW Viscous Damper Response

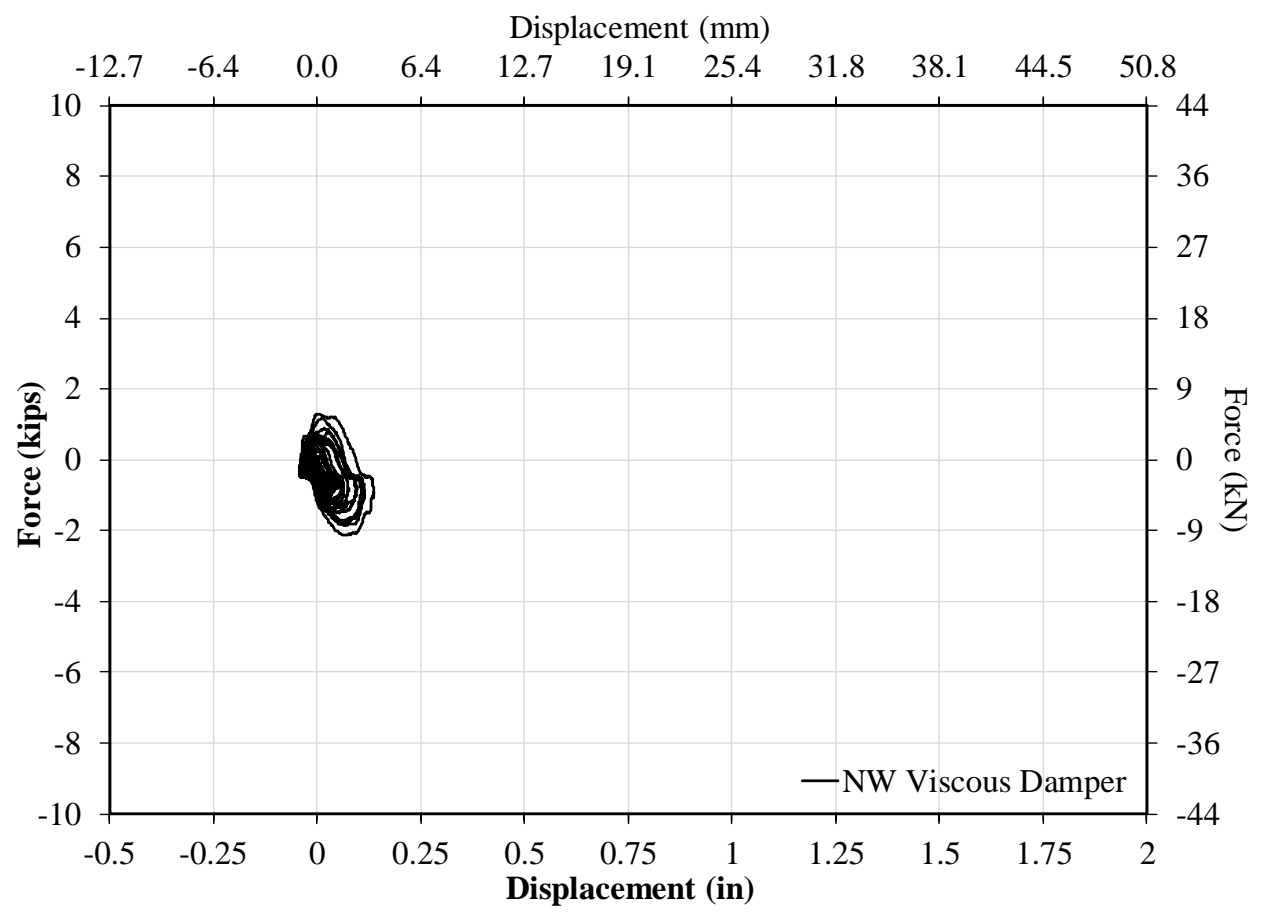

Figure 26-6: 20\% 0.5g IEEE693 NW Viscous Damper Response 


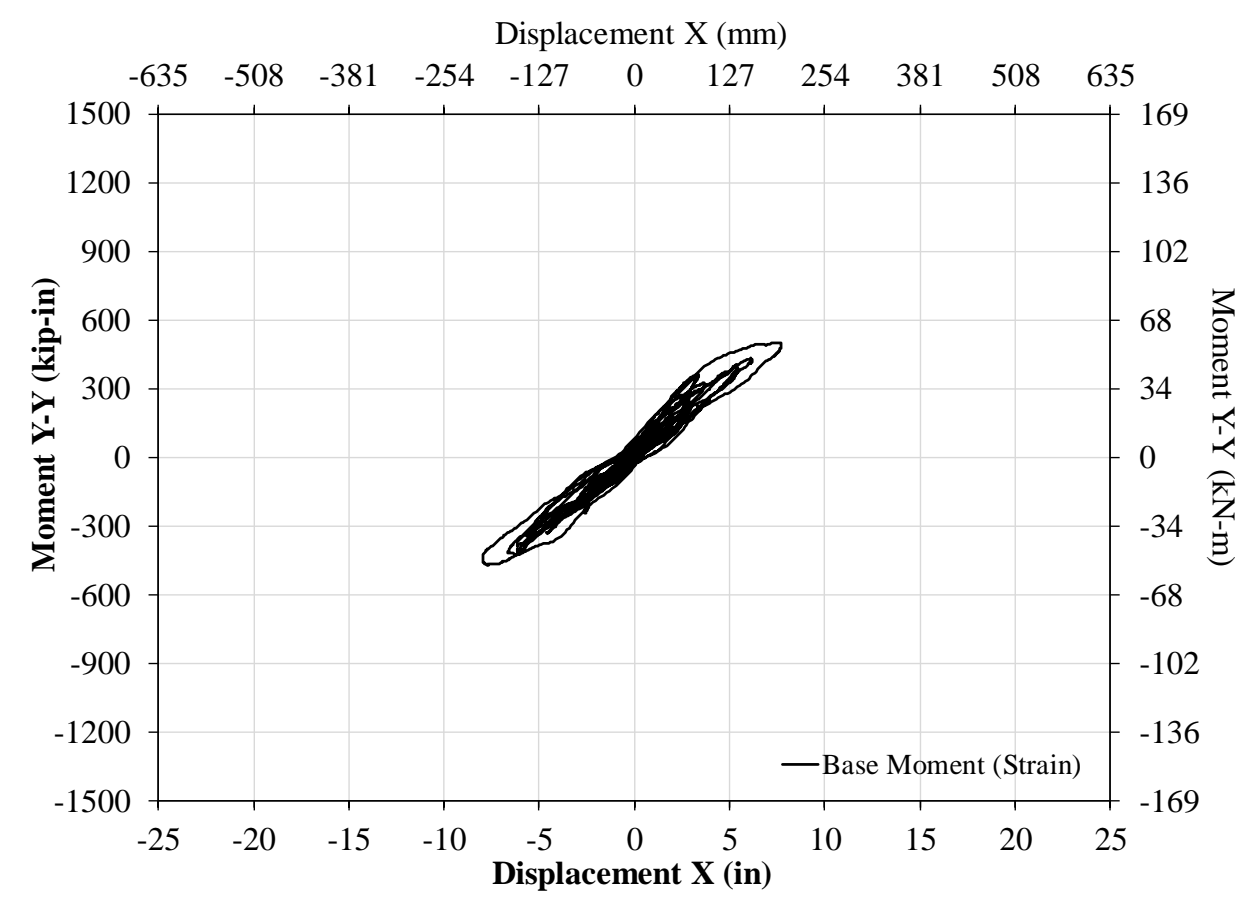

Figure 26-7: 50\% 0.5g IEEE693 X-System Response w/ Viscous Devices

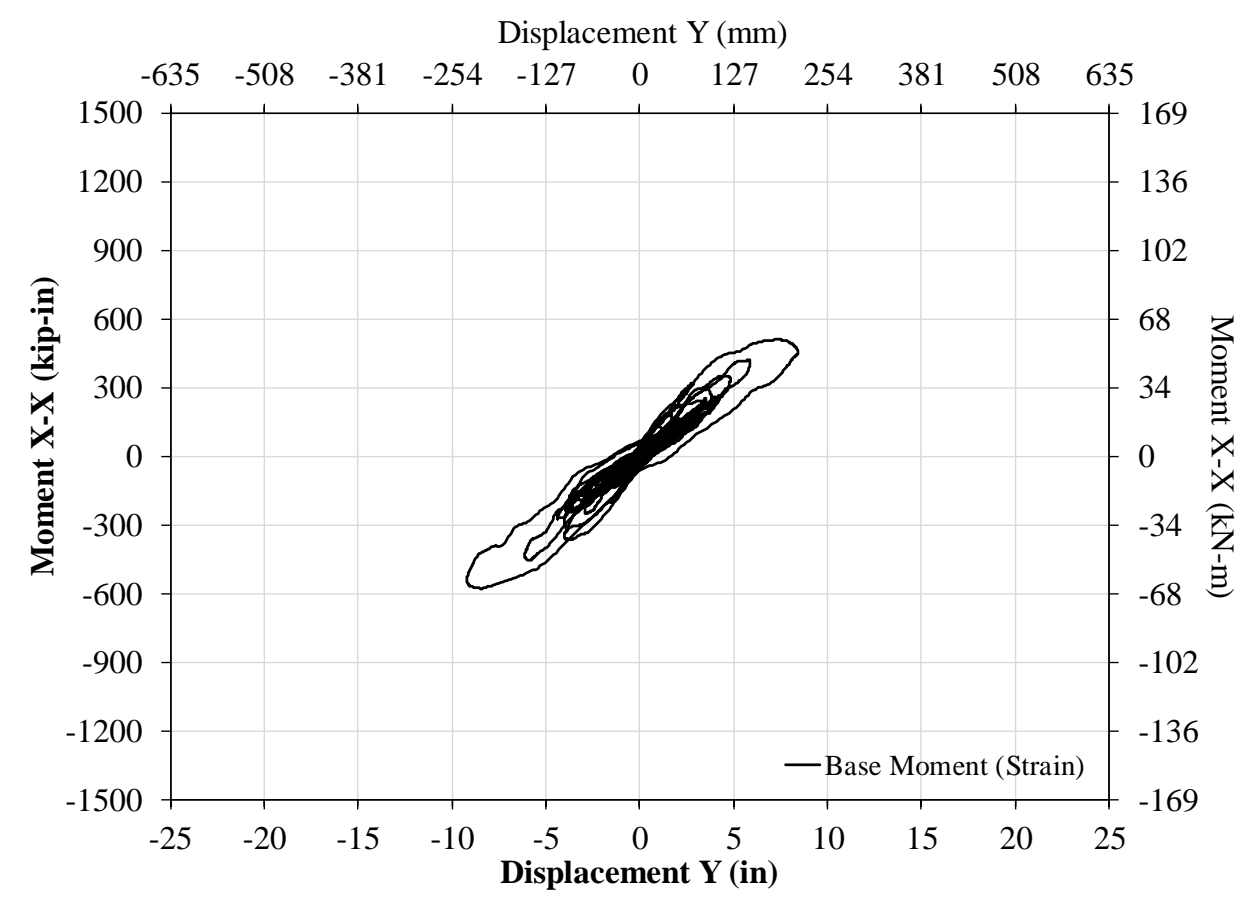

Figure 26-8: 50\% 0.5g IEEE693 Y-System Response w/ Viscous Devices 


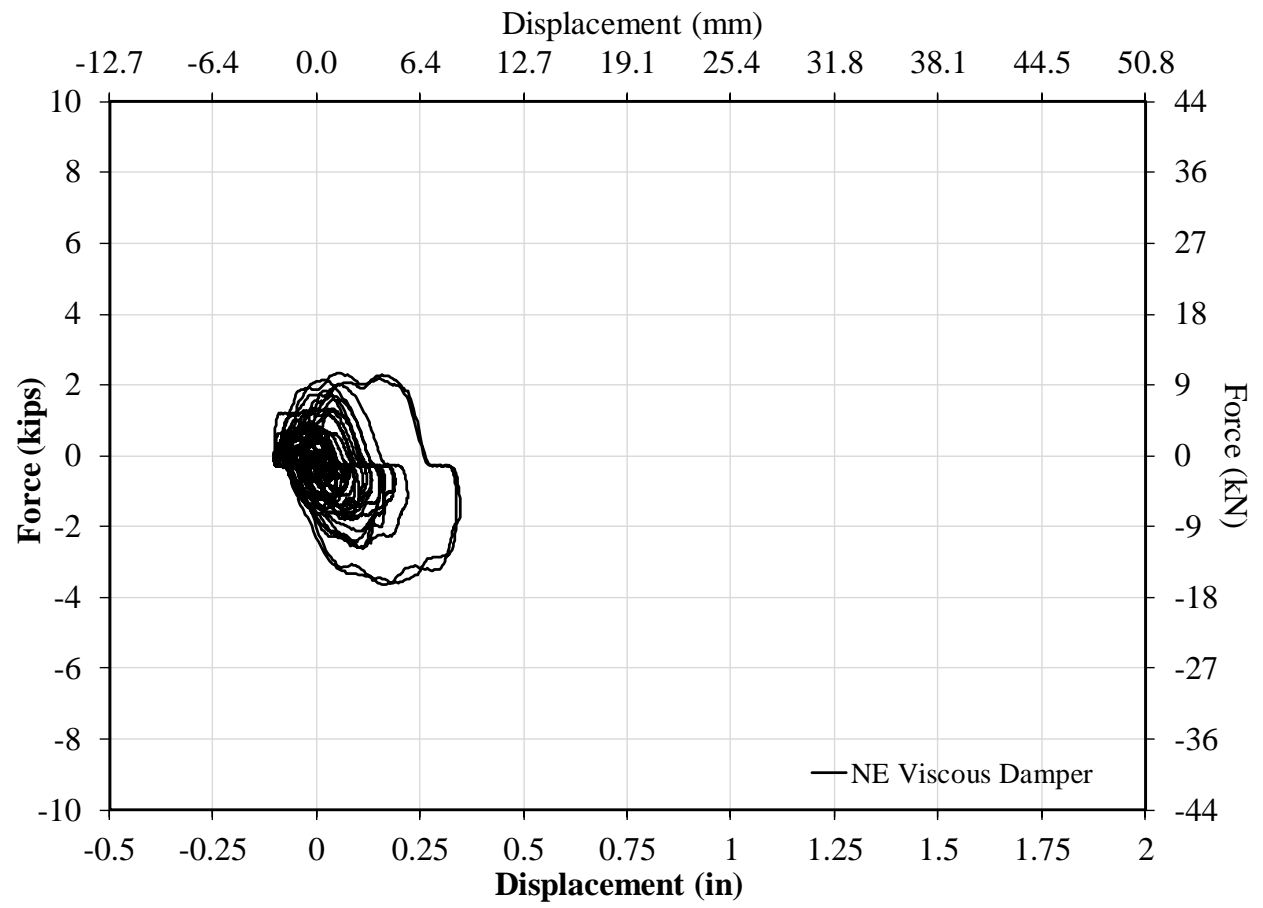

Figure 26-9: 50\% 0.5g IEEE693 NE Viscous Damper Response

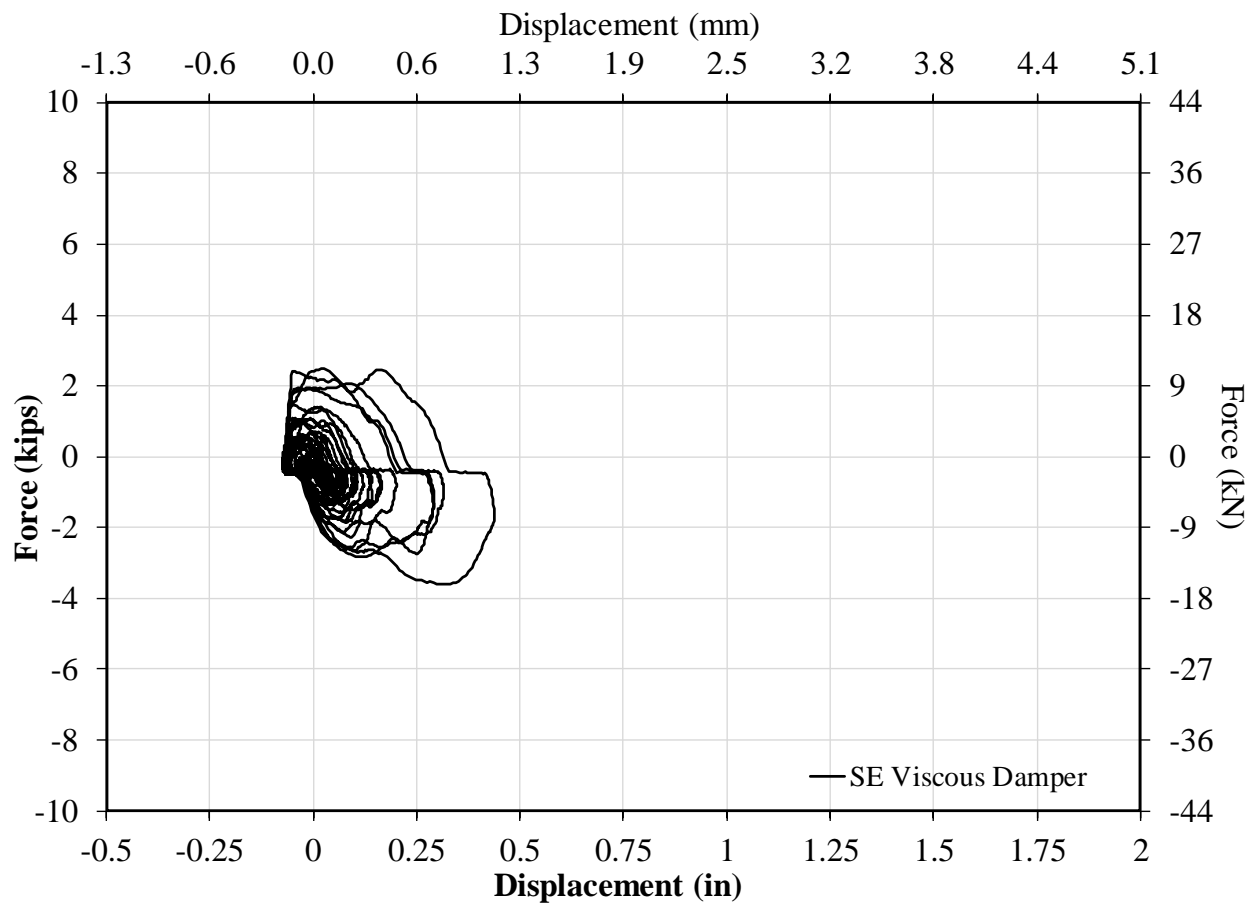

Figure 26-10: 50\% 0.5g IEEE693 SE Viscous Damper Response 


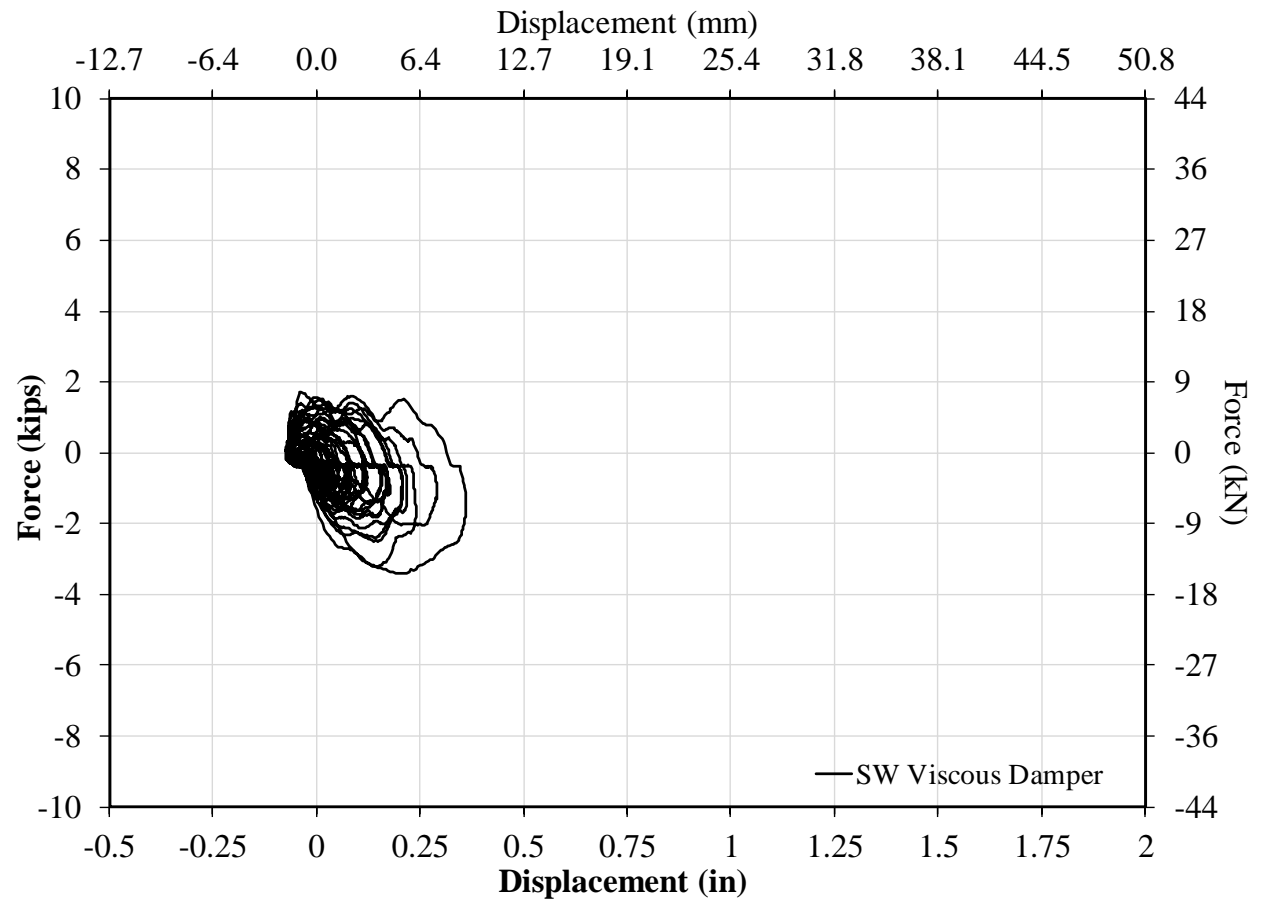

Figure 26-11: 50\% 0.5g IEEE693 SW Viscous Damper Response

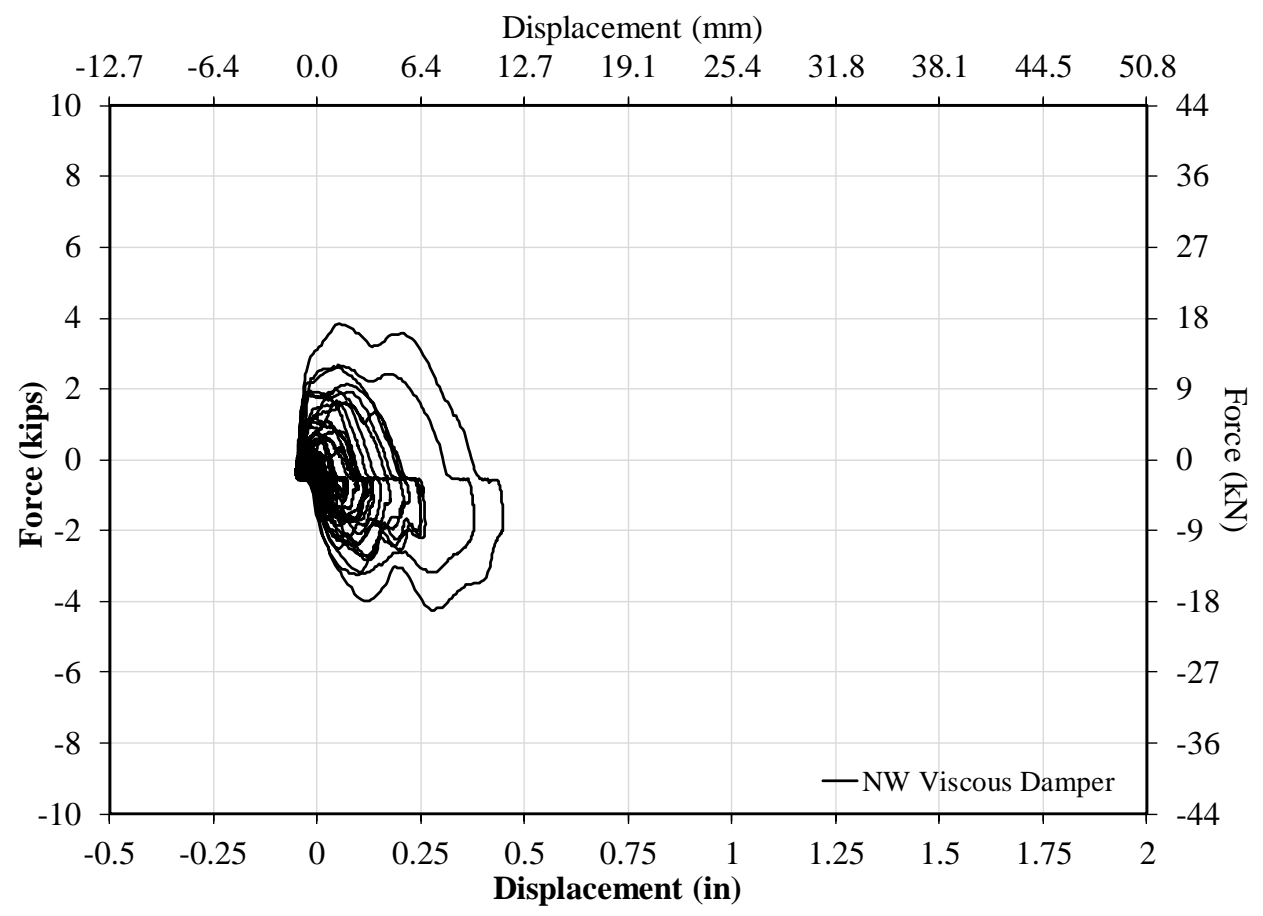

Figure 26-12: 50\% 0.5g IEEE693 NW Viscous Damper Response 


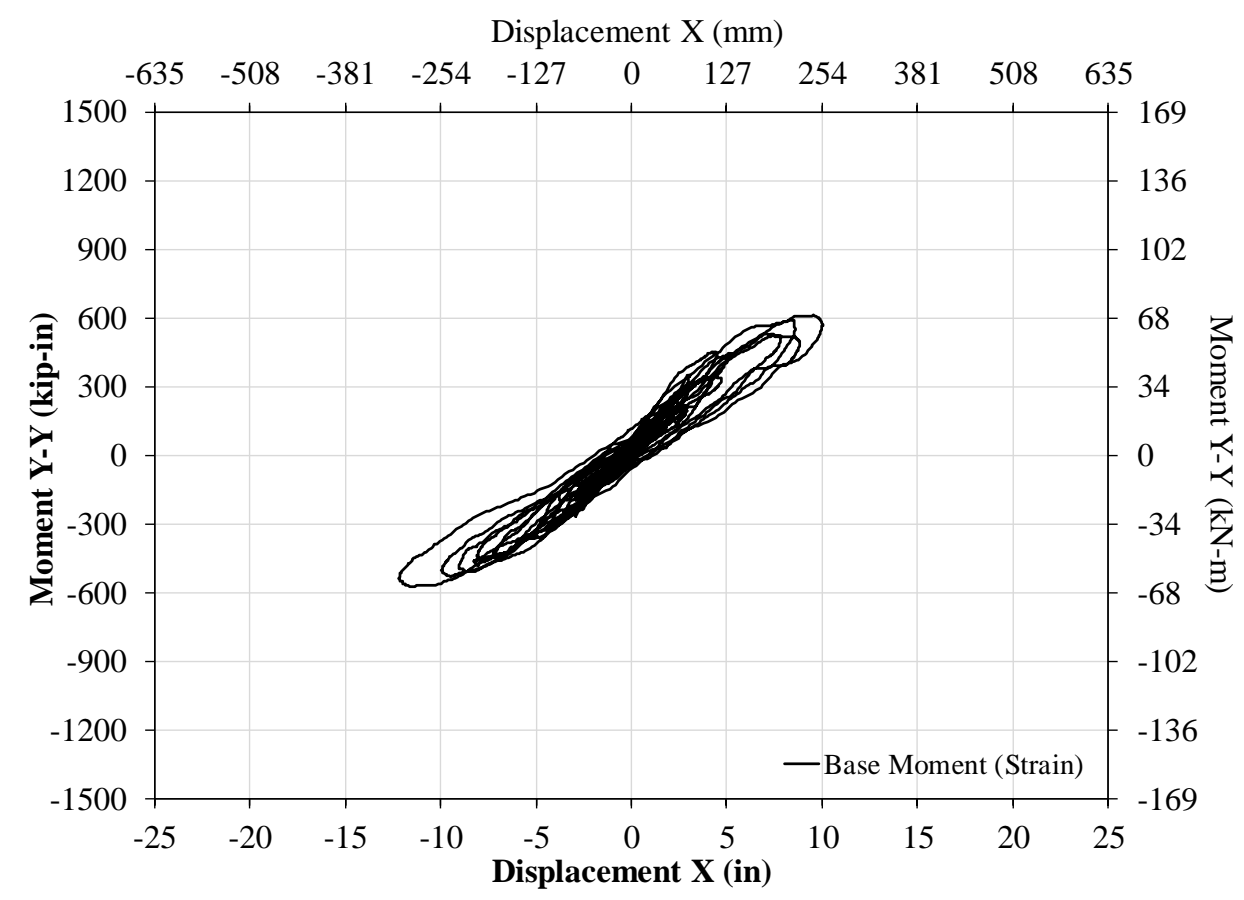

Figure 26-13: 75\% 0.5g IEEE693 X-System Response w/ Viscous Devices

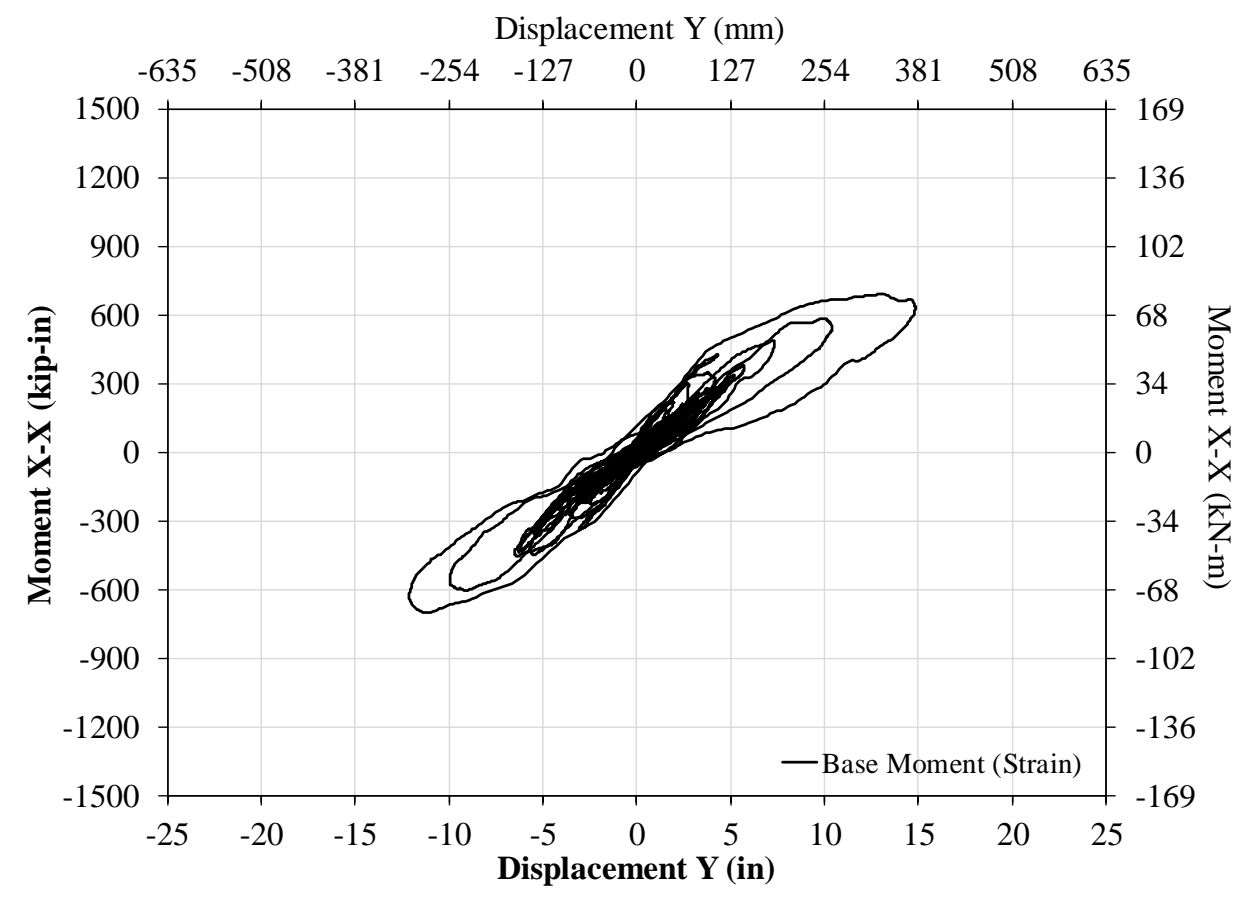

Figure 26-14: 75\% 0.5g IEEE693 Y-System Response w/ Viscous Devices 


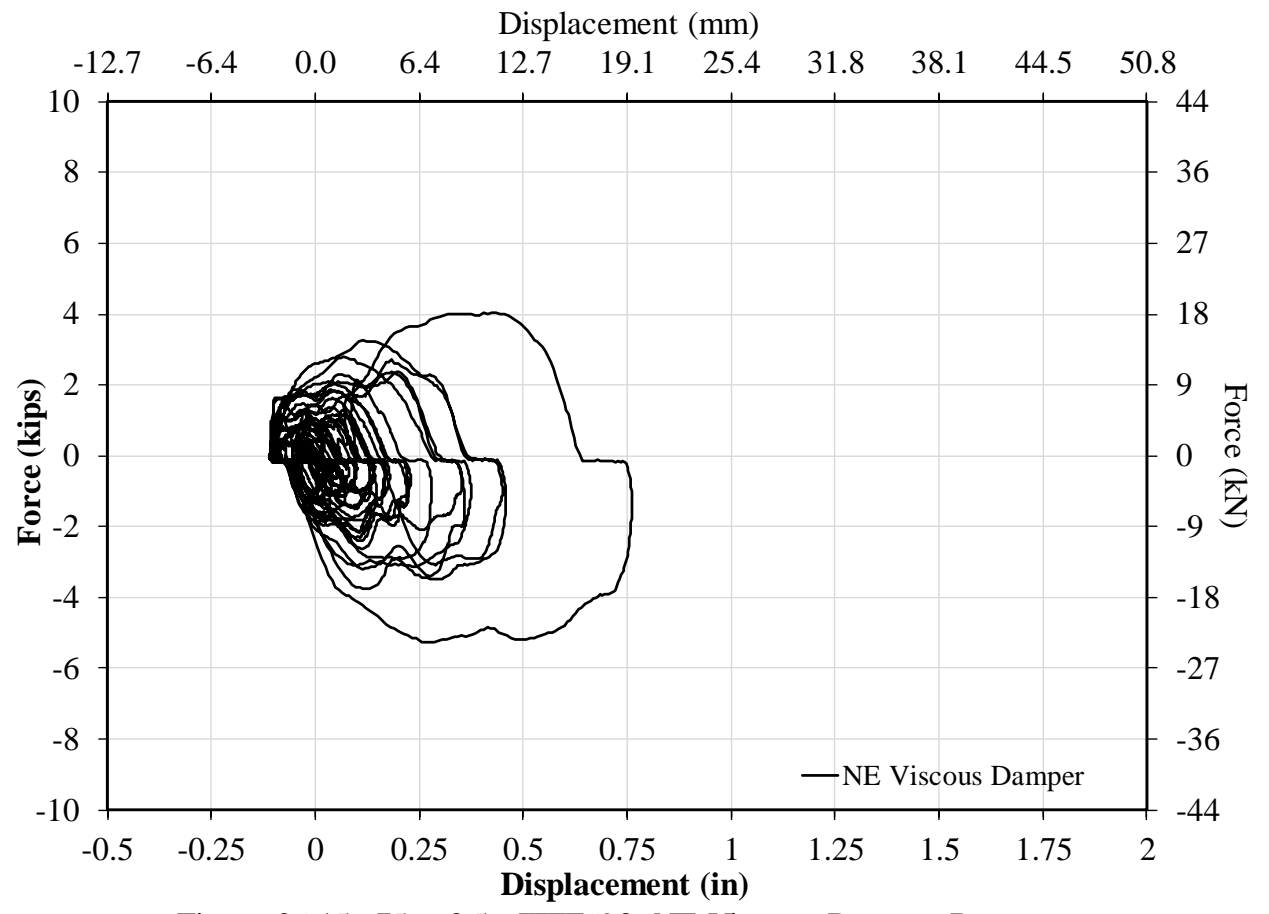

Figure 26-15: 75\% 0.5g IEEE693 NE Viscous Damper Response

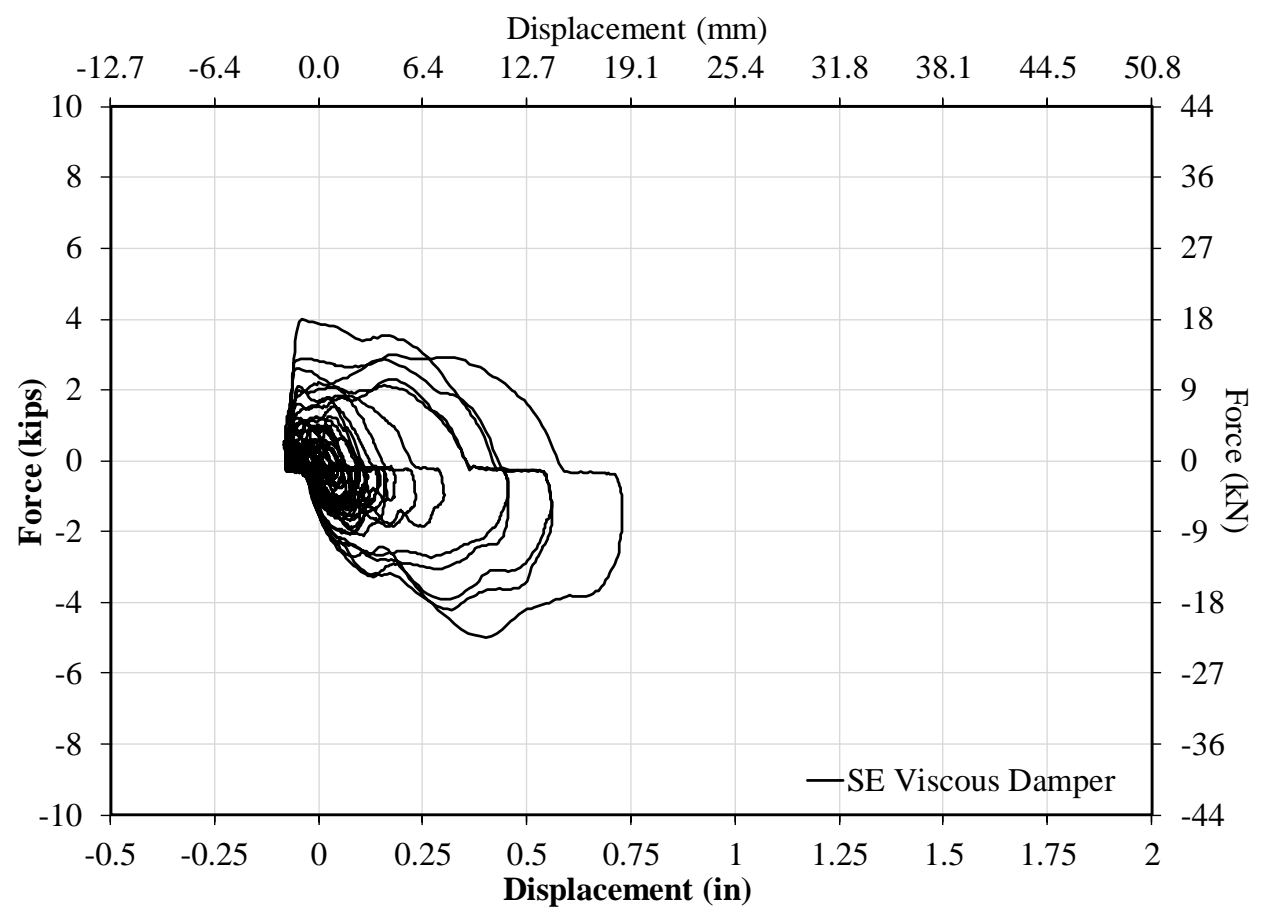

Figure 26-16: 75\% 0.5g IEEE693 SE Viscous Damper Response 


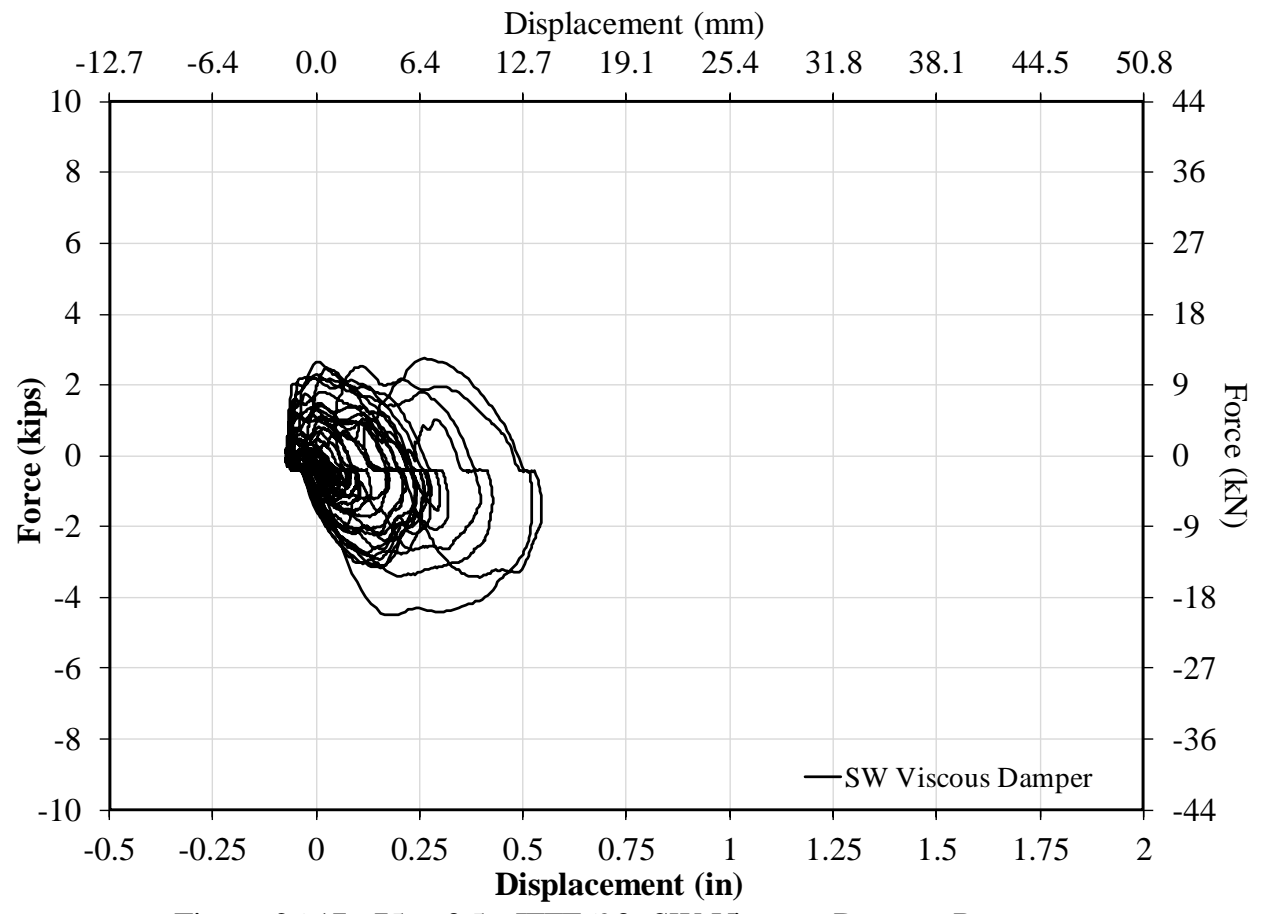

Figure 26-17: 75\% 0.5g IEEE693 SW Viscous Damper Response

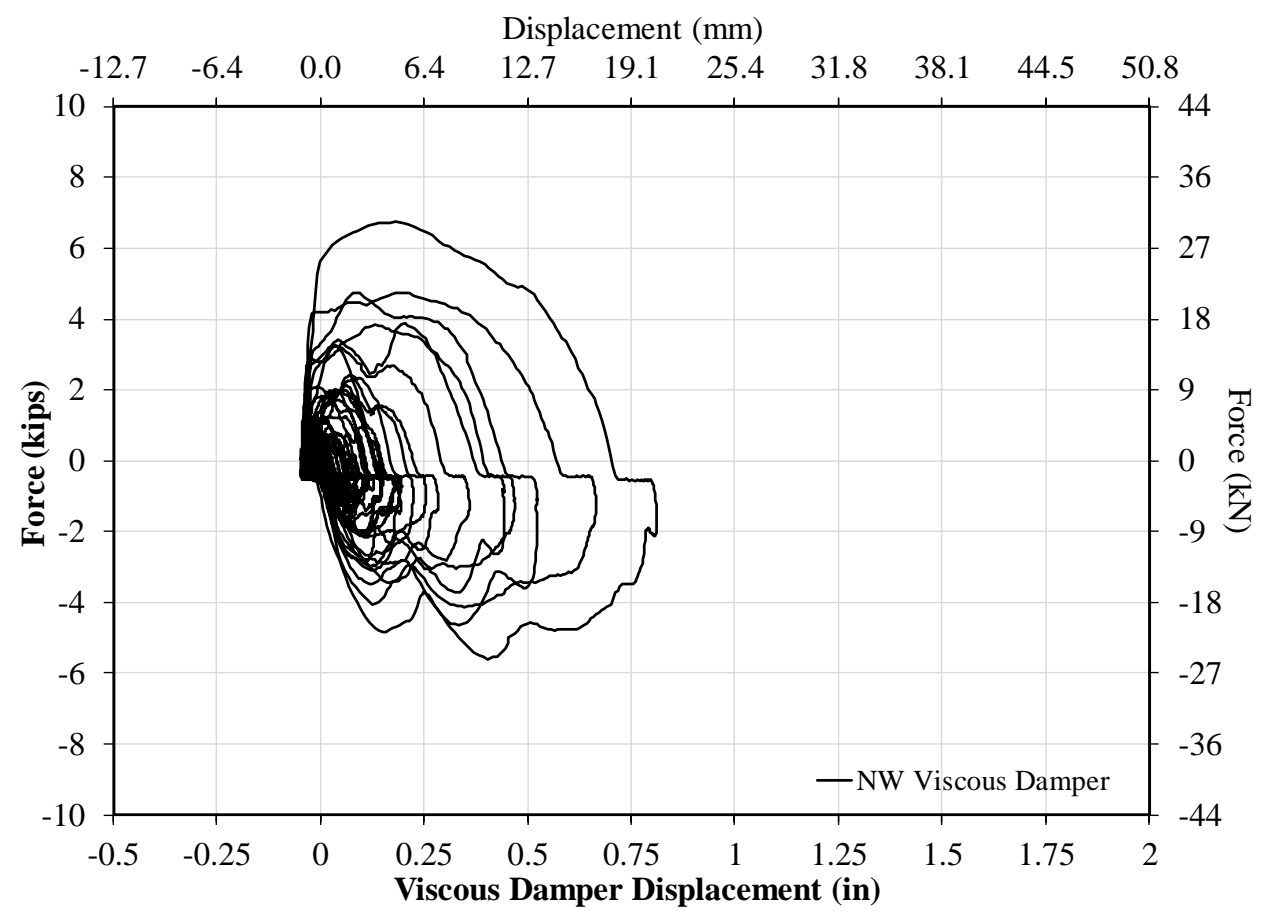

Figure 26-18: 75\% 0.5g IEEE693 NW Viscous Damper Response 


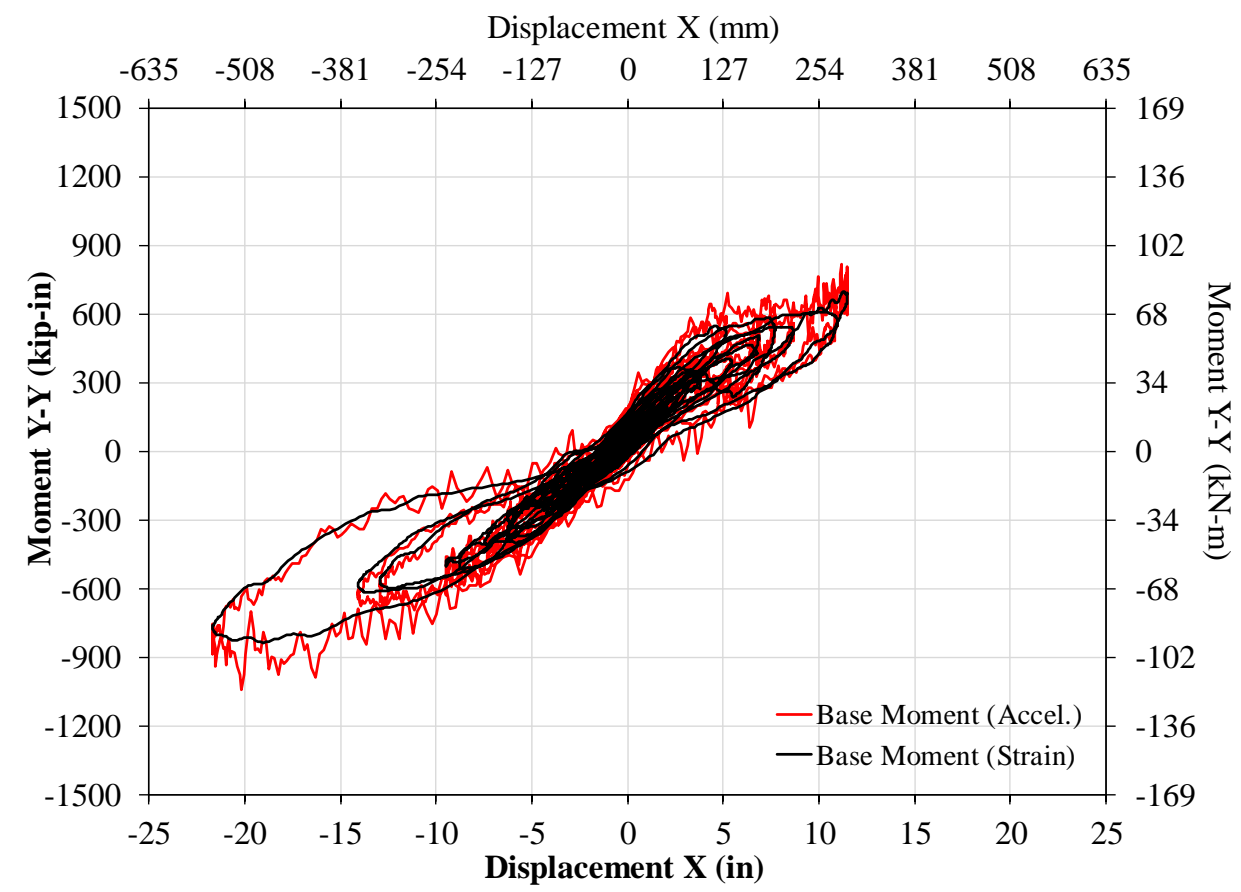

Figure 26-19: 100\% 0.5g IEEE693 X-System Response w/ Viscous Dampers

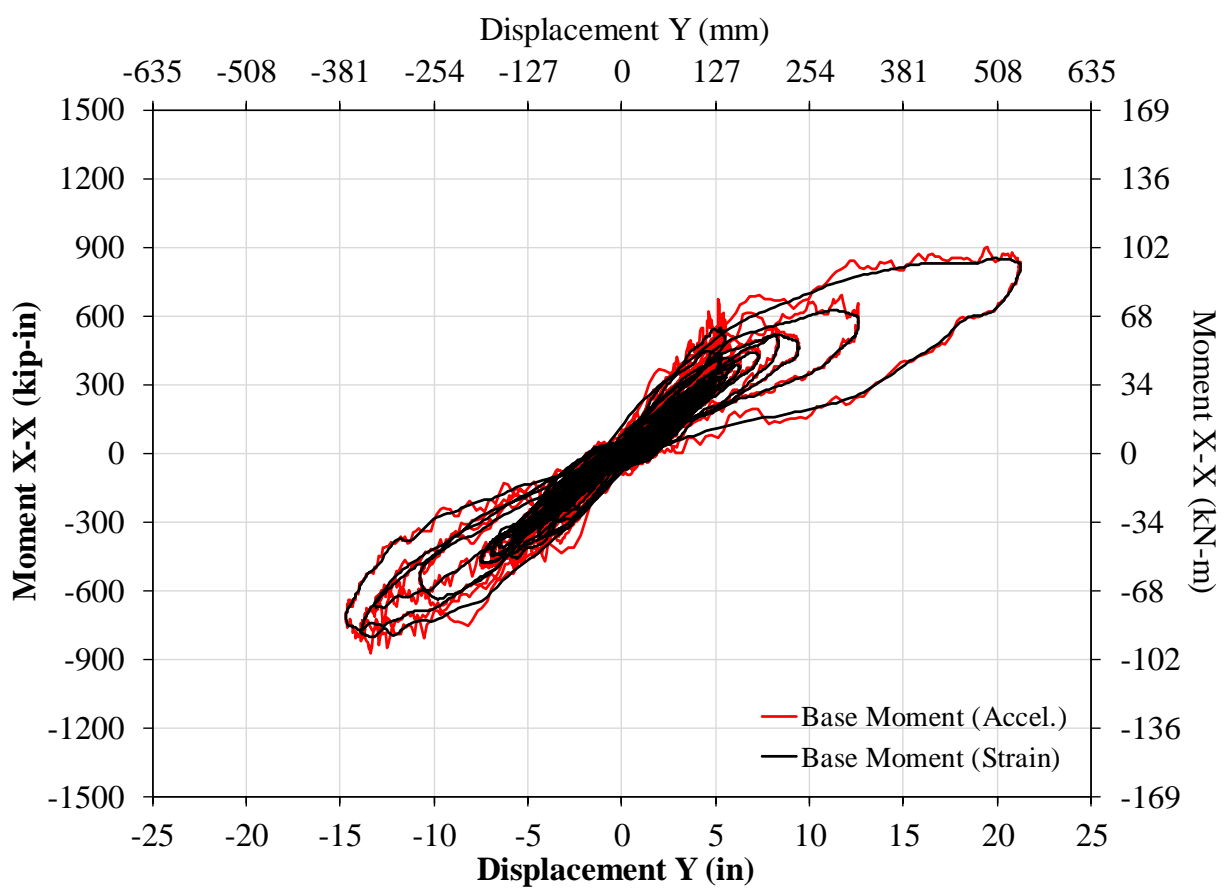

Figure 26-20: 100\% 0.5g IEEE693 Y-System Response w/ Viscous Dampers 


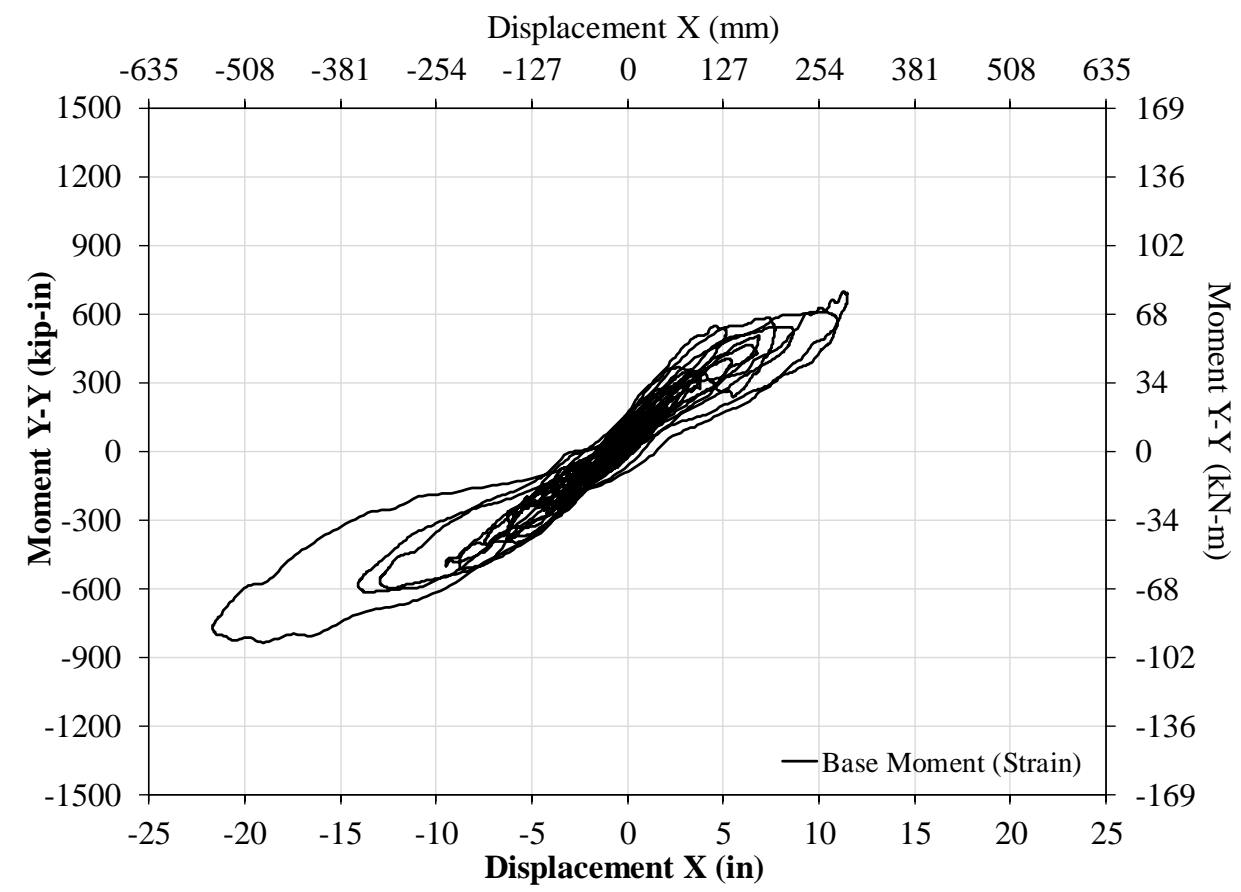

Figure 26-21: 100\% 0.5g IEEE693 X-System Response w/ Viscous Dampers

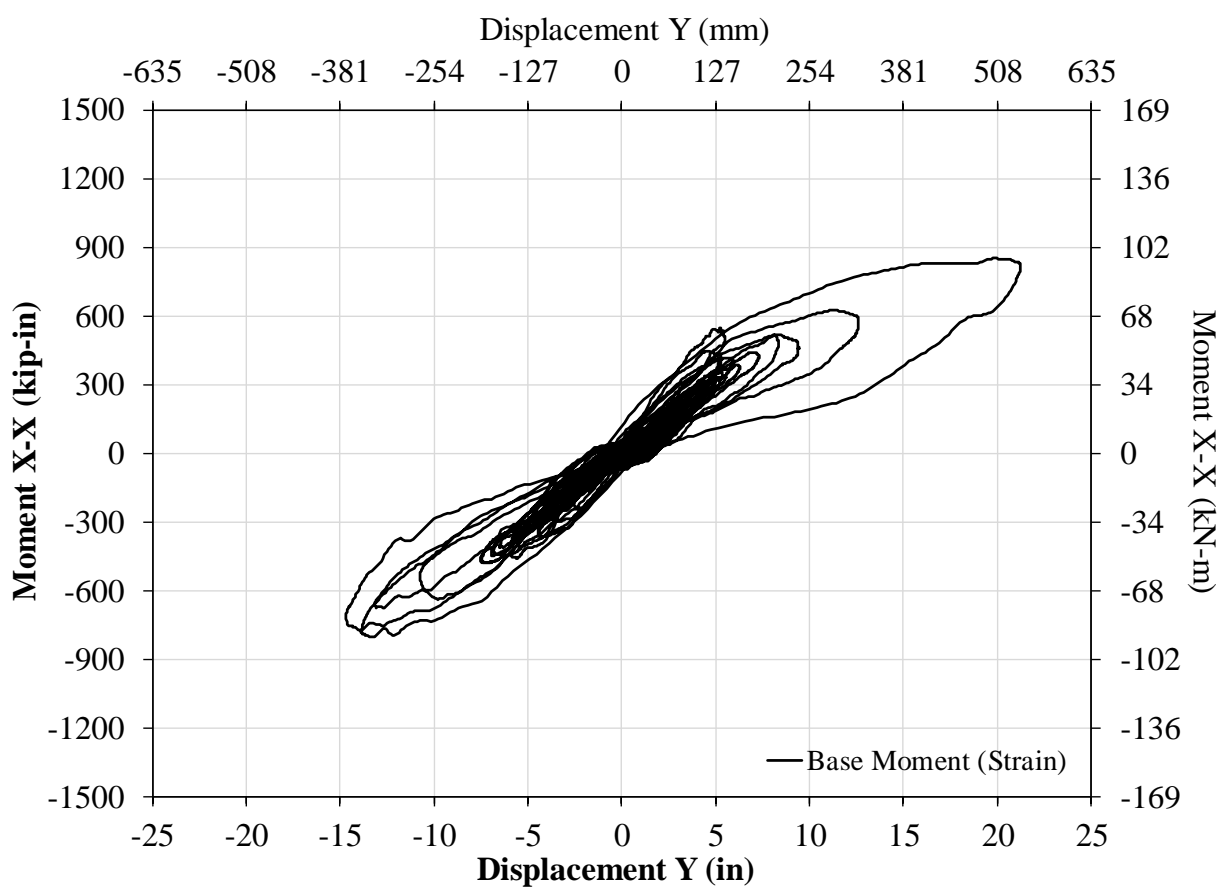

Figure 26-22: 100\% 0.5g IEEE693 Y-System Response w/ Viscous Dampers 


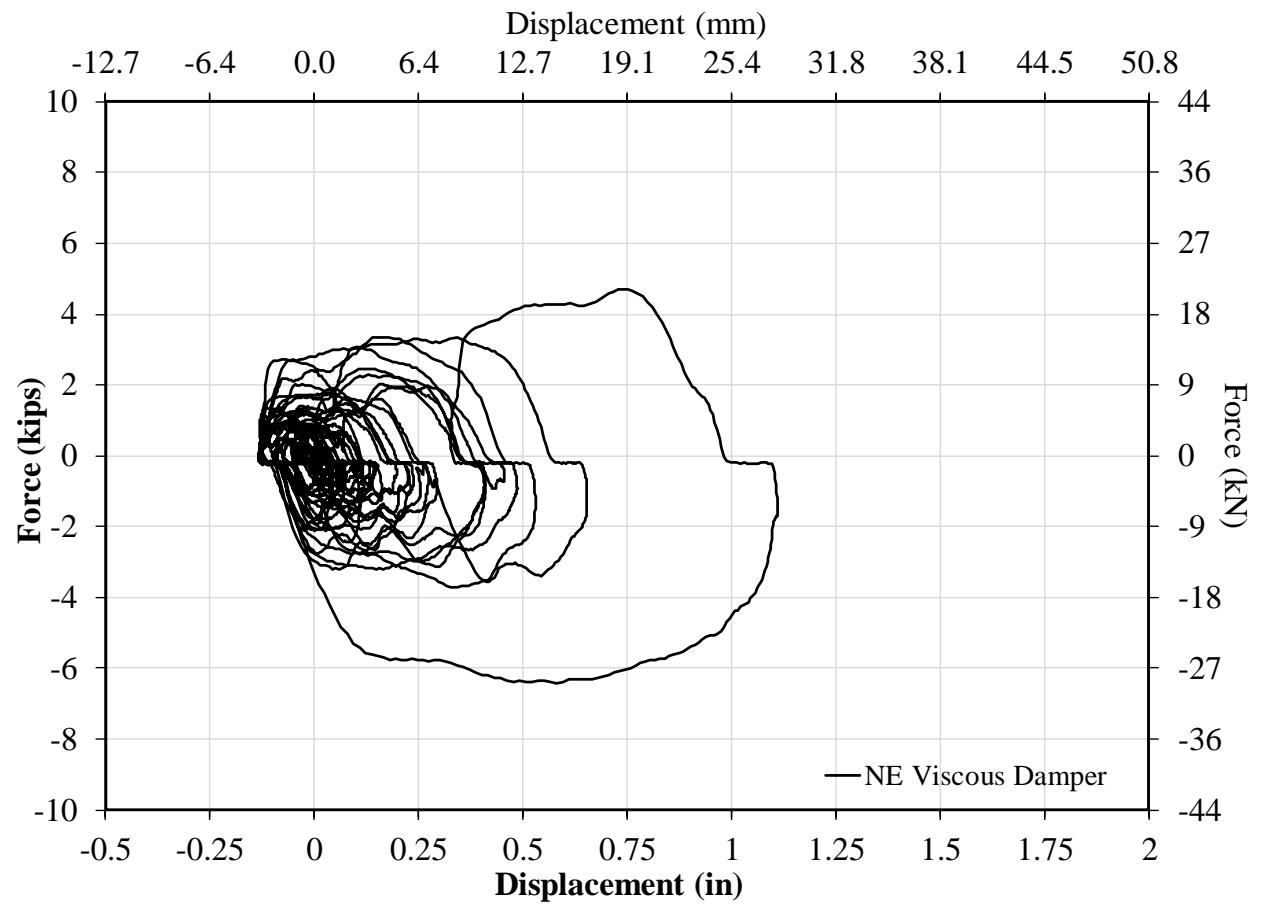

Figure 26-23: 100\% 0.5g IEEE693 NE Viscous Damper Response

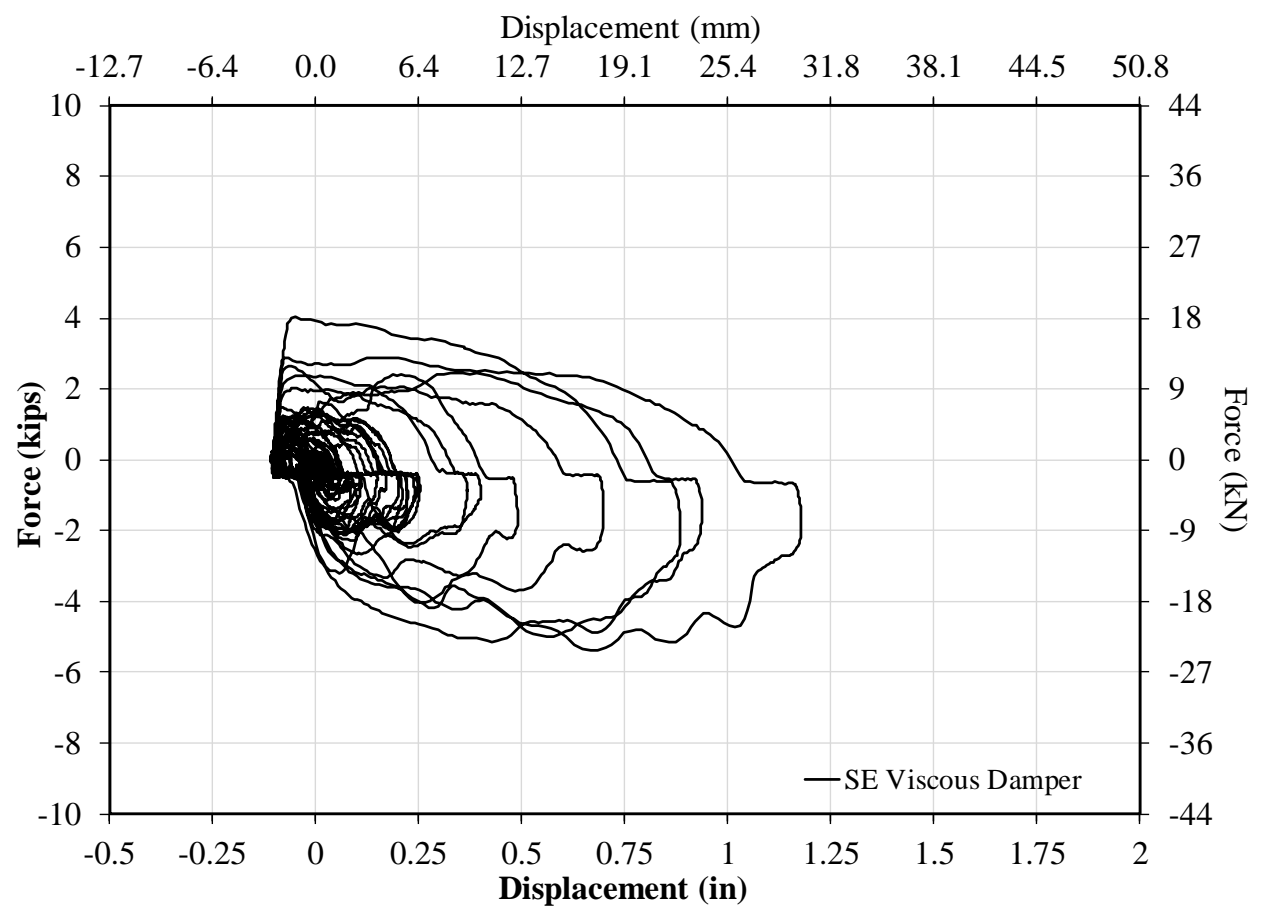

Figure 26-24: 100\% 0.5g IEEE693 SE Viscous Damper Response 


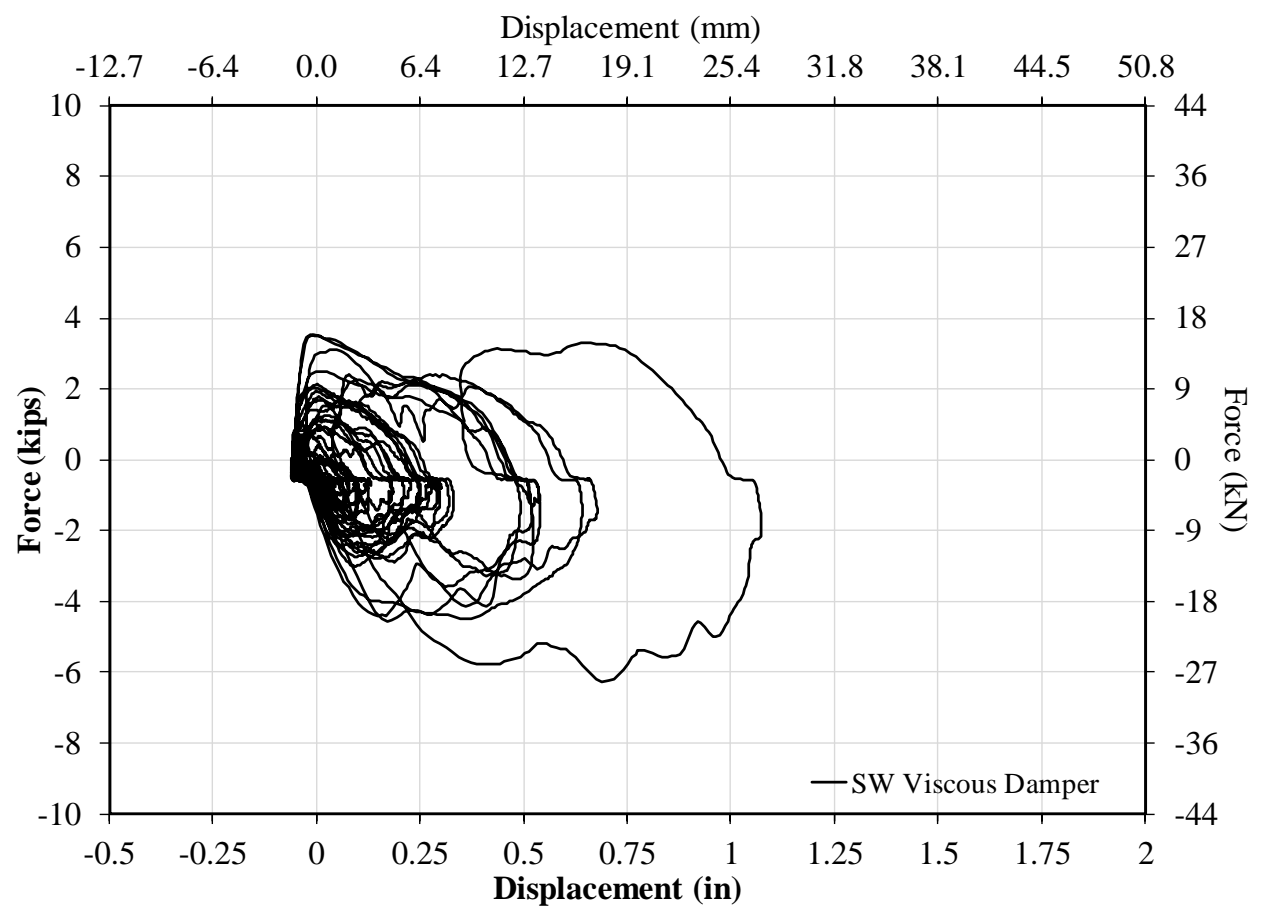

Figure 26-25: 100\% 0.5g IEEE693 SW Viscous Damper Response

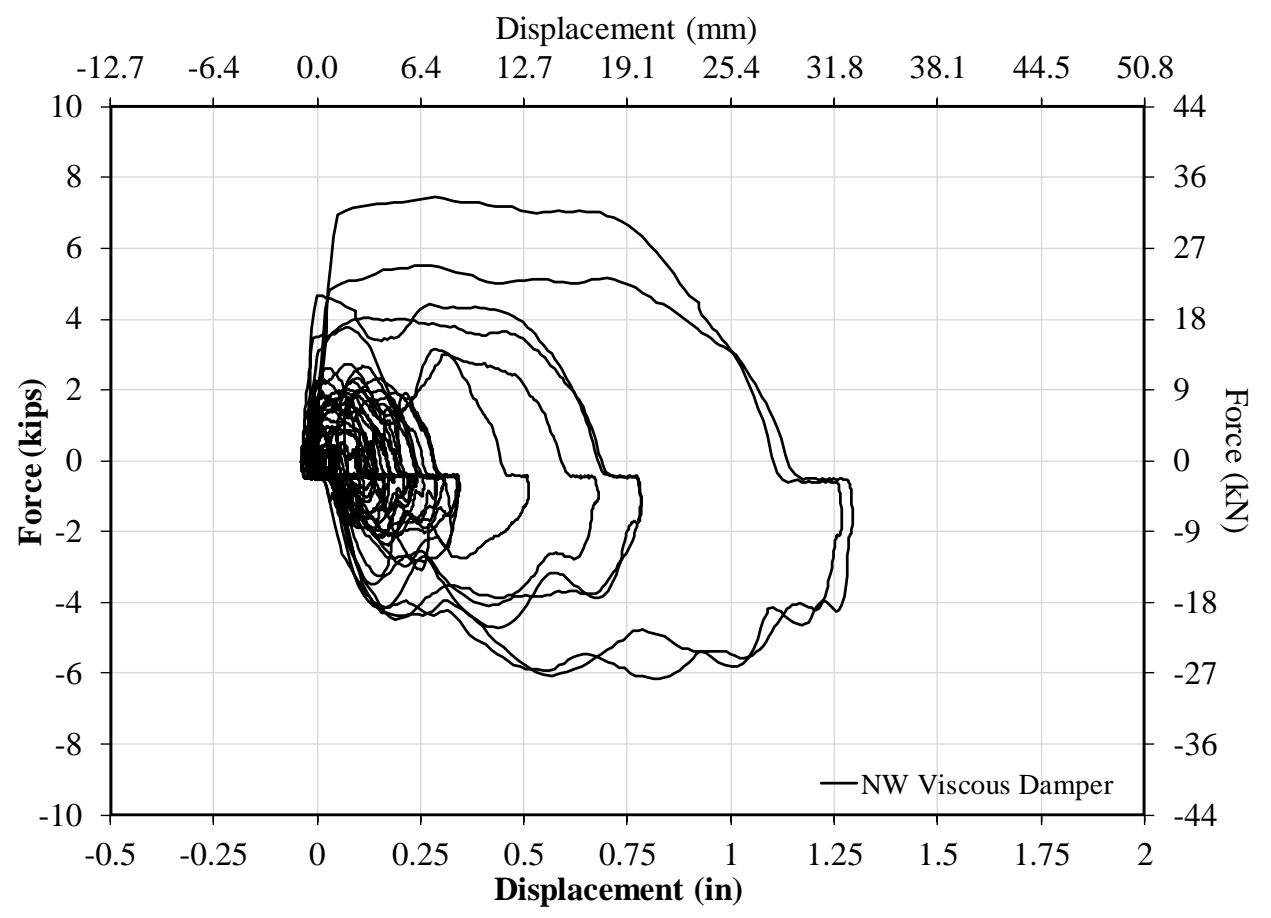

Figure 26-26: 100\% 0.5g IEEE693 NW Viscous Damper Response 


\subsection{APPENDIX I}

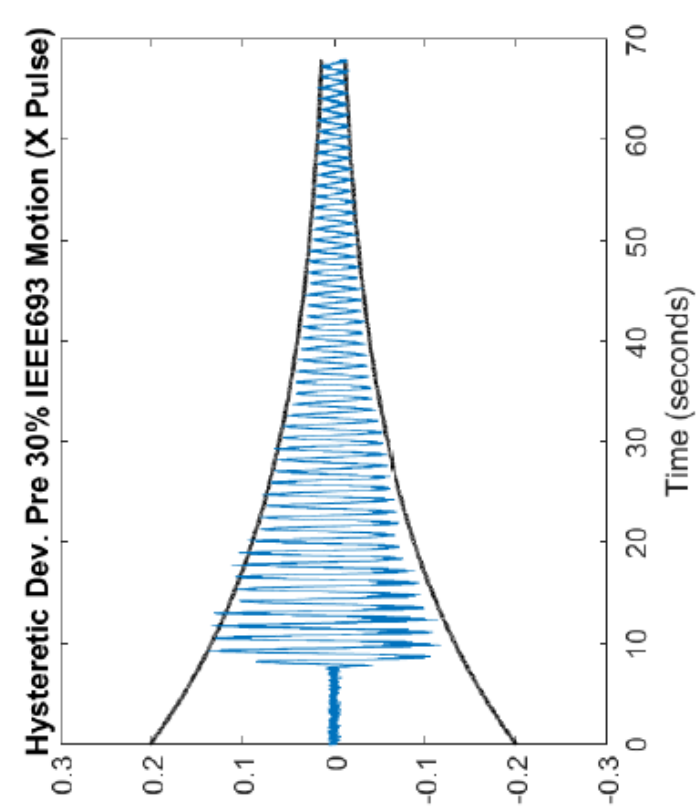

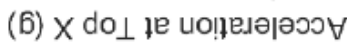

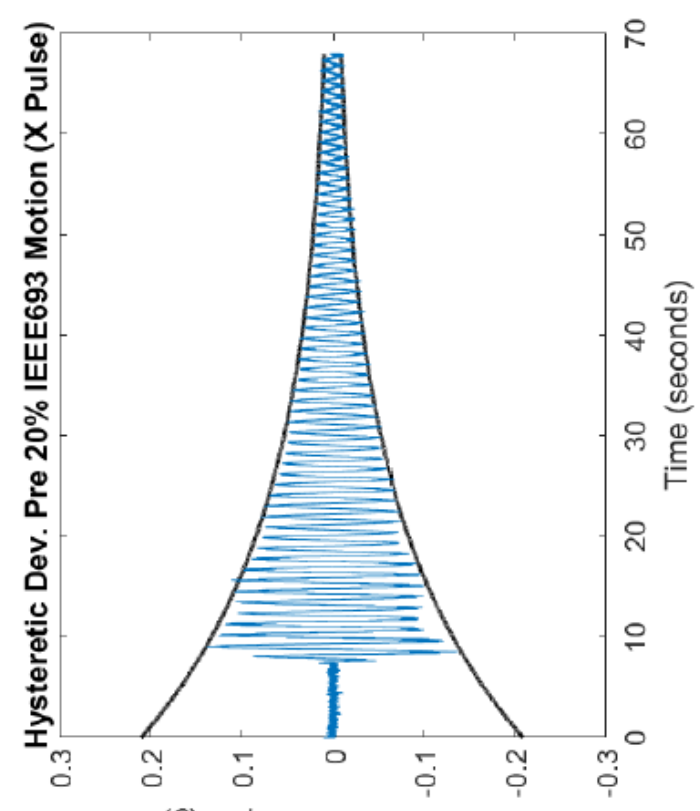

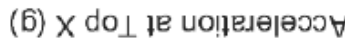

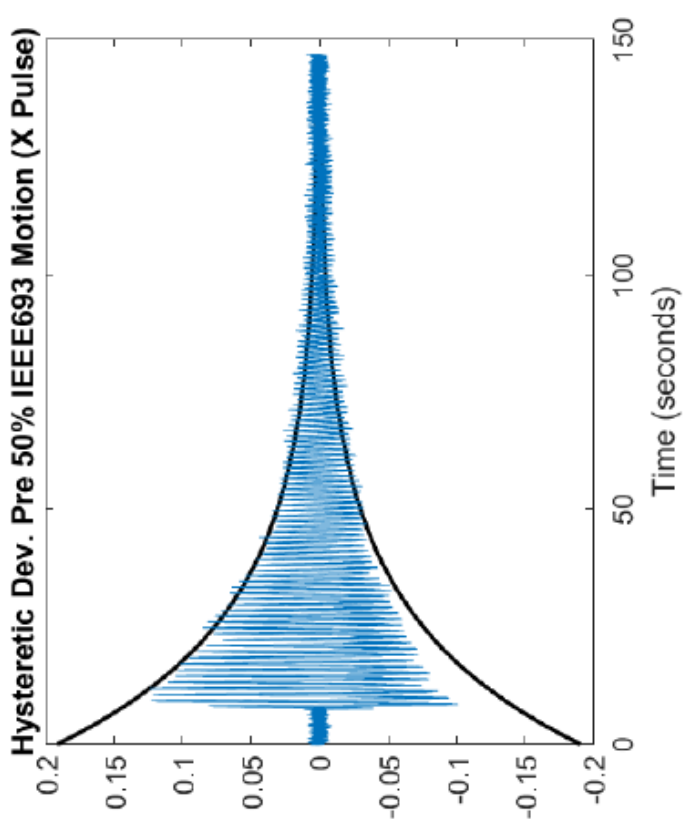

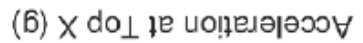

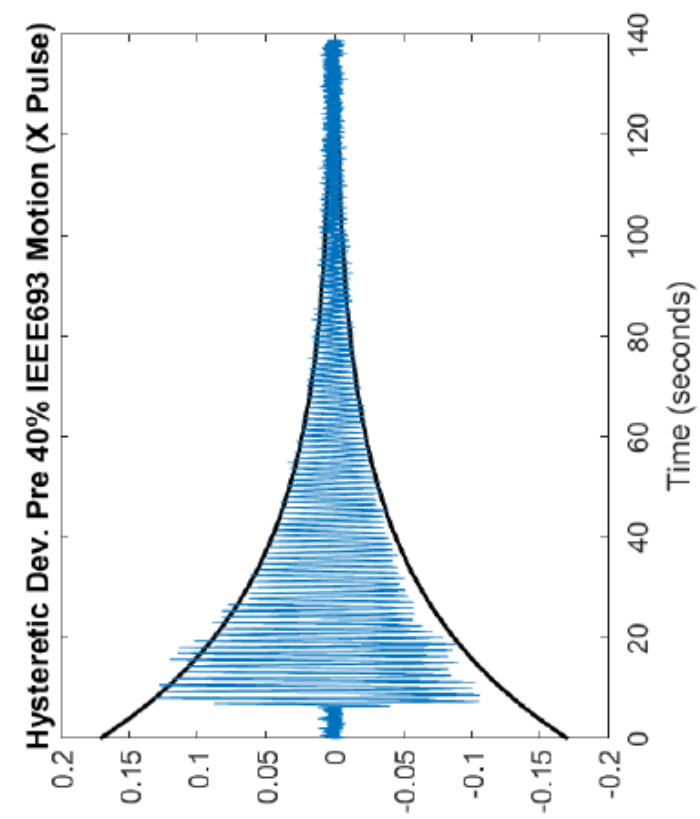

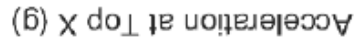

Figure 27-1: Hysteretic Device Retrofit X-Damping 20-50\% 


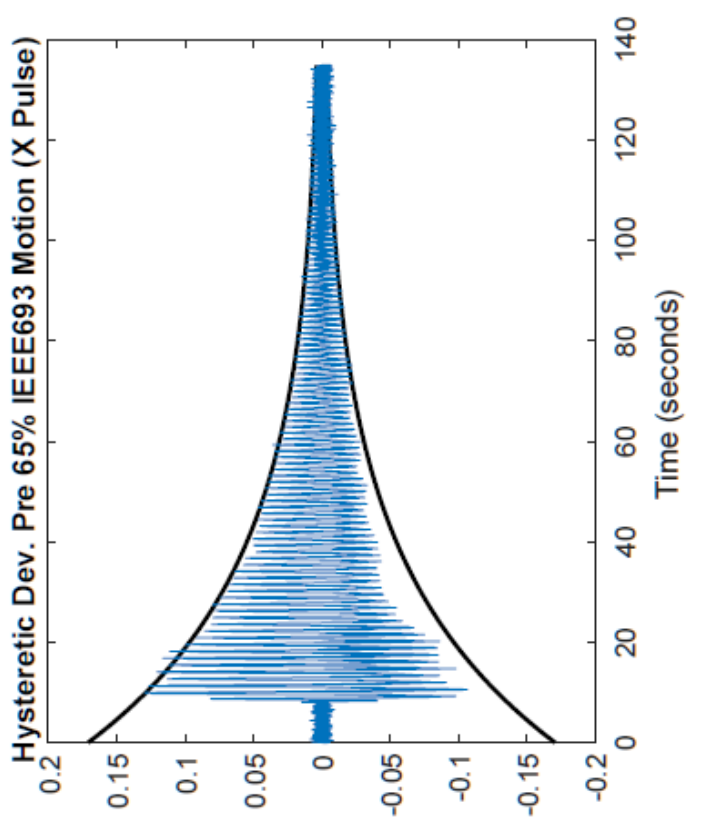

(6) $\mathrm{X}$ do $\perp$ je uo!̣eıə|əวэ $\forall$

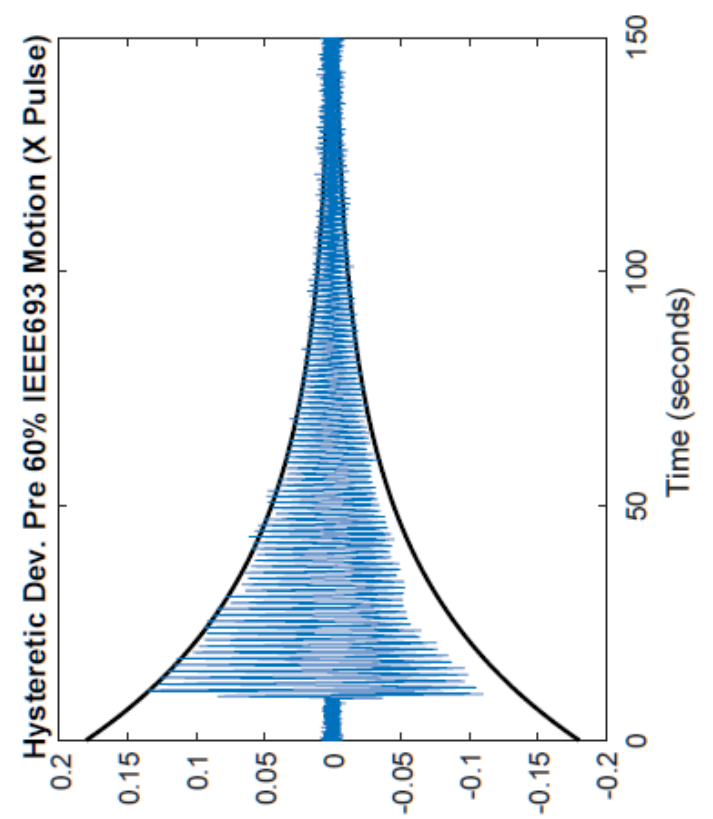

(6) $\mathrm{X}$ do $\perp$ je uọ̣e

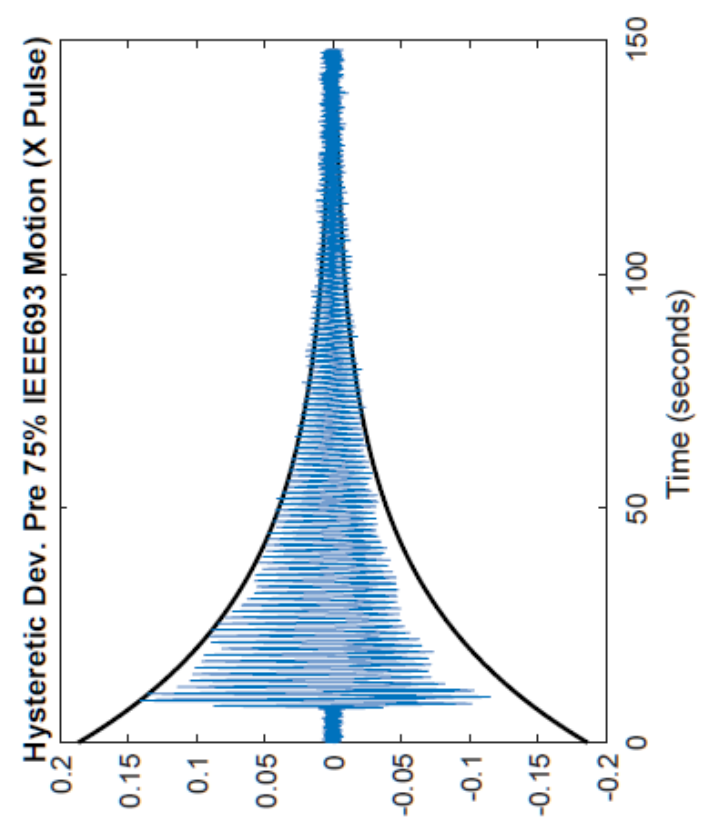

(6) $X$ do $\perp$ te uo!̣eıə|әวэ $\forall$

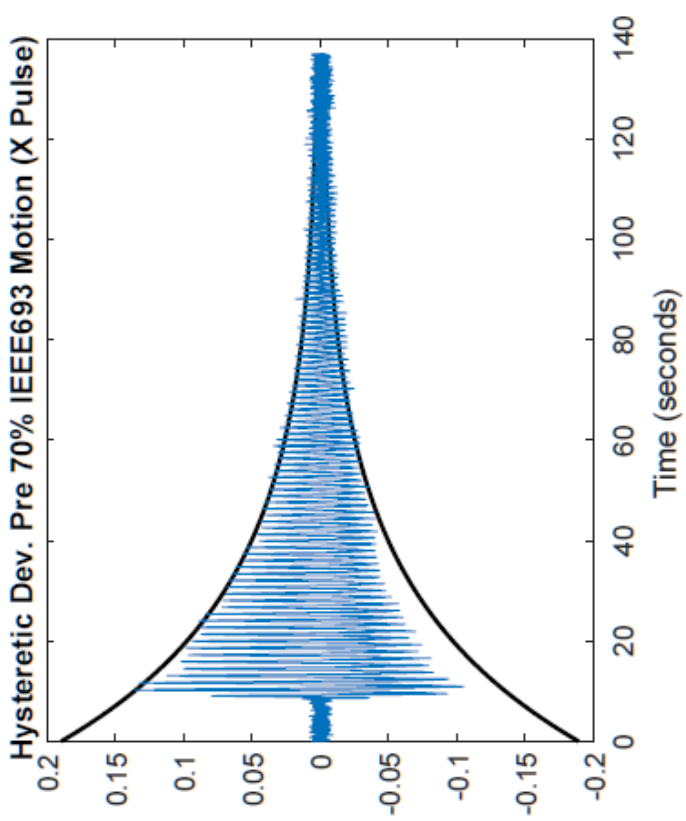

(6) $\mathrm{X}$ do $\perp$ te uo!̣eıə|әวэ

Figure 27-2: Hysteretic Device Retrofit X-Damping 60-75\% 


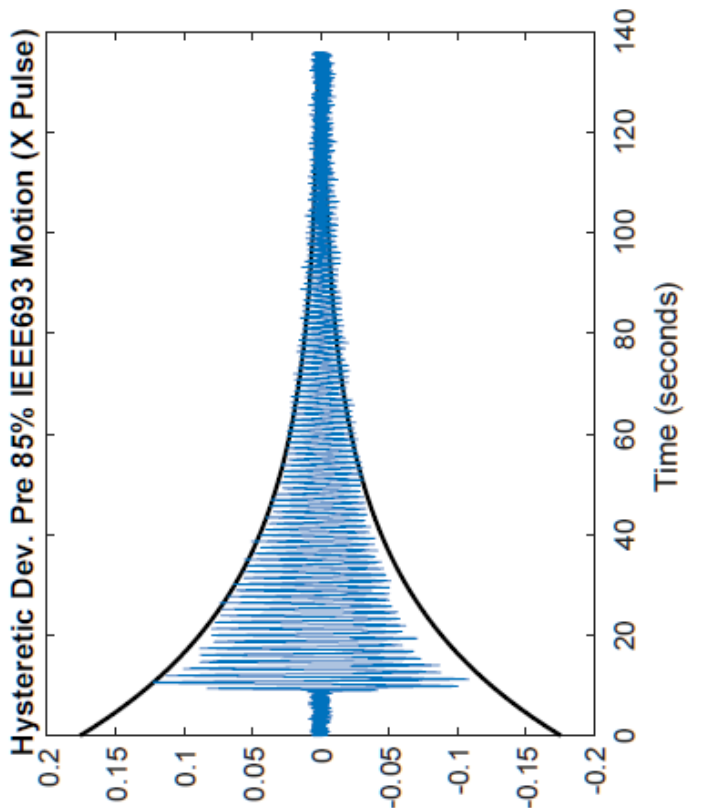

(6) $\mathrm{X}$ do $\perp$ te uo!̣e

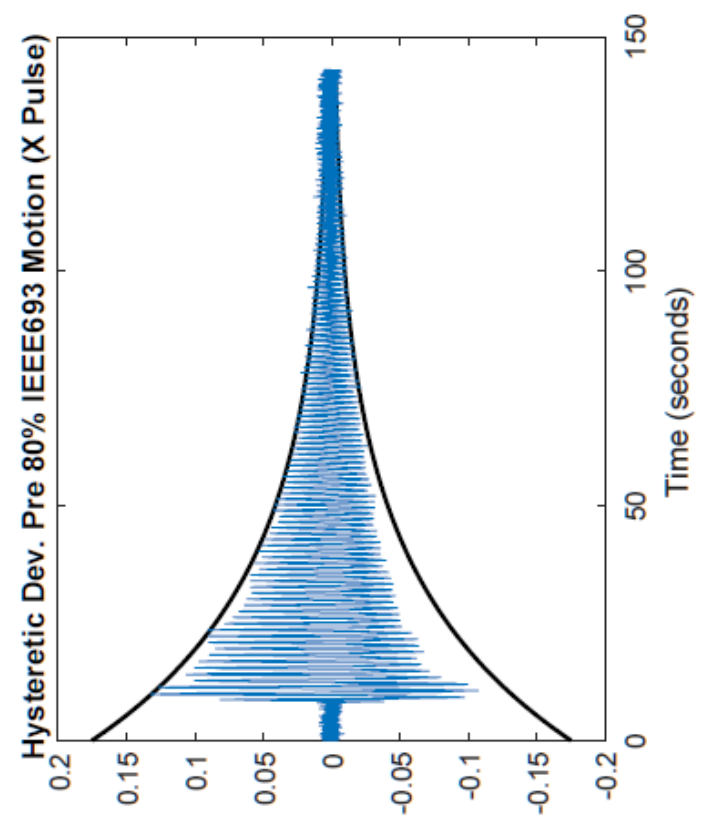

(6) $X$ do $\perp$ je uo!̣e

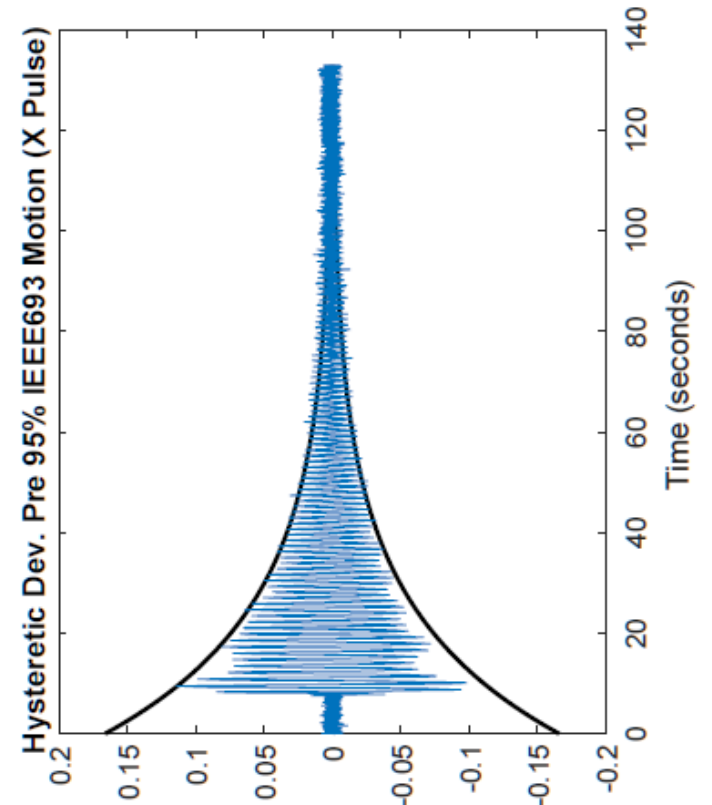

(6) $X$ do $\perp$ je uo!̣e

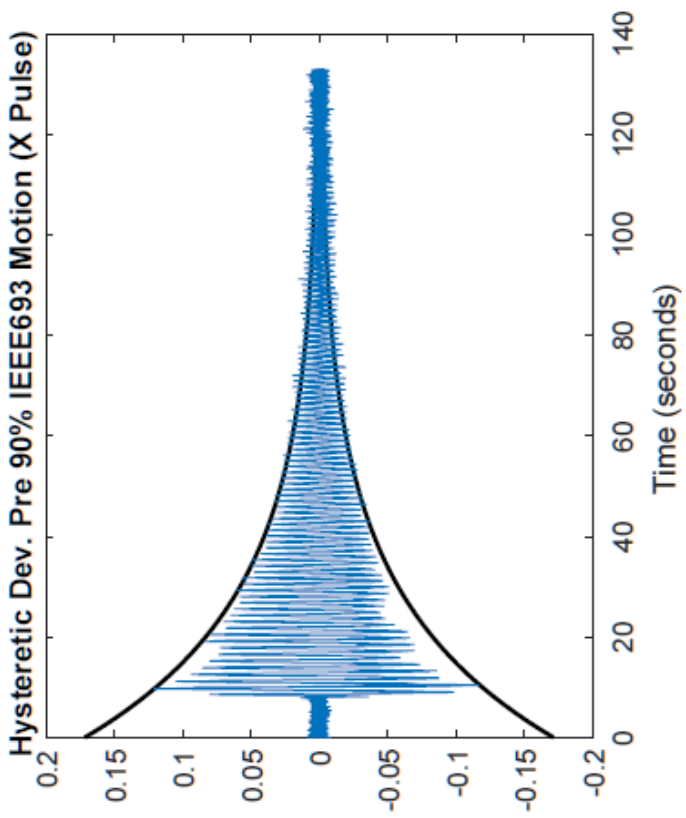

(6) $\mathrm{X}$ do $\perp$ je uoṇeıәәэว

Figure 27-3: Hysteretic Device Retrofit X-Damping 80-95\% 


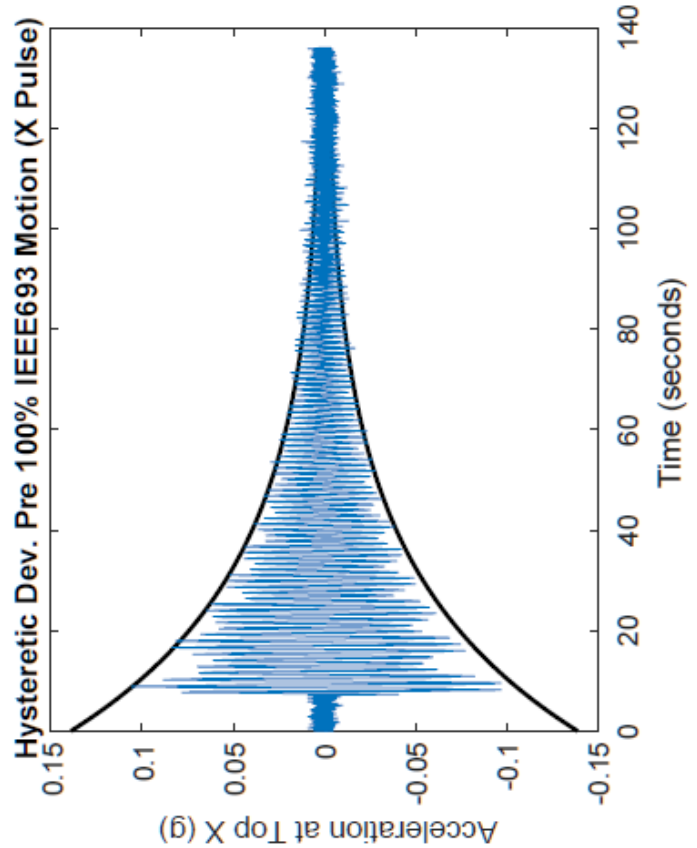

Figure 27-4: Hysteretic Device Retrofit X-Damping 100\% 


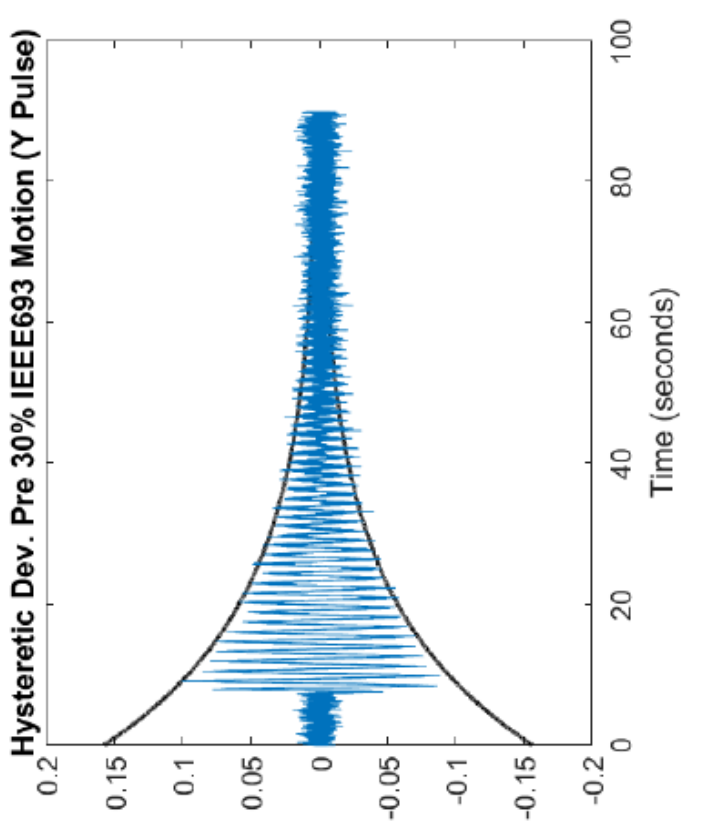

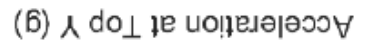

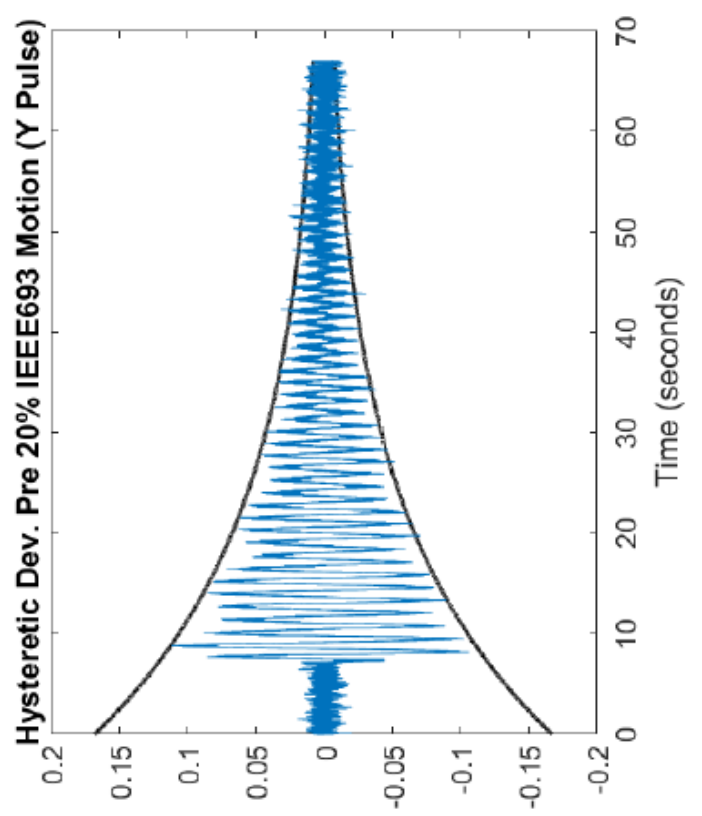

(6) $\wedge$ do $\perp$ te uo!̣eมə|əวэ $\forall$

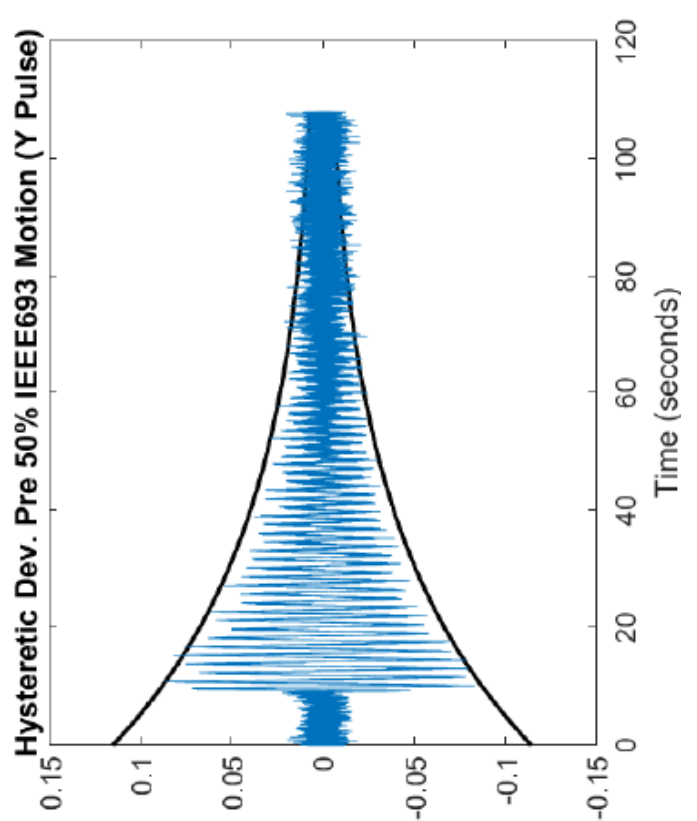

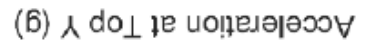

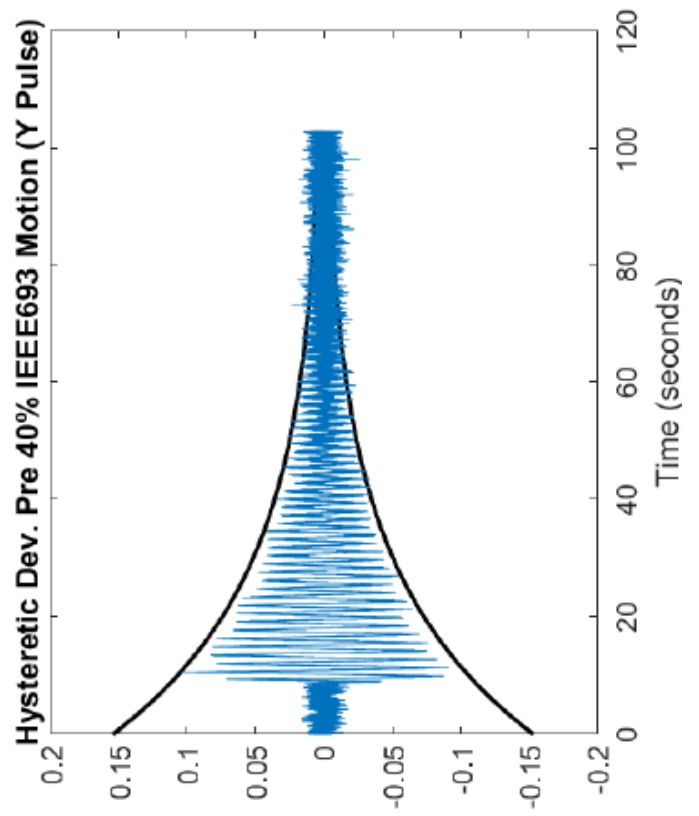

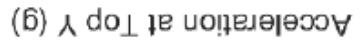

Figure 27-5: Hysteretic Device Retrofit Y-Damping 20-50\% 


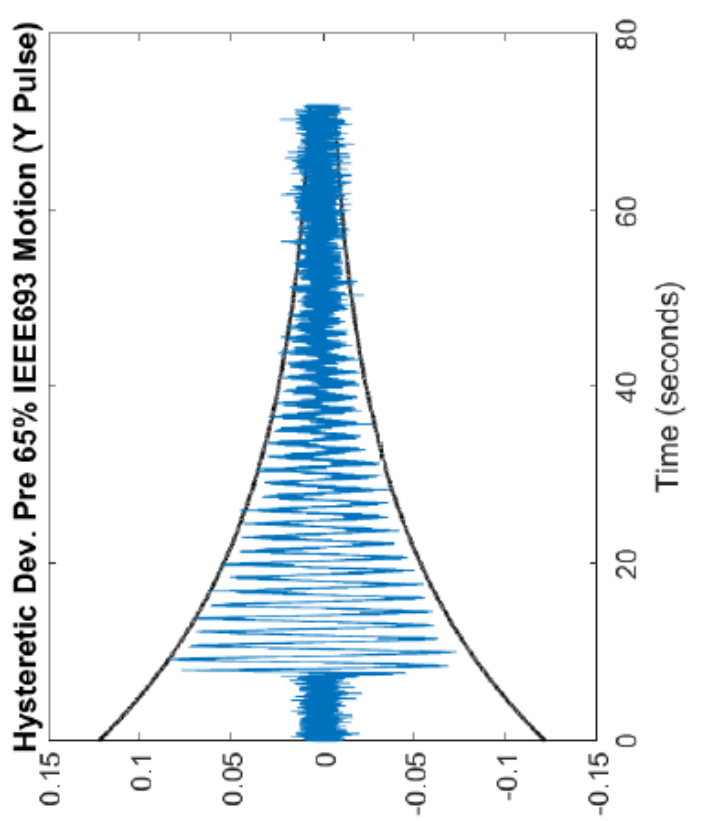

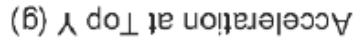

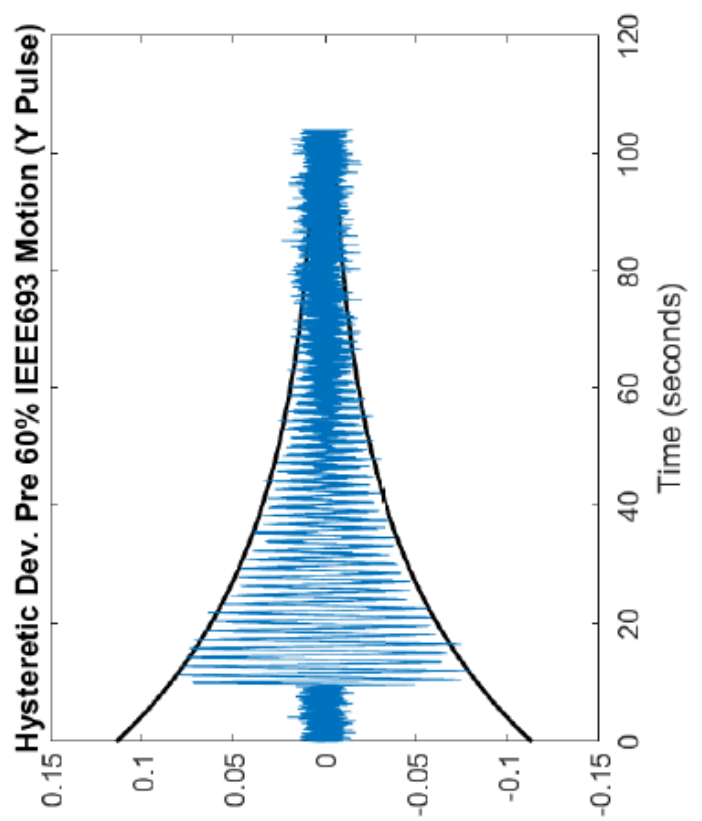

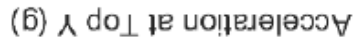

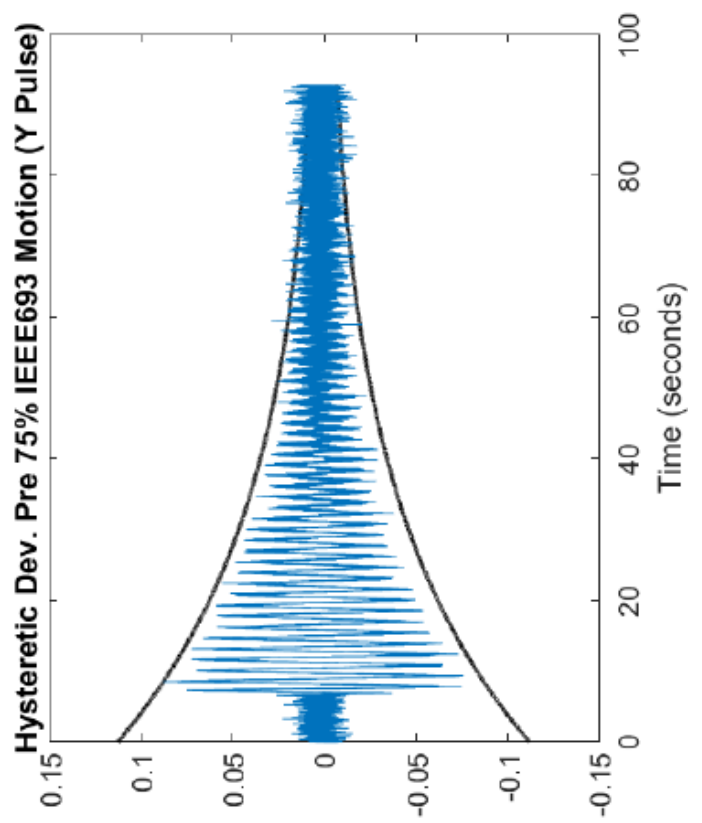

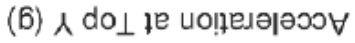

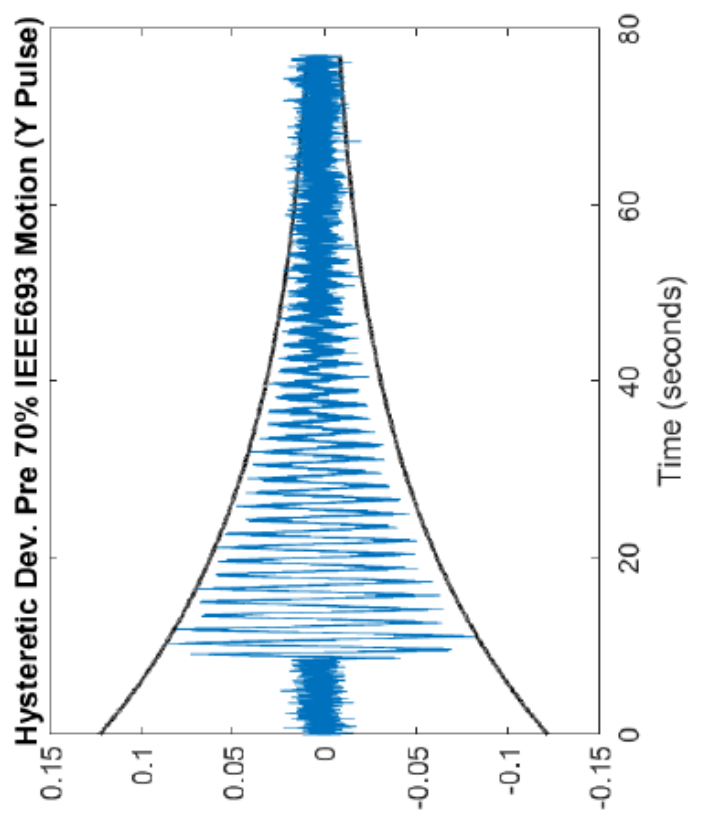

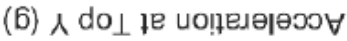

Figure 27-6: Hysteretic Device Retrofit Y-Damping 60-75\% 


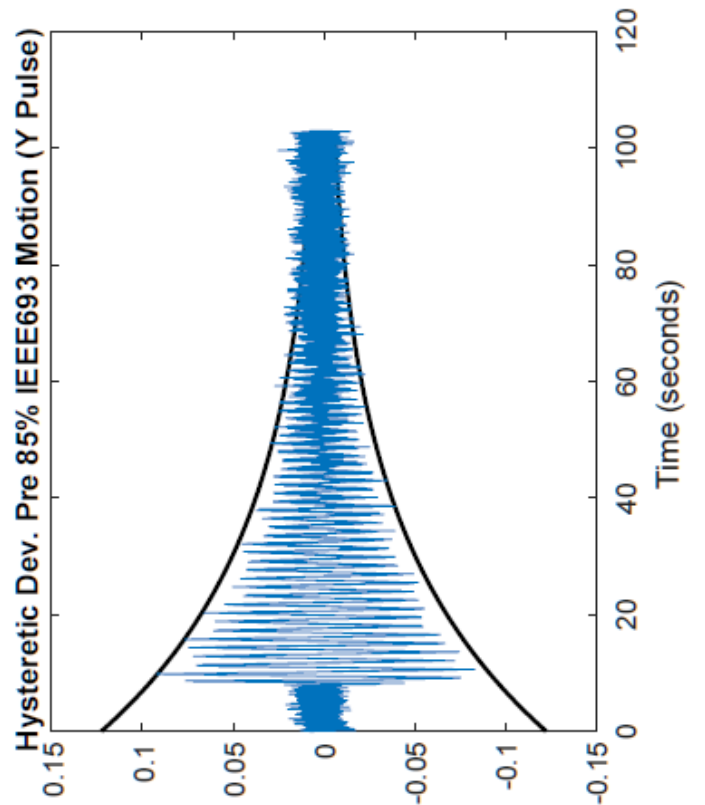

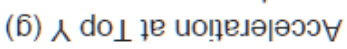

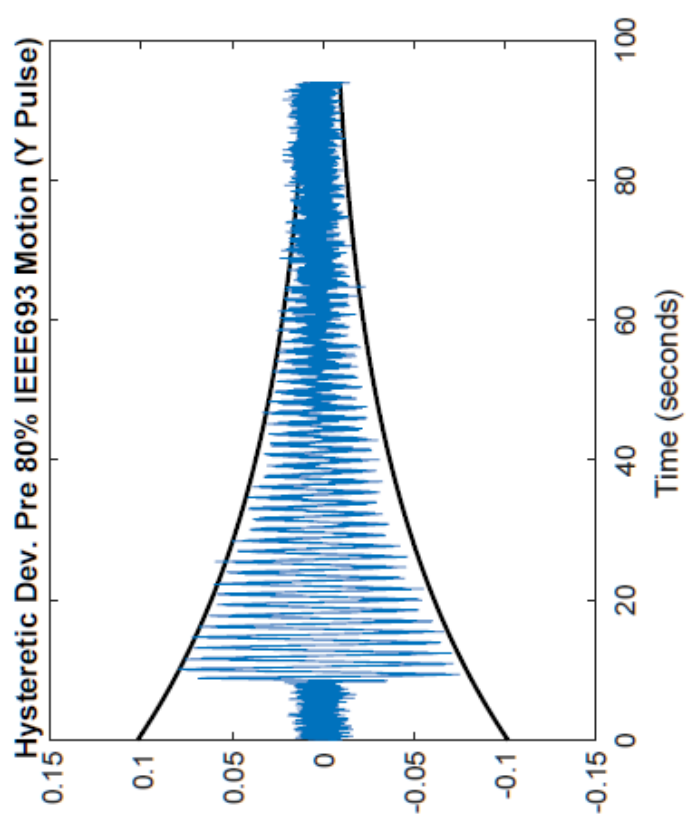

(6) $\lambda$ do $\perp$ te uo!̣e

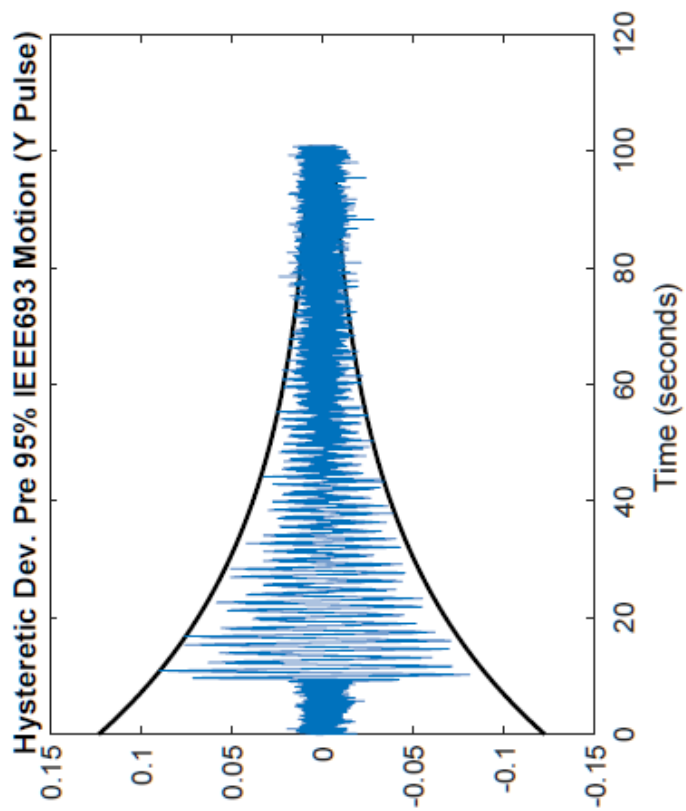

(6) $\curlywedge$ do $\perp$ je uoṇe

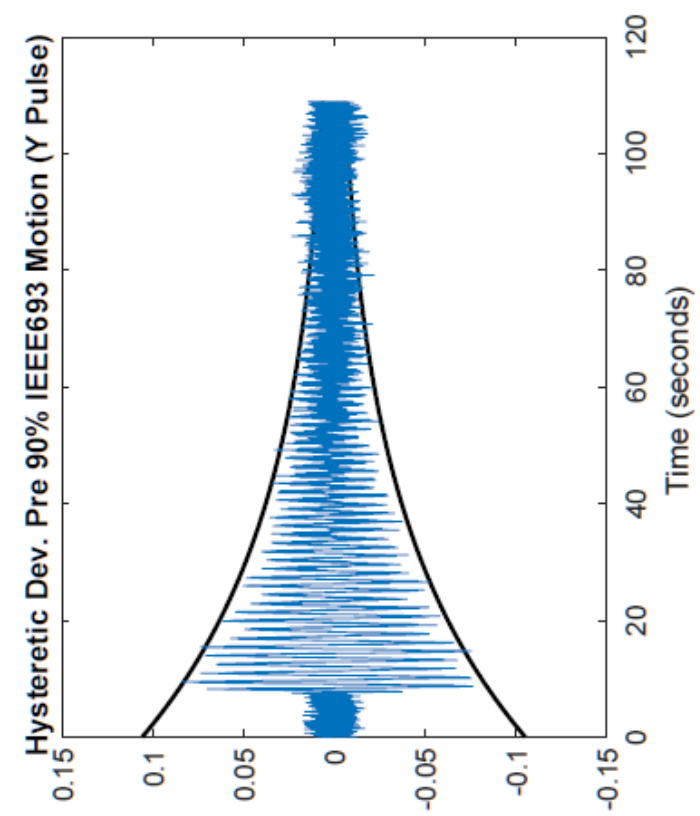

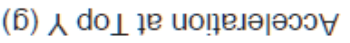

Figure 27-7: Hysteretic Device Retrofit Y-Damping 80-95\% 


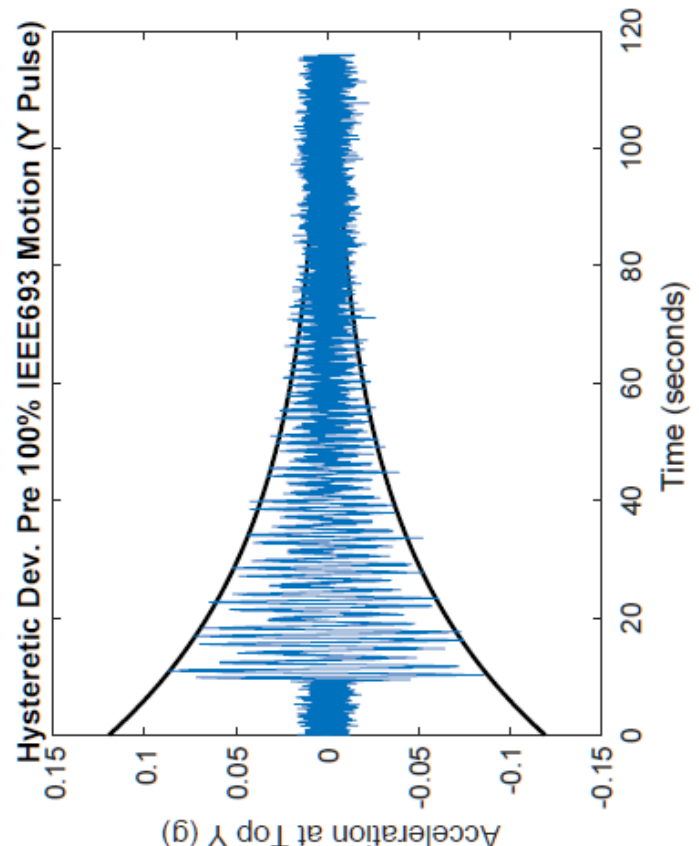

Figure 27-8: Hysteretic Device Retrofit Y-Damping 100\% 


\subsection{APPENDIX J}

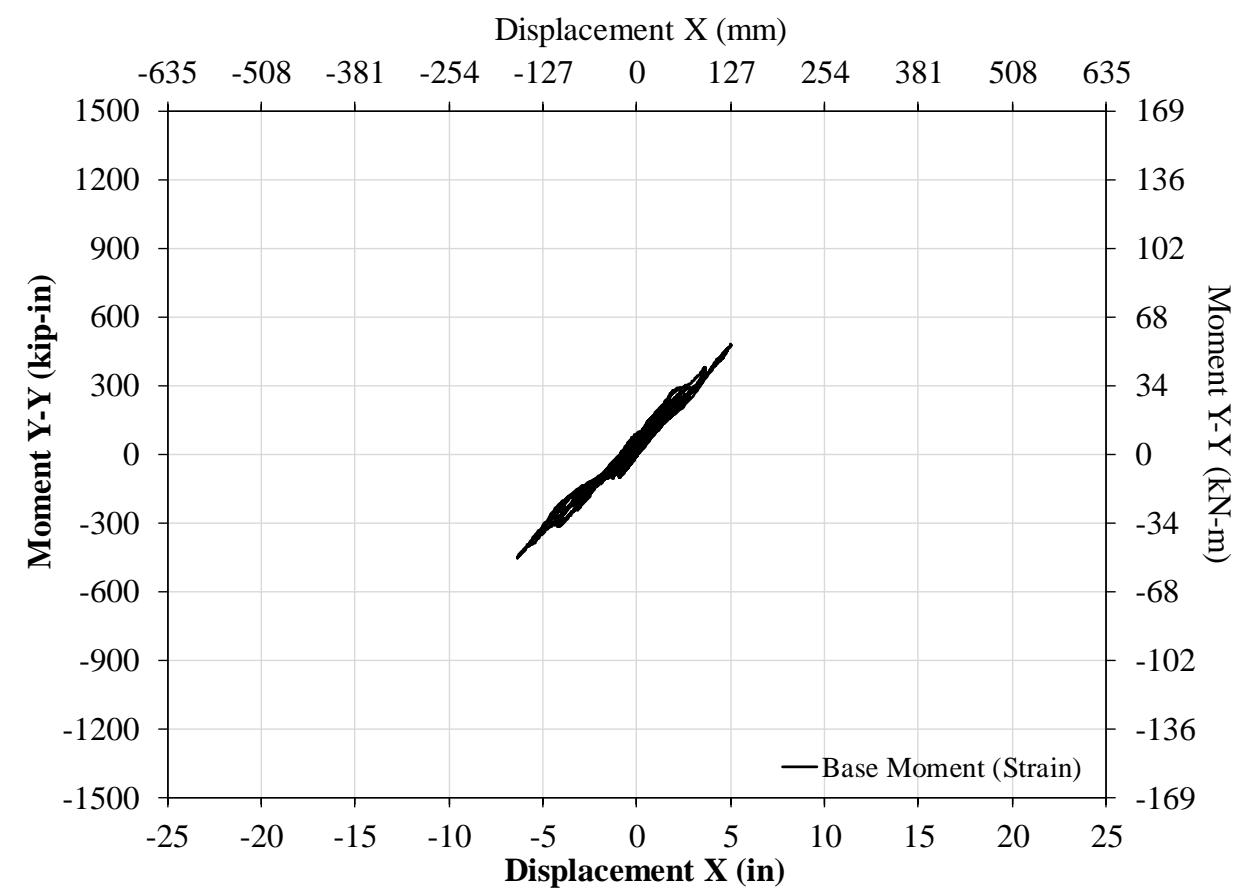

Figure 28-1: 20\% 0.5g IEEE693 X-System Response w/ Hysteretic Device

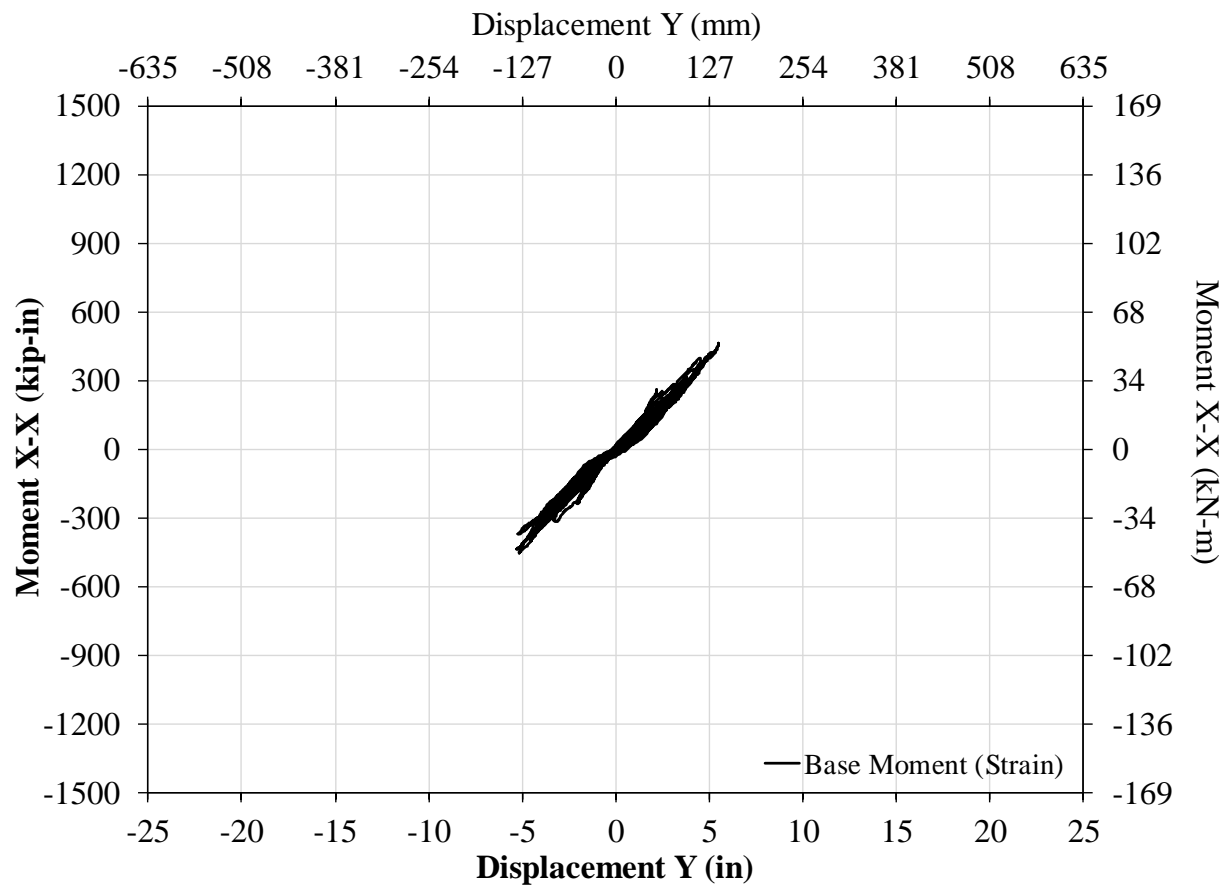

Figure 28-2: $20 \%$ 0.5g IEEE693 X-System Response w/ Hysteretic Device 


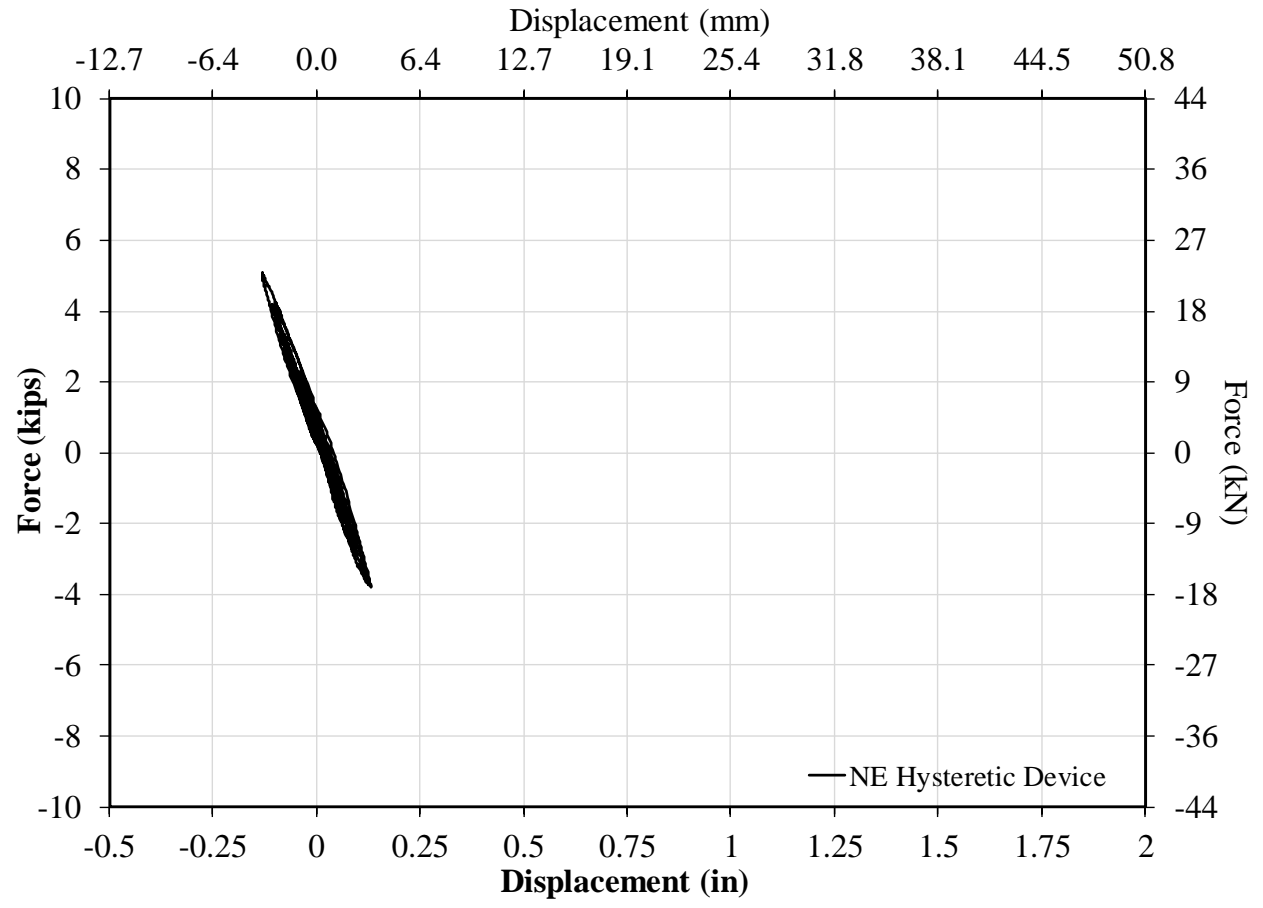

Figure 28-3: 20\% 0.5g IEEE693 NE Hysteretic Device Response

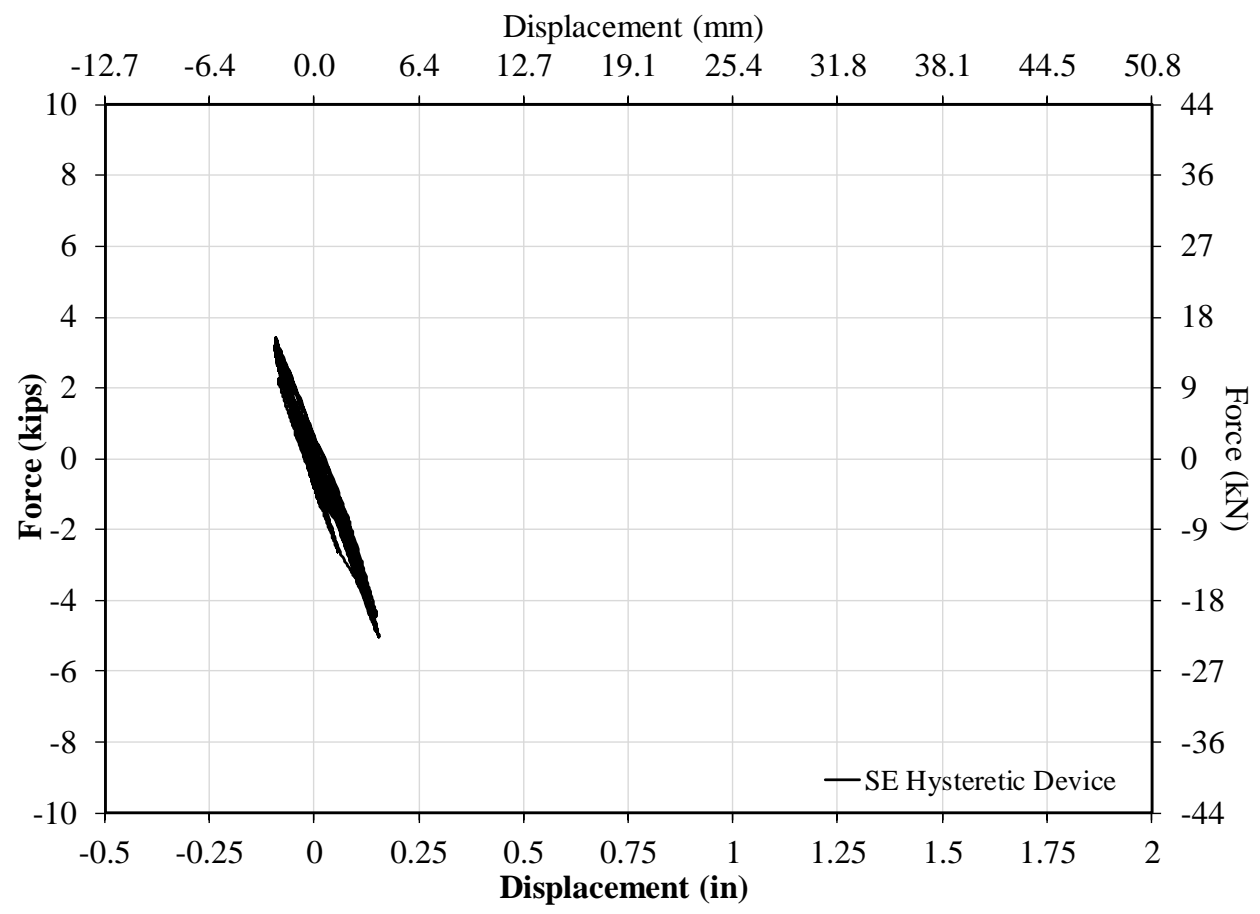

Figure 28-4: 20\% 0.5g IEEE693 SE Hysteretic Device Response 


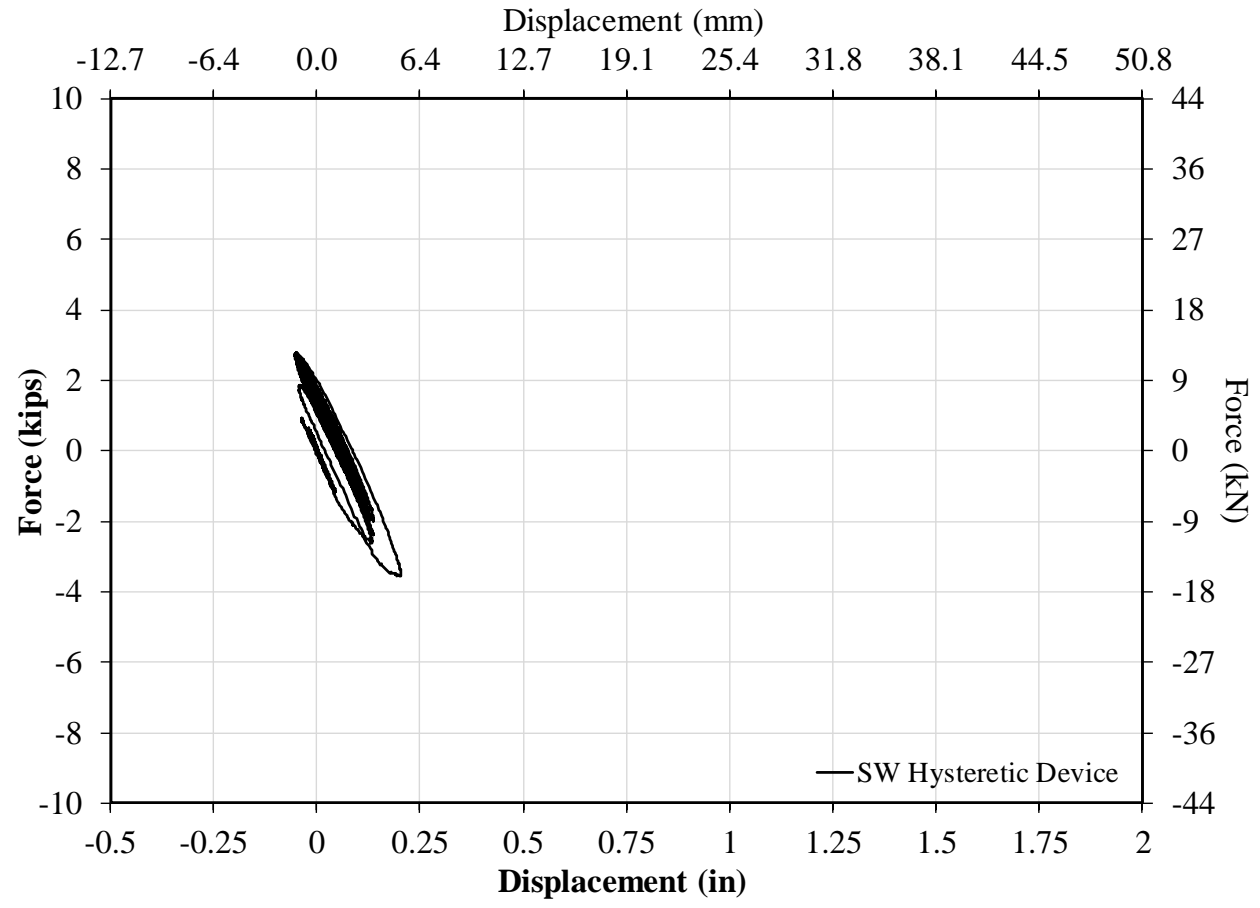

Figure 28-5: 20\% 0.5g IEEE693 SW Hysteretic Device Response

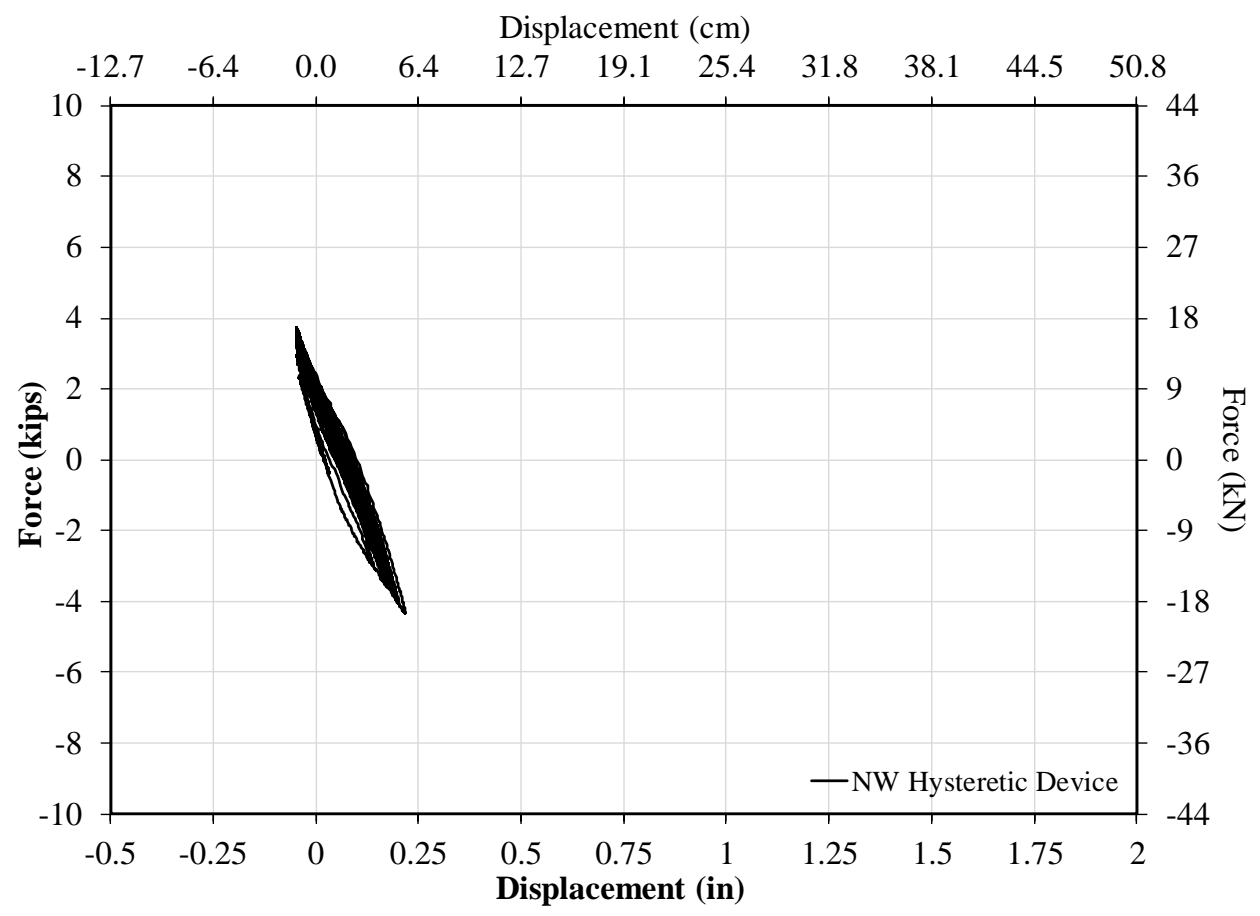

Figure 28-6: 20\% 0.5g IEEE693 NW Hysteretic Device Response 


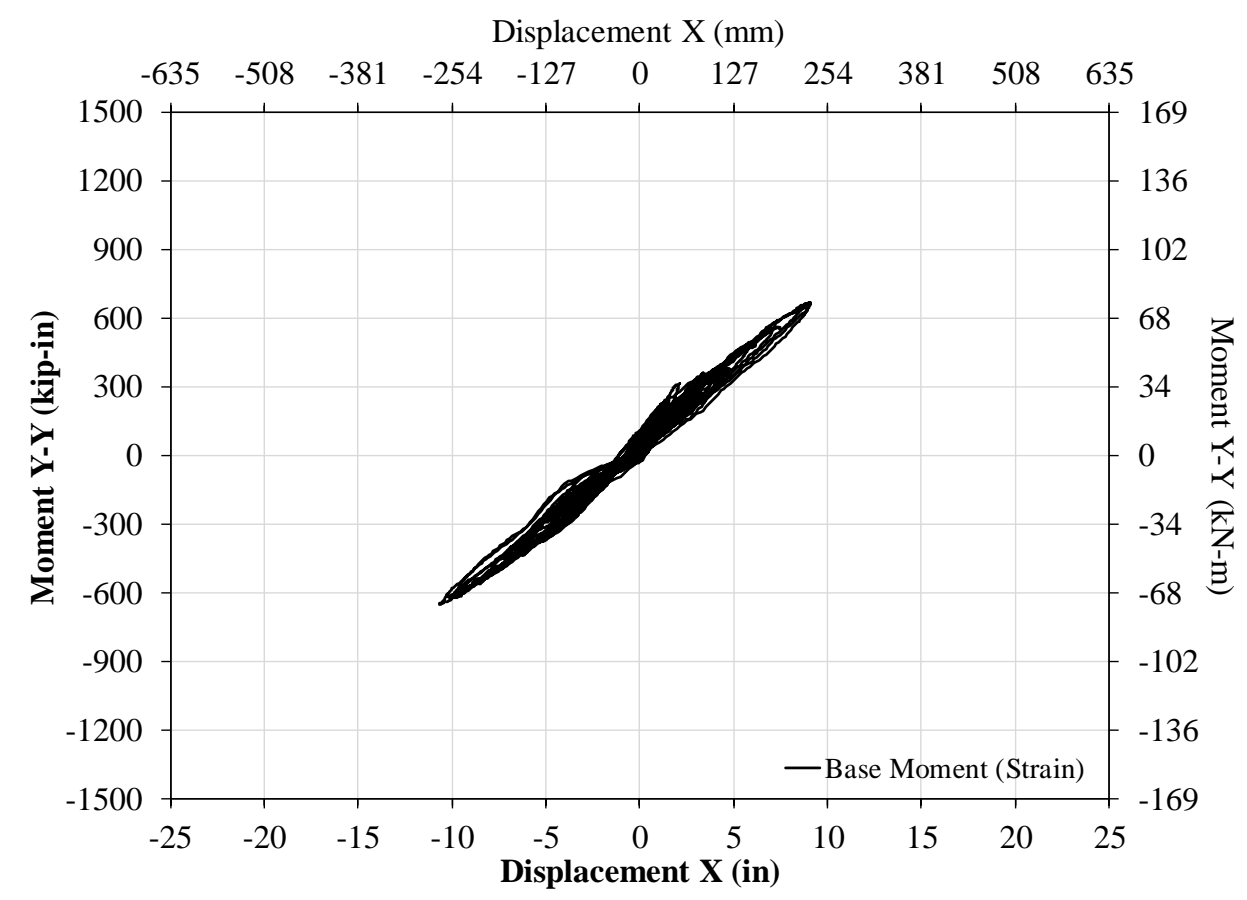

Figure 28-7: 50\% 0.5g IEEE693 X-System Response w/ Hysteretic Device

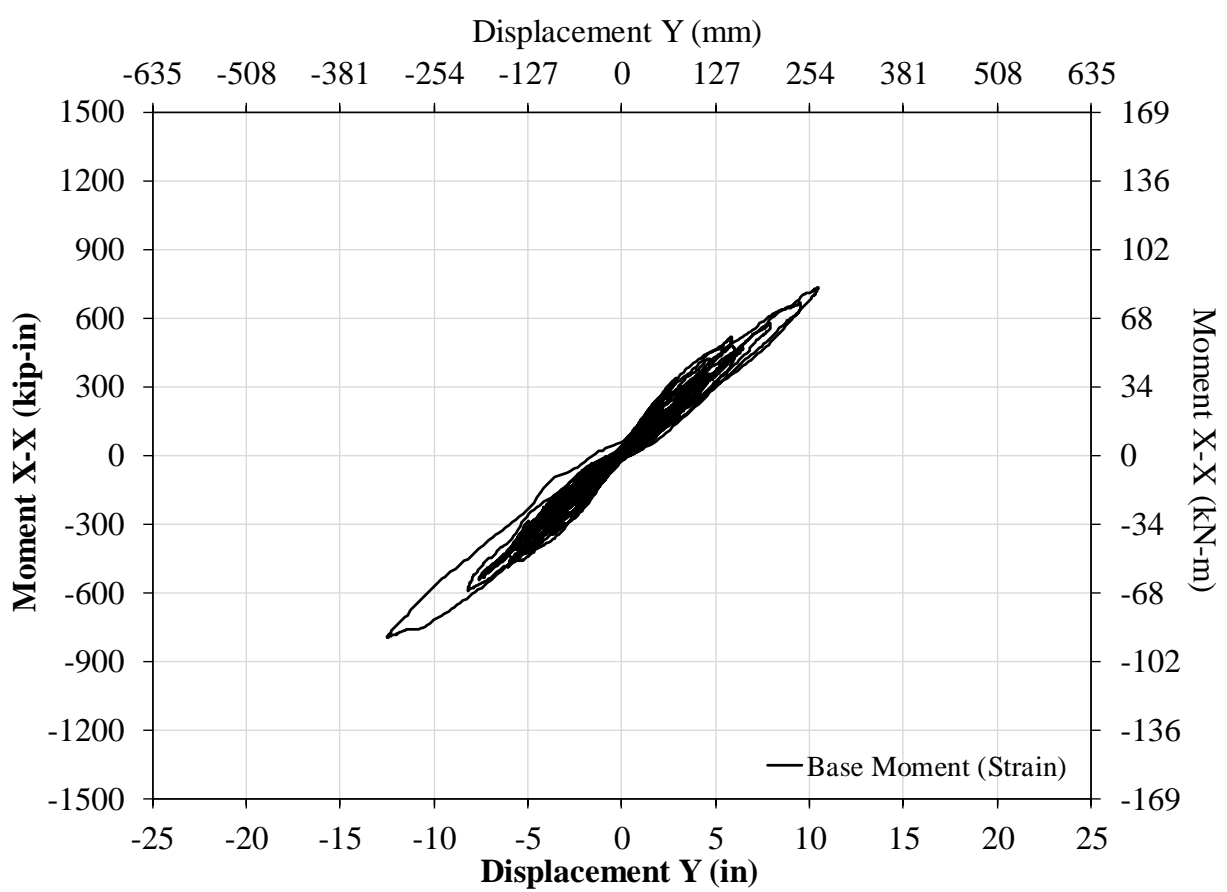

Figure 28-8: 50\% 0.5g IEEE693 Y-System Response w/ Hysteretic Device 


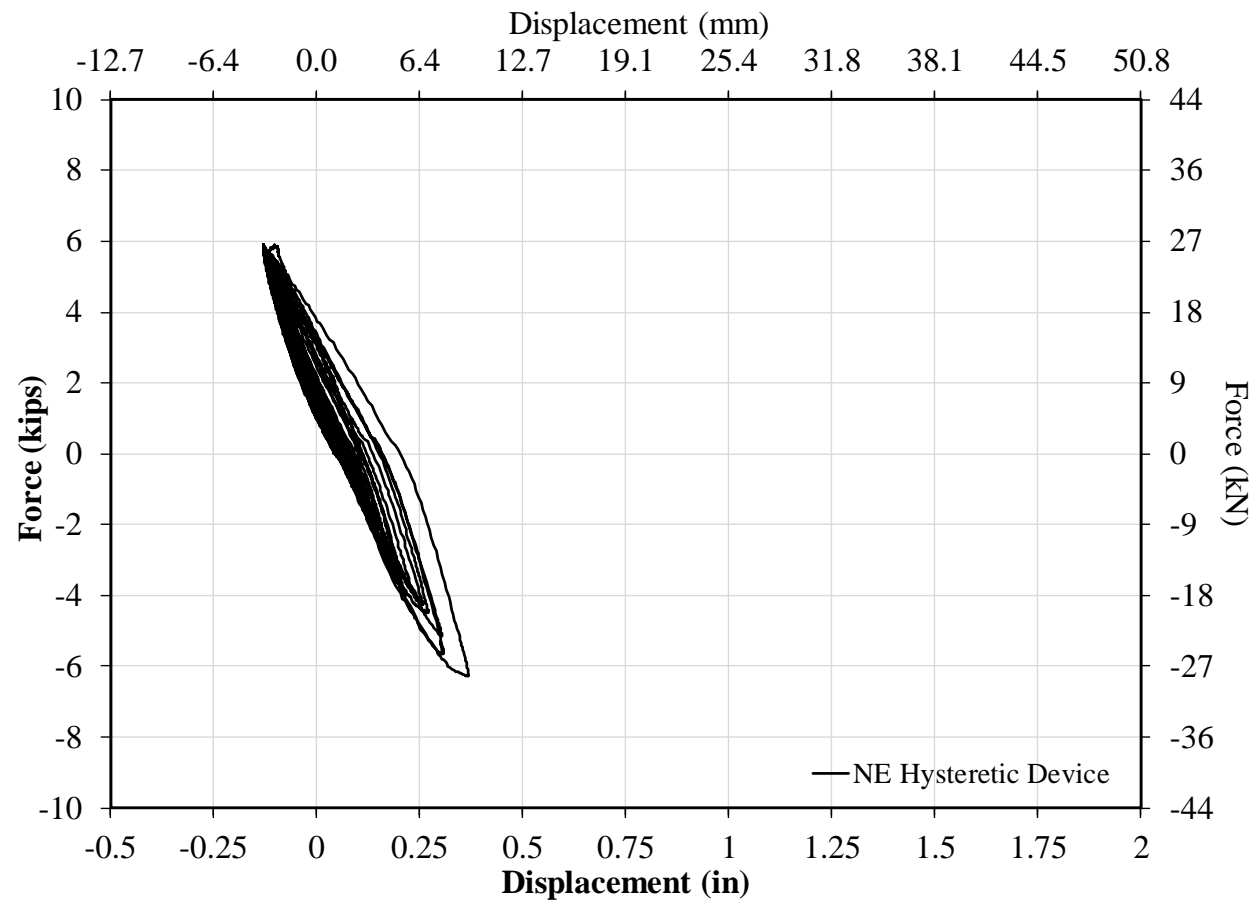

Figure 28-9: 50\% 0.5g IEEE693 NE Hysteretic Device Response

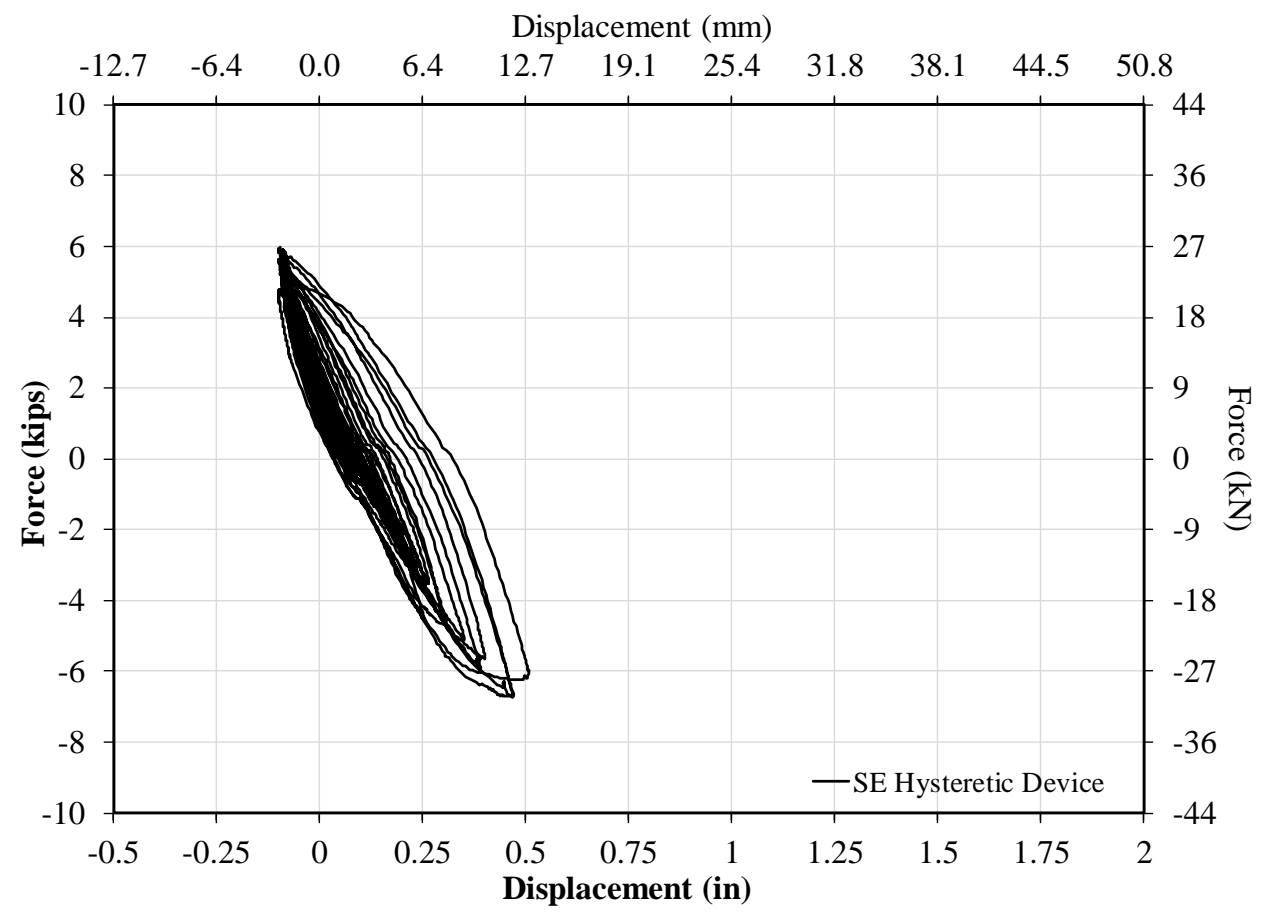

Figure 28-10: 20\% 0.5g IEEE693 SE Hysteretic Device Response 


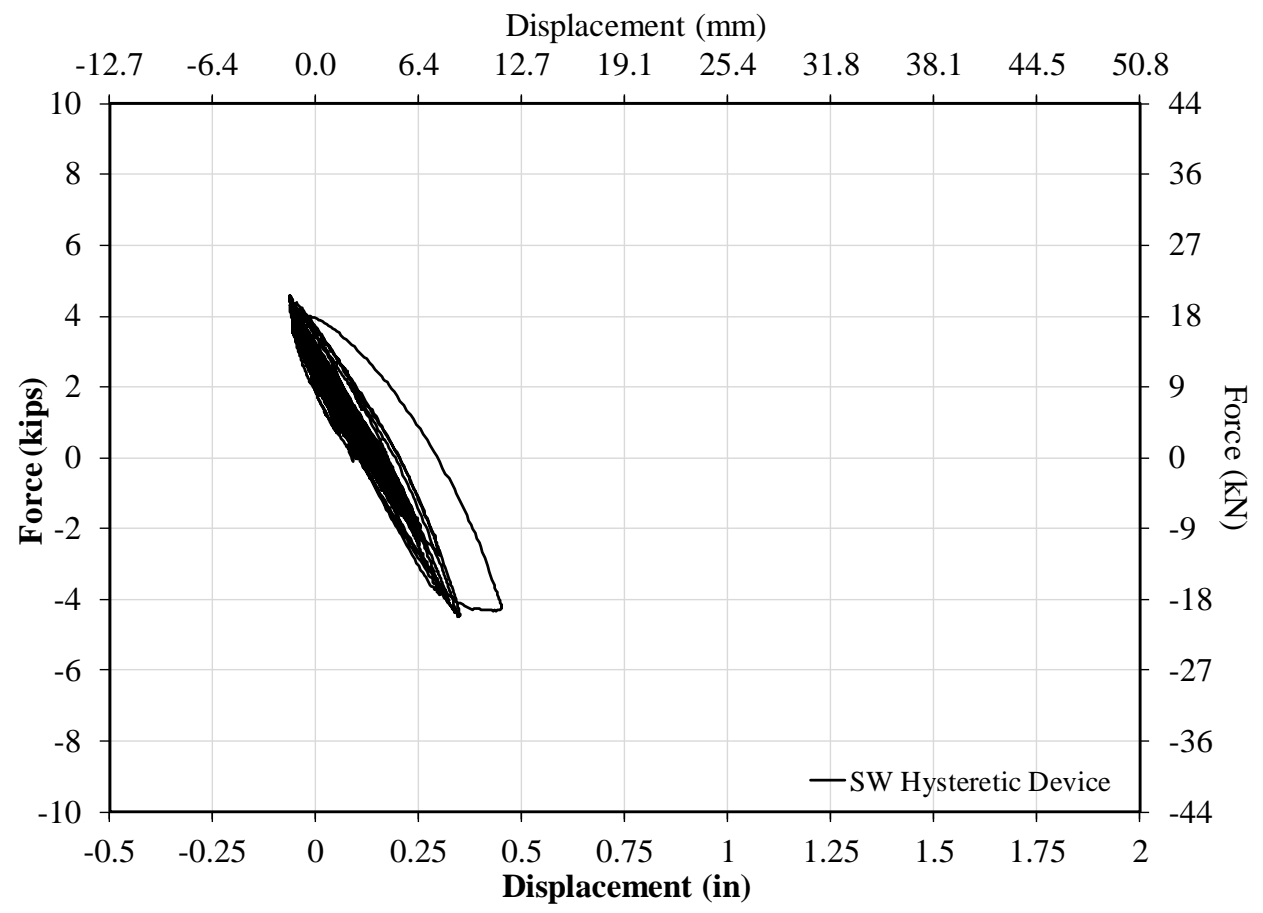

Figure 28-11: 50\% 0.5g IEEE693 SW Hysteretic Device Response

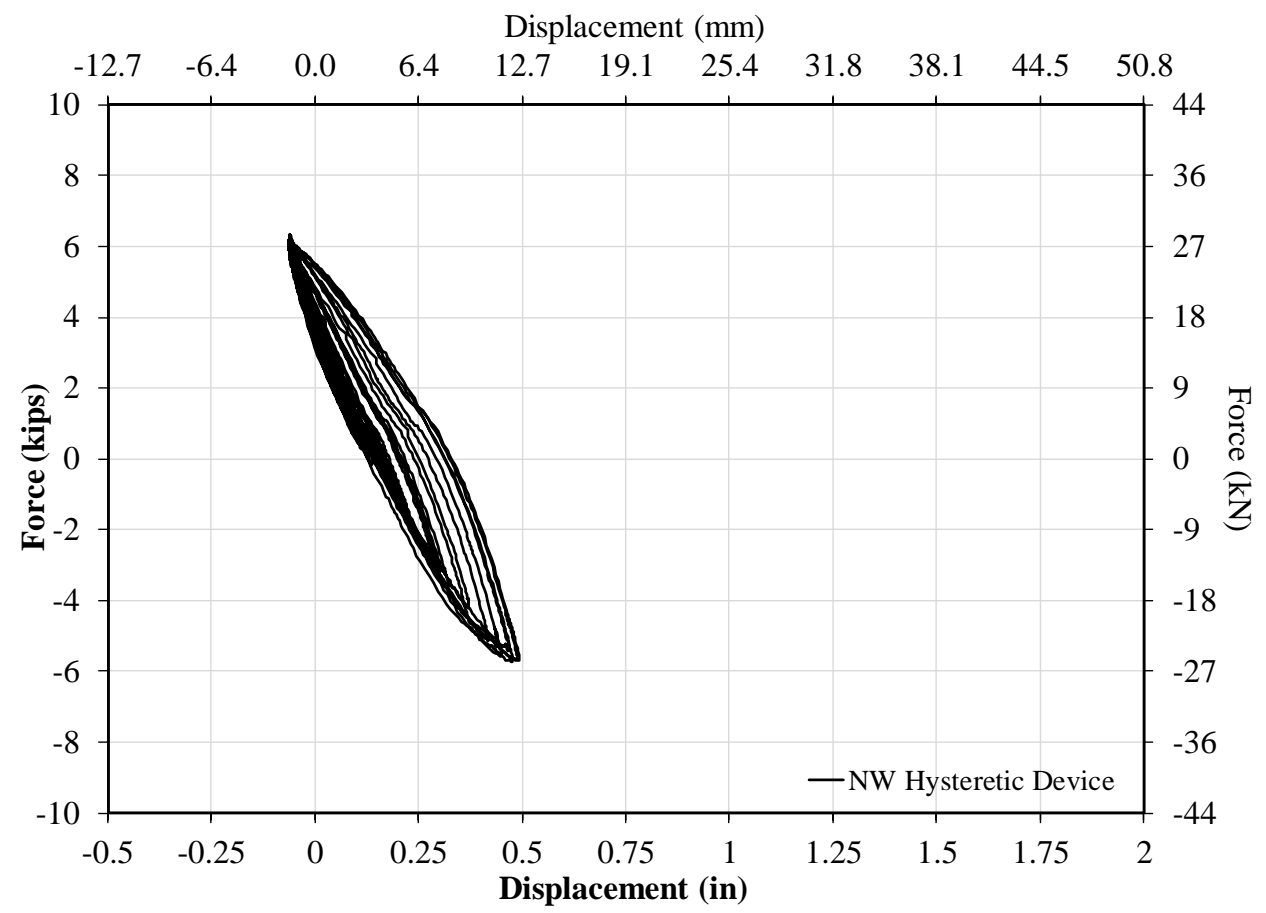

Figure 28-12: 50\% 0.5g IEEE693 NW Hysteretic Device Response 


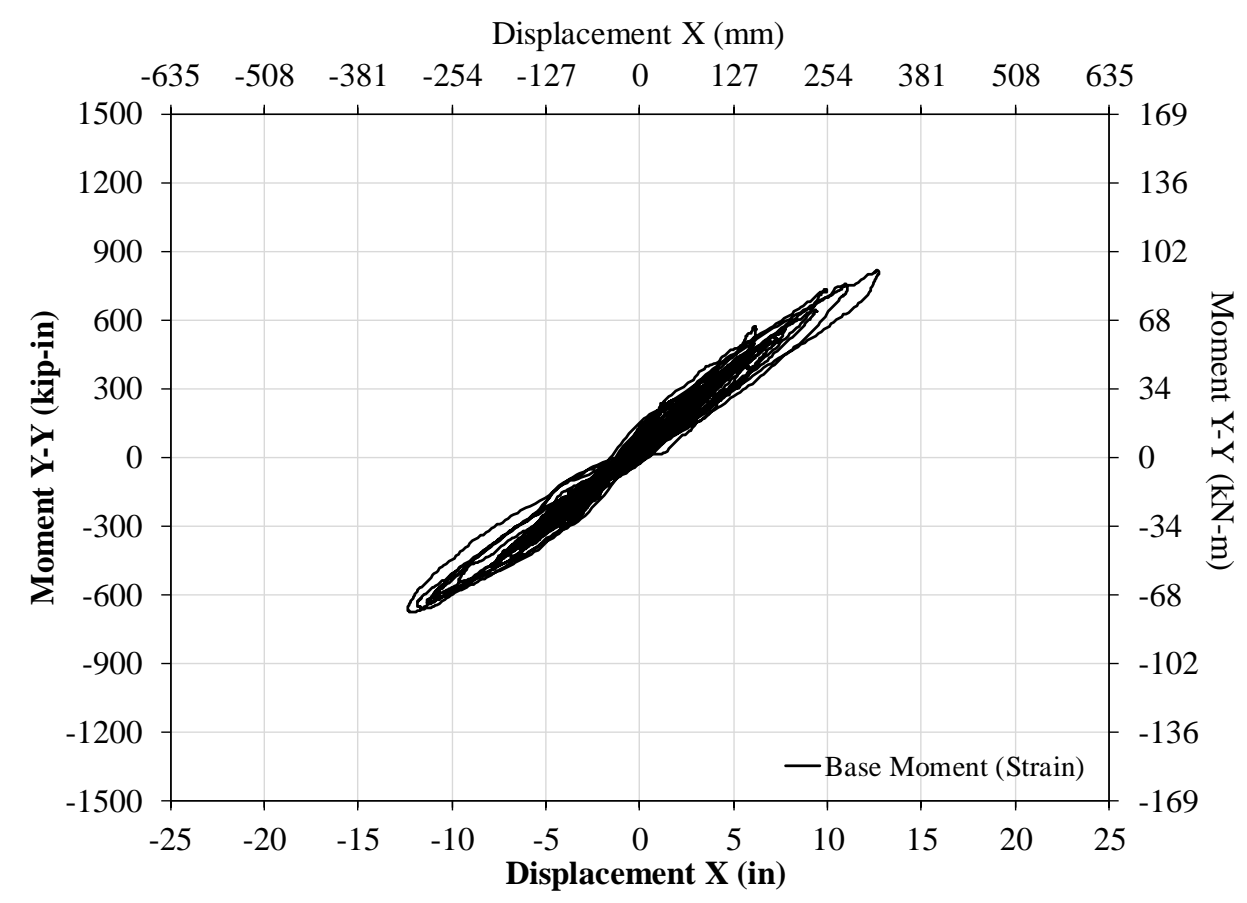

Figure 28-13: 75\% 0.5g IEEE693 X-System Response w/ Hysteretic Device

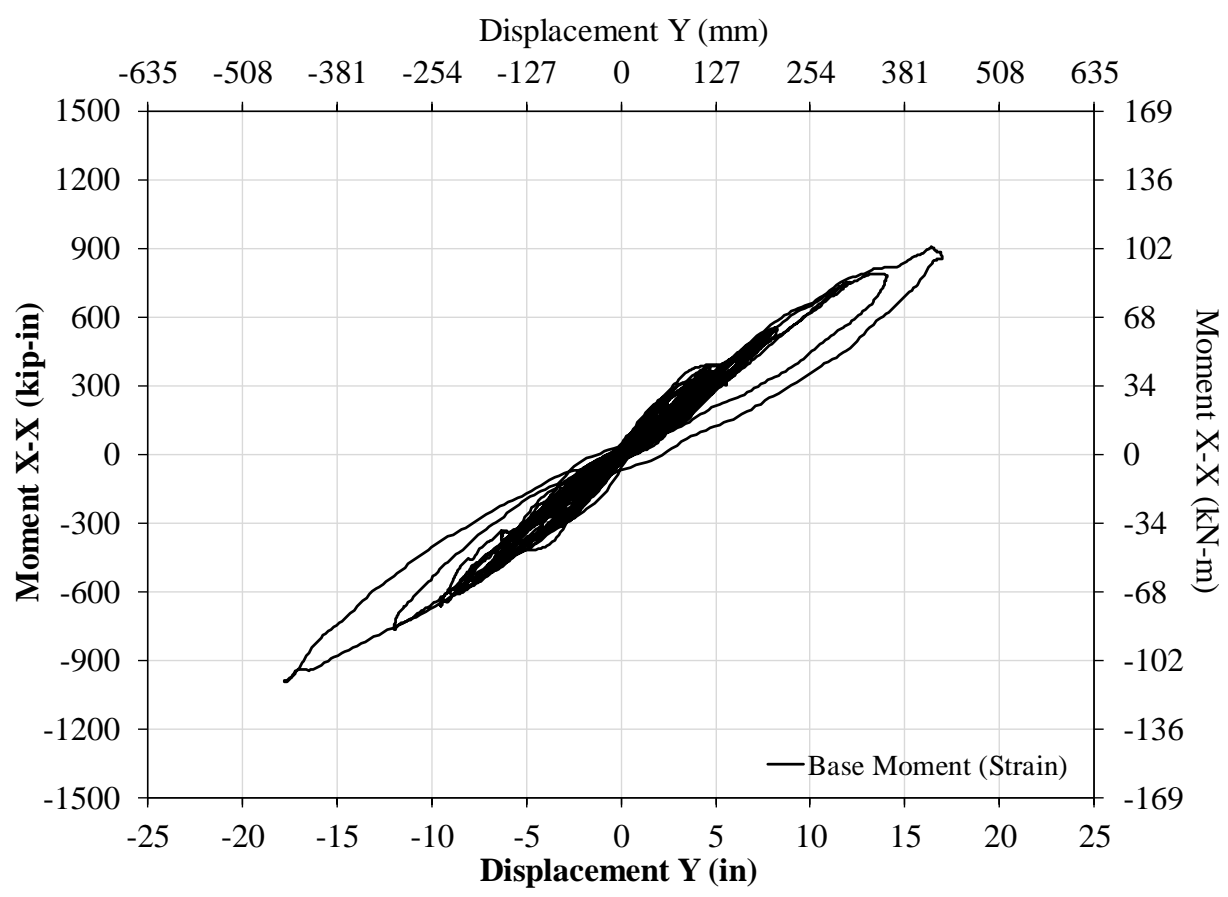

Figure 28-14: 75\% 0.5g IEEE693 Y-System Response w/ Hysteretic Device 


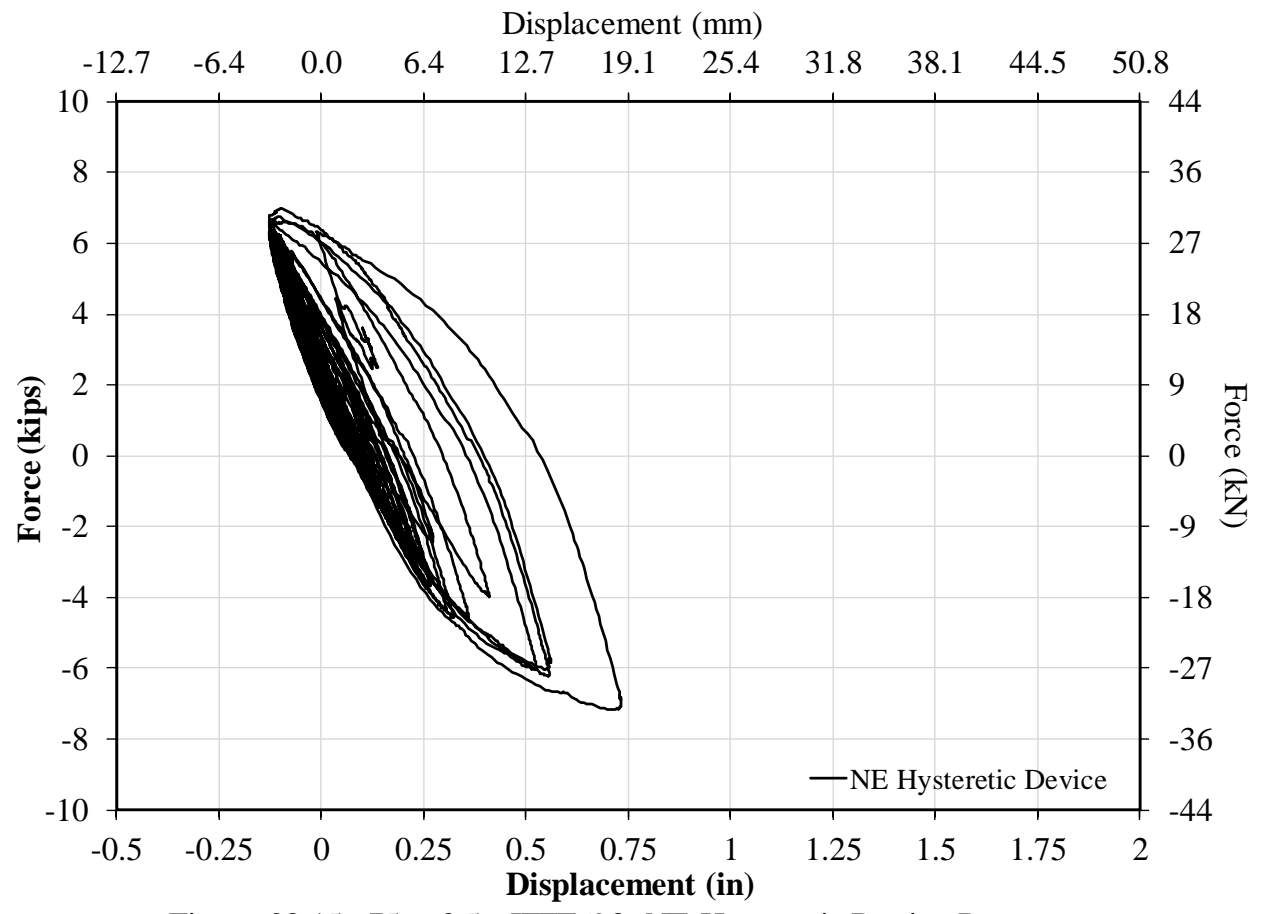

Figure 28-15: 75\% 0.5g IEEE693 NE Hysteretic Device Response

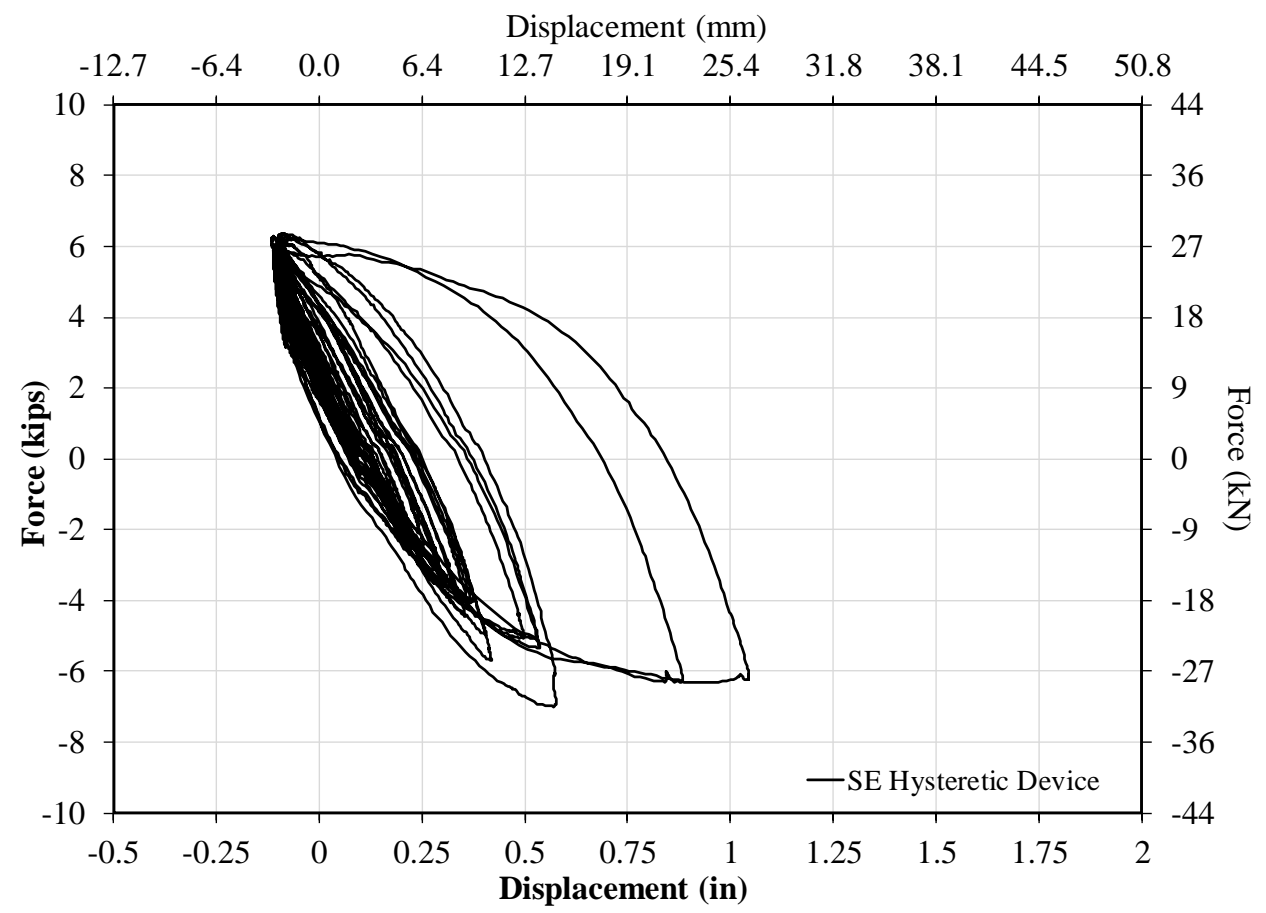

Figure 28-16: 75\% 0.5g IEEE693 SE Hysteretic Device Response 


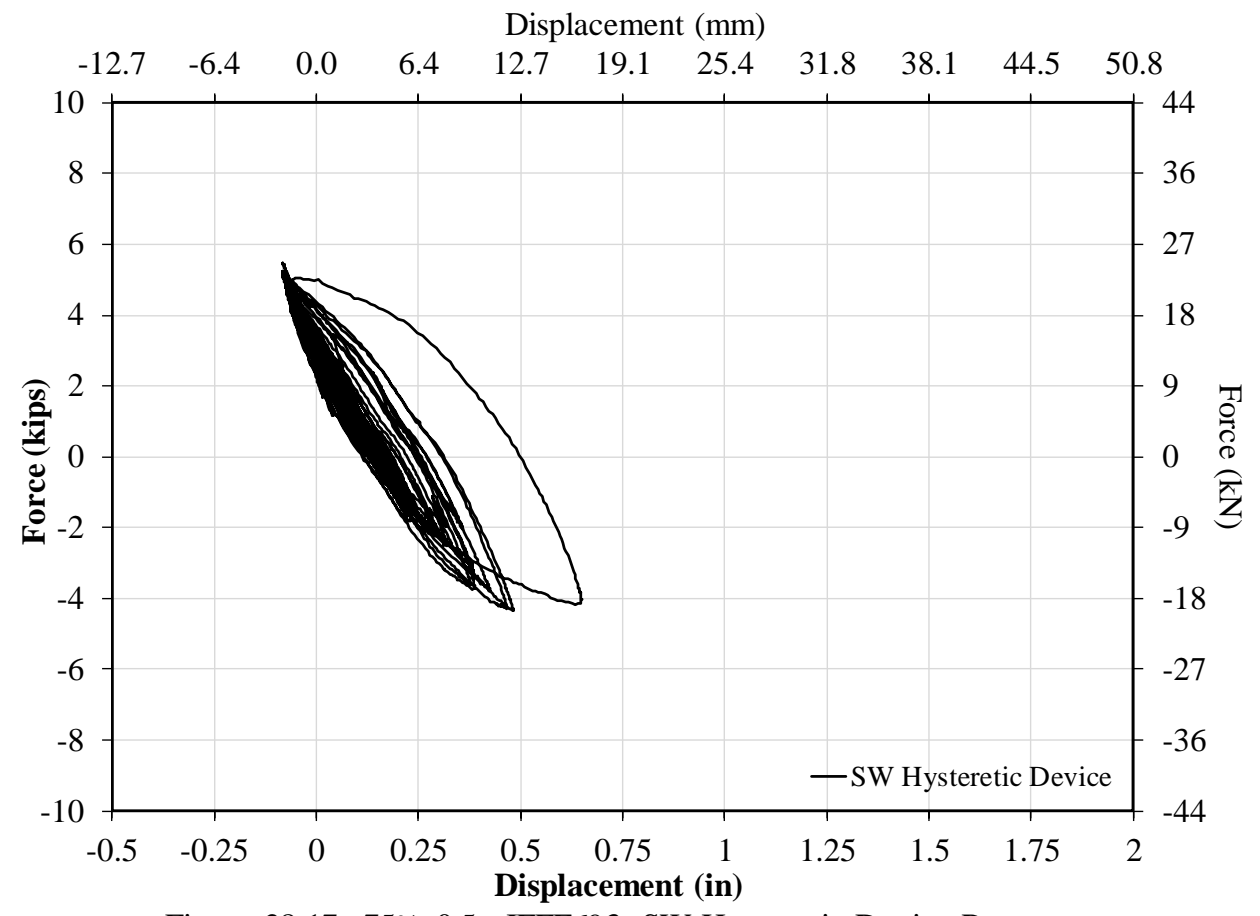

Figure 28-17: 75\% 0.5g IEEE693 SW Hysteretic Device Response

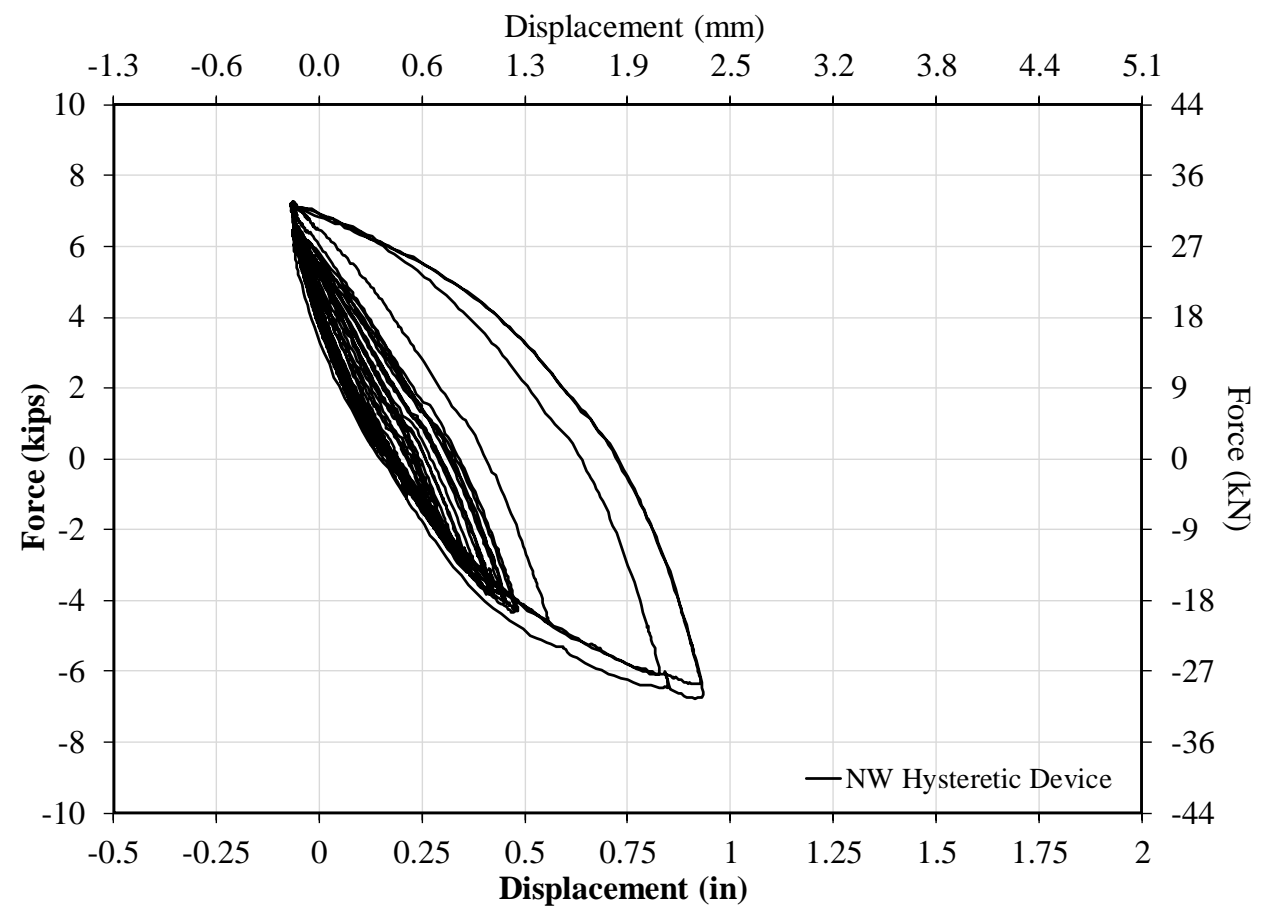

Figure 28-18: 75\% 0.5g IEEE693 NW Hysteretic Device Response 


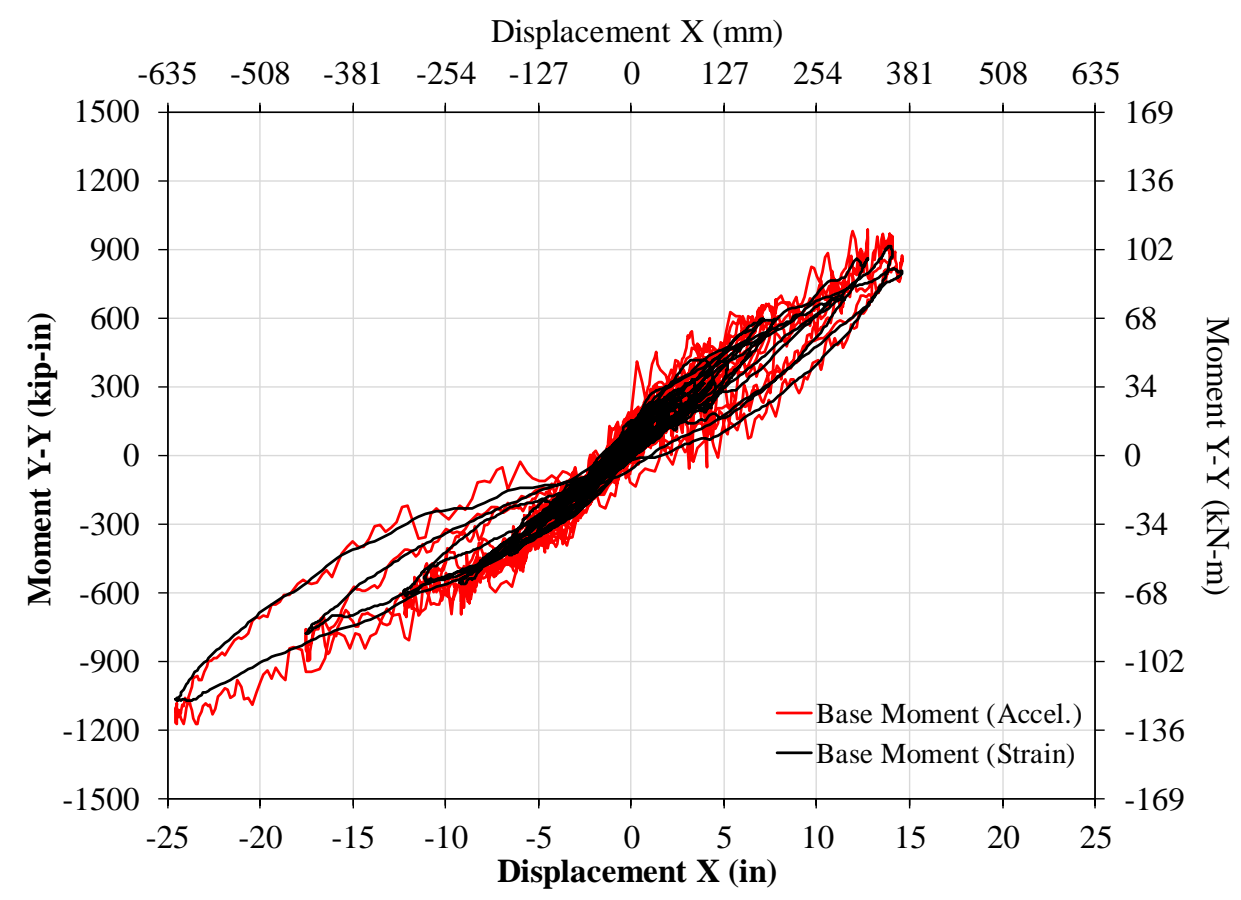

Figure 28-19: 100\% 0.5g IEEE693 X-System Response w/ Hysteretic Device

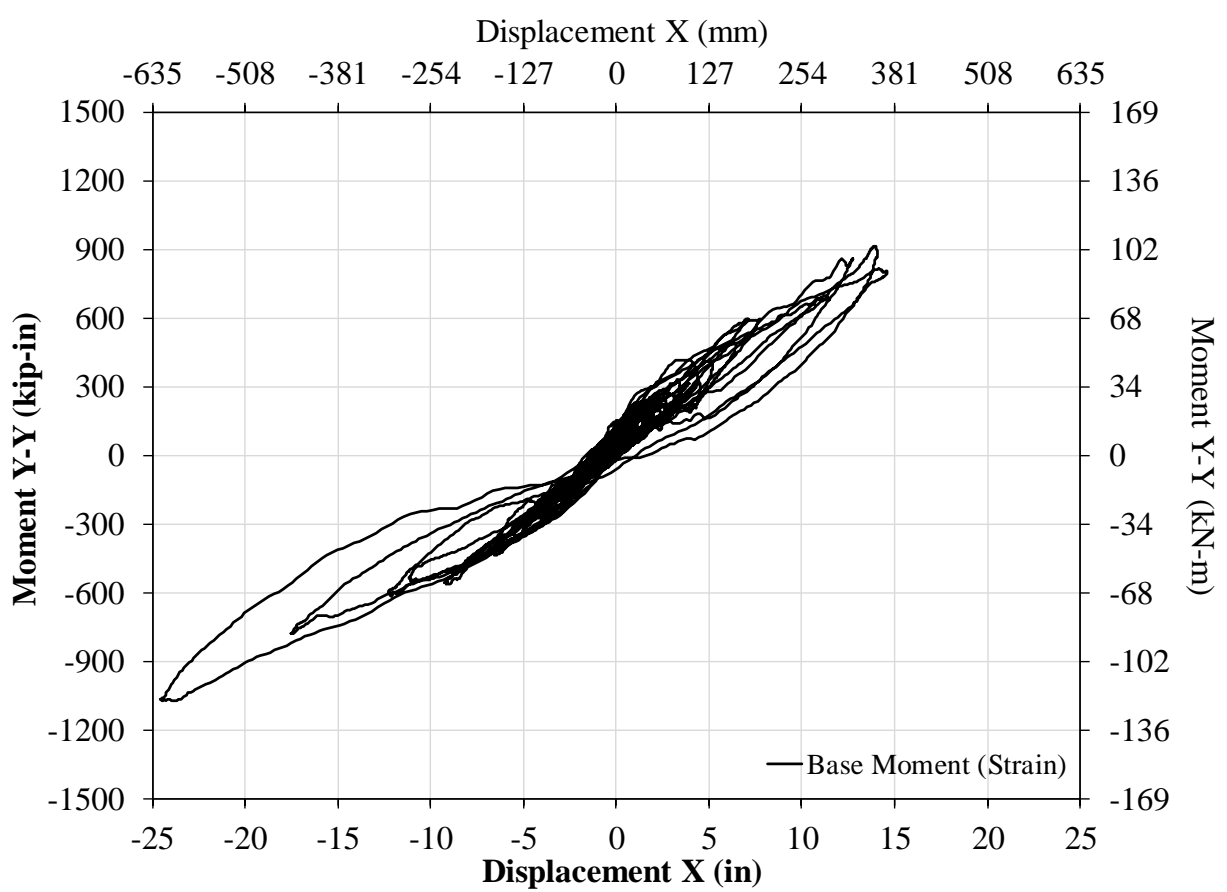

Figure 28-20: 100\% 0.5g IEEE693 X-System Response w/ Hysteretic Device 


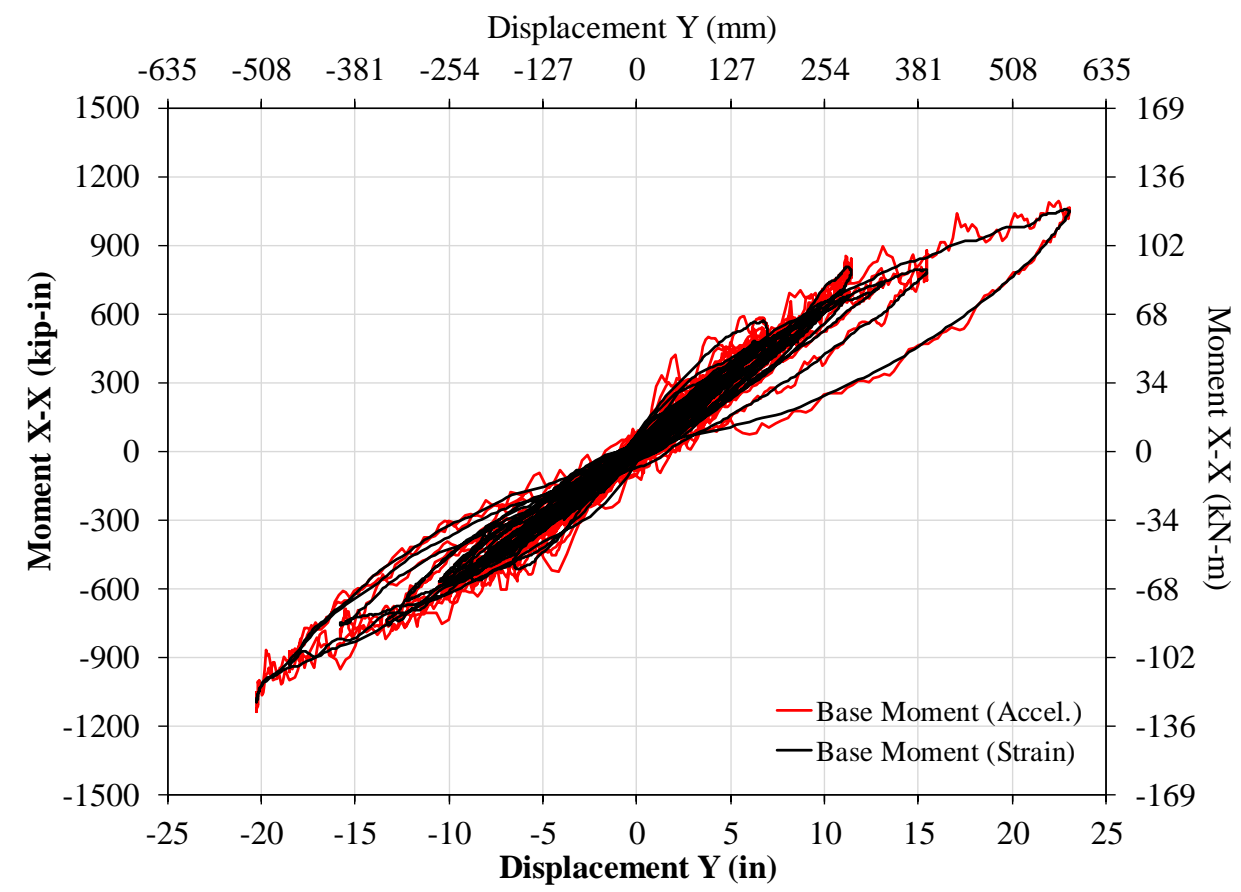

Figure 28-21: 100\% 0.5g IEEE693 Y-System Response w/ Hysteretic Device

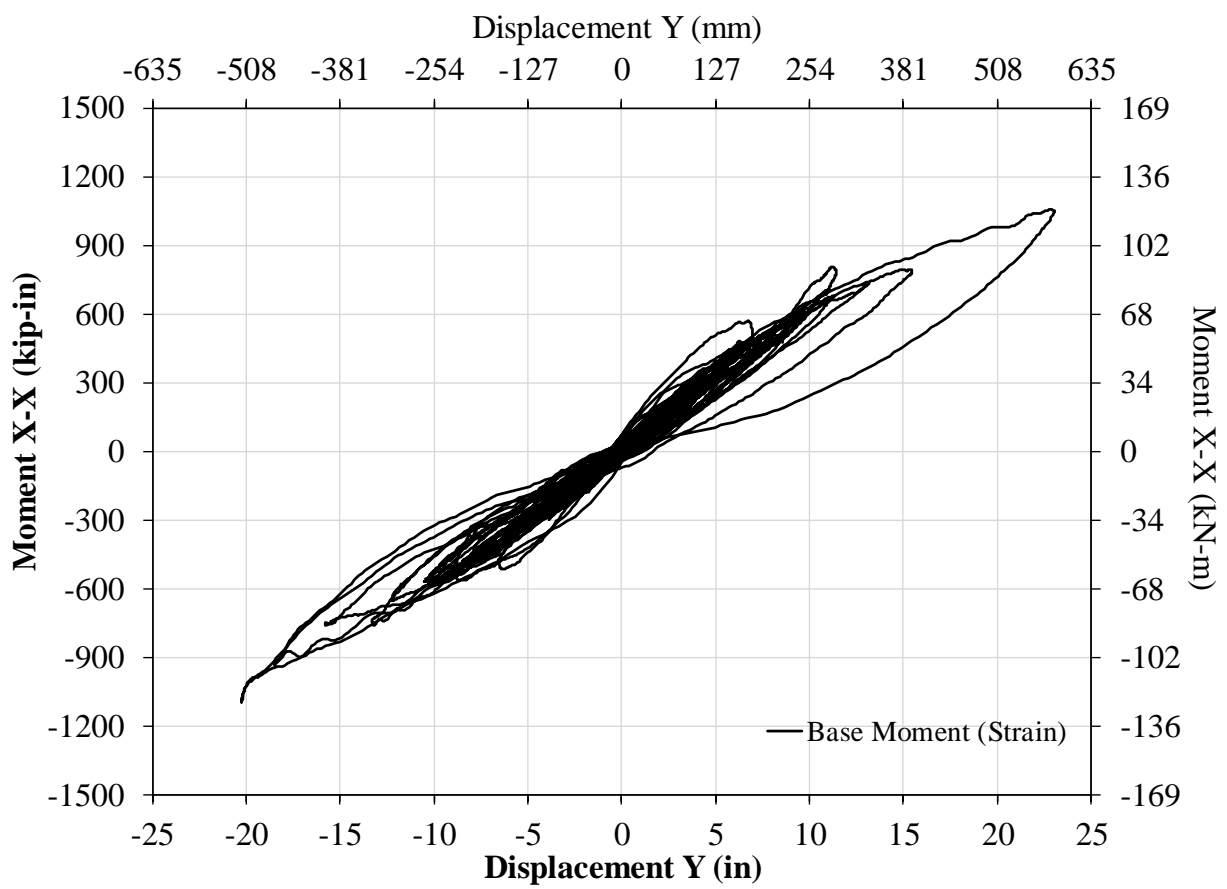

Figure 28-22: 100\% 0.5g IEEE693 Y-System Response w/ Hysteretic Device 


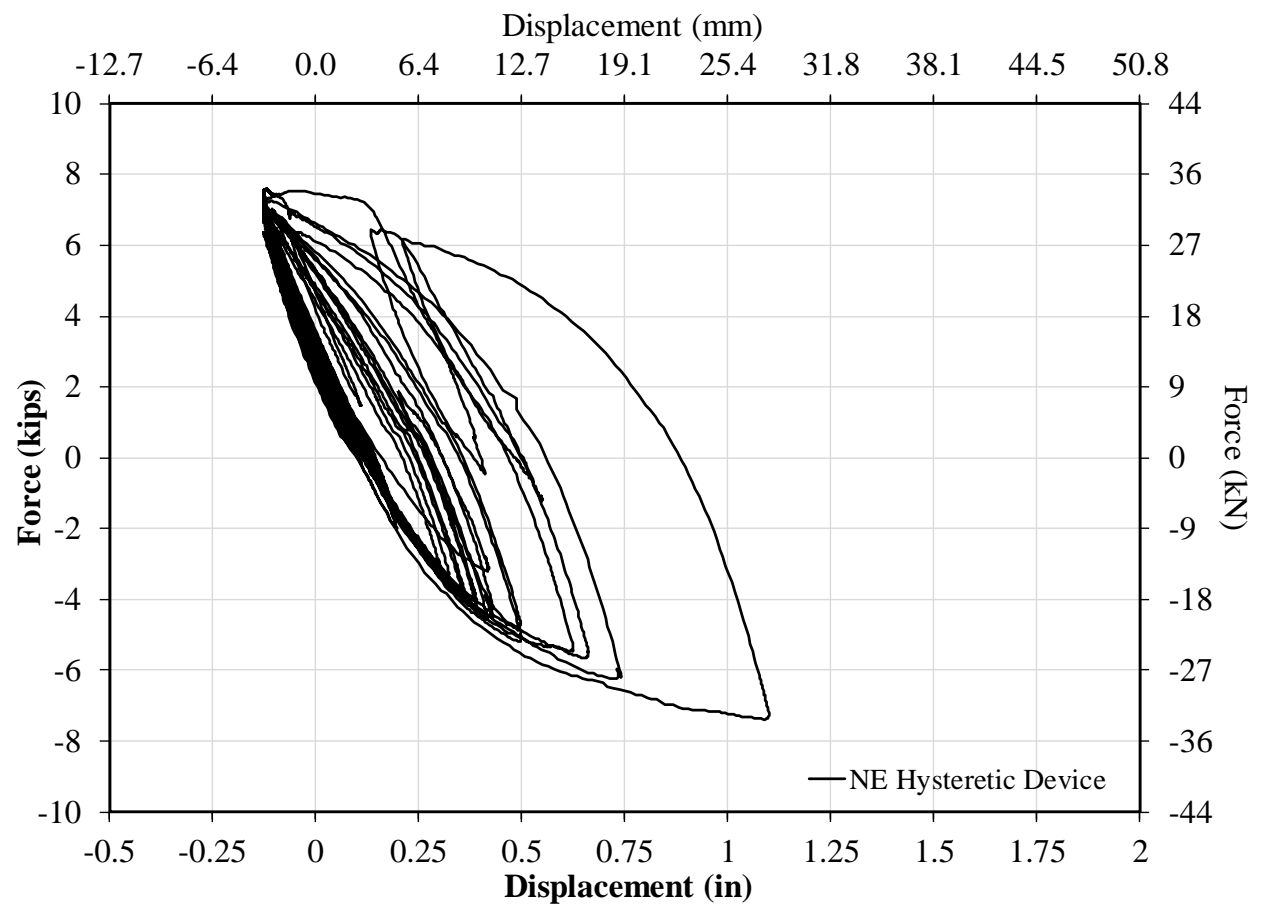

Figure 28-23: $100 \%$ 0.5g IEEE693 NE Hysteretic Device Response

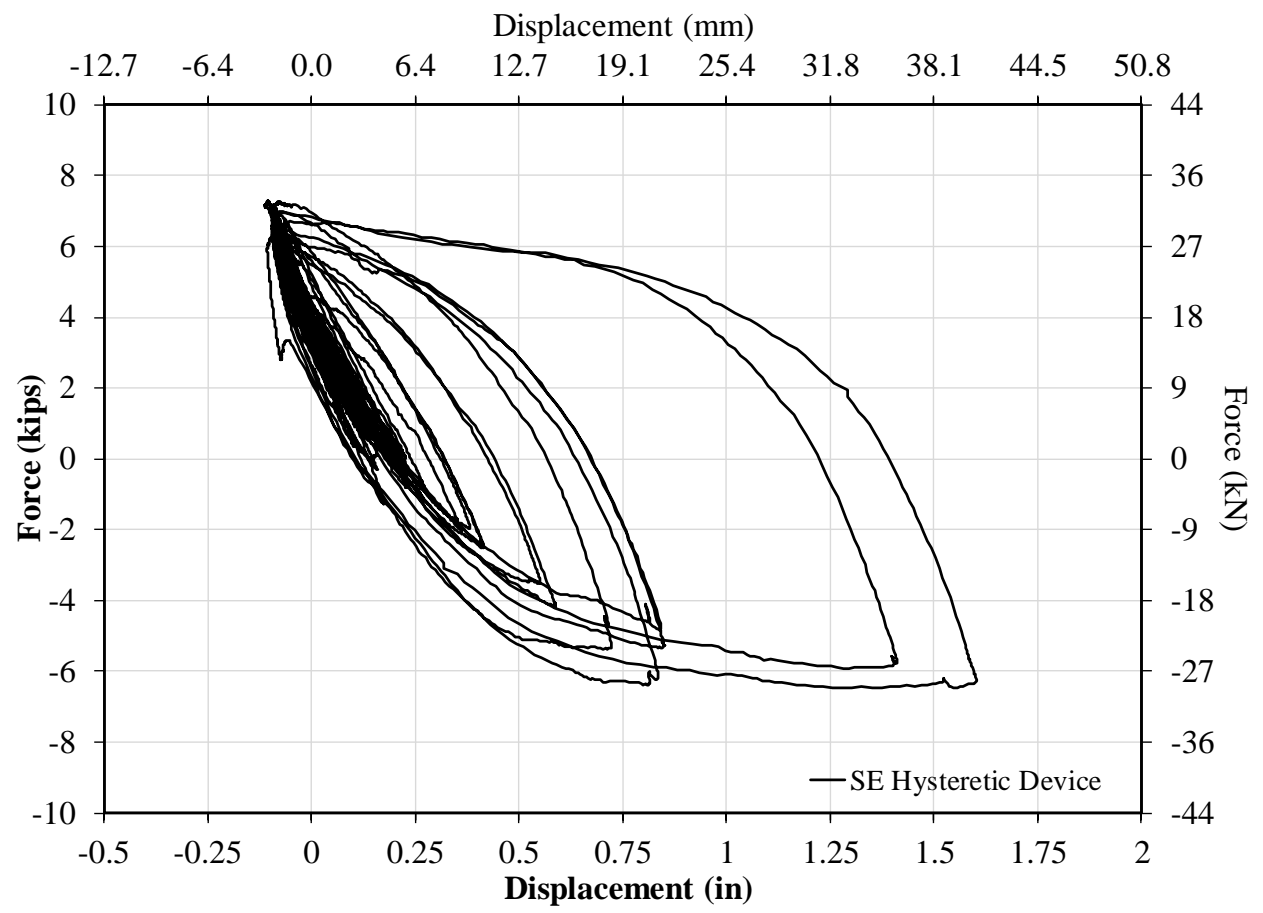

Figure 28-24: 100\% 0.5g IEEE693 SE Hysteretic Device Response 


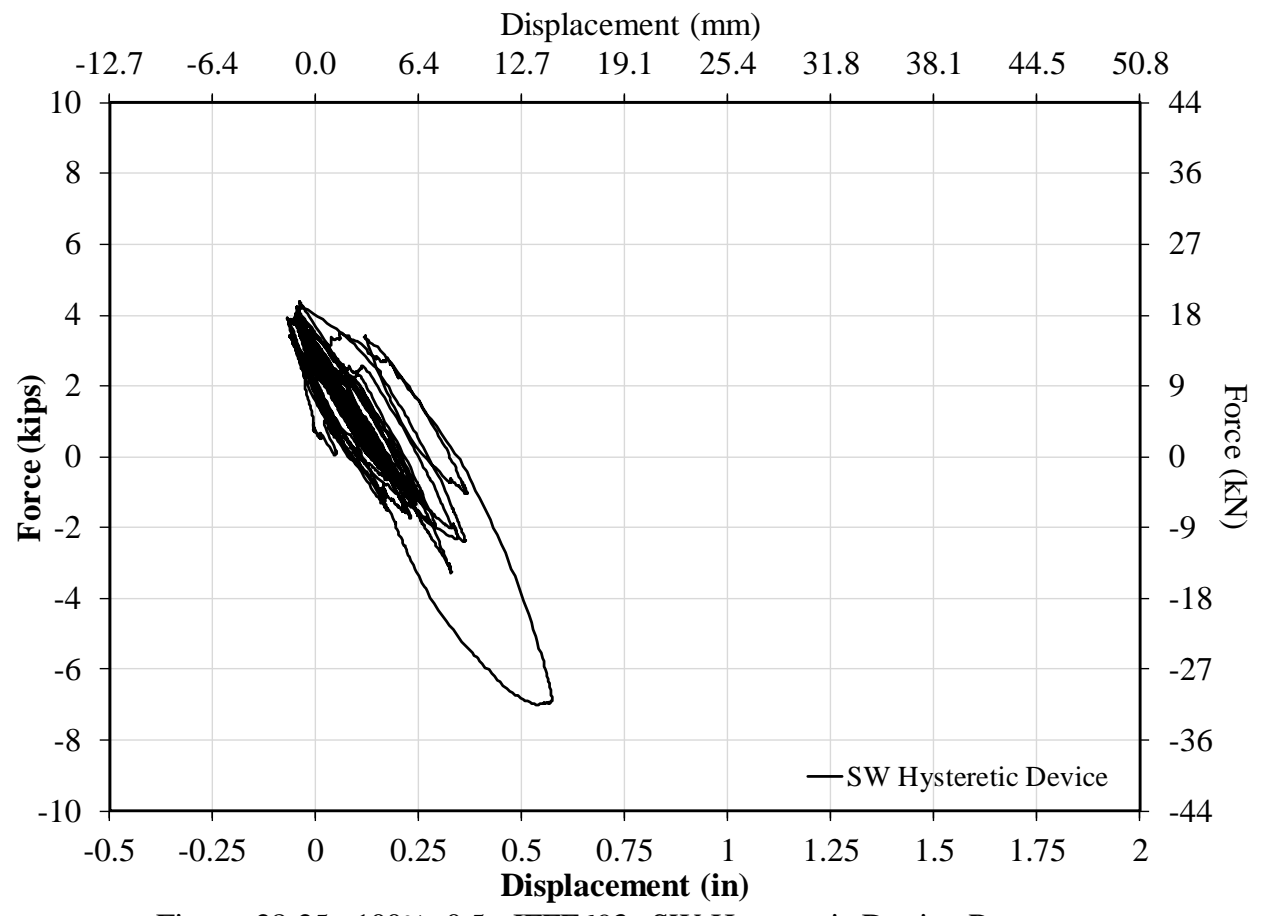

Figure 28-25: 100\% 0.5g IEEE693 SW Hysteretic Device Response

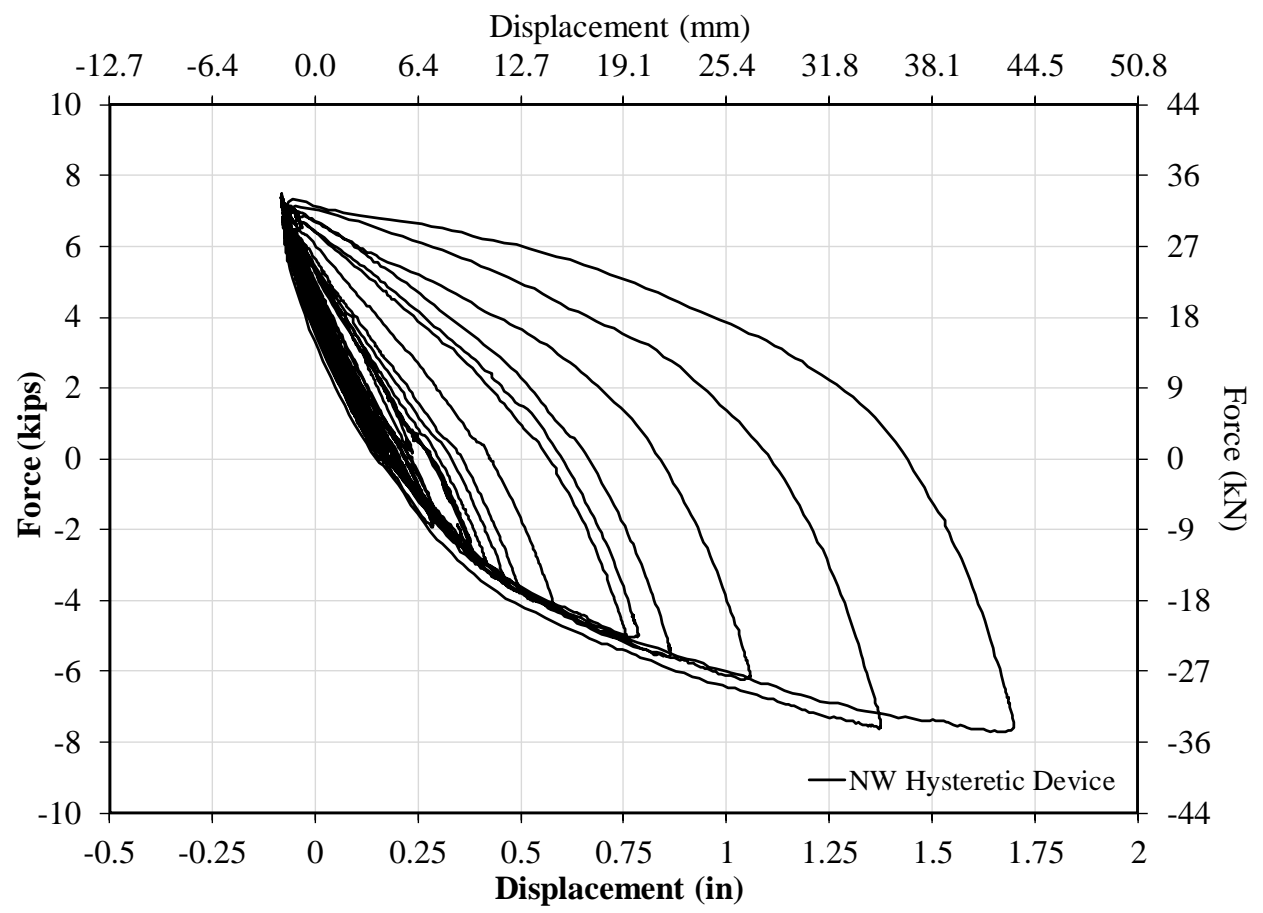

Figure 28-26: 100\% 0.5g IEEE693 NW Hysteretic Device Response 


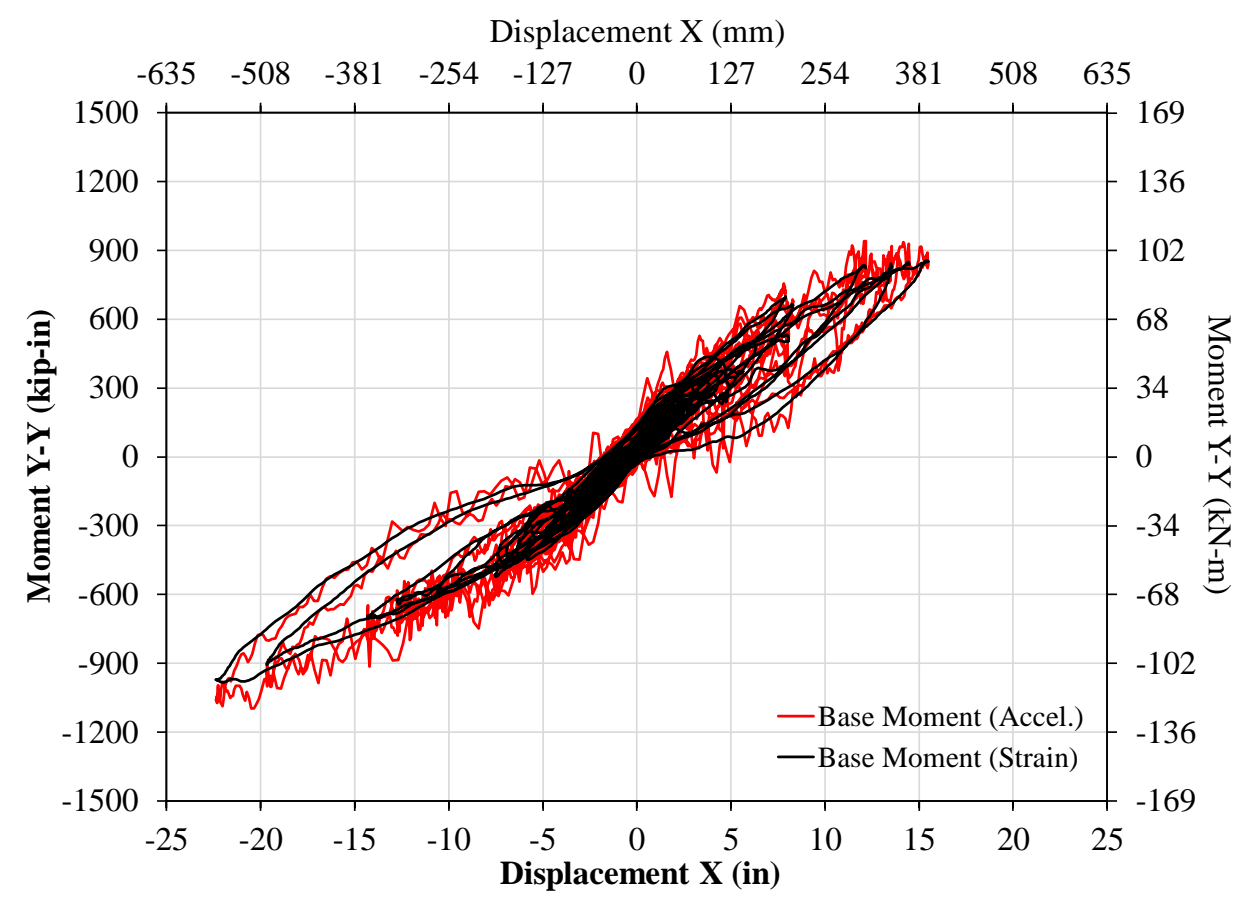

Figure 28-27: 100\% 0.5g IEEE693 X-System Response w/ Virgin Hysteretic Device

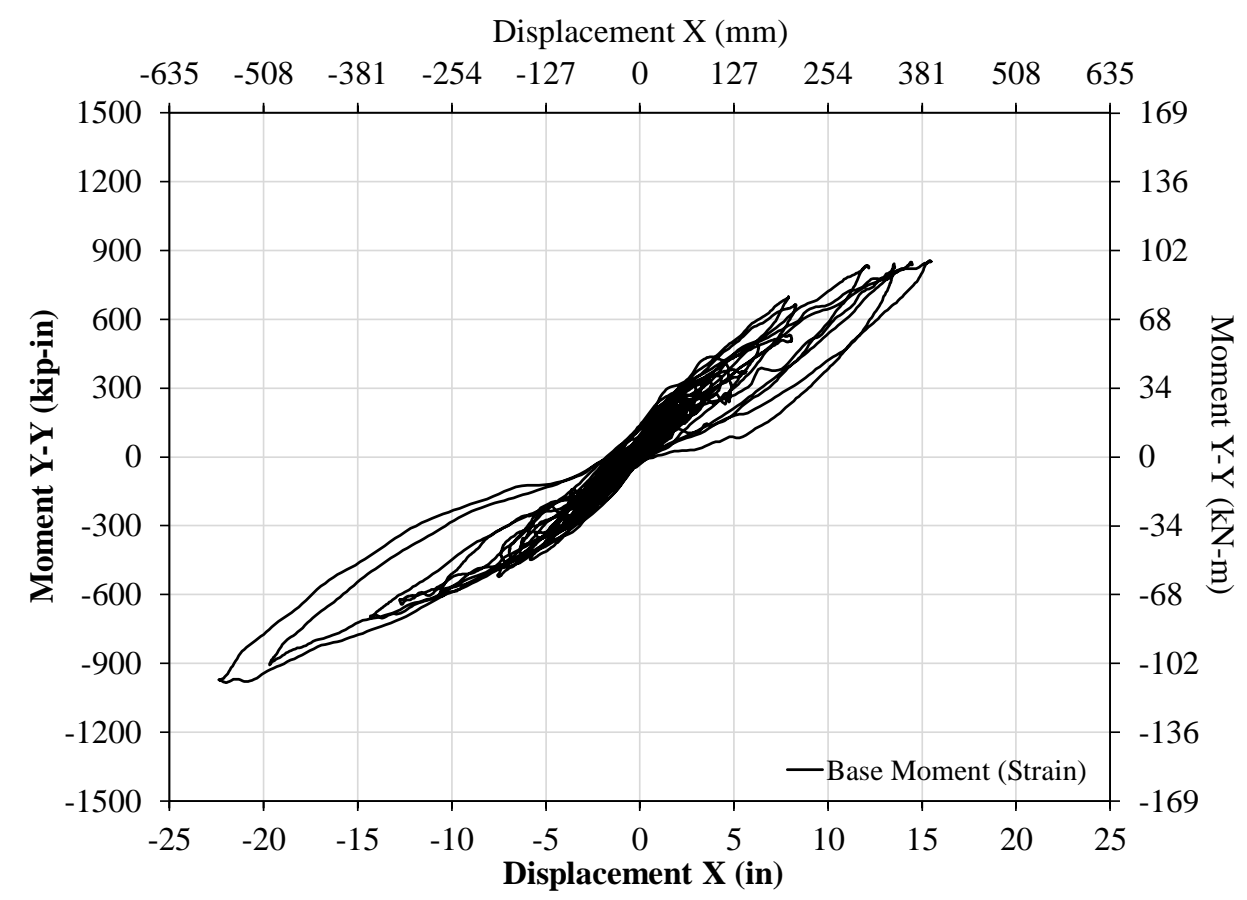

Figure 28-28: 100\% 0.5g IEEE693 X-System Response w/ Virgin Hysteretic Device 


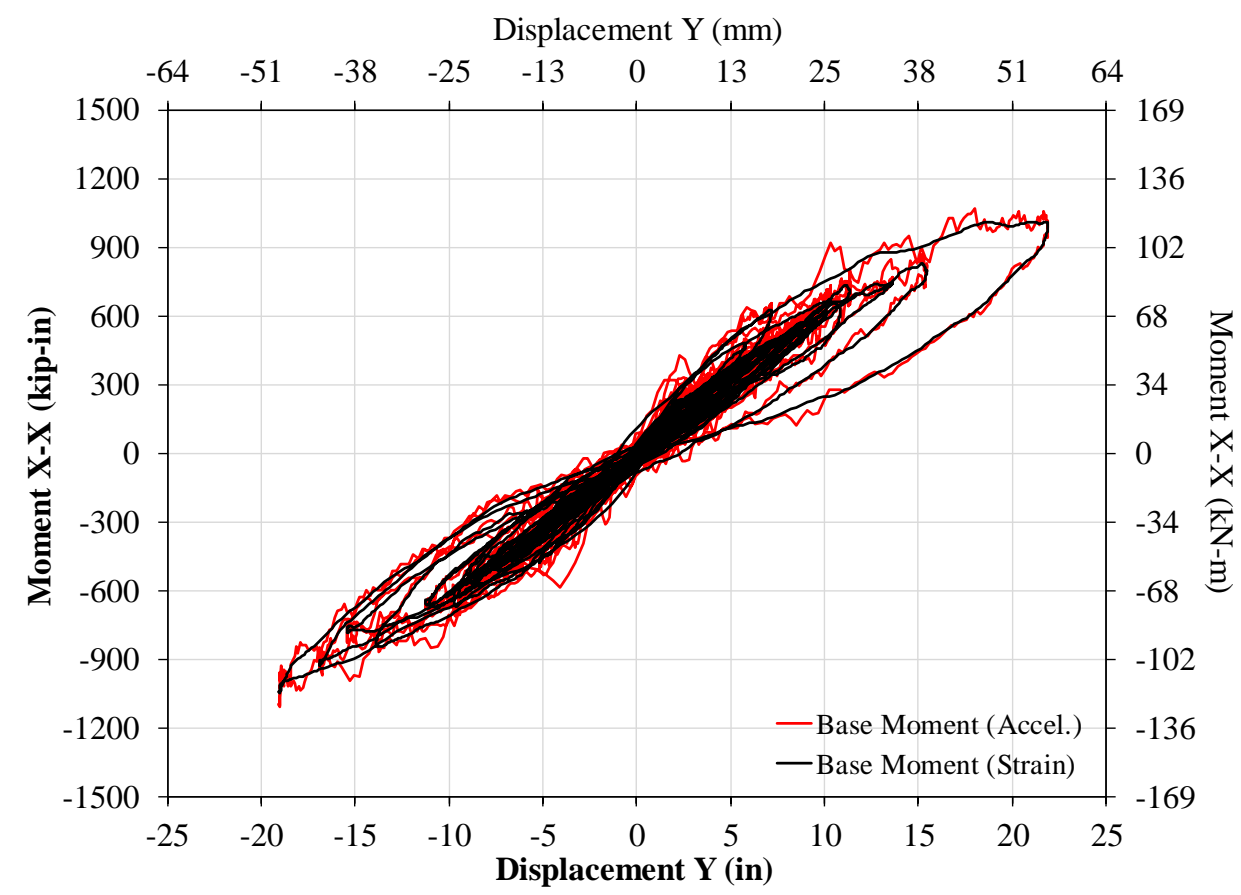

Figure 28-29: 100\% 0.5g IEEE693 Y-System Response w/ Virgin Hysteretic Device

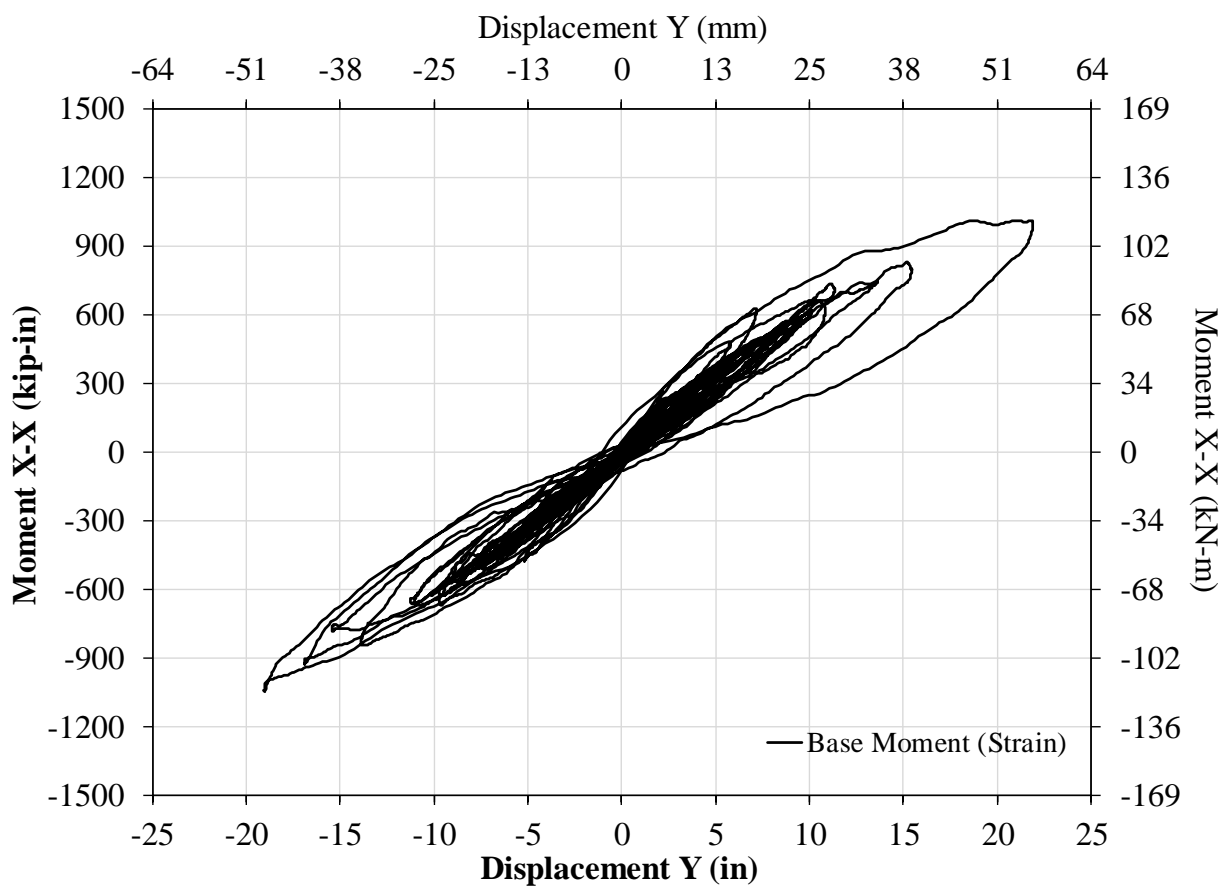

Figure 28-30: 100\% 0.5g IEEE693 Y-System Response w/ Virgin Hysteretic Device 


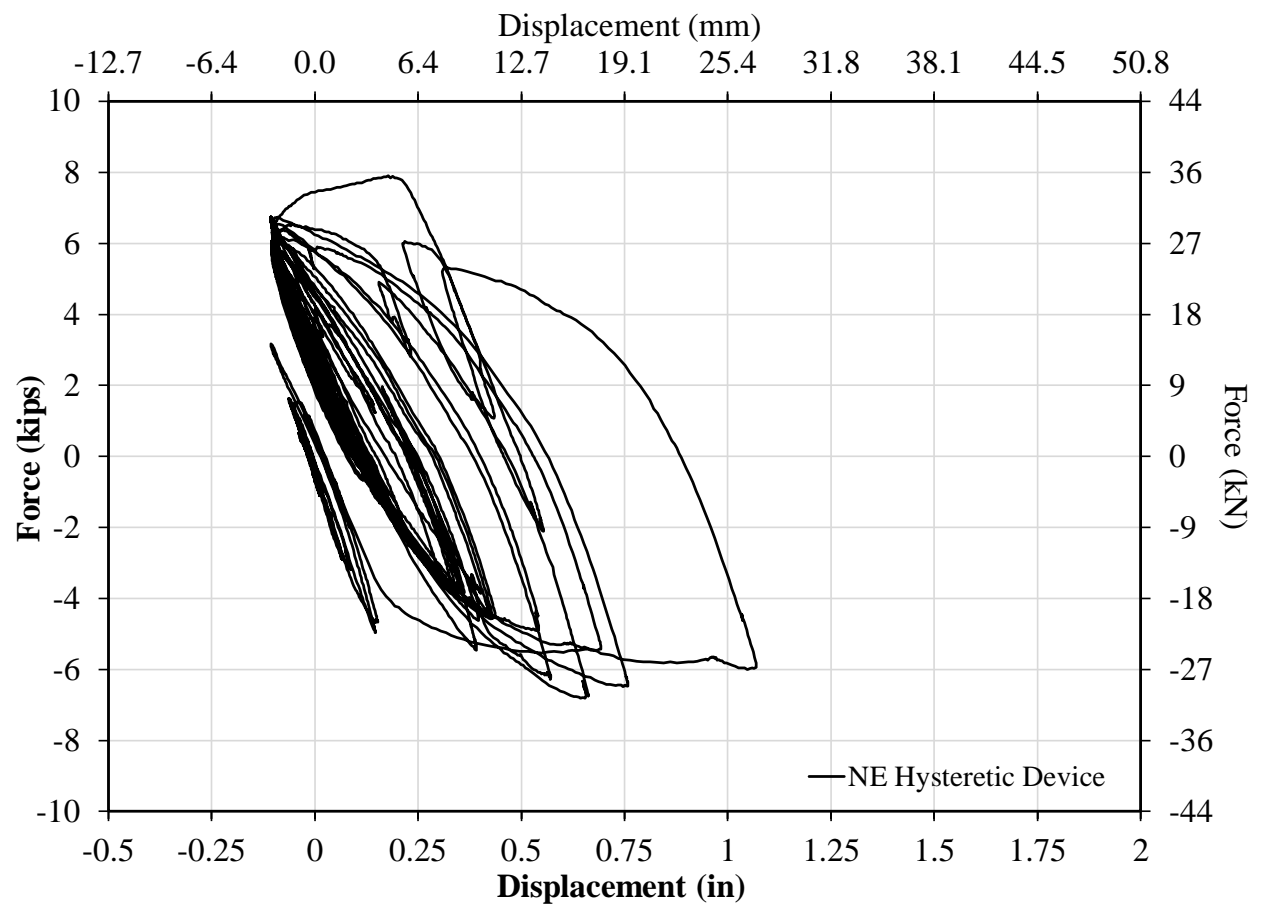

Figure 28-31: 100\% 0.5g IEEE693 NE Virgin Hysteretic Device Response

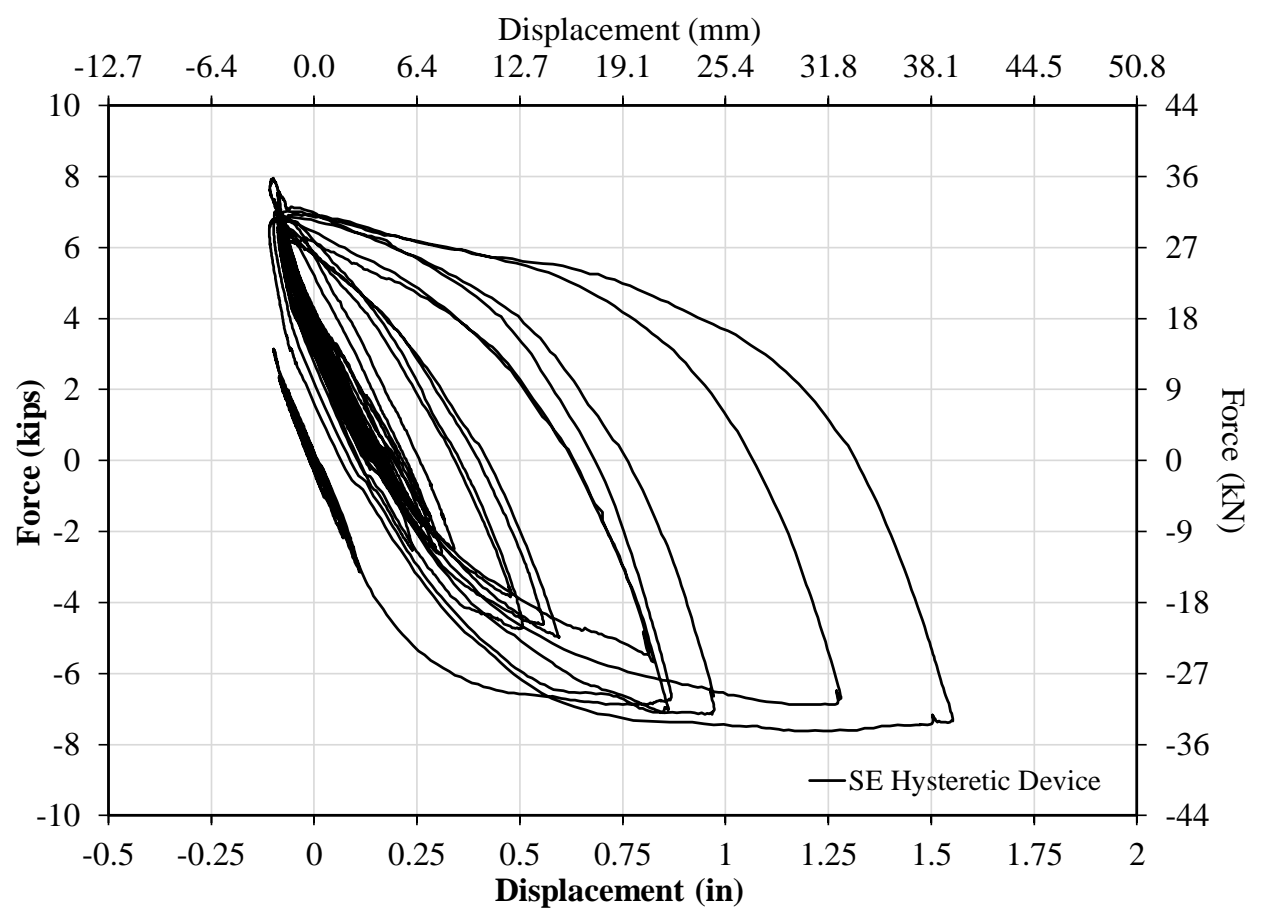

Figure 28-32: 100\% 0.5g IEEE693 SE Virgin Hysteretic Device Response 


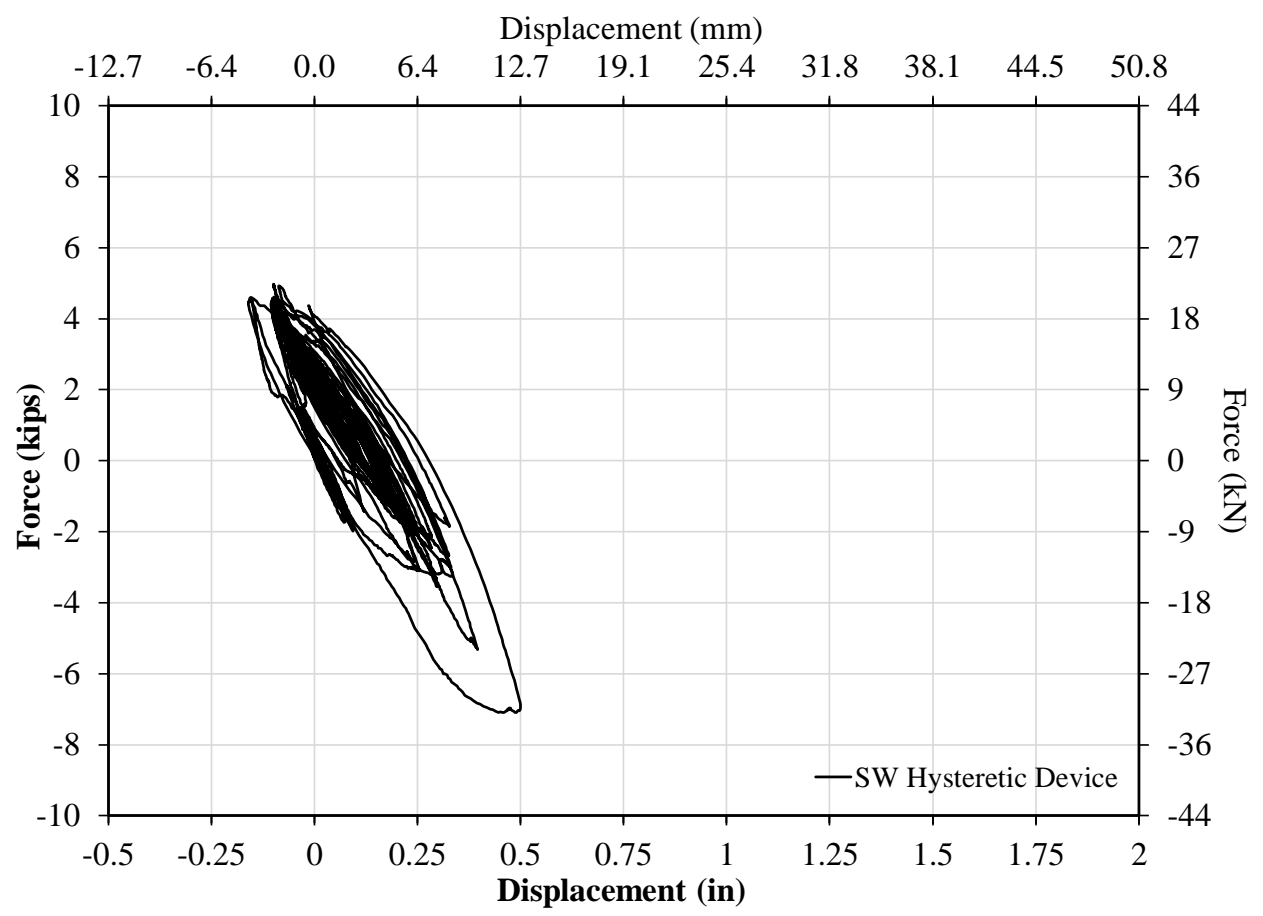

Figure 28-33: 100\% 0.5g IEEE693 NE SW Virgin Hysteretic Device Response

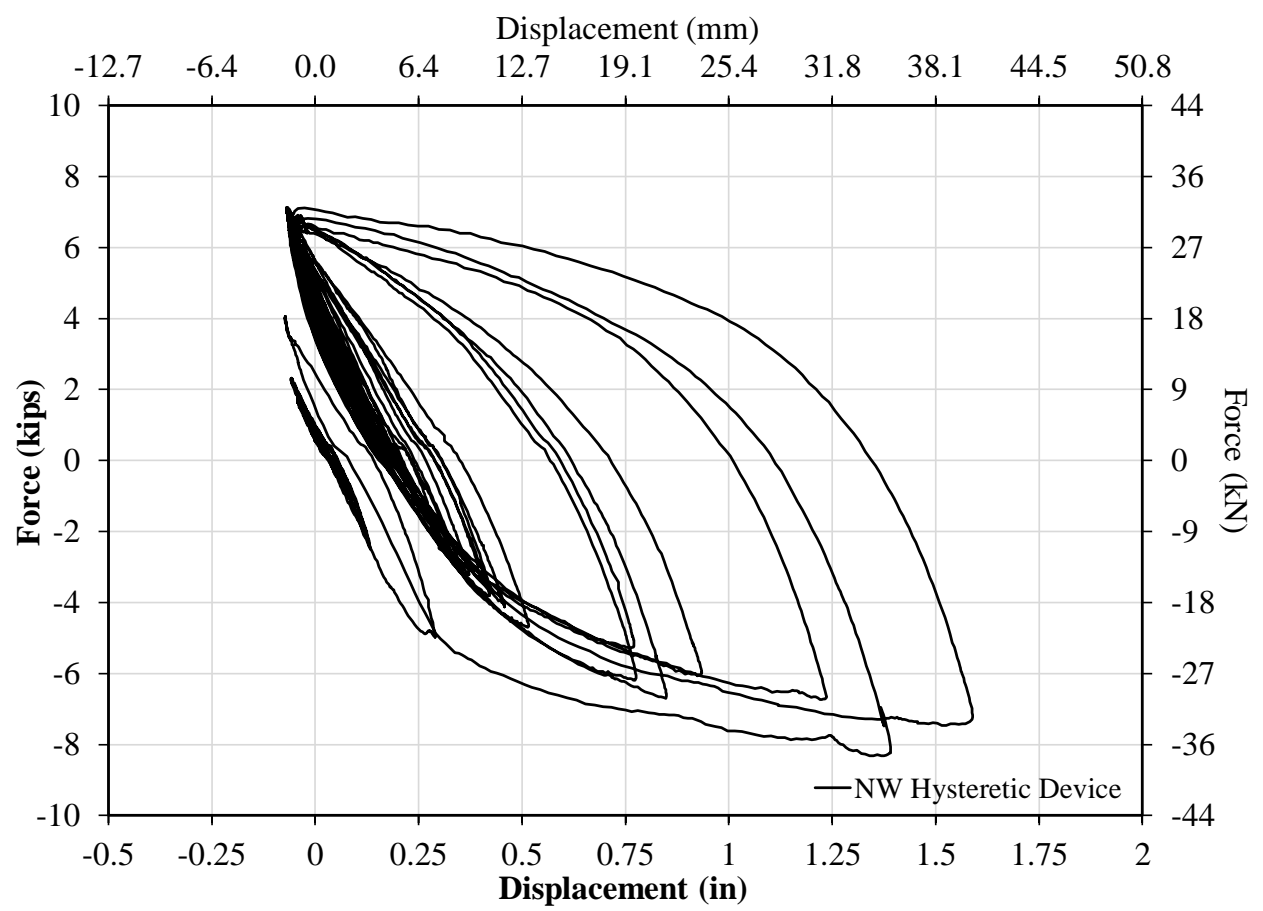

Figure 28-34: 100\% 0.5g IEEE693 NW Virgin Hysteretic Device Response 


\subsection{APPENDIX K}

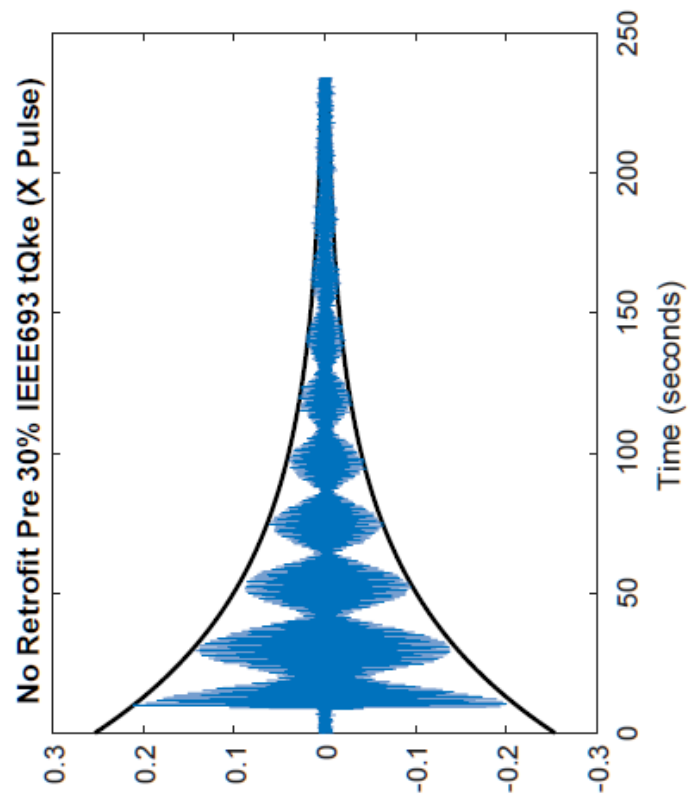

(6) $X$ do $\perp$ te uo!̣eıә|әวэ $\forall$

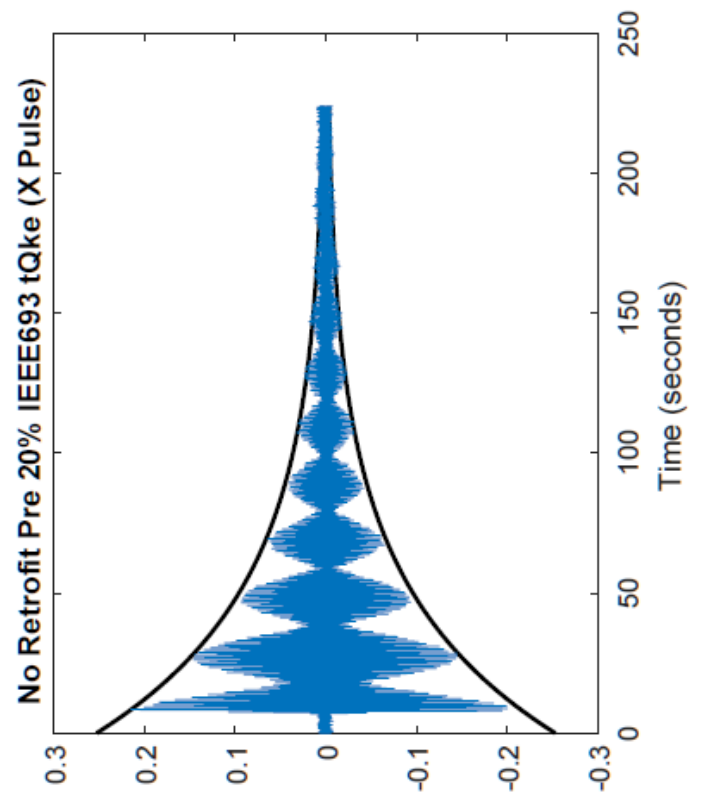

(6) $X$ do $\perp$ je uo!̣eגə|әэว $\forall$

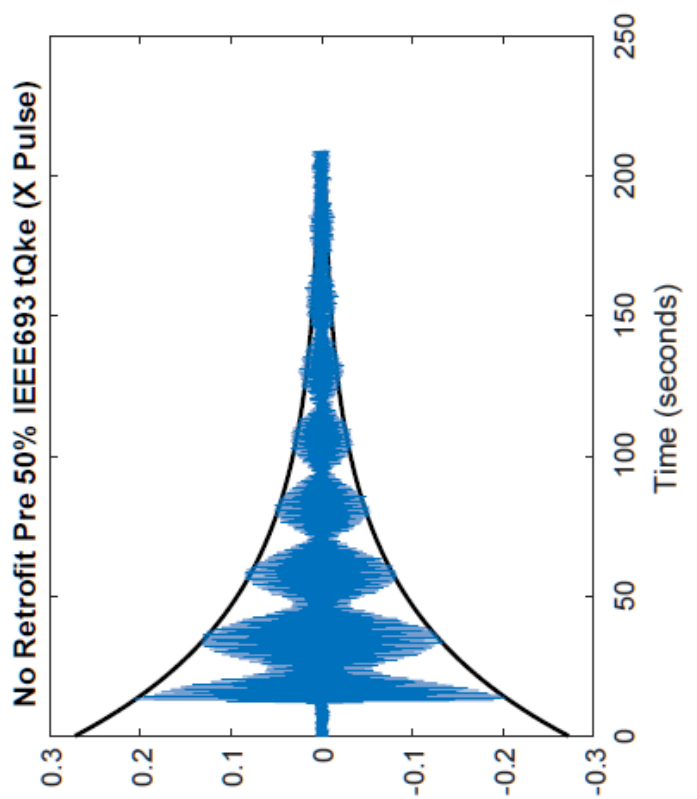

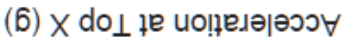

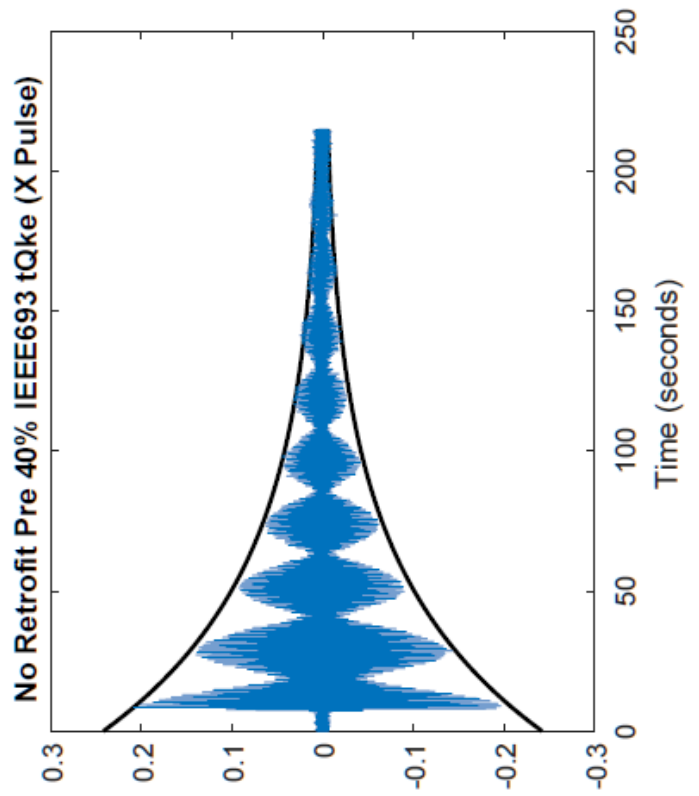

(6) $X$ do $\perp$ te uo!̣eлә|әэว $\forall$

Figure 29-1: Non- Retrofitted X-Damping 20-50\% 


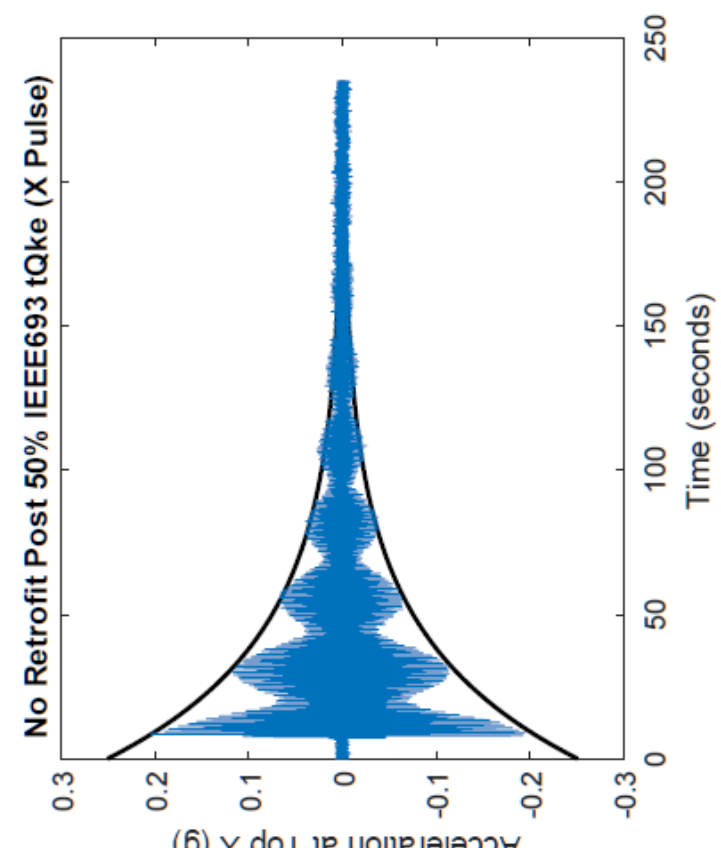

(6) $X$ do $\perp$ le uo!̣eגə|əวว

Figure 29-2: Non- Retrofitted X-Damping 50\% 


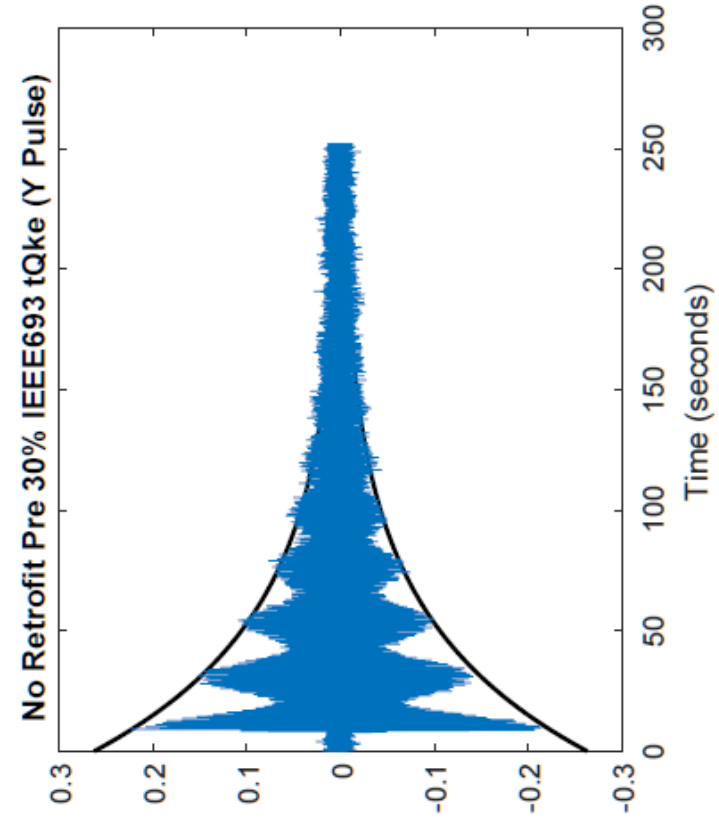

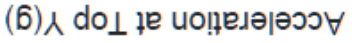

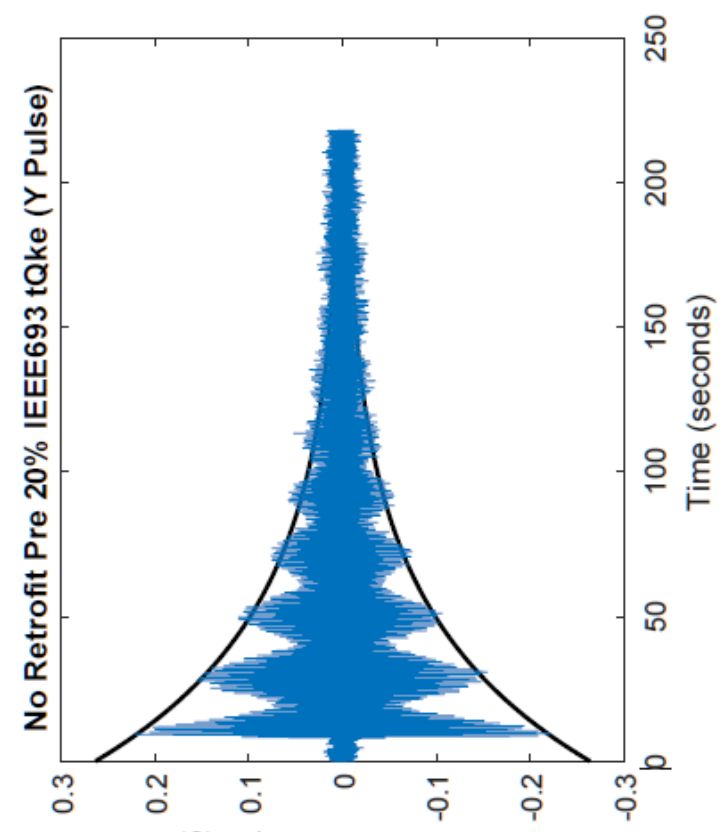

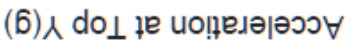

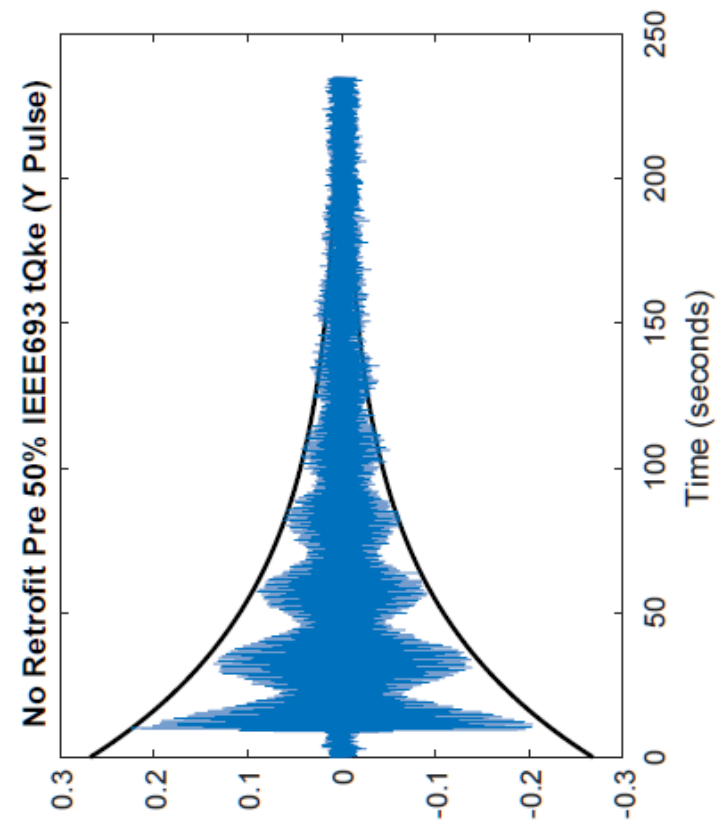

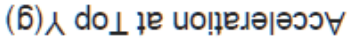

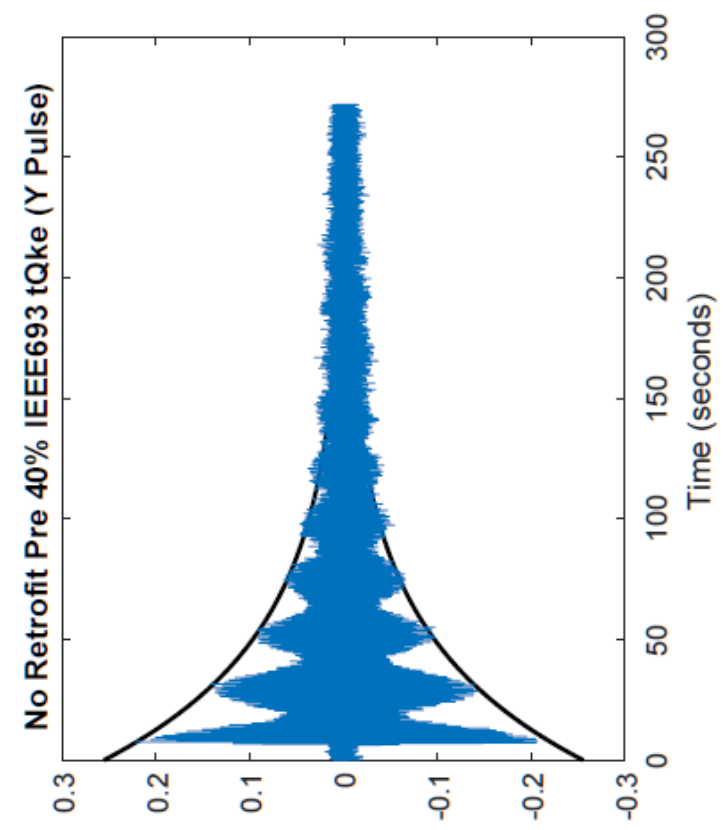

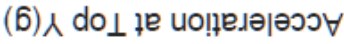

Figure 29-3: Non-Retrofitted Y-Damping 20-50\% 


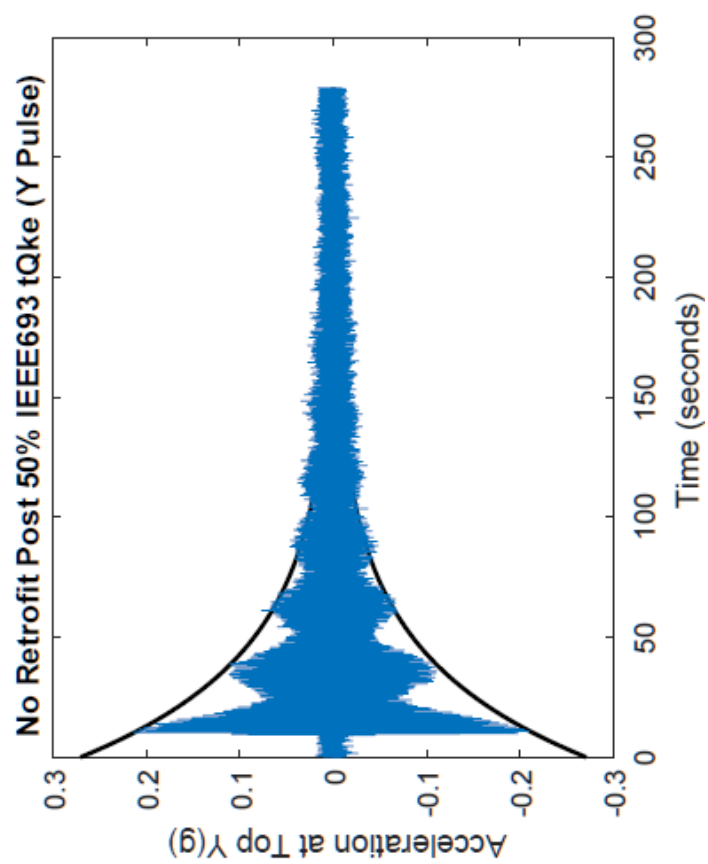

Figure 29-4: Non- Retrofitted Y-Damping 50\% 


\subsection{APPENDIX L}

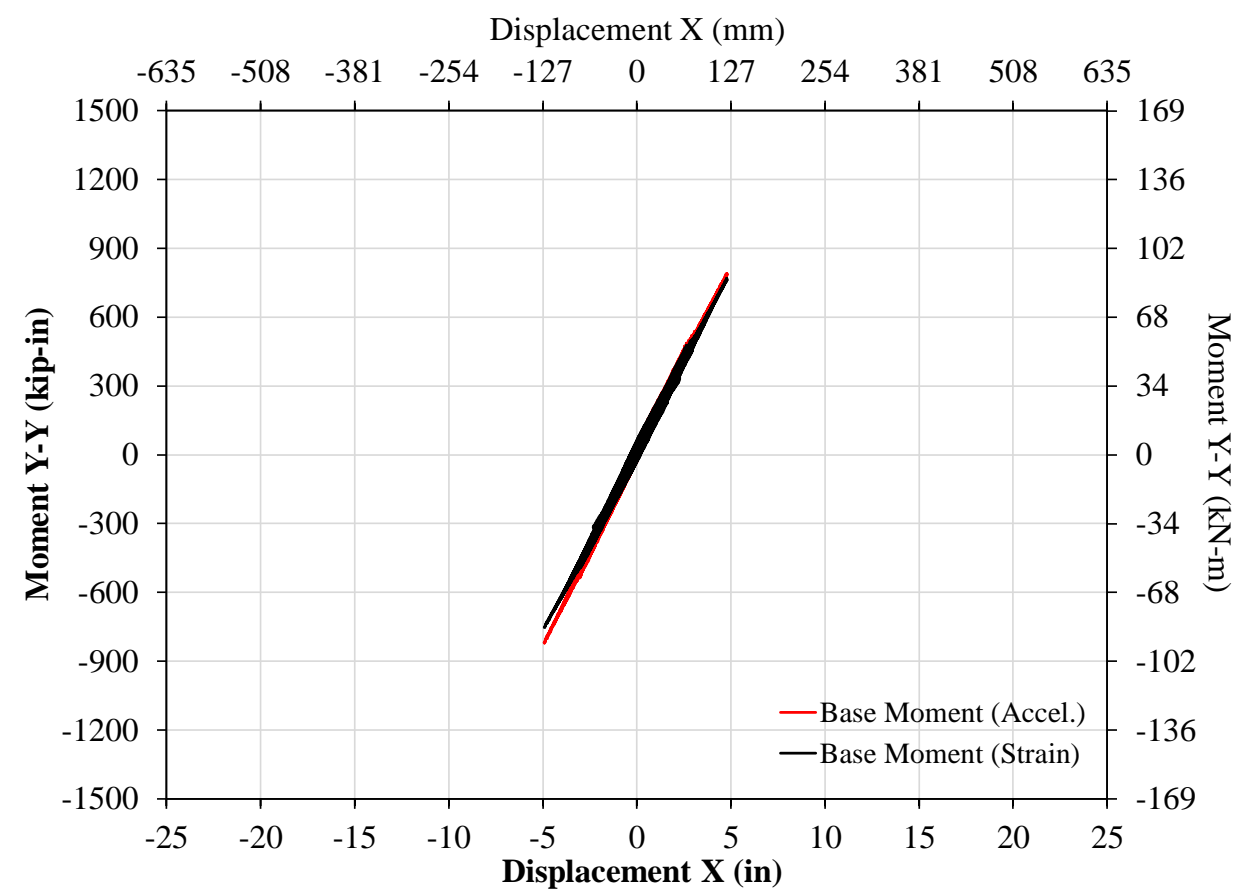

Figure 30-1: 20\% 0.5g IEEE693 Non-Retrofitted X-System Response

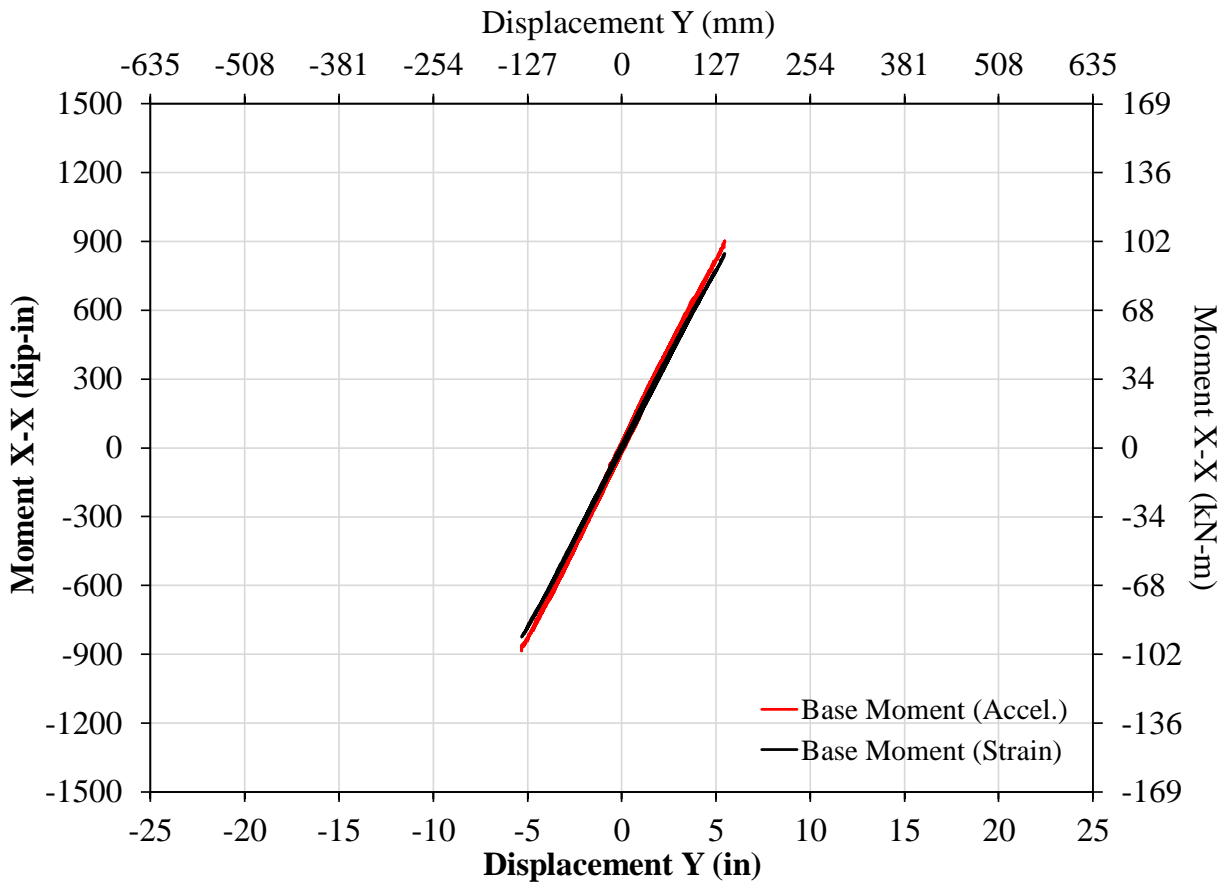

Figure 30-2: $20 \%$ 0.5g IEEE693 Non-Retrofitted Y-System Response 


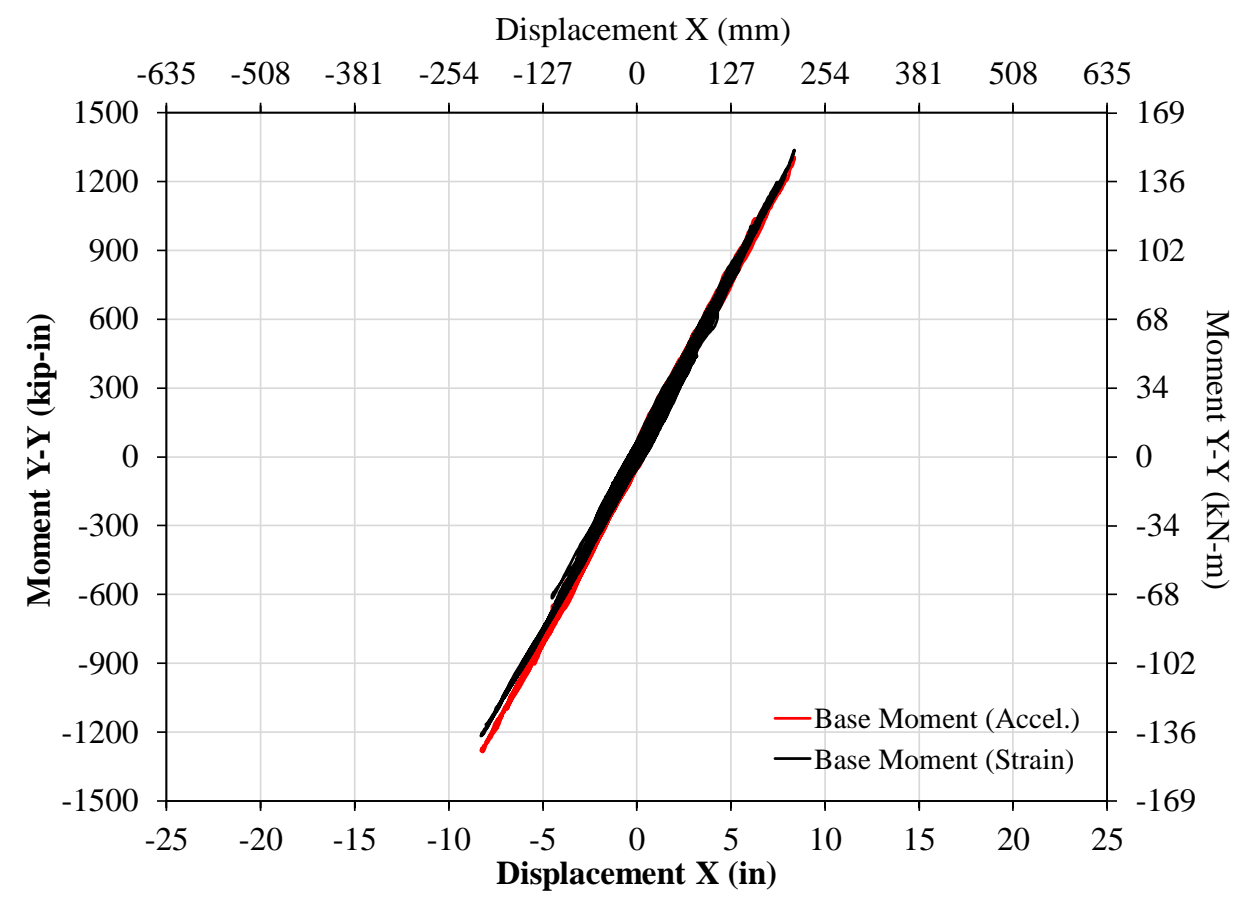

Figure 30-3: 40\% 0.5g IEEE693 Non-Retrofitted X-System Response

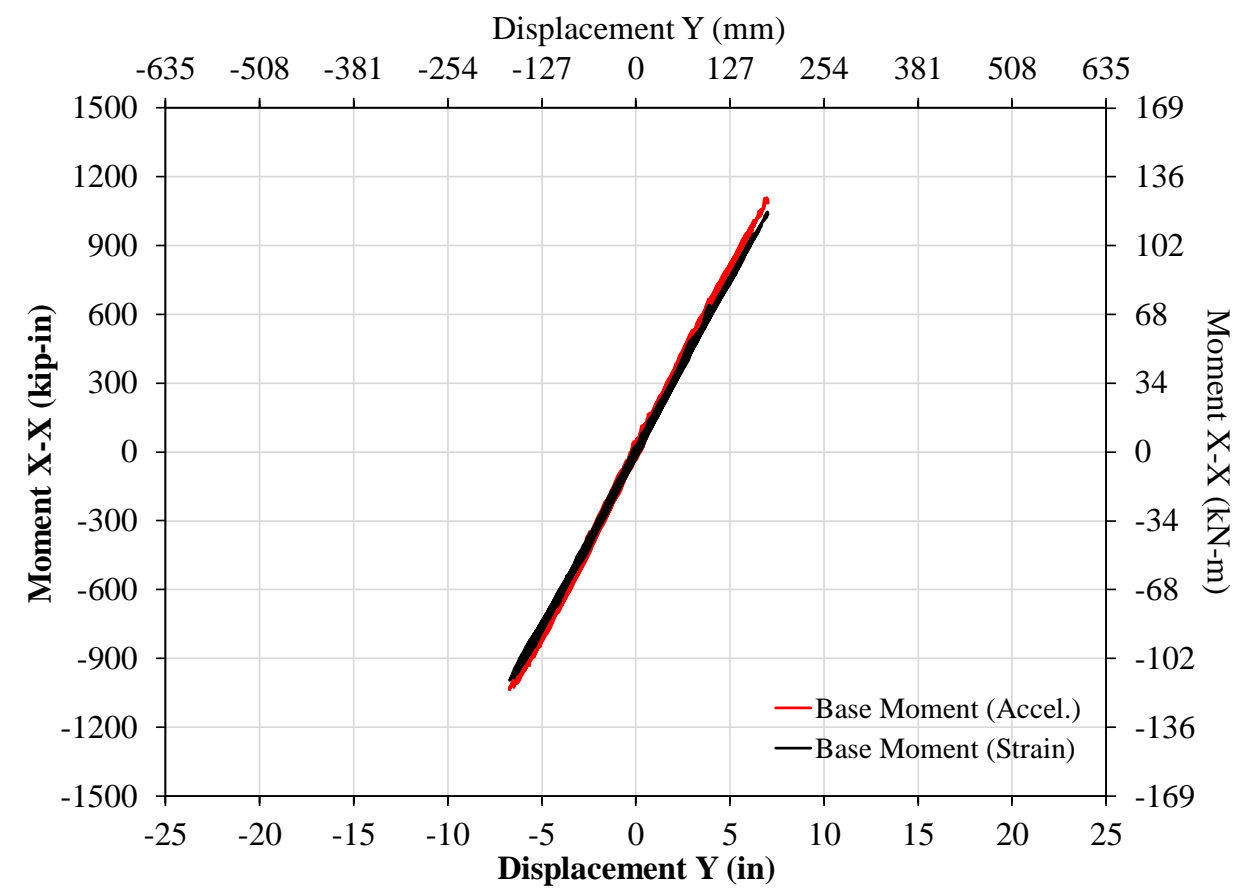

Figure 30-4: 40\% 0.5g IEEE693 Non-Retrofitted Y-System Response 